
Herausgeber

Tom Lahmer

Timon Rabczuk

Carsten Könke

ISM-Bericht 2/2021

ISSN: 1610-7381

Institut für Strukturmechanik

Fakultät Bauingenieurwesen

Bauhaus-Universität Weimar

Marienstraße 15

99423 Weimar

Germany

Tel. + +493643584504

Fax. +493643 584514

http://www.uni-weimar.de/ism 


\title{
Coupling between Shell and Generalized Beam Theory (GBT) elements
}

\author{
DISSERTATION
}

zur Erlangung des akademischen Grades

Doktor-Ingenieur (Dr.-Ing.)

an der Fakultät Bauingenieurwesen

der Bauhaus-Universität Weimar

\author{
vorgelegt von \\ M.Sc. Marcelo José Bianco \\ Geboren am 19. März 1979 in São Paulo, Brasilien
}

Mentor: Prof. Dr.-Ing. habil. Carsten Könke (interner Doktorand) 
Gutachter:

1. Prof. Dr.-Ing. habil. Carsten Könke

2. Prof. Dr.-Ing. habil. Frank Werner

3. Prof. Dr. rer.nat. habil. Klaus Gürlebeck

4. Prof. Dr. Nuno Silvestre

Erteilung der Promotion am 30. November 2020 


\section{EHRENWÖRTLICHE ERKLÄRUNG}

Ich erkläre hiermit ehrenwörtlich, dass ich die vorliegende Arbeit ohne unzulässigeHilfe Dritter und ohne Benutzung anderer als der angegebenen Hilfsmittel angefer-tigt habe. Die aus anderen Quellen direkt oder indirekt übernommenen Daten undKonzepte sind unter Angabe der Quelle gekennzeichnet.

Bei der Auswahl und Auswertung folgenden Materials haben mir die nachstehendaufgeführten Personen in der jeweils beschriebenen Weise entgeltlich/unentgeltlichgeholfen:

Keine.

Weitere Personen waren an der inhaltlich-materiellen Erstellung der vorliegendenArbeit nicht beteiligt. Insbesondere habe ich hierfür nicht die entgeltliche Hilfe vonVermittlungsbzw. Beratungsdiensten (Promotionsberater oder anderer Personen) inAnspruch genommen. Niemand hat von mir unmittelbar oder mittelbar geldwerteLeistungen für Arbeiten erhalten, die im Zusammenhang mit dem Inhalt der vorge-legten Dissertation stehen.

Die Arbeit wurde bisher weder im In- noch im Ausland in gleicher oder ähnlicherForm einer anderen Prüfungsbehörde vorgelegt.

Ich versichere ehrenwörtlich, dass ich nach bestem Wissen die reine Wahrheit gesagtund nichts verschwiegen habe.

Weimar, den 30.09.2019

Signature

Marcelo José Bianco 



\section{List of Published Articles}

[Art.1]Bianco, M. J., Könke, C., Habtemariam, Zabel, V. (2018). Exact finite element formulation in generalized beam theory. International Journal of Advanced Structural Engineering, 10-3, pp 295-323. DOI https://doi.org/10.1007/s40091-018-0199-8

[Art.2]Bianco, M. J., Könke, C., Habtemariam, Zabel, V. (2019). Analysis of warping and distortion transmission in mixed shell-GBT (generalized beam theory) models. International Journal of Advanced Structural Engineering, 11-1, pp 109-126. DOI https://doi.org/10.1007/s40091-019-0221-

\section{List of Presentations and Published Proceedings}

[Proc.1]Bianco, M. J., Könke, C., Habtemariam, Zabel, V. (2016). Exact Stiffness Matrix in Generalized Beam Theory - Proceedings of the 7th international conference on coupled instabilities in metal structures - Section 8-40

[Proc.2]Bianco, M. J., Könke, C., Habtemariam, Zabel, V. (2018). Coupling between Shell and Generalized Beam Theory (GBT) elements - Proceedings of the 8th International Conference on Thin Walled Structure - Section 8-40

[Proc.3]Bianco, M. J., Könke, C., Habtemariam, Zabel, V. (2018). Coupling between Shell and Generalized Beam Theory (GBT) elements - Forschungskolloquium 2018 Grasellenbach - 4-10

[Proc.4]Bianco, M. J., Könke, C., Habtemariam, Tartaglione, F., Zabel, V. (2019). Complementary shear and transversal elongation modes in Generalized Beam Theory (GBT) for thin-walled circular cross-sections - Advances in Engineering Materials, Structures and Systems: Innovations, Mechanics and Applications: Proceedings of the 7th International Conference on Structural Engineering, Mechanics and Computation (SEMC 2019) Section - 11-1 



\section{Acknowledgement}

I would like to express my deepest gratitude to my supervisor Univ.-Prof. Dr.-Ing. habil. Carsten Könke and Dr.-Ing. habil. Volkmar Zabel. Their guidance and observations were crucial to the development of this study. Without them, I would still be lost in the darkness of the maze of doubt and incomprehension where lies the subject of this Phd Dissertation.

Also, I would like to thank Cnpq - (Conselho Nacional de Desenvolvimento Científico e Tecnológico - Development National Council for Scientific and Technological) Brazil - for the financial support of my studies at Bauhaus-Universität Weimar, and Ricardo Bozza, who allowed the tranquility away from my technical responsibilities in Brazil.

Finally, with all my heart, I extoll my family for their endless encouragement, and the light of my life, Juliana Guarany da Cunha Santos, who helped me with more than just the English review, but also with love and support during these five years of studies. 



\section{Kurzfassung}

Seit einigen Jahrzenten hat sich die Finite Elemente Methode in Statik und Dynamik als Standardverfahren etabliert. Für viele klassische Problemstellungen bietet diese Methode eine schnellere und flexiblere Lösung als der analytische Ansatz. Damit werden Prognosen für komplizierte Ingenieurprobleme möglich, für die es früher keine Lösung gab.

Obwohl die Finite Elemente Methode ein sehr robustes Verfahren ist, führte sie zu neuen Fragestellungen. Diese Probleme kann man in zwei Hauptgruppen einteilen: Zum einen spielt die Computerleistung eine zentrale Rolle, zum anderen wirft die Interpretation der digitalen Lösung oft Fragen auf.

Zeitgleich mit der Entwicklung der Finiten Elemente Methode für numerischen Lösungen ist eine Theorie zwischen Balkentheorie und Schalentheorie entstanden: die Verallgemeinerte Technische Biegetheorie, VTB. Diese Theorie ermöglicht nicht nur eine systematische und analytisch klare Darstellung von komplizierten Tragwerksproblemen, sondern bietet auch den Vorteil eines kompakten und eleganten Lösungsverfahrens, das zur Verbesserung der Rechenzeit führen kann.

Leider erlangte die VTB bislang auf internationaler Ebene nur wenig Bekanntheit. Dies ist vor allem darauf zurückzuführen, dass in den ersten Jahren ausschließlich Veröffentlichung zur Theorie in deutscher Sprache erfolgten. Erst in den letzten Jahren hat sich die VTB allmählich zu einem breiten Forschungsthema entwickelt. Dabei ist die Entwicklung von linearen und nichtlinearen Analysen hervorzuheben.

Außerdem wird die VTB bislang ausschließlich isoliert betrachtet. Obgleich vor Kurzem die Lösungen der VTB Finite-Elemente-Methode anwenden, sind die Verbindung von den VTB Finiten Element zu anderen Elementen (Schalen, Volumenelemente) kein Gegenstand der bisherigen Forschung. Damit ist das Hauptziel dieser Dissertation die Kopplung von VTB und Schalen- bzw. Membranelementen zu implementieren und dabei die Vorteile beider Ansätze zu nutzen: die Flexibilität der Schalenelemente sowie die gute Performance der VTB bezüglich der Rechenzeit.

Ausgehend von den Grundlagen der VTB zeigt diese Dissertation auf, wie durch Trennung der Variablen zwei Bereiche der Berechnung definiert werden: der Querschnittsbereich und der Längsachsenbereich. Daher kann die Finite-Elemente Methode nicht nur im Querschnittsbereich angewendet werden, sondern es kann auch ein exakter Finite-Elemente Ansatz unter Verwendung der VTB in Längsrichtung entwickelt werden.

Die Querschnittsanalyse wird durch Lösung des quadratischen Eigenwertproblems unter Anwendung der Trennung von Platten- und Membranverhalten durchgeführt. Dies führt zu einer exakten Darstellung der Deformationsmoden sowie der reduzierten quadratischen Eigenwertproblems.

Bezüglich der Longitudinalrichtung werden in dieser Dissertation neue Elementfor- 
mulierungen basierend auf hyperbolischen und trigonometrischen Ansatzfunktionen entwickelt. Obwohl die Formulierung dieser Funktionen nicht trivial ist, steht damit ein rekursives Verfahren zur Verfügung, das unter Verwendung von periodischen Ableitungen eine systematische Entwicklung der Steifigkeitsmatrizen erlaubt.

Außerdem ermöglichen die Ansatzfunktionen eine Diskretisierung in Einzelelemente und sorgen für ein geglättetes Spannungsfeld.

Ausgehend von diesen Herleitungen wird die Verbindung von VTB und Schalenelementen in einem gemischten Model entwickelt, was das Hauptziel dieser Dissertation darstellt. Basierend auf dem Verschiebungsfeld werden Kopplungsgleichungen unter Verwendung des Master-Slave-Modells formuliert. Dies führt dazu, dass strukturelle Verbindungen und Gelenke mit Schalenelementen, Balken jedoch mit VTB Elementen modelliert werden können.

Als Nebeneffekt sorgen die Kopplungsgleichungen unter Anwendung der VTB für eine Begrenzung des Verschiebungsfeldes der Schalenelemente, insbesondere in der Umgebung des Kopplungsquerschnitts.

In einer linearen Analyse sind diese Effekte fast unmerklich, wohingegen sie in der nichtlinearen Analyse zu kumulativen Fehlern führen. Die Anwendung der gemischten VTB-Schalen-Modellierung in nichtlinearen Analysen wird am Ende dieser Dissertation diskutiert. 


\section{Abstract}

In the last decades, Finite Element Method has become the main method in statics and dynamics analysis in engineering practice. For current problems, this method provides a faster, more flexible solution than the analytic approach. Prognoses of complex engineer problems that used to be almost impossible to solve are now feasible.

Although the finite element method is a robust tool, it leads to new questions about engineering solutions. Among these new problems, it is possible to divide into two major groups: the first group is regarding computer performance; the second one is related to understanding the digital solution.

Simultaneously with the development of the finite element method for numerical solutions, a theory between beam theory and shell theory was developed: Generalized Beam Theory, GBT. This theory has not only a systematic and analytical clear presentation of complicated structural problems, but also a compact and elegant calculation approach that can improve computer performance.

Regrettably, GBT was not internationally known since the most publications of this theory were written in German, especially in the first years. Only in recent years, GBT has gradually become a fertile research topic, with developments from linear to non-linear analysis.

Another reason for the misuse of GBT is the isolated application of the theory. Although recently researches apply finite element method to solve the GBT's problems numerically, the coupling between finite elements of GBT and other theories (shell, solid, etc) is not the subject of previous research. Thus, the main goal of this dissertation is the coupling between GBT and shell/membrane elements. Consequently, one achieves the benefits of both sides: the versatility of shell elements with the high performance of GBT elements.

Based on the assumptions of GBT, this dissertation presents how the separation of variables leads to two calculation's domains of a beam structure: a cross-section modal analysis and the longitudinal amplification axis. Therefore, there is the possibility of applying the finite element method not only in the cross-section analysis, but also the development for an exact GBT's finite element in the longitudinal direction.

For the cross-section analysis, this dissertation presents the solution of the quadratic eigenvalue problem with an original separation between plate and membrane mechanism. Subsequently, one obtains a clearer representation of the deformation mode, as well as a reduced quadratic eigenvalue problem.

Concerning the longitudinal direction, this dissertation develops the novel exact elements, based on hyperbolic and trigonometric shape functions. Although these functions do not have trivial expressions, they provide a recursive procedure that allows periodic derivatives to systematise the development of stiffness matrices. Also, these shape functions enable a single-element discretisation of the beam structure and ensure 
a smooth stress field.

From these developments, this dissertation achieves the formulation of its primary objective: the connection of GBT and shell elements in a mixed model. Based on the displacement field, it is possible to define the coupling equations applied in the masterslave method. Therefore, one can model the structural connections and joints with finite shell elements and the structural beams and columns with GBT finite element.

As a side effect, the coupling equations limit the displacement field of the shell elements under the assumptions of GBT, in particular in the neighbourhood of the coupling cross-section.

Although these side effects are almost unnoticeable in linear analysis, they lead to cumulative errors in non-linear analysis. Therefore, this thesis finishes with the evaluation of the mixed GBT-shell models in non-linear analysis. 


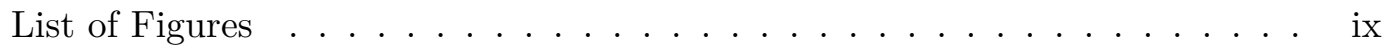

List of Tables . . . . . . . . . . . . . . . . xiv

Nomenclature . . . . . . . . . . . . . . . . . . xviii

1 Introduction 1

1.1 Motivation . . . . . . . . . . . . . . . . 1

1.2 Literature Review and historical development of GBT . . . . . . . . 2

1.3 Objectives of the dissertation . . . . . . . . . . . . 4

1.4 Dissertation organization $\ldots \ldots \ldots \ldots \ldots$

2 Generalized Beam Theory Assumptions 7

2.1 Hypotheses of Generalized Beam Theory . . . . . . . . . . . . . . 7

2.1.1 Discretization of the thin-walled beam cross-section as a combi-

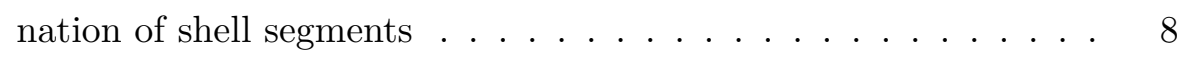

2.1 .2 Kinematic assumptions . . . . . . . . . . . . . 8

2.1.3 Separation of variables to describe the displacement field. . . . . 12

2.1.4 A linear combination of orthogonal deformation-shape function describes any possible displacement in a cross-section . . . . . . 13

2.2 Variational Formulation in Generalized Beam Theory . . . . . . . . . . . 14

2.2.1 Internal strain energy according to GBT . . . . . . . . . . . 14

2.2.2 External load potential energy according to GBT . . . . . . . . 16

2.2 .3 Equilibrium by Hamilton's principle . . . . . . . . . . . . 18

2.3 Stress field in GBT: generalized internal forces . . . . . . . . . . . . 19

3 GBT's analysis of thin-walled hollow circular cross-section 25

3.1 GBT's analysis of thin-walled hollow circular cross-section . . . . . . . . 25

3.1.1 GBT's displacement field for thin-walled hollow circular cross-

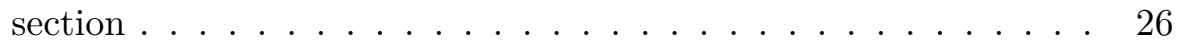


3.1.2 transverse displacement due to Poisson's effect in membrane be-

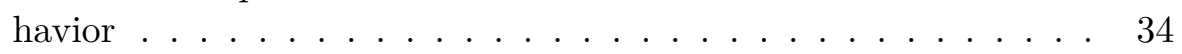

3.1.3 External loads in thin-walled hollow circular cross-section according to GBT . . . . . . . . . . . . . . . . . . 34

3.1.4 Stress field and internal forces for thin-walled hollow circular cross-section according to GBT . . . . . . . . . . 35

4 GBT's analysis of a generic segmented thin-walled cross-section 41

4.1 Cross-section semi-discretization . . . . . . . . . . . . . . 44

4.2 Transverse Stiffness matrices _. . . . . . . . . . . . . 47

4.3 Identification and elimination of singular modes . . . . . . . . . . . . . 54

4.4 Membrane, Plate and Membrane-Plate modes . . . . . . . . . . . . . 54

4.4.1 Membrane mode and constraints . . . . . . . . . . . . 55

$4.4 .2 \quad$ Plate modes and constraints . . . . . . . . . . . . . . 59

4.4.3 Mode 2, 3 and 4 - Pure longitudinal bending and Torsion . . . . 63

4.5 Quadratic eigenvalue solution . . . . . . . . . . . . . 67

4.5.1 Symmetric and anti-symmetric modes . . . . . . . . . . . . 67

4.5.2 Linearization and quadratic pencil . . . . . . . . . . . . 68

4.5.3 Recover of the eigenvectors in the original coordinate system . . 69

5 GBT's finite element in longitudinal direction 71

5.1 Review of the applied solution methods in GBT's ordinary differential equations . . . . . . . . . . . . . . . . . 72

5.2 Shape function assortment and transverse deformation mode classification 73 5.2.1 Classification of GBT's deformation modes concerning the ordinary differential equation . . . . . . . . . . . . 74

5.2.2 Classification of GBT's deformation modes concerning the solution of ordinary differential equations . . . . . . . . 75

5.2.3 Shape functions based on homogeneous solutions in GBT analysis 76

5.2.4 Shape functions based on non-homogeneous solutions in GBT

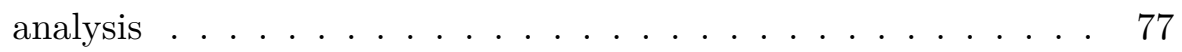

5.2.5 Non-Homogeneous solution for distortion with warping - case A with clamped-clamped boundary conditions . . . . . . . 78

5.2.6 Simplification of the high-order derivatives and stiffness matrices for coupled modes . . . . . . . . . . . . . . 87

5.2.7 Non-Homogeneous solution for distortion with warping - case A with hinged-hinged boundary conditions . . . . . . . . . 90

5.2.8 Non-Homogeneous solution for distortion with warping - case A with clamped-hinged boundary conditions . . . . . . . . . . . . 91

5.3 Numerical Examples . . . . . . . . . . . . . . . . . . . . . 95

5.3.1 Cross-section analysis and load's mode participation . . . . . . 95

5.3.2 Finite element solution in the longitudinal direction . . . . . . . 96

5.3 .3 Analysis of the displacement field . . . . . . . . . . . . . . 99

5.3 .4 Analysis of the stress field . . . . . . . . . . . . . . . 106 
$\begin{array}{lll}6 & \text { GBT's applied in frame beam structures } & 117\end{array}$

6.1 Pure GBT beam frame analysis . . . . . . . . . . . . . . . . . 118

6.1.1 Internal support mechanism . . . . . . . . . . . . . . . . . . 119

6.1 .2 Inverse-clamp mechanism . . . . . . . . . . . . . . . . 120

6.1.3 Coupling of different high modes due to connections . . . . . . . 121

6.1 .4 Clamp mechanism . . . . . . . . . . . . . . . . . . 123

6.2 Connections obtained from coupling between GBT and shell elements . 124

6.2.1 Definition of master and slaver degrees of freedom . . . . . . . . 125

6.2.2 Master-slave relationships based on GBT's modes . . . . . . . . . 126

6.2.3 Coupled stiffness matrix and external load vector . . . . . . . . . 130

6.3 Numerical example of coupling GBT and shell elements . . . . . . . . . 130

6.3.1 Setup of finite element and coupling matrices . . . . . . . . . . 131

6.3.2 Finite element solution . . . . . . . . . . . . . . . . . . . . 134

6.3.3 Analysis of displacement field . . . . . . . . . . . . . . 135

6.3.4 Analysis of stress field . . . . . . . . . . . . . . . . . . 140

7 Non-linear analysis of mixed Shell-GBT models $\quad 147$

7.1 Introduction - Brief historical development of non-linear GBT . . . . . 148

7.2 Geometric stiffness in GBT . . . . . . . . . . . . . . . . 149

7.2.1 Principle of Virtual Works applied in non-linear GBT . . . . . . 149

7.2.2 Variation of longitudinal internal strain energy . . . . . . . . . . 152

7.2.3 Variation of transversal internal strain energy . . . . . . . . . . . 154

7.2.4 Linear and Quadratic Initial Stresses Stiffness - Longitudinal Direction . . . . . . . . . . . . . 156

7.2.5 Linear and Quadratic Initial Stresses Stiffness - Transversal Direction . . . . . . . . . . . . . . . . . . 159

7.2.6 Linear and Quadratic Initial Displacements Stiffness - Longitudinal Direction . . . . . . . . . . . . . . . . 162

7.2.7 Linear and Quadratic Initial Displacements Stiffness - transversal Direction . . . . . . . . . . . . . 166

7.3 Internal forces evaluation in non-linear GBT . . . . . . . . . . . 168

7.4 Properties of GBT's coupling tensors . . . . . . . . . . . . . . 171

7.5 Development of geometric stiffness matrices in GBT . . . . . . . . . 172

7.5.1 Simplification of the multiplication among several shape functions 172

7.5.2 Setup of GBT's non-linear stiffness matrices . . . . . . . . . . . 173

7.6 Numerical example of non-linear analysis in coupling GBT and shell elements . . . . . . . . . . . . . . . . . . . . . 175

7.6.1 Setup of the mixed GBT-shell finite element model . . . . . . . 176

7.6.2 Finite element solution . . . . . . . . . . . . . . . . . . . 179

7.6 .3 Analysis of displacement field . . . . . . . . . . . . . 180

$\begin{array}{lll}8 & \text { Conclusion and future research } & 187\end{array}$

8.1 Conclusion . . . . . . . . . . . . . . . . . . 187

8.2 Future research . . . . . . . . . . . . . . . . . . . . . . 189 
A Appendix A: Numerical example - GBT's analysis of a generic segmented thinwalled cross-section

A.1 Numerical example . . . . . . . . . . . . . . . . . . . . . . . . . 205

A.1.1 Step 1 - Setup of Cross-section stiffness matrices . . . . . . . . 205

A.1.2 Step 2 - transverse elongation constrains . . . . . . . . . . 215

A.1.3 Step 3 - Pure elongation membrane mode . . . . . . . . . . . 218

A.1.4 Step 4 - Membrane shear strain constraint . . . . . . . . . . . . 220

A.1.5 Step 5 - Pure plate modes . . . . . . . . . . . . . . . 220

A.1.6 Step 6 - Membrane-plate modes . . . . . . . . . . . . . . . . . . 222

A.1.7 Step 7 - Pure longitudinal bending modes and Vlasov mode . . . 225

A.1.8 Step 8 - Symmetric and anti-symmetric modes . . . . . . . . 228

A.1.9 Step 9 - Quadratic eigenvalue problem . . . . . . . . . . . . . 229

A.1.10 Step 10 - eigenvectors in original coordinate system . . . . . . . 230

A.1.11 Step 11 - Analysis of pure plate modes . . . . . . . . . . . . 235 


\section{List of Figures}

1.1 Nodal decomposition of a concentrated load in a column according to Vlasov . . . . . . . . . . . . . . . . . . . . . .

2.1 Global and local coordinate systems: a) in a generic segmented crosssection; b) in a hollow circular cross-section . . . . . . . . . . . 8

2.2 Types of Distortion: a) transverse bending distortion due to the variation of transverse bending moments; b) transverse bending distortion due to the variation of transverse forces; c) transverse elongation distortion. . . 9

2.3 Membrane shear flow in a infinitesimal segment of a cross-section. . . . 10

2.4 Longitudinal orthogonal modes shapes of a generic segment cross-section. 14

2.5 Local longitudinal equilibrium of a cross-section segment. . . . . . . . . 22

3.1 Coordinate system in a thin-walled hollow circular cross-section . . . . . 26

3.2 The angle of tangent displacement $\vartheta$ : (a) relationship according to the angle $\theta$ and a constant angle; (b) relationship according to the radius $r+t$ and a constant angle . . . . . . . . . . . 27

3.3 transverse deformation shape modes of a thin-walled circular hollow section according to GBT. . . . . . . . . . . . . . . 32

3.4 transverse deformation of the additional modes of uniform radial elongation and torsion. . . . . . . . . . . . . . . 33

3.5 Absence of Poisson's effect from the longitudinal into the transverse bending moment: the behavior of a plane shell structure, as shown in a), is maintained in a gradually curved cross-section, as shown in b). This behavior is also maintained in a fully circular cross-section, as shown in c). . . . . . . . . . . . . . . . . 36

3.6 Equilibrium in a infinitesimal segment of a thin-walled circular hollow section, according to GBT. . . . . . . . . . . . . 
3.7 Axial shell's equilibrium in a infinitesimal segment of a thin-walled circular hollow section, according to GBT. . . . . . . . . . . . . . . 39

4.1 Nodal nomenclature of a generic segmented cross-section . . . . . . . . . 41

4.2 cross-section classification: a) and b) open unbranched; c) closed unbranched; d) open branched; e) closed branched . . . . . . . . . . . . . 42

4.3 Independent unit warping functions . . . . . . . . . . . . . . . . 42

4.4 An element of a segment in the local coordinate system . . . . . . . . . 44

4.5 transverse bending moment conditions for membrane-plate modes. This bending moment is null in the end segments. Also, at the internal nodes, the equilibrium must be satisfied without any external load. . . . . . . . 61

4.6 Radius vector: a) according to classical Vlasov Beam Theory; b) according to GBT's identification . . . . . . . . . . . . . . . . 64

5.1 Element for Non-homogeneous solution with 6 degrees of freedom . . . . 78

5.2 Thin-walled circular hollow section under a linear projected force; a) Elevation; b) cross-section; c) Force and area's projection in local coordinate system. . . . . . . . . . . . . . . . . . . . 95

5.3 Generalized displacement of the radial mode, ${ }^{a} V$, (in $\left.\mathrm{mm}\right) \quad \ldots . . .100$

5.4 Generalized displacement of mode $5,{ }^{5} V$, (in $\left.\mathrm{mm}\right) \ldots . . .100$

5.5 Generalized displacement of mode $7,{ }^{7} V$, (in $\left.\mathrm{mm}\right) \ldots . . \ldots 100$

5.6 Generalized displacement of mode $11,{ }^{11} \mathrm{~V}$, (in $\mathrm{mm}$ ) . . . . . . . . 100

5.7 Generalized displacement of mode $15,{ }^{15} \mathrm{~V}$, (in $\mathrm{mm}$ ) . . . . . . . . 101

5.8 Generalized warping displacement of the radial mode, ${ }^{a} V^{\prime}$, (in $\left.\mathrm{m} / \mathrm{m}\right)$. . 101

5.9 Generalized warping displacement of mode $5,{ }^{5} V^{\prime}$, (in $\left.\mathrm{m} / \mathrm{m}\right) \quad \ldots . .101$

5.10 Generalized warping displacement of mode $7,{ }^{7} V^{\prime}$, (in $\left.\mathrm{m} / \mathrm{m}\right) \quad \ldots . .101$

5.11 Generalized warping displacement of mode $11,{ }^{11} V^{\prime}$, (in $\left.\mathrm{m} / \mathrm{m}\right) \quad \ldots . .102$

5.12 Generalized warping displacement of mode $15,{ }^{15} V^{\prime}$, (in $\left.\mathrm{m} / \mathrm{m}\right)$. . . . . . 102

5.13 Results of top cross-section deformation: the GBT solution is obtained by the summation of all modal deformation factors. The solution achieved from shell elements is also presented. . . . . . . . . . . . . . . 103

5.14 Results of longitudinal displacement at top cross-section: Comparison between the solutions of GBT and shell elements. . . . . . . . . . . . . . 104

5.15 Results of cross-section deformation at $\mathrm{x}=1 \mathrm{~m}$ : Comparison among the GBT solutions with/without Poisson effect and shell elements. . . . . . 104

5.16 Results of longitudinal displacement of the cross-section at $\mathrm{x}=1 \mathrm{~m}$ : Comparison between the solutions of GBT and shell elements. . . . . . . . 105

5.17 Generalized Internal force of radial mode, ${ }^{a} W,\left(\right.$ in $\left.\mathrm{MN}^{*} \mathrm{~m}\right)$. . . . . . 106

5.18 Generalized Internal force of radial mode, ${ }^{a} W^{\prime}$, (in MN) . . . . . . . . 106

5.19 Generalized Internal force of mode $5,{ }^{5} W$, (in $\left.\mathrm{MN}^{*} \mathrm{~m}\right)$. . . . . . . . 106

5.20 Generalized Internal force of mode $5,{ }^{5} W^{\prime}$, (in MN) . . . . . . . . . 107

5.21 Generalized Internal force of mode $7,{ }^{7} \mathrm{~W}$, (in $\left.\mathrm{MN}^{*} \mathrm{~m}\right) \quad \ldots . .107$

5.22 Generalized Internal force of mode $7,{ }^{7} W^{\prime}$, (in MN) $\ldots \ldots \ldots$. . . . 107

5.23 Generalized Internal force of mode $11,{ }^{11} \mathrm{~W}$, (in $\left.\mathrm{MN}^{*} \mathrm{~m}\right)$. . . . . . . . 107

5.24 Generalized Internal force of mode $11,{ }^{11} W^{\prime}$, (in MN) . . . . . . . . . 108 
5.25 Generalized Internal force of mode $15,{ }^{15} W$, (in $\left.\mathrm{MN}^{*} \mathrm{~m}\right)$. . . . . . . 108

5.26 Generalized Internal force of mode $15,{ }^{15} W^{\prime}$, (in MN) . . . . . . . . . 108

5.27 Longitudinal bending moment, $M_{x}$, in cross-section at x=1m . . . . . . 109

5.28 transverse bending moment, $M_{\theta}$, in cross-section at $\mathrm{x}=1 \mathrm{~m} \ldots \ldots . . .109$

5.29 Twist moment, $M_{\theta x}$, in cross-section at x=1m . . . . . . . . . . . . 110

5.30 Shear forces, $Q_{x}$, in cross-section at x=1m . . . . . . . . . . 111

5.31 Shear forces, $Q_{\theta}$, in cross-section at $\mathrm{x}=1 \mathrm{~m} \ldots \ldots \ldots \ldots$. . . . . . 112

5.32 Membrane force, $N_{x}$, in cross-section at $\mathrm{x}=1 \mathrm{~m} \ldots \ldots \ldots . . \ldots 113$

5.33 Membrane force, $N_{\theta}$, in cross-section at $\mathrm{x}=1 \mathrm{~m} \ldots \ldots \ldots . . \ldots 114$

5.34 Membrane force, $N_{\theta x}$, in cross-section at $\mathrm{x}=1 \mathrm{~m} \ldots \ldots . \ldots . \ldots 114$

6.1 Type of connections to clarify the kinematic behavior in high modes . . 119

6.2 Elementary node to describe the kinematic behavior of the connection, which involves high deformation modes. . . . . . . . . . . . . . . 119

6.3 Kinematic mechanism of box connection; a) the stress compatibility; b) and c) local bending moments in the flanges of beam and column;

d)resume of the kinematic interpretation . . . . . . . . . . . 120

6.4 Kinematic mechanism of inverse distortion transmission; a) undeformed structure under a bi-torsion internal force (the connection rod is in red); b) deformed structure with inverse transmission. . . . . . . . . . . . 121

6.5 Kinematic mechanism of non-stiffener connection; a) warping deformation of the web; b) and c) local torsional and bending moments in the flanges of beam and column; d) distortion shaped in the column's cross section.

6.6 Kinematic mechanism of diagonal connection; a) bi-moment equilibrium as nodal equilibrium; b) and c) bending moments in the stiffener and in the flanges of beam and column; d) Left view of the nodal moment equilibrium. . . . . . . . . . . . . . .

6.7 Diagonal connection with the effects of torsion inertia of flanges and stiffener resulting in a box-diagonal connection. . . . . . . . . . . . 124

6.8 Example of coupling of shell and GBT models: a)Hollow circular crosssection between; b)Shell nodes in the middle of GBT's cross-section segments . . . . . . . . . . . . . . . . . . 126

6.9 Thin-walled circular hollow section under a linear projected force and a coupled shell element segment; a) elevation; b) cross-section in GBT's domain; c) cross-section in shell's domain at the coupling point . . . . . 131

6.10 Membrane element local displacement definition . . . . . . . . . . . . . 131

6.11 Results of top cross-section: transversal displacement of mixed shellGBT element model. The sum of all modal deformation factors, at the top point, leads to the GBT solution. The solutions achieved from the full shell element models are also presented. . . . . . . . . . . . . 137

6.12 Comparison of results among mixed shell-GBT and fully shell models at top cross-section: longitudinal displacement. . . . . . . . . . . . . 138 
6.13 Comparison of results among mixed shell-GBT and fully shell models at the coupling cross-section: transversal displacement. . . . . . . . . . 138

6.14 Comparison of results among mixed shell-GBT and fully shell models at the coupling cross-section: longitudinal displacement. . . . . . . . . . 139

6.15 Comparison of results among mixed shell-GBT and fully shell models at point $x=525 \mathrm{~mm}$ : longitudinal bending moment, $M_{x} \ldots \ldots \ldots \ldots$

6.16 Comparison of results among mixed shell-GBT and fully shell models at point $x=525 \mathrm{~mm}$ : transversal bending moment, $M_{\theta} \ldots \ldots \ldots$. . . . 141

6.17 Comparison of results among mixed shell-GBT and fully shell models at point $x=525 \mathrm{~mm}$ : twist moment, $M_{\theta x}$. . . . . . . . . . . . . 142

6.18 Comparison of results among mixed shell-GBT and fully shell models at point $x=525 \mathrm{~mm}$ : longitudinal shear force, $Q_{x} \ldots \ldots \ldots \ldots$. . . . 143

6.19 Comparison of results among mixed shell-GBT and fully shell models at point $x=525 \mathrm{~mm}$ : transversal shear force, $Q_{\theta} \ldots \ldots \ldots \ldots$. . . . 143

6.20 Comparison of results among mixed shell-GBT and fully shell models at point $x=525 \mathrm{~mm}$ : longitudinal membrane force, $N_{x} \ldots \ldots \ldots \ldots$. . . 144

6.21 Comparison of results among mixed shell-GBT and fully shell models at point $x=525 \mathrm{~mm}$ : shear membrane force, $N_{\theta x} \ldots \ldots \ldots \ldots \ldots$

6.22 Comparison of results among mixed shell-GBT and fully shell models at point $x=525 \mathrm{~mm}$ : transversal membrane force, $N_{\theta} \ldots \ldots \ldots \ldots$

7.1 Fiber elongation of a thin-walled beam segment . . . . . . . . . . . . 149

7.2 Coupling structure among thin-walled circular hollow section and two open thin-walled cross-sections: a)frontal elevation; b)connection's frontal elevation; c)top view; d)beams' discretization; e)connections left view; f) shell model's perspective. . . . . . . . . . . . . . . . . . 176

7.3 Results of longitudinal displacement, without mean value, of mixed shellGBT and full shell-181 model: Linear analysis - Load Case 1. . . . . . . 181

7.4 Results of longitudinal displacement, without mean value, of mixed shellGBT and full shell-181 model: Non-linear analysis - Load Case 1. . . . . 182

7.5 Results of transversal displacement of mixed shell-GBT and full shell-181 model: Linear analysis - Load Case 1. . . . . . . . . . . . . . . . 182

7.6 Results of transversal displacement of mixed shell-GBT and full shell-181 model: Non-linear analysis - Load Case 1. . . . . . . . . . . . . . . 183

7.7 Results of longitudinal displacement of mixed shell-GBT and full shell181 model: Linear analysis - Load Case 2. . . . . . . . . . . . . . . . 184

7.8 Results of longitudinal displacement, without mean value, of mixed shellGBT and full shell-181 model: Non-linear analysis - Load Case 2. . . . . 184

7.9 Results of transversal displacement of mixed shell-GBT and full shell-181 model: Linear analysis - Load Case 2. . . . . . . . . . . . . . . 185

7.10 Results of transversal displacement, without mean value, of mixed shellGBT and full shell-181 model: Non-linear analysis - Load Case 2. . . . . 185

A.1 Concrete Roof extracted from R. Schadt [136]. . . . . . . . . . . . 205

A.2 transverse modal displacements: modes 1 to $4 \ldots \ldots . . \ldots 231$ 
A.3 transverse modal displacements: modes 5 to 7 . . . . . . . . . . . 232

A.4 transverse modal displacements of plate symmetric modes: 1 to 4 . . . 239

A.5 transverse modal displacements of plate anti-symmetric modes: 5 to 9 . 240 


\section{List of Tables}

3.1 Orthogonal deformation shape modes of circular hollow cross-section according to GBT . . . . . . . . . . . . . . . . . . 31

3.2 Additional orthogonal deformation shape modes of circular hollow crosssection: a - uniform axial elongation; $\mathrm{t}$ - uniform torsion . . . . . . . . 33

4.1 Properties and nomination of singular modes according to GBT . . . . . 54

5.1 Ordinary Differential Equation of GBT . . . . . . . . . . . . 75

5.2 Example 5.1 - cross-section and external load modal decomposition . . . 96

5.3 Example 5.1 - Modal classification . . . . . . . . . . . . . . . 96

5.4 Mean differences, in \%, and standard deviation (SD) of displacement field between GBT and shell elementsk models at point $x=L / 2 \ldots$. . 103

5.5 GBT displacements at $\mathrm{x}=1 \mathrm{~m} \ldots \ldots$. . . . . . . . . . . . . 104

5.6 Mean differences, in \%, and standard deviation (SD) of displacement field between GBT and shell elementsk models at point $x=1 \mathrm{~m} \ldots$. . . . 105

$5.7 M_{x}$ : Mean differences, (in table's upper-right side), and their standard deviation (in table's lower-left side) among all models . . . . . . . . . . 109

$5.8 M_{\theta}$ : Mean differences, (in table's upper-right side), and their standard deviation (in table's lower-left side) among all models . . . . . . . . . . 110

$5.9 M_{\theta x}$ : Mean differences, (in table's upper-right side), and their standard deviation (in table's lower-left side) among all models . . . . . . . . . . 111

$5.10 Q_{x}$ : Mean differences, (in table's upper-right side), and their standard deviation (in table's lower-left side) among all models . . . . . . . . . . 112

$5.11 Q_{\theta}$ : Mean differences, (in table's upper-right side), and their standard deviation (in table's lower-left side) among all models . . . . . . . . . . 112

$5.12 N_{x}$ : Mean differences, (in table's upper-right side), and their standard deviation (in table's lower-left side) among all models . . . . . . . . . . 113 
$5.13 N_{\theta}$ : Mean differences, (in table's upper-right side), and their standard deviation (in table's lower-left side) among all models . . . . . . . . . . 113

$5.14 N_{\theta x}$ : Mean differences, (in table's upper-right side), and their standard deviation (in table's lower-left side) among all models . . . . . . . . . . 115

6.1 Setup of degrees of freedom in mixed membrane-GBT model . . . . . . 133

6.2 Solution of GBT's degrees of freedom, in mm . . . . . . . . . . 135

6.3 Shell elements' degrees of freedom, in mm, on global coordinate system 136

6.4 Mean differences, in \%, and standard deviation (SD) of displacement field between GBT and shell element models at the top point . . . . . . 137

6.5 Mean differences, in \%, and standard deviation (SD) of displacement field between GBT and shell elementsk models at the point $x=1 \mathrm{~m} \ldots 139$

6.6 Longitudinal bending moment, $M_{x}$, at $x=525 \mathrm{~mm}$ : mean differences (in the table's upper-right side) and their standard deviation (in the table's lower-left side) among all models . . . . . . . . . . . . . . . . . 140

6.7 Transversal bending moment, $M_{\theta}$, at $x=525 \mathrm{~mm}$ : mean differences (in the table's upper-right side) and their standard deviation (in the table's lower-left side) among all models . . . . . . . . . . . . . . . . . . 141

6.8 Twist bending moment, $M_{\theta x}$, at $x=525 \mathrm{~mm}$ : mean differences (in the table's upper-right side) and their standard deviation (in table's lowerleft side) among all models . . . . . . . . . . . . . . . . . . . . . 142

6.9 Longitudinal plate's shear, $Q_{x}$, at $x=525 \mathrm{~mm}$ : mean differences (in the table's upper-right side) and their standard deviation (in the table's lower-left side) among all models . . . . . . . . . . . . . . . . . . . . 142

6.10 Transversal plate's shear, $Q_{\theta}$, at $x=525 \mathrm{~mm}$ : mean differences (in the table's upper-right side) and their standard deviation (in the table's lower-left side) among all models . . . . . . . . . . . . . . . . . . 144

6.11 Longitudinal membrane force, $N_{x}$, at $x=525 \mathrm{~mm}$ : mean differences (in the table's upper-right side) and their standard deviation (in the table's lower-left side) among all models. . . . . . . . . . . . . . . . . . . . . . 144

6.12 Shear membrane force, $N_{\theta x}$, at $x=525 \mathrm{~mm}$ : mean differences (in the table's upper-right side) and their standard deviation (in the table's lower-left side) among all models. . . . . . . . . . . . . . .

6.13 Membrane tangential force, $N_{\theta}$, at $x=525 \mathrm{~mm}$ : mean differences (in the table's upper-right side) and their standard deviation (in the table's lower-left side) among all models . . . . . . . . . . . . . . . . . 146

7.1 Summary of virtual work of the initial stress in the incremental stages in the longitudinal direction. . . . . . . . . . . . . . . . 159

7.2 Summary of virtual work of the initial stress in the incremental stages in the transversal direction. . . . . . . . . . . . . . . . . . 162

7.3 Summary of virtual work of the initial displacement in the incremental stages in the longitudinal direction. . . . . . . . . . . . . 166

7.4 Summary of virtual work of the initial displacement in the incremental stages in transversal direction. . . . . . . . . . . . . 168 
7.5 Generalized properties: hollow circular cross-section . . . . . . . . . 177

7.6 Generalized properties: Beams' cross-section . . . . . . . . . . . . . . . . 177

7.7 Accumulative modal displacements . . . . . . . . . . . . . . . . . . . 180

7.8 Mean differences, in \%, and standard deviation (SD) of displacement field between mixed GBT-Shell and Shell-181 model - linear analysis load case $1 \ldots \ldots$. . . . . . . . . . . . . . . 181

7.9 Mean differences, in \%, and standard deviation (SD) of displacement field between mixed GBT-Shell and Shell-181 model - Non-linear analysis load

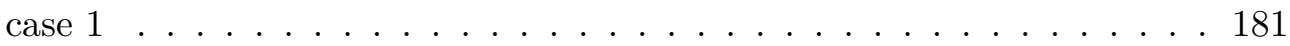

7.10 Mean differences, in \%, and standard deviation (SD) of displacement field between mixed GBT-Shell and Shell-181 model - linear analysis load case $2 \ldots \ldots \ldots \ldots \ldots$

7.11 Mean differences, in \%, and standard deviation (SD) of displacement field between mixed GBT-Shell and Shell-181 model - Non-linear analysis load

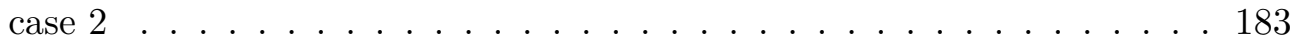

A.1 Generalized cross-section properties . . . . . . . . . . . . . . 233

A.2 Generalized cross-section properties due to coupling modes . . . . . . . 234

A.3 Longitudinal generalized stiffness, including plate's longitudinal strain stiffness . . . . . . . . . . . . . . . . . . . . . 234

A.4 Generalized cross-section properties - without $[C]^{P}$ and QEP . . . . . . 234

A.5 Differences among the eigenvectors from the proposed approach and [136]235 
Abbreviations

CAE Computer Aided Engineering

DSM Direct Strength Method

eq. equation

FEM Finite Element Method

GBT Generalized Beam Theory

QEP Quadratic Eigenvalue Problem

General notations

$\alpha \quad$ hyperbolic amplification term in GBT modes with dominant distortion behavior

$\beta \quad$ trigonometric amplification term in GBT modes with dominant distortion behavior

$\chi \quad$ Transversal perpendicular rotation of the cross-section's segments

$\boldsymbol{\delta}() \quad$ Variatio notation

$\varepsilon \quad$ Biot strain

$\eta \quad$ trigonometric amplification term in GBT modes with dominant torsion behavior

$\gamma \quad$ Biot shear strain

$\int \quad$ Integral on the cross-section area

$\int \quad$ Integral along the beam length

$\int_{L} \quad$ Integral along the beam length 
$\int_{V} \quad$ Integral over the beam volume

$\imath \quad$ trigonometric and trigonometric amplification term in GBT critical torsiondistortion behavior

$\kappa \quad$ plate's curvature

$\lambda \quad$ Eigenvalues

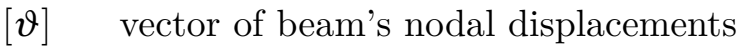

$\left.\left[S h_{N H a c c}\right]\right]$ Completeness coefficient matrices for hyperbolic-trigonometric shape function in clamped-clamped boundary conditions for GBT modes with dominant distortion

$\left[T x_{N H a}\right]$ variable vector of hyperbolic-trigonometric shape functions for GBT modes with dominant distortion

[I] Second order tensor of inertia of bending

$[\mathbb{V}] \quad$ trial transverse displacement vector

$[\Upsilon] \quad$ Kernel transverse stiffness matrix

$[I] \quad$ Identity matrix

[k] Stiffness matrix

$\left[S h_{H e 1 c c}\right]$ Completeness coefficient matrices for linear Hermitian shape function in clampedclamped boundary conditions

$\left[S h_{H e 3 c c}\right]$ Completeness coefficient matrices for cubic Hermitian shape function in clampedclamped boundary conditions

[T] Transformation matrix

$\left[T_{c}\right] \quad$ Multi-freedom constraint transformation matrix

[TS1] Linear interpolation matrix for the displacements of the cross-section's segments

[TS3] Cubic interpolation matrix for the displacements of the cross-section's segments

$\left[T s_{\mathrm{He} 1}\right]$ Variable vector of linear Hermitian shape function

$\left[T s_{H e 3}\right]$ Variable vector of cubic Hermitian shape function

$[U] \quad$ Modal shape-transformation matrix

$\mu \quad$ Poisson's ratio

$\omega \quad$ Vlasov's sectorial area

$\overline{G D}$ equivalent term of shear stiffness

$\phi \quad$ Longitudinal rotation 
$\Pi \quad$ Total energy

$\sigma \quad$ Biot stress

$\tau \quad$ shear stress

$\theta \quad$ angular direction in polar coordinate system; segment's angle in generic segmented cross-section

$v \quad$ Transversal rotation of the cross-section's segments

$\rho \quad$ element length/height ratio

$\vartheta \quad$ Variation of the angle in hollow circular cross-sections

$\zeta \quad$ hyperbolic amplification term in GBT modes with dominant torsion behavior

$B \quad$ Generic moment of inertia, related to transverse strains

C Generic moment of inertia, related to longitudinal strains

D Generic moment of inertia, related to shear strains

E Young's modulus

F integral of longitudinal amplification function of distributed load

$f \quad$ longitudinal amplification function of distributed load

G shear modulus

$J_{\#} \quad$ constants in ordinary differential equations

$K \quad$ Plate's stiffness

$l_{s} \quad$ element/segment length

$m \quad$ natural number in trignometric functions

$M_{\theta} \quad$ Generalized transverse bending moment in circular hollow cross-section

$M_{s} \quad$ Generalized transverse bending moment

$M_{x} \quad$ Generalized longitudinal bending moment

$m_{\#} \quad$ stiffness term of membrane element

$M_{T x} \quad$ Generalized torsion moment

$M_{x \theta} \quad$ Generalized twist bending moment in circular hollow cross-section

MS Generalized first moment of inertia

$n \quad$ number of cross-section nodes

$N_{\theta} \quad$ Internal membraner force in transverse direction of hollow circular cross-sections

$N_{x} \quad$ Internal longitudinal membrane force in longitudinal direction 
$N_{x \theta} \quad$ Internal membrane shear force in longitudinal direction

$P \quad$ concentrated load

$q \quad$ distributed load

$Q_{\theta} \quad$ Internal shear force in transverse direction of hollow circular cross-sections

$Q_{x} \quad$ Internal shear force in longitudinal direction

$r \quad$ middle-line radius for hollow circular cross-section;

$r(s) \quad$ radius vector according to Vlasov beam theory

$s \quad$ local coordinate system - transverse direction aligned to segment

$t \quad$ local coordinate system - transverse direction perpendicular to segment

$t_{h} \quad$ thickness of a thin-walled segment/hollow circular cross-section

$u \quad$ local displacement - in longitudinal beam direction

$u_{g} \quad$ global displacement - in longitudinal beam direction

$u_{v} \quad$ global displacement - in the direction of beam's width

$u_{w} \quad$ global displacement - in the direction of beam's height

$U_{\text {int }} \quad$ Internal strain energy

$V \quad$ longitudinal amplification function

$v \quad$ local displacement - in transverse direction aligned to segment

$V_{\text {ext }} \quad$ External load potential energy

W $\quad$ Generalized internal force

w local displacement - in transverse direction perpendicular to segment

X global coordinate system - longitudinal beam direction

$x \quad$ local coordinate system - longitudinal beam direction

$Y \quad$ global coordinate system - in the direction of beam's width

Z global coordinate system - in the direction of beam's height

${ }^{i j k l} \mathbb{S}_{u w 4}$ Fourth order coupling tensor of GBT's variation of longitudinal and transversal displacement modes

${ }^{i j k l} \mathbb{S}_{u w 4}$ Fourth order coupling tensor of GBT's variation of longitudinal displacement modes

${ }^{i j k l} \mathbb{X}_{4} \quad$ Fourth order coupling tensor of GBT's transversal mode deformations

${ }^{i j k} \mathbb{V}_{X 4}^{l}$ Vector in mode 1 due to initial stages in modes $\mathrm{i}, \mathrm{j}$ and $\mathrm{k}$

xxii 
${ }^{i j k} \mathbb{X}_{3}$ Third order coupling tensor of GBT's transversal mode deformations

${ }^{i j} \mathbb{V}_{S 4}^{k l} \quad$ Stiffness matrix between modes $\mathrm{k}$ and $\mathrm{l}$ due to the of initial stage of modes i and $\mathrm{j}$

${ }^{i j} \mathbb{V}_{S X 4}^{k l}$ Stiffness matrix between the longitudinal variation of modes $\mathrm{k}$ and $\mathrm{l}$ due to the initial stage of modes $i$ and $j$

${ }^{i j} \mathbb{V}_{X 3}^{k} \quad$ Vector in mode $\mathrm{k}$ due to initial stages in modes $\mathrm{i}$ and $\mathrm{j}$

${ }^{i j} \mathbb{V}_{X 4}^{k l} \quad$ Stiffness matrix between modes $\mathrm{k}$ and $\mathrm{l}$ due to the initial stage of modes $\mathrm{i}$ and $\mathrm{j}$

${ }^{i j} \mathbb{V}_{X S 4}^{k l}$ Stiffness matrix between modes $\mathrm{k}$ and $\mathrm{l}$ due to the longitudinal variation of initial stage of modes $\mathrm{i}$ and $\mathrm{j}$

${ }^{i} \mathbb{V}_{3 \text { uw }}^{j k}$ Stiffness matrix between the longitudinal variation of modes $\mathrm{k}$ and $\mathrm{j}$ due to the initial stage of modes $\mathrm{i}$

${ }^{i} \mathbb{V}_{3 u}^{j k} \quad$ Stiffness matrix between the longitudinal variation of modes $\mathrm{j}$ and $\mathrm{k}$ due to the initial stage of modes i

${ }^{i} \mathbb{V}_{X 3}^{j k} \quad$ Stiffness matrix between modes $\mathrm{j}$ and $\mathrm{k}$ due to the initial stage of mode $\mathrm{i}$

lower-right indexes

()$_{a}$ and ()$_{b}$ indeces for subgroups related to symmetric or anti-symmetric modes

(), indicates partial derivatives

()$_{f} \quad$ final beam node notation

()$_{i} \quad$ initial beam node notation

()$_{+} \quad$ Complementary function in for shear and transverse elongation in membrane behavior

()$_{g} \quad$ indicator of variable in global coordinate system

()$_{h} \quad$ term based on Hermitian shape functions

()$_{l} \quad$ indicator of variable in local coordinate system

() $\quad$ index of the cross-section node

()$_{\text {GBT cs }}$ GBT coordinate system

()$_{G B T}$ index for GBT variable

()$_{S c p} \quad$ indicator of coupled matrices

()$_{\text {Scs }} \quad$ Shell element coordinate system

()$_{\text {seg }}$ index for cross-section segment

()$_{\text {shell }}$ index for shell variable

()$_{\text {Suc }}$ indicator of uncoupled matrices 
lower-left indexes

$i() \quad$ index of matrix transformation

$i()$ and ${ }_{i i}()$ indeces for conjugated modes

Matrices indexes

[〜] decoupled matrix index

upper-right indexes

- $\quad$ notation of initial state in non-linear analysis

()$^{\mu} \quad$ indicates Poisson's effect due to constitutive relationship

()$^{M} \quad$ indicates membrane component

()$^{P} \quad$ indicates plate component

()$^{M c}$ constant membrane function

()$^{\star} \quad$ middle line tangent direction in hollow circular cross-sections

()$^{e l} \quad$ single element index

()$^{M M}$ index of Membrane-Membrane coupling behavior

()$^{M P} \quad$ index of Membrane-Plate coupling behavior

()$^{P M} \quad$ index of Plate-Membrane coupling behavior

()$^{P P} \quad$ index of Plate-Plate coupling behavior

()$^{s t} \quad$ whole structural's domain index

$+\quad$ notation of incremental state in non-linear analysis

$H \quad$ indicates high mode components

$M P \quad$ indicates membrane-plate components

upper-left indexes

a index of uniform radial elongation

${ }^{i}() \quad$ index of GBT mode

$t \quad$ index of uniform torsion mode 


\section{Introduction}

\subsection{Motivation}

There are two major motivations in this dissertation. The first one concerns the economic and sustainable feature of thin-walled beam structures. Such structures lead to minimal material consumption, and they are created from a wide variety of materials, from the traditional metal elements, like steel and aluminum, to state of the art of fibers, such as carbon fiber and fiberglass.

However, in the last decades, thin-walled beams have been showing a unique behavior, especially when compared to traditional thick beams. Distortion, warping, local and distortion buckling are some examples of inherent effects in this type of structure.

The prediction of these unusual effects brings to the second major motivation here: find an understanding of physical behavior, as well as a practical design approach in thin-walled beam structures.

Although Computer Aided Engineering, CAE, has been providing a powerful and robust tool for structural design for thin-walled beams, many results have neither a clear physical understanding nor a direct correlation with traditional formulations. In this scenario, models based on the Finite Element Method, FEM, more specific shell elements, have been widely used in the last four decades. Smoothly colorful graphs showing the displacement and stress fields in a thin-walled beam, with total conformity to mechanics of materials, have been obtained. However, if these results are compared to classical beam theories, it stands out that the FEM modeling solution is beyond the capacity of traditional beam analysis.

This procedure drives into two main inconveniences: the first one is the computational effort necessary to solve it; the second one is the labor effort in modeling all shell elements.

Although computer performance has improved over the last decades, a FEM shell 
modeling of thin-walled beam leads to a substantial amount of redundant information. Therefore, much of computer effort spent to solve a thin-walled beam model, based on shell elements, is used to solve nearly similar nodal outcomes.

Concerning the practical modeling issue, the main problem is not in the generation of the initial finite element mesh in the structural analysis. Mesh generation algorithms have the capacity to overcome this problem. However, a structural design approach works with several tries, i.e., for each try, it involves changing further than wall thickness. Changes in thin-walled cross-sections require a new generation of finite element meshes, or at least an update of the original ones. Since the mesh of a beam is usually linked to the mesh of a neighbor structural component, this neighbor mesh must be regenerated as well, which brings it to a general model mesh regeneration. This question is even more highlighted in the scenario of Building Information Modelling, BIM, in which versatility in model changing is one of the main focus of this new design approach. 1D beam elements are still a friendly and easily modeling manner in this environment.

Moreover, the analytical interpretation of results obtained from FEM shell modeling is quite elaborated. As an outcome, this analysis provides the stress fields in the thinwalled beam. However, structural design codes are widely based on internal forces. Thus, one must still integrate the stress field over the cross-section domain.

Unfortunately, if one tries to rebuild the stress field based on the internal forces, then one does not reach the same results of the initial stress field. In other words, the internal forces due to traditional beam theories, such as Bernoulli-Euler, Timoshenko[170] and Vlasov beam theories, cannot provide a full description of mechanic behavior of a thinwalled beam[41, 178].

Consequently, thin-walled beam structures require an advanced theory that has the benefits of easy analysis, processing, and modeling of beam theories and the accurate results of shell theory.

The seeking for the above requirements leads to major theories: Finite Strip Method [43, 85, 134] and Generalized Beam Theory,GBT. This last one is the main focus of study in this dissertation.

\subsection{Literature Review and historical development of GBT}

Generalized Beam Theory, GBT, or in original in German "Verallgemeinerte Technische Biegetheorie" is a numerical approach that was initially developed to solve open thinwalled prismatic beams by Richard Schardt in Darmstadt, Germany [135, 136, 141, $142]$.

According to Schardt, [46], GBT is a natural extension of Vlasov Beam Theory, [176]. It was only a matter of time for Vlasov to reach a similar formulation of GBT. Unfortunately, his early death stopped his researches. 
In fact, when Vlasov solved the non-uniform torsion for thin-walled beams, he brought a new view over beam elements: beyond the six degrees of freedom, which are concerning the rigid body motion, a prismatic beam has an extra one, the seventh degree of freedom, that is concerning the warping of the cross-section. This extra deformation shape is related to a new internal force, which Vlasov named as Bi-moment.

To simply illustrate and prove this idea, Vlasov used a nodal force decomposition of an "I" profile under a concentrated load in one of its corners, as shown below:

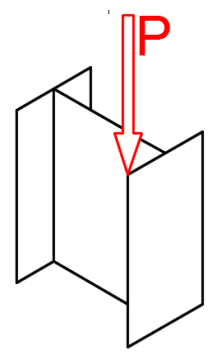

a

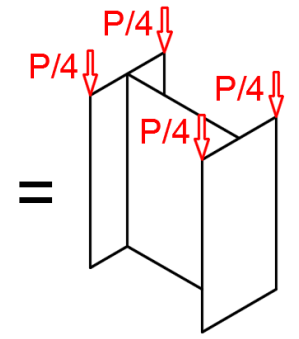

b

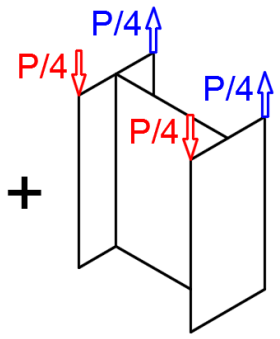

C

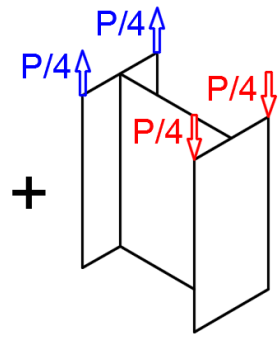

d

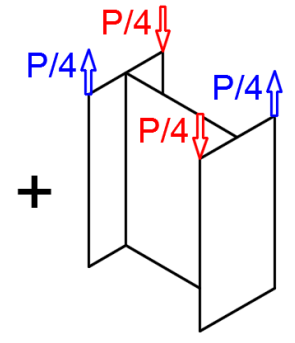

e

Figure 1.1: Nodal decomposition of a concentrated load in a column according to Vlasov

According to the classical Bernoulli-Euler Beam Theory, this column is under an oblique compression that can be described as a linear combination of the nodal forces in configurations $\mathrm{b}$ until $\mathrm{d}$ in the above picture. However, the sum over these nodal forces does not lead the initial force as shown in picture 1.1.a. An extra deformation of cross-section rises: the Warping. Also, it leads to a new internal force, the Bi-moment. They are, respectively, an extra degree of freedom and internal force of beams. Therefore, a 3D space beam has seven degrees of freedom[129]: six concerning the rigid body motions and one concerning the warping deformation.

Schardt, in his turn, extended this example to cross-section with five, or more, nodes. The wonder of how to build the nodal force decomposition, in these cases, leads to GBT principles.

Over a time of more than twenty years, Schardt developed GBT with his co-workers[133, $135,140,174]$. As a result, in 1989, he published the only book concerning the subject [136]. This book explains all linear analysis in GBT, with particular attention to the cross-section analysis in the cases of circular, tubular and open cross-sections.

During this period, GBT has its first publication in English, made by J.M.Davies, [49], in 1986. He is the pioneer of applying FEM in GBT. Davies is also responsible for GBT's disclosure outside Germany.

Later in 1994, Schardt publishes his publications in English language, [137, 138], in which the focus are the non-linear analysis and the buckling from the coupling among different deformations modes.

In 1998, J.M.Davies publishes a book section about GBT,[46]. Here, he introduces GBT in the linear and non-linear analysis. 
At the end of the '90s, Dinar Camotim, from the Technical University of Lisbon, started his group of GBT studies [149, 150]. Together with other researchers such as Nuno Silvestre, Rodrigo Gonçalves and Rui Bebiano, they have become the most expressive research group of GBT in the latest years. With more than 100 publications in the last 17 years, they have been pushing the boundaries of GBT. As a few examples of their progress, one can mention:

- developments in linear analysis: open-cross section applications [75, 76, 155], generic branched open cross-section analysis [50, 78], inclusion of shear lag [89], shear deformations in GBT [64, 145, 160], 3D frame connection behavior [11, 13, 15], cross-validation with Finite Strip Method [53, 72, 161], vibration [31, 156, 158, 159] and dynamic analysis [24] and the development of the GBT software analysis GBTUL [23];

- developments in buckling and post-buckling analysis [33, 66, 153, 154] and the effects of initial shear stress in buckling coupling [6, 14, 65, 157].

- developments in non-linear analysis: i) extension for non-linear physical behavior $[67,69,79]$; ii) study of large deformation [108] and rotations in GBT; iii) analysis of initial imperfections [109, 111, 112].

Recently, in the last five years, GBT started to spread out around the world. In Italy and Australia, a joined group of GBT studies, coordinated respectively by G. Piccardo and G. Ranzi[127, 169], has been progress in the dynamic analysis, including shear effects. In Romanian, Nedelcu et.al. developed studies about buckling identification modes based on GBT [122] and taper beams[120], especially concerning conical shell analysis $[116,121]$.

In Denmark, M.J. Andreassen, [97, 98, 99, 100], developed a unique approach to obtain the deformation modes in GBT that systematizes this extensive and characteristic GBT's analysis.

\subsection{Objectives of the dissertation}

The presented dissertation concerns the study and development of Generalized Beam Theory, GBT, based on Finite Element Method. It does not only review this theory, but it also contributes to several points, as listed below:

- Cross-section analysis: Based on recent GBT cross-section analysis of [97], this dissertation proposes some modifications and improvements in this procedure. As a result, one obtains a division among pure membrane, pure plate, and membrane-plate modes. This separation is useful in the study of non-linear coupling;

- Linear analysis: It develops an exact finite element solution. Especial attention is given to the ordinary differential equation of GBT, which leads to the deformation mode classification and its respective shape functions. The conception of Completeness Coefficient Matrix is a feature issue to overcome the numerical difficulties, which rise from the most typical shape functions applied in GBT; 
- Modeling: One of the significant novel points of this dissertation is the coupling between shell and GBT finite elements. This novel approach uses the definitions of GBT's deformation shapes as the basis of the coupling equation between these two types of elements. As a result, it creates a mixed shell-GBT finite element model;

- Buckling Analysis: Last, but not least, this dissertation presents a buckling analysis of the mixed shell-GBT finite element model. The development of this analysis is not limited to the usual linear initial stress stiffness components, but also studies the quadratic ones. It develops the linear and quadratic initial displacement stiffness components as well, and it shows the possible couplings between membrane and plate behaviours. Moreover, this analysis reuses the exact formulation in linear analysis for the coupling between longitudinal compression and the transverse high modes deformation.

\subsection{Dissertation organization}

The dissertation presents the above topics in a total of 8 chapters. The first one is this introduction.

Initially, Chapter 2 presents a review of the development and principles of GBT. Starting from the kinematic assumptions, this chapter shows how GBT is a natural extension of Vlasov beam theory. Furthermore, it introduces the main features of GBT analysis: i) the separation of variables of the displacement field in longitudinal and transverse domains; ii) the orthogonal deformation modes concept.

Based on these features, Chapter 2 develops the variational formulation of internal and external energies in GBT, leading not only to the fundamental equation of equilibrium of this theory, but also highlighting the idea of generalized cross-section properties.

As a first cross-section analysis, Chapter 3 studies the thin-walled circular hollow crosssection. Although this type of cross-section is a particular case of GBT's application, it has a unique characteristic of avoiding the laborious analysis of the cross-section by the quadratic and generalized eigenvalue problems. Thus, one can have a faster introduction and overview of the potential of GBT.

Chapter 4 presents the analysis of a generic segmented thin-walled cross-section. Based on the systematic approach formulated by Andreassen et al. ([97], [99], [98] and [100]), this chapter presents an alternative approach to split the cross-section modal deformation shapes into the pure membrane, pure plate and membrane-plate modes. Also, at the appendix, a full example compares the results of this alternative analysis with the classical Schardt analysis.

Chapter 5 develops the exact finite element solution for GBT in the linear analysis. Based on the particular solution of GBT ordinary differential equation, this chapter proposes a complementary mode classification. In order to assort the correct shape function, this complementary classification evaluates the ratio among the generalized cross-section properties. This chapter also introduces the Completeness Coefficient Ma- 
trix, showing the practical application for this approach. A presented example details the application of this exact finite element and compares the results of displacement and stress fields with an entirely shell finite element model.

Chapter 6 builds the coupling between GBT and shell elements. This mixed shell-GBT model has an appropriate application in practical modeling problems, especially concerning steel structures. This modeling of connection is usually made by shell elements and the structural frame by beam elements. This chapter develops the coupling equation for this application based on the deformation modes of GBT. The example in this chapter provides not only the implementation of the mixed shell-GBT model, but also it compares the results to an entirely shell finite element model.

Chapter 7 is related to non-linear analyses of the mixed shell-GBT model. It highlights how the mode deformation decomposition, used in the linear analysis, can lead to the coupling tensors of modes in the non-linear analysis. The physical meaning of these tensors is priceless and maps all possible coupling effects among the linear modes of GBT. Thus, the implementation and unique procedures of GBT in non-linear cases are detailed here. Moreover, Chapter 7 extends the application of the coupling tensor to develop the initial displacement stiffness matrix and a consistent internal force formulation in non-linear GBT. As an outcome, the example of this chapter shows the limitations of the mixed shell-GBT model in non-linear analysis, especially concerning the results of the longitudinal displacements.

As a conclusion of this dissertation, Chapter 8 resumes and highlights the main contributions. Also, this chapter presents ideas for future works. 


\section{Generalized Beam Theory Assumptions}

This chapter presents a detailed review of the Generalized Beam Theory's assumptions. Initially, the Vlasov beam theory's assumption and kinematic model is introduced, in order to provide a natural development of GBT as an extension of this beam theory.

This chapter describes the two main features of GBT that are particularly relevant for this study. The first one is the approach of separation of variables, which receives a particular modification in its application in GBT. As for the second main feature, the idea of orthogonal deformation shapes stands out.

Furthermore, the application of the variational formulation reaches the GBT's differential equation and its boundary conditions. As an outcome, one obtains the description of generalized cross-section properties, which condensates the GBT's ideas.

\subsection{Hypotheses of Generalized Beam Theory}

GBT has three main hypotheses:

- Discretization of the thin-walled beam cross-section as a combination of shells segments;

- Separation of variables to describe the displacement field;

- A linear combination of orthogonal deformation-shape functions describes the displacement in a cross-section.

Every hypothesis presented here involves other particular assumptions, as detailed in the next subsections. 


\subsubsection{Discretization of the thin-walled beam cross-section as a com- bination of shell segments}

As mentioned before, Generalized Beam Theory is an extension of Vlasov's Thin-Walled Beam Theory Vlasov [176]. They share the same basic principle, which is the description of any thin-walled beam as a combination of $2 \mathrm{D}$ element segments, as shown below:
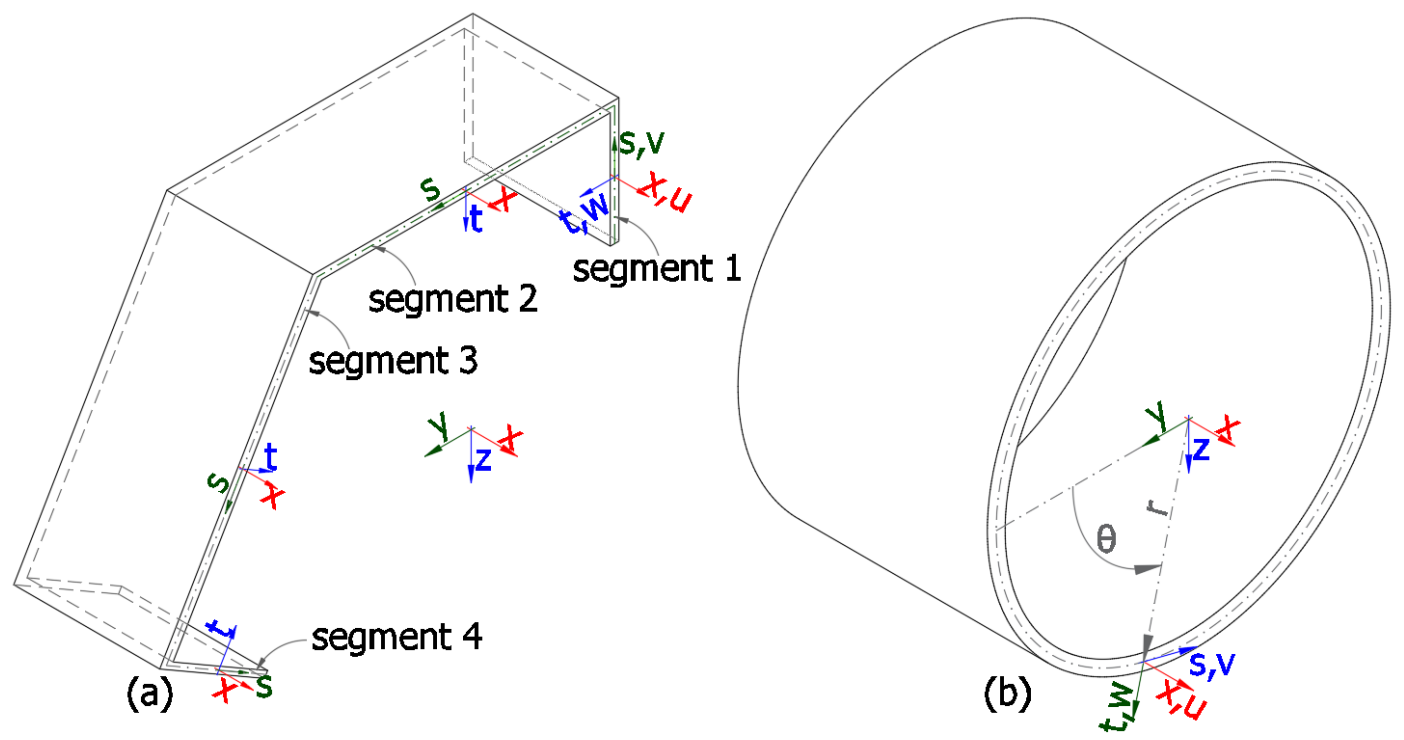

Figure 2.1: Global and local coordinate systems: a) in a generic segmented crosssection; b) in a hollow circular cross-section

In both theories, the cross-section's geometry is described in a convenient local coordinate system $(x, s, t)$ instead of the global coordinate system (X, Y, Z). Similarly, the local displacement $(u, v, w)$ describes the displacement field. The global displacements $\left(u_{g}, v_{g}, w_{g}\right)$ are useful in the cross-section analysis, as presented later in Chapter 4.

Concerning the particular case of a hollow circular cross-section, it has only one segment, represented by polar coordinates, as illustrated in figures 2.1.b.

\subsubsection{Kinematic assumptions}

In order to understand the extension of kinematic assumptions of GBT from Vlasov Beam Theory, it is important to briefly review the kinematic hypotheses of Vlasov Beam Theory, and subsequently how GBT affects them.

Kinematic assumptions of Vlasov Beam Theory

Vlasov Beam Theory uses a composition of simple beams to describe the thin-walled beams. These simple beams have partial membrane and plate behaviors. It is partial membrane behavior because it is assumed no transverse elongation and no shear contribution for longitudinal equilibrium. It is also partial plate behavior since it uses only 
the shear plate deformation. Therefore, there is not any transverse, neither longitudinal curvature in each composing beam. Consequently, Vlasov Beam Theory has three main kinematic assumntions. as listed below:
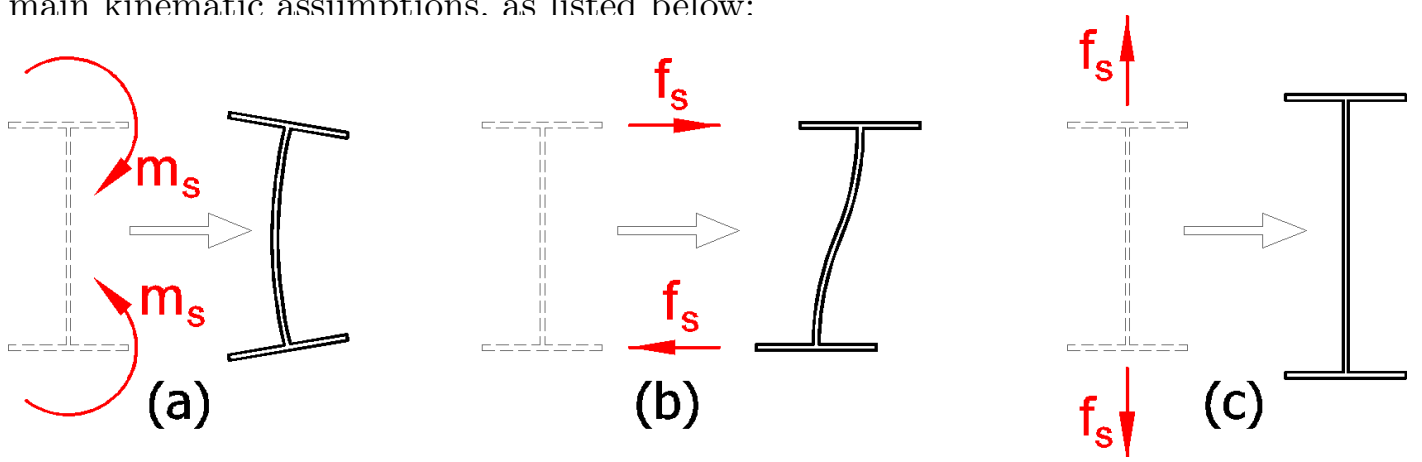

Figure 2.2: Types of Distortion: a) transverse bending distortion due to the variation of transverse bending moments; b) transverse bending distortion due to the variation of transverse forces; c) transverse elongation distortion.

- $1^{\text {st }}$ Constraining of transverse bending in each segment of the cross-section. Therefore, the beam's cross-section has no transverse distortion. Figures 2.2.a and 2.2.b clarify this type of constrained deformation;

$\cdot 2^{\text {nd }}$ Constraining of transverse elongation:

$$
\varepsilon_{s}^{M}(x, s)=v_{, s}(x, s)=0
$$

where, the superscript ()$^{M}$ and comma subscript (), indicate a membrane term and partial derivative, respectively.

It results in no transverse elongation distortion in each segment of the cross-section. Figure 2.2.c shows this specific type of cross-section distortion.

- $3^{\text {rd }}$ Assumption of no shear contribution in the total longitudinal equilibrium: This assumption conciliates the hypothesis of no shear deformation from the Bernoulli-Euler beam theory with Bredt's shear flow, in closed cross-section under uniform torsion. To illustrate this longitudinal equilibrium, figure 2.3 shows an infinitesimal part of a segment, which is under shear stress $\tau_{x s}$

The last kinematic assumption leads to the following restriction:

$$
\int_{s} \tau_{x s, s}^{M}(x, s) t_{h} \mathrm{~d} s=0
$$

where, $t_{h}$ and $\tau$ represent the thickness of the segment and the shear stress, respectively.

It is interesting to observe that the restriction in equation 2.2 allows a local shear contribution in the longitudinal equilibrium of a cross-section's section segment, as shown in figure 2.3.b, but it does not allow the shear contribution in the total longitudinal 


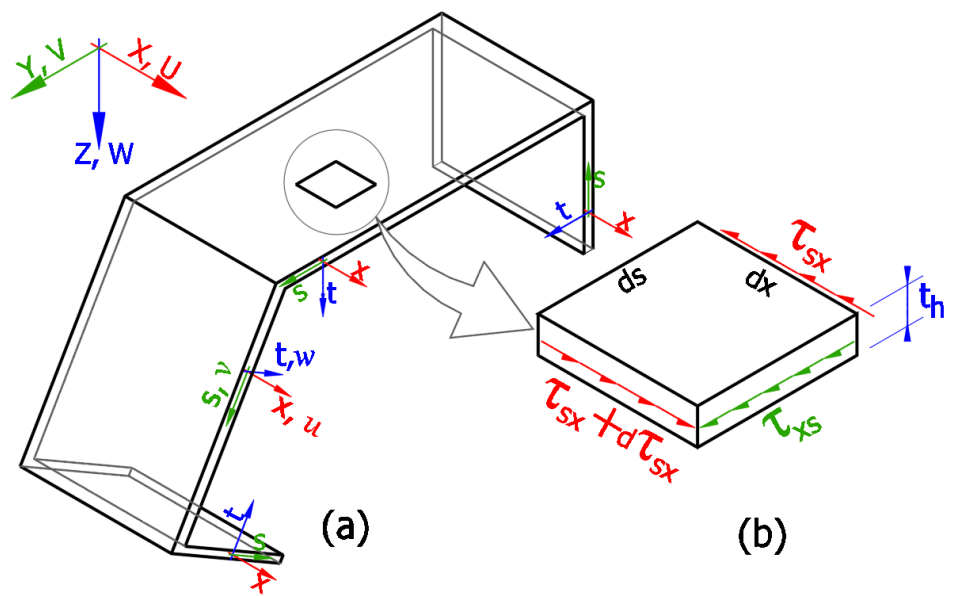

Figure 2.3: Membrane shear flow in a infinitesimal segment of a cross-section.

equilibrium of the whole cross-section, as shown in figure 2.3.a. Therefore, the Grashof's method, which is used in Bernoulli-Euler beam theory to obtain the shear stress distribution in the cross-section, is applied in Vlasov beam theory. This observation is reviewed later in the analysis of GBT's stress field.

Thus, the membrane shear strain, $\gamma_{x s}^{M}$ :

$$
\gamma_{x s}^{M}(x, s)=u_{, s}(x, s)+v_{, x}(x, s)
$$

together with the simple constitutive relationship: $\tau_{x s}^{M}(x, s)=G \gamma_{x s}^{M}(x, s)$, where $G$ is the shear modulus, into eq. 2.2 reaches the following constraint expression:

$$
\int_{s} G\left[u_{, s s}(x, s)+v_{, x s}(x, s)\right] t_{h} \mathrm{~d} s=0
$$

This expression can be fulfilled by Bernoulli-Euler beam theory as well as by the Bredt's constant shear flow assumption. In Bernoulli-Euler beam theory, the assumption of no shear deformation leads to equality:

$$
u_{, s}(x, s)=-v_{, x}(x, s)
$$

Therefore, each membrane's segment remains plane, with no longitudinal distortion for any deformation field. However, it is possible to have a different partial derivative of longitudinal displacement in function of the local system s: $\left.u_{(}, s\right)$ among the segments, i.e. for two distinguished segments $\mathrm{i}$ and $\mathrm{j}$, it is possible to have: $u_{\text {segmenti,s }} \neq u_{\text {segment } j, s}$. It is interesting to note that the cross-section, as a total, might not necessarily remain plane. This hypothesis allows Vlasov Beam Theory to describe the warping in crosssection due to non-uniform torsion.

However, the uniform torsion in closed cross-section cannot be studied under this assumption, since it involves deformation due to constant shear flow. To overcome this 
limitation, one assumes the Bredt's constant shea flow, which has the following definition of shear strain:

$$
\gamma_{x s}^{M c}(x, s)=u_{, s}(x, s)+v_{, x}(x, s)=0+v_{, x}^{M c}(x)
$$

Here, the superscript ()$^{M c}$ indicates a constant membrane function. One can observe that his expression is complementary to Bernoulli-Euler shear assumption, eq. 2.5, in the constrained relationship given in eq. 2.4.

\section{Kinematic assumptions in GBT}

In Generalized Beam Theory, the beam's segments have a full plate behavior. Consequently, the transverse bending distortion due to variation of transverse bending moments and forces (figure 2.2.a and 2.2.b respectively) can be studied. Here, Generalized Beam Theory relaxes the first hypothesis of the Vlasov Beam Theory presented above.

Consequently, the description of longitudinal, transverse and shear strains is:

- Longitudinal strains: This strain has two components: the membrane one, which is constant in thickness dimension, and the plate one, which is concerning the local curvature in the longitudinal direction, therefore a linear function in thickness dimension:

$$
\varepsilon_{x}(x, s, t)=\varepsilon_{x}^{M}(x, s)+\varepsilon_{x}^{P}(x, s, t)=u_{, x x}(x, s)+w_{, x x}(x, s) t
$$

Here, the superscript ()$^{P}$ indicates a plate term, and $t$ is the variable in thickness dimensions, as showed in local coordinate system in figure 2.2 .

- transverse strains: The Vlasov's assumption of no transverse elongation, is partially maintained in Generalized Beam Theory. The elongation of the cross-section middle line is still neglected. I.e., there is no membrane elongation in this direction. However, the elongation due to the transverse bending is allowed; in other words, the transverse elongation is a linear function in the thickness dimension:

$$
\varepsilon_{s}(x, s, t)=\varepsilon_{s}^{M}(x, s)+\varepsilon_{s}^{P}(x, s, t)=0+w_{, s s}(x, s) t
$$

- Shear strains: similar to Vlasov beam theory, the shear strains has two components: the constant membrane flow and a shear strain due to plate behavior:

$$
\gamma_{x s}(x, s, t)=\gamma_{x s}^{M c}(x, s)+\gamma_{s x}^{P}(x, s, t)=v_{, x}^{M c}(x)+2 w_{, x s}(x, s) t
$$

Also, GBT has an additional modification in Vlasov's assumption. Although GBT uses the same simplified constitutive relationship for membrane behavior:

$$
\left[\begin{array}{l}
\sigma_{x}^{M} \\
\sigma_{s}^{M} \\
\tau_{x s}^{M}
\end{array}\right]=\left[\begin{array}{ccc}
E & 0 & 0 \\
0 & E & 0 \\
0 & 0 & G
\end{array}\right]\left[\begin{array}{c}
\varepsilon_{x}^{M} \\
\varepsilon_{s}^{M} \\
\gamma_{x s}^{M}
\end{array}\right]
$$


GBT does not adopt this assumption for plate behavior. It uses the full constitutive relationship:

$$
\left[\begin{array}{c}
\sigma_{x}^{P} \\
\sigma_{s}^{P} \\
\tau_{x s}^{p}
\end{array}\right]=\frac{E}{1-\mu^{2}}\left[\begin{array}{ccc}
1 & \mu & 0 \\
\mu & 1 & 0 \\
0 & 0 & \frac{1-\mu}{2}
\end{array}\right]\left[\begin{array}{c}
\varepsilon_{x}^{P} \\
\varepsilon_{s}^{P} \\
\gamma_{x s}^{P}
\end{array}\right]
$$

Here, $E$ and $\mu$ represent the Young's Modulus and the Poisson's ratio, respectively. These assumptions in constitutive relationships guarantee that there is no changing in equations eq. 2.1 and eq. 2.2 for the middle line of the cross-section. As shown later, these two equations can link the longitudinal displacement with the transverse displacement.

\subsubsection{Separation of variables to describe the displacement field.}

One of the Schardt's outstanding ideas is the application of separation of variables in displacements field functions $u, v$ and $w$. In this approach [84, 130], one function has the variable concerning displacements on cross-section middle line $s$; and a second function has the variable in longitudinal direction, $x$ :

$$
\begin{gathered}
u^{M}(x, s)=u(s) V_{, x}(x) \\
v^{M}(x, s)=v(s) V(x) \\
w^{M}(x, s)=w(s) V(x)
\end{gathered}
$$

where $V(x)$ is an amplitude function. This function is the same for three displacements: $u, v$ and $w$. It is important to note that the first derivative, $V_{, x}(x)$, in eq. 2.12 is not arbitrary. As it will be shown later on, it is necessary to link the longitudinal displacement in the cross-section, $u(x, s)$, with the transverse displacements: $v(x, s)$ and $w(x, s)$ in order to avoid shear energy deformation. Also, this derivative can be alternatively interpreted by the following perspectives:

- The transverse displacements are fully described in the same plane of the cross-section, while the longitudinal displacement is perpendicular to cross-section's plane;

- A cross-section is a beam with an infinitesimal length.

Another consequence of this hypothesis is the highlight of the cross-section functions, $u(s), v(s)$ and $w(s)$, which leads to the generalized cross-section properties, and the dependent amplification function ${ }^{1} V(x)$ that can be solved as a beam problem.

Also, the sum of membrane and plate parts gives the total displacement at a generic point in the cross-section:

$$
\begin{gathered}
u(x, s, t)=u^{M}(x, s)+u^{P}(x, s, t)=[u(s)-t w(s)] V_{, x}(x) \\
v(x, s, t)=v^{M}(x, s)+v^{P}(x, s, t)=\left[v(s)-t w_{, s}(s)\right] V(x) \\
w(x, s)=w^{M}(x, s)+w^{P}(x, s)=w(s) V(x)+0
\end{gathered}
$$

\footnotetext{
${ }^{1}$ Gonçalves et al. $[124,125,126]$ showed that the dependency between the cross-section functions and the longitudinal amplification function is not valid for a curved beam's axis
} 


\subsubsection{A linear combination of orthogonal deformation-shape func- tion describes any possible displacement in a cross-section}

Similar to other mathematical approaches to solve partial differential equations, the separation of variables of GBT leads to a system of ordinary differential equations that can be expressed in an orthogonal basis. Thus, each equation can be solved independently. Hence, equations 2.12, 2.13 and 2.14 can be rewritten as:

$$
\begin{gathered}
u(x, s)=\sum_{i=1}^{n} u(s)^{i} V_{, x}(x) \\
v(x, s)=\sum_{i=1}^{n} v(s)^{i} V(x) \\
w(x, s)=\sum_{i=1}^{n} w(s)^{i} V(x) \\
\text { with the null inner product for any } i \neq j \quad\left\{\begin{array}{l}
\int_{s}^{i} u(s)^{j} u(s) \mathrm{d} s=0 \\
\int_{s}^{i} u(s)^{j} u(s) \mathrm{d} s=0 \\
\int_{s}^{i} w(s)^{j} w(s) \mathrm{d} s=0
\end{array}\right.
\end{gathered}
$$

Where, the left-upper indexes indicates the GBT mode. This feature not only simplifies the solving of beam problem, but also brings a clear representation of the physical meaning in each deformation shape. It also allows the simple superposition among these deformation shapes.

Moreover, by the linear combinations of orthogonal deformation shape functions, GBT can be understood as a natural sequence of beam theories: starting from the classical Bernoulli-Euler, followed by the Vlasov, and reaching GBT itself. Therefore, BernoulliEuler beam theory is a particular case of Vlasov beam theory and this one is a particular case of GBT.

The generic segmented cross-section, shown in figure 2.1.a, illustrates this feature of GBT and its orthogonal modes:

As one can note, the first mode is the simple axial extension; the second and third ones are the major and minor bending (Bernoulli-Euler theory), respectively; the fourth one is the torsion and warping mode (Vlasov beam theory); the fifth one is a distortion mode of GBT.

It is important to emphasize that the orthogonality of GBT happens only in the linear analysis, after the solution of a quadratic eigenvalue problem. In the non-linear analysis, the GBT equations are once again coupled. However, in this case, as showed in the non-linear chapter, GBT can provide a clear systematic map of the coupling among the modes due to the initial displacement/stress. 


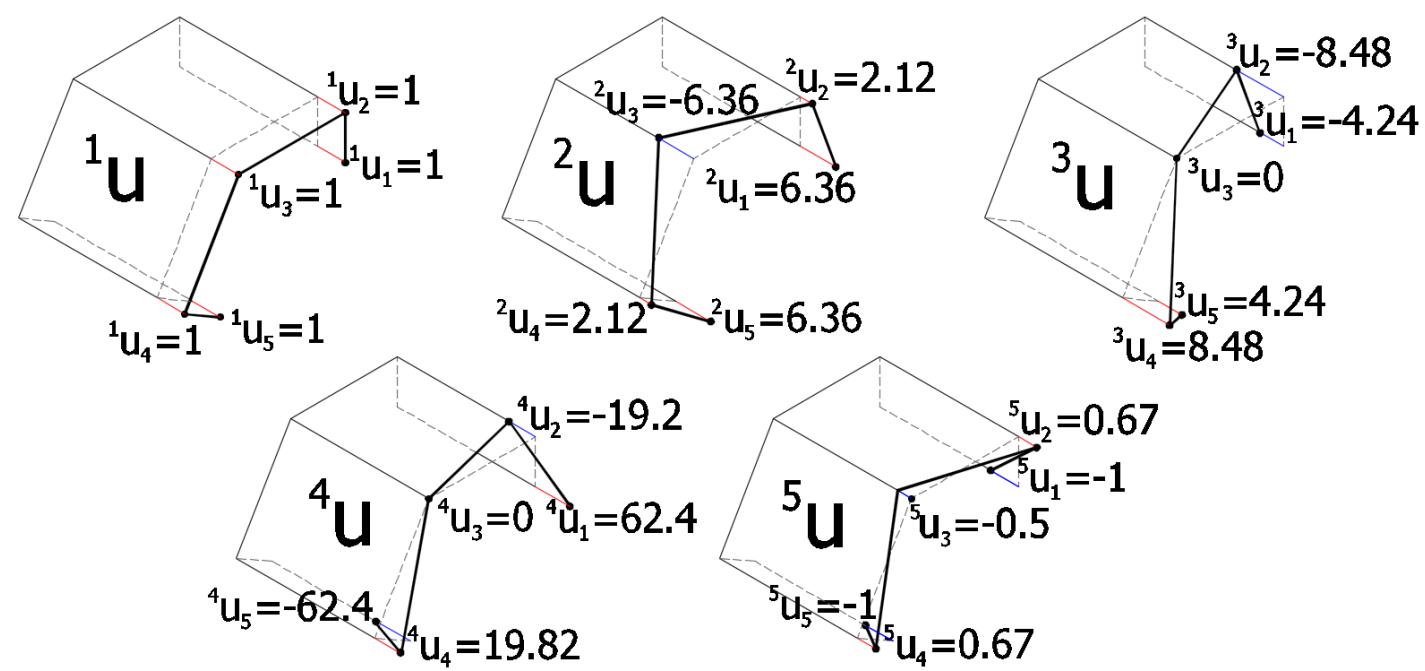

Figure 2.4: Longitudinal orthogonal modes shapes of a generic segment cross-section.

\subsection{Variational Formulation in Generalized Beam Theory}

Based on the hypothesis and assumptions described in the previous section, the Variational Formulation is applied to highlight the generic cross-section properties, as well as the GBT differential equation and its boundary conditions. One obtains it from the minimization of the total energy's functional:

$$
\begin{gathered}
\Pi=U_{i n t}-V_{e x t} \\
\delta \Pi=\delta U_{i n t}-\delta V_{\text {ext }}=0
\end{gathered}
$$

Where $\Pi$ is the total energy, $U_{\text {int }}$ the internal strain energy and $V_{\text {ext }}$ the external load potential energy. The next subsections develop each term according to GBT.

\subsubsection{Internal strain energy according to GBT}

According to GBT's assumptions, the superposition of membrane and plate strains lead to internal strain energy. However, it is necessary to keep in mind that for membrane strains there is the absent of transverse and non-constant shear contributions. Hence, the functional of internal strain energy in GBT is:

$$
U_{i n t}=\int_{V}\left[\int_{\varepsilon_{x}^{M}} \sigma_{x}^{M} \mathrm{~d} \varepsilon_{x}^{M}+\int_{\varepsilon_{x}^{P}} \sigma_{x}^{P} \mathrm{~d} \varepsilon_{x}^{P}+\int_{\gamma_{x s}^{M c}} \tau_{x s}^{M c} \mathrm{~d} \gamma_{x s}^{M c}+\int_{\gamma_{x s}^{P}} \tau_{x s}^{P} \mathrm{~d} \gamma_{x s}^{P}+\int_{\varepsilon_{s}^{P}} \sigma_{s}^{P} \mathrm{~d} \varepsilon_{s}^{P}\right] \mathrm{d} V
$$

Using the simplified constitutive relation, eq. 2.10, to the membrane part, and the fully constitutive relation, eq. 2.11, to the plate part, the functional leads to: 


$$
\begin{aligned}
U_{\text {int }}=\int_{V} \frac{E}{2}\left(\varepsilon_{x}^{M}\right)^{2}+\frac{E}{1-\mu^{2}} & {\left[\frac{\left(\varepsilon_{x}^{P}\right)^{2}}{2}+\mu \varepsilon_{x}^{P} \varepsilon_{s}^{P}\right]+} \\
& +\frac{G}{2}\left[\left(\gamma_{x s}^{M c}\right)^{2}+\left(\gamma_{x s}^{P}\right)^{2}\right]+\frac{E}{1-\mu^{2}}\left[\frac{\left(\varepsilon_{s}^{P}\right)^{2}}{2}+\mu \varepsilon_{x}^{P} \varepsilon_{s}^{P}\right] \mathrm{d} V
\end{aligned}
$$

And its variation concerning the internal strain energy is:

$$
\begin{aligned}
\delta U_{\text {int }}=\int_{V} E \varepsilon_{x}^{M} \delta \varepsilon_{x}^{M}+\frac{E}{1-\mu^{2}}\left[\varepsilon_{x}^{P}+\mu \varepsilon_{s}^{P}\right] \delta \varepsilon_{x}^{P}+ \\
\qquad G\left[\gamma_{x s}^{M c} \delta \gamma_{x s}^{M c}+\gamma_{x s}^{P} \delta \gamma_{x s}^{P}\right]+\frac{E}{1-\mu^{2}}\left[\varepsilon_{s}^{P} \delta \varepsilon_{s}^{P}+\mu \varepsilon_{x}^{P}\right] \delta \varepsilon_{s}^{P} \mathrm{~d} V
\end{aligned}
$$

From the GBTs definition of the displacement functions (equations 2.12, 2.13 and 2.14), one rewrites the strains equations $2.7,2.9$ and 2.30 in the form:

$$
\begin{gathered}
\varepsilon_{x}(x, s, t)=\varepsilon_{x}^{M}(x, s)+\varepsilon_{x}^{P}(x, s, t)=[u(s)-t w(s)] V_{, x x}(x) \\
\gamma_{x s}^{P}(x, s, t)=2 t w_{, s}(s) V_{, x}(x) \\
\gamma_{x s}^{M c}(x, s)=v^{c}(s) V_{, x}(x) \\
\varepsilon_{s}^{P}(x, s, t)=-t w_{, s s}(s) V(x)
\end{gathered}
$$

Since the terms $u(s)$ and $v(s)$ are predefined, their variations are obtained by the amplification function: $\delta V(x)$. Therefore, the variation of strain according to GBT is:

$$
\begin{gathered}
\delta \varepsilon_{x}(x, s, t)=\delta \varepsilon_{x}^{M}(x, s)+\delta \varepsilon_{x}^{P}(x, s, t)=[u(s)-t w(s)] \delta V_{, x x}(x) \\
\delta \gamma_{x s}^{P}(x, s, t)=2 t w_{, s}(s) \delta V_{, x}(x) \\
\delta \gamma_{x s}^{M c}(x, s)=v^{M c}(s) \delta V_{, x}(x) \\
\delta \varepsilon_{s}^{P}(x, s, t)=-t w_{, s s}(s) \delta V(x)
\end{gathered}
$$

These expressions, together with the orthogonal modes assumption (equations 2.18, $2.19,2.20$ and 2.21 ), leads to the follow expression:

$$
\begin{aligned}
& \delta U_{\text {int }}=\sum_{i=1}^{n} \int_{V}\left[E^{i} u^{2}(s)+\frac{E}{1-\mu}\left(t^{i} w(s)\right)^{2}\right]{ }^{i} V_{, x x}(x) \delta^{i} V_{, x x}(x) \mathrm{d} V+ \\
& \int_{V} \frac{\mu E}{1-\mu} t^{2 i} w_{, s s}(s)^{i} w(s)^{i} V(x) \delta^{i} V_{, x x}(x) \mathrm{d} V+G \int_{V}\left[\left(2 t^{i} w_{, s}(s)\right)^{2}+{ }^{i} v^{M c}(s)^{2}\right]{ }^{i} V_{, x}(x) \delta^{i} V_{, x}(x) \mathrm{d} V \\
& +\frac{E}{1-\mu^{2}} \int_{V}\left(t^{i} w_{, s s}(s)\right)^{2}{ }^{i} V(x) \delta^{i} V(x) \mathrm{d} V+\int_{V} \frac{\mu E}{1-\mu} t^{2 i} w_{, s s}(s)^{i} w(s) \delta^{i} V(x)^{i} V_{, x x}(x) \mathrm{d} V
\end{aligned}
$$


The integrals in eq. 2.35 can have their integration domain over the volume split into cross-sections' area and longitudinal domains. Consequently, the generic cross-section properties stand out:

$$
\begin{aligned}
\delta U_{\text {int }}=\sum_{i=1}^{n} & \left(E^{i} C^{M}+K^{i} C^{P}\right) \int_{L} V_{, x x}(x) \delta^{i} V_{, x x}(x) \mathrm{d} x+G\left({ }^{i} D^{M c}+{ }^{i} D^{P}\right) \int_{L}{ }^{i} V_{, x} \delta^{i} V_{, x}(x) \mathrm{d} x+ \\
& +K^{i} B \int_{L}{ }^{i} V(x) \delta^{i} V(x) \mathrm{d} x+\mu K^{i} D^{\mu} \int_{L}{ }^{i} V(x) \delta^{i} V_{x x}(x)+\delta^{i} V(x)^{i} V_{x x}(x) \mathrm{d} x
\end{aligned}
$$

where ${ }^{i} C^{M}$ and ${ }^{i} C^{P}$ are the generic moments of inertia, related to longitudinal strains, in membrane and plate behaviors, respectively; ${ }^{i} D^{M c},{ }^{i} D^{P}$ and ${ }^{i} D^{\mu}$, are the generic shear inertias due to the constant membrane shear flow, the plate's shear inertia and the effect of Poisson coupling, $\mu$, as shear inertia, respectively; ${ }^{i} B$ is the generic transverse bending inertia; $K$ is the plate's stiffness. These terms are given by:

$$
\begin{gathered}
{ }^{i} C^{M}=t_{h} \int_{s} u(s)^{2} \mathrm{~d} s \\
{ }^{i} C^{P}=\int_{s}{ }^{i} w(s)^{2} \mathrm{~d} s \\
{ }^{i} D^{M c}=t_{h} \int_{s}{ }^{i} v^{c}(s)^{2} \mathrm{~d} s \\
{ }^{i} D^{P}=\frac{t_{h}^{3}}{3} \int_{s}{ }^{i} w_{, s}(s)^{2} \mathrm{~d} s \\
K=\frac{E t_{h}^{3}}{12\left(1-\mu^{2}\right)} \\
{ }^{i} B=\int_{s}{ }^{i} w_{, s s}^{2}(s) \mathrm{d} s \\
{ }^{i} D^{\mu}=\int_{s}{ }^{i} w_{, s s}(s)^{i} w(s) \mathrm{d} s
\end{gathered}
$$

\subsubsection{External load potential energy according to GBT}

GBT considers the external load as a distributed force in the local coordinate system. Hence, it is considered as a vector field (with the components $q_{x}, q_{v}$ and $q_{w}$ in the local coordinate system) in function of the dimensions $x$ and $s$ :

$$
[q(x, s)]^{T}=\left[\begin{array}{lll}
q_{x}(x, s) & q_{v}(x, s) & q_{w}(x, s)
\end{array}\right]
$$

Similar to the displacement field, one represents the above components as separation of variables:

$$
q_{x}(x, s)=q_{x}(s) f_{x}(x)
$$




$$
\begin{gathered}
q_{v}(x, s)=q_{v}(s) f_{v}(x) \\
q_{w}(x, s)=q_{w}(s) f_{w}(x)
\end{gathered}
$$

Similarly, one can represent the concentrated nodal loads at the initial and final beam's nodes in the local coordinate system. With the index $i$ and $f$, these nodal forces are given by:

$$
\begin{aligned}
& {\left[P_{i}(s)\right]^{T}=\left[\begin{array}{lll}
P_{i x}(s) & P_{i v}(s) & P_{i w}(s)
\end{array}\right]} \\
& {\left[P_{f}(s)\right]^{T}=\left[\begin{array}{lll}
P_{f x}(s) & P_{f v}(s) & P_{f w}(s)
\end{array}\right]}
\end{aligned}
$$

The inner product of these functions and their respective displacement leads to the external load potential energy:

$$
V_{e x t}=\int_{s} \int_{L}[q(x, s)]^{T}[u] \mathrm{d} x\left[P_{i}(s)\right]^{T}\left[u_{i}\right]+\left[P_{f}(s)\right]^{T}\left[u_{f}\right] \mathrm{d} s
$$

where $[u]$ is the displacement field vector: $[u]=[u(x, s) v(x, s) \quad w(x, s)]^{T}$ and the vectors $\left[u_{i}\right]$ and $\left[u_{f}\right]$ are the displacement fields at the initial and final nodes respectively. Introducing in the above equation expressions from eq. 2.44 until eq. 2.49, one reaches:

$$
\begin{aligned}
& V_{\text {ext }}=\int_{s} \int_{L}\left[q_{x}(s) f_{x}(x) \quad q_{v}(s) f_{v}(x) \quad q_{w}(s) f_{w}(x)\right]\left[\begin{array}{c}
u(x, s) \\
v(x, s) \\
w(x, s)
\end{array}\right] \mathrm{d} x+ \\
& +\left[\begin{array}{lll}
P_{i x}(s) & P_{i v}(s) & P_{i w}(s)
\end{array}\right]\left[\begin{array}{c}
u(x=i, s) \\
v(x=i, s) \\
w(x=i, s)
\end{array}\right]+\left[\begin{array}{lll}
P_{f x}(s) & P_{f v}(s) & P_{f w}(s)
\end{array}\right]\left[\begin{array}{c}
u(x=f, s) \\
v(x=f, s) \\
w(x=f, s)
\end{array}\right] \mathrm{d} s
\end{aligned}
$$

With the GBT's representation of the displacement fields as a summation of orthogonal modes, equations 2.18, 2.19 and 2.20 , it is possible to notice that the integral over local dimension $s$ is in fact an inner product of deformation orthogonal modes and the load vector. Consequently, the expression of external load is a sum of orthogonal load modes as well. For instance, for the distributed load, one obtains:

$$
\begin{aligned}
& { }^{i} q_{x}(s)=\int_{s} q_{x}(s)^{i} u(s) \mathrm{d} s \\
& { }^{i} q_{v}(s)=\int_{s} q_{v}(s)^{i} v(s) \mathrm{d} s \\
& { }^{i} q_{w}(s)=\int_{s} q_{w}(s)^{i} w(s) \mathrm{d} s
\end{aligned}
$$

The load vectors of nodal concentrated in eq. 2.51 follow the same approach. Thus, the evaluation of eq. 2.51 leads to the representation of the external load potential energy, 
according to GBT:

$$
\begin{array}{r}
V_{e x t}=\sum_{i=1}^{n}\left[\int_{L}{ }^{i} q_{x}(s) f_{x}(x){ }^{i} V_{, x}(x)+{ }^{i} q_{v}(s) f_{v}(x){ }^{i} V(x)+{ }^{i} q_{w}(s) f_{w}(x){ }^{i} V(x) \mathrm{d} x+\right. \\
\left.+{ }^{i} P_{x i}{ }^{i} V_{i, x}+{ }^{i} P_{x f}{ }^{i} V_{f, x}+{ }^{i} P_{v i}{ }^{i} V_{i}+{ }^{i} P_{v f}{ }^{i} V_{f}+{ }^{i} P_{w i}{ }^{i} V_{i}+{ }^{i} P_{w f}{ }^{i} V_{f}\right]
\end{array}
$$

And its variation $[63,117]$ is:

$$
\begin{array}{r}
\delta V_{e x t}=\sum_{i=1}^{n}\left[\int_{L}{ }^{i} q_{x}(s) f_{x}(x) \delta^{i} V_{, x}(x)+{ }^{i} q_{v}(s) f_{v}(x) \delta^{i} V(x)+{ }^{i} q_{w}(s) f_{w}(x) \delta^{i} V(x) \mathrm{d} x+\right. \\
\left.+{ }^{i} P_{x i} \delta^{i} V_{i, x}+{ }^{i} P_{x f} \delta^{i} V_{f, x}+{ }^{i} P_{v i} \delta^{i} V_{i}+{ }^{i} P_{v f} \delta^{i} V_{f}+{ }^{i} P_{w i} \delta^{i} V_{i}+{ }^{i} P_{w f} \delta^{i} V_{f}\right]
\end{array}
$$

\subsubsection{Equilibrium by Hamilton's principle}

Introducing the variations of internal strain energy eq. 2.36 and the external potential energy eq. 2.56 into Hamilton's principle presented in eq. 2.23, one obtains:

$$
\begin{aligned}
& 0=\sum_{i=1}^{n}[\left(E^{i} C^{M}+K^{i} C^{P}\right) \int_{L}{ }^{i} V_{, x x}(x) \delta^{i} V_{, x x}(x) \mathrm{d} x+G\left({ }^{i} D^{M c}+{ }^{i} D^{P}\right) \int_{L}{ }^{i} V_{, x} \delta^{i} V_{, x}(x) \mathrm{d} x+ \\
&+K^{i} B \int_{L}{ }^{i} V(x) \delta^{i} V(x) \mathrm{d} x+\mu K^{i} D^{\mu} \int_{L}{ }^{i} V(x) \delta^{i} V_{x x}(x)+\delta^{i} V(x){ }^{i} V_{x x}(x) \mathrm{d} x+ \\
&-\int_{L}{ }^{i} q_{x}(s) f_{x}(x) \delta^{i} V_{, x}(x)+{ }^{i} q_{v}(s) f_{v}(x) \delta^{i} V(x)+{ }^{i} q_{w}(s) f_{w}(x) \delta^{i} V(x) \mathrm{d} x+ \\
&\left.-{ }^{i} P_{x i}{ }^{i} \delta V_{i, x}-{ }^{i} P_{x f}{ }^{i} \delta V_{f, x}-{ }^{i} P_{v i}^{i} \delta V_{i}+{ }^{i} P_{v f}{ }^{i} \delta V_{f}-{ }^{i} P_{w i}^{i} \delta V_{i}-{ }^{i} P_{w f}{ }^{i} \delta V_{f}\right]
\end{aligned}
$$

Integrating twice, by parts, the first integral and the first term in the fourth integral, one finds:

$$
\begin{gathered}
\int_{L}{ }^{i} V_{, x x}(x) \delta^{i} V_{, x x}(x) \mathrm{d} x=\left[{ }^{i} V_{, x x}(x) \delta^{i} V_{, x}(x)\right]_{i}^{f}-\left[{ }^{i} V_{, x x x}(x) \delta^{i} V(x)\right]_{i}^{f}+\int_{L}{ }^{i} V_{, x x x x}(x) \delta^{i} V(x) \mathrm{d} x \\
\int_{L}{ }^{i} V(x) \delta^{i} V_{, x x}(x) \mathrm{d} x=\left[{ }^{i} V(x) \delta^{i} V_{, x}(x)\right]_{i}^{f}+\left[{ }^{i} V_{, x}(x) \delta^{i} V(x)\right]_{i}^{f}+\int_{L}{ }^{i} V_{, x x}(x) \delta^{i} V(x) \mathrm{d} x
\end{gathered}
$$

Also, integrating once, by parts, the second integral in eq. 2.36 and the first term in eq. 2.56:

$$
\begin{array}{r}
G \int_{L}\left({ }^{i} D^{M c}+{ }^{i} D^{P}\right)^{i} V_{, x}(x) \delta^{i} V_{, x}(x) \mathrm{d} x=\left[G\left({ }^{i} D^{M c}+{ }^{i} D^{P}\right)^{i} V_{, x}(x) \delta^{i} V(x)\right]_{i}^{f}+ \\
-\int_{L} G\left({ }^{i} D^{M c}+{ }^{i} D^{P}\right)^{i} V_{, x x}(x) \delta^{i} V(x) \mathrm{d} x
\end{array}
$$




$$
\int_{L}^{i} q_{x}(s) f_{x}(x) \delta^{i} V_{, x}(x) \mathrm{d} x=\left[{ }^{i} q_{x}(s) F_{x}(x) \delta^{i} V_{, x}(x)\right]_{i}^{f}-\int_{L}^{i} q_{x}(s) F_{x}(x) \delta^{i} V(x) \mathrm{d} x
$$

Where $F_{x}(x)$ is the integral of the amplification function of the distributed load, $f_{x}(x)$, over the beam domain. These results in eq. 2.57 lead to:

$$
\begin{gathered}
0=\int_{L}\left[\left(E^{i} C^{M}+K^{i} C^{P}\right){ }^{i} V_{, x x x x}(x)-\left(G\left({ }^{i} D^{M c}+{ }^{i} D^{P}\right)-2 \mu K^{i} D^{\mu}\right){ }^{i} V_{, x x}(x)+K^{i} B^{i} V(x)+\right. \\
\left.{ }^{i} q_{x}(s) F_{x}(x)-{ }^{i} q_{v}(s) f_{v}(x)-{ }^{i} q_{w}(s) f_{w}(x)\right] \mathrm{d} x \delta^{i} V(x)-\left({ }^{i} P_{v i}+{ }^{i} P_{w i}\right){ }^{i} \delta V_{i}-\left({ }^{i} P_{v f}+{ }^{i} P_{w f}\right)^{i} \delta V_{f}+ \\
\left.-{ }^{i} P_{x i}^{i} \delta V_{i, x}-{ }^{i} P_{x f}{ }^{i} \delta V_{f, x}+\left[\left(\left(E^{i} C^{M}+K^{i} C^{P}\right)\right)^{i} V_{, x x}(x)+2 \mu K^{i} V(x)\right) \delta^{i} V_{, x}(x)\right]_{i}^{f}+ \\
{\left[\left(\left(G\left({ }^{i} D^{M c}+{ }^{i} D^{P}\right)-2 \mu K^{i} D^{\mu}\right)^{i} V_{, x}(x)-\left(E^{i} C^{M}+K^{i} C^{P}\right){ }^{i} V_{, x x x}(x)-{ }^{i} q_{x}(s) F_{x}(x)\right) \delta^{i} V(x)\right]_{i}^{f}}
\end{gathered}
$$

Here, the equilibrium and boundary conditions stand out. Taking into account that the functional eq. 2.62 is null for any arbitrary functions of longitudinal amplification, $\delta^{i} V(x)$, the parentheses terms of the integral must be zero, which leads to the nonhomogeneous differential equation of equilibrium in GBT:

$$
\begin{array}{r}
\left(E^{i} C^{M}+K^{i} C^{P}\right)^{i} V_{, x x x x}(x)-\left(G\left({ }^{i} D^{M c}+{ }^{i} D^{P}\right)-2 \mu K^{i} D^{\mu}\right)^{i} V_{, x x}(x)+K^{i} B^{i} V(x)= \\
{ }^{i} q_{v}(s) f_{v}(x)+{ }^{i} q_{w}(s) f_{w}(x)-{ }^{i} q_{x}(s) F_{x}(x)
\end{array}
$$

Furthermore, the remaining terms are related to boundary conditions:

$$
\begin{gathered}
{\left[\left(\left(G\left({ }^{i} D^{M c}+{ }^{i} D^{P}\right)-2 \mu K^{i} D^{\mu}\right)^{i} V_{, x}(x)-\right.\right.} \\
\left(E^{i} C^{M}+K^{i} C^{P}\right)^{i} V_{, x x x}(x)+ \\
\left.\left.-{ }^{i} q_{x}(s) F_{x}(x)-{ }^{i} P_{v i}-{ }^{i} P_{w i}\right) \delta^{i} V(x)\right]_{x=i}=0 \\
\left.\left[\left(\left(E^{i} C^{M}+K^{i} C^{P}\right)\right)^{i} V_{, x x}(x)+2 \mu K^{i} V(x)-{ }^{i} P_{x i}\right) \delta^{i} V_{, x}(x)\right]_{x=i}=0 \\
{\left[\left(\left(G\left({ }^{i} D^{M c}+{ }^{i} D^{P}\right)-2 \mu K^{i} D^{\mu}\right)^{i} V_{, x}(x)-\left(E^{i} C^{M}+K^{i} C^{P}\right){ }^{i} V_{, x x x}(x)+\right.\right.} \\
\left.\left.-{ }^{i} q_{x}(s) F_{x}(x)-{ }^{i} P_{v f}-{ }^{i} P_{w f}\right) \delta^{i} V(x)\right]_{x=f}=0 \\
{\left[\left(\left(E^{i} C^{M}+K^{i} C^{P}\right)^{i} V_{, x x}(x)+2 \mu K^{i} V(x)-{ }^{i} P_{x f}\right) \delta^{i} V_{, x}(x)\right]_{x=f}=0}
\end{gathered}
$$

Equations 2.64 and 2.66 represent the boundary conditions for the transverse displacement and forces at the initial and final nodes, respectively. Meanwhile, equations 2.65 and 2.67 are the boundary conditions for the longitudinal forces and displacement at these nodes. Since these equations involve generalized internal and external forces, special attention is given for this subject in the next subsection.

\subsection{Stress field in GBT: generalized internal forces}

Using orthogonal deformation modes to describe the displacement field also has its benefits in representing the stress field. It can be expressed by a simple superposition of stresses due to generalized internal forces. 
The definition of orthogonal generalized internal forces is based on the inner product of the total stress in a cross-section and the deformation mode of the stress direction. For instance, the longitudinal generalized internal force, ${ }^{i} W_{x}(x)$, of a particular mode $i$ is expressed in the form:

$$
{ }^{i} W_{x}(x)=\int_{A} \sigma_{x}(x, s, t){ }^{i} u(s, t) \mathrm{d} A
$$

With this representation, GBT automatically contains the usual internal forces of a rod and Bernoulli-Euler beam: if ${ }^{i} u(s, t)=1$, then one obtains the normal internal force; if ${ }^{i} u(s, t)=y$ or ${ }^{i} u(s, t)=z$, where $y$ and $z$ are the main directions of moment of inertia, then one obtains the internal bending moments. Also, the Vlasov's bi-moment is already in this representation, which is reached if ${ }^{i} u(s, t)=\omega$, where $\omega$ is the sectorial area.

Carrying on the evaluation of the above expression, the extra internal forces of GBT stand out. Also, it is necessary to remember that each orthogonal deformation mode is itself a linear superposition of two orthogonal deformation behaviors of membrane and plate, as shown in eq. 2.27. Thus, with the constitutive assumptions in equations 2.10 and 2.11, the generalized internal force can always be decomposed in membrane and plate parts:

$$
\begin{gathered}
{ }^{i} W_{x}^{M}(x)=E \int_{A}{ }^{i} u^{M}(s) \sum_{j=1}^{n} \varepsilon_{x}^{M}(x, s) \mathrm{d} A \\
{ }^{i} W_{x}^{P}(x)=\frac{E}{1-\mu} \int_{A}{ }^{i} u^{P}(s, t) \sum_{j=1}^{n}\left[{ }^{j} \varepsilon_{x}^{P}(x, s, t)+\mu^{j} \varepsilon_{s}^{P}(x, s, t)\right] \mathrm{d} A
\end{gathered}
$$

The strain definitions in eq. 2.27 and the principle of orthogonal modes, eq. 2.21 lead the expressions above into:

$$
\begin{array}{r}
{ }^{i} W_{x}^{M}(x)=E t_{h} \int_{s}{ }^{i} u^{M}(s)^{2} \mathrm{~d} s^{i} V_{, x x}(x)=E^{i} C^{M i} V_{, x x}(x) \\
{ }^{i} W_{x}^{P}(x)=\frac{E t_{h}^{3}}{12(1-\mu)}\left[\int_{s}{ }^{i} w(s)^{2} \mathrm{~d} s^{i} V_{, x x}(x)+\mu \int_{s}{ }^{i} w(s)^{i} w_{, s s}(s) \mathrm{d} s^{i} V(x)\right]= \\
=K\left[{ }^{i} C^{P i} V_{, x x}(x)+\mu^{i} D^{\mu i} V(x)\right]
\end{array}
$$

Again, one can note that eq. 2.71 includes the particular cases of Bernoulli-Euler bending moment and Vlasov's bi-moment.

Meanwhile, eq. 2.72 can easily express the internal bending moment of a particular mode only based on the respective modal amplification function ${ }^{i} V(x)$ and its second derivative, ${ }^{i} V_{, x x}(x)$.

Also, GBT can express the longitudinal stress in function of the generalized internal forces that are evaluated from the constitutive relations for membrane and plate, eq. 2.10 and eq. 2.11 , respectively:

$$
{ }^{i} \sigma_{x}(x, s, t)={ }^{i} \sigma_{x}^{M}(x, s)+{ }^{i} \sigma_{x}^{P}(x, s, t)
$$


The evaluation of ${ }^{i} \sigma_{x}^{M}(x, s)$ leads to:

$$
{ }^{i} \sigma_{x}^{M}(x, s)=E^{i} \varepsilon_{x}^{M}(x, s)=E^{i} u(s){ }^{i} V_{, x x}(x)=\frac{{ }^{i} W(x)}{{ }^{i} C}{ }^{i} u(s)
$$

One can note that eq. 2.74 has as particular cases the well-known expressions of longitudinal stress due to i) normal force, if ${ }^{i} C$ is the area and ${ }^{i} u=1$; ii) bending moments , if ${ }^{i} C$ is the moment of inertia ${ }^{i} u$ is the respective main direction $y$ or $z$; iii) bi-moment, if ${ }^{i} C$ is the warping constant and ${ }^{i} u$ is the sectorial area.

And the evaluation of ${ }^{i} \sigma_{x}^{P}(x, s)$ leads to longitudinal stress of the generalized plate's bending moment:

${ }^{i} \sigma_{x}^{P}(x, s, t)=\frac{E}{1-\mu}\left[{ }^{i} \varepsilon_{x}^{P}(x, s, t)+\mu^{i} \varepsilon_{s}^{P}(x, s, t)\right]=\frac{-E t}{1-\mu}\left[{ }^{i} w(s)^{i} V_{, x x}(x)+\mu^{i} w_{, s s}(s)^{i} V(x)\right]$

The generalized internal shear forces have a similar evaluation. This internal force is a generalization of the torsion moment, which is indicated here as $M_{T x}$. To obtain it, it is necessary to apply the inner product of the shear stress, eq. 2.28 and eq. 2.29, and the respective transverse displacement $v(s, t)$ :

$$
\begin{array}{r}
\left.{ }^{i} M_{T x}(x)=\int_{A} \tau_{x s}(x, s, t)\right)^{i} v(s, t) \mathrm{d} A=\int_{A}\left(\tau_{x s}^{M}(x, s)+\tau_{x s}^{P}(x, s, t)\right)\left({ }^{i} v^{M c}(s)-2 t^{i} w_{, s}(s)\right) \mathrm{d} A= \\
=G \int_{A}\left({ }^{i} v^{M c}(s)-2 t^{i} w_{, s}(s)\right)^{2} \mathrm{~d} A^{i} V_{, x}(x)
\end{array}
$$

Since the membrane and plate parts are orthogonal between themselves, each term inside of the above integral leads directly to the generic shear inertia due to the constant shear flown and plate's shear inertia, eq. 2.39 and eq. 2.40, respectively. Hence, one describes the generalized internal torsion moment as:

$$
{ }^{i} M_{T x}(x)={ }^{i} M_{T x}^{M c}(x)+{ }^{i} M_{T x}^{P}(x)=G^{i} D^{M c}{ }^{i} V_{, x}(x)+G^{i} D^{P i} V_{, x}(x)
$$

Similar to the longitudinal stress, one recovers the shear stress from the generalized torsion moment based on i) the superposition of shear due to constant shear flow in membrane and plate's behavior; ii) the constitutive relationship:

$$
{ }^{i} \tau_{x s}(x, s, t)={ }^{i} \tau_{x s}^{M c}(x)+{ }^{i} \tau_{x s}^{P}(x, s, t)
$$

The evaluation of ${ }^{i} \tau_{x s}^{M c}(x, s)$ leads to:

$$
{ }^{i} \tau_{x s}^{M c}(x)=G \gamma_{x s}^{M c}(x)=G v^{M c i} V_{, x}(x)=\frac{{ }^{i} M_{T x}^{M c}(x)}{{ }^{i} D^{M c}}{ }^{i} v^{M c}
$$

One can recognize, in the above equation, the well-known expression of shear stress in a hollow circular cross-section if ${ }^{i} v^{M c}=r$ (where $r$ is the middle-line radius).

The evaluation of the plate part leads to the expression:

$$
{ }^{i} \tau_{x s}^{P}(x, s)=G^{i} \gamma_{x s}^{P}(x, s)=G t^{i} w_{, s}(s){ }^{i} V_{, x}(x)=\frac{{ }^{i} M_{T x}^{P}(x)}{{ }^{i} D^{P}} t^{i} w_{, s}(s)
$$


In the particular case of ${ }^{i} w_{, s}(s)=1$, one achieves the shear stress due to torsion moment in thin-walled beams.

Unfortunately, no traditional beam theory studies the transverse cross-section bending. Consequently, the generalized internal force of transverse bending, $M_{s}(x)$, does not have any direct connection to a well-known expression. Nevertheless, this unique internal force is evaluated here for further applications:

$$
{ }^{i} M_{s}(x)=\int_{A} \sigma_{s}^{P}(x, s, t) t^{i} w_{, s s}(s) \mathrm{d} A=\frac{E}{1-\mu} \int_{A}\left[\mu^{i} \varepsilon_{x}^{P}(x, s, t)+{ }^{i} \varepsilon_{s}^{P}(x, s, t)\right] t^{i} w_{, s s}(s) \mathrm{d} A
$$

With the definition of longitudinal and transverse strains, given in equations 2.27 and 2.30, the evaluation of the above expression reaches the representation of the transverse bending moment only as a function along the beam's longitudinal axis:

$$
\begin{array}{r}
{ }^{i} M_{s}(x)=\frac{E t_{h}^{3}}{12(1-\mu)}\left[\int \mu_{s} \mu^{i} w(s)^{i} w_{, s s}(s) \mathrm{d} s^{i} V_{, x x}(x)+\int_{s}{ }_{s} w_{, s s}^{2}(s) \mathrm{d} s^{i} V(x)\right]= \\
=K\left[\mu^{i} D^{\mu i} V_{, x x}(x)+{ }^{i} B^{i} V(x)\right]
\end{array}
$$

As mentioned before, in subsection 2.1.2, the shear stress analysis of GBT's modes, which has no shear strain deformation, requires the Grashof's method. Thus, the local longitudinal equilibrium of a cross-section's segment obtains the shear stress distribution, as shown in figure 2.5:

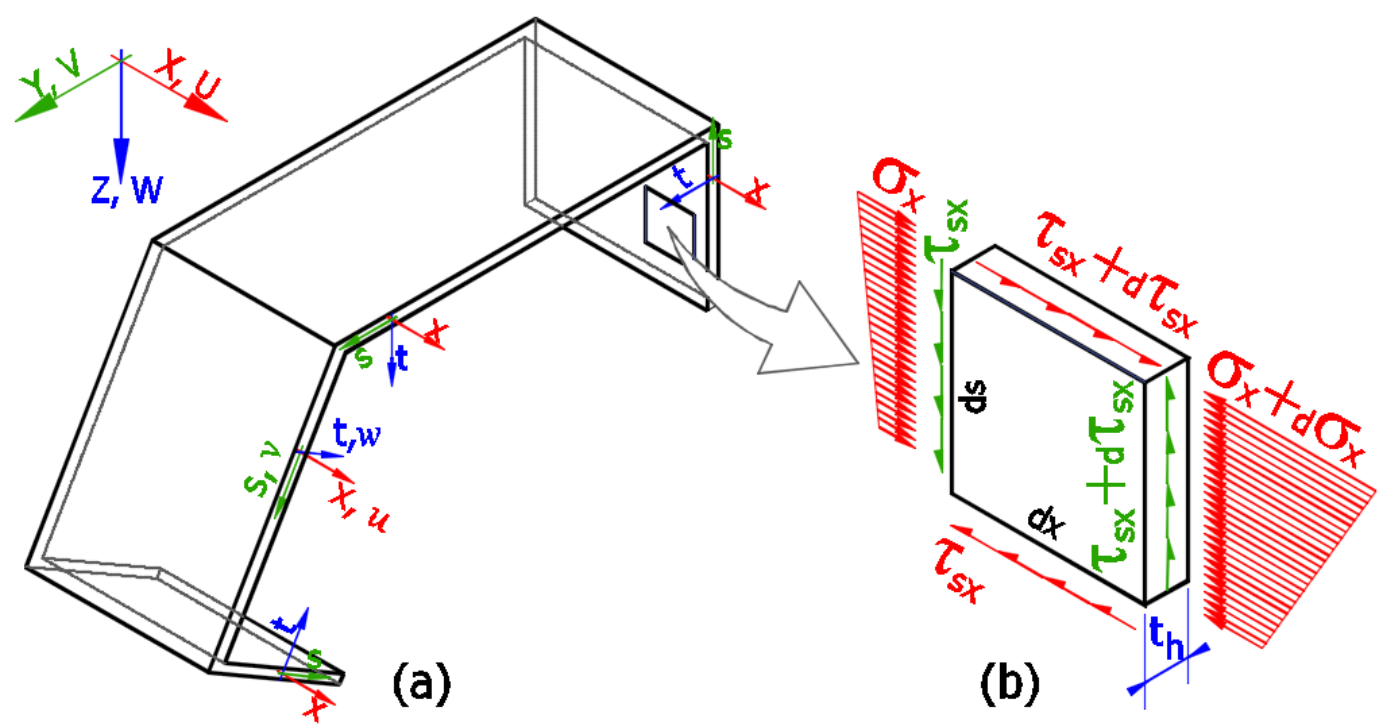

Figure 2.5: Local longitudinal equilibrium of a cross-section segment.

The longitudinal equilibrium in figure 2.5.b leads to:

$$
\mathrm{d} \sigma_{x}^{M} \mathrm{~d} s=\mathrm{d} \tau_{x s}^{M} \mathrm{~d} x
$$


Since the longitudinal stress, $\sigma_{x}$, is described as a linear combination of orthogonal modes, the shear stress can follow it as well. Thus, the introduction of eq. 2.74 in eq. 2.83 leads to:

$$
{ }^{i} \tau_{x s, s}^{M}(x, s)=\frac{{ }^{i} W_{, x}(x)}{{ }^{i} C} u(s)
$$

Where ${ }^{i} W_{, x}(x)$ is the generalized internal shear force. The integration of the above expression achieves the GBT's general definition of the membrane's shear stress:

$$
{ }^{i} \tau_{x s}^{M}(x, s)=\frac{{ }^{i} W_{, x}(x)}{{ }^{i} C} \int_{s i}^{s f}{ }^{i} u(s) \mathrm{d} s+{ }^{i} \tau_{x s}^{M c}(x)
$$

In this expression, the constant shear stress, ${ }^{i} \tau_{x s}^{M c}(x)$, has been already analyzed in the eq 2.79. Also, the integral over the segment's domain is nothing more than the generalized first moment of inertia, named here as ${ }^{i} M S$ :

$$
{ }^{i} M S=\int_{s i}^{s f} u(s) \mathrm{d} s
$$

Until this point, this study presents the variational formulation and the analysis of displacement and stress fields in GBT. However, the functions to describe the orthogonal deformation mode shapes are still open. The approach to obtain these functions is one of the main characteristics of GBT. The next two chapters develop the cases of circular hollow and segmented cross-sections, respectively. 
This chapter has the hollow circular cross-section as a first example in GBT. Since this cross-section leads directly to an orthogonal solution, one avoids the long approach of cross-section analysis, which is typical of segmented profiles. Therefore, one can keep the focus on the general ideas of GBT.

Initially, this chapter studies and reviews the state-of-art in hollow circular cross-section according to GBT. Also, it emphasizes the representation of deformation shapes as a Fourier-Series [136, 148]. Thus, GBT quickly and clearly describes the mechanical behaviors of ovalization and warping.

One finds in this chapter the analysis of displacement and stress fields. The transverse displacement receives a minor contribution, which includes the Poisson's effect on membrane behavior. Furthermore, this chapter demonstrates that the transversal bending moment, $M_{\theta}(x, \theta)$ presented in the original work of Richardt Schardt [136], requires an adjustment.

\subsection{GBT's analysis of thin-walled hollow circular cross-section}

This particular cross-section, which has a wide range of application (from pipe-lines systems to structural frames[18]), can directly illustrate the behaviors and characteristics of the generalized cross-section properties. In fact, due to axisymmetric property, the hollow circular cross-section can avoid the quadratic eigenvalue problem of GBT, as well as all the steps to reach this problem[136, 146].

The analysis of this cross-section starts in the study of the displacement field that highlights the relationships among displacements in each direction. 


\subsubsection{GBT's displacement field for thin-walled hollow circular cross- section}

To develop the displacement field for thin-walled hollow circular cross-section, some changes concerning the generalized strain and displacements, presented in section 2.1.1, are necessary as the polar coordinates are now required.

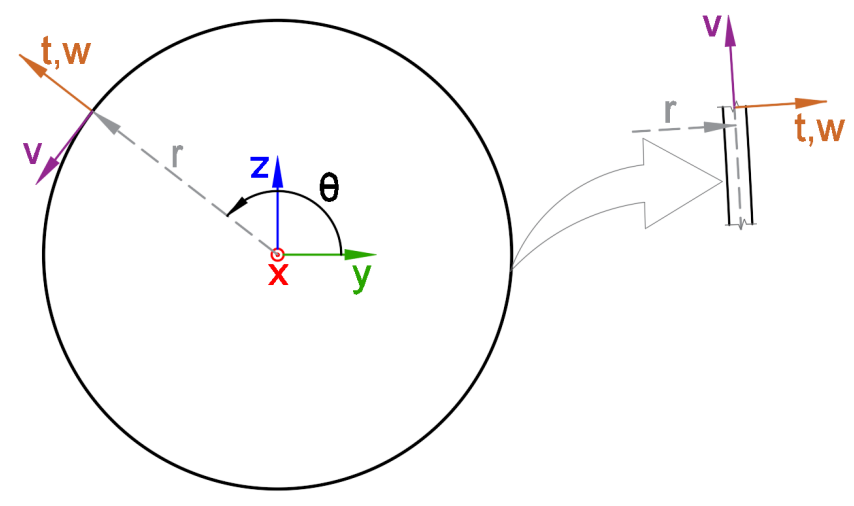

Figure 3.1: Coordinate system in a thin-walled hollow circular cross-section

The hollow circular cross-section, presented in figure 2.1 and 3.1, has the same relations of longitudinal displacement and strains as the segmented cross-section. I.e., the equations $2.15,2.18$ and 2.27 are still the same, only with the changes from variables $s$ to $\theta$ :

$$
\begin{gathered}
u(x, \theta, t)=u^{M}(x, \theta)+u^{P}(x, \theta, t)=[u(\theta)-t w(\theta)] V_{, x}(x) \\
u(x, \theta)=\sum_{i=1}^{n}{ }^{i} u(\theta){ }^{i} V_{, x}(x) \\
\varepsilon_{x}(x, \theta, t)=[u(\theta)-t w(\theta)] V_{, x x}(x)
\end{gathered}
$$

Moreover, the radial displacement, $w(x, \theta)$, keeps the same property as shown in eq. 2.20.

$$
w(x, \theta)=\sum_{i=1}^{n}{ }^{i} w(\theta){ }^{i} V(x)
$$

The significant difference in hollow circular cross-section is concerning the way to obtain the tangent and angular displacements, as well as the angular strain. It is convenient to divide the development of these displacements and strains in three steps:

The first step is the evaluation of tangent displacement, which has two components: i) the membrane middle line term; ii) the plate term:

$$
v(x, \theta, t)=v^{M}(x, \theta, t)+v^{P}(x, \theta, t)=\frac{r+t}{r} v^{\star}(x, \theta)-\frac{t}{r} w_{, \theta}(x, \theta)
$$


Here, $v^{\star}(x, \theta)$ represents the middle line elongation in tangent direction. This function is described in GBT's manner, as a summation of orthogonal modes:

$$
v^{\star}(x, \theta)=\sum_{i=1}^{n}{ }^{i} v^{\star}(\theta){ }^{i} V(x)
$$

The second step is the evaluation of angular strain, that in polar coordinates $\varepsilon_{\theta}$ is obtained not by the variation of perimeter's length, $v(x, \theta, t)$, but from the variation of the angle that described it, defined here as $\vartheta(x, \theta, t)$. Take the assumption that $\vartheta(x, \theta, t)$ is always a small angle, as shown in figure 3.2.a, one can consider its perimeter as the tangent displacement:

$$
\vartheta(x, \theta, t)=\frac{v(x, \theta, t)}{r+t}
$$

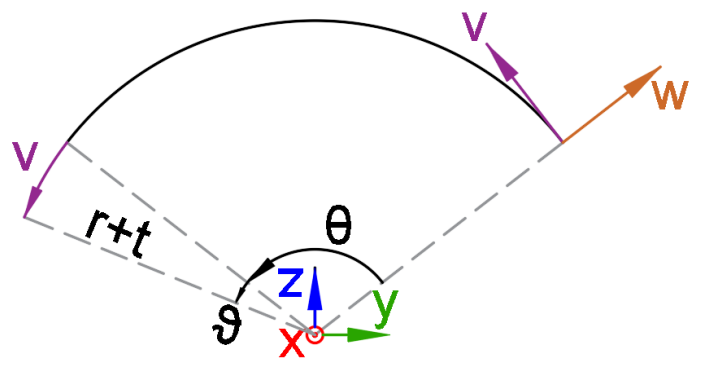

(a)

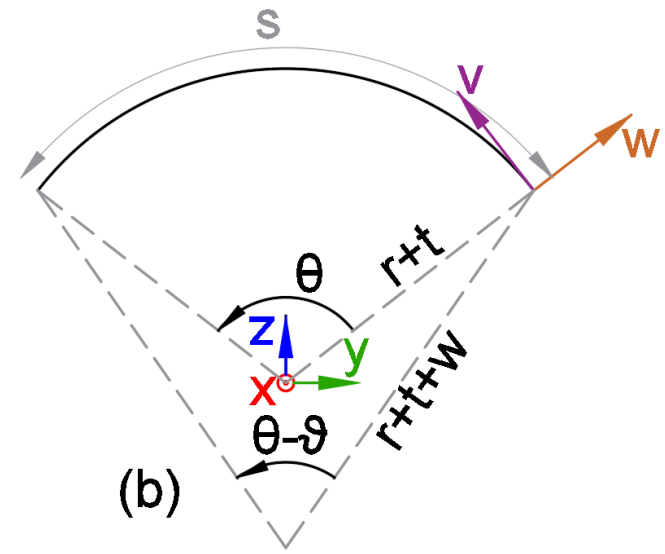

Figure 3.2: The angle of tangent displacement $\vartheta$ : (a) relationship according to the angle $\theta$ and a constant angle; (b) relationship according to the radius $r+t$ and a constant angle

However, it is important to note that $\vartheta(x, \theta, t)$ not only happens if there is a changing in the perimeter displacement, $v(x, \theta)$, but it also occurs in the case without a perimeter displacement and a changing of the radius, due to radial displacement $w(x, \theta)$. As shown in figure 3.2.b, a constant perimeter can lead to the following relationship:

$$
(r+t) \theta=(r+t+w(x, \theta))(\theta-\vartheta(x, \theta, t))
$$

which leads to:

$$
\vartheta(x, \theta, t)=\theta\left(\frac{w(x, \theta)}{r+t+w(x, \theta)}\right)
$$

With the assumption that the radial displacement, $w(x, \theta)$, is much smaller than the radial position $r+t$, one simplifies the above expression. Hence, one achieves the general definition of the angular description of the tangent displacement by the sum of the above expression with eq. 3.7:

$$
\vartheta(x, \theta, t)=\frac{1}{r+t}[v(x, \theta)+\theta(w(x, \theta))]
$$


The third step is the evaluation of the angular strain itself. Following the definition of the angular strain, $\varepsilon_{\theta}(x, \theta, t)=\vartheta_{, \theta}(x, \theta, t)$, one obtains from eq. 3.10:

$$
\varepsilon_{\theta}(x, \theta, t)=\frac{1}{r+t}\left[v_{, \theta}(x, \theta)+w(x, \theta)+\theta w_{, \theta}(x, \theta)\right]
$$

Here, the last term is much smaller than the other ones. Therefore, it is possible to reach the simplified expression:

$$
\varepsilon_{\theta}(x, \theta, t)=\frac{1}{r+t}\left[v_{, \theta}(x, \theta)+w(x, \theta)\right]
$$

Introducing eq. 3.5 in this expression, one obtains:

$$
\varepsilon_{\theta}(x, \theta, t)=\frac{1}{r}\left[v_{, \theta}^{\star}(x, \theta)-\frac{t}{r} \frac{r}{r+t} w, \theta \theta(x, \theta)+\frac{r}{r+t} w(x, \theta)\right]
$$

A Taylor Series over the variable $t$, truncated at the second term, leads to the following expression [102]:

$$
\frac{r}{r+t} \approx 1-\frac{t}{r}
$$

Therefore, eq. 3.5 becomes:

$$
\varepsilon_{\theta}(x, \theta, t)=\frac{v_{, \theta}^{\star}(x, \theta)+w(x, \theta)}{r}-t \frac{w(x, \theta)+w_{, \theta \theta}(x, \theta)}{r^{2}}+t^{2} \frac{w_{, \theta \theta}(x, \theta)}{r^{3}}
$$

Since in thin-walled structures the ratio $t^{2} / r^{3}$ is tiny when compared with the other terms, it is possible to neglect the last term in the above expression. Thus, it stands out that the first and second terms in eq. 3.15 are the strains related to membrane and plate behavior, respectively. Following the GBT's assumption of no membrane elongation, one achieves the below constraint relationship between the tangent and radial displacements:

$$
v_{, \theta}^{\star}(x, \theta)=-w(x, \theta)
$$

And the final expression of angular strain is:

$$
\varepsilon_{\theta}(x, \theta, t)=-t \frac{w(x, \theta)+w_{, \theta \theta}(x, \theta)}{r^{2}}
$$

Concerning the shear strains, its assumptions remain unchanged from the case of segmented cross-sections: i) the membrane behavior has shear strains only due to constant shear flow, ii) the plate behavior has the consideration of its fully shear strains.

To analyze the consequence of these assumptions, one considers initially the generic definition of shear strain, in eq. 2.3 , but now in polar coordinates:

$$
\gamma_{x \theta}(x, \theta, t)=\frac{u_{, \theta}(x, \theta, t)}{r+t}+v_{, x}(x, \theta, t)
$$

Introducing equations 3.1 and 3.5 into above expression, one obtains:

$$
\gamma_{x \theta}(x, \theta, t)=\frac{r}{r+t} \frac{u_{, \theta}(x, \theta)-t w_{, x \theta}(x, \theta)}{r}+\frac{r+t}{r} v_{, x}^{\star}(x, \theta)-\frac{t}{r} w_{, x \theta}(x, \theta)
$$


And the Taylor Series simplification, given in eq. 3.14, leads to:

$$
\begin{aligned}
\gamma_{x \theta}(x, \theta, t)=\frac{u_{, \theta}(x, \theta)+r v_{, x}^{\star}(x, \theta)}{r}-t \frac{u_{, \theta}(x, \theta)-r v_{, x}^{\star}(x, \theta)+2 r w_{, x \theta}(x, \theta)}{r^{2}} & +t^{2} \frac{w_{, x \theta}(x, \theta)}{r^{2}}
\end{aligned}
$$

Similar to angular strain, $\varepsilon_{\theta}$, the third term in the expression above is tiny when compared to the other two. Also, one can recognize that the first and second terms are the shear strain due to membrane and plate behaviors, respectively. Here, these two types of shear strains are studied separately, since they are complementary to each other.

For the constant shear flow in membrane behavior, there is only a constant function, $v^{M c}$, to describe the tangential displacement. In fact, the Bredt's shear flow assumption requires an inexistent warping function, ${ }^{i} u(s)$, and no transverse displacement ${ }^{i} w(s)$.

$$
\gamma_{x \theta}^{M c}(x)=v^{M c}(x)+\frac{t}{r} v^{M c}(x)
$$

The assumption of inexistent of membrane's shear strain isolates the plate's shear part. Thus, one can extract the relationship:

$$
u_{, \theta}(x, \theta)=-r v_{, x}^{\star}(x, \theta)
$$

And the final expression of plate's shear strain is:

$$
\gamma_{x \theta}^{P}(x, \theta, t)=-2 t \frac{w_{, x \theta}(x, \theta)-v_{, x}^{\star}(x, \theta)}{r}
$$

It is important to highlight two points: the first one concerns the orthogonality between shear strains from membrane and plate behaviors. These strains are already orthogonal and complementary in their assumptions and definitions; the second one is how the expressions in equations 3.16 and 3.22 together with the separation of variables, resume the kinematic assumptions of GBT in a circular hollow cross-section:

$$
v^{\star}(\theta)=-\frac{u_{, \theta}(\theta)}{r}
$$

$$
w(\theta)=\frac{u_{, \theta \theta}(\theta)}{r}
$$

To obtain the cross-section properties of a hollow circular, it is necessary to evaluate once more the variation of internal energy, eq. 2.26, with the definition of strains as 
expressed in equations $3.3,3.20$ and 3.23 :

$$
\begin{aligned}
& \delta U_{\text {int }}=\sum_{i=1}^{n} \int_{V}\left[E^{i} u^{2}(\theta)+\frac{E}{1-\mu}\left(t^{i} w(\theta)\right)^{2}\right]{ }^{i} V_{, x x}(x) \delta^{i} V_{, x x}(x) \mathrm{d} V+ \\
&+\int_{V} \frac{\mu E}{1-\mu} t^{2} \frac{{ }^{i} w(\theta)+{ }^{i} w_{, \theta \theta}(\theta)}{r^{2}} w(\theta) V(x) \delta V_{, x x}(x) \mathrm{d} V+ \\
&+G \int_{V}\left[\left(v^{c}+\frac{t}{r} v^{c}\right)^{2}+\left(2 t \frac{{ }^{i} w_{\theta}(\theta)-{ }^{i} v^{\star}(\theta)}{r}\right)^{2}\right]{ }^{i} V_{, x}(x) \delta^{i} V_{, x}(x) \mathrm{d} V+ \\
&+\frac{E}{1-\mu^{2}} \int_{V}\left(t \frac{{ }^{i} w(\theta)+{ }^{i} w_{, \theta \theta}(\theta)}{r^{2}}\right)^{2}{ }^{i} V(x) \delta^{i} V(x) \mathrm{d} V+ \\
&+\int_{V} \frac{\mu E}{1-\mu} t^{2} \frac{{ }^{i} w(\theta)+{ }^{i} w_{, \theta \theta}(\theta)}{r^{2}} w(\theta) \delta V(x) V_{, x x}(x) \mathrm{d} V
\end{aligned}
$$

From the first above integral, one obtains the generalized moment of inertia, which has two parts: membrane and plate. They are the same expressions as eq. 2.37 and eq. 2.38 , but in polar coordinates:

$$
\begin{aligned}
{ }^{i} C^{M} & =r t_{h} \oint{ }^{i} u^{2}(\theta) \mathrm{d} \theta \\
{ }^{i} C^{P} & =r \oint{ }^{i} w^{2}(\theta) \mathrm{d} \theta
\end{aligned}
$$

The fourth integral is related to the generic inertia of transverse bending:

$$
{ }^{i} B=r \oint\left(\frac{{ }^{i} w(\theta)+{ }^{i} w_{, \theta \theta}(\theta)}{r^{2}}\right)^{2} \mathrm{~d} \theta
$$

The first and second terms in the third integral are the generic inertia of shear, due to constant shear flow in the wall's thickness and the plate's shear inertia, respectively:

$$
\begin{gathered}
{ }^{i} D^{M c}=t_{h}\left(r+\frac{t_{h}^{2}}{3 r}\right) \oint\left({ }^{i} v^{M c}\right)^{2} \mathrm{~d} \theta \\
{ }^{i} D^{P}=\frac{r}{3} t_{h} \oint \oint\left(\frac{{ }^{i} w_{, \theta}(\theta)-{ }^{i} v^{\star}(\theta)}{r}\right)^{2} \mathrm{~d} \theta
\end{gathered}
$$

Lastly, the second - or the fifth - integral provides the generic inertia of shear due to the Poison effect of coupling longitudinal and transverse strains:

$$
{ }^{i} D^{\mu}=r \oint \frac{{ }^{i} w(\theta)+{ }^{i} w_{, \theta \theta}(\theta)}{r^{2}} w(\theta) \mathrm{d} \theta
$$

These expressions of the generic properties of the hollow circular cross-section are the key to obtain the displacement functions. Together with equations 3.24 and 3.25, they 
lead to the orthogonal conditions for the function ${ }^{i} u(\theta)$ and its derivation until the fourth order:

$$
\oint^{i} u(\theta){ }^{j} u(\theta) \mathrm{d} \theta=0 \text { for } i \neq j
$$

And equations 3.29 and 3.31 give the additional orthogonal condition:

$$
\begin{array}{r}
\oint\left({ }^{i} u_{, \theta \theta}(\theta)+{ }^{i} u_{, \theta \theta \theta \theta}(\theta)\right)\left({ }^{j} u_{, \theta \theta}(\theta)+{ }^{j} u_{, \theta \theta \theta \theta}(\theta)\right) \mathrm{d} \theta=0 \text { for } i \neq j \\
\oint\left({ }^{i} u_{, \theta \theta \theta}(\theta)+{ }^{i} u_{, \theta}(\theta)\right)\left({ }^{j} u_{, \theta \theta \theta}(\theta)+{ }^{j} u_{, \theta}(\theta)\right) \mathrm{d} \theta=0 \text { for } i \neq j
\end{array}
$$

A direct solution leads to trigonometric functions: $\sin (m \theta)$ and $\cos (m \theta)$, where $m$ is a natural number. The GBT's deformation modes in thin-walled circular hollow crosssection are, in fact, a decomposition of the displacement field in a Fourier-Series. Also, it is important to note that none of these deformations modes have a constant shear flow in membrane component.

To maintain the consistency of a right-hand coordinate system, as shown in figure. 3.1, the cosine terms receive a negative product. The table below resumes the deformation shape modes:

Table 3.1: Orthogonal deformation shape modes of circular hollow cross-section according to GBT

\begin{tabular}{ccccc}
\hline $\mathrm{i}$ & $\mathrm{m}$ & ${ }^{i} u$ & ${ }^{i} v^{\star}$ & ${ }^{i} w$ \\
\hline 1 & 0 & 1 & 0 & 0 \\
2 & 1 & $r \sin (\theta)$ & $-\cos (\theta)$ & $-\sin (\theta)$ \\
3 & 1 & $-r \cos (\theta)$ & $-\sin (\theta)$ & $\cos (\theta)$ \\
4 & 2 & $r \sin (2 \theta)$ & $-2 \cos (2 \theta)$ & $-2^{2} \sin (2 \theta)$ \\
5 & 2 & $-r \cos (2 \theta)$ & $-2 \sin (2 \theta)$ & $2^{2} \cos (2 \theta)$ \\
$\vdots$ & $\vdots$ & $\vdots$ & $\vdots$ & $\vdots$ \\
$2 \mathrm{~m}$ & $\mathrm{~m}$ & $r \sin (m \theta)$ & $-m \cos (m \theta)$ & $-m^{2} \sin (m \theta)$ \\
$2 \mathrm{~m}+1$ & $\mathrm{~m}$ & $-r \cos (m \theta)$ & $-m \sin (m \theta)$ & $m^{2} \cos (m \theta)$ \\
\hline
\end{tabular}

Below, some deformation shape modes are plotted to emphasize the outcomes of GBT concerning physical meaning: i) the lower modes are the traditional elongation and Euler-Bernoulli beam behaviors; ii) the high modes represent cross-section's ovalizations: Here, it should be noted that the some modal shapes obtained in GBT, such as $i=4,5,8$, or 9 , are similar to the von Karman analysis and its application in elbow elements $[20,166]$. Here, GBT can be realized as an extension and generalization of von Karman concepts [177]. Also, one can observe, from the deformation shape modes in figure 3.3, that not only the case of uniform torsion is absent, but also the case of the uniform transverse elongation. These modes are not presented directly in Richard Schardt's original work [136]. However, Nuno Silvestre presents this extension [36, 147] as a natural sequence, with some particular observations. 


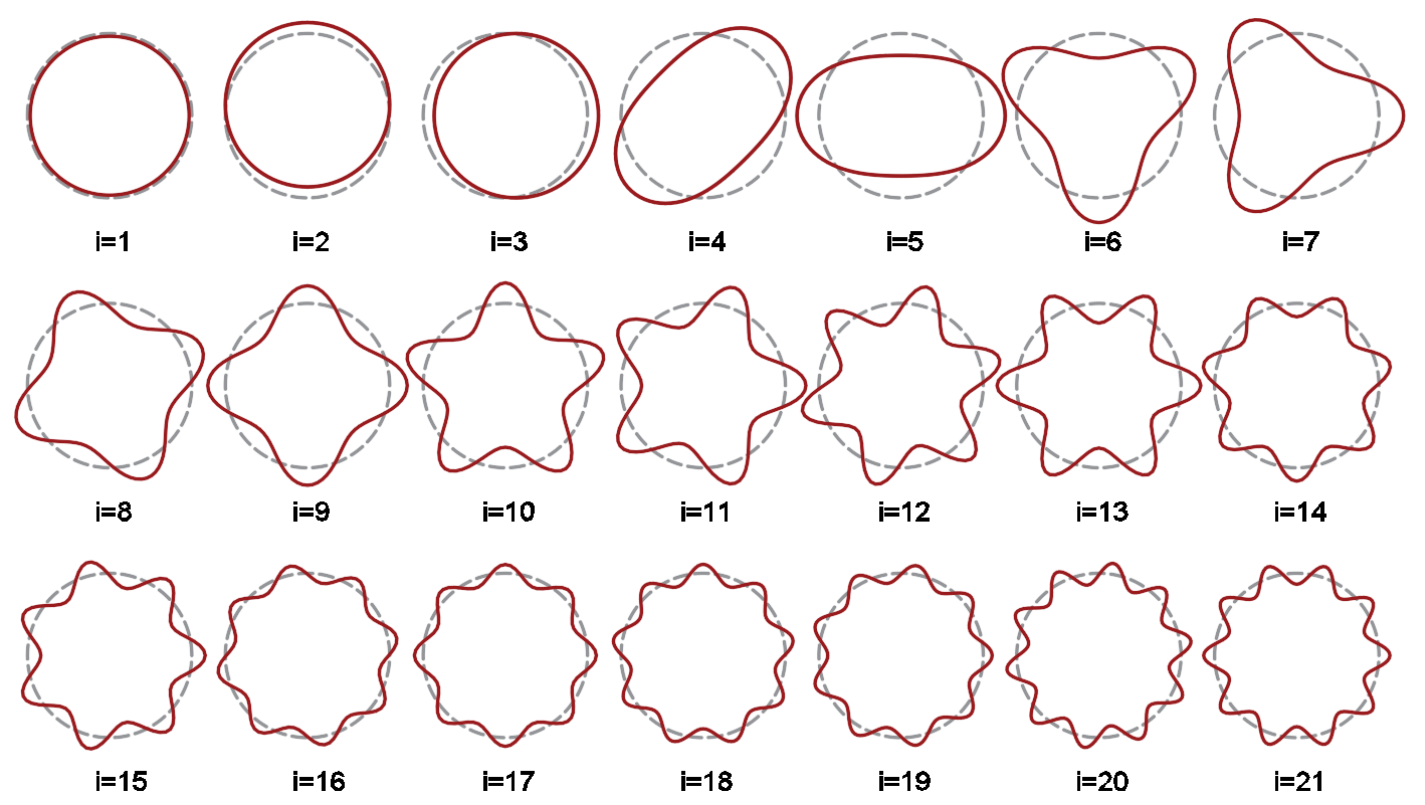

Figure 3.3: transverse deformation shape modes of a thin-walled circular hollow section according to GBT.

The uniform torsion mode leads directly to Bredt's constant shear flow, which is free of warping. As already mentioned, this deformation mode have neither longitudinal, $u(\theta)$, nor radial, $w(\theta)$, displacements. The tangent displacement must be a constant and, according to Bredt's torsion hypothesis, must also be in proportion of radius: ${ }^{t} v^{M c}=r$. Here, the superscript t represent this particular mode.

Moreover, this case waives the hypothesis of absence of shear deformation in the middleline. Therefore, the membrane's shear strain expression, eq. 3.21, leads to:

$$
{ }^{t} \gamma_{x \theta}^{M c}(x, t)=\left(r+t_{h}\right){ }_{h}^{t} V_{, x}(x)
$$

So, the shear inertia from constant shear flow, eq. 3.30, leads to the well-known torsional moment of inertia of a hollow circular:

$$
{ }^{t} D^{M c}=2 \pi r^{3} t+\pi \frac{r}{6} t_{h}^{3}
$$

Usually, due to the small dimension of the thickness, $t_{h}$, the second term is neglected.

The case of uniform transverse elongation requires another exception. This deformation shape mode violates the assumption of absence in membrane angular strain, $\varepsilon_{\theta}$. Nevertheless, similar to the case of uniform torsion, the simplified constitutive relationship for membrane, given in eq. 2.10, avoid any coupling between this mode and others, especially the uniform axial elongation mode, $i=1$. As a result, one includes the uniform transverse elongation in GBT formulation without further modifications.

The table below presents this deformation shape mode function, as well as the case of uniform torsion. Here, the superscript a represents the uniform axial elongation: 
Table 3.2: Additional orthogonal deformation shape modes of circular hollow crosssection: a - uniform axial elongation; $\mathrm{t}$ - uniform torsion

\begin{tabular}{cccc}
\hline $\mathrm{i}$ & ${ }^{i} u$ & ${ }^{i} v^{\star}$ & ${ }^{i} w$ \\
\hline $\mathrm{a}$ & 0 & 0 & 1 \\
$\mathrm{t}$ & 0 & $\mathrm{r}$ & 0 \\
\hline
\end{tabular}

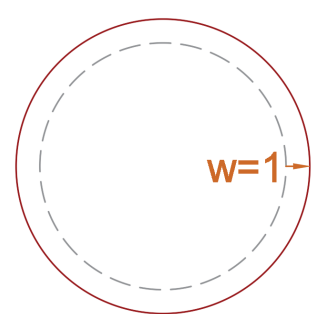

$\mathrm{i}=\mathrm{a}$

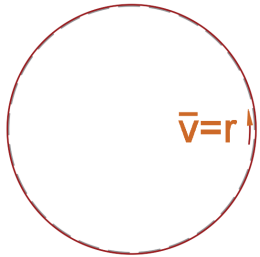

$\mathrm{i}=\mathrm{t}$

Figure 3.4: transverse deformation of the additional modes of uniform radial elongation and torsion.

The radial uniform deformation shape functions can be introduced directly in equations $3.27,3.28,3.29,3.30,3.31$ and 3.32 with no extra effort.

As a resume, one can obtain the following generic cross-section properties for the hollow circular:

$$
\begin{aligned}
& { }^{i} C^{M}=\left\{\begin{array}{l}
0 \text { for } i=t \\
0 \text { for } i=a \\
2 \pi r t_{h} \text { for } i=1 \\
\pi t_{h} r^{3} \text { for } i>1
\end{array}\right. \\
& { }^{i} C^{P}=\left\{\begin{array}{l}
0 \text { for } i=t \\
2 \pi r \text { for } i=a \\
0 \text { for } i=1 \\
\pi r m^{4} \text { for } i>1
\end{array}\right. \\
& { }^{i} B=\left\{\begin{array}{l}
0 \text { for } i=t \text { and } i=1 \\
2 \pi / r \text { for } i=a \\
\pi m^{4} / r^{3}\left(m^{2}-1\right)^{2} \text { for } i>1
\end{array}\right. \\
& { }^{i} D^{M c}=\left\{\begin{array}{l}
2 \pi r^{3} t_{h}+\pi r / 6 t_{h}^{3} \text { for } i=t \\
0 \text { other cases }
\end{array}\right. \\
& { }^{i} D^{P}=\left\{\begin{array}{l}
\frac{\pi}{3 r} t_{h}^{3} m^{2}\left(m^{2}-1\right)^{2} \text { for } i>1 \\
0 \text { for other cases }
\end{array}\right.
\end{aligned}
$$




$$
{ }^{i} D_{\mu}=\left\{\begin{array}{l}
\frac{\pi}{r} m^{4}\left(1-m^{2}\right) \text { for } i>1 \\
0 \text { for other cases }
\end{array}\right.
$$

\subsection{2. transverse displacement due to Poisson's effect in membrane behavior}

Although GBT does not include the Poisson's effect in membrane behavior, as presented in the simplified constitutive relationship in eq. 2.10, in the case of thin-walled hollow circular cross-section it is possible to include this effect.

According to Poisson's principle, the variation of transverse and longitudinal strains is:

$$
\mu=-\frac{\partial \varepsilon_{\theta}}{\partial \varepsilon_{x}}
$$

In hollow circular cross-section, it implies in proportion between longitudinal and radial elongations. Therefore, if one introduces the longitudinal strain, given in eq. 3.3, into the above expression, then the expression of transverse membrane strain due to Poisson's effect stands out:

$$
\varepsilon_{\theta}^{\mu}=-\mu u(\theta) V_{, x x}(x)
$$

Comparing the above expression to the membrane part of eq. 3.12 and considering the middle line of the cross-section, $t=0$, one builds the following relationship:

$$
w^{\mu}(x, \theta)=-r \mu u(\theta) V_{, x x}(x)
$$

Here, the index $\mu$ indicates the Poisson's effect origin of the displacement. One can express the above equation directly as a function of radial displacement. From eq. 3.25 and the definition of deformation modes given in table. 3.1, one obtains:

$$
w^{\mu}(x, \theta)=\mu \frac{r^{2}}{m^{2}} w(\theta) V_{, x x}(x)
$$

The contribution of this particular displacement is relevant in the regions of the beam, where it involves high longitudinal stress. The analysis of displacement in the numerical example in Chapter 5 clearly shows it.

\subsubsection{External loads in thin-walled hollow circular cross-section ac- cording to GBT}

After the evaluation of displacement field and cross-section properties of a thin-walled hollow circular, one can build a system of homogeneous ordinary differential equation. However, the majority of practical problems lead to a non-homogeneous system, i.e., it involves external loads, which need special treatment by the approach of separation of variables:

$$
\begin{aligned}
& p_{x}(x, \theta)=f_{x}(x) q_{x} \\
& p_{v}(x, \theta)=f_{v}(x) q_{v}
\end{aligned}
$$




$$
p_{w}(x, \theta)=f_{w}(x) q_{w}
$$

Furthermore, it is necessary to perform a modal decomposition, achieved by the inner product of the deformation modes (listed in tables 3.1 and 3.2) and the functions of external load:

$$
\begin{gathered}
{ }^{i} q_{x}=-r \oint q_{x}(\theta)^{i} u(\theta) \mathrm{d} \theta \\
{ }^{i} q_{v}=r \oint q_{v}(\theta)^{i} v^{\star}(\theta) \mathrm{d} \theta \\
{ }^{i} q_{w}=r \oint q_{w}(\theta){ }^{i} w(\theta) \mathrm{d} \theta
\end{gathered}
$$

These functions are the external loads for each deformation mode. They lead to the nonhomogeneous ordinary differential equation of GBT for hollow circular cross-sections. Moreover, it is interesting to observe that each expression above represents a coefficient of Fourier Series, where half of the period is $\pi[83,136]$. Therefore, one can express the external loads in equations $3.48,3.49$ and 3.50 as:

$$
\begin{gathered}
p_{x}(x, \theta)=f_{x}(x) q_{x}(\theta)=-f_{x}(x)\left[\frac{{ }^{1} q_{x}}{\pi r}+\sum_{m=1}^{n} \frac{{ }^{2 m} q_{x}}{\pi r^{3}}{ }^{2 m} u(\theta)-\frac{{ }^{2 m+1} q_{x}}{\pi r^{3}}{ }^{2 m+1} u(\theta)\right] \\
p_{v}(x, \theta)=f_{v}(x) q_{v}(\theta)=-f_{v}(x)\left[\sum_{i=2}^{n} \frac{{ }^{i} q_{v}}{\pi r m^{2}}{ }^{i} v^{\star}(\theta)\right] \\
p_{w}(x, \theta)=f_{w}(x) q_{w}(\theta)=-f_{x}(x)\left[\sum_{m=1}^{n} \frac{{ }^{2 m} q_{w}}{\pi r m^{4}}{ }^{2 m} w(\theta)-\frac{{ }^{2 m+1} q_{w}}{\pi r m^{4}}{ }^{2 m+1} w(\theta)\right]
\end{gathered}
$$

These representations of the external loads are particularly useful to obtain the internal membrane forces, as shown next.

\subsubsection{Stress field and internal forces for thin-walled hollow circular cross-section according to GBT}

Among the benefits of using GBT in thin-walled hollow circular cross-section's analysis, the extraction of internal forces and their consequent stress fields is a highlight. GBT provides a clear set of internal forces due to membrane and plate behaviors, which lead to a smooth and continuum stress field.

Since the internal forces due to membrane behavior require the internal cross-section equilibrium that involves the plate's internal forces, one must evaluate the plate's internal force initially.

Thus, the curvatures in longitudinal direction, $\kappa_{x}$, as well as the one in transverse direction, $\kappa_{\theta}$, are extracted from equations 3.3 and 3.17 , respectively. In these expressions the curvatures are the factors multiplied by the thickness dimension $t$ :

$$
{ }^{i} \kappa_{x}(x, \theta)=w(\theta) V_{, x x}(x)
$$




$$
{ }^{i} \kappa_{\theta}(x, \theta)=\frac{{ }^{i} w(\theta)+{ }^{i} w_{, \theta \theta}(\theta)}{r^{2}}{ }^{i} V(x)
$$

Base on the constitutive relationship of plate behavior, eq. 2.11 and on Kirchhoff-Love plate theory, one achieves the internal bending moment:

$$
\begin{aligned}
& { }^{i} M_{x}(x, \theta)=-K\left[{ }^{i} \kappa_{x}(x, \theta)+\mu^{i} \kappa_{\theta}(x, \theta)\right] \\
& { }^{i} M_{\theta}(x, \theta)=-K\left[\mu^{i} \kappa_{x}(x, \theta)+{ }^{i} \kappa_{\theta}(x, \theta)\right]
\end{aligned}
$$

Where $K$ is the plate stiffness, given in eq. 2.41.

It is relevant to observe that the positive and negative signals in the above expressions are due to the local coordinate system, as shown in figure. 3.1.

Furthermore, there is an important correction concerning the the internal forces of thin-walled hollow cross-section based on the original work of Richardt Schardt [136]: the transverse bending moment $M_{\theta}(x, \theta)$ has no contribution from the Poisson's effect of the longitudinal bending moment $M_{x}(x, \theta)$. This behavior is a result of the absence of transverse curvature constraints along the longitudinal direction of the beam. I.e., one can assume that the thin-walled hollow cross-section is a plane shell with support conditions only in one direction, figure 3.5.a. In this configuration, a longitudinal bending moment generates no transverse bending moment due to Poisson's effect, since there are no support conditions in the longitudinal direction. This behavior is maintained in a curved cross-section, as shown in figure 3.5.b, and it is also maintained in gradually curved cross-section until reaching the full circumference, figure 3.5.c

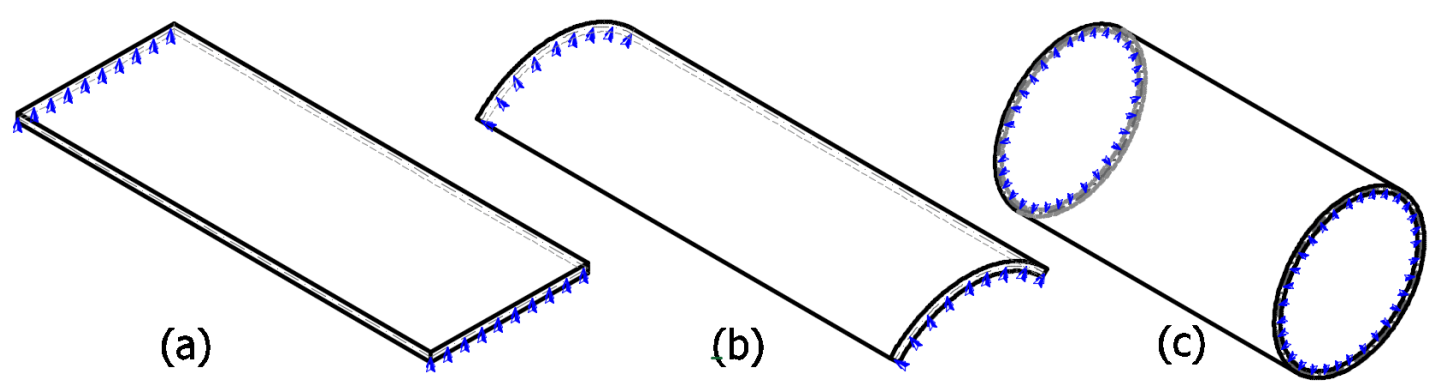

Figure 3.5: Absence of Poisson's effect from the longitudinal into the transverse bending moment: the behavior of a plane shell structure, as shown in a), is maintained in a gradually curved cross-section, as shown in b). This behavior is also maintained in a fully circular cross-section, as shown in c).

Inserting equations 3.57 and 3.58 into equations 3.59 and 3.60 respectively, together with the sum of all modes, leads to the total longitudinal and transverse internal bending moments:

$$
M_{x}(x, \theta)=-K \sum_{i=2}^{n}{ }^{i} w(\theta){ }^{i} V_{, x x}(x)+\mu \frac{{ }^{i} w(\theta)+{ }^{i}{ }_{, \theta \theta}(\theta)}{r^{2}}{ }^{i} V(x)
$$




$$
M_{\theta}(x, \theta)=-K \sum_{i=2}^{n} \frac{{ }^{i} w(\theta)+{ }^{i} w_{, \theta \theta}(\theta)}{r^{2}}{ }^{i} V(x)
$$

One obtains the internal twisting moment in a similar way. The fraction in eq. 3.23 provides the curvature of twist:

$$
{ }^{i} \kappa_{x \theta}(x, \theta)=\frac{{ }^{i} w_{, \theta}(\theta)-{ }^{i} v^{\star}(\theta)}{r}{ }^{i} V_{, x}(x)
$$

Inserting this curvature into internal twisting moment, according to Kirchhoff-Love theory:

$$
{ }^{i} M_{x \theta}(x, \theta)=-K(1-\mu){ }^{i} \kappa_{x \theta}(x, \theta)
$$

The sum in all relevant modes leads to the expression of GBT's internal twisting moment in the hollow circular's wall:

$$
M_{x \theta}(x, \theta)=-K \frac{1-\mu}{r} \sum_{i=2}^{n}\left[{ }^{i} w_{, \theta}(\theta)-{ }^{i} v^{\star}(\theta)\right]{ }^{i} V_{, x}(x)
$$

The derivatives of internal plate moments lead straight forward to internal shear forces, $Q$ :

$$
\begin{aligned}
Q_{x}(x, \theta) & =-M_{x, x}(x, \theta)-\frac{1}{r} M_{x \theta, \theta}(x, \theta) \\
Q_{\theta}(x, \theta) & =-M_{x \theta, x}(x, \theta)-\frac{1}{r} M_{\theta, \theta}(x, \theta)
\end{aligned}
$$

Therefore, by inserting equations 3.61, 3.62 and 3.65 into the above expression, one achieves the GBT's representation of internal shear forces due to plate behavior in each direction of hollow circular's wall:

$$
\begin{aligned}
& Q_{x}(x, \theta)=K \sum_{i=1}^{n}{ }^{i} w(\theta){ }^{i} V_{, x x x}(x)+\left(\mu\left({ }^{i} w(\theta)+{ }^{i} v_{, \theta}^{\star}(\theta)\right)+{ }^{i} w_{, \theta \theta}(\theta)-{ }^{i} v_{, \theta}^{\star}(\theta)\right) \frac{{ }^{i} V_{, x}(x)}{r^{2}} \\
& Q_{\theta}(x, \theta)=\frac{K}{r} \sum_{i=1}^{n}(1-\mu)\left({ }^{i} w_{, \theta}(\theta)-{ }^{i} v^{\star}(\theta)\right){ }^{i} V_{, x x}(x)+\frac{{ }^{i} w_{, \theta \theta \theta}(\theta)+{ }^{i} w_{, \theta}(\theta)}{r^{2}}{ }^{i} V(x)
\end{aligned}
$$

After the analysis of internal forces due to plate behavior, it is possible to evaluate all membrane's internal forces.

Starting from the simplest case, longitudinal internal force, one can obtain it from the membrane's longitudinal strain (given in the first term in eq. 3.3):

$$
{ }^{i} \varepsilon_{x}(x, \theta)={ }^{i} u(\theta){ }^{i} V_{, x x}(x)
$$

The integration of above expression over the membrane's thickness, together with GBT's constitutive law for membrane, eq. 2.10, and the sum of all relevant modes lead to:

$$
N_{x}(x, \theta)=E t_{h} \sum_{i=1}^{n}{ }^{i} V_{, x x}(x){ }^{i} u(\theta)
$$


Unfortunately, the other two internal forces due to membrane behavior cannot be obtained directly from the relationship between stress and strains, as used up to here. The assumptions of null transverse elongation, $\varepsilon_{\theta}=0$, and the lack of participation of shear stress in the total longitudinal equilibrium of the cross-section, invalidate any attempt to obtain the stresses and internal forces due to these two kinds of membrane's strains.

To overcome this difficulty, one can reach these two internal forces by the equilibrium of a cross-section's segment. For instance, the longitudinal equilibrium of segment, shown in the figure 3.6, leads to the membrane's shear force:

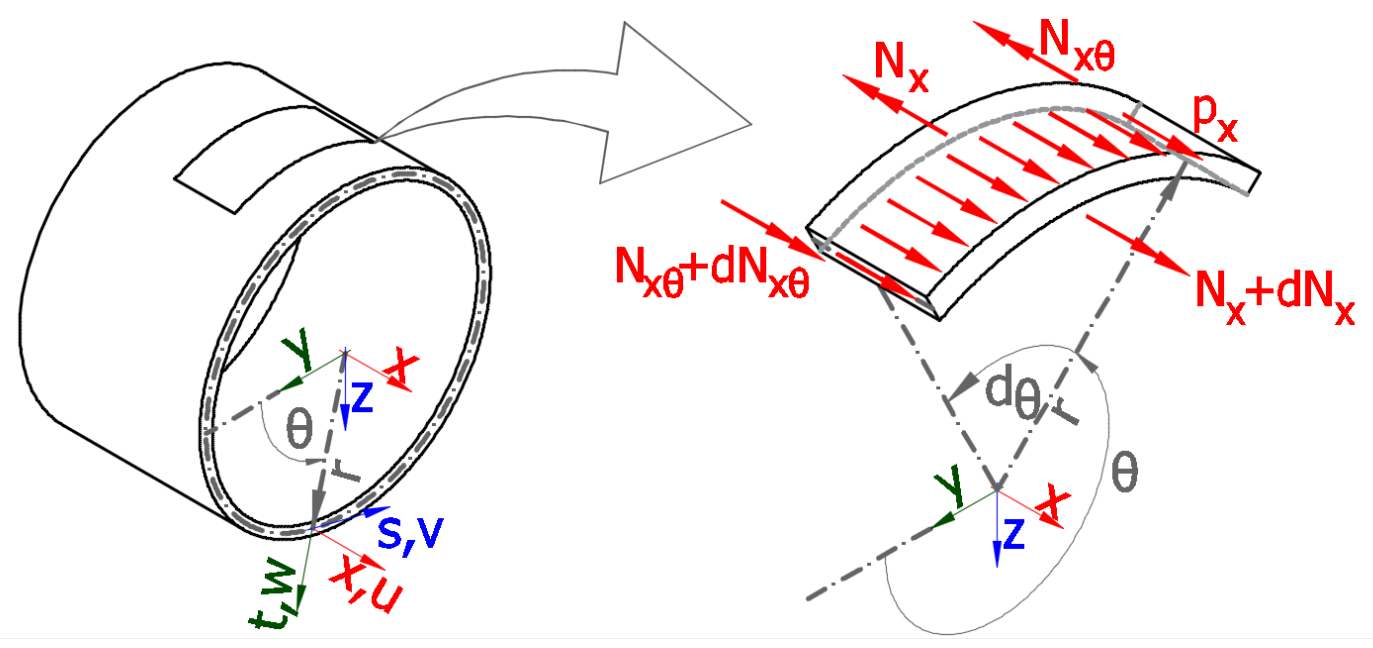

Figure 3.6: Equilibrium in a infinitesimal segment of a thin-walled circular hollow section, according to GBT.

$$
N_{x, x}(x, \theta)+\frac{1}{r} N_{x \theta, \theta}(x, \theta)+p_{x}(\theta)=0
$$

Inserting eq. 3.71 and the modal representation of the longitudinal external load, given in eq. 3.54, into the above equation, and integrating over the angular domain, lead to:

$$
\begin{aligned}
N_{\theta x}(x, \theta)={ }^{t} N_{\theta x}(x)+2 f_{x}(x){ }^{1} q_{x}+r \sum_{m=1}^{n}\left[\left(E t_{h}{ }^{2 m} V_{, x x x}(x)-f_{x}(x) \frac{{ }^{2 m} q_{x}(\theta)}{\pi r^{3}}\right) \frac{{ }^{2 m} u_{, \theta}(s)}{m^{2}}+\right. \\
\left.+\left(E t_{h}{ }^{2 m+1} V_{, x x x}(x)+f_{x}(x) \frac{{ }^{2 m+1} q_{x}(\theta)}{\pi r^{3}}\right) \frac{{ }^{2 m+1} u_{, \theta}(s)}{m^{2}}\right]
\end{aligned}
$$

In the evaluation above, the following property of trigonometric functions is used:

$$
\int{ }^{i} u(\theta) \mathrm{d} \theta=-\frac{{ }^{i} u_{, \theta}(s)}{m^{2}}
$$

Also, the constant ${ }^{t} N_{\theta x}$ is nothing more than the internal shear force due to uniform torsion mode, [147]. 
Following the same approach, one can obtain the angular internal force, $N_{\theta}(x, \theta)$, from the axial shell's equilibrium concerning the axial load $q_{w}(\theta)$, as shown in figure 3.7:

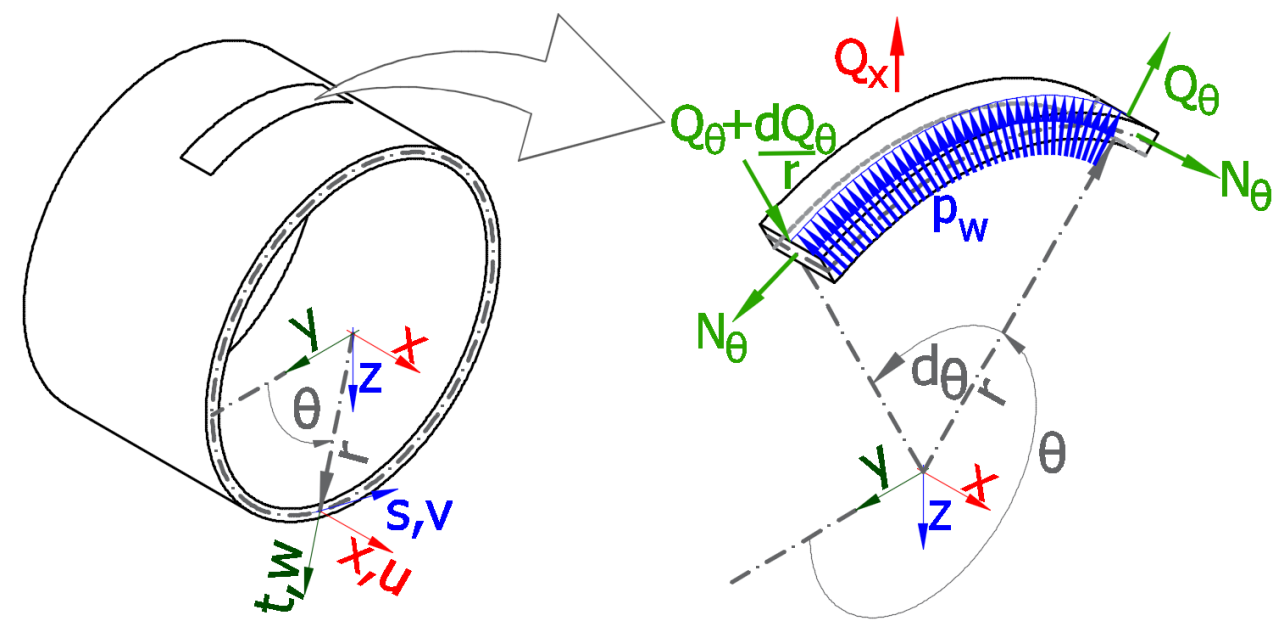

Figure 3.7: Axial shell's equilibrium in a infinitesimal segment of a thin-walled circular hollow section, according to GBT.

$$
\frac{N_{\theta}(x, \theta)}{r}+Q_{x, x}(x, \theta)+\frac{Q_{\theta, \theta}(x, \theta)}{r}-p_{w}(x, \theta)=0
$$

The evaluation of the above expression leads to:

$$
\begin{array}{r}
N_{\theta}(x, \theta)=N_{\theta}^{A}+r f_{w}(x) q_{w}(\theta)-K \sum_{i=2}^{n} r^{i} w(\theta){ }^{i} V_{, x x x x}(x)+\frac{{ }^{i} w_{, \theta \theta \theta \theta}(\theta)+{ }^{i} w_{, \theta \theta}(\theta)}{r^{3}}{ }^{i} V(x)+ \\
\left(\mu\left({ }^{i} w(\theta)-{ }^{i} w_{, \theta \theta}(\theta)\right)+2\left({ }^{i} v_{, \theta}(\theta)(\mu-1)+{ }^{i} w_{, \theta \theta}(\theta)\right)\right) \frac{{ }^{i} V_{, x x}(x)}{r}
\end{array}
$$

The first term in the above expression is the tangent force due to the radial mode, [147].

Chapter 5 presents an example of GBT's analysis of circular hollow cross-section. Also, this example discusses and identifies which modes are more relevant in a given problem of thin-walled structure analysis. 


\section{GBT's analysis of a generic segmented thin-walled cross-section}

This chapter presents, perhaps, the most relevant characteristic and arduous procedure in GBT: a generic cross-section analysis. In fact, this procedure is the major actor not only in Richard Schardt's book, [136], but it is also the main focus for many publications concerning GBT: P. Leach [104], Silvestre et al. [50], Miranda et al. [113], Ranzi et al. [127, 169], Andreassen et al. [97, 98, 99] and Bebiano et.al. [27, 29].

A common point among these works concerns the description of cross-section as a group of straight segments, whom each segment has a natural node in each extreme. Also, the segments can or cannot have intermediate nodes, known as internal nodes. Moreover, there are the branched nodes, which are natural nodes related to three segments or more. Figure 4.1 illustrates these nodes.
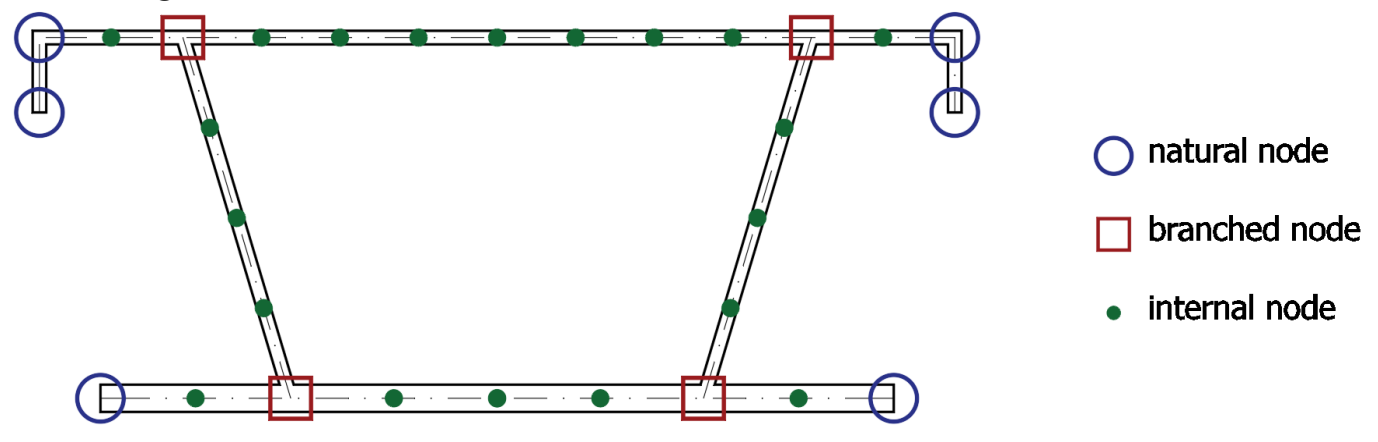

Figure 4.1: Nodal nomenclature of a generic segmented cross-section

Initially, Schardt elaborated the whole cross-section formulation based on a mixed variation of internal energy. On one hand, it uses the virtual displacement to obtain the transverse stiffness matrices due to elongation $[C]$ and shear $[D]$; on another hand, it uses the virtual internal forces, i.e., a force method, to find the transverse bending stiffness matrix, $[B]$, from the inverse of its flexible matrix. 


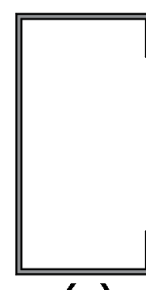

(a)

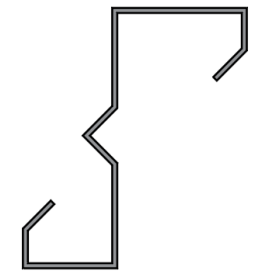

(b)

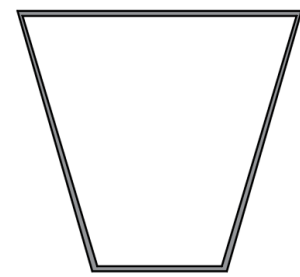

(c)

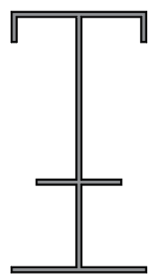

(d)

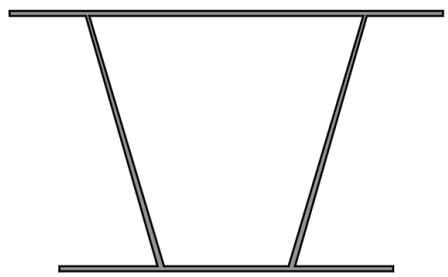

(e)

Figure 4.2: cross-section classification: a) and b) open unbranched; c) closed unbranched; d) open branched; e) closed branched

This method can solve opened and closed unbranched cross-sections, as shown in figure 4.2.a, 4.2.b and 4.2.c. These cross-section shapes have a unique feature, in which the number of natural nodes is the same as warping functions. This feature occurs if the membrane part is the only one considered in the energy of longitudinal extension. Consequently, Schardt's simplified longitudinal energy assumption leads to arbitrary and independent unit warping functions to set up the problem of cross-section analysis, as illustrated below in figure 4.3:

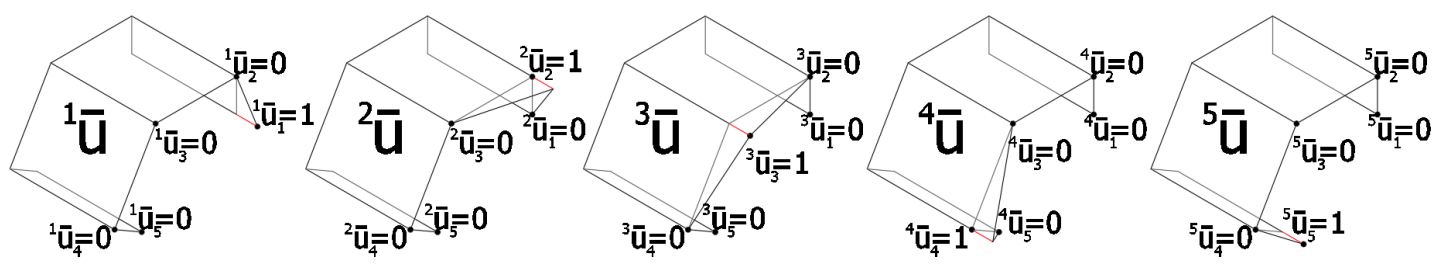

Figure 4.3: Independent unit warping functions

The unit warping functions are grouped in a nodal warping displacement matrix, $[U]$. Each line represents the longitudinal displacement of a natural node of the cross-section, whilst each column represents an orthogonal mode shape. Initially, this matrix is an identity matrix, $[I]$. The outcome from this setup is a system of non-orthogonal ordinary differential equations:

$$
[C]\left\{V_{, x x x x}\right\}-[D]\left\{V_{, x x}\right\}+[B]\{V\}=0
$$

Here, each coupled cross-section stiffness matrix, $[C],[D]$ and $[B]$, is understood on the orthonormal basis $[U]$. In order to uncouple this system, Schardt's method applies a generic linear eigenvalue problem, which brings an almost decoupled orthogonal system of ordinary differential equation:

$$
[\tilde{C}]\left\{V_{, x x x x}\right\}-[\tilde{D}]\left\{V_{, x x}\right\}+[\tilde{B}]\{V\}=0
$$

where $[\tilde{C}]$ and $[\tilde{B}]$ main diagonal matrices and $[\tilde{D}]$ is a non-diagonal matrix, in which the off-diagonal terms are usually much smaller than the diagonal terms. This condition is especially found in open cross-section, obtained from:

$$
[\tilde{C}]=[\tilde{U}]^{T}[C][\tilde{U}]
$$




$$
\begin{aligned}
& {[\tilde{D}]=[\tilde{U}]^{T}[D][\tilde{U}]} \\
& {[\tilde{B}]=[\tilde{U}]^{T}[B][\tilde{U}]}
\end{aligned}
$$

The new orthogonal base, $[\tilde{U}]$, describes at each natural node how much is the longitudinal displacement in each orthogonal deformation mode.

Unfortunately, some difficulties rise in classical Schardt's method:

- For cross-section with a relative stiff transverse stiffness matrix, $[D]$, such as closed cross-sections $[68,73,74]$, the approximation of almost decoupled system of differential equations cannot be held. Although Schardt presented an alternative approach in the orthogonalization process to minimize the coupled effect (which involves the solution of the generic linear eigenvalue problem between the matrices $[C]$ and $[D]$ ), it still presents imprecise results;

- For branched cross-sections, the classical Schardt's method requires the compatibility of transverse displacements among three (or more) segments. Thus, the hypothesis of null membrane shear deformation by the force method leads to an indeterminate system, which has more natural nodes than warping functions, [114]. Consequently, the setup of analysis cannot start from independent unit warping functions, but it is necessary to select a convenient warping function to do so, [50, 115].

To overcome the second difficulty, Dinis et al., [50], replaced the force method for the transverse bending stiffness matrix, $[B]$, with the displacement method. Although this alternative handles any branched cross-section, it still requires an initial selection of convenient warping functions, which is an undesired feature in a general and unified approach. Ranzi et al. achieved a directly approach [128], which not only unifies the analysis of different types of cross-sections, but also uses only one step in the generalized eigenvalue problem.

A relevant contribution in cross-section analysis was archived by J.M.Andreassen and J.Jönsson, [97, 98, 99], who developed a semi-discretized approach entirely based on the variation of internal strain energy. This approach not only describes any crosssection under the GBT's initial assumptions of null membrane's shear and transverse elongation, but also uses a quadratic eigenvalue solution to reach set of cross-section stiffness matrices. Moreover, this approach has the benefits to be unified, general and easy to implement in computer codes, since it is a directly extension of J.Jönsson's Finite Element Method to determine general cross-section properties [94].

Later, Gonçalves et al., [27], developed a similar, but independent, approach. Some benefits from it stand out: i) the cross-section analysis involves the additional modes of membrane's shear and transverse elongation; ii) a particular treatment is carried out for closed multi-cells cross-sections [80]. However, as a disadvantage, this procedure requires a long sequence of solutions of generic eigenvalues problems.

From the features of each approach, this dissertation presents an alternative one: a discretized cross-section analysis, based on segment element that uses the internal strain 
energy of membrane and/or plate in a systematic manner to lead to orthogonal membrane/plate modes. In other words, this alternative approach can separate the orthogonal deformation modes into three groups: i) pure membrane behavior; ii) pure plate behavior; iii) coupled membrane-plate behavior. These features, as presented later in Chapter 7, are useful in non-linear analysis.

\subsection{Cross-section semi-discretization}

The alternative approach proposed here follows the semi-discretization found in the works of Gonçalves et.al, [27, 82] and specially J.M.Andreassen and J.Jönsson - [97]. These works describe a cross-section, as shown in figure 4.1, in an element assessment. Each segment is viewed as one and each element has two nodes: the initial, $s=-l_{s} / 2$; and the final node, $s=l_{s} / 2$. Here, $l_{s}$ is the element/segment length in local coordinate svstem. as shown in figure. 4.4.

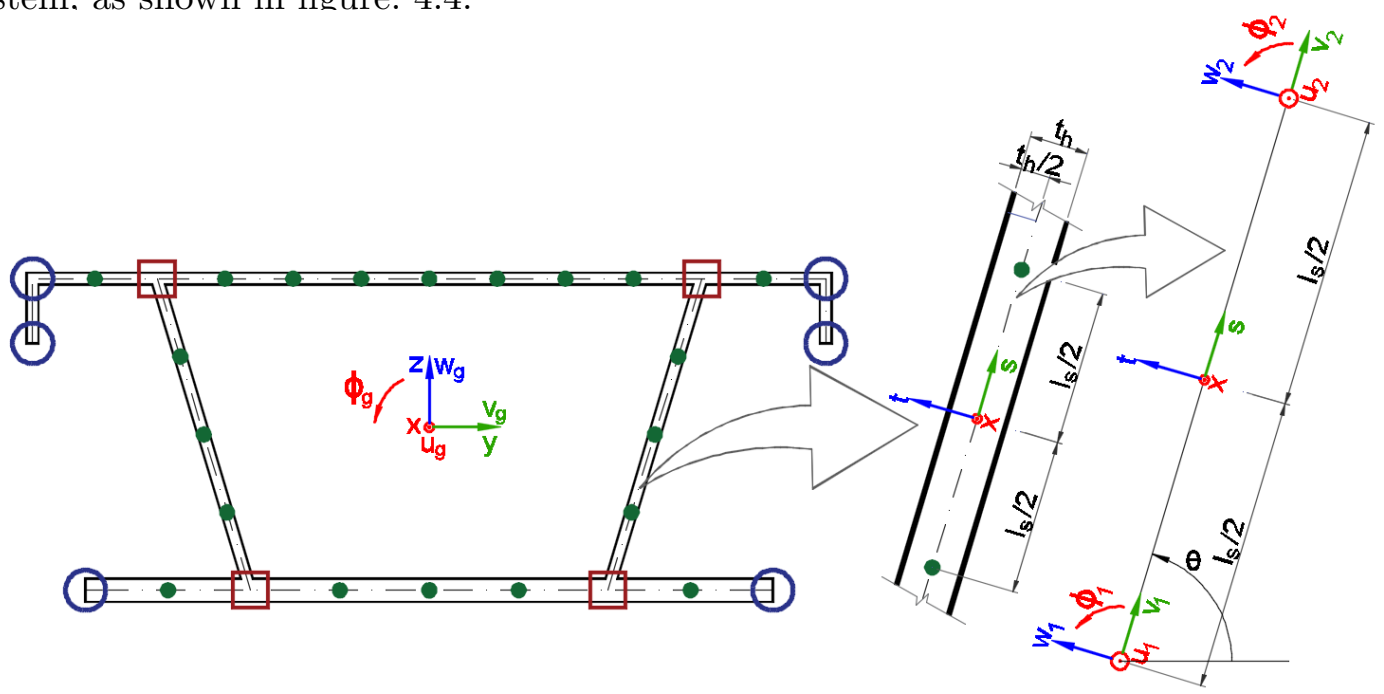

Figure 4.4: An element of a segment in the local coordinate system

Both the initial and final nodes have four degrees of freedom. Therefore, each element has eight degrees of freedom. Also, it is possible to observe that from four degrees of freedom per node, one is related to the longitudinal rotation, $\phi$, and three are related to the displacements.

It is essential to observe that, in GBT's perspective point of view, each element's degree of freedom represents a deformation mode shape. Therefore, each element could express up to eight orthogonal deformation mode shapes. However, the assumptions of no segment elongation and shear strains limit to six deformation mode shapes per element.

To evaluate a displacement at an arbitrated point inside the element, $-l_{s} / 2 \leq s \leq l_{s} / 2$, this approach uses the Hermitian shape functions to describe the displacement functions of a particular deformation mode $i$ as: ${ }^{i} u_{h}(s),{ }^{i} v_{h}(s)$ and ${ }^{i} w_{h}(s)$. Here, the subindex $h$ 
indicates the type of interpolation function.

In the cases of longitudinal and transverse displacement functions, $u_{h}(s)$ and $v_{h}(s)$ respectively, one uses linear functions with $C^{0}$ continuity between elements nodes:

$$
\begin{aligned}
& { }^{i} u_{h}(s)=\left[\begin{array}{ll}
s & 1
\end{array}\right] \frac{1}{2 l_{s}}\left[\begin{array}{cc}
-2 & 2 \\
l_{s} & l_{s}
\end{array}\right]^{i}\left[\begin{array}{l}
u_{1} \\
u_{2}
\end{array}\right]=\left[T s_{H e 1}\right]\left[S h_{H e 1 c c}\right]^{i}\left[u_{h}\right] \\
& { }^{i} v_{h}(s)=\left[\begin{array}{ll}
s & 1
\end{array}\right] \frac{1}{2 l_{s}}\left[\begin{array}{cc}
-2 & 2 \\
l_{s} & l_{s}
\end{array}\right]^{i}\left[\begin{array}{l}
v_{1} \\
v_{2}
\end{array}\right]=\left[T s_{H e 1}\right]\left[S h_{H e 1 c c}\right]^{i}\left[v_{h}\right]
\end{aligned}
$$

Also, for the transverse displacement in the thickness direction, it receives Hermitian cubic shape functions as an interpolation function. Therefore, a $C^{1}$ continuity between elements is archived:

$$
{ }^{i} w_{h}(s)=\frac{\left[\begin{array}{llll}
s^{3} & s^{2} & s & 1
\end{array}\right]}{8 l_{s}^{3}}\left[\begin{array}{cccc}
16 & -16 & 8 l_{s} & 8 l_{s} \\
0 & 0 & -4 l_{s}^{2} & 4 l_{s}^{2} \\
-12 l_{s}^{2} & 12 l_{s}^{2} & -2 l_{s}^{3} & -2 l_{s}^{3} \\
4 l_{s}^{3} & 4 l_{s}^{3} & l_{s}^{4} & -l_{s}^{4}
\end{array}\right]^{i}\left[\begin{array}{c}
w_{1} \\
w_{2} \\
\phi_{1} \\
\phi_{2}
\end{array}\right]=\left[T s_{H e 3}\right]\left[S h_{H e 3 c c}\right]\left[\begin{array}{l}
{[} \\
i\left[w_{h}\right] \\
{\left[\phi_{h}\right]}
\end{array}\right]
$$

In expressions 4.6, 4.7 and 4.8, the Hermitian shape functions are split in three components: i) the variable vector $\left[T s_{\mathrm{He} 1}\right]$ or $\left[T s_{\mathrm{He} 3}\right]$, which are functions of distance $s$. Here, the subindex $\mathrm{He}$ indicates a Hermitian shape function and the digit 1 or 3 names the type of function linear or cubic, respectively; ii) $\left[S h_{H e 1 c c}\right]$ and $\left[S h_{H e 3 c c}\right]$ are the completeness coefficient matrices obtained from the completeness requirements of the shape functions. The subindex $c c$ indicates the local boundary conditions of the element: clamped-clamped. In Chapter 5, there will be more details on these matrices; iii) a nodal amplification vector of the deformation mode shape, ${ }^{i}\left[u_{h}\right]$ or ${ }^{i}\left[v_{h}\right]$ or $\left[{ }^{i}\left[w_{h}\right]{ }^{i}\left[\phi_{h}\right]\right]$, which describes the amount of deformation of each degree of freedom for each deformation mode shape.

Although, in a first moment, the split representation of shape functions seems to be awkward, it can easily handle much more complex shape functions, such as trigonometric and/or hyperbolic. Moreover, in non-linear analysis, this representation tracks a systematic approach, as shown later in Chapter 7 .

There are seven interesting observations about this discretization of the displacement field:

$1^{s t}$ : The principle of completeness in the finite element method is the initial orthonormal basis of GBT's deformation mode shapes. It is necessary to keep in mind that each shape function is multiplied only by one term of the nodal amplification vector: a unit value in this term and null in all other terms, which specifies a unit vector in an orthonormal base. For instance, the transverse perpendicular displacement function 
above, ${ }^{i} w_{h}(s)$, has the two first unit vectors of its basis as:

$$
{ }^{1} \bar{w}_{h}(s)=\left[T s_{H e 3}\right]\left[S h_{H e 3 c c}\right]\left[\begin{array}{l}
1 \\
0 \\
0 \\
0
\end{array}\right] ; \quad{ }^{2} \bar{w}_{h}(s)=\left[T s_{H e 3}\right]\left[S h_{H e 3 c c}\right]\left[\begin{array}{l}
0 \\
1 \\
0 \\
0
\end{array}\right]
$$

Consequently, all deformation mode shapes related to transverse perpendicular displacement and rotation of one element can be described as an orthonormal base:

$$
\bar{w}_{h}^{e l}(s)=\left[T s_{H e 3}\right]\left[S h_{H e 3 c c}\right]\left[\begin{array}{cccc}
1 & 0 & 0 & 0 \\
0 & 1 & 0 & 0 \\
0 & 0 & 1 & 0 \\
0 & 0 & 0 & 1
\end{array}\right]=\left[T s_{H e 3}\right]\left[S h_{H e 3 c c}\right][I]
$$

Where $[I]$ is the identity matrix. Naturally, one can apply this representation in the other displacement functions:

$$
\begin{aligned}
& {\overline{u_{h}}}^{e l}(s)=\left[T s_{H e 1}\right]\left[S h_{H e 1 c c}\right]\left[\begin{array}{ll}
1 & 0 \\
0 & 1
\end{array}\right]=\left[T s_{H e 1}\right]\left[S h_{H e 1 c c}\right][I] \\
& {\overline{v_{h}}}^{e l}(s)=\left[T s_{H e 1}\right]\left[S h_{H e 1 c c}\right]\left[\begin{array}{ll}
1 & 0 \\
0 & 1
\end{array}\right]=\left[T s_{H e 1}\right]\left[S h_{H e 1 c c}\right][I]
\end{aligned}
$$

$2^{\text {nd }}$ : Any cross-section deformation mode shape can be defined by the global nodal amplification vector ${ }^{i}\left[U_{h}\right]$ :

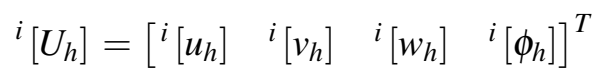

where the sub-vectors $\left[u_{h}\right],\left[v_{h}\right],\left[w_{h}\right]$ and $\left[\phi_{h}\right]$ are each one an sub-orthonormal basis of the respective displacement sort:

$$
\begin{gathered}
{ }^{i}\left[u_{h}\right]=\left[\begin{array}{lll}
{ }^{i} u_{g 1} & { }^{i} u_{g 2} & { }^{i} u_{g 3} \ldots
\end{array}\right]^{T} \\
\left.{ }^{i} v_{h}\right]=\left[\begin{array}{lll}
{\left[{ }^{i} v_{g 1}\right.} & { }^{i} v_{g 2} & { }^{i} v_{g 3} \ldots
\end{array}\right]^{T} \\
{ }^{i}\left[w_{h}\right]=\left[\begin{array}{llll}
{ }^{i} w_{g 1} & { }^{i} w_{g 2} & { }^{i} w_{g 3} & \ldots
\end{array}\right]^{T} \\
{ }^{i}\left[\phi_{h}\right]=\left[\begin{array}{llll}
{ }^{i} \phi_{g 1} & { }^{i} \phi_{g 2} & { }^{i} \phi_{g 3} & \ldots
\end{array}\right]^{T}
\end{gathered}
$$

Here, the subindex $g_{g \#}$ indicates the global coordinate system and the number of the node in the cross-section discretization. Hence, the initial orthonormal basis of analysis of a whole cross-section is defined by:

$$
\left[\bar{U}_{h}\right]_{n x n}=[I]_{n x n}
$$

where $n$ is the total degree of freedoms of the cross-section. Furthermore, the solution of eigenvalue problem will transform $\left[\bar{U}_{h}\right]$ into $\left[\tilde{U}_{h}\right]$, which can be understood as the matrix 
of eigenvectors, or as the transformation matrix, of ordinary differential equations, as given in equations $4.3,4.4$ and 4.5 .

$3^{r d}$ : The linear interpolation function for longitudinal displacement, $u_{h}(s)$, expresses the exact assumption of GBT's membrane displacement;

$4^{\text {th }}$ : The transverse interpolation displacement function, $v_{h}(s)$, in the element direction $s$, allows a linear elongation in element middle-line. This elongation violates the classical GBT's assumption of null membrane elongation, $\varepsilon_{s}=0$. Hence, some constraints are necessary for this function. Nevertheless, it stands out an opportunity for further relaxation of traditional GBT's assumptions;

$5^{\text {th }}$ The perpendicular transverse interpolation function, based on vectors $w_{h}(s)$ and $\phi_{h}(s)$, describes exactly a plate deformation according to Lowe-Kirchoff plate theory;

$6^{\text {th }}$ The assumption of no membrane's contribution from shear stress in the longitudinal equilibrium of the cross-section can be represented by the constant part of the function $v_{h}(s)$. Therefore, there is coupling between the displacement function $u_{h}(s)$ and $v_{h}(s)$;

$7^{\text {th }}$ Although in the local coordinate system the transverse interpolation displacement functions $w_{h}(s)$ and $v_{h}(s)$ are orthogonal and decoupled between themselves, they are coupled in a global coordinate system of the whole cross-section.

From the last two observations, one can conclude that it is necessary to build a set of global displacement functions to archive the cross-section analysis and its general properties.

\subsection{Transverse Stiffness matrices}

Since the semi-discretization allows strains and displacements beyond the GBT's kinematic assumptions, it is necessary to consider these extra effects and then constrain them to recover the traditional GBT's kinematic assumptions.

Consequently, the total internal strain energy functional, eq. 2.24, must have all terms of plate and membrane. With the above extra effects, one can obtain the following expression of a single element:

$$
U_{i n t}^{e l}=\int_{V^{e l}}\left[\int_{\varepsilon_{x}^{M}} \sigma_{x}^{M} \mathrm{~d} \varepsilon_{x}^{M}+\int_{\varepsilon_{x}^{P}} \sigma_{x}^{P} \mathrm{~d} \varepsilon_{x}^{P}+\int_{\gamma_{x s}^{M}} \tau_{x s}^{M} \mathrm{~d} \gamma_{x s}^{M}+\int_{\gamma_{x s}^{P}} \tau_{x s}^{P} \mathrm{~d} \gamma_{x s}^{P}+\int_{\varepsilon_{s}^{M}} \sigma_{s}^{M} \mathrm{~d} \varepsilon_{s}^{M}+\int_{\varepsilon_{s}^{P}} \sigma_{s}^{P} \mathrm{~d} \varepsilon_{s}^{P}\right] \mathrm{d} V^{e l}
$$

Here, the superscript ( $)^{e l}$ indicates a single element term.

Similar to M.Andreassen and J.Jönsson, the simplified constitutive relation, eq. 2.10, is adopted for both membrane and plate parts. Hence, the functional above leads 
to:

$$
U_{i n t}^{e l}=\int_{V^{e l}} \frac{E}{2}\left[\left(\varepsilon_{x}^{M}\right)^{2}+\left(\varepsilon_{x}^{P}\right)^{2}\right]+\frac{G}{2}\left[\left(\gamma_{x s}^{M}\right)^{2}+\left(\gamma_{x s}^{P}\right)^{2}\right]+\frac{E}{2}\left[\left(\varepsilon_{s}^{M}\right)^{2}+\left(\varepsilon_{s}^{P}\right)^{2}\right] \mathrm{d} V^{e l}
$$

And its variation concerning the internal strain energy is given by:

$$
\delta U_{i n t}^{e l}=\int_{V^{e l}} E\left[\varepsilon_{x}^{M} \delta \varepsilon_{x}^{M}+\varepsilon_{x}^{P} \delta \varepsilon_{x}^{P}\right]+G\left[\gamma_{x s}^{M} \delta \gamma_{x s}^{M}+\gamma_{x s}^{P} \delta \gamma_{x s}^{P}\right]+E\left[\varepsilon_{s}^{M} \delta \varepsilon_{s}^{M}+\varepsilon_{s}^{P} \delta \varepsilon_{s}^{P}\right] \mathrm{d} V^{e l}
$$

In the expression above, one can introduce the displacement functions given in equations 4.10, 4.11 and 4.12. Also, similar to the case of hollow circular cross-section, the integrals over the volume are split in cross-section and longitudinal domains, which leads to:

$$
\begin{gathered}
\delta U_{\text {int }}^{e l}=\int_{s} E\left[t_{h} u_{h}^{e l}(s)^{2}+\frac{t_{h}^{3}}{12} w_{h}^{e l}(s)^{2}\right] \mathrm{d} s \int_{L} V_{, x x}(x) \delta V_{, x x}(x) \mathrm{d} x+ \\
+\int_{s} G\left[t_{h} u_{h, s}^{e l}(s)^{2}+2 t_{h} u_{h, s}^{e l}(s) v_{h}^{e l}(s)+t_{h} v_{h}^{e l}(s)^{2}+\frac{t_{h}^{3}}{3} w_{h, s}^{e l}(s)^{2}\right] \mathrm{d} s \int_{L} V_{, x}(x) \delta V_{, x}(x) \mathrm{d} x+ \\
+\int_{s} E\left[t_{h} v_{h, s}^{e l}(s)^{2}+\frac{t_{h}^{3}}{12} w_{h, s s}^{e l}(s)^{2}\right] \mathrm{d} s \int_{L} V(x) \delta V(x) \mathrm{d} x
\end{gathered}
$$

Here, the sum notation is no longer necessary, since the displacement functions already have all orthonormal deformation mode shapes of one element.

Each term of each integral over the element length, $s$, generates a transverse stiffness matrix. Starting with the membrane's longitudinal strain energy (first term in the first integral), one reaches:

$$
\int_{s} E t u_{h}^{e l}(s)^{2} \mathrm{~d} s=E t\left[S h_{H e 1 c c}\right]^{T} \int_{s}\left[T s_{H e 1}\right]^{T}\left[T s_{H e 1}\right] \mathrm{d} s\left[S h_{H e 1 c c}\right]
$$

As shown above, only the variable vectors $\left[T s_{\mathrm{Hel}}\right]^{T}$ and $\left[T s_{\mathrm{Hel}}\right]$, which are function of $s$, are evaluated in the integral. Thus, one reaches a kernel transverse stiffness matrix:

$$
\left[\Upsilon_{H e 1}\right]=\int_{s}\left[T s_{H e 1}\right]^{T}\left[T s_{H e 1}\right] \mathrm{d} s=\frac{l_{s}}{12}\left[\begin{array}{cc}
l_{s}^{2} & 0 \\
0 & 12
\end{array}\right]
$$

And the cross-section stiffness matrix due to membrane's longitudinal strain is:

$$
k_{u u}^{M \sigma_{x}}=E t_{h}\left[S h_{H e 1 c c}\right]^{T}\left[\Upsilon_{H e 1}\right]\left[S h_{H e 1 c c}\right]=l_{s} \frac{E t_{h}}{6}\left[\begin{array}{ll}
2 & 1 \\
1 & 2
\end{array}\right]
$$

Since the displacement functions for longitudinal and transverse element's directions, $u_{h}^{e l}(s)$ and $v_{h}^{e l}(s)$ respectively, share the same shape functions, the evaluation of third 
term of the second integral in eq. 4.22 requires no additional effort. Thus, the crosssection stiffness matrices due to membrane's shear strains from the transverse displacement, $v(s)$, are:

$$
k_{v v}^{M \tau}=G t_{h}\left[S h_{H e 1 c c}\right]^{T}\left[\Upsilon_{H e 1}\right]\left[S h_{H e 1 c c}\right]=l_{s} \frac{G t_{h}}{6}\left[\begin{array}{ll}
2 & 1 \\
1 & 2
\end{array}\right]
$$

Following this procedure, one can evaluate the first term in the second integral, as well as the first term in the third integrals, which are based on the first derivative of shape functions. Therefore, it requires the first derivative of the variable vector:

$$
\left[T s_{H e 1, s}\right]=\left[\begin{array}{ll}
1 & 0
\end{array}\right]
$$

which leads to following kernel stiffness matrix:

$$
\left[\Upsilon_{H e 1, s, s}\right]=\int_{s}\left[T s_{H e 1, s}\right]^{T}\left[T s_{H e 1, s}\right] \mathrm{d} s=\left[\begin{array}{cc}
l_{s} & 0 \\
0 & 0
\end{array}\right]
$$

Here, the index ()$_{\text {s.s }}$ indicates that the kernel stiffness matrix is based on two first derivative of the variable vector. Subsequently, the cross-section stiffness matrix, due to membrane's shear strains from the longitudinal displacement, $u(s)$, is:

$$
k_{u u}^{M \tau}=G t_{h}\left[S h_{H e 1 c c}\right]^{T}\left[\Upsilon_{H e 1, s, s}\right]\left[S h_{H e 1 c c}\right]=\frac{G t_{h}}{l_{s}}\left[\begin{array}{cc}
1 & -1 \\
-1 & 1
\end{array}\right]
$$

With the same kernel stiffness and completeness coefficient matrices, the cross-section stiffness matrix, due to membrane's transverse strains has a similar result:

$$
k_{v v}^{M \sigma_{s}}=E t_{h}\left[S h_{H e 1 c c}\right]^{T}\left[\Upsilon_{H e 1, s, s}\right]\left[S h_{H e 1 c c}\right]=\frac{E t_{h}}{l_{s}}\left[\begin{array}{cc}
1 & -1 \\
-1 & 1
\end{array}\right]
$$

The last cross-section stiffness matrix, due to membrane behavior, concerns the shear strain energy between the longitudinal and transverse displacements, which stands out from the second term of the second integral in eq. 4.22. This term requires an observation: the product of variable vectors, $u_{h, s}^{e l}(s) v_{h}^{e l}(s)$, is not commutative, but it leads to transpose matrices. Consequently, this stiffness matrix is not locally symmetric. However, it is out of the main diagonal of the global cross-section stiffness matrix, i.e., the transpose pair of matrices are symmetric from each other in the final global stiffness matrix.

Hence, to evaluate them, one must find first the kernel stiffness matrix from a variable vector and its first derivative, and vice-versa:

$$
\begin{gathered}
{\left[\Upsilon_{H e 1, s}\right]=\int_{s}\left[T s_{H e 1, s}\right]^{T}\left[T s_{H e 1}\right] \mathrm{d} s=\left[\begin{array}{cc}
0 & l_{s} \\
0 & 0
\end{array}\right]} \\
{\left[\Upsilon_{H e 1, s}\right]^{T}=\int_{s}\left[T s_{H e 1}\right]^{T}\left[T s_{H e 1, s}\right] \mathrm{d} s=\left[\begin{array}{ll}
0 & 0 \\
l_{s} & 0
\end{array}\right]}
\end{gathered}
$$


With the respective completeness coefficient matrix for longitudinal and transverse displacements, the cross-section stiffness matrix due to the membrane's shear strain from longitudinal-transverse displacements is:

$$
k_{u v}^{M \tau}=G t_{h}\left[S h_{H e 1 c c}\right]_{u}^{T}\left[\Upsilon_{H e 1, s}\right]\left[S h_{H e 1 c c}\right]_{s}=\frac{G t_{h}}{2}\left[\begin{array}{cc}
-1 & -1 \\
1 & 1
\end{array}\right]
$$

Here, the temporary indexes $u$ and ${ }_{v}$ emphasize the correct order of completeness coefficient matrices. Following this evaluation, one can find the transpose related crosssection stiffness matrix: membrane's shear strain of coupling transverse-longitudinal displacements:

$$
k_{v u}^{M \tau}=G t_{h}\left[S h_{H e 1 c c}\right]_{v}^{T}\left[\Upsilon_{H e 1, s}\right]^{T}\left[S h_{H e 1 c c}\right]_{u}=\frac{G t_{h}}{2}\left[\begin{array}{ll}
-1 & 1 \\
-1 & 1
\end{array}\right]
$$

After the analysis of each membrane term, the next evaluations are the plate terms. Starting with the second term in the first integral in eq. 4.22 , which represents the cross-section stiffness matrix due to the plate's longitudinal strain, one must initially find the kernel stiffness matrix for this case:

$$
\left[\Upsilon_{H e 3}\right]=\int_{s}\left[T s_{H e 3}\right]^{T}\left[T s_{H e 3}\right] \mathrm{d} s=\frac{l_{s}}{4}\left[\begin{array}{cccc}
l_{s}^{6} / 112 & 0 & l_{s}^{4} / 20 & 0 \\
& l_{s}^{4} / 20 & 0 & l_{s}^{2} / 3 \\
& & l_{s}^{2} / 3 & 0 \\
s y m . & & & 4
\end{array}\right]
$$

Thus, the cross-section stiffness matrix for this term is:

$$
\begin{aligned}
k^{P \sigma_{x}}=E \frac{t_{h}^{3}}{12}\left[S h_{H e 3 c c}\right]^{T}\left[\Upsilon_{H e 3}\right]\left[S h_{H e 3 c c}\right]= \\
\left.E \frac{t_{h}^{3}}{5040} l_{s}\left[\begin{array}{cccc}
156 & 54 & 22 l_{s} & -13 l_{s} \\
& 156 & 13 l_{s} & -22 l_{s} \\
& & 4 l_{s}^{2} & -3 l_{s}^{2} \\
S y m . & & & 4 l_{s}^{2}
\end{array}\right]=E\left[\begin{array}{l}
{\left[k_{w w}^{P \sigma_{x}}\right]} \\
{\left[k_{\phi w}^{P \sigma_{x}}\right.}
\end{array}\right]\left[\begin{array}{c}
k_{w \phi}^{P \sigma_{x}} \\
k_{\phi \phi}^{P \sigma_{x}}
\end{array}\right]\right]
\end{aligned}
$$

Here, in order to highlight the respective degree of freedom - transverse displacements $w(s)$ or the longitudinal rotation $\phi(s)$ - the plate's stiffness matrix is subdivided.

The next plate's term concerns the shear strain, last term of the second integral in eq. 4.22 , requires the first derivative of cubic Hermitian's variable vector:

$$
\left[T s_{H e 3, s}\right]=\left[\begin{array}{llll}
3 s^{2} & 2 s & 1 & 0
\end{array}\right]
$$

Which leads to the following kernel and cross-section stiffness matrices, respectively:

$$
\left[\Upsilon_{H e 3, s, s}\right]=\int_{s}\left[T s_{H e 3, s}\right]^{T}\left[T s_{H e 3, s}\right] \mathrm{d} s=l_{s}\left[\begin{array}{cccc}
9 l_{s}^{4} / 80 & 0 & l_{s}^{2} / 4 & 0 \\
& l_{s}^{2} / 3 & 0 & 0 \\
& & 1 & 0 \\
s y m . & & & 0
\end{array}\right]
$$




$$
\begin{gathered}
k^{P \tau}=G \frac{t_{h}^{3}}{3}\left[S h_{H e 3 c c}\right]^{T}\left[\Upsilon_{H e 3, s, s}\right]\left[S h_{H e 3 c c}\right]= \\
\left.\frac{G t_{h}^{3}}{180 l_{s}}\left[\begin{array}{cccc}
72 & -72 & 6 l_{s} & 6 l_{s} \\
& 72 & -6 l_{s} & -6 l_{s} \\
& & 8 l_{s}^{2} & -2 l_{s}^{2} \\
S y m . & & & 8 l_{s}^{2}
\end{array}\right]=G\left[\begin{array}{l}
{\left[k_{w w}^{P \tau}\right]} \\
{\left[k_{\phi w}^{P \tau}\right.}
\end{array}\right]\left[\begin{array}{c}
k_{w \phi}^{P \tau} \\
k_{\phi \phi}^{P \tau}
\end{array}\right]\right]
\end{gathered}
$$

The last cross-section stiffness matrix is due to plate's transverse strain elongation, $k_{w w}^{P \sigma_{s}}$. One obtains it from the evaluation of the second term in the last integral of eq. 4.22, and it requires the second derivative of cubic Hermitian's variable vector:

$$
\left[T s_{H e 3, s, s}\right]=\left[\begin{array}{llll}
6 s & 2 & 0 & 0
\end{array}\right]
$$

Following the same procedure of the previous cases, one reaches the kernel and crosssection stiffness matrices for plate's transverse elongation strains, respectively:

$$
\left[\Upsilon_{H e 3, s s, s s}\right]=\int_{s}\left[T s_{H e 3, s s}\right]^{T}\left[T s_{H e 3, s s}\right] \mathrm{d} s=l_{s}\left[\begin{array}{cccc}
3 l_{s}^{2} & 0 & 0 & 0 \\
& 4 & 0 & 0 \\
& & 0 & 0 \\
s y m . & & & 0
\end{array}\right]
$$

$$
\begin{aligned}
k^{P \sigma_{s}}=E \frac{t_{h}^{3}}{12}\left[S h_{H e 3 c c}\right]^{T} & {\left[\Upsilon_{H e 3, s s, s s}\right]\left[S h_{H e 3 c c}\right] } \\
& =\frac{E t_{h}^{3}}{l_{s}^{3}}\left[\begin{array}{cccc}
1 & -1 & l_{s} / 2 & l_{s} / 2 \\
& 1 & -l_{s} / 2 & -l_{s} / 2 \\
& & l_{s}^{2} / 3 & l_{s}^{2} / 6 \\
S y m . & & l_{s}^{2} / 3
\end{array}\right]=E\left[\begin{array}{l}
{\left[k_{w w}^{P \sigma_{s}}\right]} \\
{\left[k_{\phi w}^{P \sigma_{s}}\right]}
\end{array}\left[\begin{array}{c}
k_{w \phi}^{P \sigma_{s}} \\
{\left[k_{\phi \phi}^{P \sigma_{s}}\right.}
\end{array}\right]\right.
\end{aligned}
$$

All these cross-section stiffness matrices can be grouped in the order of degrees of freedom as exposed in eq. 4.13. I.e. first, the longitudinal displacement of the membrane, $u(s)$; second, the transverse displacement in the segment direction, $v(s)$; third, the perpendicular segments transverse displacement, $w(s)$; and fourth, the longitudinal rotation, $\phi(s)$. Following this arrangement, the internal strain energy of a single element, 
eq. 4.22 , is expressed in a matrix form:

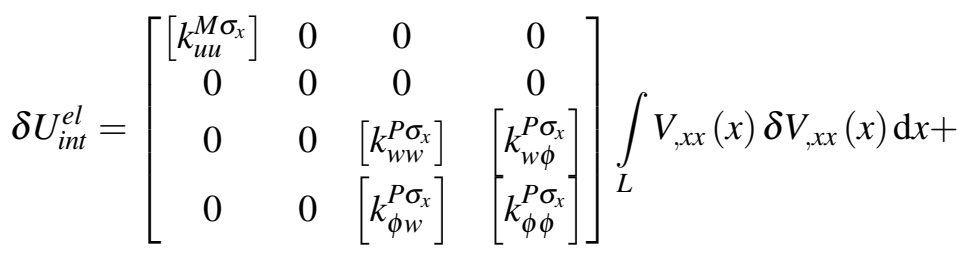

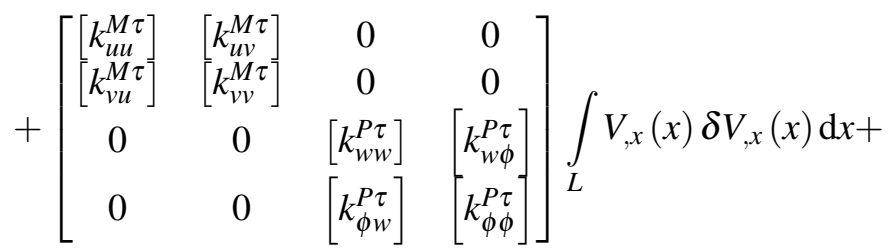

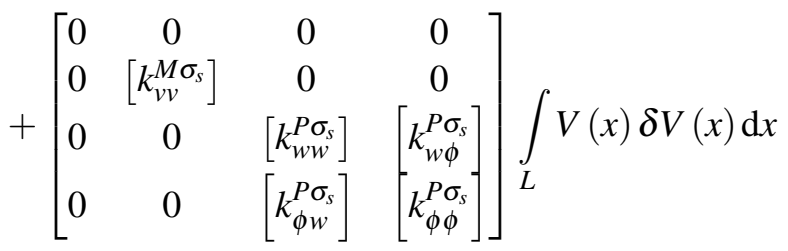

As one achieves the variation of internal strain energy for a single element, it is possible to assemble the total variation of internal strain energy of the cross-section. However, the elements do not usually share the same local coordinate system. Therefore, for the plate's stiffness matrices, a usual matrix transformation, $\left[T^{P}\right]$, is required to convert the coordinate system from element's local one into the global cross-section:

$$
\left[T^{P}\right]=\left[\begin{array}{cccccc}
\cos (\theta) & 0 & \sin (\theta) & 0 & 0 & 0 \\
0 & \cos (\theta) & 0 & \sin (\theta) & 0 & 0 \\
-\sin (\theta) & 0 & \cos (\theta) & 0 & 0 & 0 \\
0 & -\sin (\theta) & 0 & \cos (\theta) & 0 & 0 \\
0 & 0 & 0 & 0 & 1 & 0 \\
0 & 0 & 0 & 0 & 0 & 1
\end{array}\right]
$$

Here, $\left[T^{P}\right]$ has the coordinate order of $v(s), w(s)$ and a rotation angle $\theta$, as shown in figure. 4.4. Since the displacement $v(s)$ and $w(s)$ are in the same plane, there is a coupling between these displacements for non-aligned elements. For instance, this transformation in sub-matrix $\left[k_{v v}^{P \sigma_{x}}\right]$ leads to:

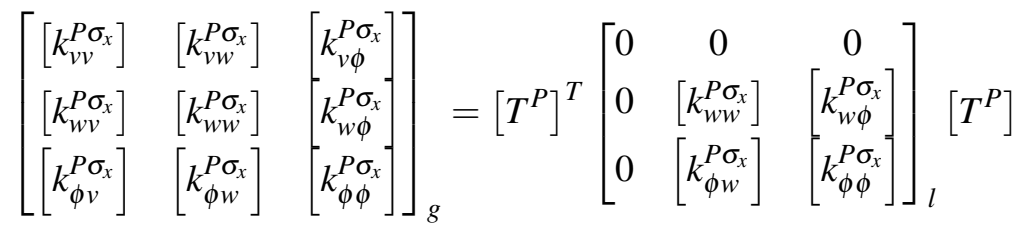

Where the indexes $g$ and ${ }_{l}$ indicate the basis of the coordinate systems global and local, respectively.

Although the above transformation is usual in structural analysis, the coordinate transformation for the membrane's stiffness matrices is unique. It involves the longitudinal displacement $u(s)$ instead of the longitudinal rotation $\phi(s)$ : 


$$
\left[T^{M}\right]=\left[\begin{array}{cccccc}
1 & 0 & 0 & 0 & 0 & 0 \\
0 & 1 & 0 & 0 & 0 & 0 \\
0 & 0 & \cos (\theta) & 0 & \sin (\theta) & 0 \\
0 & 0 & 0 & \cos (\theta) & 0 & \sin (\theta) \\
0 & 0 & -\sin (\theta) & 0 & \cos (\theta) & 0 \\
0 & 0 & 0 & -\sin (\theta) & 0 & \cos (\theta)
\end{array}\right]
$$

Consequently, in the global coordinate system of the cross-section there is a coupling between the membrane's displacements $u(s)$ and $w(s)$, that does not exist in the local coordinate system of each segment. As an example, the transformation of transverse stiffness matrix due to the membrane's shear strain is:

$$
\left[\begin{array}{lll}
{\left[k_{u u}^{M \tau}\right]} & {\left[k_{u v}^{M \tau}\right]} & {\left[k_{u w}^{M \tau}\right]} \\
{\left[k_{v u}^{M \tau}\right]} & {\left[k_{v v}^{M \tau}\right]} & {\left[k_{v w}^{M \tau}\right]} \\
{\left[k_{w u}^{M \tau}\right]} & {\left[k_{w v}^{M \tau}\right]} & {\left[k_{w w}^{M \tau}\right]}
\end{array}\right]_{G l}=\left[T^{M}\right]^{T}\left[\begin{array}{ccc}
{\left[k_{u u}^{M \tau}\right]} & {\left[k_{u v}^{M \tau}\right]} & 0 \\
{\left[k_{v u}^{M \tau}\right]} & {\left[k_{v v}^{M \tau}\right]} & 0 \\
0 & 0 & 0
\end{array}\right]_{L o}\left[T^{M}\right]
$$

After the transformation from local to global coordinates systems, one can assemble each element's matrix into a total cross-section stiffness matrix, leading to:

$$
\begin{aligned}
& \delta U_{i n t}^{s t}=\left[\begin{array}{cccc}
{\left[k_{u u}^{M \sigma_{x}}\right]} & 0 & 0 & 0 \\
0 & {\left[k_{v v}^{P \sigma_{x}}\right]} & {\left[k_{v w}^{P \sigma_{x}}\right]} & {\left[k_{v \phi}^{P \sigma_{x}}\right.} \\
0 & {\left[k_{w v}^{P \sigma_{x}}\right]} & {\left[k_{w w}^{P \sigma_{x}}\right]} & {\left[k_{w \phi}^{P \sigma_{x}}\right.} \\
0 & {\left[k_{\phi v}^{P \sigma_{x}}\right]} & {\left[k_{\phi w}^{P \sigma_{x}}\right]} & {\left[k_{\phi \phi}^{P \sigma_{x}}\right.}
\end{array}\right] \int_{s t} V_{, x x}(x) \delta V_{, x x}(x) \mathrm{d} x+ \\
& +\left[\begin{array}{cccc}
{\left[k_{u u}^{M \tau}\right]} & {\left[k_{u v}^{M \tau}\right]} & {\left[k_{u w}^{M \tau}\right]} & 0 \\
{\left[k_{v u}^{M \tau}\right]} & {\left[k_{v v}^{M \tau}\right]+\left[k_{v v}^{P \tau}\right]} & {\left[k_{v w}^{M \tau}\right]+\left[k_{v w}^{P \tau}\right]} & {\left[k_{v \phi}^{P \tau}\right]} \\
{\left[k_{w u}^{M \tau}\right]} & {\left[k_{w v}^{M \tau}\right]+\left[k_{w v}^{P \tau}\right]} & {\left[k_{w w}^{M \tau}\right]+\left[k_{w w}^{P \tau}\right]} & {\left[k_{w \phi}^{P \tau}\right.} \\
0 & {\left[k_{\phi v}^{P \tau}\right]} & {\left[k_{\phi w}^{P \tau}\right]} & {\left[k_{\phi \phi}^{P \tau}\right]}
\end{array}\right] \int_{s t} V_{, x}(x) \delta V_{, x}(x) \mathrm{d} x+ \\
& +\left[\begin{array}{cccc}
0 & 0 & 0 & 0 \\
0 & {\left[k_{v v}^{M \sigma_{s}}\right]+\left[k_{v v}^{P \sigma_{s}}\right]} & {\left[k_{v w}^{M \sigma_{s}}\right]+\left[k_{v w}^{P \sigma_{s}}\right]} & {\left[k_{v \phi}^{P \sigma_{s}}\right.} \\
0 & {\left[k_{w v}^{M \sigma_{s}}\right]+\left[k_{w v}^{P \sigma_{s}}\right]} & {\left[k_{w w}^{M \sigma_{s}}\right]+\left[k_{w w}^{P \sigma_{s}}\right]} & k_{w \phi}^{P \sigma_{s}} \\
0 & {\left[k_{\phi v}^{P \sigma_{s}}\right]} & {\left[k_{\phi w}^{P \sigma_{s}}\right]} & {\left[k_{\phi \phi}^{P \sigma_{s}}\right]}
\end{array}\right] \int_{s t} V(x) \delta V(x) \mathrm{d} x
\end{aligned}
$$

where the index st indicates the whole domain of structural beam.

The matrices above are named as: longitudinal stiffness of the cross-section, $[C]$; shear stiffness of the cross-section, $[D]$; transverse stiffness of the cross-section, $[B]$. In each one of these matrices, there are some singularities, listed below:

- Matrix $[C]$ : As shown in eq. 4.43, there is not a term concerning the longitudinal stiffness of a single element due to a transverse displacement in the element direction. Thus, there is a singularity in this matrix for a chain of aligned elements. 
- Matrix $[D]$ : Since the cross-section's deformation modes concerning i) uniform elongation, $i=1$; ii and iii) main directions of bending, $i=2$ and $i=3$, these modes have no shear strains. Thus, the matrix $[D]$ has three singularities concerning these three cross-section's rigid body movements that must be identified and isolated.

- Matrix $[B]$ : For the transverse stiffness matrix of the cross-section, there are four singularities. In fact, all four deformation modes concerning rigid body movements have no transverse distortion.

\subsection{Identification and elimination of singular modes}

Once all transverse stiffness matrices are set up, it is necessary to perform the elimination of unique modes that have no cross-section shear stiffness matrix, $[D]$. This elimination avoids singularities in GBT's quadratic eigenvalue problem.

Moreover, since these modes do not involve all derivatives in expression eq. 4.22 , it is necessary to identify and address them to the correct differential equation solution and the respective traditional beam theory.

The table below presents these singular modes and clarifies the GBT's nomination and traditional beam theory concerning them:

Table 4.1: Properties and nomination of singular modes according to GBT

\begin{tabular}{cccccc}
\hline Mode number & {$[\bar{C}]$} & {$[\bar{D}]$} & {$[\bar{B}]$} & Description & Beam Theory \\
\hline 1 & $\checkmark$ & - & - & Pure longitudinal elongation & rod's theory \\
2 & $\checkmark$ & - & - & Major bending & Euler-Bernoulli \\
3 & $\checkmark$ & - & - & Minor bending & Euler-Bernoulli \\
4 & $\checkmark$ & $\checkmark$ & - & Non-uniform torsion & Vlasov \\
t & - & $\checkmark$ & - & Uniform torsion & St-Venant-Bredt \\
$>5$ & $\checkmark$ & $\checkmark$ & $\checkmark$ & Warping and Distortion & GBT \\
\hline
\end{tabular}

Each mode in the above table demands an analysis step that involves not only a basis transformation, but also requires a particular constraining expression for each beam theory. The sequence of steps follows the order of steps above.

\subsection{Membrane, Plate and Membrane-Plate modes}

Up to this point, the development of the cross-section analysis follows a procedure similar to $[97,98,99,100]$. However, from this point on, the presented alternative approach starts to diverge.

Here, the main idea consists in the splitting of eq. 4.48 in three parts: i) the internal energy, involving only membrane behavior such as the first row/column in each matrix of eq. 4.48 ; ii) the internal energy, involving only plate behavior such as the last 
row/column in each matrix of eq. 4.48; iii) the internal energy, involving membrane and plate behaviors simultaneously, found in the second and third row/columns of the matrices of eq. 4.48 .

The benefit of this splitting approach is that not only highlights the singularities, but also brings a clear classification of the deformation mode: i) membrane; ii) plate; iii) membrane and plate.

The next sub-sections detail the filtering process of each internal energy behavior and the necessary constraints due to GBT's assumptions.

\subsubsection{Membrane mode and constraints}

The singular and plural words in the title of this section already indicate the outcome: due to traditional GBT's assumptions, membrane modes that involve transverse elongation and shear strains are constrained. Consequently, only one mode of pure membrane behavior remains, which is the longitudinal elongation.

Initially, one must apply the constraint concerning the transverse elongation, which is also required in plate behavior. Thus, one imposes the eigenvector for a uniform longitudinal displacement demanding an orthogonalization process afterwards. Finally, based on the remained membrane stiffness matrices, one extracts the constraint relationship to avoid the membrane shear energy. These steps are detailed below.

Transverse elongation constraint:

This constraint condition is directly obtained by the imposition of a uniform transverse displacement $v_{l 1}=v_{l 2}$ in the element local coordinate system. In vectorial representation, one represents this coupling equation in a more convenient and general form:

$$
\left[\begin{array}{llll}
1 & -1 & 0 & 0
\end{array}\right]\left[\begin{array}{l}
v_{1} \\
v_{2} \\
w_{1} \\
w_{2}
\end{array}\right]_{l}=0
$$

With this representation, it is possible to obtain the coupling equation in the global coordinate system by a standard rotation operation:

$$
\left[\begin{array}{llll}
1 & -1 & 0 & 0
\end{array}\right]\left[\begin{array}{cccc}
\cos (\theta) & 0 & \sin (\theta) & 0 \\
0 & \cos (\theta) & 0 & \sin (\theta) \\
-\sin (\theta) & 0 & \cos (\theta) & 0 \\
0 & -\sin (\theta) & 0 & \cos (\theta)
\end{array}\right]\left[\begin{array}{c}
v_{1} \\
v_{2} \\
w_{1} \\
w_{2}
\end{array}\right]_{g}=0
$$

Thus, one assembles a total global coupling matrix based on transverse elongation coupling equation of all elements - this coupling matrix leads to a multi-freedom constraint approach. Among the possible approaches, the present cross-section analysis selects the master-slave method because it not only reduces the number of degrees of freedom of the cross-section in $n-1$ (where $n$ is the number of segments in the cross-section), but it 
also allows the definition of the master degree of freedoms. This definition is necessary especially for extreme natural node, which must have its degrees of freedoms defined as slave ones in order to avoid singularities in the stiffness matrices.

As an outcome, this constraint step reaches a transformation matrix among all transverse displacements $v_{i}$ and $w_{i}$ of the cross-section: $\left[{ }_{1} U\right]_{2 m x 2 m-n+1}$, where the subindex $m$ indicates the number of cross-sections' nodes and the left subindex is necessary to distinguish the present transformation among the several ones that will be used.

One remark is necessary: for free end segments, the transverse displacement of the extreme node has no energy contribution in any cross-section stiffness matrix. Hence, the respective degree of freedom must be vanished by the elimination of the correspondent row in the constraint matrix $\left[{ }_{1} U\right]$.

It is possible to confirm the values of the constraint matrix $\left[{ }_{1} U\right]$ when applied in the transverse stiffness matrix concerning only the membrane behavior, $[B]^{M}$. As result, one must obtain a reduced order and null matrix $\left[{ }_{1} B\right]^{M}$ as:

$$
\left.\left[{ }_{1} B\right]^{M}={ }_{1} U\right]_{2 m \times 2 m-n+1}^{T}\left[\begin{array}{ll}
{\left[k_{v v}^{M \sigma_{s}}\right]} & {\left[k_{v w}^{M \sigma_{s}}\right.} \\
{\left[k_{w v}^{M \sigma_{s}}\right]} & {\left[k_{w w}^{M \sigma_{s}}\right.}
\end{array}\right]_{2 m \times 2 m}\left[{ }_{1} U\right]_{2 m \times 2 m-n+1}=0
$$

Since matrix $\left[{ }_{1} B\right]^{M}$ is null, it requires no further transformation. Following the above transformation, one achieves the cross-section shear stiffness matrix of membrane behavior:

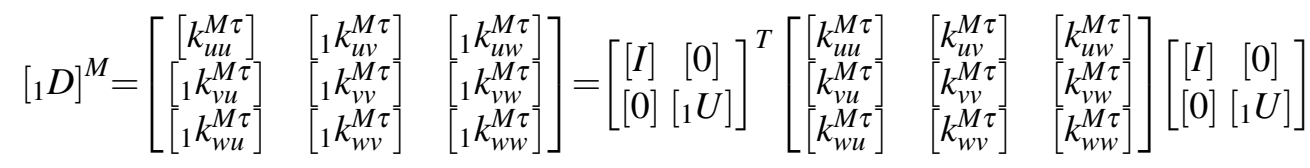

Here, the transformation matrix, $\left[{ }_{1} U\right]$, receives an identity matrix $[I]$ to handle the stiffness terms related to transverse and longitudinal displacements, $\left[k_{u u}^{M \tau}\right]$ and $\left[k_{u v}^{M \tau}\right]$ and their respective transposes. Hence, this identity matrix has the rank of mxm, which represents the total number of degrees of freedom of longitudinal displacement.

Concerning the cross-section longitudinal stiffness matrix of membrane behavior, $[C]^{M}$, one realizes that this stiffness matrix requires no transformation, since it has only terms which involve only longitudinal displacement. Therefore, one obtains:

$$
\left[{ }_{1} C\right]^{M}=[C]^{M}
$$

Pure elongation membrane mode:

Before the setup of shear membrane strain constraint, one must isolate and eliminate the pure membrane elongation mode, since it has no shear effect.

The pure longitudinal elongation has a trivial displacement field: a unit longitudinal displacement without transverse displacement. Consequently, the eigenvector of this mode concerning the longitudinal displacement is:

$$
{ }^{1}[U]_{m}=\left[\begin{array}{llll}
{ }^{1} u_{g 1}=1 & { }^{1} u_{g 2}=1 & { }^{1} u_{g 3}=1 & \ldots
\end{array}\right]^{T}
$$


Based on this eigenvector, one can use a new non-orthogonal basis for the longitudinal displacement degrees of freedom, which is an identity matrix that the first column receives the terms of vector ${ }^{1}[U]$ concerning the longitudinal displacement, ${ }^{1} u_{g i}$.

$$
\left[{ }_{2} U\right]_{m x m}=\left[\begin{array}{lc}
{ }^{1}[U]_{1 x n}^{T} & 0 \\
{[I I]_{n-1 x n-1}}
\end{array}\right]=\left[\begin{array}{ccccc}
1 & 0 & 0 & 0 & \ldots \\
1 & 1 & 0 & 0 & \ldots \\
1 & 0 & 1 & 0 & \ldots \\
\vdots & \vdots & \vdots & \vdots & \ddots
\end{array}\right]
$$

Alternatively, the notation of Von Neumann, [123], can express the setup of this basis. Such notation is useful in the next steps:

$$
\left.{ }_{2} U\right]_{n x n}=\operatorname{Subst}\left(\begin{array}{c}
\mathrm{Col}_{1} \\
{ }^{1}[U]
\end{array}\right)[I]_{n x n}
$$

Converting the matrices $\left[{ }_{1} C\right]$ and $\left[{ }_{1} D\right]$ into this new basis, one reaches:

$$
\begin{aligned}
& {\left[{ }_{2} C\right]^{M}=\left[{ }_{2} U\right]^{T}\left[{ }_{1} C\right]^{M}\left[{ }_{2} U\right]} \\
& \left.\left.{ }_{2} D\right]^{M}=\left[{ }_{2} U\right]^{T}\left[{ }_{1} D\right]^{M}{ }_{{ }_{2}} U\right]
\end{aligned}
$$

The outcome from this changing of basis is the achievement of cross-section's longitudinal stiffness, $E A$, in the correspondent term of matrix $\left[{ }_{2} C_{a}\right]$, which is the first value in the first row.

Since the pure axial elongation has not any shear stiffness contribution, the basis transformation also brings out null values in the first row and column of the shear stiffness matrix, $\left[{ }_{2} D_{a}\right]$. Therefore, after this transformation, the differential equation of pure axial elongation concerns only the longitudinal stiffness matrix, which is not yet orthogonalized with the other longitudinal degrees of freedoms. The matrix $\left.{ }_{2} \mathrm{C}\right]$ has non-null values out of diagonal in the first row and column.

There are two procedures to overcome this lack of orthogonality in the cross-section analysis.

The first one, developed by Andreassen et al. [97], consists in removing the row and column concerning the pure axial elongation by a coupling equation. One defines this coupling equation as null internal energy in the first row of $\left.{ }_{2} \mathrm{C}\right]$ for any displacement field:

$$
\left[\begin{array}{llll}
{ }_{2} C_{1,1} & { }_{2} C_{1,2} & \cdots & { }_{2} C_{1, n}
\end{array}\right]\left[\begin{array}{c}
u_{g 1} \\
u_{g 2} \\
\vdots \\
u_{g m}
\end{array}\right]=0
$$

Based on this coupling equation, it is possible to use the Master-Slave method once more. The slave term for this method is the first longitudinal degree of freedom. Thus, one achieves a new transformation matrix $\left.{ }_{3} U\right]$. It removes the pure elongation membrane mode from the cross-section stiffness matrices without any side effect:

$$
\left[{ }_{3} C\right]^{M}=\left[{ }_{3} U\right]^{T}\left[{ }_{2} C\right]^{M}\left[{ }_{3} U\right]
$$




$$
\left[{ }_{3} D\right]^{M}=\left[{ }_{3} U\right]^{T}\left[{ }_{2} D\right]^{M}\left[{ }_{3} U\right]
$$

The second procedure consists in a partial simultaneous orthogonalization of the longitudinal degrees of freedom of matrices $\left.{ }_{2} C\right]$ and $\left[{ }_{2} D\right]$, in order to obtain a basis from which the pure longitudinal elongation is entirely orthogonal in respect to other deformation modes.

This can be done by using Falk-Langemeyer method, [58], as Richard Schardt did in his works. It is important to note that the Falk-Langemeyer method is applied only in the off-diagonal terms of the first rows of matrices $\left.{ }_{2} C\right]$ and $\left.{ }_{2} D\right]$, since one searches only an independent ordinary differential equation for the longitudinal elongation. Consequently, the partial application of Falk-Langemeyer method, together with the elimination of the first longitudinal degree of freedom, leads also to the transformation matrix $\left.{ }_{3} U\right]$, used in equations 4.61 and 4.60 .

Finally, it is necessary to make a final remark concerning the pure axial elongation. Mode 1 abolishes the GBT's manner to express the longitudinal displacement function with the first derivative of the amplification function, $V_{, x}(x)$. Instead, the function $V(x)$ defines the $V_{, x}(x)$ of this mode:

$$
{ }^{1} u(x, s, t)={ }^{1} u(s, t){ }^{1} V(x)
$$

The insertion of this modification, together with the full orthogonalization among the stiffness matrices into eq. 4.48, leads to the well-known expression of internal strain energy of a rod:

$$
{ }^{1} \delta U_{i n t}^{e l}=E^{1} C \int_{L}{ }^{1} V_{, x}(x){ }^{1} \delta V_{, x}(x) \mathrm{d} x
$$

Membrane shear strain constraint:

The assumption of no contribution from the shear strain energy in the membrane behavior of the cross-section, eq. 2.2, is represented in matrix form by the sub-matrices of $\left[{ }_{3} D\right]^{M}$ :

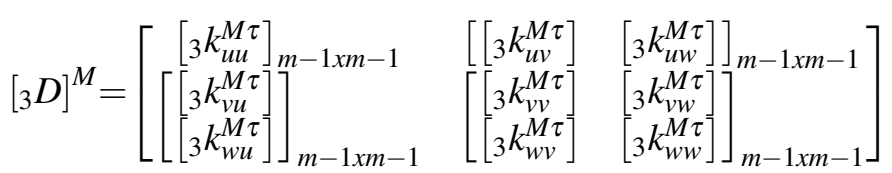

From the first row in eq. 4.64, one achieves the following relationship for non-shear membrane energy:

$$
\left[{ }_{3} k_{u u}^{M \tau}\right]_{m-1 x m-1}\left[\begin{array}{c}
u_{g 1} \\
u_{g 2} \\
\vdots \\
u_{g m-1}
\end{array}\right]+\left[\left[{ }_{3} k_{u v}^{M \tau}\right] \quad\left[{ }_{3} k_{u w}^{M \tau}\right]\right]_{m-1 x m-1}\left[\begin{array}{c}
v_{g 1} \\
v_{g 2} \\
\vdots \\
w_{g 1} \\
w_{g 2} \\
\vdots
\end{array}\right]=0
$$


Therefore, one reaches a relationship of the slave transverse displacements $v$ and $w$ in respect to master degrees of freedom for the longitudinal displacement, $u$ :

$$
\left[\begin{array}{c}
v_{g 1} \\
v_{g 2} \\
\vdots \\
w_{g 1} \\
w_{g 2} \\
\vdots
\end{array}\right]=-\left[\left[{ }_{3} k_{u v}^{M \tau}\right] \quad\left[{ }_{3} k_{u w}^{M \tau}\right]\right]^{-1}\left[{ }_{3} k_{u u}^{M \tau}\right]\left[\begin{array}{c}
u_{g 1} \\
u_{g 2} \\
\vdots \\
u_{g m-1}
\end{array}\right]
$$

Thus, one obtains the transformation matrix concerning the membrane shear constraint, $\left[{ }_{4} U\right]$ :

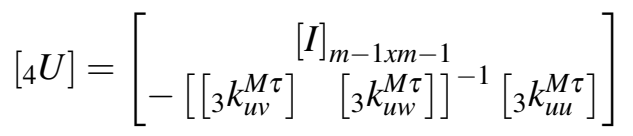

Similar to the transverse elongation constrain, it is possible to confirm the values of the constraint matrix $\left[{ }_{4} U\right]$ when applied in the transverse shear membrane stiffness matrix, $\left[{ }_{3} D\right]^{M}$. As a result, one must obtain a reduced order and null matrix $\left[{ }_{4} D\right]^{M}$ as:

$$
\left[{ }_{4} D\right]^{M}=\left[{ }_{4} U\right]^{T}\left[{ }_{3} D\right]^{M}\left[{ }_{4} U\right]=0
$$

Consequently, the membrane behavior has only the stiffness matrix concerning the longitudinal strain elongation:

$$
\left[{ }_{4} C\right]^{M}=\left[{ }_{4} U\right]^{T}\left[{ }_{3} C\right]^{M}\left[{ }_{4} U\right]
$$

This stiffness matrix is necessary for the analysis of membrane-plate modes, requiring the participation of stiffness of the plate behavior.

\subsubsection{Plate modes and constraints}

Similar to the membrane stiffness matrices, one must split the modes of plate behavior into two groups: the first one concerning pure plate behavior; the second one concerning the mixed membrane-plate behavior.

Pure plate modes:

The pure plate modes have no stiffness contribution from the membrane behavior. I.e., if the application of the pure plate's modal eigenvectors into the stiffness matrices of membrane behavior, then the outcome stiffness must be zero. Example of this mode are the torsion stiffness of angle profiles [52].

The stiffness from the longitudinal strains satisfies the above condition directly. In fact, the displacement degrees of freedom of plate behavior has no longitudinal displacement terms and the matrix $[C]^{M}$ is solely related to this type of degrees of freedom behavior. 
The stiffness from transverse elongation strains, $[B]^{M}$, also disappears. The transverse elongation constraint, which is mandatory in plate behavior, eliminates any possibility of participation of the displacement field of plates in this membrane's stiffness type.

Lastly, the stiffness from shear strains, $[D]^{M}$, must be null for any transverse displacement field that involves the transverse degrees of freedom of membrane behavior.

The above limitations lead to remaining degrees of freedom of longitudinal rotation, $\phi$, and the orthogonal transverse displacement at the extreme node of free end segments. Thus, the transformation matrix to obtain the pure plate modes, $\left.{ }_{5} U\right]$, is nothing less than a regular constraint matrix: an identity matrix, which the removing of the columns related to the degrees of freedom of non-pure plate behavior.

As a result, one obtains the following stiffness matrices:

$$
\begin{aligned}
& \left.\left.\left[{ }_{1} C\right]^{P}={ }_{5} U\right]^{T}\left[\begin{array}{ccc}
{\left[k_{v v}^{P \sigma_{x}}\right]} & {\left[k_{v w}^{P \sigma_{x}}\right]} & {\left[k_{v \phi}^{P \sigma_{x}}\right]} \\
{\left[k_{w v}^{P \sigma_{x}}\right]} & {\left[k_{w w}^{P \sigma_{x}}\right]} & k_{w \phi}^{P \sigma_{x}} \\
{\left[k_{\phi v}^{P \sigma_{x}}\right]} & {\left[k_{\phi w}^{P \sigma_{x}}\right]} & {\left[k_{\phi \phi}^{P \sigma_{x}}\right]}
\end{array}\right]{ }_{5} U\right] \\
& \left.\left.\left[{ }_{1} D\right]^{P}={ }_{5} U\right]^{T}\left[\begin{array}{l}
{\left[k_{v v}^{P \tau}\right]\left[k_{v w}^{P \tau}\right]\left[k_{v \phi}^{P \tau}\right]} \\
{\left[k_{w v}^{P \tau}\right]\left[k_{w w}^{P \tau}\right]\left[k_{w \phi}^{P \tau}\right]} \\
{\left[k_{\phi v}^{P \tau}\right]\left[k_{\phi w}^{P \tau}\right]\left[k_{\phi \phi}^{P \tau}\right]}
\end{array}\right]{ }_{5} U\right]
\end{aligned}
$$

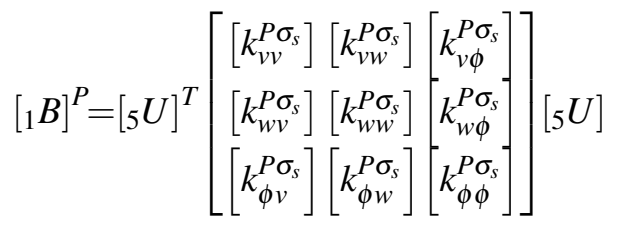

To obtain the modal deformation modes and the generic cross-section properties due to plate behavior, one must solve a quadratic eigenvalue of the three matrices above. This solution and its spectral analysis are presented later, together with the analysis of membrane-plate modes.

Transformation to obtain membrane-plate modes:

The outcome of this subsection is the constraint transformation matrix that highlights the stiffness matrices of membrane-plate modes from the total plate's stiffness matrices.

Here, the key point is the analysis of the transverse bending moment, ${ }^{i} M_{s}(x)=K^{i} B^{i} V(x)$, given in eq. 2.82. For membrane-plate modes, this transverse bending moment must fulfill the following requirements:

i) there is no transverse bending moment in segments with a free end; 
ii) all internal/branched nodes must be in equilibrium with their respective transverse bending moments of the neighbor segments.

Figure. 4.5 illustrates a generic opened cross-section with a possible ${ }^{i} M_{s}(x)$ distribution for membrane-plate modes.

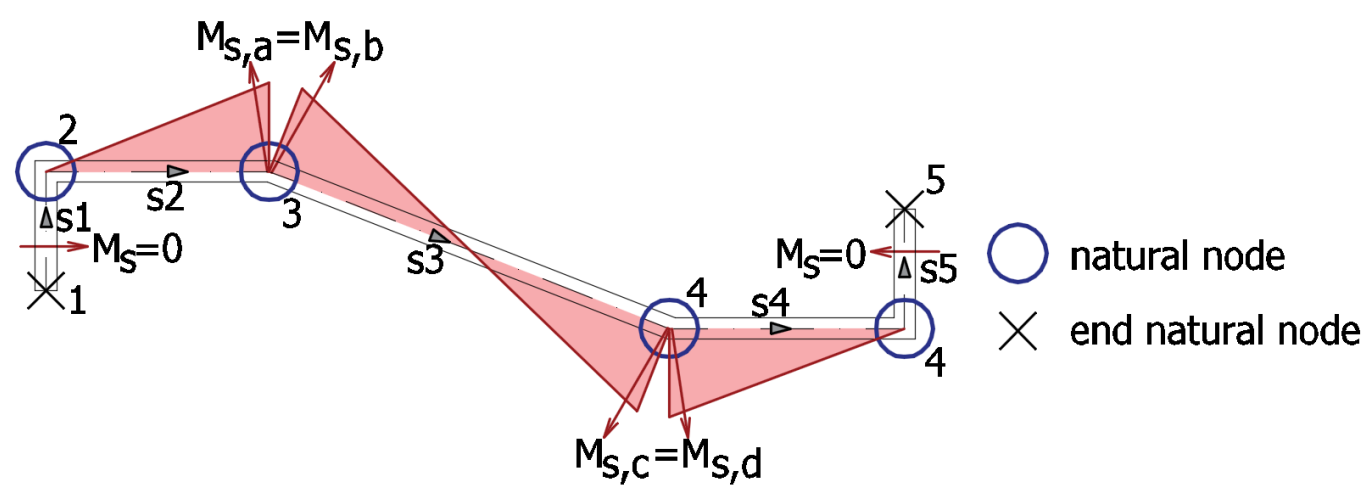

Figure 4.5: transverse bending moment conditions for membrane-plate modes. This bending moment is null in the end segments. Also, at the internal nodes, the equilibrium must be satisfied without any external load.

One can express the above requirements directly by the plate's transverse stiffness matrix of each segment. For instance, an end segment one uses the last two rows of stiffness matrix given in eq. 4.42 to define the null transverse bending moment along with it:

$$
\left[\begin{array}{l}
0 \\
0
\end{array}\right]=\left[\begin{array}{l}
M_{s 1} \\
M_{s 2}
\end{array}\right]_{l}=\frac{E t_{h}^{3}}{l_{s}^{3}}\left[\begin{array}{llll}
l_{s} / 2 & -l_{s} / 2 & l_{s}^{2} / 3 & l_{s}^{2} / 6 \\
l_{s} / 2 & -l_{s} / 2 & l_{s}^{2} / 6 & l_{s}^{2} / 3
\end{array}\right]_{l o}\left[\begin{array}{l}
w_{1} \\
w_{2} \\
\phi_{1} \\
\phi_{2}
\end{array}\right]_{l}
$$

The above expression still requires a transformation from local to global coordinate system in order to insert it fully cross-section coupling matrix.

Concerning the master-slave degree of freedom definition, one realizes that the degrees of freedom of pure plate behavior modes, defined previously, must now be defined as slave ones. Thus, the modes of pure plate and membrane-plate behaviors are complementary to each other.

For an internal node, the stiffness matrices of the segments, whose the degrees of freedom relate to this node, give the compatibility among the transverse bending moments. As an example, node 3 in figure. 4.5 has the compatibility between the transverse bending moments of the final and initial nodes segment $s 2$ and $s 3$, respectively. Therefore, the coupling equation is based on the last row of the transverse stiffness matrix of the segment $s 2$ and the penultimate row of the $s 2$ 's stiffness matrix. In the global coordinate 
system, one achieves:

$$
\frac{E t_{h}^{3}}{l_{s 2}^{3}}\left[\left[k_{\phi v, s 2}^{P \sigma_{s}}\right]\left[k_{\phi w, s 2}^{P \sigma_{s}}\right]\left[k_{\phi \phi, s 2}^{P \sigma_{s}}\right]\right]\left[\begin{array}{c}
v_{2} \\
v_{3} \\
w_{2} \\
w_{3} \\
\phi_{2} \\
\phi_{3}
\end{array}\right]^{T}=\frac{E t_{h}^{3}}{l_{s 3}^{3}}\left[\left[k_{\phi v, s 3}^{P \sigma_{s}}\right]\left[k_{\phi w, s 3}^{P \sigma_{s}}\right]\left[k_{\phi \phi, s 3}^{P \sigma_{s}}\right]\right]\left[\begin{array}{c}
v_{3} \\
v_{4} \\
w_{3} \\
w_{4} \\
\phi_{3} \\
\phi_{4}
\end{array}\right]^{T}
$$

Performing and collecting these coupling equations among all cross-section segments, one reaches the transformation matrix $\left.{ }_{6} U\right]$, which extracts the membrane-plate behavior from the total plate stiffness matrices:

$$
\begin{aligned}
& \left.\left.{ }_{2} C\right]^{P}={ }_{6} U\right]^{T}\left[\begin{array}{ccc}
{\left[k_{v v}^{P \sigma_{x}}\right]} & {\left[k_{v w}^{P \sigma_{x}}\right]} & {\left[k_{v \phi}^{P \sigma_{x}}\right.} \\
{\left[k_{w v}^{P \sigma_{x}}\right]} & {\left[k_{w w}^{P \sigma_{x}}\right]} & k_{w \phi}^{P \sigma_{x}} \\
{\left[k_{\phi v}^{P \sigma_{x}}\right]} & {\left[k_{\phi w}^{P \sigma_{x}}\right]} & {\left[k_{\phi \phi}^{P \sigma_{x}}\right]}
\end{array}\right]{ }_{\left.{ }_{6} U\right]}
\end{aligned}
$$

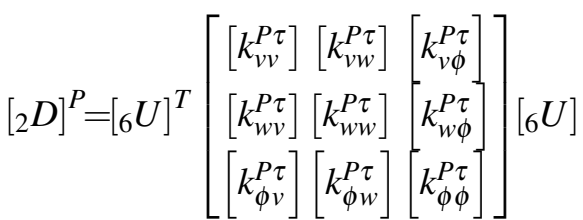

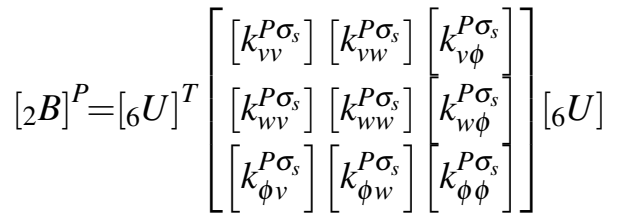

Since plate theory has no stiffness due to elongation in the segment direction, the constraint relationship used to eliminate the transverse membrane elongation is required here as well. Thus, one obtains:

$$
\begin{aligned}
& {\left[{ }_{3} C\right]^{P}=\left[{ }_{1} U\right]^{T}\left[{ }_{2} C\right]^{P}\left[{ }_{1} U\right]} \\
& {\left[{ }_{3} D\right]^{P}=\left[{ }_{1} U\right]^{T}\left[{ }_{2} D\right]^{P}\left[{ }_{1} U\right]} \\
& \left.\left[{ }_{3} B\right]^{P}=\left[{ }_{1} U\right]^{T}\left[{ }_{2} B\right]^{P}{ }_{1} U\right]
\end{aligned}
$$

To merge the plate and membrane stiffness matrices, one must transform the three stiffness matrices shown above from the transverse displacements $v$ and $w$ to the longitudinal displacement $u$. This transformation is already defined in eq. 4.66. Thus, one has:

$$
\left[{ }_{7} U\right]=-\left[\left[{ }_{3} k_{u v}^{M \tau}\right] \quad\left[{ }_{3} k_{u w}^{M \tau}\right]\right]^{-1}\left[{ }_{3} k_{u u}^{M \tau}\right]
$$

And plate stiffness matrices have a new transformation:

$$
\left[{ }_{4} C\right]^{P}=\left[{ }_{7} U\right]^{T}\left[{ }_{3} C\right]^{P}\left[{ }_{7} U\right]
$$




$$
\begin{aligned}
& {\left[{ }_{4} D\right]^{P}=\left[{ }_{7} U\right]^{T}\left[{ }_{3} D\right]^{P}\left[{ }_{7} U\right]} \\
& \left.\left[{ }_{4} B\right]^{P}=\left[{ }_{7} U\right]^{T}\left[{ }_{4} B\right]^{P}{ }_{\left.{ }_{7} U\right]} U\right]
\end{aligned}
$$

Hence, a simple superposition of membrane and plates stiffness matrices leads to the complete stiffness matrices of membrane-plate behavior:

$$
\begin{gathered}
{\left[{ }_{1} C\right]^{M P}=\left[{ }_{4} C\right]^{M}+\left[{ }_{4} C\right]^{P}} \\
{\left[{ }_{1} D\right]^{M P}=\left[{ }_{4} D\right]^{M}+\left[{ }_{4} D\right]^{P}=\left[{ }_{4} D\right]^{P}} \\
{\left[{ }_{1} B\right]^{M P}=\left[{ }_{1} B\right]^{M}+\left[{ }_{4} B\right]^{P}=\left[{ }_{4} B\right]^{P}}
\end{gathered}
$$

The above expressions use the assumptions of null shear and transverse elongation of membrane behavior, $\left[{ }_{4} D\right]^{M}=\left[{ }_{1} B\right]^{M}=0$.

\subsubsection{Mode 2, 3 and 4 - Pure longitudinal bending and Torsion}

As one obtains the stiffness matrices of membrane-plate behavior, it is necessary to identify the modes related to rigid-body transverse displacements: two related to bending, modes 2 and 3; one related to torsion, mode 4 . The combined identification of these modes is particularly useful in the assessment of the major/minor inertia axis and the shear center.

The pure longitudinal bending modes, which are related to Bernoulli-Euler beam's hypothesis, have no shear and transverse distortion cross-section stiffness contribution, $\left[{ }^{2,3} D\right]=0$ and $\left[{ }^{2,3} B\right]=0$, respectively. Consequently, one must extract these modes before the solution of GBT's quadratic eigenvalue problem.

Fortunately, the extraction of these modes is straightforward: two unit displacement vectors, one in each transverse direction, express the longitudinal bending modes. These unit displacement vectors need to be orthogonal to each other, but not necessarily in the main direction of bending. Thus, the simplest forms of these vectors are based on the global coordinates $\mathrm{Y}$ and $\mathrm{Z}$, as shown in figure 4.4:

$$
\begin{aligned}
& { }^{2}[\mathbb{V}]=\left[\begin{array}{llllll}
{ }^{2} v_{g 1}=1 & { }^{2} v_{g 2}=1 & \ldots & { }^{2} w_{g 1}=0 & { }^{2} w_{g 2}=0 & \ldots
\end{array}\right]^{T} \\
& { }^{3}[\mathbb{V}]=\left[\begin{array}{llllll}
{ }^{3} v_{g 1}=0 & { }^{3} v_{g 2}=0 & \ldots & { }^{3} w_{g 1}=1 & { }^{w} w_{g 2}=1 & \ldots
\end{array}\right]^{T}
\end{aligned}
$$

Since previous constraint operations removed several degrees of freedom, the above vectors require only the master degrees of freedom.

Other initial trial representations of mode 2 and mode 3 can be assumed. It is only necessary to be aware that they must be consistent with the right-hand coordinate system. Since both vectors are in the orthogonal basis of the transverse displacements $v$ and $w$, it is necessary to convert them to the longitudinal basis $u$. This transformation is directly obtained by the pre-multiplication by matrix $\left.{ }_{7} U\right]^{-1}$. Thereby, these two vectors can now compose a new transformation matrix:

$$
\left[{ }_{8} U\right]=\left[{ }_{7} U\right]^{-1}\left[{ }^{2}[\mathbb{V}] \quad{ }^{3}[\mathbb{V}]\right]
$$


which leads to the second order tensor of inertia of bending, [I]:

$$
[\mathbb{I}]=\left[{ }_{8} U\right]^{T}\left[{ }_{1} C\right]^{M P}\left[{ }_{8} U\right]
$$

Thus, a standard eigenvalue problem [173] leads to the major/minor bending direction:

$$
[[\mathbb{I}]-\lambda[I]]\left[u_{g}\right]=0
$$

Which the eigenvectors of major/minor directions are:

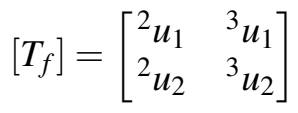

Thus, one reaches longitudinal displacements for mode 2 and 3:

$$
\left.{ }^{2}[U]{ }^{3}[U]\right]=\left[T_{f}\right]\left[{ }_{8} U\right]
$$

Before the application of the above vectors as a matrix transformation, it is convenient to achieve the shear center, from which the identification is based on radius vector of longitudinal rotation, $r(s)$.

According to Vlasov beam theory, the radius vector is the distance from the shear center until the perpendicular point in the segment middle line, as shown in figure 4.6.a. However, in order to achieve the transverse displacement in each node, the radius vector's definition is related to the nodes. I.e., the nodal radius vector is decomposed in terms of the global coordinate system, as shown in figure 4.6.b, and the node's transverse displacements are perpendicular to their respective radius vector, according to a right-hand system. Thus, the proportion of the radius vector defines the magnitude of transverse displacement.
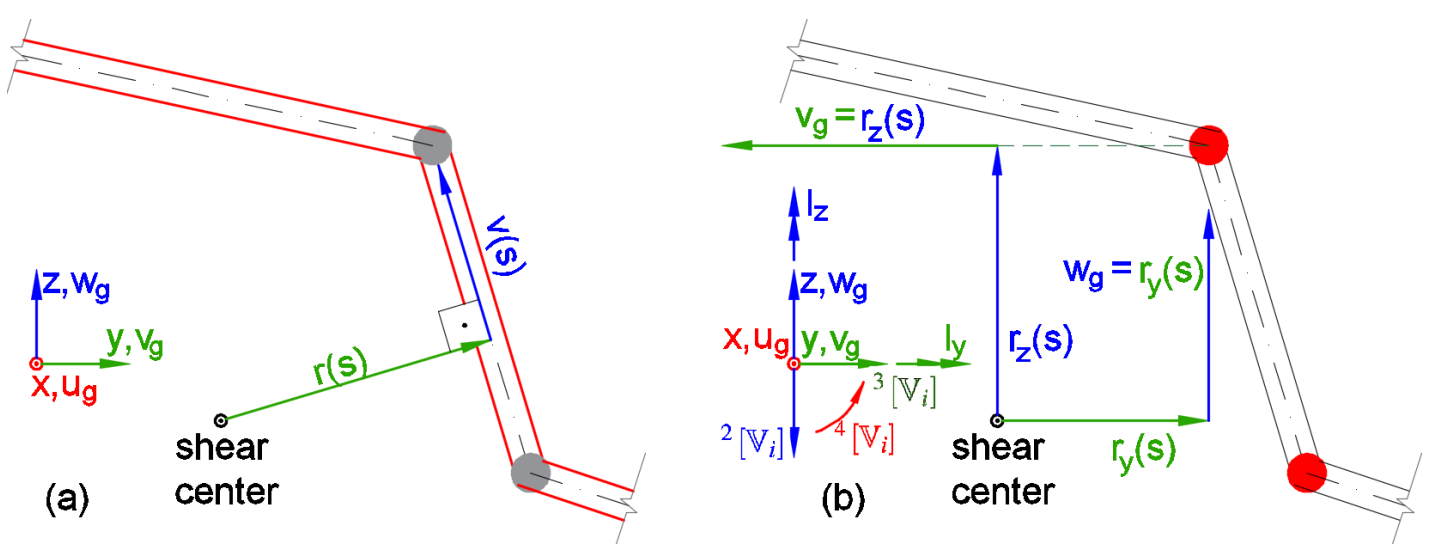

Figure 4.6: Radius vector: a) according to classical Vlasov Beam Theory; b) according to GBT's identification

Following this definition, one can set up a trial displacement vector for the torsion mode. Initially, any point of cross-section's plan can receive the trial shear center, and 
one adopts a temporary coordinate system. It is not required from this temporary coordinate system to be in the same basis of the principal moments of inertia. It is only necessary to obey a right-hand system. However, there is an important detail concerning the orientation's consistency: the radius vector has its coordinate system defined in the directions of the transverse displacements due to longitudinal bending moments. The example in figure 4.6.b, that uses a temporary coordinate system in the global coordinate system, clarifies this definition: i) a positive bending around the global coordinate axis $\mathrm{Y}$ has the transverse displacement in the global direction $-Z$. Therefore, this direction defines the origin of the longitudinal rotation angle $\phi_{g}$; ii) a positive bending around the global coordinate axis $\mathrm{Z}$ has the transverse displacement in the global direction $+Y$. This direction defines a right-hand system concerning the origin of the longitudinal angle $\phi_{g}$.

After the definition of the initial trial shear center, the temporary coordinate system and the origin of the longitudinal rotation angle, one obtains an initial trial transverse displacement vector for mode 4 :

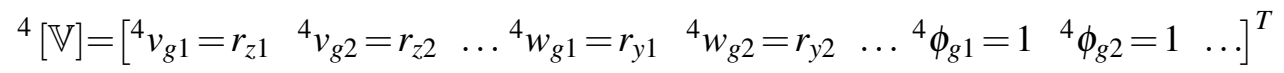

Similar to the trial vectors for bending modes, the master degrees of freedom, which remain after the previous constraint operations, are enough to represent the trial vector of mode 4. The transformation $\left[{ }_{7} U\right]^{-14}[\mathbb{V}]$ converts this vector into longitudinal displacements. Together with the vectors in eq. 4.94, it is possible to build a new transformation matrix:

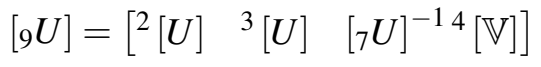

As in the case of eq. 4.91, it provides a new second order tensor [173] that has not only the bending stiffness terms in principal directions, but also the Vlasov's warping stiffness terms concerning these directions:

$$
\left[\mathbb{I}_{\text {Vlasov }}\right]=\left[{ }_{9} U\right]^{T}\left[{ }_{1} C\right]^{M P}\left[{ }_{9} U\right]=E\left[\begin{array}{ccc}
I_{22} & 0 & I_{2 \omega} \\
0 & I_{33} & I_{3 \omega} \\
I_{\omega 2} & I_{\omega 3} & I_{\omega \omega}
\end{array}\right]
$$

where, $I_{\omega \omega}$ is the inertia of warping, or usually namely constant of warping. Also, $I_{2 \omega}$ and $I_{3 \omega}$ are the product of sectorial inertia. According to Vlasov, [176], the radius vector between the trial and real shear centers is obtained by:

$$
r v_{c s}=I_{2 \omega} / I_{22}
$$

$$
r w_{c s}=I_{3 \omega} / I_{33}
$$

From this radius vector, one achieves longitudinal displacement vector of mode 4 :

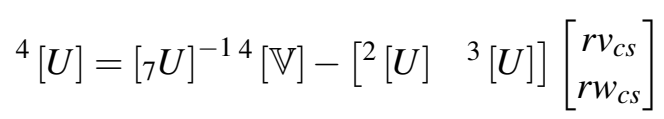

The collection of ${ }^{2}[U],{ }^{3}[U]$ and ${ }^{4}[U]$ leads to transformation matrix $\left[{ }_{10} U\right]$ :

$$
\left[{ }_{10} U\right]_{n-1 x n-1}=\operatorname{Subst}\left({ }_{2} \operatorname{Cols}_{1,2,3}{ }_{[U]}^{3}[U]{ }_{[U]}\right)[I]_{n-1 x n-1}
$$


This transformation stands out the null shear and transverse stiffness of Bernoulli-Euler modes:

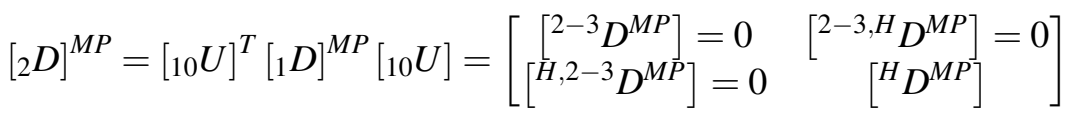

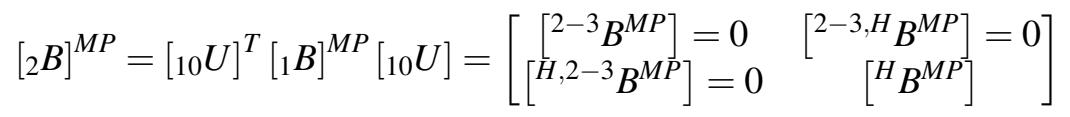

Here, the upper-left index ${ }^{H}$ is used to indicate any mode higher than 3.

Although the transformation matrix $\left[{ }_{10} U\right]$ leads to the warping, major and minor moments of inertia, this transformation matrix does not reach a full decoupled longitudinal stiffness matrix:

$$
\left[{ }_{2} C\right]^{M P}=\left[{ }_{10} U\right]^{T}\left[{ }_{1} C\right]^{M P}\left[{ }_{10} U\right]=\left[\begin{array}{cc}
{\left[{ }^{2-3} C^{M P}\right]} & {\left[{ }^{2-3, H} C^{M P}\right]} \\
{\left[{ }^{2} C^{M P}\right]} & {\left[{ }^{H} C^{M P}\right]}
\end{array}\right]
$$

From the first row of sub-matrices in the above expression, one achieves the following multi-freedom constraint:

$$
\left[{ }^{2-3} C^{M P}\right]\left[\begin{array}{l}
u_{1} \\
u_{2}
\end{array}\right]+\left[{ }^{2-3, H} C^{M P}\right]\left[\begin{array}{c}
u_{3} \\
u_{4} \\
\vdots
\end{array}\right]=\left[\begin{array}{l}
0 \\
0
\end{array}\right]
$$

Hence, this multi-freedom constraint leads to a transformation matrix, which not only uncouples the Bernoulli-Euler modes, but also isolates the higher modes:

$$
\left[{ }_{11} U\right]=\left[\begin{array}{c}
-\left[{ }^{2-3} C^{M P}\right]^{-1}\left[{ }^{2-3, H} C^{M P}\right] \\
{[I]_{m-3 x m-3}}
\end{array}\right]
$$

Now, it is possible to extract the orthogonal ordinary differential equation of BernoulliEuler modes from modes 2 and 3, leading to:

$$
{ }^{2} \delta U_{\text {int }}=E^{2} C \int_{L}{ }^{2} V_{, x x}(x)^{2} \delta V_{, x x}(x) \mathrm{d} x \quad(4.107){ }^{3} \delta U_{\text {int }}=E^{3} C \int_{L}^{3} V_{, x x}(x)^{3} \delta V_{, x x}(x) \mathrm{d} x
$$

It is essential to highlight that mode 4 is still coupled to the other higher modes. The eigenvector obtained in ${ }^{4}[U]$ is not a general solution $[95,96]$, which requires the solution of quadratic eigenvalue problem based on the cross-section stiffness matrices of membrane-plate behavior:

$$
\begin{aligned}
& {\left[{ }_{3} C\right]^{P M}=\left[{ }_{11} U\right]^{T}\left[{ }_{2} C\right]^{P M}\left[{ }_{11} U\right]} \\
& \left.\left[{ }_{3} D\right]^{M P}=\left[{ }_{11} U\right]^{T}\left[{ }_{2} D\right]^{M P}{ }_{{ }_{11} U}\right] \\
& {\left[{ }_{3} B\right]^{M P}=\left[{ }_{11} U\right]^{T}\left[{ }_{2} B\right]^{M P}{ }_{\left.{ }_{11} U\right]}}
\end{aligned}
$$




\subsection{Quadratic eigenvalue solution}

\subsubsection{Symmetric and anti-symmetric modes}

Before the development of the quadratic eigenvalue problem, it is interesting to shed light on one property concerning the solution used by Schardt, [136], and its effect on separation of symmetric and anti-symmetric modes.

Schardt did not solve the quadratic eigenvalue. Instead, he applied Falk-Langemeyer method, [58], to solve a generic eigenvalue problem concerning only the matrices $[C]$ and $[B]$. Thus, $[D]$ was never a simple diagonal matrix. Schardt advocated for this solution, since the off-diagonal terms of matrix $[D]$ are usually small and do not affect the final solution, especially for open thin-walled cross-sections.

Similar to the cross-section analysis developed by J.M.Andreassen and J.Jönsson, [97, 98, 99], here the solution is addressed directly to the quadratic eigenvalue problem. However, the traditional approach of Schardt is still useful: for symmetric, or at least mono-symmetric cross-sections, the Falk-Langemeyer method applied in the matrices $[C]$ and $[B]$ highlights the separation of symmetric and anti-symmetric modes in matrix $[D]$. Hence, the Falk-Langemeyer leads to transformation matrix $\left[{ }_{12} U\right]$. Following the previous transformations, one obtains:

$$
\begin{aligned}
& {\left[{ }_{4} C\right]^{P M}=\left[{ }_{12} U\right]^{T}\left[{ }_{3} C\right]^{P M}\left[{ }_{12} U\right]} \\
& {\left[{ }_{4} D\right]^{M P}=\left[{ }_{12} U\right]^{T}\left[{ }_{3} D\right]^{M P}\left[{ }_{12} U\right]} \\
& {\left[{ }_{4} B\right]^{M P}=\left[{ }_{12} U\right]^{T}\left[{ }_{3} B\right]^{M P}\left[{ }_{12} U\right]}
\end{aligned}
$$

In matrix $\left[{ }_{4} D\right]^{M P}$ one can verify a "chess-pattern" in the off-diagonal values. I.e., nonnull values are interleaved with null values. Based on this feature, one re-order the rows and columns by a new transformation matrix, $\left[{ }_{13} U\right]$, which leads to the following representation:

$$
\begin{aligned}
& \left.\left[{ }_{5} C\right]^{M P}=\left[\begin{array}{cc}
{\left[{ }_{5} C_{a}^{M P}\right]} & {[0]} \\
{[0]} & {\left[{ }_{5} C_{b}^{M P}\right]}
\end{array}\right]=\left[{ }_{13} U\right]^{T}\left[{ }_{4} C\right]^{M P}{ }_{13} U\right] \\
& {\left[{ }_{5} D\right]^{M P}=\left[\begin{array}{cc}
{\left[{ }_{5} D_{a}^{M P}\right]} & {[0]} \\
{[0]} & {\left[{ }_{5} D_{b}^{M P}\right]}
\end{array}\right]=\left[{ }_{13} U\right]^{T}\left[{ }_{4} D\right]^{M P}\left[{ }_{13} U\right]} \\
& {\left[{ }_{5} B\right]^{M P}=\left[\begin{array}{cc}
{\left[{ }_{5} B_{a}^{M P}\right]} & {[0]} \\
{[0]} & {\left[{ }_{5} B_{b}^{M P}\right]}
\end{array}\right]=\left[{ }_{13} U\right]^{T}\left[{ }_{4} B\right]^{M P}\left[{ }_{13} U\right]}
\end{aligned}
$$

Here, the sub-indexes $a$ and ${ }_{b}$ indicate the subgroups related to symmetric or antisymmetric modes. Since mode 4 is anti-symmetric, the subgroup that has this mode is the anti-symmetric one.

Other benefits of this transformation are: i) the reduction size of the quadratic eigenvalue problem; ii) the simplification in the selection of conjugated eigenvectors, in the case of two real roots obtained in the quadratic eigenvalue problem.

This transformation is also applied to pure plate behavior modes. 


\subsubsection{Linearization and quadratic pencil}

As one achieves the most compact form of the quadratic eigenvalue problem of GBT:

$$
Q(\lambda) u:=\left(\lambda^{2}[C]-\lambda[D]+[B]\right)[u]=0
$$

Then, it is possible to linearize it by a quadratic pencil transformation, such as

$$
L(\lambda ; C, D, B) z:=\left(\left[\begin{array}{cc}
-[D] & {[C]} \\
{[C]} & {[0]}
\end{array}\right] \lambda+\left[\begin{array}{cc}
{[B]} & {[0]} \\
{[0]} & -[C]
\end{array}\right]\right)[z]=0
$$

where:

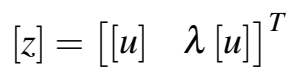

The first row of eq. 4.119 has the definition of the GBT's quadratic eigenvalue problem and the second row of eq. 4.119 is set up to achieve the so-called structure preserving transformations. As clarified and presented in [62], several other possibilities exist in the linearization of the quadratic eigenvalue problem. For GBT, that leads invariably to a set of complex and real eigenvectors.

It is important to note that, different from the general eigenvalue problem, in which a single eigenvector leads to a decoupled ordinary differential equation, in the quadratic eigenvalue problem there are two eigenvectors to each ordinary differential equation. In other words, the quadratic eigenvalue problem shows that the high order generalized inertias ${ }^{i} C,{ }^{i} D$ and ${ }^{i} B$ have two possible conjugated modes, here named $\left[{ }_{i} u\right]$ and $[i i u]$.

For complex eigenvector, the identification of conjugated modes is straight forward, since conjugated modes share the same real part of the eigenvector and different values in the imaginary part. However, if there is more than four real eigenvectors, the definition of each pair of conjugated eigenvectors is arbitrary. I.e., any possible combination will lead to an equivalent orthogonal system. The only exception is the Vlasov torsion mode, which has almost the same real eigenvectors. One identifies the first eigenvector of this mode based on the respondent null eigenvalue. Then, it is possible to recognize the second eigenvector by the almost unit solution of the inner product to the first eigenvector.

Thus, the conjugated transformation achieves the cross-section matrices:

$$
\begin{aligned}
& \left.\left[{ }_{6} C\right]^{M P}=\frac{1}{2}\left[{ }_{i} U\right]^{T}\left[{ }_{5} C\right]^{M P}\left[{ }_{i i} U\right]+\left[{ }_{i i} U\right]^{T}\left[{ }_{5} C\right]^{M P}\left[{ }_{i} U\right]\right] \\
& {\left[{ }_{6} D\right]^{M P}=\frac{1}{2}\left[\left[{ }_{i} U\right]^{T}\left[{ }_{5} D\right]^{M P}\left[{ }_{i i} U\right]+\left[{ }_{i i} U\right]^{T}\left[{ }_{5} D\right]^{M P}\left[{ }_{i} U\right]\right]} \\
& {\left[{ }_{6} B\right]^{M P}=\frac{1}{2}\left[\left[{ }_{i} U\right]^{T}\left[{ }_{5} B\right]^{M P}\left[{ }_{i i} U\right]+\left[{ }_{i i} U\right]^{T}\left[{ }_{5} B\right]^{M P}\left[{ }_{i} U\right]\right]}
\end{aligned}
$$

One realizes from equations 4.121, 4.122 and 4.123, that the imaginary part of complex eigenvector has vanished.

Although these three matrices lead to the generalized properties of the cross-section, none of them are necessarily diagonal matrices [103]. Thus, it implies in a residual coupling among the deformation modes. 


\subsubsection{Recover of the eigenvectors in the original coordinate sys- tem}

After the achievement of the solution of quadratic eigenvalue problem, one must transform the resulting eigenvectors back to the original coordinate system. Therefore, one must apply the sequence of matrix transformations in backward order.

For instance, the eigenvectors of modes 2 and 3 require the following sequence of multiplication:

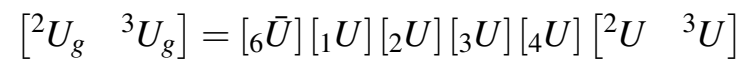

Since the matrix $\left[{ }_{6} U\right]$ has no terms related to the degrees of freedom of longitudinal displacement, it receives an identity matrix on these degrees of freedom and $\left[{ }_{6} \bar{U}\right]$ represents this adjustment.

For membrane-plate modes, the backward transformation is a bit longer:

$$
\left[U_{i, a, \text { real }}\right]=\left[{ }_{6} \bar{U}\right]\left[{ }_{1} U\right]\left[{ }_{2} U\right]\left[{ }_{3} U\right]\left[{ }_{4} U\right]\left[{ }_{10} U\right]\left[{ }_{11} U\right]\left[{ }_{12} U\right]\left[{ }_{13} U\right]\left[\begin{array}{c}
{\left[U_{i}^{M P}\right]} \\
{[0]}
\end{array}\right]
$$

The imaginary part and the second eigenvector follows the same transformation.

Concerning the pure plate behavior modes, one reaches the following transformation:

$$
\left[U_{i, a, \text { real }}\right]=\left[{ }_{5} U\right]\left[{ }_{12} U^{P}\right]\left[{ }_{13} U^{P}\right]\left[\begin{array}{c}
{\left[U_{i}^{P}\right]} \\
{[0]}
\end{array}\right]
$$

The last observation concerns mode 1 . In a practical point of view, this mode does not demand any transformation because none of the transformations affect its trivial definition as a unit longitudinal displacement with no transverse displacement, as presented in the first columns of matrix $\left[{ }_{2} U\right]$, eq. 4.55 .

A detailed example, presented at the appendix, clarifies not only this transformation but also the whole procedure presented here. 


\section{GBT's finite element in longitudinal direction}

After the cross-section analysis developed in Chapters 3 and 4, each deformation shape will lead to an ordinary differential equation, something entirely independent of the other deformation shapes in the linear analysis. Thus, this chapter is dedicated to study and to obtain a solution of each ordinary differential equation of each deformation mode.

Initially, it is presented a short review of the methods applied in the solution of GBT's differential equations. Starting from the Finite Differences Method, used by R. Schardt [131, 139], until the recent developments in Finite Element Method, FEM.

Also, this chapter presents a novel approach in the Finite Element Method to reach the exact solution in GBT problems. Among the benefits of this novel approach, one can list: i) it avoids longitudinal discretization; ii) it reduces the numerical and computation effort to obtain the GBT' solution; iii) it leads to smoother displacement and stress fields; iv) for constant internal loads, it also leads to the exact solution in non-linear analysis.

In order to obtain the exact solution, there are two new concepts that need to be presented.

The first one concerns the assortment of the GBT's ordinary differential equation. There is not only one type of ordinary differential equation in GBT, but there are several of them. It is interesting to note that this classification is not only requested by a mathematical point of view, but also has a mechanical interpretation, as it will be discussed later.

The second one is related to the development of the exact stiffness matrix in the Finite Element Method. Since GBT involves sophisticated functions in its analytical solutions, a substantial mathematical effort is required to reach the shape functions and the exact stiffness matrix. Even by computational symbolic analysis, these results are hard to be found. To overcome this difficulty, it is presented here the concept of developing 
the exact stiffness matrix by the new matrices: Completeness Coefficient Matrix and Kernel Stiffness Matrix.

A final and detailed example clarifies the novel approach proposed here. Besides, it compares the pros and cons of the novel approach and the one based on the well-known Hermitian shape functions.

\subsection{Review of the applied solution methods in GBT's ordi- nary differential equations}

In the original works of Richard Schardt and his co-workers, the ordinary differential equations were evaluated by the finite difference method [131, 139]. Although this method has a clear and direct numerical solution, it has been replaced by the finite element method (FEM) in structural analysis, owing to the well-known versatility of this method [151].

The first application of FEM in GBT was performed by J.M.Davies [49], who proposed the use of an exact element. However, the formulation presented in this element has some controversial points: i) there is not a clear development of the stiffness matrix. Davies does not present a variational formulation and a detailed study of the internal strain energy. Instead, he only presents an adaptation from a beam on elastic foundation problem into GBT analysis; ii) he does not include the stiffness components of transverse bending in the complete stiffness matrix, i.e., the transverse bending inertia, ${ }^{i} B$, is neglected. Therefore, high deformation mode, which presents a high distortion and a lower amount of warping, have coarse results; iii) the shape function used in the formulation is based on the homogeneous solution, a linear independent function of the most usual load functions, such as constant loading distribution.

In 2001, N. Silvestre and D. Camotim [151] developed a finite element based on Hermitian shape functions. This method has been extensively applied in many studies and analyses, such as [152], [81], [39], [2], and [3]. Although this type of element has good convergence and allows easy implementation of the stiffness matrix, it does not reach an exact solution and it also requires longitudinal discretization.

Here, it is essential to observe the longitudinal discretization in GBT. Since GBT requires a high number of degrees of freedom in each node, if a beam structure also requires a high number of elements and nodes to be modeled, then the total number of degrees of freedom and unknowns of GBT will be no different from the total amount needed in a usual shell elements modeling. Thus, one must avoid or at least minimize the longitudinal discretization in order to obtain the high computational performance of GBT [77].

Moreover, the generalized internal force obtained from Hermitian shape functions presented a coarse result due to the higher order derivatives that are necessary to achieve it. These issues affect the non-linear analysis, i.e. the initial stress stiffness matrix [35]. 
In this case, an even higher longitudinal discretization is required.

As an alternative to overcome these coarse results in higher order problems, Duan [55] presents the formulation of a B-splines-based GBT, which provides continuity between two adjacent elements. However, this alternative still requires longitudinal discretization.

Recently, Gonçales and Eisenberger, [25, 26, 28], developed an exact finite element solution for the ordinary differential equation of GBT based on Power Series Method. In this approach, an exact solution is obtained not by a predefined and parametric stiffness matrix, but by a posterior numerical stiffness matrix, using the recursive property of Power Series. Thus, for each ordinary differential equation of each deformation mode of GBT, this approach requires an iterative solution of a system of linear equations. As each beam element has four degrees of freedom, the system of the linear equation has the same size. Furthermore, one repeats the iterative-recursive approach until the limit of the computational numerical precision.

On one hand, the stiffness matrix, based on Power Series, reaches the exact solution and it requires any longitudinal discretization. On another hand, it involves an extra numerical effort to obtain the computational exact stiffness matrix.

In order to avoid this extra numerical effort and obtain a predefined exact stiffness matrix, this dissertation presents a novel approach, based on two concepts. The first one is the classification of GBT's ordinary differential equation, involving not only a mathematical assortment, but also a mechanical behavior. The second concept is the separation of shape functions and stiffness matrix into two major terms: a kernel one, which is a set of functions obtained from the analytical solution of the ordinary differential equation; and a coefficient completeness matrix, imposed by the Completeness Principle of Finite Element Method.

In next sections, these two concepts will be detailed.

\subsection{Shape function assortment and transverse deformation mode classification}

The development of shape functions that describes the exact displacement field of GBT is initially based on the analytical solution of the ordinary differential equation of each GBT's deformation mode.

It is important to note that, in GBT's high deformation modes, the ordinary differential equation can lead to different types of solution based on the value of generic crosssection properties: ${ }^{i} C,{ }^{i} D$ and ${ }^{i} B$. Here, the terms related to ${ }^{i} V_{, x x}(x)$ in eq. 2.63 are simplified into a single equivalent term of shear stiffness as:

$$
\overline{G D}=G\left({ }^{i} D^{M c}+{ }^{i} D^{P}\right)-2 \mu K^{i} D^{\mu}
$$


Thus, it is indispensable to perform a modal classification concerning not only the type of respective ordinary differential equation, but also its solution. Each one of these classifications are presented in the following subsections.

\subsubsection{Classification of GBT's deformation modes concerning the ordinary differential equation}

As presented in the previous chapters, the lower deformation modes, involving rigid body movements of the cross-section, have a well-known related ordinary differential equation. However, for the high modes, their related ordinary differential equations are unusual in structural analysis.

One can divide these ordinary differential equations for high modes into four types, whose nomenclature is based on kinematic behavior:

- Distortion with warping: This type of deformation shape involves all stiffness terms of the cross-section: ${ }^{i} C,{ }^{i} D$ and ${ }^{i} B$.

$$
E^{i} C^{i} V_{, x x x x}(x)-{ }^{i} \overline{G D}^{i} V_{, x x}(x)+K^{i} B^{i} V(x)={ }^{i} q(x)
$$

Schardt studied only this case in his initial work, [136]. Since there is longitudinal stress in this type of deformation modes, they are relevant in nonlinear analysis;

- Distortion without warping: This particular deformation shape has no longitudinal strain or stress. Therefore, the only displacement is concerning the transverse displacement, which is a combination of longitudinal rotation of the same segments and transverse bending of other segments of the cross-section. The ordinary differential equation is:

$$
-{ }^{i} \overline{G D}^{i} V_{, x x}(x)+K^{i} B^{i} V(x)={ }^{i} q(x)
$$

- Local distortion with warping: This unique deformation shape happens only in specific cross-section, such as the radial elongation of hollow circular cross-section, as presented at the end of subsection 3.1.1. Usually, the longitudinal displacement and stress are only due to plate behavior. The ordinary differential equation for this case is:

$$
E^{i} C^{i} V_{, x x x x}(x)+K^{i} B^{i} V(x)={ }^{i} q(x)
$$

- Local distortion without warping: The simplest ordinary differential equation occurs in this case of deformation mode, which has no longitudinal displacement or stress and no type of transverse displacement due to longitudinal rotation or pure transverse displacement, i.e., there is only transverse bending in cross-section's segments. The ordinary differential equation is:

$$
K^{i} B^{i} V(x)={ }^{i} q(x)
$$

Table 5.1 lists each GBT's ordinary differential equation, as well as the usual ordinary differential equations of rod, Euler-Bernoulli, Vlasov and Bredt-Saint Venant torsion beam theories: 
Table 5.1: Ordinary Differential Equation of GBT

\begin{tabular}{cc}
\hline Kinematic behavior & Diff. Equation \\
\hline Pure radial elongation & $E^{1} C^{1} V_{, x x}(x)={ }^{1} q(x)$ \\
Major bending & $E^{2} C^{2} V_{, x x x x}(x)={ }^{2} q(x)$ \\
Minor bending & $E^{3} C^{3} V_{, x x x}(x)={ }^{3} q(x)$ \\
Non-uniform torsion & $E^{4} C^{4} V_{, x x x x}(x)-G^{4} D^{4} V_{, x x}(x)={ }^{4} q(x)$ \\
Uniform torsion & $-G^{4} D^{4} V_{, x x}(x)={ }^{4} q(x)$ \\
distortion with warping & $E^{i} C^{i} V_{, x x x x}(x)-{ }^{i} G D^{i} V_{, x x}(x)+{ }^{i} B^{i} V(x)={ }^{i} q(x)$ \\
distortion without warping & $-{ }^{i} \bar{G} D^{i} V_{, x x}(x)+{ }^{i} B^{i} V(x)={ }^{i} q(x)$ \\
local distortion with warping & $E^{i} C^{i} V_{, x x x}(x)+{ }^{i} B^{i} V(x)={ }^{i} q(x)$ \\
local distortion without warping & ${ }^{i} B^{i} V(x)={ }^{i} q(x)$ \\
\hline
\end{tabular}

\subsubsection{Classification of GBT's deformation modes concerning the solution of ordinary differential equations}

All ordinary differential equations presented in table 5.1 have only one possible case of solution, i.e., in each one of these differential equations, the homogeneous solution leads to a function that is the same, independently of the value of cross-section properties ${ }^{i} C,{ }^{i} D$ and ${ }^{i} B$.

There is only one exception: the case of distortion with warping, in which different values of cross-section properties can lead to different types of homogeneous solution.

In fact, the homogeneous part of the ordinary differential equation is:

$$
E^{i} C^{i} V_{, x x x x}(x)-{ }^{i} \overline{G D}^{i} V_{, x x}(x)+{ }^{i} B^{i} V(x)=0
$$

And it has the characteristic equation: $E C^{i} \lambda^{4}-G^{i} D \lambda^{2}+{ }^{i} B=0$, with the following possible roots:

$$
\lambda_{1,2,3,4}= \pm \sqrt{\frac{{ }^{i} \overline{G D}}{2 E^{i} C} \pm \sqrt{\left(\frac{{ }^{i} \overline{G D}}{2 E^{i} C}\right)^{2}-\frac{K^{i} B}{E^{i} C}}}
$$

The internal square root can classify not only the mathematical type of numerical root, but also the main stiffness component in a transverse deformation mode of the crosssection (i.e. torsion or distortion). Therefore, one can derive the following cases:

- Case A: Dominant distortion mode if $\left(\frac{{ }^{i} \overline{G D}}{2 E^{i} C}\right)^{2}<\frac{K^{i} B}{E^{i} C}$ i.e. ${ }^{i} \overline{G D}<2 \sqrt{K^{i} B E^{i} C}$;

Deformation modes, in this case, have the main contribution in total transverse stiffness from the transverse bending of each wall in the cross-section. This case is the most relevant. In fact, it is the most usual case in GBT, and it was the basic case studied in Schardt's work [136]. With two real and one complex conjugate roots, the solution of the homogeneous differential equation is:

$$
V(x)=J_{1} \cosh (\alpha x) \cos (\beta x)+J_{2} \cosh (\alpha x) \sin (\beta x)+J_{3} \sinh (\alpha x) \sin (\beta x)+J_{4} \sinh (\alpha x) \cos (\beta x)
$$


where: $J_{1}, J_{2}, J_{3}$ and $J_{4}$ are constants found by the boundary conditions; and:

$$
\begin{aligned}
& \alpha=\sqrt{\sqrt{\frac{K^{i} B}{4 E^{i} C}}+\frac{{ }^{i} \overline{G D}}{4 E^{i} C}} \\
& \beta=\sqrt{\sqrt{\frac{K^{i} B}{4 E^{i} C}}-\frac{{ }^{i} \overline{G D}}{4 E^{i} C}}
\end{aligned}
$$

- Case B: Dominant torsion mode if $\left(\frac{{ }^{i} \overline{G D}}{2 E^{i} C}\right)^{2}>\frac{K^{i} B}{E^{i} C}$ i.e. ${ }^{i} \overline{G D}>2 \sqrt{K^{i} B E^{i} C}$;

In this case, torsion of each wall in the cross-section is the main contributor in the total transverse stiffness for deformation modes. This case occurs in closed rectangular crosssection [175] and opened cross-sections, especially when the extremity walls are much thicker than the internal walls. With four real roots, the solution of the homogeneous differential equation is:

$$
V(x)=J_{1} \cosh (\zeta x)+J_{2} \sinh (\zeta x)+J_{3} \sinh (\eta x)+J_{4} \cosh (\eta x)
$$

where:

$$
\begin{gathered}
\zeta=\sqrt{\frac{{ }^{i} \overline{G D}}{2 E^{i} C}+\sqrt{\left(\frac{i \overline{G D}}{2 E^{i} C}\right)^{2}-\frac{K^{i} B}{E^{i} C}}} \\
\eta=\sqrt{\frac{{ }^{i} \overline{G D}}{2 E^{i} C}-\sqrt{\left(\frac{{ }^{i} \overline{G D}}{2 E^{i} C}\right)^{2}-\frac{K^{i} B}{E^{i} C}}}
\end{gathered}
$$

- Case C: Critical Torsion-Distortion mode if $\left(\frac{i \overline{G D}}{2 E^{i} C}\right)^{2}=\frac{K^{i} B}{E^{i} C}$ i.e. ${ }^{i} \overline{G D}=2 \sqrt{K^{i} B E^{i} C}$;

In this particular case, torsion and transverse bending of each wall in the cross-section have the same contribution in the total transverse stiffness for deformation modes. This case is the border between the two previously presented cases. With two pairs of identical real roots, the solution of the homogeneous differential equation is:

$$
\begin{gathered}
V(x)=J_{1} x \cosh (l x)+J_{2} x \sinh (\imath x)+J_{3} \cosh (l x)+J_{4} \sinh (\imath x) \\
\text { with: } \imath=\sqrt{\frac{i \overline{G D}}{2 E^{i} C}}
\end{gathered}
$$

\subsubsection{Shape functions based on homogeneous solutions in GBT analysis}

After the classification of possible types of ordinary differential equations, as well as the possible types of solutions of them, one can set up shape function based on the homogeneous solution. 
From table 5.1, the ordinary differential equations concerning the pure longitudinal elongation and uniform torsion have a first order polynomial homogeneous solution:

$$
V(x)=J_{1} x+J_{2} \quad \text { for modes } 1 \text { and } 5
$$

And the major and minor bending moments (modes 2 and 3 ) have a third order polynomial homogeneous solution:

$$
V(x)=J_{1} x^{3}+J_{2} x^{2}+J_{3} x+J_{4} \quad \text { for modes } 2 \text { and } 3
$$

These polynomial functions lead to the well-known Hermitian shape functions.

However, other deformation modes do not have a polynomial solution. For instance, the non-uniform torsion (mode 4) has the homogeneous solution:

$$
V(x)=J_{1} \cosh (\sqrt{2} \imath x)+J_{2} \sinh (\sqrt{2} \imath x)+J_{3} x+J_{4}
$$

where $l$ is given in eq. 5.15. Therefore, the Hermitian shape functions cannot reach the exact solution directly. The exact solution of this mode requires a shape function based on hyperbolic functions that can be found in [107].

The same question arises in other deformation modes, such as distortion with and without warping and local distortion with warping, whose shape functions are based on trigonometric-hyperbolic functions, as presented in equations 5.2, 5.3 and 5.4.

At this point, one can formulate the shape functions based on a homogeneous solution, leading to exact solutions for these modes of GBT. However, there is an issue about distortion modes, which is absent the rod, Euler-Bernoulli and Vlasov deformation modes: the usual external loading functions of beams are linear independents of the homogeneous solution. Since the usual loading in the structural analysis are constant or linear functions in respect to the longitudinal direction of the beam, equations 5.2, 5.3 and 5.4 do not include these loading functions.

Consequently, it is not interesting for GBT's distortion modes, in a practical point of view, to obtain the shape functions based on the homogeneous solution. Instead, one can go directly to the shape functions from the non-homogeneous solution. This also includes the case of a homogeneous solution.

\subsubsection{Shape functions based on non-homogeneous solutions in GBT analysis}

In non-homogeneous differential equations, the general solution is a combination between the homogeneous and a particular solution that represents an external load function. In the most common structural analysis, external load functions are constants or linear functions linearly independent from the homogeneous solutions of equations 5.2, 5.3 and 5.4. Therefore, the function of the exact solution of non-homogeneous equation needs six terms, four from the boundary conditions - $J_{1}, J_{2}, J_{3}$, and $J_{4}$-, for the non-polynomial functions and two extra terms due to loading distribution $-J_{5} x+J_{6}$. 
For instance, the non-homogeneous solution for distortion with warping for these type of loads is:

$$
\begin{aligned}
V(x)=J_{1} \cosh (\alpha x) \cos (\beta x)+J_{2} \cosh (\alpha x) \sin (\beta x) & +J_{3} \sinh (\alpha x) \sin (\beta x)+ \\
& +J_{4} \sinh (\alpha x) \cos (\beta x)+J_{5} x+J_{6}
\end{aligned}
$$

which leads to three nodes for each element, with two degrees of freedom per node. Further, for convenience of symmetric and anti-symmetric properties of trigonometric function, the initial node is chosen in $x=-\frac{L}{2}$ and the final node as $x=\frac{L}{2}$, as shown in figure 5.1.

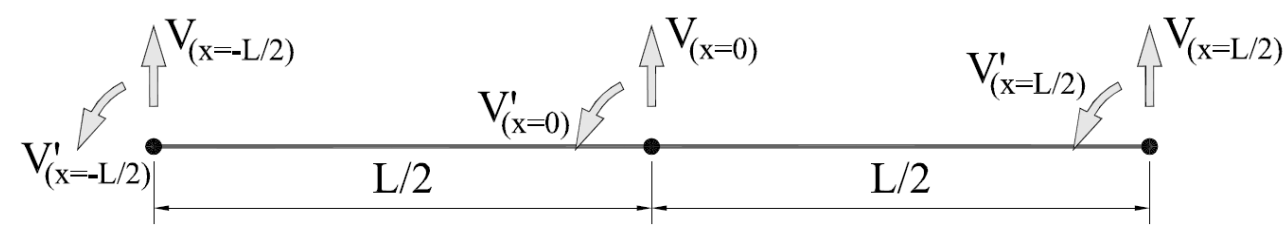

Figure 5.1: Element for Non-homogeneous solution with 6 degrees of freedom

In this proposed approach, all elements' formulations are based on this element with an extra node. Nevertheless, one can note that a static contraction can avoid this extra node.

In the next paragraphs, the exact shape functions and their respective stiffness matrices are developed for the GBT modes, for different cases of solutions and also for a variety of boundary conditions.

\subsubsection{Non-Homogeneous solution for distortion with warping - case A with clamped-clamped boundary conditions}

Starting with the shape functions of case A, dominant distortion mode, in clampedclamped $^{1}$ boundary conditions. It is necessary to satisfy the completeness principle: each interpolation function corresponds to a nodal displacement (transverse or longitudinal). Moreover, each interpolation function must have a unitary value for its respective nodal displacement and vanish for other displacements and nodes [167]. For instance, the first shape function, $S h_{\mathrm{Hacc} 1}{ }^{2}$ which interpolates the initial generalized displacement, is based on:

$$
\begin{aligned}
& S h_{\text {Hacc } 1}=J_{11} \cosh (\alpha x) \cos (\beta x)+J_{21} \cosh (\alpha x) \sin (\beta x)+ \\
& \quad+J_{31} \sinh (\alpha x) \sin (\beta x)+J_{41} \sinh (\alpha x) \cos (\beta x)+J_{51} x+J_{61}
\end{aligned}
$$

\footnotetext{
${ }^{1}$ The term clamped-clamped boundary conditions is an analog of bending moment. It refers to a beam with longitudinal and transverse restraints in both initial and final nodes

${ }^{2}$ The index $\mathrm{Hacc}_{1}$ indicates that the shape functions are based on Hyperbolic-Trigonometric functions $(\mathrm{H})$, in clamped-clamped boundary conditions (cc) and it concerns the first function (1) of the set of orthogonal functions.
} 
Where $J_{i j}$ is the $i^{\text {nd }}$ constant value of shape function $\mathrm{j}$. The constants above must satisfy the following conditions:

$$
\begin{gathered}
\operatorname{Sh}_{\text {Hacc } 1}\left(x=\frac{-L}{2}\right) 1 \therefore J_{11} \cosh \left(\frac{-\alpha L}{2}\right) \cos \left(\frac{-\beta L}{2}\right)+J_{21} \cosh \left(\frac{-\alpha L}{2}\right) \sin \left(\frac{-\beta L}{2}\right)+ \\
+J_{31} \sinh \left(\frac{-\alpha L}{2}\right) \sin \left(\frac{-\beta L}{2}\right)+J_{41} \sinh \left(\frac{-\alpha L}{2}\right) \cos \left(\frac{-\beta L}{2}\right)-J_{51} \frac{L}{2}+J_{61}=1 \\
\operatorname{Sh}_{\text {Hacc } 1}(x=0)=0 \therefore J_{11}+J_{61}=0
\end{gathered}
$$

$$
\begin{aligned}
\operatorname{Sh}_{\text {Hacc } 1}(x= & \left.\frac{L}{2}\right)=0 \therefore J_{11} \cosh \left(\frac{\alpha L}{2}\right) \cos \left(\frac{\beta L}{2}\right)+J_{21} \cosh \left(\frac{\alpha L}{2}\right) \sin \left(\frac{\beta L}{2}\right)+ \\
& +J_{31} \sinh \left(\frac{\alpha L}{2}\right) \sin \left(\frac{\beta L}{2}\right)+J_{41} \sinh \left(\frac{\alpha L}{2}\right) \cos \left(\frac{\beta L}{2}\right)+J_{51} \frac{L}{2}+J_{61}=0
\end{aligned}
$$

$$
\begin{gathered}
\operatorname{Sh}_{\text {Hacc } 1, x}\left(x=\frac{-L}{2}\right)=0 \therefore J_{11}\left(\alpha \sinh \left(\frac{-\alpha L}{2}\right) \cos \left(\frac{-\beta L}{2}\right)-\beta \cosh \left(\frac{-\alpha L}{2}\right) \sin \left(\frac{-\beta L}{2}\right)\right)+ \\
J_{21}\left(\alpha \sinh \left(\frac{-\alpha L}{2}\right) \sin \left(\frac{-\beta L}{2}\right)+\beta \cosh \left(\frac{-\alpha L}{2}\right) \cos \left(\frac{-\beta L}{2}\right)\right)+ \\
J_{31}\left(\alpha \cosh \left(\frac{-\alpha L}{2}\right) \sin \left(\frac{-\beta L}{2}\right)+\beta \sinh \left(\frac{-\alpha L}{2}\right) \cos \left(\frac{-\beta L}{2}\right)\right)+ \\
+J_{41}\left(\alpha \cosh \left(\frac{-\alpha L}{2}\right) \cos \left(\frac{-\beta L}{2}\right)-\beta \sinh \left(\frac{-\alpha L}{2}\right) \sin \left(\frac{-\beta L}{2}\right)\right)+J_{51}=0 \\
\operatorname{Sh}_{\text {Hacc } 1, x}(x=0)=0 \therefore J_{21} \beta+J_{41} \alpha+J_{51}=0
\end{gathered}
$$

$$
\begin{aligned}
& \operatorname{Sh}_{H a c c 1, x}\left(x=\frac{L}{2}\right)=0 \therefore J_{11}\left(\alpha \sinh \left(\frac{\alpha L}{2}\right) \cos \left(\frac{\beta L}{2}\right)-\beta \cosh \left(\frac{\alpha L}{2}\right) \sin \left(\frac{\beta L}{2}\right)\right)+ \\
& +J_{21}\left(\alpha \sinh \left(\frac{\alpha L}{2}\right) \sin \left(\frac{\beta L}{2}\right)+\beta \cosh \left(\frac{\alpha L}{2}\right) \cos \left(\frac{\beta L}{2}\right)\right)+ \\
& +J_{31}\left(\alpha \cosh \left(\frac{\alpha L}{2}\right) \sin \left(\frac{\beta L}{2}\right)+\beta \sinh \left(\frac{\alpha L}{2}\right) \cos \left(\frac{\beta L}{2}\right)\right)+ \\
& +J_{41}\left(\alpha \cosh \left(\frac{\alpha L}{2}\right) \cos \left(\frac{\beta L}{2}\right)-\beta \sinh \left(\frac{\alpha L}{2}\right) \sin \left(\frac{\beta L}{2}\right)\right)+J_{51}=0
\end{aligned}
$$

The conditions presented above can also be expressed in a matrix form that can be applied for the other interpolation functions in this case:

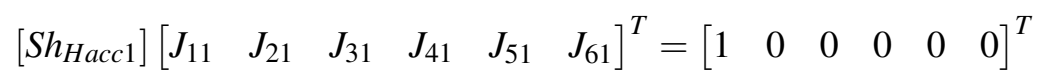


With: $\left[S h_{\text {Hacc1 } 1}\right]=$

$$
\left[\begin{array}{cccccc}
c h_{\frac{L}{2}} c_{\frac{L}{2}} & -c h_{\frac{L}{2}} s_{\frac{L}{2}} & s h_{\frac{L}{2}} s_{\frac{L}{2}} & -s h_{\frac{L}{2}} c_{\frac{L}{2}} & -\frac{L}{2} & 1 \\
-\alpha s h_{\frac{L}{2}} c_{\frac{L}{2}}+\beta c h_{\frac{L}{2}} s_{\frac{L}{2}} & \alpha s h_{\frac{L}{2}} s_{\frac{L}{2}}+\beta c h_{\frac{L}{2}} c_{\frac{L}{2}} & -\alpha c h_{\frac{L}{2}} s_{\frac{L}{2}}+\beta s h_{\frac{L}{2}} c_{\frac{L}{2}} & \alpha c h_{\frac{L}{2}} c_{\frac{L}{2}}-\beta s h_{\frac{L}{2}} s_{\frac{L}{2}} & 1 & 0 \\
c h_{\frac{L}{2}} c_{\frac{L}{2}} & c h_{\frac{L}{2}} s \frac{L}{2} & s h_{\frac{L}{2}} s \frac{L}{2} & s h_{\frac{L}{2}} c_{\frac{L}{2}} & \frac{L}{2} & 1 \\
\alpha s h_{\frac{L}{2}} c \frac{L}{2}-\beta c h_{\frac{L}{2}} s_{\frac{L}{2}} & \alpha s h_{\frac{L}{2}} s \frac{L}{2}+\beta c h_{\frac{L}{2}} c_{\frac{L}{2}} & \alpha c h_{\frac{L}{2}} s \frac{L}{2}-\beta s h_{\frac{L}{2}} c_{\frac{L}{2}} & \alpha c h_{\frac{L}{2}} c_{\frac{L}{2}}-\beta s h_{\frac{L}{2}} s \frac{L}{2} & 1 & 0 \\
1 & 0 & 0 & 0 & 0 & 1 \\
0 & \beta & 0 & \alpha & 1 & 0
\end{array}\right]
$$

Where: $c h_{\frac{L}{2}}=\cosh \left(\frac{\alpha L}{2}\right), \operatorname{sh} \frac{L}{2}=\sinh \left(\frac{\alpha L}{2}\right), c_{\frac{L}{2}}=\cos \left(\frac{\beta L}{2}\right)$, and $s_{\frac{L}{2}}=\sin \left(\frac{\beta L}{2}\right)$. The matrix in eq. 5.28 is symbolically inverted, leading to the values of the six constants of the first shape function. It is interesting to observe that the only difference among the six shape functions is the vector on the right side of eq. 5.27. This vector will be: $\left[\begin{array}{llllll}0 & 1 & 0 & 0 & 0 & 0\end{array}\right]^{T},\left[\begin{array}{llllll}0 & 0 & 1 & 0 & 0 & 0\end{array}\right]^{T},\left[\begin{array}{llllll}0 & 0 & 0 & 1 & 0 & 0\end{array}\right]^{T},\left[\begin{array}{llllll}0 & 0 & 0 & 0 & 1 & 0\end{array}\right]^{T}$ and $\left[\begin{array}{llllll}0 & 0 & 0 & 0 & 0 & 1\end{array}\right]^{T}$ for shape functions $2,3,4,5$ and 6 respectively. By solving the system presented above for the other five interpolation functions, it is possible to represent all constant values of the shape functions in a coefficient matrix, $S h_{N H a c c}$, called here as completeness coefficient matrix:

$$
\begin{aligned}
& {\left[S h_{N H a c c}\right]=\frac{\left[\begin{array}{cccccc}
J_{11} & J_{12} & J_{13}=J_{11} & J_{14}=-J_{12} & J_{15}=-2 J_{11} & 0 \\
J_{21} & J_{22} & J_{23}=-J_{21} & J_{24}=J_{22} & 0 & J_{26} \\
J_{31} & J_{32} & J_{33}=J_{31} & J_{34}-J_{32} & J_{35}=-2 J_{31} & 0 \\
J_{41} & J_{42} & J_{43}=-J_{41} & J_{44}=J_{42} & 0 & J_{46} \\
J_{51} & J_{52} & J_{53}=J_{51} & J_{54}-J_{52} & 0 & J_{56} \\
J_{61}=-J_{11} & J_{62}=-J_{12} & J_{63}=-J_{11} & J_{64}=J_{12} & J_{65} & 0
\end{array}\right]}{K_{N H a c c}}} \\
& \text { With: } K_{N H a c c}=2\left(\alpha \sin \left(\frac{\beta L}{2}\right)-\beta \sinh \left(\frac{\alpha L}{2}\right)\right)\left(\cos \left(\frac{\beta L}{2}\right)-\cosh \left(\frac{\alpha L}{2}\right)\right) \\
& J_{11}=\beta \sinh \left(\frac{\alpha L}{2}\right) \cos \left(\frac{\beta L}{2}\right)+\alpha \cosh \left(\frac{\alpha L}{2}\right) \sin \left(\frac{\beta L}{2}\right) \\
& J_{12}=\sinh \left(\frac{\alpha L}{2}\right) \sin \left(\frac{\beta L}{2}\right) \\
& J_{65}=\alpha \sin (\beta L)+\beta \sinh (\alpha L) \\
& J_{21}=\frac{K_{N H a c c}}{D_{S 1}} \sinh \left(\frac{\alpha L}{2}\right)\left(\beta J_{12}^{2}+\alpha J_{12}-\frac{\alpha}{4} \sinh (\alpha L) \sin (\beta L)\right)
\end{aligned}
$$

where: $D_{S 1}=\sinh ^{3}\left(\frac{\alpha L}{2}\right)\left(L\left(\alpha^{2}+\beta^{2}\right) \sin ^{2}\left(\frac{\beta L}{2}\right)+\beta \sin (\beta L)\right)+$

$$
\begin{array}{r}
-\alpha \sinh (\alpha L) \sinh \left(\frac{\alpha L}{2}\right) \sin ^{2}\left(\frac{\beta L}{2}\right)-\alpha \sinh ^{4}\left(\frac{\alpha L}{2}\right) \sin \left(\frac{\beta L}{2}\right) \sin (\beta L)+ \\
-\beta \sinh (\alpha L) \sinh ^{2}\left(\frac{\alpha L}{2}\right) \sin \left(\frac{\beta L}{2}\right)+\frac{\alpha}{4} \sinh ^{2}(\alpha L) \sin \left(\frac{\beta L}{2}\right) \sin (\beta L)
\end{array}
$$




$$
\begin{gathered}
J_{22}=J_{21} \frac{\frac{\alpha L}{2}-\sinh \left(\frac{\alpha L}{2}\right) \cos \left(\frac{\beta L}{2}\right)}{\beta J_{12}+\alpha J_{32}} \\
J_{26}=J_{21} L-2 J_{22} \\
J_{31}=\beta \cosh \left(\frac{\alpha L}{2}\right) \sin \left(\frac{\beta L}{2}\right)-\alpha \sinh \left(\frac{\alpha L}{2}\right) \cos \left(\frac{\beta L}{2}\right) \\
J_{32}=1-\cosh \left(\frac{\alpha L L}{2}\right) \cos \left(\frac{\beta L L}{2}\right) \\
J_{41}=\frac{K_{N H a c c}}{D_{S 1}} \sinh \left(\frac{\alpha L}{2}\right)\left(\alpha J_{12}^{2}-\beta J_{12}+\frac{\beta}{4} \sinh (\alpha L) \sin (\beta L)\right) \\
J_{42}=J_{41} \frac{\frac{\beta L}{2}-\cosh \left(\frac{\alpha L}{2}\right) \sin \left(\frac{\beta L}{2}\right)}{-\alpha J_{12}+\beta J_{32}} \\
J_{52}=J_{51} \frac{\alpha \operatorname{coth}\left(\frac{\alpha L}{2}\right)-\beta \cot \left(\frac{\beta L}{2}\right)}{\alpha^{2}+\beta^{2}} \\
J_{51}=-\frac{K_{N H a c c}}{D_{S 1}} \sinh \left(\frac{\alpha L}{2}\right)\left(\alpha^{2}+\beta^{2}\right) J_{12}^{2} \\
J_{46}=J_{41} L-2 J_{42} \\
J_{56}=J_{51} L-2 J_{52}+K_{N H a c c}
\end{gathered}
$$

Consequently, the generalized nodal displacements can now express the amplification function $V(x)$ :

$$
V(x)=\left[T x_{N H a}\right]\left[S h_{N H a c c}\right][\vartheta]
$$

Where $\left[T x_{N H a}\right]$ is the term vector in case $\mathrm{A}$, which is dependent of length $\mathrm{x}$, and $[\vartheta]$ is the vector of beam's nodal displacements. These two vectors are presented below:

$$
\left[T x_{H a}\right]=\left[\begin{array}{c}
\cosh (\alpha x) \cos (\beta x) \\
\cosh (\alpha x) \sin (\beta x) \\
\sinh (\alpha x) \sin (\beta x) \\
\sinh (\alpha x) \cos (\beta x) \\
x \\
1
\end{array}\right]^{T}
$$

$$
[\vartheta]=\left[\begin{array}{c}
V_{-L / 2} \\
V_{-L / 2}^{\prime} \\
V_{L / 2} \\
V_{L / 2}^{\prime} \\
V_{0} \\
V_{0}^{\prime}
\end{array}\right]
$$

The amplification function, $\mathrm{V}(\mathrm{x})$, is conveniently represented in eq. 5.46. It splits into three terms: a first vector, in eq. 5.47, only function of x; a second, in completeness coefficient matrix of boundary conditions, eq. 5.29, independent of $\mathrm{x}$; and a third vector, in eq. 5.48, which is vector of the nodal displacements. For different cases of dominant torsion and/or distortion, as well as different boundary conditions, it is only necessary to change the respective vector or/and matrix in the formulation above. 
A variation of $\mathrm{V}(\mathrm{x})$ is easily given by:

$$
\delta V(x)=\left[T x_{H a}\right]\left[S h_{H a c c}\right]
$$

Introducing equations 5.46 and 5.49 into the variational formulation presented in eq. 2.57 leads to:

- For the first integral: $\left(E^{i} C^{M}+K^{i} C^{P}\right) \int_{L}^{i} V_{, x x}(x) \delta^{i} V_{, x x}(x) \mathrm{d} x=$

$$
\begin{array}{r}
\left(E^{i} C^{M}+K^{i} C^{P}\right) \int_{-\frac{L}{2}}^{\frac{L}{2}}\left[{ }^{i}\left[T x_{N H 1, x x}\right]^{i}\left[S h_{N H a c c}\right]^{i}[\vartheta]\right]^{T}{ }^{i}\left[T x_{N H 1, x x}\right]^{i}\left[S h_{N H a c c}\right] \mathrm{d} x= \\
=\left(E^{i} C^{M}+K^{i} C^{P}\right)^{i}\left[S h_{N H a c c}\right]^{T i}\left[\Upsilon_{N H a}^{\prime \prime}\right]^{i}\left[S h_{N H a c c}\right]^{i}[\vartheta]
\end{array}
$$

The values of the integration matrix $\left[\Upsilon_{N H a}^{\prime \prime}\right]=\int_{-\frac{L}{2}}^{\frac{L}{2}}\left[T x_{N H a, x x}\right]^{T}\left[T x_{N H a, x x}\right] \mathrm{d} x$ are:

$$
\left[\Upsilon_{N H a}^{\prime \prime}\right]=\left[\begin{array}{cccccc}
\Upsilon_{H a, 11}^{\prime \prime} & 0 & \Upsilon_{H a, 13}^{\prime \prime} & 0 & 0 & 0 \\
& \Upsilon_{H a, 22}^{\prime \prime} & 0 & \Upsilon_{H a, 24}^{\prime \prime} & 0 & 0 \\
& & \Upsilon_{H a, 33}^{\prime \prime} & 0 & 0 & 0 \\
& & & \Upsilon_{H a, 44}^{\prime \prime} & 0 & 0 \\
& & & & 0 & 0 \\
\text { Sym. } & & & & & 0
\end{array}\right]
$$

where: $\Upsilon_{H a, 11}^{\prime \prime}=\frac{1}{4 \alpha \beta}\left(\beta \sinh (\alpha L)\left(\alpha^{2}\left(\alpha^{2}-3 \beta^{2}\right) \cos (\beta L)+\left(\alpha^{2}+\beta^{2}\right)^{2}\right)+\right.$

$\left.+\alpha \sin (\beta L)\left(\left(\alpha^{2}+\beta^{2}\right)^{2}+\left(\beta^{4}-3 \alpha^{2} \beta^{2}\right) \cosh (\alpha L)\right)+\alpha \beta L\left(\alpha^{4}-6 \alpha^{2} \beta^{2}+\beta^{4}\right)\right)$

$$
\begin{array}{r}
\Upsilon_{H a, 13}^{\prime \prime}=\frac{1}{4}\left(-\beta\left(\beta^{2}-3 \alpha^{2}\right) \sinh (\alpha L) \cos (\beta L)+\alpha\left(\alpha^{2}-3 \beta^{2}\right) \cosh (\alpha L) \sin (\beta L)+\right. \\
+4 \alpha \beta L(\alpha-\beta)(\alpha+\beta)) \\
\Upsilon_{H a, 22}^{\prime \prime}=-\Upsilon_{H a, 11}^{\prime \prime}+\frac{\left(\alpha^{2}+\beta^{2}\right)^{2} \sinh (\alpha L)+\alpha L\left(\alpha^{4}-6 \alpha^{2} \beta^{2}+\beta^{4}\right)}{2 \alpha} \\
\Upsilon_{H a, 24}^{\prime \prime}=\Upsilon_{H a, 13}^{\prime \prime}+2 \alpha \beta L\left(\beta^{2}-\alpha^{2}\right) \\
\Upsilon_{H a, 33}^{\prime \prime}=-\Upsilon_{H a, 11}^{\prime \prime}+\frac{\left(\alpha^{2}+\beta^{2}\right)^{2}(\alpha \sin (\beta L)+\beta \sinh (\alpha L))}{2 \alpha \beta} \\
\Upsilon_{H a, 44}^{\prime \prime}=\Upsilon_{H a, 11}^{\prime \prime}-\frac{\left(\alpha^{2}+\beta^{2}\right)^{2} \sin (\beta L)+\beta L\left(\alpha^{4}-6 \alpha^{2} \beta^{2}+\beta^{4}\right)}{2 \beta}
\end{array}
$$


Thus, one obtains the stiffness matrix due to longitudinal strains of energy from the multiplication:

$$
{ }^{i}\left[K_{c}\right]=\left(E^{i} C^{M}+K^{i} C^{P}\right)^{i}\left[S h_{H a c c}\right]^{T} i\left[\Upsilon_{H a}^{\prime \prime}\right]^{i}\left[S h_{H a c c}\right]
$$

- For the second term in the integral in eq. 2.57, one can proceed in a similar way:

$$
G\left({ }^{i} D^{M c}+{ }^{i} D^{P}\right) \int_{L}^{i} V_{, x}(x) \delta^{i} V_{, x}(x) \mathrm{d} x=G\left({ }^{i} D^{M c}+{ }^{i} D^{P}\right){ }^{i}\left[S h_{N H a c c}\right]^{T i}\left[\Upsilon_{N H a}^{\prime}\right]^{i}\left[S h_{N H a c c}\right]^{i}[\vartheta]
$$

The values of the integration matrix $\left[\Upsilon_{N H a}^{\prime}\right]=\int_{-\frac{L}{2}}^{\frac{L}{2}}\left[T x_{N H a}^{\prime}\right]^{T}\left[T x_{N H a}^{\prime}\right] \mathrm{d} x$ are given below:

$$
\left[\Upsilon_{N H a}^{\prime}\right]=\left[\begin{array}{cccccc}
\Upsilon_{H a, 11}^{\prime} & 0 & \Upsilon_{H a, 13}^{\prime} & 0 & 0 & 0 \\
& \Upsilon_{H a, 22}^{\prime} & 0 & \Upsilon_{H a, 24}^{\prime} & \Upsilon_{N H a, 25}^{\prime} & 0 \\
& & \Upsilon_{H a, 33}^{\prime} & 0 & 0 & 0 \\
& & & \Upsilon_{H a, 44}^{\prime} & \Upsilon_{N H a, 45}^{\prime} & 0 \\
& & & & L & 0 \\
\text { Sym. } & & & & & 0
\end{array}\right]
$$

where: $\Upsilon_{H a, 11}^{\prime}=\frac{1}{4 \alpha \beta}\left(\alpha \beta L\left(\beta^{2}-\alpha^{2}\right)+\beta \sinh (\alpha L)\left(\alpha^{2} \cos (\beta L)+\alpha^{2}+\beta^{2}\right)+\right.$

$$
\begin{array}{r}
\left.-\alpha \sin (\beta L)\left(\alpha^{2}+\beta^{2} \cosh (\alpha L)+\beta^{2}\right)\right) \\
\Upsilon_{H a, 13}^{\prime}=\frac{1}{4}(-2 \alpha \beta L+\beta \sinh (\alpha L) \cos (\beta L)+\alpha \cosh (\alpha L) \sin (\beta L)) \\
\Upsilon_{H a, 22}^{\prime}=-\Upsilon_{H a, 11}^{\prime}+\frac{\alpha L\left(\beta^{2}-\alpha^{2}\right)+\left(\alpha^{2}+\beta^{2}\right) \sinh (\alpha L)}{2 \alpha} \\
\Upsilon_{H a, 24}^{\prime}=\Upsilon_{H a, 13}^{\prime}+\alpha \beta L \\
\Upsilon_{H a, 33}^{\prime}=-\Upsilon_{H a, 11}^{\prime}-\frac{\left(\alpha^{2}+\beta^{2}\right)(\alpha \sin (\beta L)-\beta \sinh (\alpha L))}{2 \alpha \beta} \\
\Upsilon_{H a, 44}^{\prime}=\Upsilon_{H a, 11}^{\prime}+\frac{\beta L\left(\alpha^{2}-\beta^{2}\right)+\left(\alpha^{2}+\beta^{2}\right) \sin (\beta L)}{2 \beta} \\
\Upsilon_{N H a, 25}^{\prime}=2 \cosh \left(\frac{\alpha L}{2}\right) \sin \left(\frac{\beta L}{2}\right) \\
\Upsilon_{N H a, 45}^{\prime}=2 \sinh \left(\frac{\alpha L}{2}\right) \cos \left(\frac{\beta L}{2}\right)
\end{array}
$$

The stiffness matrix due to shear strains can be obtained from the multiplication:

$$
{ }^{i}\left[K_{d}\right]=G\left({ }^{i} D^{M c}+{ }^{i} D^{P}\right){ }^{i}\left[S h_{N} H c c\right]^{T i}\left[\Upsilon_{N H a}^{\prime}\right]{ }^{i}\left[S h_{N} H c c\right]
$$


- the third term in the integral in eq. 2.57 follows the same procedure:

$$
K^{i} B \int_{L}{ }^{i} V(x) \delta^{i} V(x) \mathrm{d} x=K^{i} B^{i}\left[S h_{H a c c}\right]^{T}{ }^{i}\left[\Upsilon_{H a}\right]^{i}\left[S h_{H a c c}\right]^{i}[\vartheta]
$$

The values of the integration matrix $\left[\Upsilon_{N H a}\right]=\int_{-\frac{L}{2}}^{\frac{L}{2}}\left[T x_{N H a}\right]^{T}\left[T x_{N H a}\right] \mathrm{d} x$ are given below:

$$
\left[\Upsilon_{N H a}\right]=\left[\begin{array}{cccccc}
\Upsilon_{H a, 11} & 0 & \Upsilon_{H a, 13} & 0 & 0 & \Upsilon_{N H a, 16} \\
& \Upsilon_{H a, 22} & 0 & \Upsilon_{H a, 24}=\Upsilon_{H a, 13} & \Upsilon_{N H a, 25} & 0 \\
& & \Upsilon_{H a, 33} & 0 & 0 & \Upsilon_{N H a, 36} \\
& & & \Upsilon_{H a, 44} & \Upsilon_{N H a, 45} & 0 \\
\text { Sym. } & & & & \frac{L^{3}}{12} & 0 \\
& & & & & L
\end{array}\right]
$$

$$
\begin{aligned}
& \text { where: } \Upsilon_{H a, 11}=\frac{1}{4 \alpha \beta\left(\alpha^{2}+\beta^{2}\right)}\left(\alpha\left(\alpha^{2}+\beta^{2}\right)(\beta L+\sin (\beta L))+\right. \\
& \left.+\beta \sinh (\alpha L)\left(\alpha^{2} \cos (\beta L)+\alpha^{2}+\beta^{2}\right)+\alpha \beta^{2} \cosh (\alpha L) \sin (\beta L)\right) \\
& \Upsilon_{H a, 13}=\frac{\alpha \cosh (\alpha L) \sin (\beta L)-\beta \sinh (\alpha L) \cos (\beta L)}{4\left(\alpha^{2}+\beta^{2}\right)} \\
& \Upsilon_{H a, 22}=-\Upsilon_{H a, 11}+\frac{\alpha L+\sinh (\alpha L)}{2 \alpha} \\
& \Upsilon_{H a, 33}=-\Upsilon_{H a, 11}+\frac{\alpha \sin (\beta L)+\beta \sinh (\alpha L)}{2 \alpha \beta} \\
& \Upsilon_{H a, 44}=\Upsilon_{H a, 11}-\frac{\sin (\beta L)-\beta L}{2 \beta} \\
& \Upsilon_{N H a, 16}=\frac{\alpha \Upsilon_{N H a, 45}^{\prime}+\beta \Upsilon_{N H a, 25}^{\prime}}{\alpha^{2}+\beta^{2}} \\
& \Upsilon_{N H a, 25}=\Upsilon_{N H a, 25}^{\prime} \frac{\frac{-\beta L}{2}\left(\alpha^{2}+\beta^{2}\right) \cot \left(\frac{\beta L}{2}\right)+\alpha \tanh \left(\frac{\alpha L}{2}\right)\left(\frac{L}{2}\left(\alpha^{2}+\beta^{2}\right)+2 \beta \cot \left(\frac{\beta L}{2}\right)\right)-\alpha^{2}+\beta^{2}}{\left(\alpha^{2}+\beta^{2}\right)^{2}} \\
& \Upsilon_{N H a, 36}=\frac{\alpha \Upsilon_{N H a, 25}^{\prime}-\beta \Upsilon_{N H a, 45}^{\prime}}{\alpha^{2}+\beta^{2}} \\
& \Upsilon_{N H a, 45}=\Upsilon_{N H a, 45}^{\prime} \frac{\frac{\beta L}{2}\left(\alpha^{2}+\beta^{2}\right) \tan \left(\frac{\beta L}{2}\right)+\alpha \operatorname{coth}\left(\frac{\alpha L}{2}\right)\left(\frac{L}{2}\left(\alpha^{2}+\beta^{2}\right)-2 \beta \tan \left(\frac{\beta L}{2}\right)\right)-\alpha^{2}+\beta^{2}}{\left(\alpha^{2}+\beta^{2}\right)^{2}}
\end{aligned}
$$

Thus, one obtains the stiffness matrix due to transverse distortion from:

$$
{ }^{i}\left[K_{b}\right]=K^{i} B^{i}\left[S h_{N H a c c}\right]^{T i}\left[\Upsilon_{N H a}\right]^{i}\left[S h_{N H a c c}\right]
$$


- the fourth term in the integral in eq. 2.57 has a unique property. It involves the shape function and its second derivative, as well as its commutative product. Each stiffness matrix is not symmetric, but they are transposed from each other. This leads to the final symmetric matrix in the summation:

$$
\mu K^{i} D^{\mu} \int_{L}{ }^{i} V(x) \delta^{i} V(x) \mathrm{d} x=\mu K^{i} D^{\mu i}\left[S h_{H a c c}\right]^{T i}\left[\Upsilon_{N H a}^{\prime \prime}{ }^{\prime}\right]^{i}\left[S h_{H a c c}\right]^{i}[\vartheta]
$$

The values of the integration matrix $\left[\Upsilon_{N H a}^{\prime \prime}{ }^{\prime}\right]=\int_{-\frac{L}{2}}^{\frac{L}{2}}\left[T x_{N H a}^{\prime \prime}\right]^{T}\left[T x_{N H a}\right]+\left[T x_{N H a}\right]^{T}\left[T x_{N H a}^{\prime \prime}\right] \mathrm{d} x$ are:

$$
\left[\Upsilon_{N H a}^{\prime \prime}{ }^{\prime}\right]=\left[\begin{array}{cccccc}
\Upsilon_{H a, 11}^{\prime \prime},{ }^{\prime} & 0 & \Upsilon_{H a, 13}^{\prime \prime}, & 0 & 0 & \Upsilon_{N H a, 16^{\prime \prime},}^{\prime} \\
& \Upsilon_{H a, 22}^{\prime \prime},{ }^{\prime \prime} & 0 & \Upsilon_{H a, 24}^{\prime \prime}, & \Upsilon_{N H a, 25}^{\prime \prime}, & 0 \\
& & \Upsilon_{H a, 33}^{\prime \prime}, & 0 & 0 & \Upsilon_{N H a, 36}^{\prime \prime}, \\
& & & \Upsilon_{H a, 44}^{\prime \prime}, & \Upsilon_{N H a, 45}^{\prime \prime}, & 0 \\
\text { Sym. } & & & & 0 & 0 \\
& & & & & 0
\end{array}\right]
$$

where: $\Upsilon_{H a, 11}^{\prime \prime},{ }^{\prime}=\frac{1}{2 \alpha \beta}\left[\beta \sinh (\alpha L)\left(\alpha^{2} \cos (\beta L)+\alpha^{2}-\beta^{2}\right)-\alpha \beta^{2} \cosh (\alpha L) \sin (\beta L)+\right.$

$$
\begin{aligned}
& \Upsilon_{H a, 13}^{\prime \prime},{ }^{\prime}= \frac{1}{2}(2 \alpha \beta L+\beta \sinh (\alpha L) \cos (\beta L)+\alpha \cosh (\alpha L) \sin (\beta L)) \\
& \Upsilon_{H a, 22}^{\prime \prime},{ }^{2}= \frac{\left(\alpha^{2}-\beta^{2}\right)(\alpha L+\sinh (\alpha L))}{\alpha}-\Upsilon_{H a, 11}^{\prime \prime},{ }^{\prime} \\
& \Upsilon_{H a, 24}^{\prime \prime},{ }^{\prime}=\Upsilon_{H a, 13}^{\prime \prime},{ }^{\prime}-2 \alpha \beta L \\
& \Upsilon_{H a, 33}^{\prime \prime},{ }^{\prime}= \Upsilon_{H a, 22}^{\prime \prime},{ }^{\prime}+\frac{\left(\alpha^{2}-\beta^{2}\right)(\beta L-\sin (\beta L))}{\beta} \\
& \Upsilon_{H a, 44}^{\prime \prime},{ }^{\prime}= \Upsilon_{H a, 11}^{\prime \prime},{ }^{\prime}-\frac{\left(\alpha^{2}-\beta^{2}\right)(\beta L+\sin (\beta L))}{\beta} \\
& \Upsilon_{N H a, 16}^{\prime \prime},{ }^{\prime}=\alpha \Upsilon_{N H a, 45}^{\prime}-\beta \Upsilon_{N H a, 25}^{\prime} \Upsilon_{N H a, 36}^{\prime \prime},{ }^{\prime}=\beta \Upsilon_{N H a, 45}^{\prime}+\alpha \Upsilon_{N H a, 25}^{\prime} \\
& \Upsilon_{N H a, 25}^{\prime \prime},=\alpha L \sinh \left(\frac{\alpha L}{2}\right) \sin \left(\frac{\beta L}{2}\right)+\beta L \cosh \left(\frac{\alpha L}{2}\right) \cos \left(\frac{\beta L}{2}\right)-\Upsilon_{N H a, 25}^{\prime} \\
& \Upsilon_{N H a, 45}^{\prime \prime},{ }^{\prime}=\alpha L \cosh \left(\frac{\alpha L}{2}\right) \cos \left(\frac{\beta L}{2}\right)-\beta L \sinh \left(\frac{\alpha L}{2}\right) \sin \left(\frac{\beta L}{2}\right)-\Upsilon_{N H a, 45}^{\prime}
\end{aligned}
$$


Similar to the other stiffness matrices, the one due to Poisson effect in plate behavior can be obtained from the multiplication:

$$
{ }^{i}\left[K_{\mu}\right]=\mu K^{i} D^{\mu i}\left[S h_{N H a c c}\right]^{T i}\left[\Upsilon_{N H a}^{\prime \prime}{ }^{\prime}\right]^{i}\left[S h_{N H a c c}\right]
$$

The total stiffness matrix can be expressed by the combination of equations. 5.58, 5.69, 5.81 and 5.94, as:

$$
\begin{array}{r}
{ }^{i}\left[K_{N H a c c}\right]={ }^{i}\left[S h_{N H a c c}\right]^{T}\left[\left(E^{i} C^{M}+K^{i} C^{P}\right){ }^{i}\left[\Upsilon_{N H a}^{\prime \prime}\right]+G\left({ }^{i} D^{M c}+{ }^{i} D^{P}\right){ }^{i}\left[\Upsilon_{N H a}^{\prime}\right]+\right. \\
\left.+K^{i} B^{i}\left[\Upsilon_{N H a}\right]+\mu K^{i} D^{\mu i}\left[\Upsilon_{N H a}^{\prime \prime},{ }^{\prime}\right]\right]^{i}\left[S h_{N H a c c}\right]
\end{array}
$$

Here, it is important to highlight that the integration matrices $\Upsilon_{N H a}, \Upsilon_{N H a}^{\prime}, \Upsilon_{N H a}^{\prime \prime}$ and $\left[\Upsilon_{N H a}^{\prime \prime}{ }^{\prime}{ }^{\prime}\right.$ ] [given respectively in equations 5.71, 5.60, 5.51 and 5.83] are not dependent on the boundary conditions. Therefore, the completeness coefficient matrix, which expresses the boundary conditions, can be understood as matrix transformation of a kernel stiffness matrix:

$$
\begin{aligned}
{ }^{i}\left[K_{N H a}\right]=\left[\left(E^{i} C^{M}+K^{i} C^{P}\right){ }^{i}\left[\Upsilon_{N H a}^{\prime \prime}\right]+G\left({ }^{i} D^{M c}\right.\right. & \left.+{ }^{i} D^{P}\right){ }^{i}\left[\Upsilon_{N H a}^{\prime}\right]+ \\
& \left.+K^{i} B^{i}\left[\Upsilon_{N H a}\right]+\mu K^{i} D^{\mu i}\left[\Upsilon_{N H a}^{\prime \prime}{ }^{\prime}\right]\right]
\end{aligned}
$$

The total stiffness matrix of clamped-clamped of case A can be expressed by:

$$
{ }^{i}\left[K_{N H a c c}\right]={ }^{i}\left[S h_{N H a c c}\right]^{T i}\left[K_{N H a}\right]^{i}\left[S h_{N H a c c}\right]
$$

Force vector for case A:

As mentioned before, the main reason to use 3 nodes, with 2 degrees of freedom each, is to fulfill the inner product of the constant/linear distributed force and the variation of generalized displacement, as expressed below:

$$
\int_{L}{ }^{i} q(x) \delta^{i} V(x) \mathrm{d} x=\int_{-\frac{L}{2}}^{\frac{L}{2}}{ }^{i} q(x)^{i}\left[T x_{N H a}\right]^{i}\left[S h_{H a c c}\right] \mathrm{d} x
$$

Just like in the development of the stiffness matrix, the completeness coefficient matrix of the boundary conditions is independent of the length, therefore one can find:

$$
{ }^{i}\left[F_{\text {NHacc }}\right]={ }^{i}\left[S_{N H a c c}\right]^{T i}\left[F k_{N H a}\right]
$$

Where the components of the vector are: ${ }^{i}\left[F k_{N H a}\right]^{T}=\left[F k_{1}, F k_{2}, F k_{3}, F k_{4}, F k_{5}, F k_{6}\right]$, which in a linear load function, ${ }^{i} q=a x+b$, the expressions are:

$$
F k_{1}=\frac{2 b\left(\alpha \sinh \left(\frac{\alpha L}{2}\right) \cos \left(\frac{\beta L}{2}\right)+\beta \cosh \left(\frac{\alpha L}{2}\right) \sin \left(\frac{\beta L}{2}\right)\right)}{\alpha^{2}+\beta^{2}}
$$




$$
\begin{gathered}
F k_{2}=\frac{a}{\left(\alpha^{2}+\beta^{2}\right)^{2}}\left(\alpha \sinh \left(\frac{\alpha L}{2}\right)\left(L\left(\alpha^{2}+\beta^{2}\right) \sin \left(\frac{\beta L}{2}\right)+4 \beta \cos \left(\frac{\beta L}{2}\right)\right)+\right. \\
\left.-\cosh \left(\frac{\alpha L}{2}\right)\left(\beta L\left(\alpha^{2}+\beta^{2}\right) \cos \left(\frac{\beta L}{2}\right)+2(\alpha-\beta)(\alpha+\beta) \sin \left(\frac{\beta L}{2}\right)\right)\right) \\
F k_{3}=\frac{2 b\left(\alpha \cosh \left(\frac{\alpha L}{2}\right) \sin \left(\frac{\beta L}{2}\right)-\beta \sinh \left(\frac{\alpha L}{2}\right) \cos \left(\frac{\beta L}{2}\right)\right)}{\alpha^{2}+\beta^{2}} \\
F k_{4}=\frac{a}{\left(\alpha^{2}+\beta^{2}\right)^{2}}\left(\sinh \left(\frac{\alpha L}{2}\right)\left(\beta L\left(\alpha^{2}+\beta^{2}\right) \sin \left(\frac{\beta L}{2}\right)+2\left(\beta^{2}-\alpha^{2}\right) \cos \left(\frac{\beta L}{2}\right)\right)+\right. \\
\left.+\alpha \cosh \left(\frac{\alpha L}{2}\right)\left(L\left(\alpha^{2}+\beta^{2}\right) \cos \left(\frac{\beta L}{2}\right)-4 \beta \sin \left(\frac{\beta L}{2}\right)\right)\right) \\
F k_{5}=\frac{a L^{3}}{12} \\
F k_{6}=L
\end{gathered}
$$

As a particular case, the constant function: ${ }^{i} q=a$ eliminates the $2^{\text {nd }}, 4^{\text {th }}$ and $5^{\text {th }}$ in the above vector.

\subsubsection{Simplification of the high-order derivatives and stiffness ma- trices for coupled modes}

The smoothness of the high-order derivatives of hyperbolic-trigonometric shape functions has the numerical cost of long terms. For each multiplication term between hyperbolic and trigonometric functions, the derivative always leads to the sum of two new multiplication terms. As an example: $(\operatorname{Sinh}(\alpha x) \operatorname{Sin}(\beta x))_{, x}=\alpha \operatorname{Cosh}(\alpha x) \operatorname{Sin}(\beta x)+$ $\beta \operatorname{Sinh}(\alpha x) \operatorname{Cos}(\beta x)$. Fortunately, the resulting expression are similar to the initial expression. Thus, it is possible to rearrange the derived terms in the completeness coefficients matrix in order to reuse the kernel stiffness matrix. For instance, one can express the first derivate of the function given in eq. 5.106 as:

$$
V(x)_{, x}=\left[T x_{N H a}\right]\left[S h_{N H a}\right]_{, x}[\vartheta]
$$

where $\left[S h_{N H a}\right]_{, x}$ is completeness coefficients matrix for the first derivative. Each row is a linear combination of the rows of the ordinary completeness coefficients matrix $\left[S h_{N H a}\right]$ :

$$
\left[\begin{array}{l}
S h_{1} \\
S h_{2} \\
S h_{3} \\
S h_{4} \\
S h_{5} \\
S h_{6}
\end{array}\right]_{N H a, x}=\left[\begin{array}{c}
\beta S h_{2}+\alpha S h_{4} \\
-\beta S h_{1}+\alpha S h_{3} \\
\alpha S h_{2}-\beta S h_{4} \\
\alpha S h_{1}+\beta S h_{3} \\
0 \\
S h_{5}
\end{array}\right]_{N H a}
$$


The second derivative has a similar simplification:

$$
V(x)_{, x x}=\left[T x_{N H a}\right]\left[S h_{N H a}\right]_{, x x}[\vartheta]
$$

with:

$$
\left[\begin{array}{c}
S h_{1} \\
S h_{2} \\
S h_{3} \\
S h_{4} \\
S h_{5} \\
S h_{6}
\end{array}\right]_{N H a, x x}=\left[\begin{array}{c}
\left(\alpha^{2}-\beta^{2}\right) S h_{1}+2 \alpha \beta S h_{3} \\
\left(\alpha^{2}-\beta^{2}\right) S h_{2}-2 \alpha \beta S h_{4} \\
\left(\alpha^{2}-\beta^{2}\right) S h_{3}-2 \alpha \beta S h_{1} \\
\left(\alpha^{2}-\beta^{2}\right) S h_{4}+2 \alpha \beta S h_{2} \\
0 \\
0
\end{array}\right]_{N H a}
$$

Thus, since the vector $\left[T x_{N H a}\right]$ is the same for the shape functions and its derivatives, there is only one type of kernel stiffness matrix that can be obtained from:

$$
{ }^{i j}\left[\Upsilon_{N H a}\right]=\int_{-\frac{L}{2}}^{\frac{L}{2}}\left[T x_{N H a}\right]^{T j}\left[T x_{N H a}\right] \mathrm{d} x
$$

If " $\mathrm{i}=\mathrm{j}$ " the above expression is equal to the kernel stiffness matrix of linear analysis 5.71. Otherwise, one obtains the matrix required in the coupling between to modes:

$$
{ }^{i j}\left[\Upsilon_{N H a}\right]=\left[\begin{array}{cccccc}
{ }^{i j} \Upsilon_{H a, 11} & 0 & { }^{i j} \Upsilon_{H a, 13} & 0 & 0 & { }^{i j} \Upsilon_{N H a, 16} \\
0 & { }^{i j} \Upsilon_{H a, 22} & 0 & { }^{i j} \Upsilon_{H a, 24} & { }^{i j} \Upsilon_{N H a, 25} & 0 \\
{ }^{i j} \Upsilon_{H a, 31} & 0 & { }^{i j} \Upsilon_{H a, 33} & 0 & 0 & { }^{i j} \Upsilon_{N H a, 36} \\
0 & { }^{i j} \Upsilon_{H a, 42} & 0 & { }^{i j} \Upsilon_{H a, 44} & { }^{i j} \Upsilon_{N H a, 45} & 0 \\
0 & { }^{i j} \Upsilon_{H a, 51} & 0 & { }^{i j} \Upsilon_{H a, 54} & \frac{L^{3}}{12} & 0 \\
{ }^{i j} \Upsilon_{H a, 51} & 0 & { }^{i j} \Upsilon_{H a, 53} & 0 & 0 & L
\end{array}\right]
$$

Here, if one sets $\alpha={ }^{i} \alpha$ and $\beta={ }^{i} \beta$, then: ${ }^{i j} \Upsilon_{N H a, 16}=\Upsilon_{N H a, 16}$ eq. 5.77; ${ }^{i j} \Upsilon_{N H a, 36}=\Upsilon_{N H a, 36}$ eq. $5.79 ;{ }^{i j} \Upsilon_{N H a, 25}=\Upsilon_{N H a, 26}$ eq. $5.78 ;{ }^{i j} \Upsilon_{N H a, 45}=\Upsilon_{N H a, 46}$ eq. 5.80 ;

Else, one sets $\alpha={ }^{j} \alpha$ and $\beta={ }^{j} \beta$, then: ${ }^{i j} \Upsilon_{N H a, 61}=\Upsilon_{N H a, 16}$ eq. $5.77 ;{ }^{i j} \Upsilon_{N H a, 63}=\Upsilon_{N H a, 36}$ eq. 5.79; ${ }^{i j} \Upsilon_{N H a, 52}=\Upsilon_{N H a, 26}$ eq. $5.78 ;{ }^{i j} \Upsilon_{N H a, 54}=\Upsilon_{N H a, 46}$ eq. 5.80. And the remained terms are: 


$$
\begin{aligned}
& {\left[\begin{array}{cccc}
{ }^{i j} \Upsilon_{H a, 11} & 0 & { }^{i j} \Upsilon_{H a, 13} & 0 \\
0 & { }^{i j} \Upsilon_{H a, 22} & 0 & { }^{i j} \Upsilon_{H a, 24} \\
{ }^{i j} \Upsilon_{H a, 31} & 0 & { }^{i j} \Upsilon_{H a, 33} & 0 \\
0 & { }^{i j} \Upsilon_{H a, 42} & 0 & { }^{i j} \Upsilon_{H a, 44}
\end{array}\right]=\left[\begin{array}{cccc}
\bar{\beta} & 0 & \bar{\alpha} & 0 \\
0 & \bar{\beta} & 0 & -\bar{\alpha} \\
\bar{\alpha} & 0 & -\bar{\beta} & 0 \\
0 & -\bar{\alpha} & 0 & -\bar{\beta}
\end{array}\right] \frac{\operatorname{Cosh}\left(\bar{\alpha} \frac{L}{2}\right) \operatorname{Sin}\left(\bar{\beta} \frac{L}{2}\right)}{2\left(\bar{\alpha}^{2}+\bar{\beta}^{2}\right)}+} \\
& {\left[\begin{array}{cccc}
\bar{\alpha} & 0 & -\bar{\beta} & 0 \\
0 & \bar{\alpha} & 0 & \bar{\beta} \\
-\bar{\beta} & 0 & -\bar{\alpha} & 0 \\
0 & \bar{\beta} & 0 & -\bar{\alpha}
\end{array}\right] \frac{\operatorname{Sinh}\left(\bar{\alpha} \frac{L}{2}\right) \operatorname{Cos}\left(\bar{\beta} \frac{L}{2}\right)}{2\left(\bar{\alpha}^{2}+\bar{\beta}^{2}\right)}+\left[\begin{array}{cccc}
+ & 0 & -\bar{\alpha} & 0 \\
\beta & -\bar{\beta} & 0 & -\bar{\alpha} \\
0 & 0 & + & 0 \\
\bar{\alpha} & 0 & \beta & 0 \\
0 & \bar{\alpha} & 0 & -\stackrel{+}{\beta}
\end{array}\right] \frac{\operatorname{Cosh}\left(\bar{\alpha} \frac{L}{2}\right) \operatorname{Sin}\left(\stackrel{+}{\beta} \frac{L}{2}\right)}{2\left(\bar{\alpha}^{2}+\beta^{2}\right)}+} \\
& {\left[\begin{array}{cccc}
\bar{\alpha} & 0 & + & 0 \\
0 & -\bar{\alpha} & 0 & + \\
+ & + \\
-\beta & 0 & \bar{\alpha} & 0 \\
0 & -\stackrel{+}{\beta} & 0 & -\bar{\alpha}
\end{array}\right] \frac{\operatorname{Sinh}\left(\bar{\alpha} \frac{L}{2}\right) \operatorname{Cos}\left(\stackrel{+}{\beta} \frac{L}{2}\right)}{2\left(\bar{\alpha}^{2}+\stackrel{+}{\beta}^{2}\right)}+\left[\begin{array}{cccc}
\bar{\beta} & 0 & -\stackrel{+}{\alpha} & 0 \\
0 & \bar{\beta} & 0 & \stackrel{+}{\alpha} \\
+ & 0 & \bar{\beta} & 0 \\
\alpha & 0 & & - \\
0 & -\stackrel{+}{\alpha} & 0 & \bar{\beta}
\end{array}\right] \frac{\operatorname{Cosh}\left(\stackrel{+}{\alpha} \frac{L}{2}\right) \operatorname{Sin}\left(\bar{\beta} \frac{L}{2}\right)}{2\left(\stackrel{+}{\alpha}^{2}+\bar{\beta}^{2}\right)}+}
\end{aligned}
$$

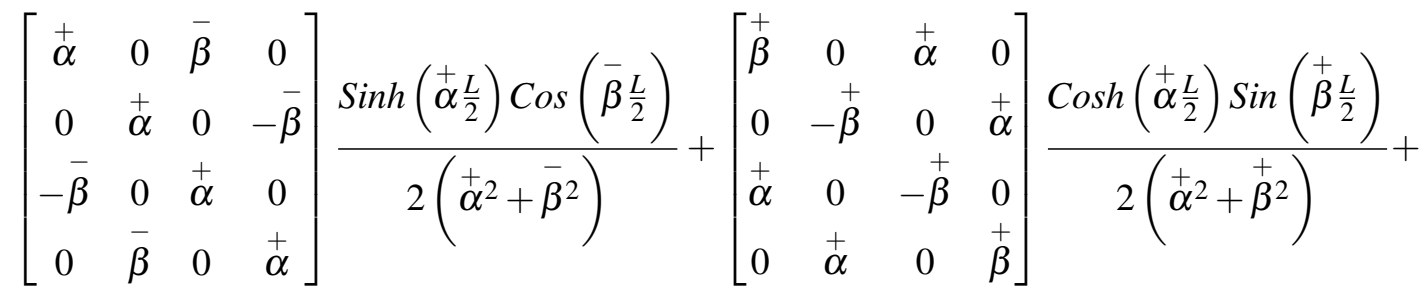

$$
\begin{aligned}
& {\left[\begin{array}{cccc}
\stackrel{+}{\alpha} & 0 & -\stackrel{+}{\beta} & 0 \\
0 & -\stackrel{+}{\alpha} & 0 & -\stackrel{+}{\beta} \\
-\stackrel{+}{\beta} & 0 & -\stackrel{+}{\alpha} & 0 \\
0 & -\stackrel{+}{\beta} & 0 & \stackrel{+}{\alpha}
\end{array}\right] \frac{\operatorname{Sinh}\left(\stackrel{+}{\alpha} \frac{L}{2}\right) \operatorname{Cos}\left(\stackrel{+}{\beta} \frac{L}{2}\right)}{2\left(\stackrel{+}{\alpha}+\stackrel{+}{\beta^{2}}\right)}}
\end{aligned}
$$

Where:

$$
\begin{aligned}
& \stackrel{+}{\alpha}={ }^{i} \alpha+{ }^{j} \alpha \\
& \bar{\alpha}={ }^{i} \alpha-{ }^{j} \alpha \\
& \stackrel{+}{\beta}={ }^{i} \beta+{ }^{j} \beta \\
& \bar{\beta}={ }^{i} \beta-{ }^{j} \beta
\end{aligned}
$$

In case of $i \neq j$, the above matrix is asymmetric. However, in a total global stiffness matrix, the coupling matrix ${ }^{i j}\left[\Upsilon_{N H a}\right]$ is completely symmetric in respect to its transpose, ${ }_{i j}^{j i}\left[\Upsilon_{N H a}\right]$. 


\subsubsection{Non-Homogeneous solution for distortion with warping - case}

\section{A with hinged-hinged boundary conditions}

When one adopts different boundary conditions at the element nodes, the advantages in presenting the stiffness matrix in the form of eq. 5.96 stand out simply by changing the completeness coefficients matrix.

For hinged-hinged ${ }^{1}$ boundary conditions, the null propriety of the first derivative of longitudinal restraint at the initial and final nodes cannot be applied. Instead, one must eliminate the generalized internal moment, which implies that the second derivative of shape function must be null at the hinged nodes.

For instance, the first two shape functions of case A, presented in eq. 5.20, must satisfy the following conditions at the initial node:

$S h_{H a h h 1}:$

$$
\begin{gathered}
S h_{\text {Hahc1 }}\left(x=-\frac{L}{2}\right)=1 \\
S h_{\text {Hahc } 1, x x}\left(x=-\frac{L}{2}\right)=0 \\
S h_{\text {Hahc1 }}(x=0)=0 \\
S h_{\text {Hahc } 1, x}(x=0)=0 \\
\operatorname{Sh}_{\text {Hahc1 }}\left(x=\frac{L}{2}\right)=0 \\
\operatorname{Sh}_{\text {Hahc } 1, x x}\left(x=\frac{L}{2}\right)=0
\end{gathered}
$$

$S h_{\text {Hahc } 2}:$

$$
\begin{gathered}
\operatorname{Sh}_{\text {Hahc } 2}\left(x=-\frac{L}{2}\right)=0 \\
S_{h_{\text {Hahc } 2, x x}\left(x=-\frac{L}{2}\right)=0} \\
\operatorname{Sh}_{\text {Hahc } 2}(x=0)=0 \\
\operatorname{Sh}_{\text {Hahc } 2, x}(x=0)=0 \\
\operatorname{Sh}_{\text {Hahc } 2}\left(x=\frac{L}{2}\right)=0 \\
\operatorname{Sh}_{\text {Hahc } 2, x x}\left(x=\frac{L}{2}\right)=0
\end{gathered}
$$

\footnotetext{
${ }^{1}$ The term hinged-hinged boundary conditions is an analog of bending moment, and it refers to a beam with longitudinal release and transverse restraint at initial and the final nodes. In GBT, a transverse membrane in a cross-section (such as a stiffener with thin thickness) provides this type of boundary condition for high modes.
} 
A matrix form can express not only the above conditions, but also other shape functions. Following the same procedure presented in the previous subsection and solving the six systems, each one with six equations, the hinged-hinged completeness coefficients matrix of boundary conditions are:

$$
\begin{aligned}
& {\left[S h_{\text {NHahh }}\right]=\frac{\left[\begin{array}{cccccc}
J_{11} & 0 & J_{13}=J_{11} & 0 & J_{15}=-2 J_{11} & 0 \\
J_{21} & 0 & J_{23}=-J_{21} & 0 & 0 & J_{26}=J_{21} L \\
J_{31} & 0 & J_{33}=J_{31} & 0 & J_{35}-2 J_{31} & 0 \\
J_{41} & 0 & J_{43}=-J_{41} & 0 & 0 & J_{46}=J_{41} L \\
J_{51} & 0 & J_{53}=J_{51} & 0 & 0 & J_{56} \\
J_{61}=-J_{11} & 0 & J_{63}=-J_{11} & 0 & J_{65} & 0
\end{array}\right]}{K_{\text {NHahh }}}} \\
& \text { With: } J_{65}=-2 \alpha \beta(\cosh (\alpha L)+\cos (\beta L)) \\
& J_{11}=\left(\beta^{2}-\alpha^{2}\right) \sinh \left(\frac{\alpha L}{2}\right) \sin \left(\frac{\beta L}{2}\right)-2 \alpha \beta \cosh \left(\frac{\alpha L}{2}\right) \cos \left(\frac{\beta L}{2}\right) \\
& K_{N H a h h}=J_{65}-2 J_{11} \\
& J_{21}=\frac{K_{N H a h h}}{D_{S 2}}\left(\left(\beta^{2}-\alpha^{2}\right) \sinh \left(\frac{\alpha L}{2}\right) \cos \left(\frac{\beta L}{2}\right)+2 \alpha \beta \cosh \left(\frac{\alpha L}{2}\right) \sin \left(\frac{\beta L}{2}\right)\right) \\
& D_{S 2}=L\left(\alpha^{2}+\beta^{2}\right)\left(\beta \sinh \left(\frac{\alpha L}{2}\right) \cos \left(\frac{\beta L}{2}\right)+\alpha \cosh \left(\frac{\alpha L}{2}\right) \sin \left(\frac{\beta L}{2}\right)\right)+J_{\text {aux }} \\
& J_{\text {aux }}=-2 \alpha \beta(\cosh (\alpha L)-\cos (\beta L)) \\
& J_{31}=\left(\alpha^{2}-\beta^{2}\right) \cosh \left(\frac{\alpha L}{2}\right) \cos \left(\frac{\beta L}{2}\right)-2 \alpha \beta \sinh \left(\frac{\alpha L}{2}\right) \sin \left(\frac{\beta L}{2}\right) \\
& J_{41}=\frac{K_{N H a h h}}{D_{S 2}}\left(2 \alpha \beta \sinh \left(\frac{\alpha L}{2}\right) \cos \left(\frac{\beta L}{2}\right)+\left(\alpha^{2}-\beta^{2}\right) \cosh \left(\frac{\alpha L}{2}\right) \sin \left(\frac{\beta L}{2}\right)\right) \\
& J_{51}=K_{N H a h h} \frac{J_{a u x}-D_{S 2}}{D_{S 2} L} \\
& J_{56}=\frac{L J_{a u x} J_{51}}{J_{\text {aux }}-D_{S 2}}
\end{aligned}
$$

By replacing $S h_{N H a c c}$ with $S h_{N H a h h}$ in eq. 5.97, one obtains the exact stiffness matrix for an element with hinged-hinged boundary conditions. The vector force in eq. 5.99 must receive the same substitution.

\subsubsection{Non-Homogeneous solution for distortion with warping - case A with clamped-hinged boundary conditions}

The completeness coefficients matrix to clamped-hinged boundary conditions can be determined in the same way of the previous case. However, due to non-symmetric boundary conditions, it leads to substantial complex and longer terms, which calls for attention to the cost of the exact solution for GBT in finite element method. 
It is important to note that, in spite of the complexity of the terms below, the stiffness matrix for these boundary conditions is only feasible with the use of completeness coefficients matrix. A direct derivation of the stiffness matrix, without the completeness coefficients matrix, will lead to extremely long terms.

By following the same procedure, as in hinged-hinged boundary conditions, one obtains the coefficient completeness matrix for the clamped-hinged boundary conditions:

$$
\left[S h_{\text {NHach }}\right]=\frac{\left[\begin{array}{cccccc}
J_{11} & J_{12} & J_{13} & 0 & J_{15} & J_{16} \\
J_{21} & J_{22} & J_{23} & 0 & J_{25} & J_{26} \\
J_{31} & J_{32} & J_{33} & 0 & J_{35} & J_{36} \\
J_{41} & J_{42} & J_{43} & 0 & J_{45} & J_{46} \\
J_{51} & J_{52} & J_{53} & 0 & J_{55} & J_{56} \\
J_{61}=-J_{11} & J_{62}=-J_{21} & J_{63}=-J_{13} & 0 & J_{65}=K_{N H a c h}-J_{15} & J_{66}=-J_{16}
\end{array}\right]}{K_{N H a c h}}
$$

To evaluate this case, the following auxiliary terms are used:

$$
\begin{gathered}
b_{1}=\left(\alpha^{2}+\beta^{2}\right) \\
b_{2}=\left(\alpha^{2}-\beta^{2}\right) \\
b_{3}=\left(3 \alpha^{2}+\beta^{2}\right) \\
b_{4}=\left(3 \alpha^{2}-\beta^{2}\right) \\
b_{5}=\left(\alpha^{2}+3 \beta^{2}\right) \\
b_{6}=\left(\alpha^{2}-3 \beta^{2}\right) \\
a_{2}=4 \sinh \left(\frac{\alpha L}{2}\right) \sin \left(\frac{\beta L}{2}\right)\left(\cos \left(\frac{\beta L}{2}\right)-\cosh \left(\frac{\alpha L}{2}\right)\right) \\
a_{1}=2 b_{1}\left(\alpha \sin \left(\frac{\beta L}{2}\right)-\beta \sinh \left(\frac{\alpha L}{2}\right)\right) \\
a_{4}=\operatorname{csch}\left(\frac{\alpha L}{2}\right) \csc \left(\frac{\beta L}{2}\right)-\operatorname{coth}\left(\frac{\alpha L}{2}\right) \cot \left(\frac{\beta L}{2}\right) \\
a_{5}=-L b_{1} b_{2} \cosh (\alpha L) \cos (\beta L) \\
a_{6}=-\beta^{2} L b_{1} \cos (\beta L) \\
a_{7}=-\alpha^{2} L b_{1} \cosh (\alpha L) \\
a_{8}=-4 \alpha \beta^{2} \sinh (\alpha L) \\
a_{9}=-4 \alpha \beta^{2} \sinh \left(\frac{\alpha L}{2}\right) \cos \left(\frac{3 \beta L}{2}\right) \\
a_{10}=-4 \alpha^{2} \beta \cosh \left(\frac{\alpha L}{2}\right) \sin \left(\frac{3 \beta L}{2}\right)
\end{gathered}
$$




$$
\begin{aligned}
& a_{11}=-2 \alpha \beta L b_{1} \sinh \left(\frac{\alpha L}{2}\right) \sin \left(\frac{\beta L}{2}\right) \\
& a_{12}=-4 \alpha^{2} \beta \cosh \left(\frac{3 \alpha L}{2}\right) \sin \left(\frac{\beta L}{2}\right) \\
& a_{13}=-4 \alpha \beta^{2} \sinh \left(\frac{3 \alpha L}{2}\right) \cos \left(\frac{\beta L}{2}\right) \\
& a_{14}=-L b_{1} b_{2} \cosh \left(\frac{\alpha L}{2}\right) \cos \left(\frac{\beta L}{2}\right) \\
& a_{15}=-2 \alpha \beta L b_{1} \sinh (\alpha L) \sin (\beta L) \\
& a_{16}=4 \alpha^{2} \beta \sin (\beta L) \\
& a_{17}=-2 \alpha b_{2} \sin \left(\frac{3 \beta L}{2}\right) \sinh \left(\frac{\alpha L}{2}\right) \\
& a_{18}=4 \alpha \beta^{2} \sin \left(\frac{\beta L}{2}\right) \cosh \left(\frac{\alpha L}{2}\right) \\
& a_{19}=4 \alpha^{2} \beta \cos \left(\frac{\beta L}{2}\right) \sinh \left(\frac{\alpha L}{2}\right) \\
& \quad a_{20}=4 \alpha^{2} \beta \sin (2 \beta L) \\
& a_{21}=-\alpha^{2} b_{1} L \cos \left(\frac{3 \beta L}{2}\right) \cosh \left(\frac{\alpha L}{2}\right) \\
& a_{22}=\beta^{2} b_{1} L \cos \left(\frac{\beta L}{2}\right) \cosh \left(\frac{3 \alpha L}{2}\right)
\end{aligned}
$$

$a_{23}=-\sinh \left(\frac{\alpha L}{2}\right)\left(4 \alpha \cosh \left(\frac{\alpha L}{2}\right)\left(b_{1} \cos (\beta L)-\alpha^{2}+4 \beta^{2} \cosh (\alpha L)\right)-\right.$

$\left.2 b_{1}\left(2 \beta \sinh \left(\frac{\alpha L}{2}\right) \sin (\beta L)+\sin \left(\frac{\beta L}{2}\right)(3 \alpha \beta L(\cos (\beta L)+1)-2 \alpha \sin (\beta L)-2 \beta \sinh (\alpha L))\right)\right)$

Therefore, the terms in eq. 5.140 are:

$$
\begin{gathered}
K_{N H a c h}=a_{23}-2\left(a_{5}+a_{6}-a_{7}-a_{15}-a_{16}\right)+3\left(\frac{a_{8}}{2}+a_{10}-a_{13}\right)+a_{11}(1-3 \cosh (\alpha L))+ \\
+a_{9}-a_{12}-a_{14}+a_{20}+a_{21}+a_{22} \\
J_{11}=a_{5}+a_{6}-a_{7}-a_{8}-a_{9}-a_{10}+a_{12}+a_{13}-a_{15}-a_{16} \\
J_{16}=\frac{a_{11}}{\alpha^{2} \beta^{2}}\left(\frac{4\left(\beta^{2} a_{7}-\alpha^{2} a_{6}\right)}{\left(L b_{1}\right)^{2}}+\frac{a_{16}-a_{8}}{4}\right) \\
J_{13}=J_{11}+a_{1} a_{2} \\
J_{15}=-2 J_{11}-a_{1} a_{2}
\end{gathered}
$$




$$
\begin{aligned}
& J_{12}=-\frac{L}{2} a_{1} a_{2}-J_{16} \\
& J_{25}=a_{3}\left(\alpha L-2 \sinh \left(\frac{\alpha L}{2}\right) \cos \left(\frac{\beta L}{2}\right)\right) \\
& J_{22}=\frac{4 \alpha \beta J_{25}}{L b_{1} a_{3}}\left(\frac{a_{11} b_{2}}{2 \alpha^{2} \beta^{2}}+\frac{2 a_{14}}{b_{2}}-\frac{a_{6}}{\beta^{2}}-\frac{a_{7}}{\alpha^{2}}\right) \\
& J_{23}=\frac{2 a_{6} b_{3}-3 b_{2} a_{11}+a_{15} b_{4}}{\beta L b_{1}}-\frac{4 \beta a_{14}\left(-2 \alpha^{4} a_{6}+L\left(\alpha \beta b_{1}\right)^{2}-\beta^{2} b_{2} a_{7}\right)}{\left(L \alpha \beta b_{1}\right)^{2} b_{2}}+ \\
& +\frac{2 \beta a_{7}}{\left(L \alpha \beta b_{1}\right)^{2}}\left(2 \alpha^{2} \beta^{2} L b_{1}-b_{4} a_{6}-2 a_{11} \beta^{2}\right)-\frac{L^{2} b_{1}\left(a_{8}+a_{16}\right)+8 a_{15}}{4 \beta L}-a_{17} \\
& J_{26}=\frac{L}{2}\left(J_{21}-J_{23}\right)-J_{22} \\
& J_{21}=-\left(J_{23}+J_{25}\right) \\
& J_{31}=-\frac{\alpha\left(a_{6}-a_{9}\right)}{\beta}+\frac{b_{3} a_{8}-b_{5} a_{16}+b_{2} a_{15}}{2 \alpha \beta}+2 \frac{\alpha \beta a_{5}}{b_{2}}-\frac{\beta a_{7}}{\alpha}+ \\
& +a_{18}\left(3+\frac{\alpha^{2}}{\beta^{2}}+2 \cos (\beta L)-2 \cosh (\alpha L)\right)+a_{19}\left(2+\frac{\beta^{2}}{\alpha^{2}}+2 \cosh (\alpha L)\right) \\
& J_{33}=J_{31}+a_{1} a_{2} a_{4} \\
& J_{32}=J_{12} a_{4} \\
& J_{36}=J_{16} a_{4} \\
& J_{35}=-\left(J_{31}+J_{33}\right) \\
& J_{43}=\frac{2}{\alpha L}\left(a_{5}+a_{6}-a_{7}+a_{14}-a_{21}-a_{22}-\frac{a_{11}}{L b_{1}}\left(\frac{a_{6}}{\beta^{2}}+\frac{a_{7}}{\alpha^{2}}-\frac{4 a_{14}}{b_{2}}\right)\right)-\frac{\beta}{\alpha} J_{23} \\
& J_{45}=-a 3\left(\beta L-2 \cosh \left(\frac{\alpha L}{2}\right) \sin \left(\frac{\beta L}{2}\right)\right) \\
& J_{42}=\frac{J_{22} J_{45}}{J_{25}} \\
& J_{46}=\frac{L}{2}\left(J_{41}-J_{43}\right)-J_{42} \\
& J_{41}=-\left(J_{45}+J_{43}\right) \\
& J_{5 i}=-\left(\beta J_{2 i}+\alpha J_{4 i}\right) \text { for: } 1 \leq i \leq 5 \\
& J_{56}=K_{\text {NHach }}-\left(\beta J_{26}+\alpha J_{46}\right)
\end{aligned}
$$

By replacing $S h_{N H a c c}$ with $S h_{N H a c h}$ in eq. 5.97, one obtains the exact stiffness matrix for an element with clamped-hinged boundary conditions. The same must be done for the vector force presented in eq. 5.99 . 


\subsection{Numerical Examples}

As a detailed numerical example of the application of the exact stiffness matrix, a thin-walled circular hollow steel cross-section, as shown in figure 5.2, is applied in a vertical cantilever structure. This structure is under a linear projected surface load, i.e., the total load applied in the structure is not a product of the surface load and the area of the surface, but the product of the surface load and the project area on the global coordinate direction z. The material parameters are: Young Modulus, $E=$ $205,000 \mathrm{~N} / \mathrm{mm}^{2}$, Poisson's ratio, $\mu=0.3$, Shear Modulus, $G=78,846.2 \mathrm{~N} / \mathrm{mm}^{2}$.

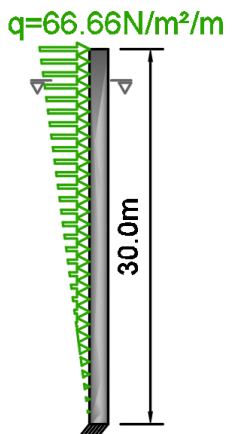

(a)

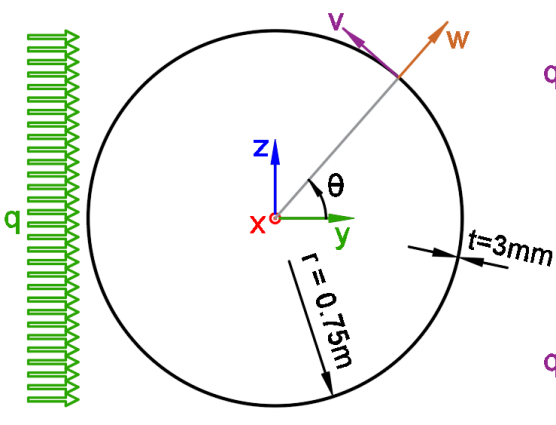

(b)

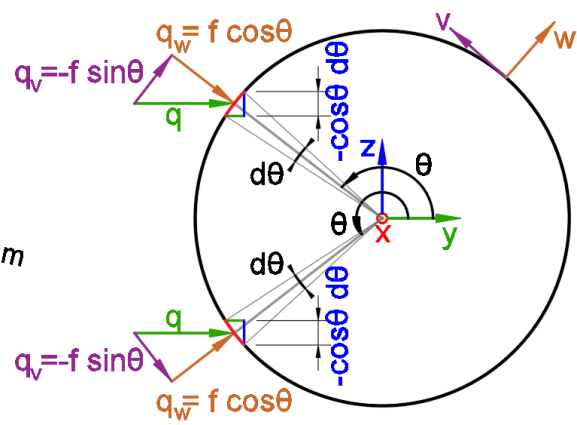

(c)

Figure 5.2: Thin-walled circular hollow section under a linear projected force; a) Elevation; b) cross-section; c) Force and area's projection in local coordinate system.

The evaluation of this problem has the following analytical steps: $1^{\text {st }}$ - Cross-section analysis and load's mode participation; $2^{\text {nd }}$ - Finite Element solution in the longitudinal direction; $3^{\text {rd }}$ and $4^{\text {th }}$ Analysis of the displacement and stress fields, respectively.

\subsubsection{Cross-section analysis and load's mode participation}

As exposed in chapter 3, the cross-section analysis of thin-walled circular hollow avoids the quadratic eigenvalue problem. Moreover, from an infinite number of orthogonal deformation modes, only a few modes are necessary to evaluate an applied problem such as the current one. The filtering technique of relevant deformation mode is performed by the modal decomposition of the external loads, which are assumed to be represented by separation of variables, as given in equations $3.48,3.49$ and 3.50 together with the inner product of the deformation modes, 3.51, 3.52 and 3.53. For instance, in the present case, the external project load can be described in the local coordinate system $(\mathrm{v}, \mathrm{w})$ as:

$$
\text { for } \pi / 2 \leq \theta \leq 3 \pi / 2 \quad\left\{\begin{array}{l}
q_{v}=q \sin (\theta) \cos (\theta) \\
q_{w}=-q \cos (\theta)^{2}
\end{array} \quad \text { and } q_{x}=0\right.
$$

In the inner products of these functions, equations 3.51, 3.52 and 3.53, the relevant modes in GBT's structural analysis stand out. Furthermore, the integral of odd trigono- 
metric functions, inside the interval $\pi / 2 \leq \theta \leq 3 \pi / 2$, are eliminated. Therefore, the even modes do not participate in this analysis, since ${ }^{\text {even }} q_{v}=q m \int_{\pi / 2}^{3 \pi / 2} \cos (\theta) \sin (\theta) \cos (m \theta) \mathrm{d} \theta=$ 0 and ${ }^{\text {even }} q_{w}=q m^{2} \int_{\pi / 2}^{3 \pi / 2} \cos (\theta)^{2} \sin (m \theta) \mathrm{d} \theta=0$. Table 3.1 present the summary of external forces modal decomposition and the respective mode cross-section properties necessary to develop the GBT solution:

Table 5.2: Example 5.1 - cross-section and external load modal decomposition

\begin{tabular}{cccccccc}
\hline Mode & $\begin{array}{c}C \\
\mathrm{~mm}^{4}\end{array}$ & $\begin{array}{c}D \\
\mathrm{~mm}^{4}\end{array}$ & $\begin{array}{c}B \\
\mathrm{~mm}^{-1}\end{array}$ & $\begin{array}{c}D^{\mu} \\
\mathrm{mm}^{-1}\end{array}$ & $\begin{array}{c}q_{v} \\
\mathrm{~N} / \mathrm{mm}^{2}\end{array}$ & $\begin{array}{c}q_{w} \\
\mathrm{~N} / \mathrm{mm}^{2}\end{array}$ & $\begin{array}{c}q_{\text {tot }} \\
\mathrm{N} / \mathrm{mm}^{2}\end{array}$ \\
\hline $\mathrm{a}$ & $11,651.51$ & 0 & $2.513-2$ & 0 & 0.000 & -2.3562 & -2.3562 \\
3 & $3,976,084,027$ & 0 & 0 & 0 & 1.000 & 2.000 & 3.000 \\
5 & $3,976,171,414$ & 1.36 & $1.072-6$ & -0.20 & -2.3562 & -4.7124 & -7.069 \\
7 & $3,976,550,088$ & 21.71 & $3.860-5$ & -2.71 & 1.800 & 3.600 & 5.400 \\
11 & $3,979,719,299$ & 542.87 & $2.681-3$ & -62.83 & -0.714 & -1.429 & -2.143 \\
15 & $3,990,065,841$ & $4,256.08$ & $4.119-2$ & -482.75 & 0.467 & 0.933 & 1.400 \\
\hline
\end{tabular}

Here, 5 modes are chosen. Among these modes, 3 and 5 are enough to solve the displacement field. However, in order to obtain some particular internal forces, especially in the neighborhood of support, higher modes are required. Since these high modes have $\mathrm{C}, \mathrm{D}$ and $\mathrm{B}$ stiffness terms, one must perform a mode classification concerning the dominant behavior, as it is in subsection 5.2.2:

Table 5.3: Example 5.1 - Modal classification

\begin{tabular}{ccccc}
\hline Mode & $G D\left(\mathrm{~N} / \mathrm{mm}^{2}\right)$ & $2 \sqrt{B E C}\left(\mathrm{~N} / \mathrm{mm}^{2}\right)$ & condition & case \\
\hline $\mathrm{a}$ & 0.00 & $7.016 \mathrm{E}+6$ & $G D<2 \sqrt{B E C}$ & $\mathrm{~A}$ \\
5 & $1.658 \mathrm{E}+5$ & $4.210 \mathrm{E}+7$ & $G D<2 \sqrt{B E C}$ & $\mathrm{~A}$ \\
7 & $2.538 \mathrm{E}+6$ & $2.526 \mathrm{E}+8$ & $G D<2 \sqrt{B E C}$ & $\mathrm{~A}$ \\
11 & $6.191 \mathrm{E}+7$ & $2.106 \mathrm{E}+9$ & $G D<2 \sqrt{B E C}$ & $\mathrm{~A}$ \\
\hline
\end{tabular}

In all GBT's modes, the dominant behavior is the distortion, which is typical in thinwalled beams. Moreover, one can apply the exact stiffness matrix approach detailed in Case A.

\subsubsection{Finite element solution in the longitudinal direction}

After obtaining and classifying the cross-section properties, the finite element approach solves the longitudinal amplification for each deformation mode. A well-known Hermitian element, with 2 nodes and 4 degrees of freedom, could solve mode 3 . However, as an opportunity to clarify the application of completeness coefficient matrix, this mode is solved here by a Hermitian element with 3 nodes and 6 degrees of freedom, such as in figure 5.1. For this element, the shape functions vector is:

$$
V(x)=\left[T x_{H e}\right]\left[S h_{H e c c}\right][\vartheta]
$$




$$
\begin{gathered}
\text { with: }\left[\begin{array}{llllll}
T x_{H e}
\end{array}\right]=\left[\begin{array}{llllll}
x^{5} & x^{4} & x^{3} & x^{2} & x & 1
\end{array}\right] \\
{[\vartheta]=\left[\begin{array}{llllll}
V_{-L / 2} & V_{-L / 2}^{\prime} & V_{L / 2} & V_{L / 2}^{\prime} & V_{0} & V_{0}^{\prime}
\end{array}\right]^{T}}
\end{gathered}
$$

The completeness coefficient matrix, $S h_{H e c c}$, can be obtained by applying the procedures showed in section 5.2.5. For the case of clamped-clamped, it results in:

$$
\left[S h_{H e c c}\right]=\left[\begin{array}{cccccc}
24 / L^{5} & 4 / L^{4} & -24 / L^{5} & 4 / L^{4} & 0 & 16 / L^{4} \\
-8 / L^{4} & -2 / L^{3} & -8 / L^{4} & 2 / L^{3} & 16 / L^{4} & 0 \\
-10 / L^{3} & -1 / L^{2} & 10 / L^{3} & -1 / L^{2} & 0 & -8 / L^{2} \\
4 / L^{2} & 1 /(2 L) & 4 / L^{2} & -1 /(2 L) & -8 / L^{2} & 0 \\
0 & 0 & 0 & 0 & 0 & 1 \\
0 & 0 & 0 & 0 & 1 & 0
\end{array}\right]
$$

The corresponding kernel stiffness matrix and force vector (for linear external force: $f=a x+b)$ for this case are, respectively:

$$
\left[\Upsilon_{H e}^{\prime \prime}\right]=\left[\begin{array}{cccccc}
25 L^{7} / 28 & 0 & 3 L^{5} / 2 & 0 & 0 & 0 \\
& 9 L^{5} / 5 & 0 & 2 L^{3} & 0 & 0 \\
& & 3 L^{3} & 0 & 0 & 0 \\
& & & 4 L & 0 & 0 \\
\text { Sym. } & & & & 0 & 0 \\
& & & & & 0
\end{array}\right] \quad(5.198) \quad\left[F_{H e}\right]=\left[\begin{array}{c}
a L^{7} / 448 \\
b L^{5} / 80 \\
a L^{5} / 80 \\
b L^{3} / 12 \\
a L^{3} / 12 \\
b L
\end{array}\right]
$$

Thus, one can build i) the effective stiffness matrix by the transformation of completeness coefficient matrix: ${ }^{3}\left[K_{c}\right]=E^{3} C^{3}\left[S h_{H e c c}\right]^{T}{ }^{3}\left[\Upsilon_{H e}^{\prime \prime}\right]^{3}\left[S h_{H e c c}\right]$, and ii) the effective external force vector: $\left[{ }^{3} F\right]={ }^{3}\left[S h_{H e c c}\right]^{T}\left[F_{H e}\right]$. Together with the boundary conditions of the null displacement and rotation in $x=-L / 2$, one addresses the system of equations to find the nodal displacements in mode 3 (units in $\mathrm{N}$ and $\mathrm{mm}$ ):

$$
\begin{array}{r}
{\left[\begin{array}{c}
1.69 E+4 \\
-3.21 E+7 \\
2.4 E+4 \\
5.14 E+7
\end{array}\right]=\left[\begin{array}{cccc}
4.39 E+3 & -2.94 E+7 & -3.09 E+3 & -4.97 E+7 \\
& 2.58 E+11 & 2.32 E+7 & 2.48 E+11 \\
& & 6.18 E+3 & 0 \\
S y m & \\
{\left[{ }^{3} V_{L / 2}\right.} & { }^{3} V_{L / 2}^{\prime} & { }^{3} V_{0} & { }^{3} V_{0}^{\prime}
\end{array}\right]^{T}=\left[\begin{array}{llll}
273.28 & 0.0124 & 93.94 & 0.0106
\end{array}\right]}
\end{array}
$$

With the nodal displacement values, eq. 5.194 and its first derivative achieve the displacement field corresponding to mode 3 at any position in the longitudinal direction.

In the sequence, it is developed the finite element analysis for GBT's high modes 5, 7, 11 and 15:

- mode 5: To build up the completeness coefficient and stiffness matrices, one must first find the values of $\alpha$ and $\beta$ :

$$
{ }^{5} \alpha=\sqrt{\sqrt{\frac{B}{4 E C}}+\frac{G D}{4 E C}}=1.13855 E-4
$$




$$
{ }^{5} \beta=\sqrt{\sqrt{\frac{B}{4 E C}}-\frac{G D}{4 E C}}=1.13401 E-4
$$

Hence, the completeness coefficient matrix is evaluated based on clamped-clamped boundary conditions, eq. 5.29:

$$
\left[S h_{\text {NHacc }}\right]=\left[\begin{array}{cccccc}
0.25 & 2,341.167 & 0.25 & -2,341.167 & -0.5 & 0.0 \\
-1.071 & -4,823.949 & 1.071 & -4,823.949 & 0.0 & -22,473.151 \\
0.318 & 1,212.475 & 0.318 & -1,212.475 & -0.637 & 0.0 \\
-0.342 & -2,639.382 & 0.342 & -2,639.382 & 0.0 & -4,994.045 \\
0.00016 & 0.848 & -0.00016 & 0.848 & 0.0 & 4.117 \\
-0.25 & -2,341.167 & -0.25 & 2,341.167 & 1.5 & 0.0
\end{array}\right]
$$

Following the presented procedure, the kernel stiffness matrices due to longitudinal stress, transverse shear, distortion and Poisson effect of the plate behavior are addressed by the respective equations $5.51,5.60,5.71$ and 5.83 :

$$
\begin{aligned}
& {\left[\Upsilon_{N H a}^{\prime \prime}\right]=10^{-11}\left[\begin{array}{cccccc}
3.048 & 0 & -0.794 & 0 & 0 & 0 \\
& 0.403 & 0 & -0.802 & 0 & 0 \\
& & 1.327 & 0 & 0 & 0 \\
& & & 4.124 & 0 & 0 \\
\text { Sym } & & & & 0 & 0 \\
& & & & & 0
\end{array}\right]} \\
& {\left[\Upsilon_{N H a}^{\prime}\right]=10^{-4}\left[\begin{array}{cccccc}
5.69 & 0 & -7.218 & 0 & 0 & 0 \\
& 11.534 & 0 & -3.344 & 56.499 E+3 & 0 \\
& & 11.843 & 0 & 0 & 0 \\
& & & 5.412 & -6.928 E+3 & 0 \\
S y m & & & & 3 E+8 & 0 \\
& & & & & 0
\end{array}\right]} \\
& {\left[\Upsilon_{H a}\right]=10^{4}\left[\begin{array}{cccccc}
1.9814 & 0 & 1.1803 & 0 & 0 & 2.1757 \\
& 6.1946 & 0 & 1.1803 & 3.709 E+4 & 0 \\
& & 4.5811 & 0 & 0 & 2.7954 \\
& & & 0.5949 & 8.088 E+3 & 0 \\
\text { Sym } & & & & 2.25 E+8 & 0 \\
& & & & & 3.0
\end{array}\right]} \\
& {\left[\Upsilon_{H a}^{\prime \prime}{ }^{\prime}{ }^{\prime}\right]=10^{-4}\left[\begin{array}{cccccc}
-6.036 & 0 & -6.682 & 0 & 0 & -7.198 \\
& 6.143 & 0 & -14.43 & 2.103+4 & 0 \\
& & 6.122 & 0 & 0 & 5.627 \\
& & & -6.054 & -9.567+4 & 0 \\
\text { Sym } & & & & 0 & 0 \\
& & & & & 0
\end{array}\right]}
\end{aligned}
$$

From eq. 5.97, these matrices lead to the exact stiffness matrix, presented below in eq. 5.209. Also, it is presented the correspondent load vector, obtained from eq. 5.99:

$$
{ }^{5}\left[K_{N H a}\right]=10^{3}\left[\begin{array}{cccccc}
6.957 & 3.394+4 & -1.022 & 5.219+3 & -2.216 & 4.563+4 \\
& 2.679+8 & -5.219+3 & 2.587+7 & -2.076+4 & 2.411+8 \\
& & 6.957 & -3.394+4 & -2.216 & -4.563+4 \\
& & & 2.679+8 & 2.076+4 & 2.411+8 \\
& & & & 1.330+1 & 0 \\
\text { Sym } & & & & & 1.171+9
\end{array}\right]
$$




$$
{ }^{5}\left[F_{\text {NHacc }}\right]^{T}=10^{4}\left[\begin{array}{llllll}
-0.9115 & -2.9 E+3 & -3.9246 & 7.416 E+3 & -5.7668 & -12.335 E+3
\end{array}\right]
$$

The boundary conditions for GBT's high modes are, in this case, the same of EulerBernoulli bending, since the longitudinal (warping) and transverse (distortion) at the initial point, $x=-L / 2$ are restrained. After this restriction, one can solve the system of linear equation to determine the other degrees of freedom:

$$
\left[\begin{array}{c}
-3.9246 \\
7.416 E+3 \\
-5.7668 \\
-12.335 E+3
\end{array}\right]=\left[\begin{array}{cccc}
0.6957 & -3.392 E+3 & -0.2216 & -4.564 E+3 \\
& 2.679 E+7 & 2.076 E+3 & 2.412+7 \\
& & 1.33 & 0 \\
\text { Sym } & & & 1.172 E+8
\end{array}\right]\left[\begin{array}{c}
{ }^{5} V_{L / 2} \\
{ }^{5} V_{L / 2}^{\prime} \\
{ }^{5} V_{0} \\
{ }^{5} V_{0}^{\prime}
\end{array}\right] \therefore\left[\begin{array}{c}
{ }^{5} V_{L / 2} \\
{ }^{5} V_{L / 2}^{\prime} \\
{ }^{5} V_{0} \\
{ }^{5} V_{0}^{\prime}
\end{array}\right]=\left[\begin{array}{c}
-13.303 \\
-4.896-4 \\
-5.788 \\
-5.228-4
\end{array}\right]
$$

- modes 7, 11, 15 and radial: These modes follow the same procedure used for mode 5 . Hence, only the final values of nodal displacements are presented here:

$$
\begin{aligned}
& { }^{a}[\vartheta]^{T}=\left[\begin{array}{llllll}
0.0 & 0.0 & -4.573 E-4 & -1.524 E-8 & -2.287 E-4 & -1.524 E-8
\end{array}\right] \\
& { }^{7}[\vartheta]^{T}=\left[\begin{array}{llllll}
0.0 & 0.0 & 2.763-1 & 9.547-6 & 1.384-1 & 9.152-6
\end{array}\right] \\
& { }^{11}[\vartheta]^{T}=\left[\begin{array}{llllll}
0.0 & 0.0 & -1.59-3 & -7.24-8 & -7.88-4 & -5.26-8
\end{array}\right] \\
& { }^{15}[\vartheta]^{T}=\left[\begin{array}{llllll}
0.0 & 0.0 & 6.71-5 & 2.53-9 & 3.35-5 & 2.23-9
\end{array}\right]
\end{aligned}
$$

In the case of radial mode, since there is not any torsion stiffness, $D=0$, it leads to a particular case, in which $\alpha=\beta$.

\subsubsection{Analysis of the displacement field}

This subsection does not only plot the generalized displacement and rotation functions, but also it compares them to the solution obtained from the usual $C^{1}$ Hermitian shape functions. Moreover, it details the cross-section transverse deformation at the extreme free point of the structure and compares it to the results obtained from a finite-element analysis based on shell elements.

The generalized displacement functions, given by eq. 5.46, are plotted below for modes a, 5, 7, and 11, in figures 5.3, 5.4, 5.5, 5.6 and 5.7, respectively. The amplification functions for cross-section warping, i.e., the longitudinal displacement, are found by the first derivative of eq. 5.46 and plotted below for the same modes in figures 5.8, 5.9, 5.10, 5.11 and 5.12 , respectively.

One can observe that the solution obtained from a single Hermitian element lacks precision, which can be overcome by finer discretization, e.g. with 10 elements as used above. Although this solution seems to have better accuracy for the displacement field for the lower order modes 5 and 7 , the first derivatives for the higher modes, such as 11,15 and the radial mode, have coarse results, especially near the support boundary condition. Further, it is important to keep in mind that this solution was obtained 


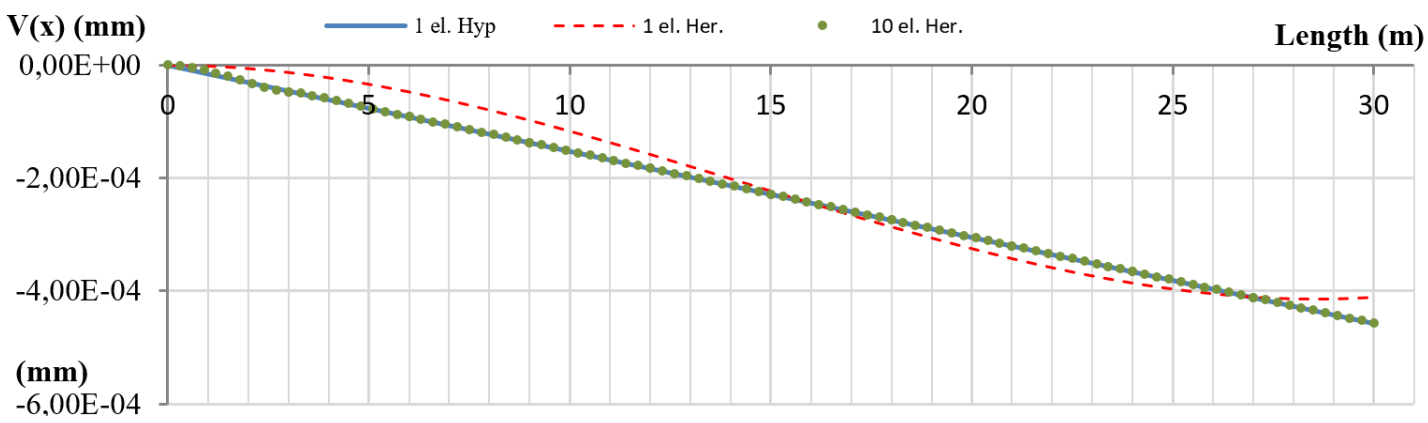

Figure 5.3: Generalized displacement of the radial mode, ${ }^{a} V$, (in $\mathrm{mm}$ )

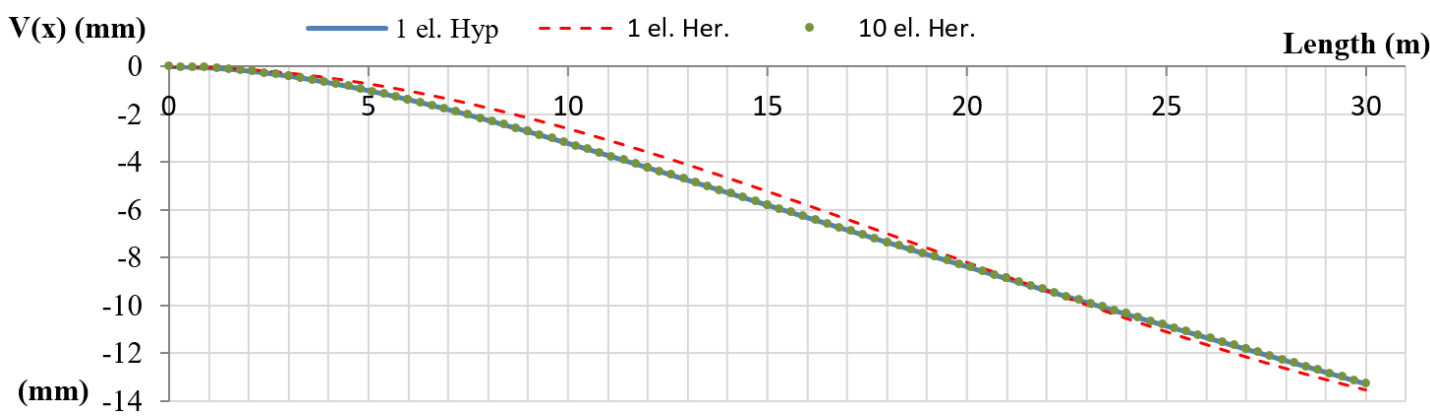

Figure 5.4: Generalized displacement of mode $5,{ }^{5} \mathrm{~V}$, (in $\mathrm{mm}$ )

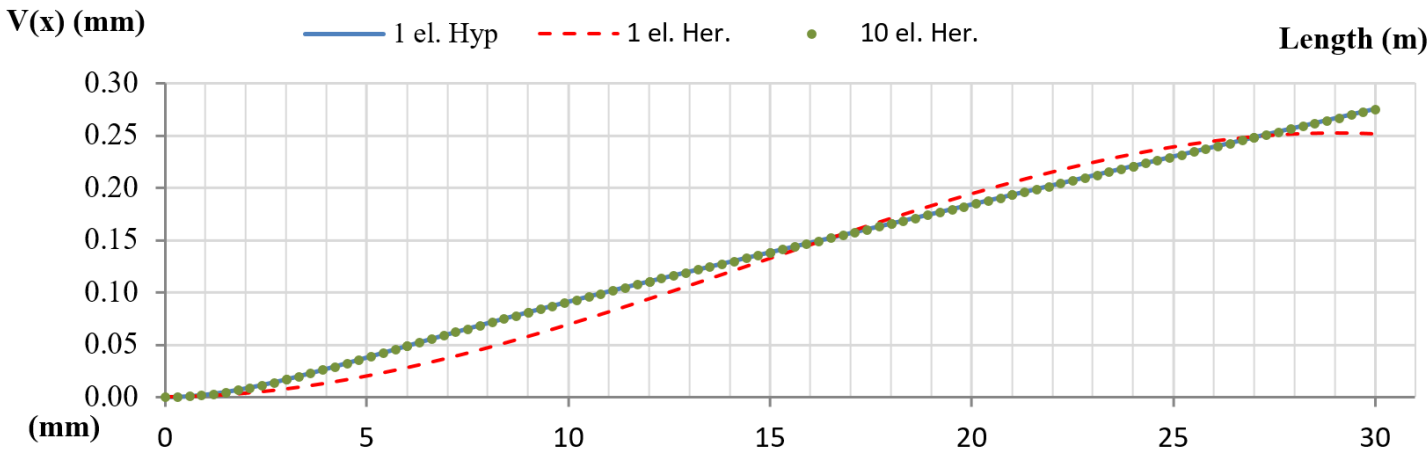

Figure 5.5: Generalized displacement of mode $7,{ }^{7} V$, (in $\mathrm{mm}$ )

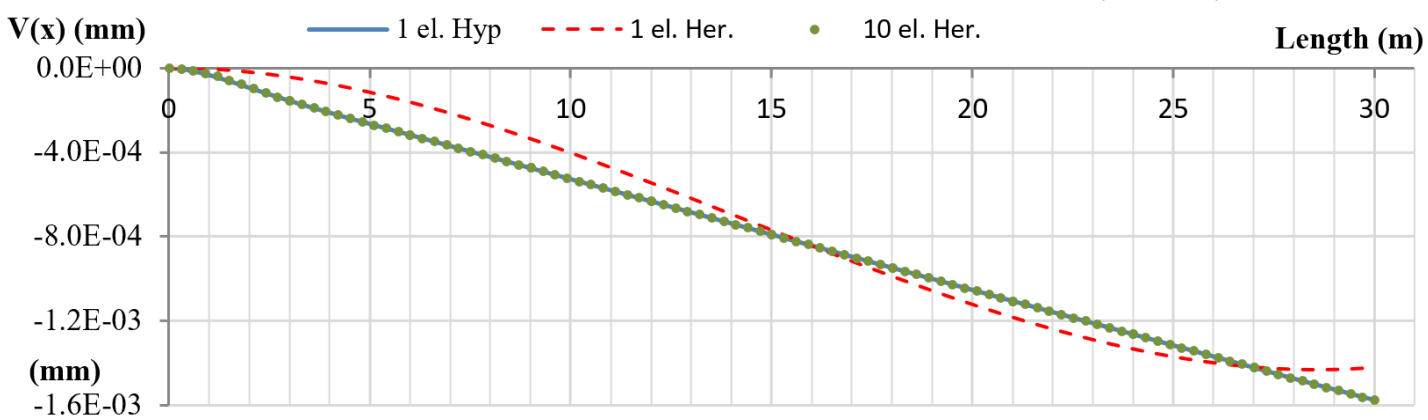

Figure 5.6: Generalized displacement of mode $11,{ }^{11} V$, (in mm)

from a linear equation system of 20 unknowns instead of a system with 4 unknowns, as proposed here by the trigonometric-hyperbolic shape functions. Moreover, the high computational performance of GBT is only reached by coarse longitudinal beam dis- 


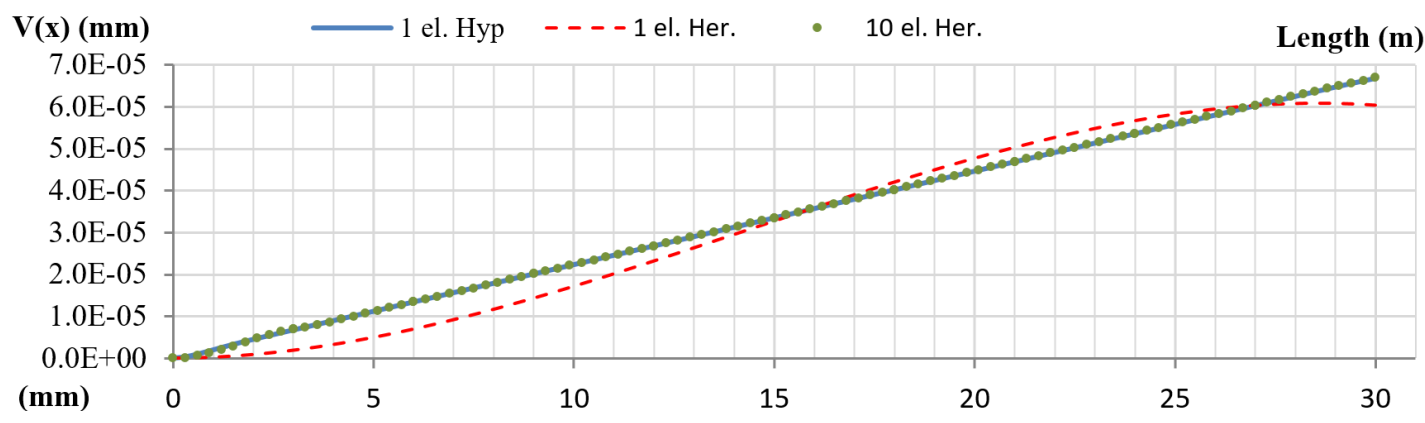

Figure 5.7: Generalized displacement of mode $15,{ }^{15} \mathrm{~V}$, (in $\mathrm{mm}$ )

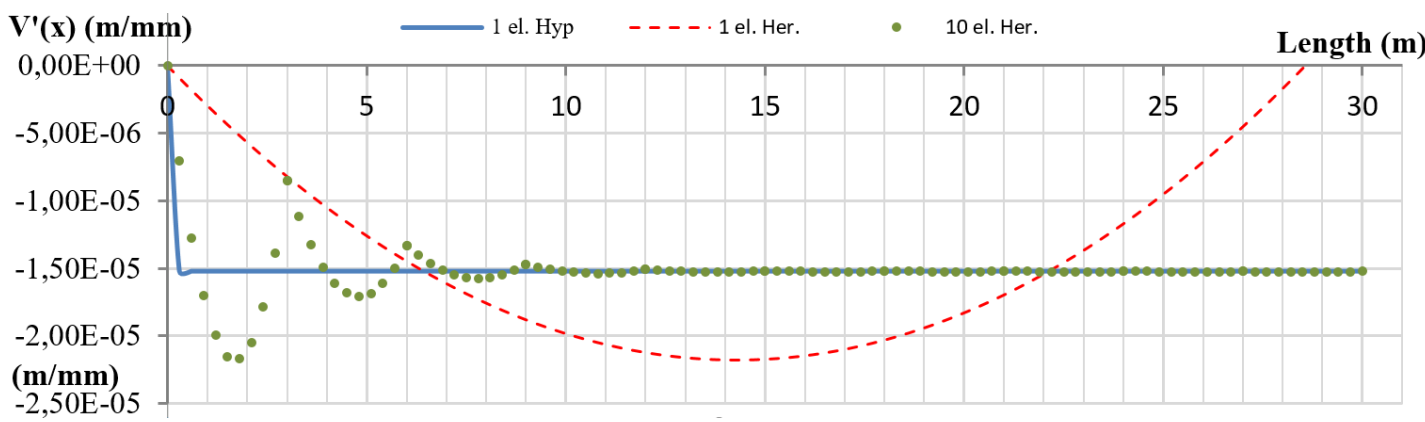

Figure 5.8: Generalized warping displacement of the radial mode, ${ }^{a} V^{\prime}$, (in $\mathrm{m} / \mathrm{m}$ )

$\mathbf{V}^{\prime}(\mathbf{x})(\mathbf{m} / \mathbf{m m}) \quad 1$ el. Hyp - - - 1 el. Her. $\quad 10 \mathrm{el}$. Her.

Length (m)

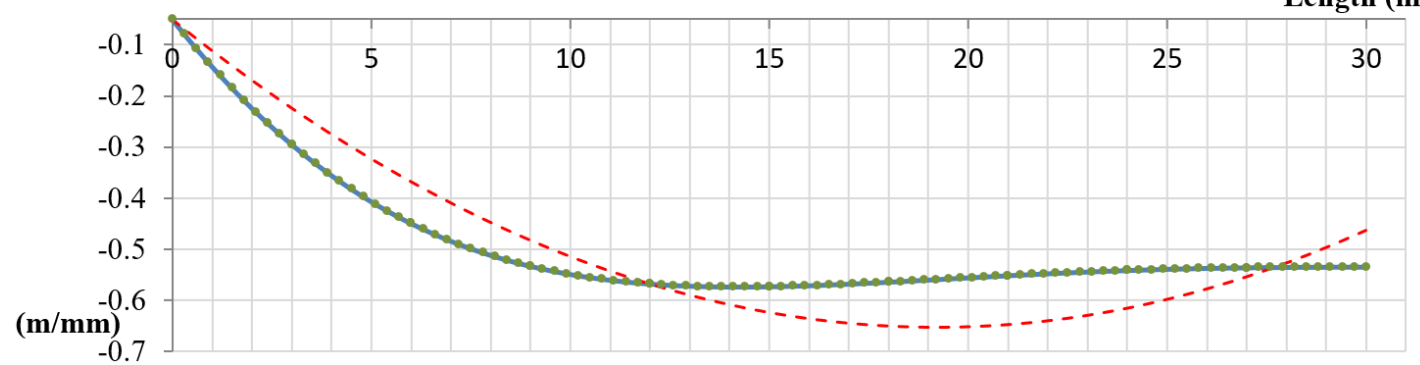

Figure 5.9: Generalized warping displacement of mode $5,{ }^{5} V^{\prime}$, (in $\left.\mathrm{m} / \mathrm{m}\right)$

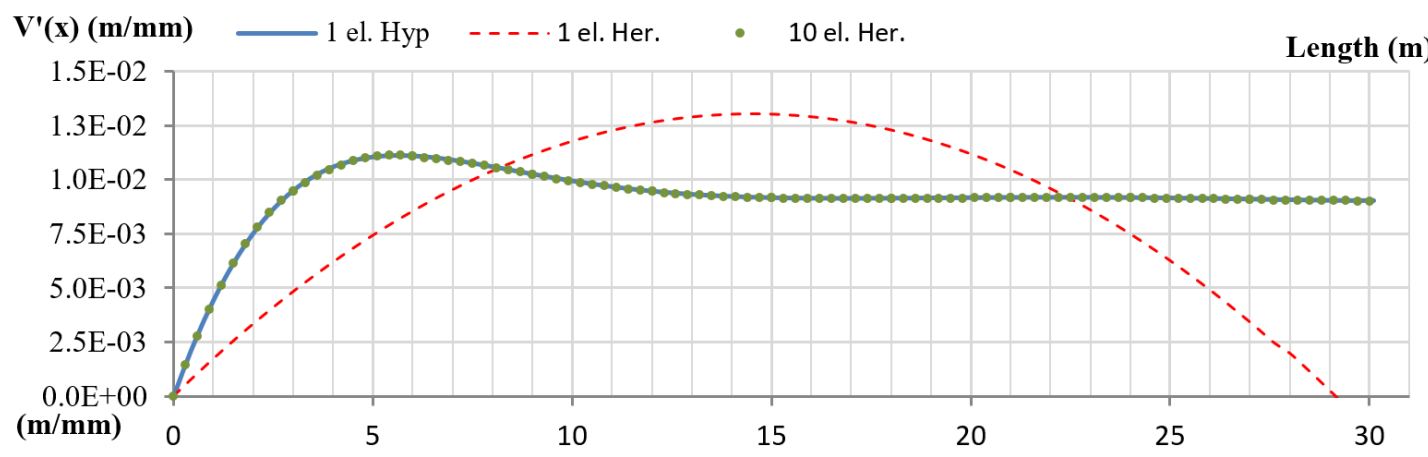

Figure 5.10: Generalized warping displacement of mode $7,{ }^{7} V^{\prime}$, (in $\mathrm{m} / \mathrm{m}$ )

cretization. If some structural analysis requires many GBT modes, it will lead to similar amount of degrees of freedom for each beam node as in the case of a cross-section modeled by shell elements. This GBT model with a high number of modes, together with 


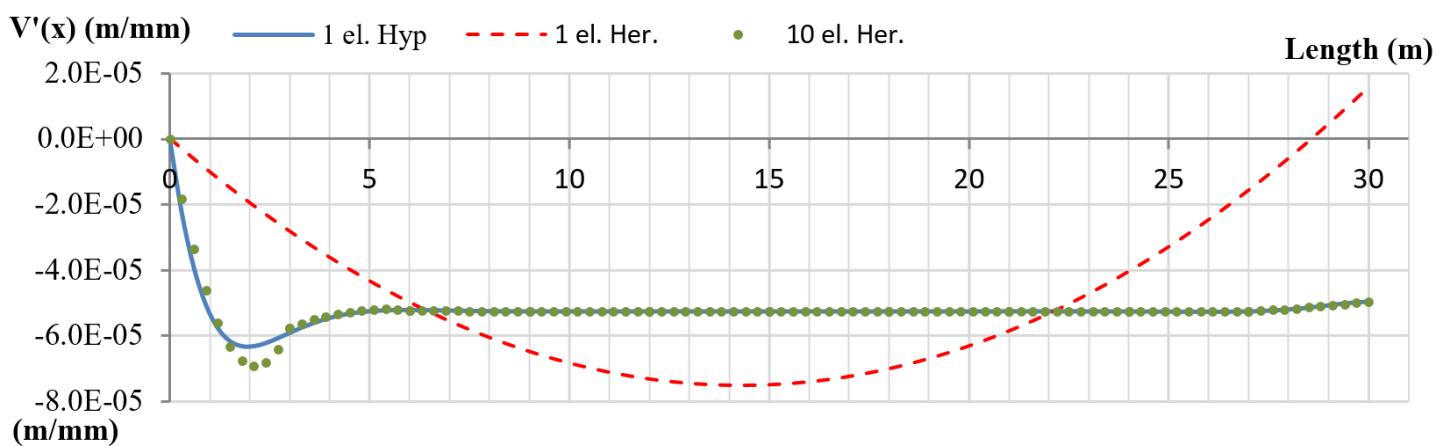

Figure 5.11: Generalized warping displacement of mode $11,{ }^{11} V^{\prime}$, (in $\mathrm{m} / \mathrm{m}$ )

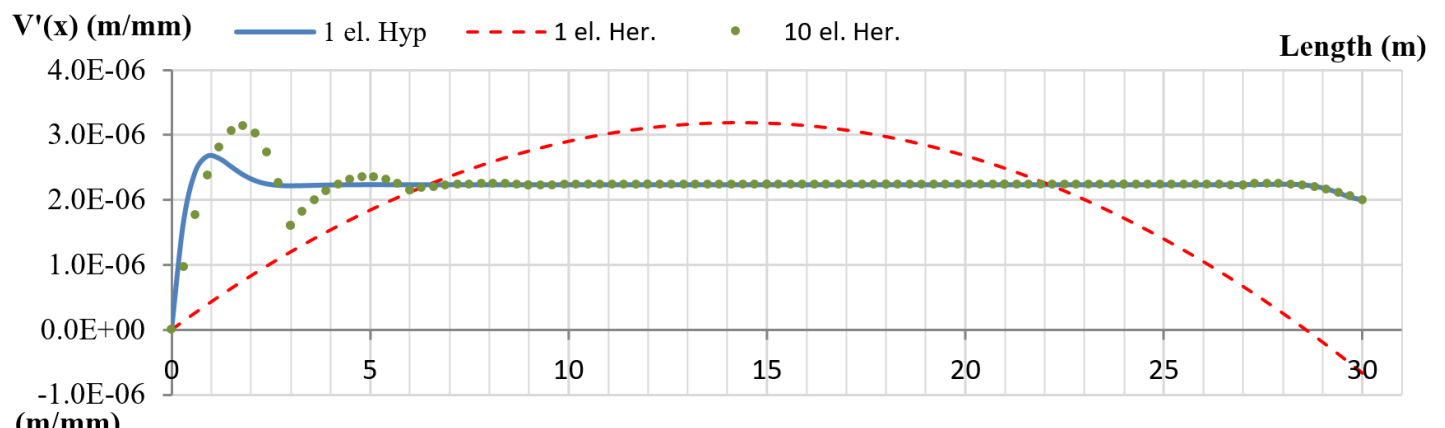

$(\mathbf{m} / \mathbf{m m})$

Figure 5.12: Generalized warping displacement of mode $15,{ }^{15} V^{\prime}$, (in $\mathrm{m} / \mathrm{m}$ )

fine longitudinal discretization, provides a total number of unknowns that is similar to a traditional shell element analysis. This case is especially applicable in non-linear analysis involving plasticity [2].

Actually, shell element models with high discretization are used in the approach to validate the GBT's models that are based on comparison of the results from both types of models $[37,38,71]$.

Here, the results using shell elements were obtained from commercial ANSYS ${ }^{\circledR}$ software, where four models based on shell elements types $63,93,181$, and 281 were developed. The discretization among these models are the same: 100 nodes for the cross-section and 600 element segments in the longitudinal direction. This leads to a total of 60,000 elements. The differences among these models are: i) the type of interpolation function applied: linear (shell-63 and shell-181) or quadratic (shell-93 and shell-281); ii) the kinematic hypotheses: Kirchhoff-Love (shell-63 and shell-93) and Mindlin-Reissner (shell-181 and shell-281) [5, 168].

The comparison of displacement fields, among these models and the GBT one, is placed in the cross-section at the free extreme, which is plotted in figures 5.13 and 5.14. The combination of radial and tangential displacements, equations 3.6 and 3.4 respectively, achieves the transverse deformation. The value of ${ }^{i} V(x)$ of each mode is taken at the node $x=L / 2$, and for each desired point on this cross-section, the values $\mathrm{v}$ and $\mathrm{w}$ are accessed in table. 3.1 . 

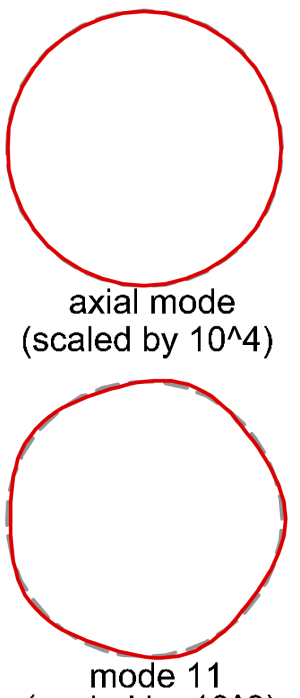

(scaled by $10^{\wedge} 3$ )
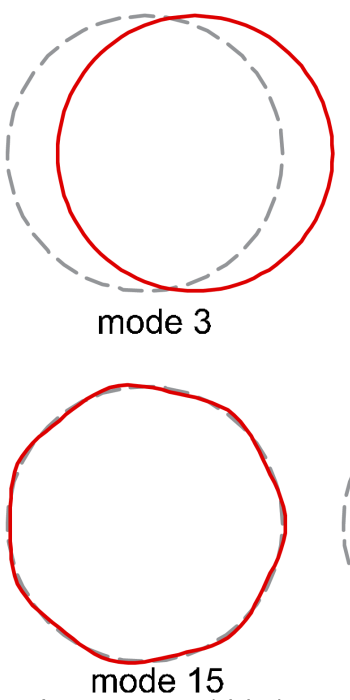

(scaled by $10^{\wedge} 4$ )
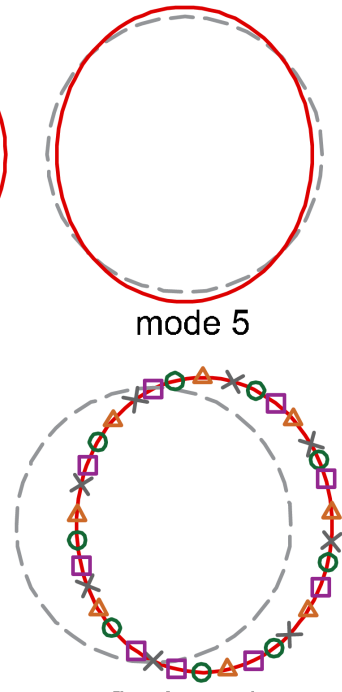

final result

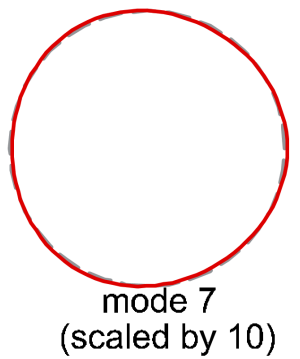

cross-section

middle wall

$=$ GBT solution

$X \times X$ shell 63

$\triangle \Delta \Delta$ shell 93

마 shell 181

৩ shell 281

Figure 5.13: Results of top cross-section deformation: the GBT solution is obtained by the summation of all modal deformation factors. The solution achieved from shell elements is also presented.

One can note in figure 5.13, that the contribution from modes 7, 11, 15 and radial are almost undetectable in the total displacement. Nevertheless, these modes are required to achieve an accurate stress field, as shown in the next subsection. Table 5.4 shows that the highest mean difference is around $0.8 \%$, which occurs in the tangential direction $v$ between GBT and Shell-63. Further, it shows that the GBT results approach the response of elements with quadratic interpolation functions.

Table 5.4: Mean differences, in \%, and standard deviation (SD) of displacement field between GBT and shell elementsk models at point $x=L / 2$

\begin{tabular}{ccccccc}
\hline element & \multicolumn{2}{c}{ u } & \multicolumn{2}{c}{ v } & \multicolumn{2}{c}{ w } \\
& diff. & SD & diff. & SD & diff. & SD \\
\hline Shell-63 & $0.13 \%$ & $0.36 \%$ & $0.78 \%$ & $0.94 \%$ & $0.4 \%$ & $1.58 \%$ \\
Shell-93 & $0.04 \%$ & $0.25 \%$ & $0.49 \%$ & $1.06 \%$ & $0.34 \%$ & $1.51 \%$ \\
Shell-181 & $0.16 \%$ & $0.41 \%$ & $0.61 \%$ & $1.08 \%$ & $0.43 \%$ & $1.62 \%$ \\
Shell-281 & $0.04 \%$ & $0.30 \%$ & $0.49 \%$ & $1.06 \%$ & $0.34 \%$ & $1.52 \%$ \\
\hline
\end{tabular}

For longitudinal displacement, the differences are even smaller. The highest mean difference is around $0.16 \%$ with respect to Shell-181. Figure. 5.14 presents a diagram of the longitudinal displacement in order to show the quality of the results:

To illustrate how the Poisson's effect, presented in sub-section 3.1.2, acts in the displacement field, figure 5.15 plots the cross-section deformation at the longitudinal position of $x=1 \mathrm{~m}$. Inserting the modal displacement, from eq. 5.211 to eq. 5.215 into eq. 5.46, one achieves the values of ${ }^{i} V(x)$ of each respective mode: 


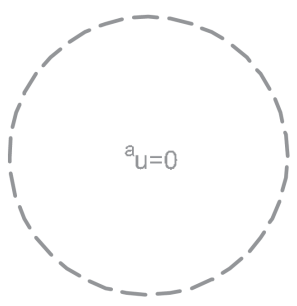

axial mode

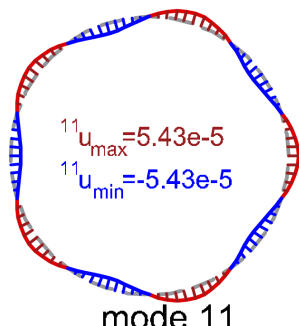

mode 11

(scaled by $10^{\wedge} 6$ )
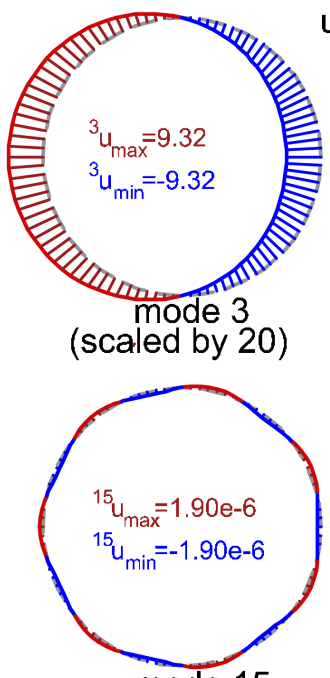

mode 15

(scaled by $10^{\wedge} 7$ )

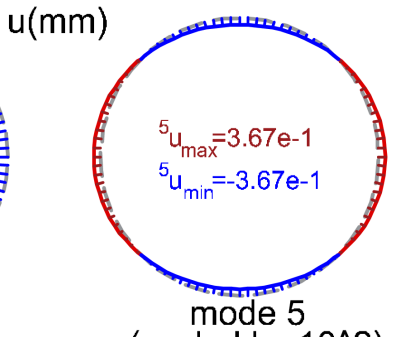

(scaled by $10^{\wedge} 2$ )

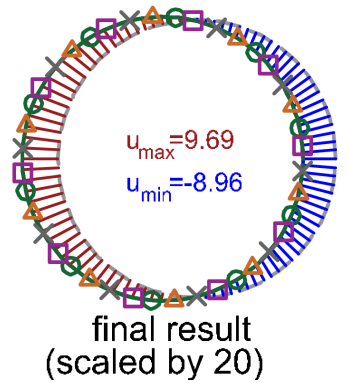

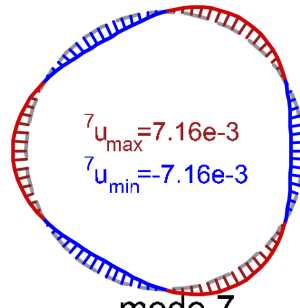

mode 7

(scaled by $10^{\wedge} 4$ )

cross-section

middle wall

$=$ GBT solution

$x \times x$ shell 63

$\Delta \Delta \Delta$ shell 93

마 shell 181

•

Figure 5.14: Results of longitudinal displacement at top cross-section: Comparison between the solutions of GBT and shell elements.

Table 5.5: GBT displacements at $\mathrm{x}=1 \mathrm{~m}$

\begin{tabular}{cccccc}
\hline${ }^{a} V$ & ${ }^{3} V$ & ${ }^{5} V$ & ${ }^{7} V$ & ${ }^{11} V$ & ${ }^{15} V$ \\
\hline$-1.524-5$ & $5.429-1$ & $-4.712-2$ & $2.331-3$ & $-3.160-5$ & $1.951-6$ \\
\hline
\end{tabular}

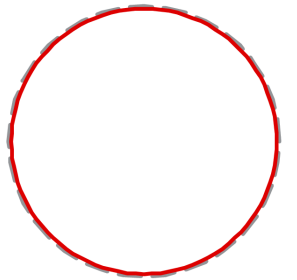

axial mode (scaled by $10^{\wedge} 6$ )

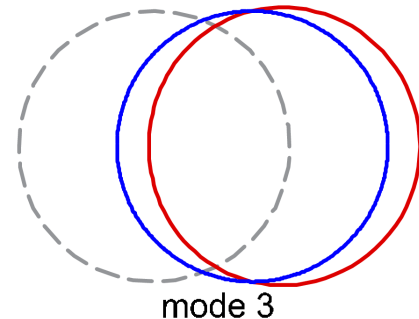

(scaled by $10^{\wedge} 3$ )

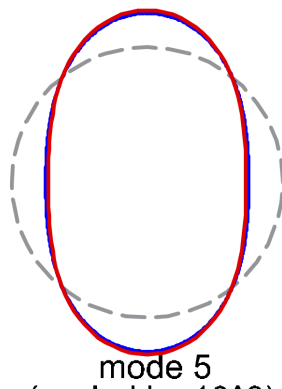

(scaled by 10^3)

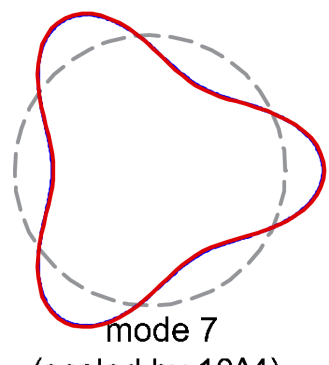

(scaled by $10^{\wedge} 4$ )

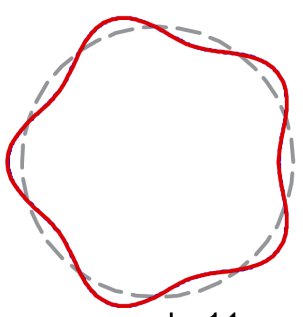

mode 11

(scaled by $10^{\wedge} 5$ )

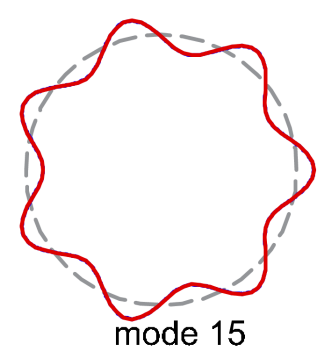

(scaled by $10^{\wedge} 6$ )

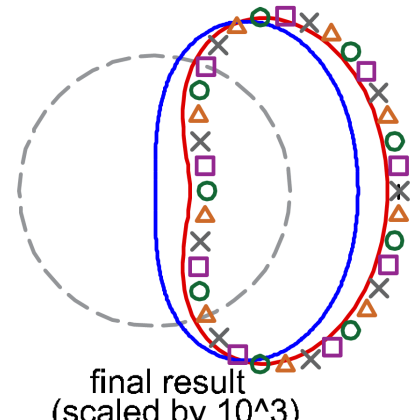

cross-section

middle wall

GBT with

Poisson's effect

GBT without

Poisson's effect

XXX shell 63

$\Delta \Delta \Delta$ shell 93

마 shell 181

॰ shell 281

Figure 5.15: Results of cross-section deformation at $\mathrm{x}=1 \mathrm{~m}$ : Comparison among the GBT solutions with/without Poisson effect and shell elements. 


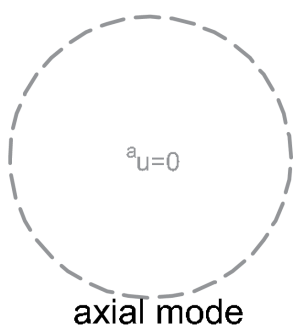

(scaled by $10^{\wedge} 6$ )

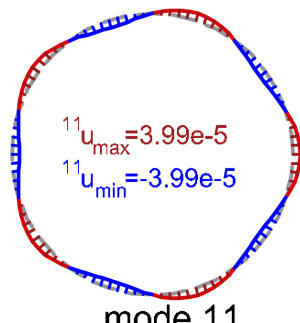

(scaled by $10^{\wedge} 6$ )

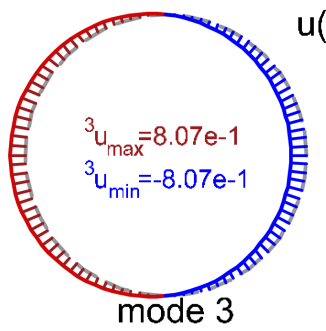

(scaled by $10^{\wedge} 2$ )

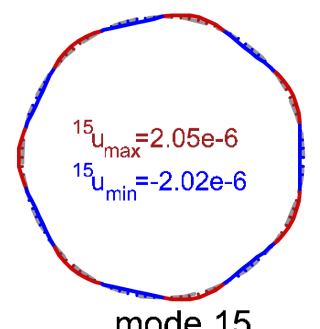

(scaled by $10^{\wedge} 7$ ) $\mathrm{u}(\mathrm{mm})$

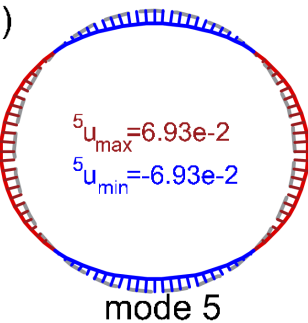

(scaled by $10^{\wedge} 3$ )

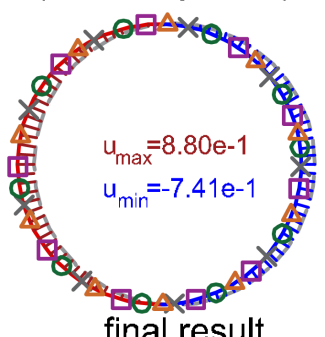

(scaled by $10^{\wedge} 2$ )

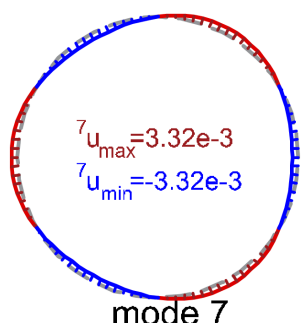

(scaled by $10^{\wedge} 4$ )

cross-section middle wall

$=$ GBT solution

$x \times x$ shell 63

$\Delta \Delta \Delta$ shell 93

마 shell 181

•• shell 281

Figure 5.16: Results of longitudinal displacement of the cross-section at $\mathrm{x}=1 \mathrm{~m}$ : Comparison between the solutions of GBT and shell elements.

Table 5.6: Mean differences, in \%, and standard deviation (SD) of displacement field between GBT and shell elementsk models at point $x=1 \mathrm{~m}$

\begin{tabular}{ccccccccc}
\hline \multirow{2}{*}{ element } & \multicolumn{2}{c}{$\mathrm{u}$} & \multicolumn{2}{c}{$\mathrm{v}$} & \multicolumn{2}{c}{ w w/o Poisson } & \multicolumn{2}{c}{ w with Poisson } \\
& diff. & SD & diff. & SD & diff. & SD & diff. & SD \\
\hline Shell 63 & $-0.14 \%$ & $0.51 \%$ & $15.74 \%$ & $8.75 \%$ & $35.06 \%$ & $38.02 \%$ & $9.7 \%$ & $8.84 \%$ \\
Shell 93 & $-0.19 \%$ & $0.44 \%$ & $13.71 \%$ & $11.68 \%$ & $35.09 \%$ & $37.87 \%$ & $9.76 \%$ & $8.3 \%$ \\
Shell 181 & $-0.19 \%$ & $0.45 \%$ & $15.73 \%$ & $8.73 \%$ & $35.09 \%$ & $37.88 \%$ & $9.75 \%$ & $8.32 \%$ \\
Shell 281 & $-0.09 \%$ & $0.59 \%$ & $13.8 \%$ & $11.7 \%$ & $35.12 \%$ & $38.11 \%$ & $9.73 \%$ & $9.12 \%$ \\
\hline
\end{tabular}

It is interesting to observe how the Poisson's effect correction approximates to the shell finite elements' solution. Table 5.6 shows a reduction in mean difference and standard deviation values, $35 \%$ and $38 \%$ respectively, to $9.7 \%$ and $8.9 \%$ if one includes the Poisson's effect into the analysis of radial displacement $w$.

Also, figure 5.15 shows that this effect decreases in high modes. The only difference between the results concerns mode 3, which indicates the cos in the assumption of no shear deformation in membrane behavior. Therefore, to improve the quality of this result, it is necessary to change the kinematic assumption of mode 3 from the traditional Bernoulli-Euler beam to Timoshenko beam theory[129, 170].

Another evidence that supports the lack of shear deformation in the transverse displacement is the longitudinal displacement of the cross-section at this point. Figure 5.16 and table. 5.6 show the differences between GBT and shell element solutions are almost imperceptible: around $0.2 \%$. 


\subsubsection{Analysis of the stress field}

As shown in section 3.1.4, one obtains the longitudinal and the shear stresses, at a particular point of one cross-section, by the superposition of all stress modal participation (equations 2.74 and 2.85, respectively). Similar to the displacement field, this subsection plots the results of the stress field obtained from the formulation of exact solution in GBT, as well as the results from the traditional Hermitian shape functions.

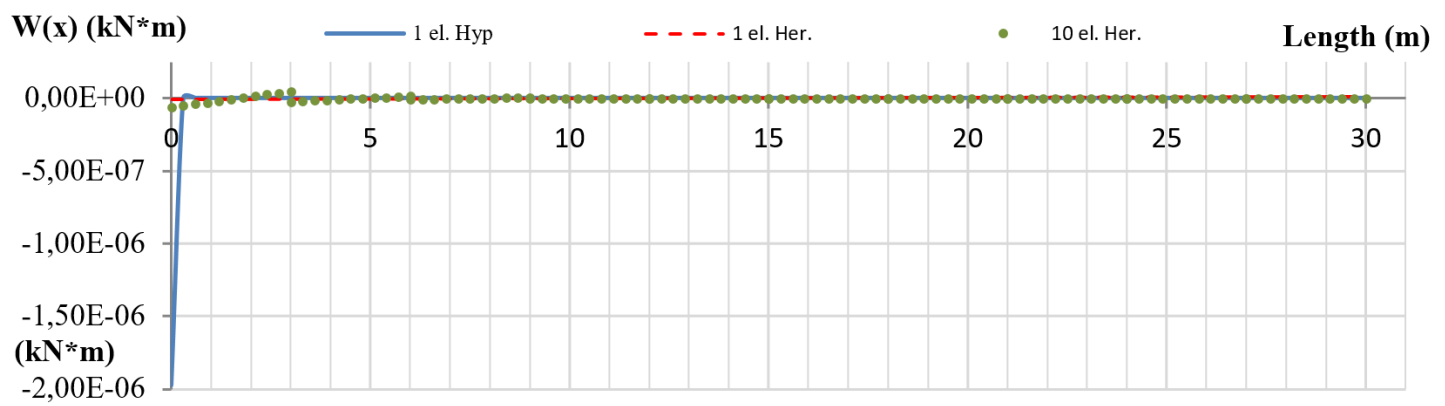

Figure 5.17: Generalized Internal force of radial mode, ${ }^{a} W$, (in $\mathrm{MN}^{*} \mathrm{~m}$ )

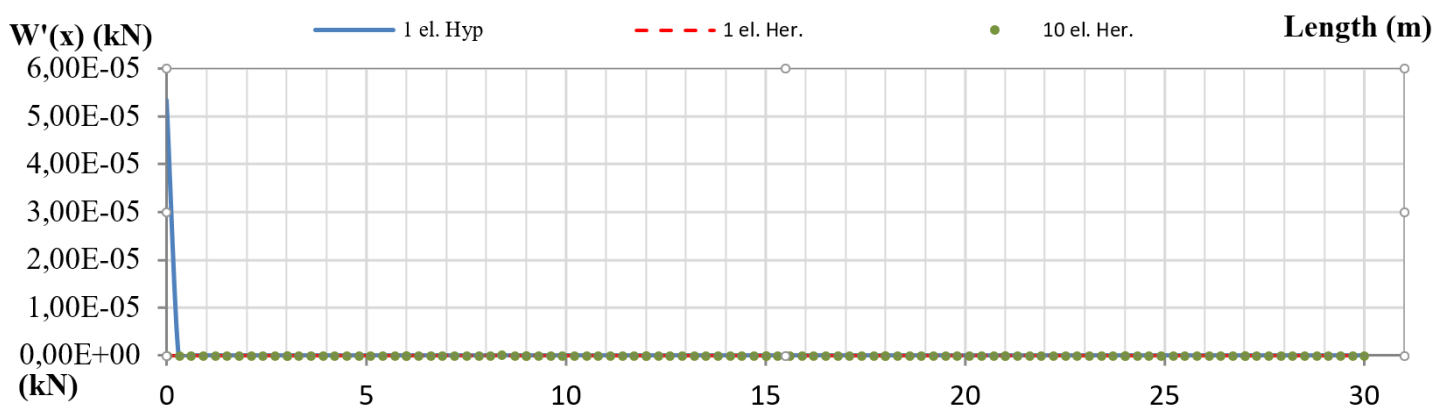

Figure 5.18: Generalized Internal force of radial mode, ${ }^{a} W^{\prime}$, (in MN)

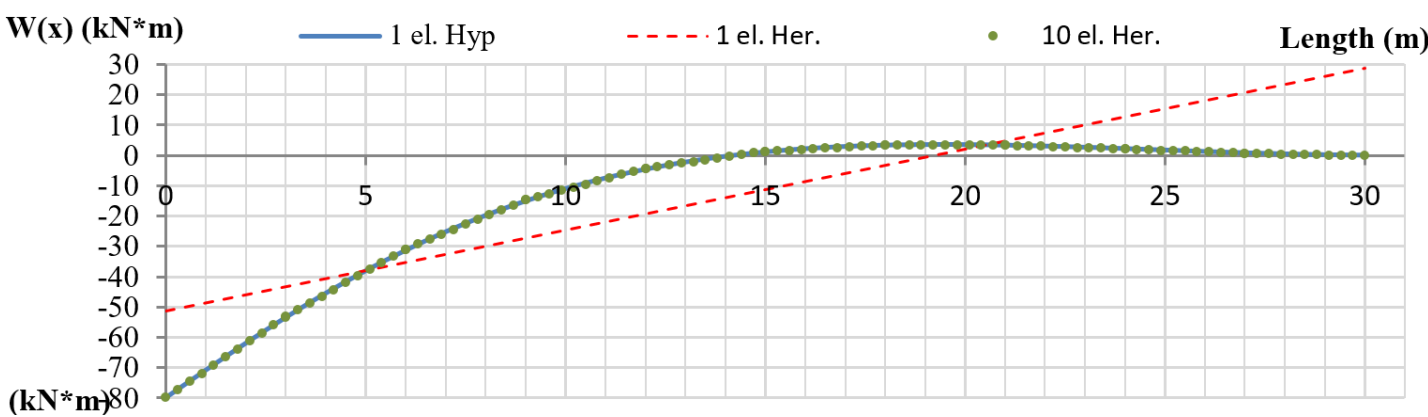

Figure 5.19: Generalized Internal force of mode $5,{ }^{5} \mathrm{~W}$, (in $\mathrm{MN}^{*} \mathrm{~m}$ )

The stress field results found from Hermitian and trigonometric-hyperbolic functions have a clear contrast. Due to the higher order derivatives required to achieve the stress fields, the Hermitian shape functions present discontinuities, especially for the generalized shear force (see figures 5.18, 5.20, 5.22 and 5.24). Since hyperbolic-trigonometric 


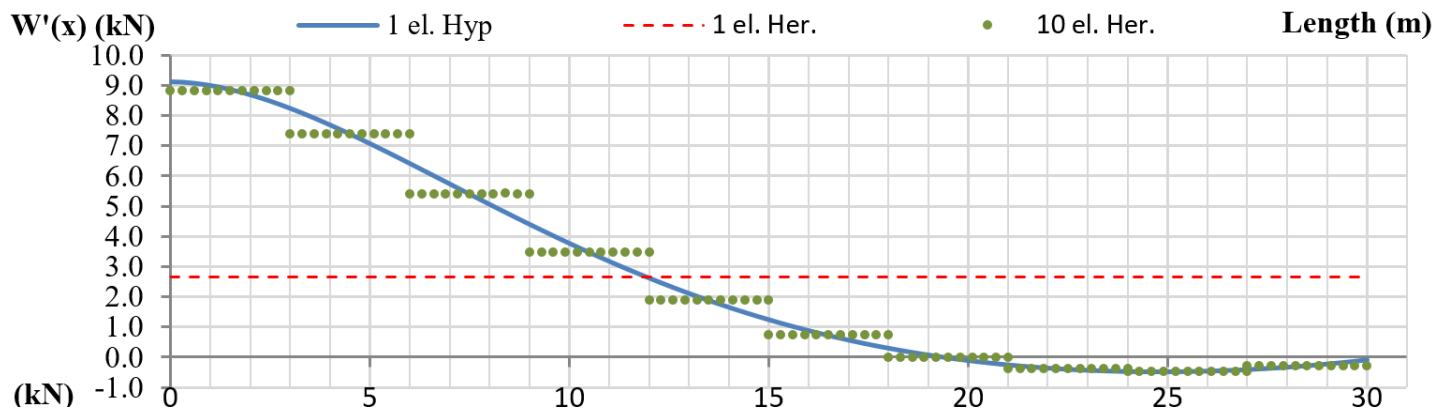

Figure 5.20: Generalized Internal force of mode $5,{ }^{5} W^{\prime}$, (in MN)

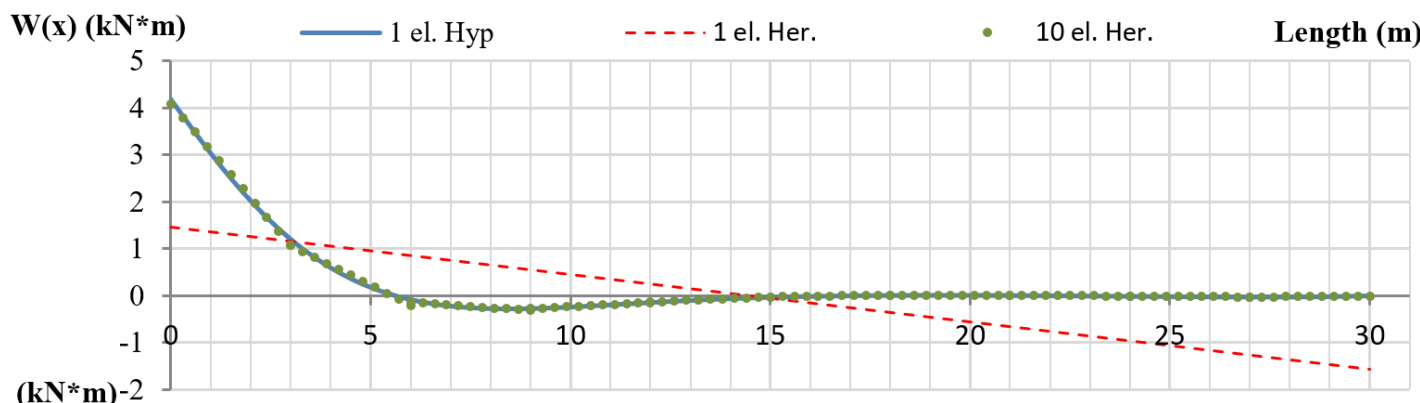

Figure 5.21: Generalized Internal force of mode $7,{ }^{7} \mathrm{~W}$, (in $\mathrm{MN}^{*} \mathrm{~m}$ )

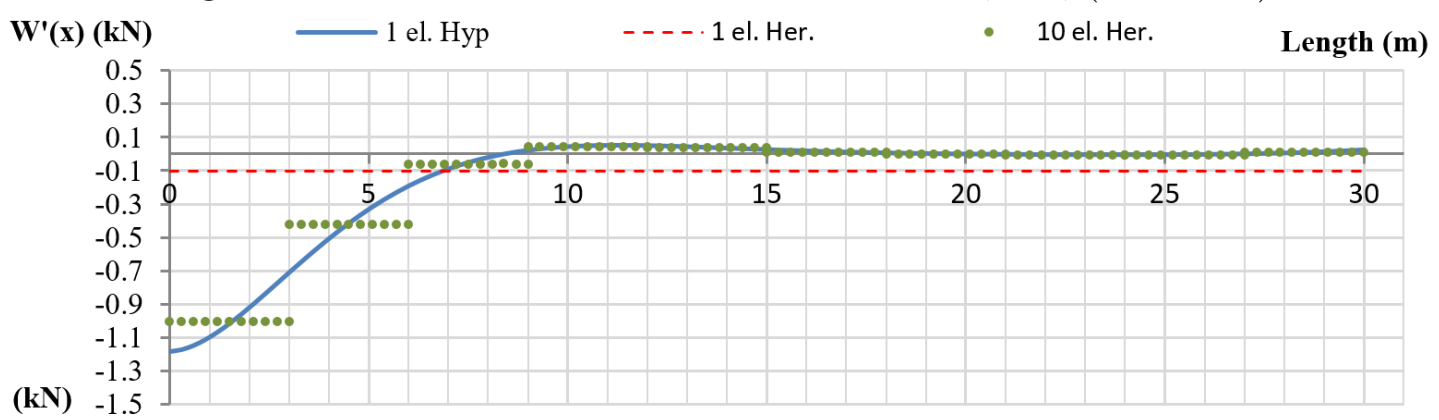

Figure 5.22: Generalized Internal force of mode $7,{ }^{7} W^{\prime}$, (in MN)

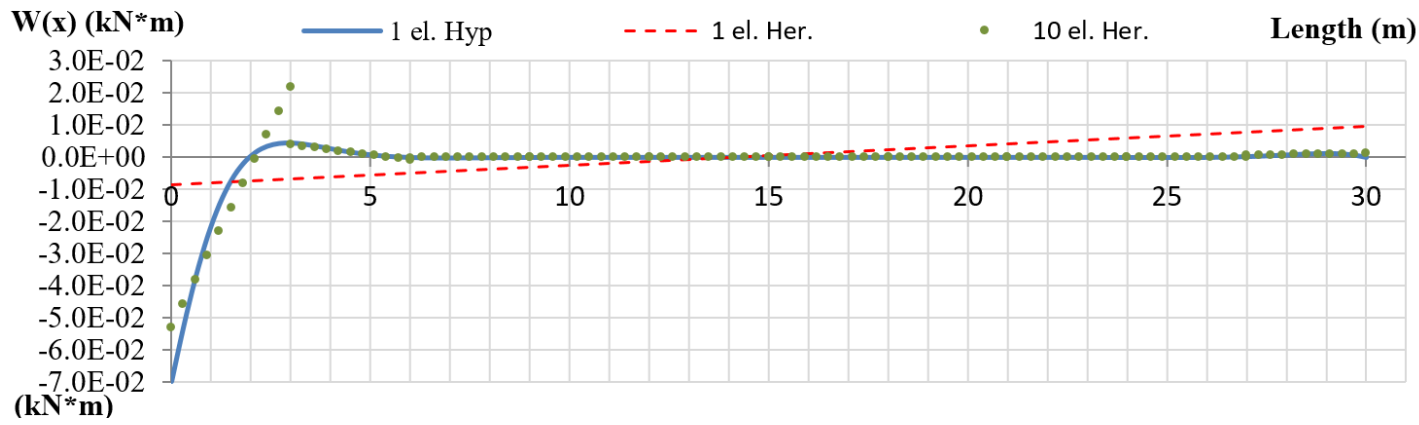

Figure 5.23: Generalized Internal force of mode $11,{ }^{11} \mathrm{~W}$, (in $\mathrm{MN}^{*} \mathrm{~m}$ )

shape functions always have derivatives, such effect does not exist. Besides, the generalized bending moments for radial and higher order modes (figures 5.17 and 5.21, respectively) present an exponential growing behavior near the support. Consequently, the Hermitian shape functions require an even higher discretization in this domain. 


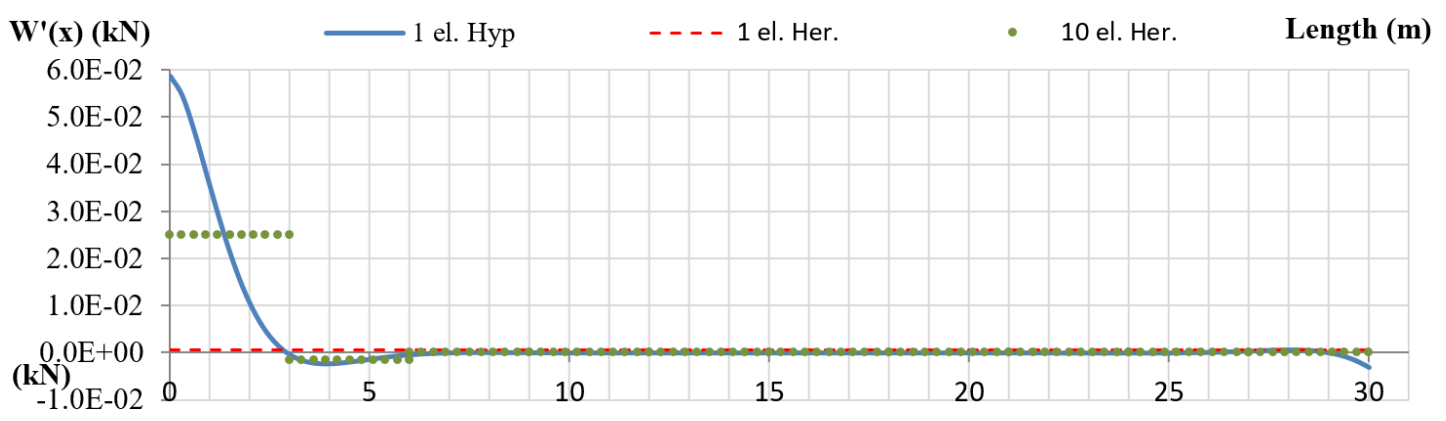

Figure 5.24: Generalized Internal force of mode $11,{ }^{11} W^{\prime}$, (in MN)

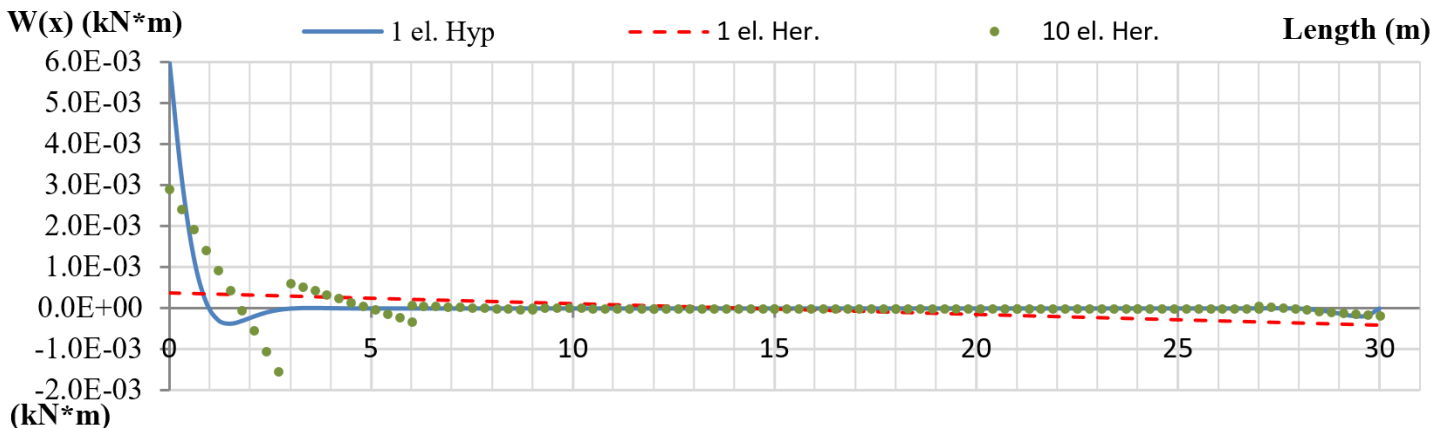

Figure 5.25: Generalized Internal force of mode $15,{ }^{15} \mathrm{~W}$, (in $\mathrm{MN}^{*} \mathrm{~m}$ )

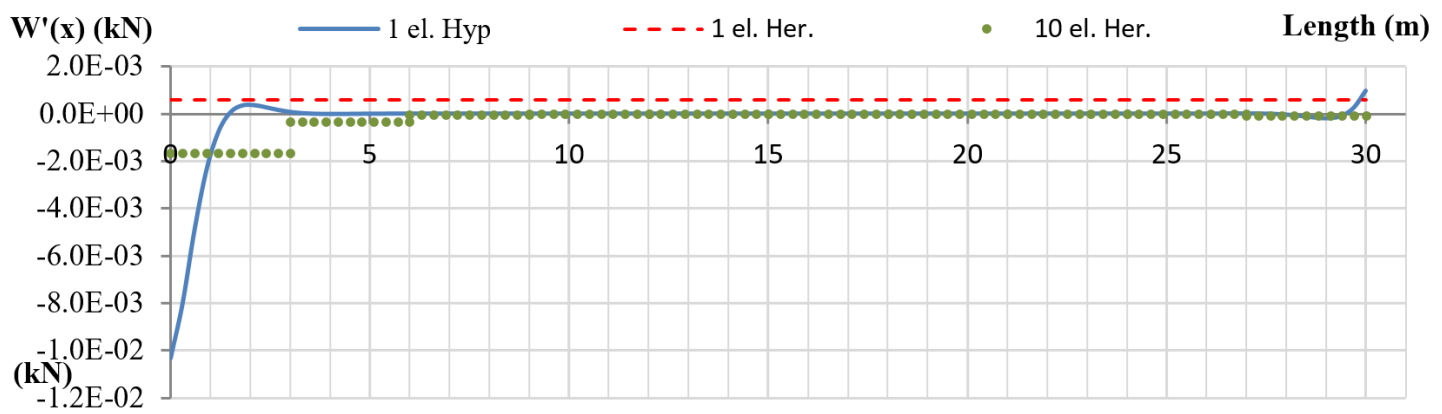

Figure 5.26: Generalized Internal force of mode $15,{ }^{15} W^{\prime}$, (in MN)

This issue of precision deficiency in the Hermitian shape function has a particular highlight in the case of circular hollow sections, whose internal forces are obtained from a combination of the higher order derivatives, as presented in equations 3.61, 3.62, 3.65, 3.66 and 3.67 .

To illustrate the quality of the results obtained from the presented GBT approach, all internal bending moments and shear forces in the cross-section at the point $x=1 \mathrm{~m}$ are plotted below alongside the results obtained from shell element models.

Figures 5.27 to 5.31 show how GBT approaches the results of the other models. It also indicates that there is no consensus among the different shell element models regarding the values of the plate's internal forces. Tables 5.7 to 5.11 present mean differences and standard deviations for all models.

For the longitudinal bending moment $M_{x}$, GBT leads to the results of Shell-93 model. 

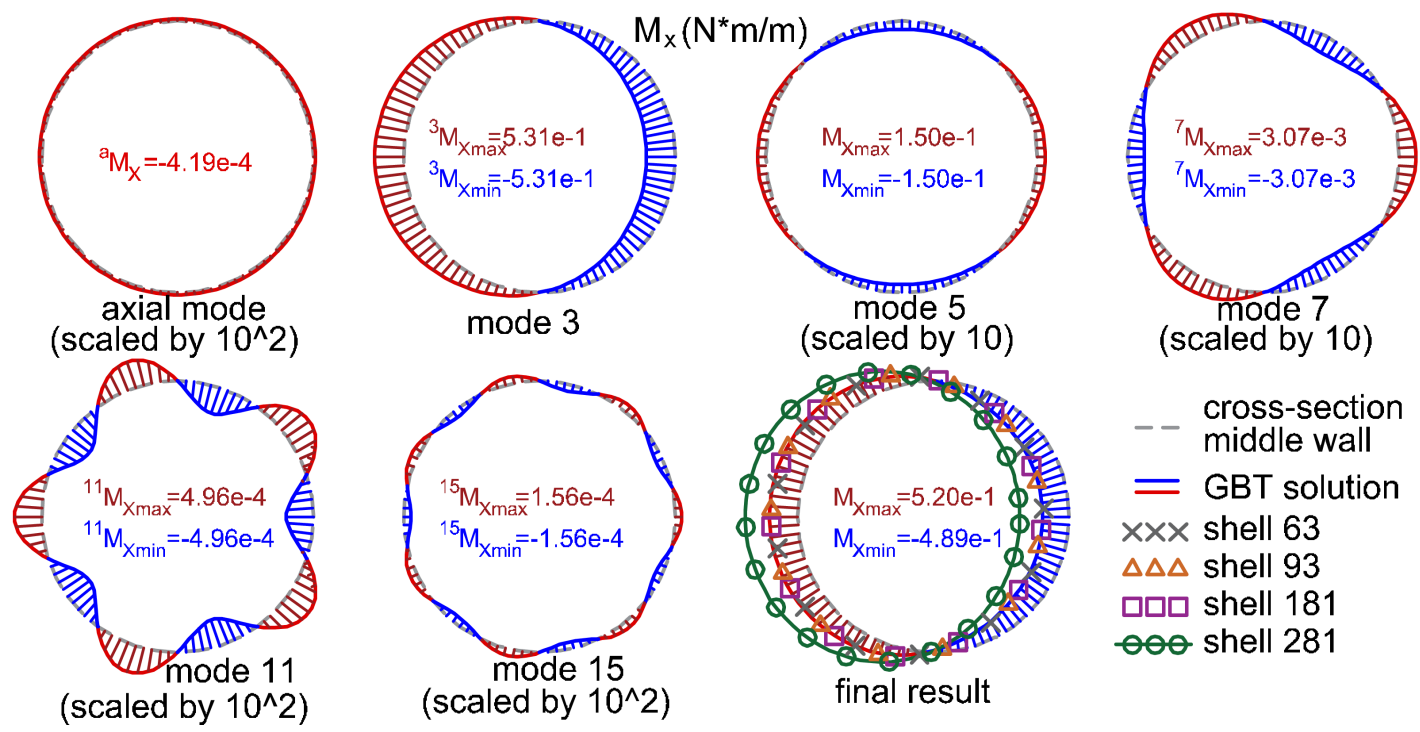

cross-section

- middle wall

$=$ GBT solution $x \times x$ shell 63 $\Delta \Delta \Delta$ shell 93

마 shell 181

॰ shell 281 (scaled by $10^{\wedge} 2$ )

Figure 5.27: Longitudinal bending moment, $M_{x}$, in cross-section at $\mathrm{x}=1 \mathrm{~m}$

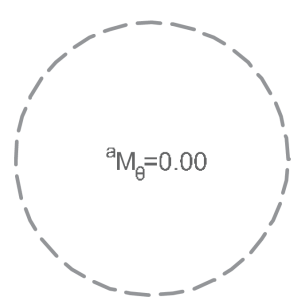

axial mode

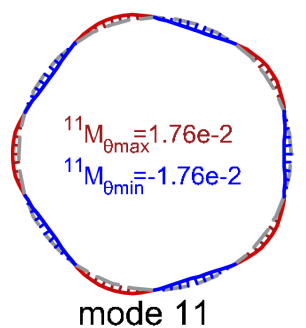

(scaled by 10)
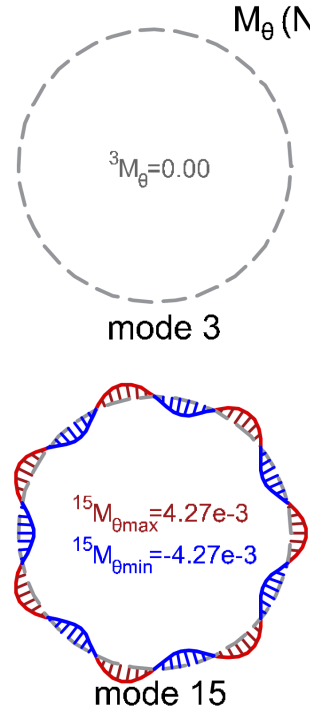

(scaled by $10^{\wedge} 2$ )
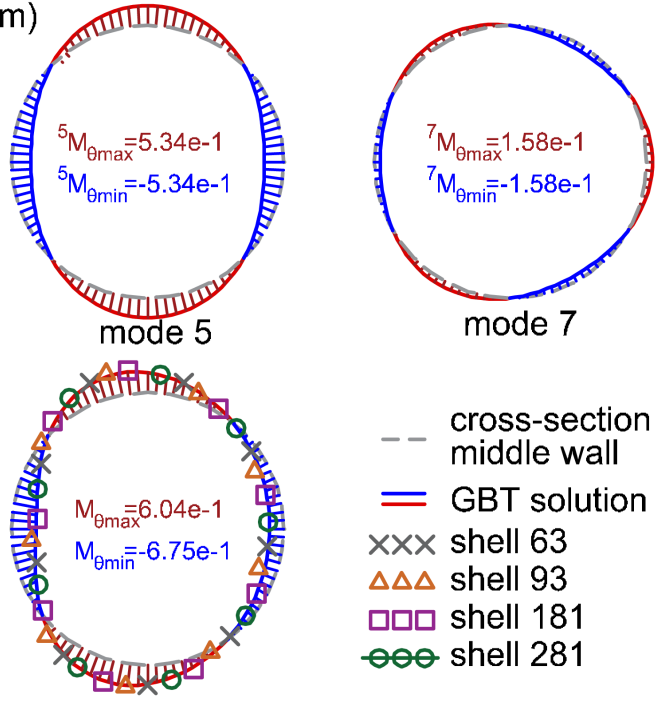

final result

Figure 5.28: transverse bending moment, $M_{\theta}$, in cross-section at $\mathrm{x}=1 \mathrm{~m}$

Table 5.7: $M_{x}$ : Mean differences, (in table's upper-right side), and their standard deviation (in table's lower-left side) among all models

\begin{tabular}{cccccc}
\hline$M_{x}$ & GBT & Shell-63 & Shell-93 & Shell-181 & Shell-281 \\
\hline GBT & - & $-6.22 \%$ & $0.31 \%$ & $-6.16 \%$ & $78.9 \%$ \\
Shell 63 & $12.24 \%$ & - & $-8.32 \%$ & $-0.05 \%$ & $93.94 \%$ \\
Shell 93 & $8.83 \%$ & $1.97 \%$ & - & $-8.27 \%$ & $77.8 \%$ \\
Shell 181 & $12.37 \%$ & $0.1 \%$ & $2.06 \%$ & - & $92.89 \%$ \\
Shell 281 & $5.62 \%$ & $18.34 \%$ & $19.76 \%$ & $22.81 \%$ & - \\
\hline
\end{tabular}



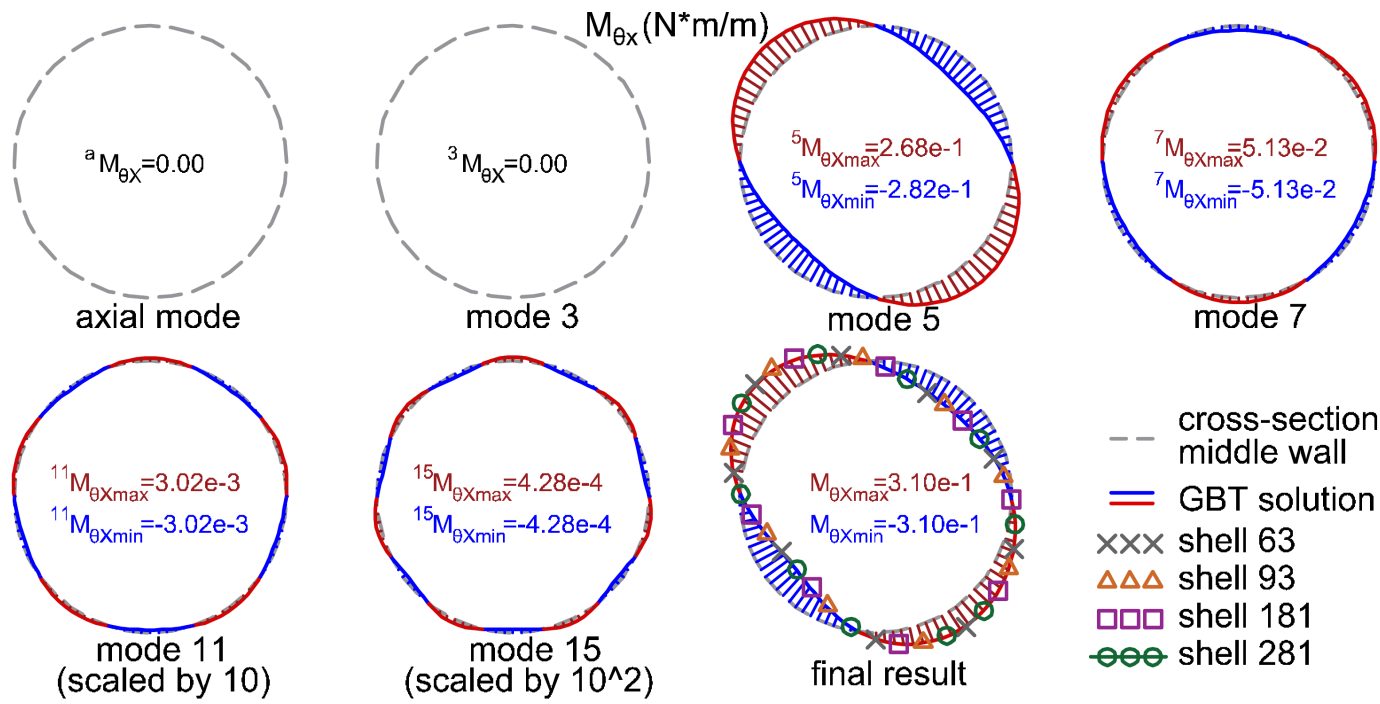

Figure 5.29: Twist moment, $M_{\theta x}$, in cross-section at $\mathrm{x}=1 \mathrm{~m}$

The mean difference is around $0.31 \%$. Shell models with a linear interpolation function, Shell-63 and Shell-181, reach almost the same result. The mean difference between these two models is $-0.05 \%$ and the standard deviation is lower than $0.1 \%$. Both models have the mean difference and standard deviation with GBT around $6.2 \%$ and $12.24 \%$, respectively. Surprisingly, Shell-281 has results much higher than the other models.

However, for the transverse bending moment $M_{\theta}$, the highest mean difference is between Shell-93 and GBT, which is around of 19.5\%. GBT results are consistent with the ones obtained from the other shells models. It is possible to observe the same behavior in twist bending moment, $M_{\theta x}$. GBT results diverge from the Shell-93 ones and converge to the other models. Nevertheless, the mean differences for this internal bending moment are lower than the other bending moments.

Table 5.8: $M_{\theta}$ : Mean differences, (in table's upper-right side), and their standard deviation (in table's lower-left side) among all models

\begin{tabular}{cccccc}
\hline$M_{\boldsymbol{\theta}}$ & GBT & Shell-63 & Shell-93 & Shell-181 & Shell-281 \\
\hline GBT & - & $7.16 \%$ & $19.48 \%$ & $7.27 \%$ & $7.27 \%$ \\
Shell 63 & $0.72 \%$ & - & $1.33 \%$ & $-0.1 \%$ & $-0.1 \%$ \\
Shell 93 & $41.03 \%$ & $37.19 \%$ & - & $-0.09 \%$ & $-0.08 \%$ \\
Shell 181 & $0.72 \%$ & $0.03 \%$ & $38.12 \%$ & - & $0.01 \%$ \\
Shell 281 & $0.71 \%$ & $0.09 \%$ & $38.09 \%$ & $0.06 \%$ & - \\
\hline
\end{tabular}

One interesting observation concerns how the GBT modal analysis provides a spectral description of the internal forces that points out the relevant kinematic behavior in a thin-walled structure. This feature leads to a clear design approach, which allows the mitigation of the significant stress' modes.

For instance, from figure 5.27, it is clear that the longitudinal bending moment is 
Table 5.9: $M_{\theta x}$ : Mean differences, (in table's upper-right side), and their standard deviation (in table's lower-left side) among all models

\begin{tabular}{cccccc}
\hline$M_{\theta x}$ & GBT & Shell-63 & Shell-93 & Shell-181 & Shell-281 \\
\hline GBT & - & $2.78 \%$ & $3.31 \%$ & $2.45 \%$ & $2.9 \%$ \\
Shell 63 & $2.93 \%$ & - & $1.13 \%$ & $1.06 \%$ & $-0.11 \%$ \\
Shell 93 & $9.77 \%$ & $9.25 \%$ & - & $-0.01 \%$ & $1.25 \%$ \\
Shell 181 & $3.78 \%$ & $5.7 \%$ & $5.75 \%$ & - & $1.19 \%$ \\
Shell 281 & $3.05 \%$ & $0.13 \%$ & $9.33 \%$ & $5.81 \%$ & - \\
\hline
\end{tabular}

mainly provided from mode 3 . On the other hand, this mode has no participation in transverse bending or twist moments, as shown in figures 5.28 and 5.29. Also, from these pictures, one can observe that modes 5 and 7 are the most relevant for transverse bending and twist moments.

Following the same representation, one can express and compare the internal shear forces, given in equations 3.66 and 3.67, with the results of shell models.
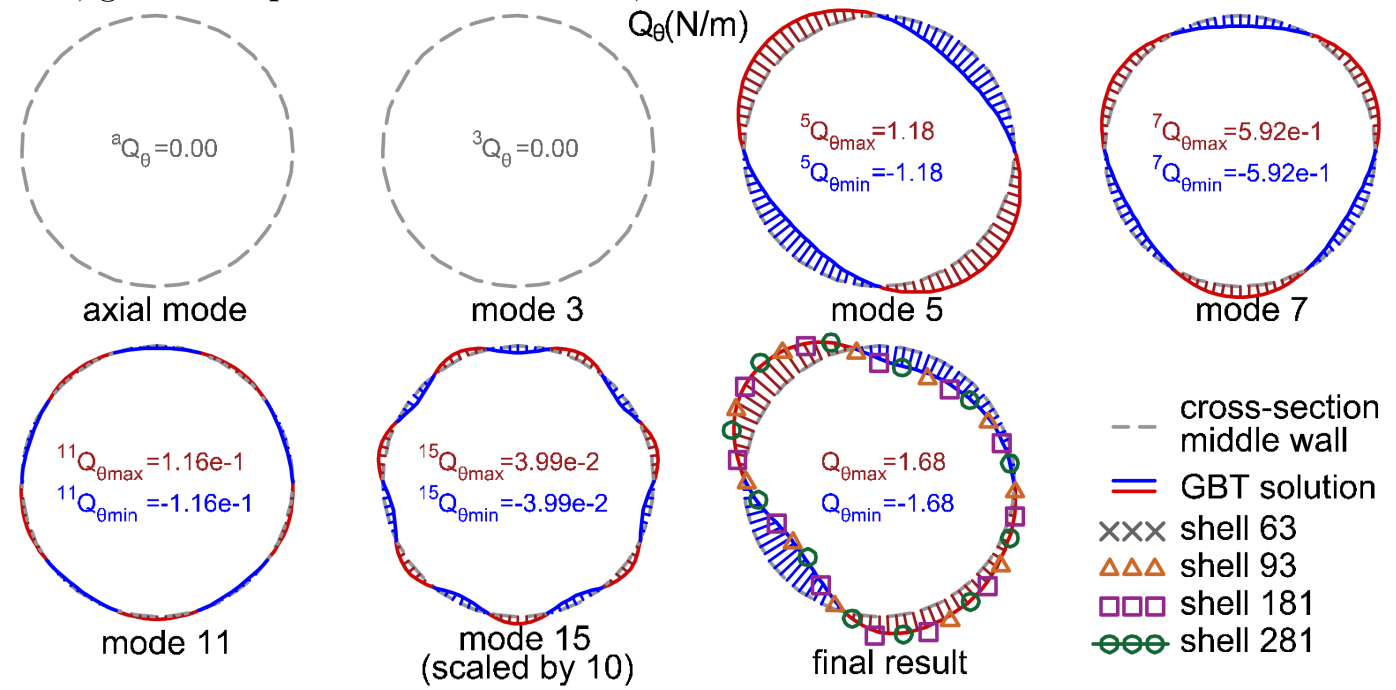

$=\mathrm{GBT}$ solution

XXX shell 63

$\Delta \Delta \Delta$ shell 93

प्वप shell 181

๑॰ shell 281

Figure 5.30: Shear forces, $Q_{x}$, in cross-section at $\mathrm{x}=1 \mathrm{~m}$

Similar to longitudinal bending moment, GBT's longitudinal shear force, $Q_{x}$, approaches the results found in Shell-93 by the mean difference value. However, if one considers the standard deviation, GBT achieves the results of Shell-281 model. Shell-181 is another highlight in this internal force. As presented in figure 5.30, the results of this shell model have not only a quantitative difference, but also a considerable qualitative difference.

On the other hand, for the transverse shear force, Shell-181 presents the lower difference from GBT's results, mean difference of $8.93 \%$ and standard deviation of $3.75 \%$.

This lack of consensus about the plate's internal forces does not happen for the internal membrane forces. Although membrane requires even higher derivatives of the amplifi- 


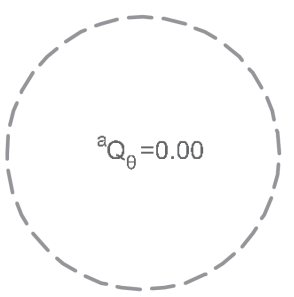

axial mode

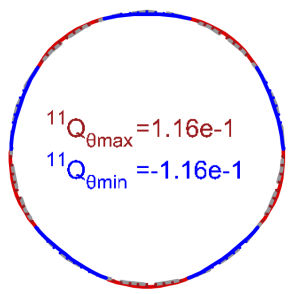

mode 11

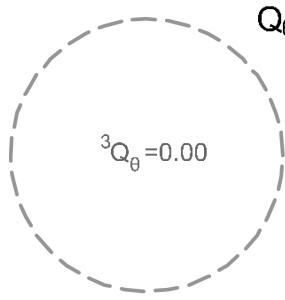

mode 3

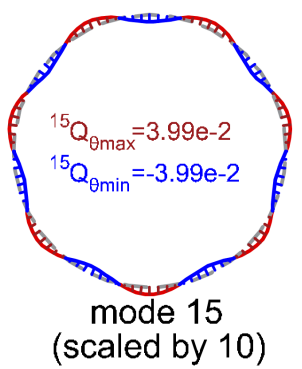

$Q_{\theta}(\mathrm{N} / \mathrm{m})$

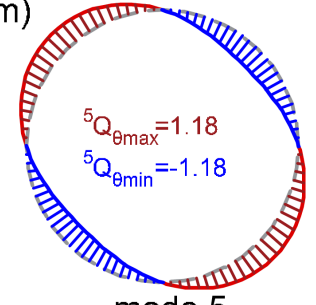

mode 5

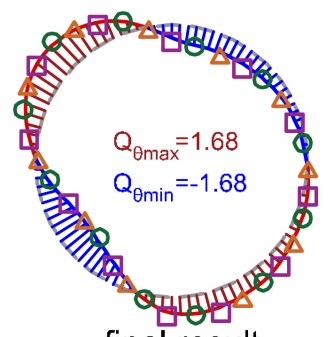

final result

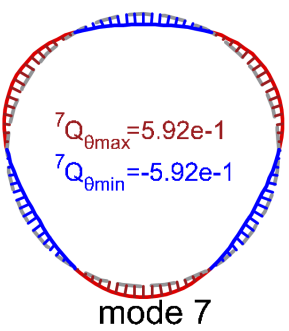

cross-section

middle wall

$=$ GBT solution

Xxx shell 63

$\Delta \Delta \Delta$ shell 93

맘 shell 181

-

Figure 5.31: Shear forces, $Q_{\theta}$, in cross-section at $\mathrm{x}=1 \mathrm{~m}$

Table 5.10: $Q_{x}$ : Mean differences, (in table's upper-right side), and their standard deviation (in table's lower-left side) among all models

\begin{tabular}{ccccc}
\hline$Q_{x}$ & GBT & Shell-93 & Shell-181 & Shell-281 \\
\hline GBT & - & $3.58 \%$ & $25.43 \%$ & $3.74 \%$ \\
Shell 93 & $11.71 \%$ & - & $-3.97 \%$ & $0.95 \%$ \\
Shell 181 & $280.16 \%$ & $350.09 \%$ & - & $-75.59 \%$ \\
Shell 281 & $5.1 \%$ & $8.08 \%$ & $73.5 \%$ & - \\
\hline
\end{tabular}

Table 5.11: $Q_{\theta}$ : Mean differences, (in table's upper-right side), and their standard deviation (in table's lower-left side) among all models

\begin{tabular}{ccccc}
\hline$Q_{\theta}$ & GBT & Shell-93 & Shell-181 & Shell-281 \\
\hline GBT & - & $15.11 \%$ & $8.93 \%$ & $10.36 \%$ \\
Shell 93 & $21.36 \%$ & - & $-2.19 \%$ & $-1.43 \%$ \\
Shell 181 & $3.75 \%$ & $19.7 \%$ & - & $2.48 \%$ \\
Shell 281 & $12.11 \%$ & $10.54 \%$ & $9.63 \%$ & - \\
\hline
\end{tabular}

cation function $V(x)$ to express the internal forces (as presented in equations 3.71, 3.73 and 3.76), the proposed GBT element and all shell models lead almost to the same solution. The internal membrane forces, in the cross-section at the point $\mathrm{x}=1 \mathrm{~m}$, show this convergence in figure 5.32 and Table 5.12, as well.

Since GBT has a high accuracy concerning the longitudinal displacement, it is not a surprise that the longitudinal membrane force reaches a high agreement to the results from shell element models. 


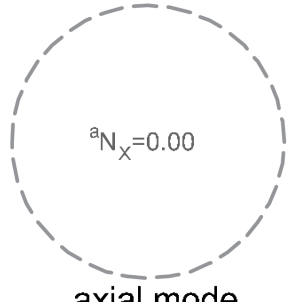

axial mode

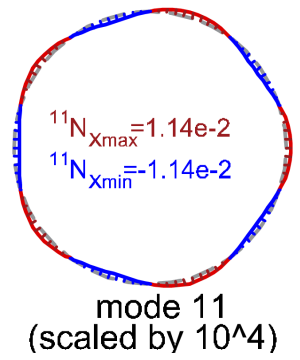

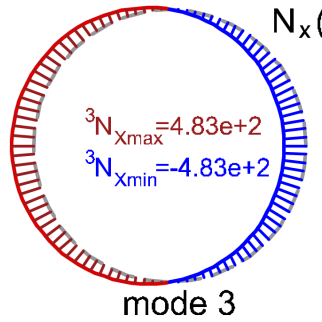

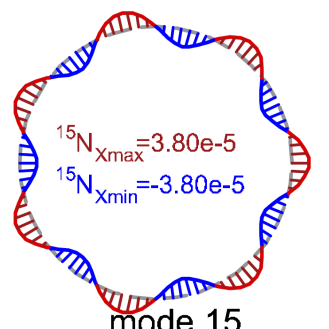

(scaled by $10^{\wedge} 7$ )

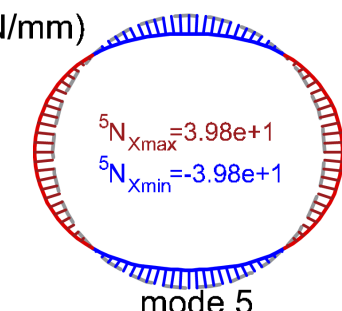

mode 5

(scaled by 10)

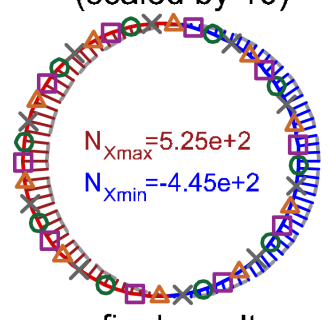

final result

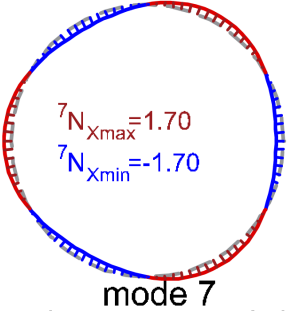

(scaled by $10^{\wedge} 2$ )

cross-section

middle wall

$=$ GBT solution

$x \times x$ shell 63

$\Delta \Delta \Delta$ shell 93

마 shell 181

๑o shell 281

Table 5.12: $N_{x}$ : Mean differences, (in table's upper-right side), and their standard deviation (in table's lower-left side) among all models

\begin{tabular}{cccccc}
\hline$N_{x}$ & GBT & Shell-63 & Shell-93 & Shell-181 & Shell-281 \\
\hline GBT & - & $0.02 \%$ & $-0.02 \%$ & $0.05 \%$ & $-0.02 \%$ \\
Shell 63 & $0.17 \%$ & - & $0.05 \%$ & $-0.03 \%$ & $0.05 \%$ \\
Shell 93 & $0.13 \%$ & $0.04 \%$ & - & $0.08 \%$ & $0.01 \%$ \\
Shell 181 & $0.19 \%$ & $0.02 \%$ & $0.06 \%$ & - & $-0.08 \%$ \\
Shell 281 & $0.13 \%$ & $0.04 \%$ & $0.01 \%$ & $0.06 \%$ & - \\
\hline
\end{tabular}

The tangential membrane force, $N_{\theta}$, reaches high accurate results as well, which the closest results concerns to model Shell-63. Moreover, this internal force requires a fourth order derivative, which is null in cubic Hermitian shape functions. It is another point that stresses the need for functions with continuous derivatives, such as the Hyperbolictrigonometric functions presented here.

Table 5.13: $N_{\theta}$ : Mean differences, (in table's upper-right side), and their standard deviation (in table's lower-left side) among all models

\begin{tabular}{cccccc}
\hline$N_{\theta}$ & GBT & Shell-63 & Shell-93 & Shell-181 & Shell-281 \\
\hline GBT & - & $0.79 \%$ & $1.11 \%$ & $0.83 \%$ & $-1.96 \%$ \\
Shell 63 & $1.81 \%$ & - & $-0.44 \%$ & $-0.08 \%$ & $3.1 \%$ \\
Shell 93 & $1.44 \%$ & $0.45 \%$ & - & $-0.4 \%$ & $-0.64 \%$ \\
Shell 181 & $1.91 \%$ & $0.14 \%$ & $0.57 \%$ & - & $2.42 \%$ \\
Shell 281 & $5.42 \%$ & $7.77 \%$ & $9.55 \%$ & $14.65 \%$ & - \\
\hline
\end{tabular}

Lastly, the GBT's membrane shear force, $N_{\theta x}$, leads almost to the same result of shell element models. 


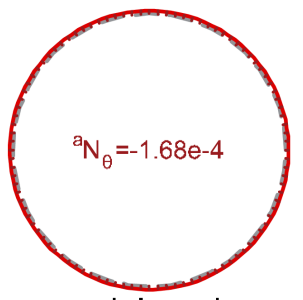

axial mode

(scaled by $10^{\wedge} 4$ )

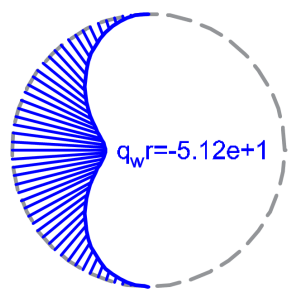

External force $q_{w}$

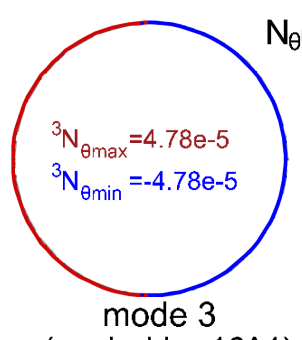

(scaled by $10^{\wedge} 4$ )

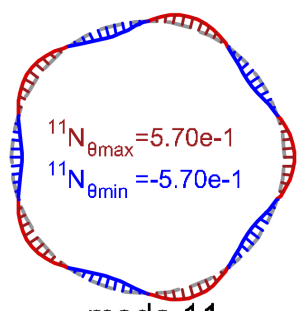

mode 11

(scaled by 10 )

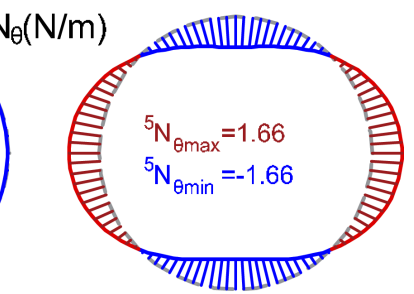

mode 5

(scaled by 10)

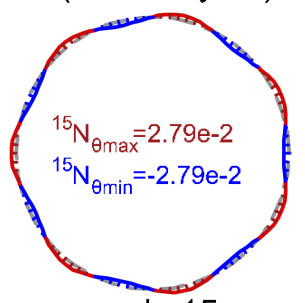

mode 15

(scaled by 10)
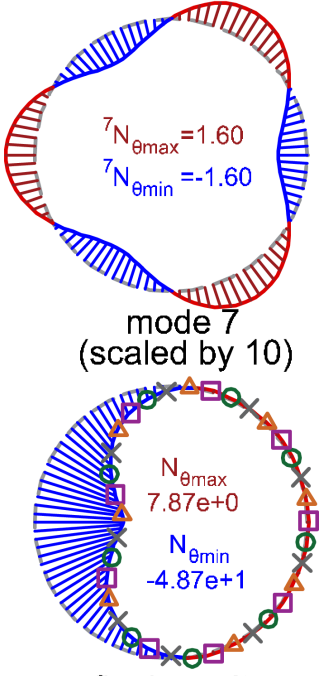

final result

\section{- - cross-section middle wall $=$ GBT solution $\begin{array}{ll}x \times x \text { shell } 63 & \text { and shell } 181 \\ \Delta \Delta \Delta \text { shell } 93 & \text { ooo shell } 281\end{array}$}

Figure 5.33: Membrane force, $N_{\theta}$, in cross-section at $\mathrm{x}=1 \mathrm{~m}$

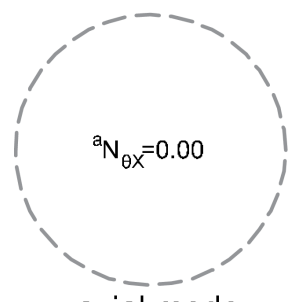

axial mode

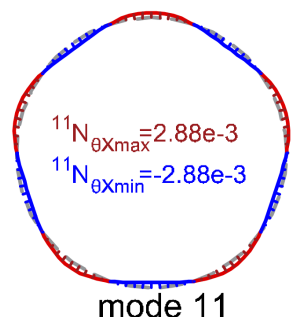

(scaled by $10^{\wedge} 3$ )
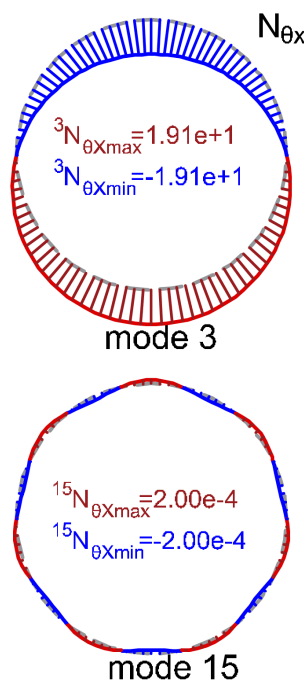

(scaled by $10^{\wedge} 4$ )
$\mathrm{N}_{\theta x}(\mathrm{~N} / \mathrm{mm})$

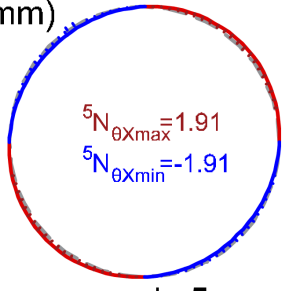

mode 5

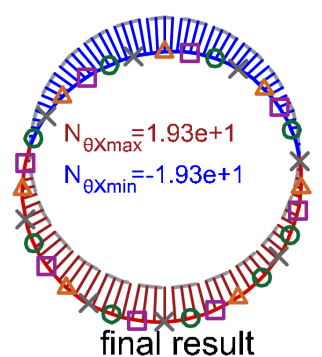

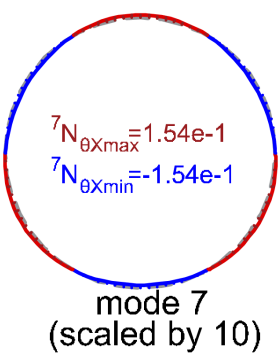

cross-section middle wall

$=\mathrm{GBT}$ solution

$x \times x$ shell 63

$\Delta \Delta \Delta$ shell 93

प्वप shell 181

॰ shell 281

Figure 5.34: Membrane force, $N_{\theta x}$, in cross-section at $\mathrm{x}=1 \mathrm{~m}$

As a conclusion from this numerical example, one verifies not only the accurate displacement and stress fields obtained by GBT, but also it stands out the low computing effort to achieve the results based on the type of element presented here. 
Table 5.14: $N_{\theta x}$ : Mean differences, (in table's upper-right side), and their standard deviation (in table's lower-left side) among all models

\begin{tabular}{cccccc}
\hline$N_{\theta x}$ & GBT & Shell-63 & Shell-93 & Shell-181 & Shell-281 \\
\hline GBT & - & $0.01 \%$ & $-0.02 \%$ & $0.01 \%$ & $-0.02 \%$ \\
Shell 63 & $0.17 \%$ & - & $0.03 \%$ & $0.01 \%$ & $0.03 \%$ \\
Shell 93 & $0.14 \%$ & $0.03 \%$ & - & $0.03 \%$ & $0 \%$ \\
Shell 181 & $0.19 \%$ & $0.02 \%$ & $0.05 \%$ & - & $-0.03 \%$ \\
Shell 281 & $0.15 \%$ & $0.02 \%$ & $0.01 \%$ & $0.04 \%$ & - \\
\hline
\end{tabular}




\section{GBT's applied in frame beam structures}

Up to this point, the linear analysis of GBT is carried out concerning only a simple structural model, which is a straight beam. Since many structures are composed by beam frames, it is necessary to handle the interaction among the beams, sharing a common node.

Here, there are two obstacles that stand out. The first one is due to the complexity in the kinematic coupling among the GBT's deformation shapes at the joint, which involves several modes simultaneously. The next section presents the bibliographic review and the state-of-art about this issue.

The second obstacle concerns the physical connection itself. In practical structural design, a substantial amount of details arises at the connection, such as stiffeners, bolts and weldings for steel structures. These details not only change the kinematic behavior of the connection, but also cannot be modeled as beam elements.

Thus, due to the high level of details in structural connections, shell and solid elements are widely applied in their structural analysis. Therefore, it is convenient to split the structural analysis into two domains: the first is the beam domain, in which GBT elements are applied; the second one is the connection domain, in which shell or solid elements are used.

This domain separation leads directly to the question of how to couple the degrees of freedom of shell and solid elements with the high order of GBT's degrees of freedom that represent the modal shape deformations.

The current chapter develops a novel application of multi-freedom constraints in a coupling method between shell and GBT elements in order to solve the issue of the previous paragraph. Additionally, it provides a detailed numerical example to illustrate the proposed method, as well as compare the result of the mixed GBT-shell models with those of pure shell models. 


\subsection{Pure GBT beam frame analysis}

The expression used here, pure GBT beam frame analysis, is the nomenclature to describe numerical models that involve solely GBT's finite elements. Although the first studies in this application were not directly related to GBT, they were concerned of warping transmission of Vlasov beam element, which one can understand as the first high mode of GBT.

Among these studies, one can highlight the following publications:

- Fujitami et al., [59, 60], indicate the capacity of warping and bi-moment to be fully transmitted independently of the angle between beams at the connection. In this publication, Fujitami et al. proposed that the warping acts as a scalar and not as a vector. Therefore, the warping is not related to the local coordinate system of each beam in a frame. As shown in the next subsection, this assumption is only correct in some particular cases;

- Tong et al., [171], are the firsts indicating the coupling between torsional-warping and the distortion of the cross-section at connections with diagonal stiffeners. Here, warping and bi-moment are treated as scalars;

- Kraus and Kindmann, [101], also treat warping as a scalar, with no relation to the coordinate system of the beam element. Moreover, the authors emphasize how the construction type of the connection is of crucial importance to understand the warping transmission. They address special attention to the rule of stiffeners, which can act as an internal support mechanism. I.e., in some particular layouts, the stiffeners eliminate the internal bi-moment by a direct link of opposite self-balanced moments. Unfortunately, the example used, the box connection, has another behavior, as presented below;

- Basaglia et al., $[8,12,16,17]$ : since these publications are the first ones to study the warping as a high mode of GBT, they are particularly important. These publications highlight not only the leading role of the layout of connection in warping transmission, but also emphasize the kinematic behavior of the internal support mechanism and coupling with distortion. However, different from Kraus and Kindmann, Basaglia et al. prove that the box type connection is not an internal support restraint, but another kinematic mechanism that occurs only in modes higher than six degrees of freedom of rigid-body motion: the inverse-clamp behavior.

To clarify how the connection layout affects the warping and distortion transmission, one can consider four particular types of connections between beams with an "I" profile: box-diagonal, box, non-stiffener and diagonal connections, as shown in figure 6.1. In fact, these types of welded steel connections are widely applied in structural design. Each one of these connections is detailed in sequence. 


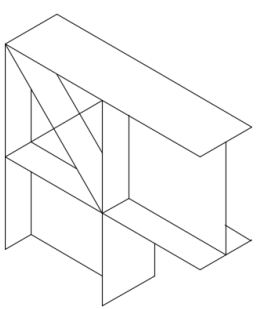

Box-Diagonal

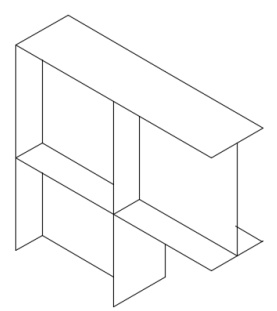

Box

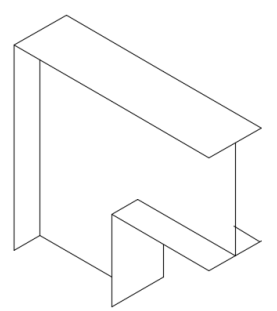

Non-Stiffener

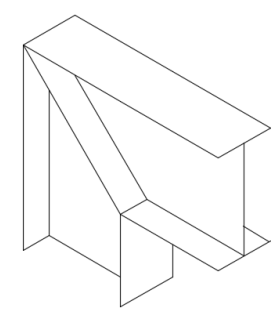

Diagonal

Figure 6.1: Type of connections to clarify the kinematic behavior in high modes

\subsubsection{Internal support mechanism}

The connection that leads to internal support behavior is the box-diagonal one. Here, the internal support mechanism is nothing more than the restraint of cross-section warping and/or distortion by stiffeners. Since all internal forces, which are beyond the traditional six degrees of freedom, have two or more opposite forces and/or bending moments in the cross-section, a stiffener component can directly link these opposite forces and/or bending moments, which will nullify them.

For instance, in the particular case of non-uniform torsion of Vlasov, the seventh degree of freedom indicates warping in the node. Different from the six traditional degrees of freedom, that can be understood as a representation of rigid-body movement at one node, the seventh degree of freedom indicates deformation at the node itself.

Following this representation, a node cannot be interpreted as a point but as an elementary volume, as shown by the elementary cube in figure $6.2^{1}$. Consequently, the warping degree of freedom, and any other GBT's high deformation mode, can be understood as the nodal deformation mode, i.e., the warping is only one of several possible deformations of the high modes at the node.
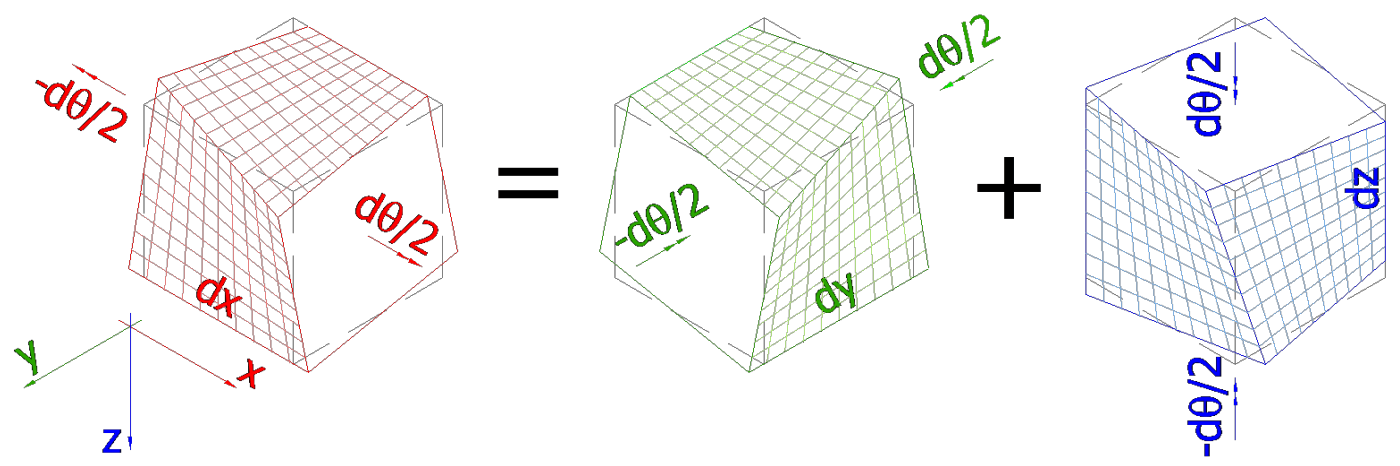

Figure 6.2: Elementary node to describe the kinematic behavior of the connection, which involves high deformation modes.

\footnotetext{
${ }^{1}$ The figure also presents the unique behavior of high modes. In the case of torsional warping, a body with positive warping in the $\mathrm{x}$ axis also can be understood as an increase of rotation around the same $\mathrm{x}$ axis in the $\mathrm{x}$ axis direction. However, it can also be described as a combination of simultaneous negative warping in the $\mathrm{y}$ and $\mathrm{z}$ directions.
} 
The remarkable property of any degree of freedom higher than the six of rigid-body movement is that it does not necessarily need an external restriction (such as building foundations) to have a support conditional. In other words, it is possible to have a support for the node's deformation only by stiffening the node. Therefore, the internal support is a node that is stiff enough to avoid the warping or distortion deformation, such as the box-diagonal connection, illustrated above.

\subsubsection{Inverse-clamp mechanism}

To explain how it is possible to transmit inverse internal forces of high modes, one can consider the simple beam-column frame under an external bi-moment as presented in figure 6.3.

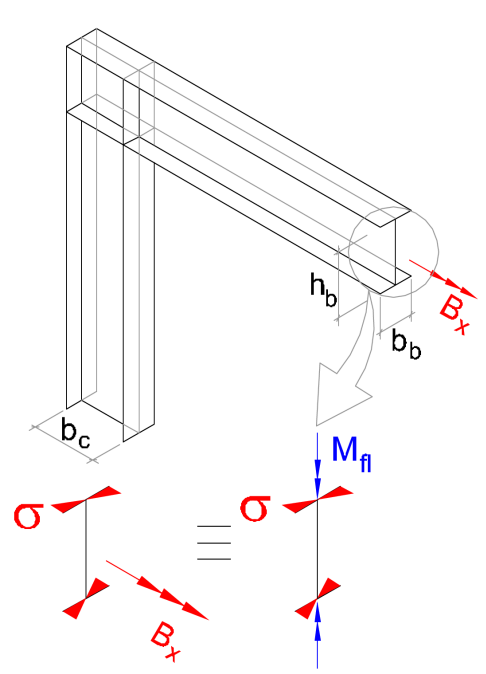

(a)

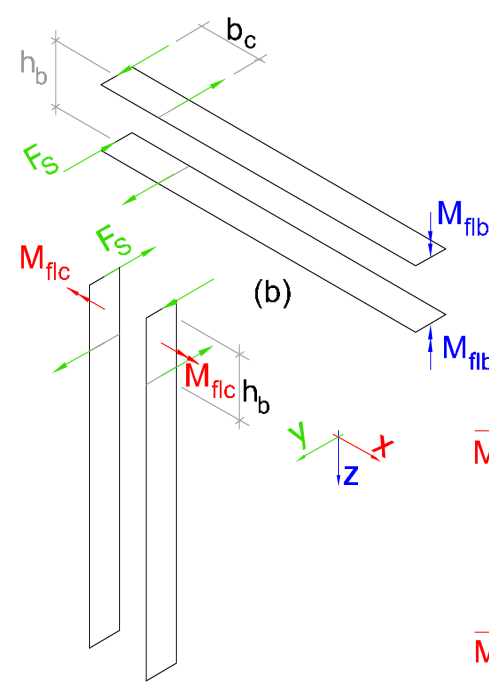

(c)

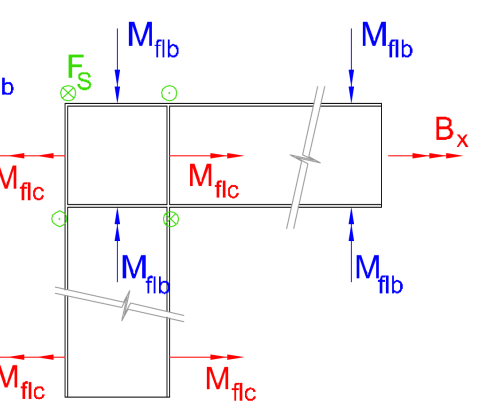

(d)

Figure 6.3: Kinematic mechanism of box connection; a) the stress compatibility; b) and c) local bending moments in the flanges of beam and column; d)resume of the kinematic interpretation

According to Vlasov's assumption that the cross-section does not remain plane in the deformation configuration, each flange can be interpreted as a single beam. Hence, the flanges can rotate independently from each other due to the low stiffness of the web.

By decoupling the upper and lower flanges from the structure shown in figure 6.3, one obtains two cantilever beams. Their supports are the two vertical stiffeners of the box connection, which lead to a binary reaction force. Since the vertical stiffeners have low values of torsion stiffness, each vertical stiffener acts as hinge support.

The reaction forces in the stiffeners, with the principle of action and reaction, act in the column's flanges as concentrated bending moments, similar to the case of the beam's flanges. However, these local bending moments in the column have an opposite direction compared to the local bending moments in the flange of the beam. For instance, in 
figure 6.3 the beam's moment vectors of the flanges are inward oriented, while in the column these vectors are outward oriented.

It is important to note that this unique property of warping transmission also exists in other high modes. As a second example, one can consider the following "I" beam under an opposite dual self-balanced torsion moment ${ }^{2}$, as illustrated in figure 6.4. In this example, the upper flange's extreme of beam $\mathrm{A}$ is linked by a rigid rod to the bottom flange's extreme of beam B. Since the rod creates a compatibility of displacement between these flanges, the vertical displacement of the upper flange will be the same as that of the bottom flange. However, the vertical displacement of the bottom flange's extreme in beam $\mathrm{B}$ is opposite that of the same point in beam A. Thus, the total distortion of beam B is opposite that of beam A. Despite the fact that this connection is not usual in current structures, it is an example of how the inverse transmission of distortion can be produced.
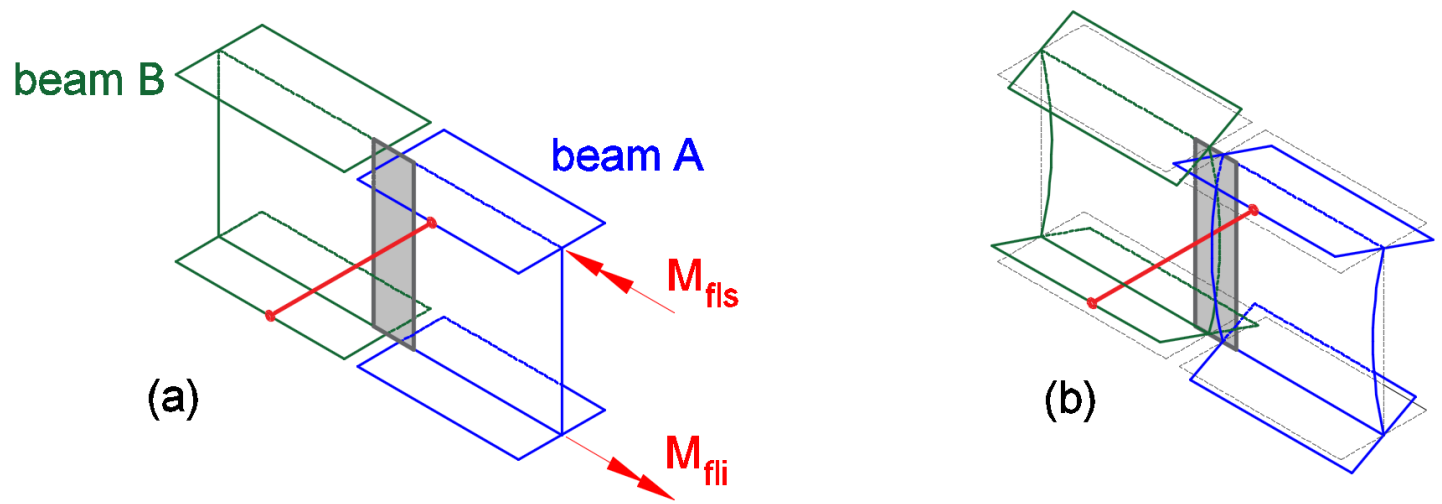

Figure 6.4: Kinematic mechanism of inverse distortion transmission; a) undeformed structure under a bi-torsion internal force (the connection rod is in red); b) deformed structure with inverse transmission.

\subsubsection{Coupling of different high modes due to connections}

The non-stiffener connection not only explains the hinge behavior of GBT's high modes, but it is also a good example of how warping and distortion can be coupled due to the connection.

Similar to the explanation of box connection, figure 6.5 shows how the flanges of the beam can be treated as cantilever beams. Furthermore, the cross-section's bi-moment can be expressed as a local bending moment of these cantilever beams.

However, in the case of non-stiffener connection, there is no reaction binary force composed by the vertical stiffener. The only restraints provided are the torsional inertia of each individual column's flange and the flexural bending stiffness of the web. Both

\footnotetext{
${ }^{2}$ The dual self-balanced torsion moment is named here as bi-torsion in analogy to the Vlasov bimoment.
} 


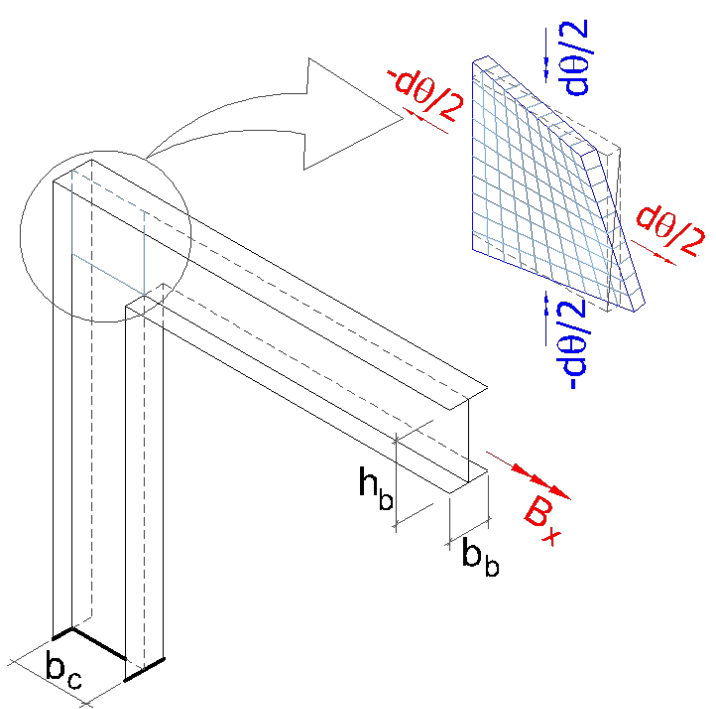

(a)

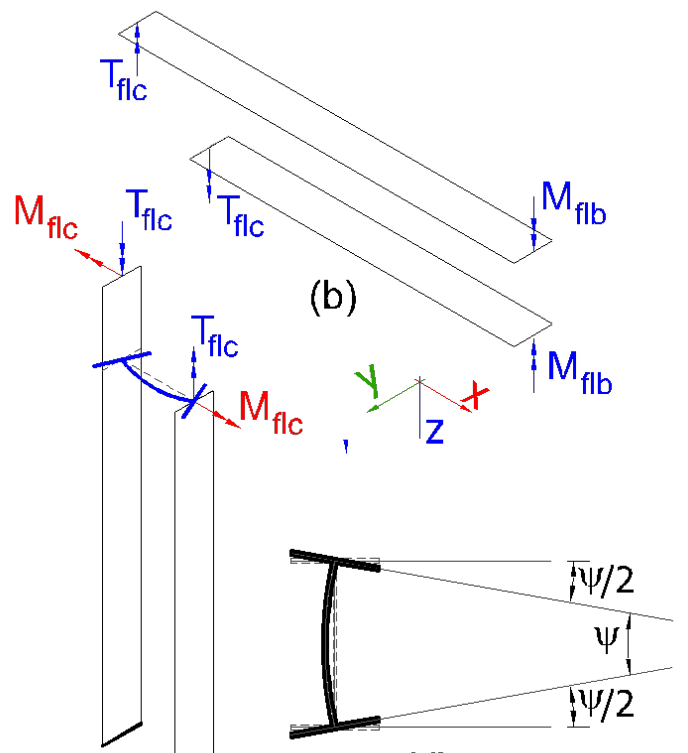

(c)

(d)

Figure 6.5: Kinematic mechanism of non-stiffener connection; a) warping deformation of the web; b) and c) local torsional and bending moments in the flanges of beam and column; d) distortion shaped in the column's cross section.

effects in general cases have low values compared to the moment of inertia of the beam's flange.

Consequently, only residual warping is transmitted among the elements. If the thicknesses of the web and the column's flange tend to zero, then the non-stiffener connection can be considered as an ideal warping hinge connection.

It is important to note two effects in this connection type. The first one is the inversion of the residual bi-moment from the beam into the column. This inversion is due to the web's warping transmission, which can be understood as an elementary slice of the cube presented in figure 6.2. Naturally, the web capacity for warping transmission is quite limited.

The second effect is the coupling of the residual transmitted warping with the distortion of the column's cross-section. The non-stiffener connection is an emblematic example of how the warping is coupled with distortion. Figures 6.5.b and c show a kinematic interpretation of how the local bending moments in the flange become local torsion moments in the column's flange. These torsion moments are self-balanced in the crosssection and can be described as bi-torsion. This distortion-torsional mode has no warping stiffness. Therefore, this mode, in this example, only involves the transversal bending and shear stiffness matrices of the cross-section. 


\subsubsection{Clamp mechanism}

The clamp mechanism behavior of high modes can be explained by the diagonal connection. The kinematic behavior of this connection, which does not invert the high modes' internal forces and displacements, can be understood based on the equilibrium of the flanges and the stiffener.

Different from the box connections, the local bending moments of the beam's flanges are not balanced by binary forces due to the reactions of the stiffeners. Instead, they are balanced by the moments' equilibrium at intersection points among i) the flanges of the column; ii) the flange of the beam; and iii) the diagonal stiffener, as the point "A" presented in figure 6.6.

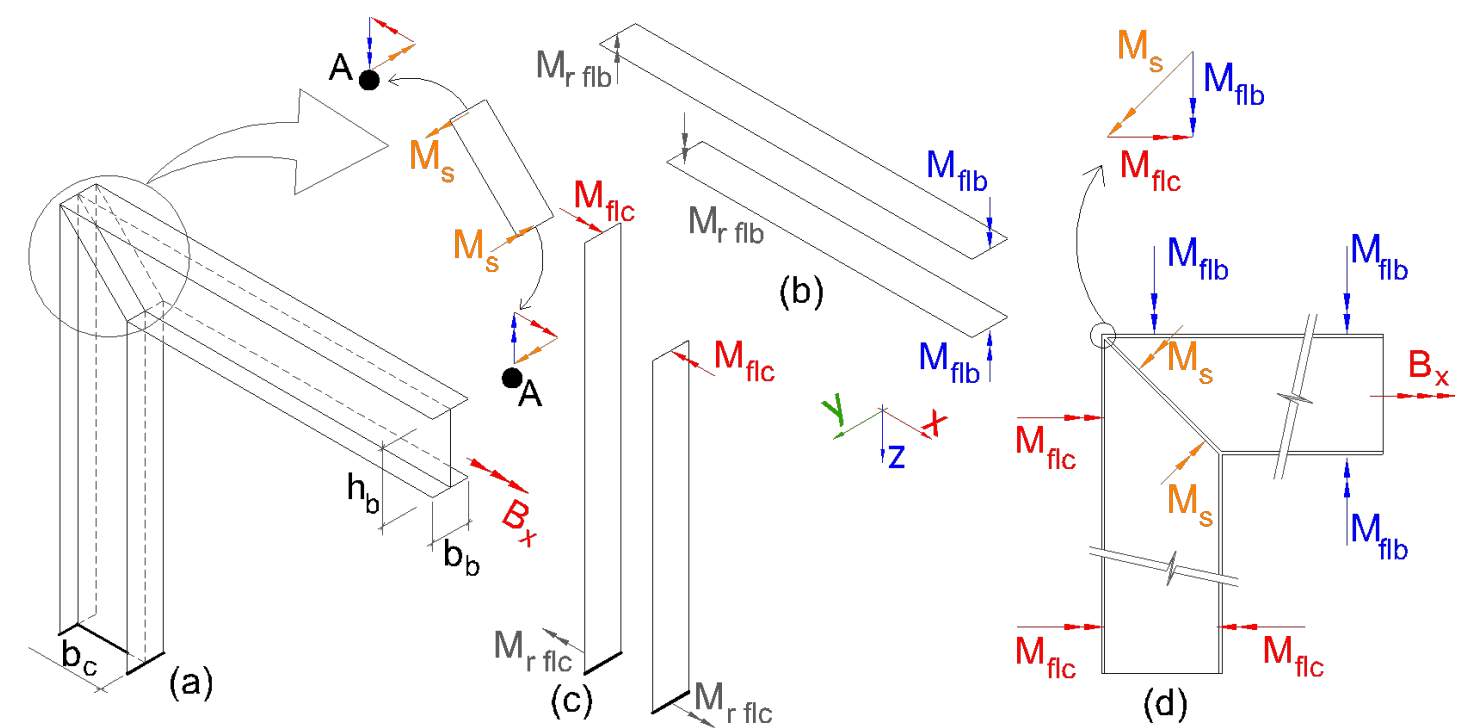

Figure 6.6: Kinematic mechanism of diagonal connection; a) bi-moment equilibrium as nodal equilibrium; b) and c) bending moments in the stiffener and in the flanges of beam and column; d) Left view of the nodal moment equilibrium.

Analogous to the case of trusses, point $\mathrm{A}$ in figure $6.6 .4 \mathrm{~b}$ and $\mathrm{c}$ has its equilibrium when the sum of the moment's vector is null. Naturally, the only difference with respect to the vectorial equilibrium approach in trusses is that the bending vectors are always perpendicular to the flanges and the stiffeners and not aligned with them.

Following this idea and by the third Newton's law, the local bending moments in the column's flanges have an "incoming" direction, such as the local bending moments in the beam's flanges. Therefore, there is no inversion of the warping and bi-moment direction.

However, this simple explanation has the assumption of null torsional inertia in the stiffener and in the flanges. If the connection has a thick stiffener and flanges, then the torsion moments vector, which is aligned with the stiffeners and flanges, will restrain the connection to warping. In other words, as perpendicular vectors with respect to 
the bending moment vectors, the torsional vectors will also restrain the connection in the stiffener's direction and not only in the perpendicular direction with respect to it as a "membrane" diagonal.

To clarify this, as shown in figure 6.7, the torsion moments can be understood as bending moments in "virtual stiffener and flanges," which are fully perpendicular to the real one. Then, a simple diagonal connection becomes a box-diagonal, an internal support condition, as explained before.
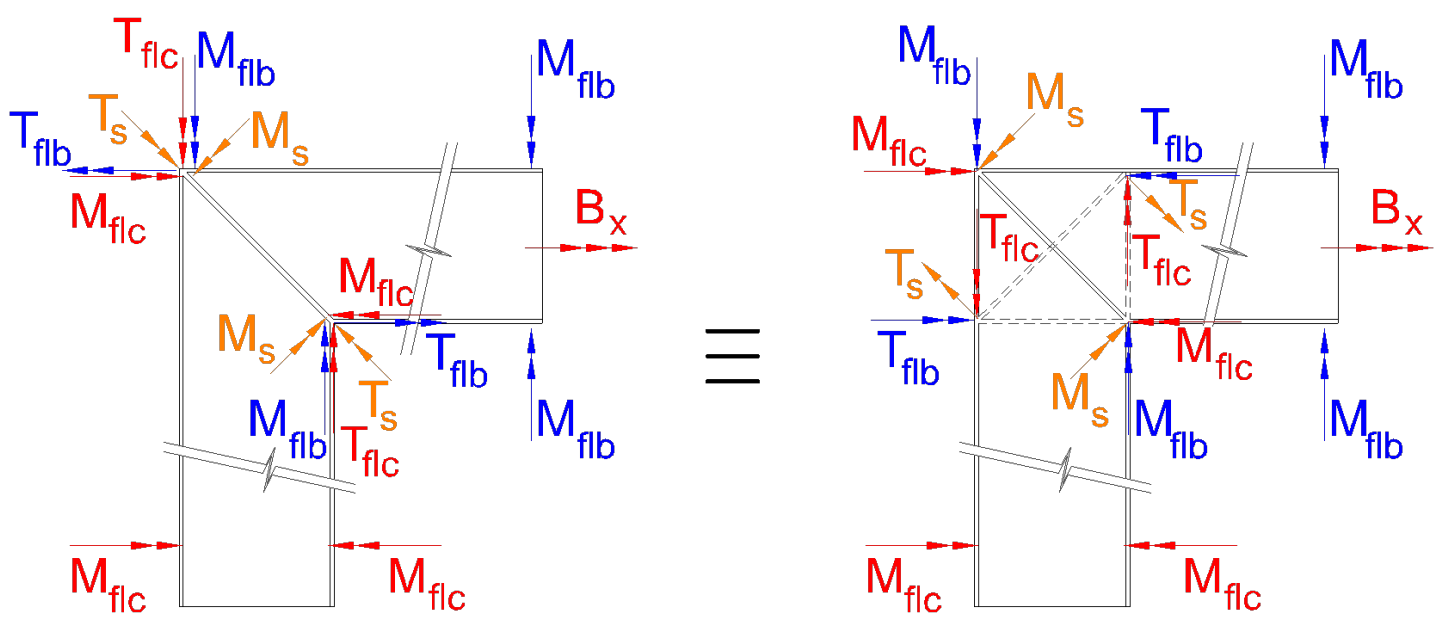

Figure 6.7: Diagonal connection with the effects of torsion inertia of flanges and stiffener resulting in a box-diagonal connection.

The final remark about this connection is the effect of the residual web's warping transmission. As explained in the previous subsection, the web has an inversed warping transmission, as well as a box-connection.

In fact, the diagonal connection is a clear example of how complex a connection's kinematic model can reach. Even more complex issues arise in the generic cross-section, in which the centers of gravity and shear are not coincident at the same point.

\subsection{Connections obtained from coupling between GBT and shell elements}

From the examples of warping transmission in the previous section, it becomes clear that the connections involving GBT's high modes are not only complex in the kinematic mechanism but also in the unique properties of these modes. In addition, a usual structure connection involves many details, such as welds, bolts, holes, and stiffeners, which require minute modeling and analysis, usually carried out by shell or solid finite elements.

Since the main field of application of GBT is thin-walled structures and the connections of these structures are often modeled by shell elements, the focus of this section is on 
the coupling between these elements and GBT elements.

The coupling approach proposed here is a novel application of Master-Slave method for beam frames and connections [92, 93]. It uses the superposition property of GBT's deformation modes to set up the relationship between the master and slaver degrees of freedom. Furthermore, these degrees of freedom are divided in two groups: shell and GBT. Each group is related to a master or slaver degree of freedom. Different from traditional applications, the presented approach predefines which group is the master and which is the slaver. These details are discussed in sequence.

\subsubsection{Definition of master and slaver degrees of freedom}

Usually, in the Master-Slaver multi-freedom constraint method, there is no rule regarding the definition of which degree of freedom is the master and which one is the slaver[172]. However, in the present implementation, this definition is predefined. Since the main focus of the proposed procedure is to build up a degree of freedom mapping of type shell-GBT or GBT-shell and avoid any kind of restraint of type GBT-GBT or shell-shell, one can classify the degree of freedom as shell or GBT group. Once this limitation and classification are defined, the next issue is which group is the master and which is the slaver.

The direct answer to this question is that the degrees of freedom of GBT are the master ones.

This choice is not arbitrary. It is based on the fact that one GBT node has a lower amount of degrees of freedom than the summation of all degrees of freedom of shell elements used to described a cross-section. For instance, one can consider the coupling of a hollow circular cross-section, as plotted in figure 6.8. This coupled cross-section has 28 shell nodes; each node has 6 degrees of freedom, giving a total of 168 degrees of freedom. This same cross-section can be described in GBT by 6 deformation modes, each one having two degrees of freedom per node (there are a few exceptions, such as the uniform torsion or the uniform longitudinal elongation deformation modes, which require just one degree of freedom per node). Consequently, GBT requires a total of 12 degrees of freedom.

Since the finite element model's response is always limited by the coarse description of the cross-section, the opposite choice, i.e., the shell's degrees of freedom as master ones, is numerically inefficient. This alternative definition requires more computational effort to reach the results of the choice mentioned in the previous paragraph.

Consequently, one can express the coupling between shell and GBT degrees of freedom as in matrix form:

$$
[\vartheta]_{\text {shell }}=\left[T_{c}\right][\vartheta]_{G B T}
$$

Where, $\left[T_{c}\right]$ is the multi-freedom constraint transformation matrix, detailed in the sequence; while $[\vartheta]_{\text {shell }}$ and $[\vartheta]_{G B T}$ are the displacement vectors of shell and GBT's degrees of freedom, respectively. 


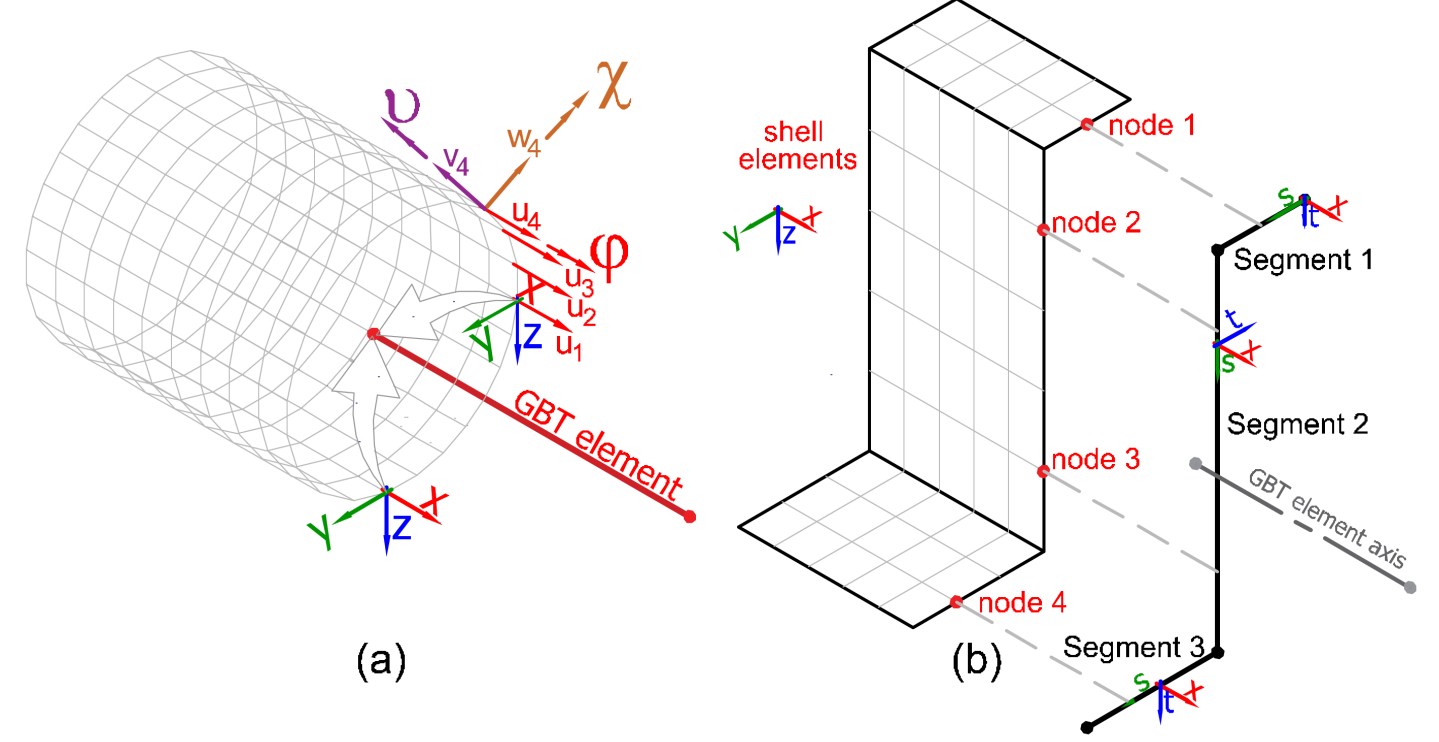

Figure 6.8: Example of coupling of shell and GBT models: a)Hollow circular crosssection between; b)Shell nodes in the middle of GBT's cross-section segments

\subsubsection{Master-slave relationships based on GBT's modes}

After the definition of the degrees of freedom's group, the next step is the setup of the multi-freedom constraint transformation matrix, $\left[T_{c}\right]$. Thus, one uses the GBT's description of displacement field as a sum of the modal displacement, equations 2.18, 2.19 and 2.20, to express the shell's nodal displacement. For instance, the longitudinal displacement of node 1, in shell element discretization showed in figure 6.8 , can be expressed as follows:

$$
u_{1, \text { shell }}={ }^{1} u_{1}{ }^{1} V(x=-L / 2)+\sum_{i=1}^{m}{ }^{i} u(s){ }^{i} V_{, x}(x)
$$

Here, the longitudinal position in beam element, $x$, is set at one of the extreme nodes: $x=-L / 2$ or $x=L / 2$, consistent with the element definition in figure 5.1. A matrix form can represent this sum, which is already the main components of multi-freedom constrain transformation matrix:

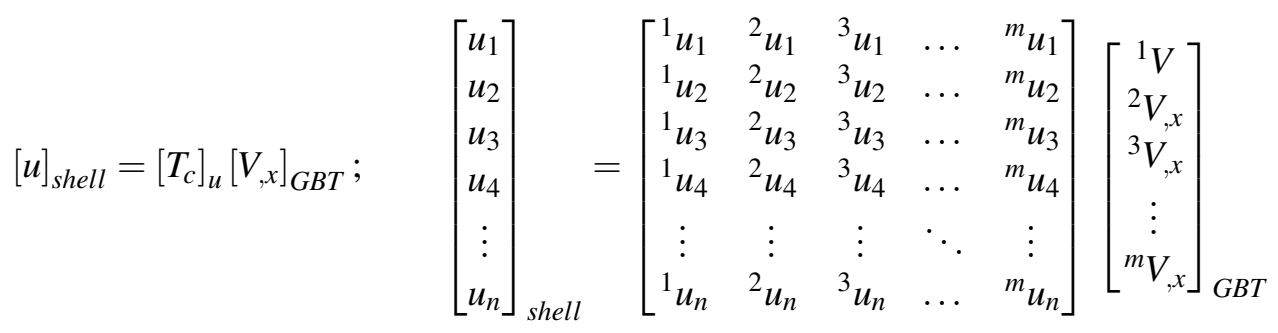

Here, the indexes $n$ and $m$ indicate the number of shell nodes and GBT modes, respectively. 
It is not necessary that the shell nodes and the GBT cross-section discretization nodes are in the same geometric position. The only restriction is the imposition that shell nodes have to be in the middle line of the cross-section walls. Since this condition is maintained, an arbitrary shell node position can be coupled with GBT modes by the interpolation functions, given in eq. 4.6. Thus, eq. 6.3 is re-order based on the segments of the cross-section:

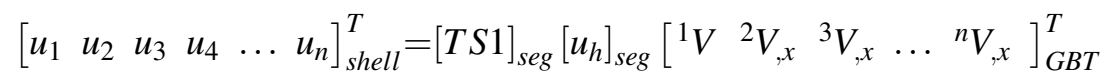

Here, $[T S 1]_{\text {seg }}$ is a matrix, in which the number of rows is the number of shell nodes and the number of columns is the number of segments of the cross-section that contains the shell nodes. This matrix is populated in each row by the linear Hermitian interpolation function of $\left[T s_{H e 1}\right]\left[S h_{H e 1 c c}\right]$ in the respective column of the segment that has the shell node. For example, in the coupling illustrated in figure 6.8.b the shell node 1 is in cross-section segment 1 , shell nodes 2 and 3 are in segment 2 , and shell node 4 is in segment 3 . For this case, one obtains the following interpolation segment matrix:

$$
[T S 1]_{\text {seg }}=\left[\begin{array}{cccc}
{\left[T s_{H e 1}\right]_{s 1}\left[S h_{H e 1 c c}\right]_{s 1}} & 0 & 0 & \ldots \\
0 & {\left[T s_{H e 1}\right]_{s 2}\left[S h_{H e 1 c c}\right]_{s 2}} & 0 & \ldots \\
0 & {\left[T s_{H e 1}\right]_{s 2}\left[S h_{H e 1 c c}\right]_{s 2}} & 0 & \ldots \\
0 & 0 & {\left[T s_{H e 1}\right]_{s 3}\left[S h_{H e 1 c c}\right]_{s 3}} & \ldots \\
\vdots & \vdots & \vdots & \ddots
\end{array}\right]
$$

Where the lower-right indexes $s 1, s 2$ and $s 3$ indicate the relative cross-section segment. The second matrix in the right-hand side of eq. $6.4,\left[u_{h}\right]_{\text {seg }}$, is nothing more than all of the modal longitudinal displacements of each segment that has the shell nodes:

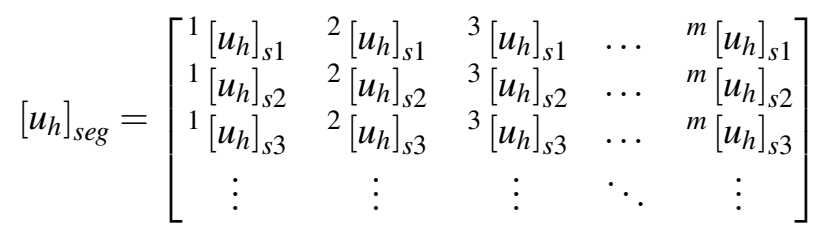

The transversal displacements $v$ and $w$ are obtained in a similar way. The only change is the absence of mode 1 (axial deformation). If the shell nodes share the same position as the GBT nodes of cross-section discretization, one finds:

$$
\begin{aligned}
& {[v]_{\text {shell }}=\left[T_{c}\right]_{v}[V]_{G B T} ; \quad\left[\begin{array}{c}
v_{1} \\
v_{2} \\
v_{3} \\
\vdots \\
v_{n}
\end{array}\right]_{\text {shell }}=\left[\begin{array}{ccccc}
2 v_{1} & 3 v_{1} & { }^{4} v_{1} & \ldots & { }^{n} v_{1} \\
2 v_{2} & 3 v_{2} & 4 v_{2} & \ldots & { }^{n} v_{2} \\
2 v_{3} & 3 v_{3} & 4 v_{3} & \ldots & { }^{n} v_{3} \\
\vdots & \vdots & \vdots & \ddots & \vdots \\
{ }^{2} v_{6} & 3 v_{6} & { }^{4} v_{6} & \ldots & { }^{n} v_{6}
\end{array}\right]\left[\begin{array}{c}
{ }^{2} V \\
{ }^{3} V \\
{ }^{4} V \\
\vdots \\
{ }^{n} V_{, x}
\end{array}\right]_{G B T}} \\
& {[w]_{\text {shell }}=\left[T_{c}\right]_{w}[V]_{G B T} ; \quad\left[\begin{array}{c}
w_{1} \\
w_{2} \\
w_{3} \\
\vdots \\
w_{n}
\end{array}\right]_{\text {shell }}=\left[\begin{array}{ccccc}
{ }^{2} w_{1} & { }^{3} w_{1} & { }^{4} w_{1} & \ldots & { }^{n} w_{1} \\
{ }^{2} w_{2} & 3 w_{2} & { }^{4} w_{2} & \ldots & { }^{n} w_{2} \\
{ }^{2} w_{3} & 3 w_{3} & { }^{4} w_{3} & \ldots & { }^{n} w_{3} \\
\vdots & \vdots & \vdots & \ddots & \vdots \\
{ }^{2} w_{6} & 3 w_{6} & { }^{4} w_{6} & \ldots & { }^{n} w_{6}
\end{array}\right]\left[\begin{array}{c}
{ }^{2} V \\
{ }^{3} V \\
{ }^{4} V \\
\vdots \\
{ }^{n} V
\end{array}\right]_{G B T}}
\end{aligned}
$$


Otherwise, the shell nodes are at another point of the cross-section segments, it is necessary to apply the interpolation functions of respective displacement, which leads to:

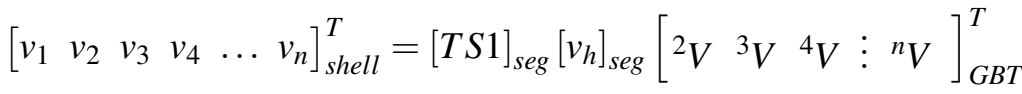

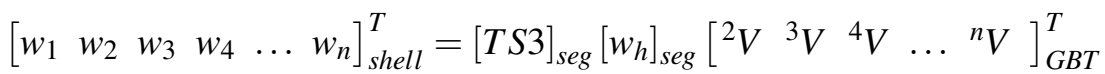

where, the interpolation segment matrices $[T S 1]_{\text {seg }}$ and $[T S 3]_{\text {seg }}$ are already presented above. The first matrix is the same of eq. 6.5 , while $[T S 3]_{\text {seg }}$ correspond to the cubic Hermitian function, given in eq. 4.8. The GBT's modal segment matrices: $\left[v_{h}\right]_{\text {seg }}$ and $\left[w_{h}\right]_{\text {seg }}$ follow the same procedure of longitudinal displacement.

Concerning the rotational degrees of freedom, one can directly setup the coupling of transversal rotation, $v$, as shown in figure 6.8.a, between shell elements and the generic segmented cross section based on eq. 2.15. From this equation, it is clear that the transversal rotation is given by:

$$
v(x, s)=w(s) V_{, x}(x)
$$

which leads to the following expression of coupling, in modal superposition:

$$
v_{\text {shell }}=\sum_{i=1}^{n}{ }^{i} w(s){ }^{i} V_{, x}(x)
$$

Thus, the transversal rotation coupling can be expressed by the multi-freedom constraint transformation matrix, $\left[T_{c}\right]_{w}$, and GBT's degrees of freedom of generalized rotation, $\left[V_{, x}\right]_{G B T}$, which are given in equations 6.8 and 6.3 , respectively:

$$
[v]_{\text {shell }}=\left[T_{c}\right]_{w}\left[V_{, x}\right]_{G B T}
$$

In the more generic case, in which the shell nodes lie at an arbitrary point in the GBT segment, the coupling expression is given by:

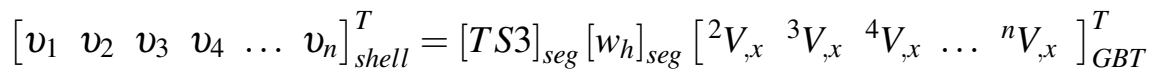

Following a similar procedure, one obtains the coupling of longitudinal rotation, $\varphi$, based on eq. 2.16:

$$
\varphi(x, s)=w_{, s}(s) V(x)
$$

If GBT's discretization and shell nodes share the same position, no interpolation of the GBT segment is necessary. Thereby, one finds:

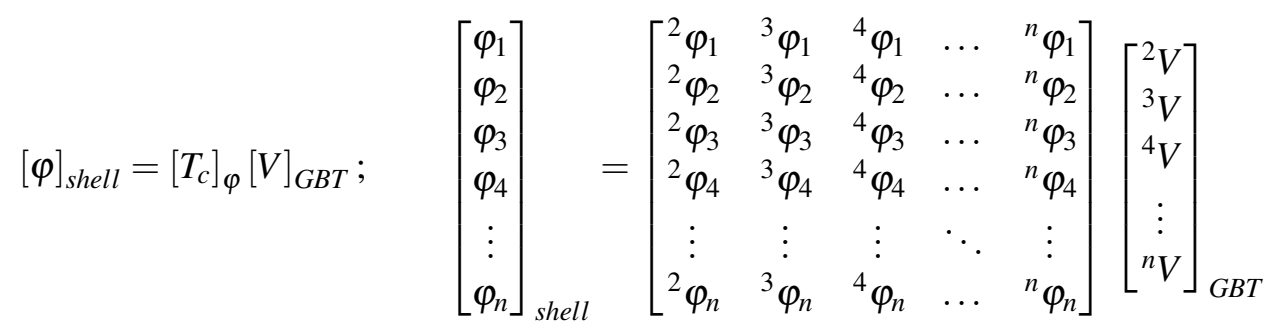


Otherwise, one expresses the coupling by the interpolated values inside of a GBT as:

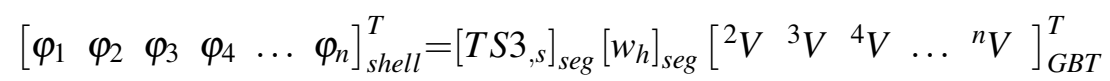

where the subindex ${ }_{,}$indicates the first derivative of the interpolation segment matrix $[T S 3]_{\text {seg }}$.

The transversal perpendicular rotational degree of freedom, $\chi$, shown in figure 6.8.a, requires special remarks.

The first one is regarding the GBT's assumption of a linear variation of the longitudinal displacement in each cross-section segment. This leads to a constant transversal perpendicular rotation in each segment. Thus, if GBT's discretization and shell nodes do not share the same position, one can reach the following expression:

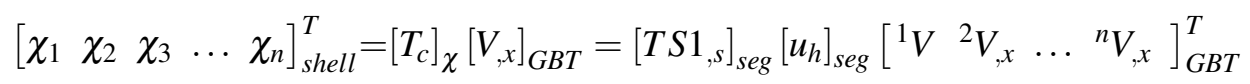

However, since each segment can have a different constant value of this rotation, this leads to a discontinuity at the GBT nodes of cross-section discretization. Consequently, if GBT's discretization and shell nodes share the same position, there are ambiguous values of GBT transversal perpendicular rotation to couple.

In a simple-minded approach, this problem can be overcome by using the average of the transversal perpendicular rotations of each segment that reach the node.

The second remark is about the type of shell element used. Many shell elements do not have this degree of freedom, named in the literature as the drilled degree of freedom. For these shell elements, there is no coupling for this transversal rotation, and all information about it vanishes.

Coupling with a different global coordinate system - the hollow circular crosssection case

It is important to observe that the approach presented up to this point considers a common coordinate system between the shell and GBT elements. Usually, this condition is found in the generic segmented cross-section, as presented in Chapter 4. However, for a hollow circular cross-section, as presented in Chapter 3, the coordinate systems are not the same, such as the local coordinate system presented in figure 3.1, which is left-handed. Therefore, coupling for the hollow circular cross-section requires an extra transformation for the displacements $v$ and $w$ :

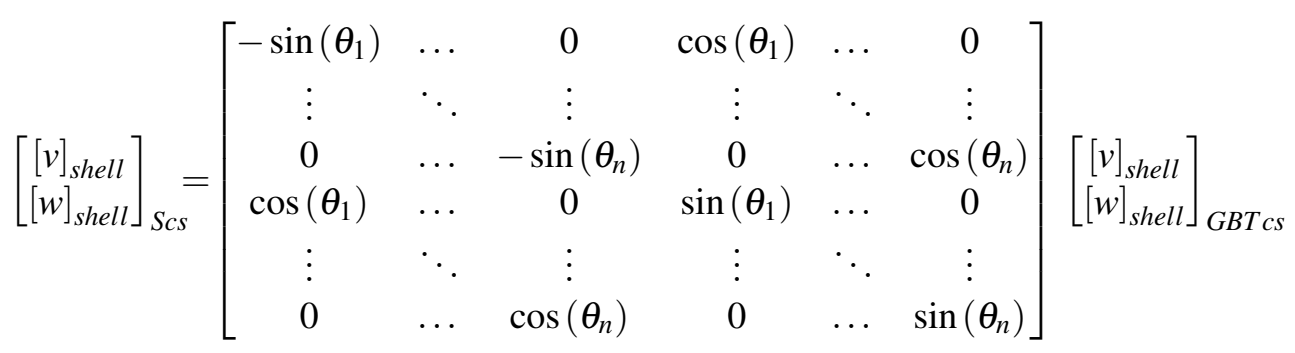


Where, $\theta_{n}$ is the angle of each shell node according to GBT's local coordinate system, as shown in figure 3.1; the subindexes $S_{c s}$ and ${ }_{\text {GBT }}$ indicate the Shell coordinate system and the GBT's coordinate system.

The transversal rotations require the same treatment:

$$
\left[\begin{array}{l}
{[v]_{\text {shell }}} \\
\left.[\chi]_{\text {shell }}\right]_{\text {Scs }}=\left[\begin{array}{cccccc}
-\sin \left(\theta_{1}\right) & \ldots & 0 & \cos \left(\theta_{1}\right) & \ldots & 0 \\
\vdots & \ddots & \vdots & \vdots & \ddots & \vdots \\
0 & \ldots & -\sin \left(\theta_{n}\right) & 0 & \ldots & \cos \left(\theta_{n}\right) \\
\cos \left(\theta_{1}\right) & \ldots & 0 & \sin \left(\theta_{1}\right) & \ldots & 0 \\
\vdots & \ddots & \vdots & \vdots & \ddots & \vdots \\
0 & \ldots & \cos \left(\theta_{n}\right) & 0 & \ldots & \sin \left(\theta_{n}\right)
\end{array}\right]\left[[v]_{\text {shell }}\right. \\
{[\chi]_{\text {shell }}}
\end{array}\right]_{\text {GBTcs }}
$$

\subsubsection{Coupled stiffness matrix and external load vector}

Once the multi-freedom constraint matrices are evaluated, the next step in the mixed shell-GBT model is the setup of coupled stiffness matrix and the external load vector. This is easily achieved by the respective matrix transformations:

$$
\begin{gathered}
{[K]_{c p}=\left[T_{c}\right]_{t o t}^{T}[K]_{u c}\left[T_{c}\right]_{t o t}} \\
{[F]_{c p}=\left[T_{c}\right]_{t o t}^{T}[F]_{u c}}
\end{gathered}
$$

where the subindexes $c p$ and $u c$ indicate the coupled and uncoupled matrices, respectively. The total coupling transformation matrix, $\left[T_{c}\right]_{t o t}$, is an identity matrix of the same rank as the uncoupled stiffness matrix, which has the columns concerning the coupled shell's degrees of freedom removed. Furthermore, the rows concerning these degrees of freedom are re-populated by the values of matrices $\left[T_{c}\right]_{u},\left[T_{c}\right]_{v},\left[T_{c}\right]_{w},\left[T_{c}\right]_{\varphi}$ and $\left[T_{c}\right]_{\chi}$. The next section shows the implementation of this matrix via a detailed example.

\subsection{Numerical example of coupling GBT and shell elements}

The structure presented in the numerical example of Chapter 5 is coupled in its base with an extension of half meter long, which has the same material and geometry properties.

One discretizes this extension in 48 nodes elements in transversal direction and 5 layers of elements in the longitudinal direction. Thus, the shell part of the model has 288 nodes and 240 shell elements. Initially, the shell element is limited to membrane behavior, which leads to 3 degrees of freedom per node. Therefore, the extension part has a total of 864 degrees of freedom. From this total, the boundary condition at the base restraints 144 degrees of freedom. Also, the multi-freedom constraint matrix restraints the same amount at the top cross-section of this prolongation. The final mixed model has 576 free degrees of freedom from the shell part. The GBT amount of degree of freedom is 36, as presented in the example of Chapter 5. 


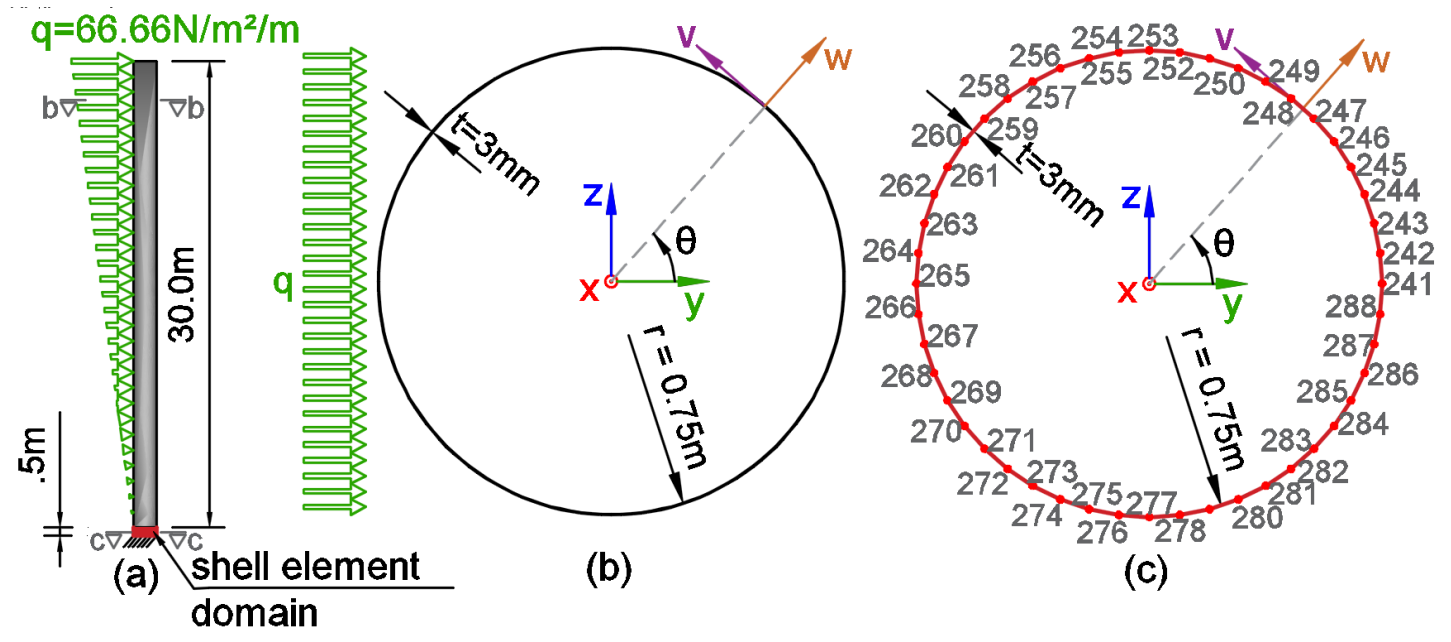

Figure 6.9: Thin-walled circular hollow section under a linear projected force and a coupled shell element segment; a) elevation; b) cross-section in GBT's domain; c) cross-section in shell's domain at the coupling point

\subsubsection{Setup of finite element and coupling matrices}

Since there is no modification in geometry and loads, the GBT's element and modes are the same used in the example of Chapter 5. Therefore, the GBT's matrices (equations 5.198 and 5.209) and load vector (equations 5.199 and 5.210) can be directly applied in this example.

Regarding the membranes, all elements are equal, which leads to the same local stiffness matrix. This matrix is presented below, as well as the plot of an isolated element:

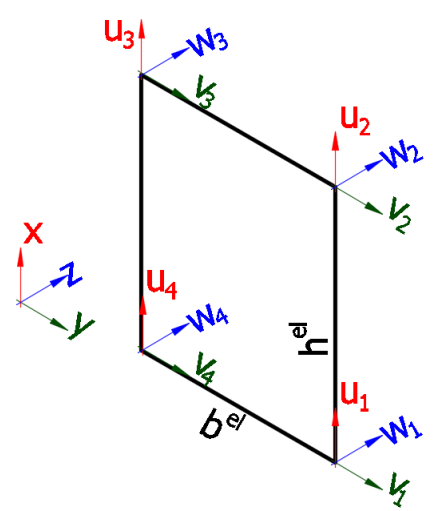

Figure 6.10: Membrane element local displacement definition 


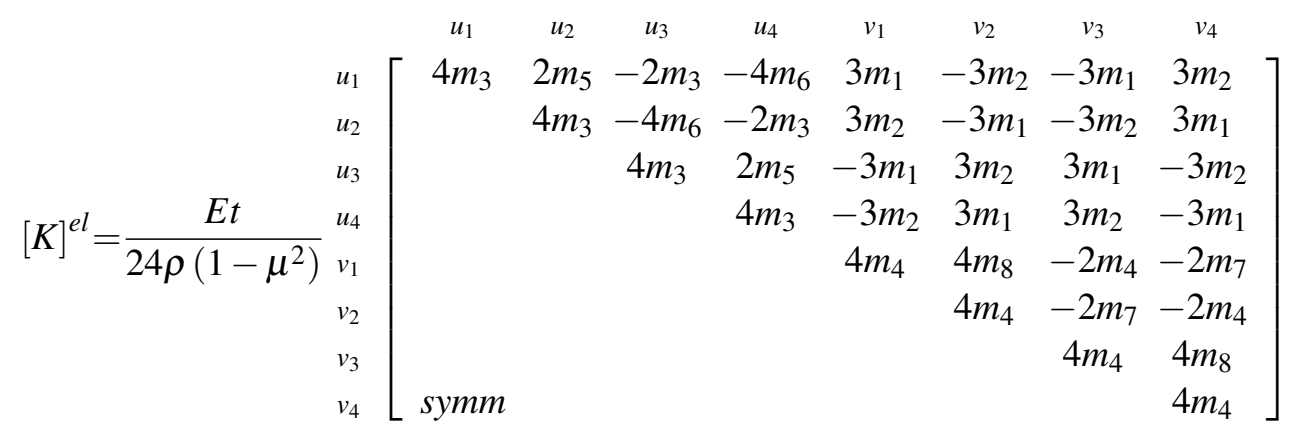

where, $\rho$ is the length/height ratio:

$$
\rho=h^{e l} / b^{e l}
$$

The matrix's terms are:

$$
\begin{gathered}
m_{1}=\rho(1+\mu) \\
m_{2}=\rho(1-3 \mu) \\
m_{3}=2+\rho^{2}(1-\mu) \\
m_{4}=2 \rho^{2}+(1-\mu) \\
m_{5}=\rho^{2}(1-\mu)-4 \\
m_{6}=\rho^{2}(1-\mu)-1 \\
m_{7}=4 \rho^{2}-(1-\mu) \\
m_{8}=\rho^{2}-(1-\mu)
\end{gathered}
$$

One can observe from this simple element that the length/height ratio is 0.98 , which involves no special issue concerning shear locking. The numerical values of the element's local stiffness matrix are (units in $\mathrm{N}$ and $\mathrm{mm}$ ):

$$
[K]^{e l}=10^{3} \begin{gathered}
u_{3} \\
u_{1} \\
u_{1} \\
v_{2} \\
v_{1} \\
v_{2} \\
v_{2} \\
v_{3}
\end{gathered}
$$

The transformation from local to global coordinate system of each element and the assembling of global stiffness matrix follow the well-known approach of finite elements $[19,91]$. The GBT's stiffness matrices are added to the global stiffness obtained from membrane elements. The resulting stiffness matrix is still uncoupled and requires the coupling procedures of the next steps. Table 6.1 resumes the setup of the degrees of freedom: 
Table 6.1: Setup of degrees of freedom in mixed membrane-GBT model

\begin{tabular}{ccccc}
\hline displacement & DOF's & Support restraint & Master DOF & Slavers DOF \\
\hline $\mathrm{u}$ & 1 to 288 & 1 to 48 & - & 241 to 288 \\
$\mathrm{~V}$ & 289 to 576 & 289 to 337 & - & 529 to 576 \\
$\mathrm{~W}$ & 577 to 864 & 577 to 625 & - & 817 to 864 \\
${ }^{3} V$ & $865 ; 867 ; 869$ & - & 865 & - \\
${ }^{3} V_{, x}$ & $866 ; 868 ; 870$ & - & 866 & - \\
${ }^{5} V$ & $871 ; 873 ; 875$ & - & 871 & - \\
${ }^{5} V_{, x}$ & $872 ; 874 ; 876$ & - & 872 & - \\
${ }^{7} V$ & $877 ; 879 ; 881$ & - & 877 & - \\
${ }^{7} V_{, x}$ & $878 ; 880 ; 882$ & - & 878 & - \\
${ }^{11} V$ & $883 ; 885 ; 887$ & - & 883 & - \\
${ }^{11} V_{, x}$ & $884 ; 886 ; 888$ & - & 884 & - \\
${ }^{15} V$ & $889 ; 891 ; 893$ & - & 889 & - \\
${ }^{15} V_{, x}$ & $890 ; 892 ; 894$ & - & 890 & - \\
${ }^{a} V$ & $895 ; 897 ; 899$ & - & 895 & - \\
${ }^{a} V_{, x}$ & $896 ; 898 ; 900$ & - & 896 & - \\
\hline
\end{tabular}

Coupling of longitudinal displacement - $u$

To couple the longitudinal displacement, $u$, one must evaluate the multi-freedom constraint transformation matrix, $\left[T_{c}\right]_{u}$, for each slaver degree of freedom with respect to each GBT mode. To do so, it is necessary to compute the modal longitudinal displacement of each slaver node, given in tables 3.1 and 3.2 , with the respective node angle $\theta$, where the $\Delta \theta$ between the nodes is $7.5^{\circ}$, as presented in figure 6.9.c. These results are introduced into eq. 6.3 , leading to:

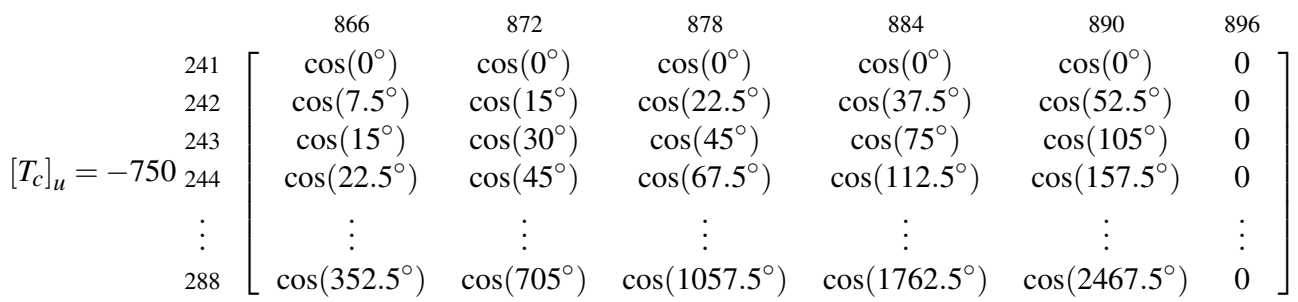

On the left and on the top, the row and column indexes indicate the slave and master degrees of freedom, respectively. Since the longitudinal displacement of GBT is consistent with the global coordinate system of the membranes, no extra transformation is required for these degrees of freedom.

Coupling of transversal displacements $-v$ and $w$

Similar to the coupling of longitudinal displacement, the transversal coupling is obtained by the multi-freedom constrain transformation matrices $\left[T_{c}\right]_{v}$ and $\left[T_{c}\right]_{w}$, which 
are based on the expression of tables 3.1 and 3.2. Therefore, one can find:

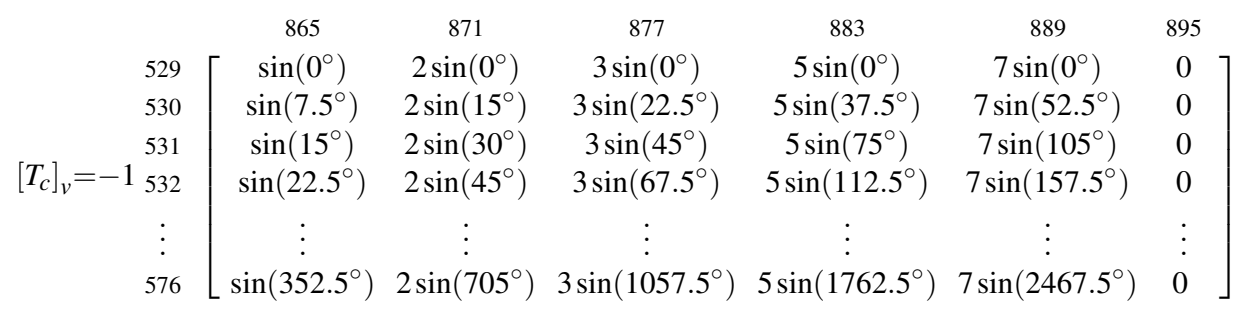

$$
\begin{aligned}
& \begin{array}{c}
\quad \\
\left.\quad T_{C}\right]_{w}= \\
817 \\
819 \\
820 \\
\vdots \\
864
\end{array}\left[\begin{array}{cccccc}
865 & 871 & 877 & 883 & 889 & 895 \\
\cos \left(0^{\circ}\right) & 4 \cos \left(0^{\circ}\right) & 9 \cos \left(0^{\circ}\right) & 25 \cos \left(0^{\circ}\right) & 49 \cos \left(0^{\circ}\right) & 1 \\
\cos \left(7.5^{\circ}\right) & 4 \cos \left(15^{\circ}\right) & 9 \cos \left(22.5^{\circ}\right) & 25 \cos \left(37.5^{\circ}\right) & 49 \cos \left(52.5^{\circ}\right) & 1 \\
\cos \left(15^{\circ}\right) & 4 \cos \left(30^{\circ}\right) & 9 \cos \left(45^{\circ}\right) & 25 \cos \left(75^{\circ}\right) & 49 \cos \left(105^{\circ}\right) & 1 \\
\cos \left(22.5^{\circ}\right) & 4 \cos \left(45^{\circ}\right) & 9 \cos \left(67.5^{\circ}\right) & 25 \cos \left(112.5^{\circ}\right) & 49 \cos \left(157.5^{\circ}\right) & 1 \\
\vdots & \vdots & \vdots & \vdots & \vdots & \vdots \\
\cos \left(352.5^{\circ}\right) & 4 \cos \left(705^{\circ}\right) & 9 \cos \left(1057.5^{\circ}\right) & 25 \cos \left(1762.5^{\circ}\right) & 49 \cos \left(2467.5^{\circ}\right) & 1
\end{array}\right]
\end{aligned}
$$

Different from the longitudinal displacement, the GBT transversal ones are not in the global membrane's coordinate system. Thereby, the transformation given in eq. 6.20 is required, which leads to:

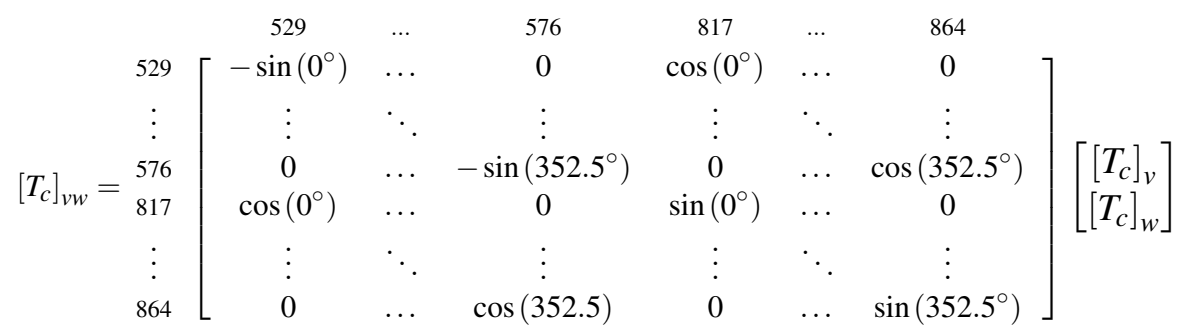

\subsubsection{Finite element solution}

Once the multi-freedom constraint transformation matrices are evaluated for each displacement direction, a total form of these matrices, $\left[T_{c}\right]_{t o t}$, can be built up.

As mentioned before, initially, an identity matrix, $I$, is defined. Its dimensions have the same number of no-support degrees of freedom, 756x756. From this matrix, all columns corresponding to slave degrees of freedom are removed, leading to the dimension $756 \times 612$. Thereafter, each component of the rows that concerns the slave degrees of freedom is replaced by the respective term from the matrices $\left[T_{c}\right]_{u}$ and $\left[T_{c}\right]_{v w}$.

With the definition of the total multi-freedom constraint transformation matrix, one can achieve the coupled free stiffness matrix $[K]_{c p}$ by eq. 6.21 , in which the dimensions are $612 \times 612$.

Since, in this example, there is no external load applied to the slave degrees of freedom, the external load vector can be directly assembled without the related rows. Alternatively, one can apply eq. 6.22. The same external load vector is reached. 
Finally, the numerical values of the degrees of freedom are achieved by a standard solver, such as the Cholesky decomposition, which is used here. Tables 6.2 and 6.3 present this numerical solution for GBT and shell elements degrees of freedom, respectively:

Table 6.2: Solution of GBT's degrees of freedom, in mm

\begin{tabular}{ccccccc}
\hline mode & $V\left(x=-\frac{L}{2}\right)$ & $V_{, x}\left(x=-\frac{L}{2}\right)$ & $V(x=0)$ & $V_{, x}(x=0)$ & $V\left(x=\frac{L}{2}\right)$ & $V_{, x}\left(x=\frac{L}{2}\right)$ \\
\hline 3 & $1.793 \mathrm{E}-1$ & $5.535 \mathrm{E}-4$ & $2.901 \mathrm{E}+2$ & $1.298 \mathrm{E}-2$ & $1.024 \mathrm{E}+2$ & $1.116 \mathrm{E}-2$ \\
5 & $-1.302 \mathrm{E}-2$ & $-4.488 \mathrm{E}-5$ & $-1.325 \mathrm{E}+1$ & $-4.788 \mathrm{E}-4$ & $-5.867 \mathrm{E}+0$ & $-5.130 \mathrm{E}-4$ \\
7 & $6.048 \mathrm{E}-4$ & $2.088 \mathrm{E}-6$ & $2.753 \mathrm{E}-1$ & $9.021 \mathrm{E}-6$ & $1.383 \mathrm{E}-1$ & $9.172 \mathrm{E}-6$ \\
11 & $-6.473 \mathrm{E}-6$ & $-2.351 \mathrm{E}-8$ & $-1.573 \mathrm{E}-3$ & $-4.965 \mathrm{E}-8$ & $-7.885 \mathrm{E}-4$ & $-5.257 \mathrm{E}-8$ \\
15 & $2.927 \mathrm{E}-7$ & $1.194 \mathrm{E}-9$ & $6.690 \mathrm{E}-5$ & $2.001 \mathrm{E}-9$ & $3.352 \mathrm{E}-5$ & $2.235 \mathrm{E}-9$ \\
axial & $7.619 \mathrm{E}-15$ & $-1.524 \mathrm{E}-8$ & $-4.573 \mathrm{E}-4$ & $-1.524 \mathrm{E}-8$ & $-2.287 \mathrm{E}-4$ & $-1.524 \mathrm{E}-8$ \\
\hline
\end{tabular}

One important remark concerns the indirect coupling among the GBT's degrees of freedom of orthogonal modes. Unlike the GBT's linear analysis, where each model is solved completely isolated from the others, this is no longer possible in the mixed model. The multi-freedom constraint transformation matrices build this indirect coupling that requires solving a relative large linear system, typical of shell element models, but on another hand can still provide the model analysis of GBT. The next subsection presents an analysis of this point.

\subsubsection{Analysis of displacement field}

To evaluate the results obtained in the mixed model above, full shell element models are used as a control parameter. Following the same approach applied in Chapter 5, one develops the shell models in the commercial software ANSYS ${ }^{\circledR}$.

To avoid any miss-understating concerning a particular property and response of shell elements, such as shear or membrane lockings and approximations for plate's shear [5, 90], the four shell models used in the previous chapter are recreated using the geometric and loading conditions of this example.

All models have the same discretization, which has 100 elements in the cross-section, and 610 segments in the longitudinal direction. In total, each model has 61,000 elements, leading to 366,600 and 1,099,200 degrees of freedom respectively for the linear and quadratic element models.

Starting at the top cross-section, the two below figures plot the comparison among all models for the transversal displacement at this point. The modal displacement fields of GBT are plotted as well.

Here, the results of transversal displacements obtained from different full shell elements models are almost the same. The highest difference is $0.588 \%$, which occurs at angle $108^{\circ}$, between the lowest value of shell types $181 \mathrm{w}=-45.391 \mathrm{~mm}$ and the highest value 
Table 6.3: Shell elements' degrees of freedom, in $\mathrm{mm}$, on global coordinate system

\begin{tabular}{|c|c|c|c|c|c|c|c|}
\hline node & $u$ & $v$ & $w$ & node & $u$ & $v$ & $w$ \\
\hline 49 & $-7.54 \mathrm{E}-02$ & $2.17 \mathrm{E}-01$ & $-2.50 \mathrm{E}-14$ & 97 & $-1.54 \mathrm{E}-01$ & $1.99 \mathrm{E}-01$ & $-5.58 \mathrm{E}-14$ \\
\hline 50 & $-7.49 \mathrm{E}-02$ & $2.14 \mathrm{E}-01$ & $2.68 \mathrm{E}-02$ & 98 & $-1.53 \mathrm{E}-01$ & $1.97 \mathrm{E}-01$ & $2.21 \mathrm{E}-02$ \\
\hline 51 & $-7.34 \mathrm{E}-02$ & $2.05 \mathrm{E}-01$ & $5.21 \mathrm{E}-02$ & 99 & $-1.50 \mathrm{E}-01$ & $1.90 \mathrm{E}-01$ & 4.31E-02 \\
\hline 52 & $-7.09 \mathrm{E}-02$ & $1.90 \mathrm{E}-01$ & $7.46 \mathrm{E}-02$ & 100 & $-1.45 \mathrm{E}-01$ & $1.78 \mathrm{E}-01$ & $6.18 \mathrm{E}-02$ \\
\hline 53 & $-6.75 \mathrm{E}-02$ & $1.71 \mathrm{E}-01$ & $9.28 \mathrm{E}-02$ & 101 & $-1.38 \mathrm{E}-01$ & $1.63 \mathrm{E}-01$ & 7.72E-02 \\
\hline 54 & $-6.30 \mathrm{E}-02$ & $1.48 \mathrm{E}-01$ & $1.06 \mathrm{E}-01$ & 102 & $-1.29 \mathrm{E}-01$ & $1.45 \mathrm{E}-01$ & $8.84 \mathrm{E}-02$ \\
\hline 55 & $-5.76 \mathrm{E}-02$ & $1.23 \mathrm{E}-01$ & $1.13 \mathrm{E}-01$ & 103 & $-1.18 \mathrm{E}-01$ & $1.26 \mathrm{E}-01$ & $9.48 \mathrm{E}-02$ \\
\hline 56 & $-5.12 \mathrm{E}-02$ & $9.73 \mathrm{E}-02$ & $1.13 \mathrm{E}-01$ & 104 & $-1.05 \mathrm{E}-01$ & $1.05 \mathrm{E}-01$ & $9.58 \mathrm{E}-02$ \\
\hline 57 & $-4.39 \mathrm{E}-02$ & $7.23 \mathrm{E}-02$ & $1.06 \mathrm{E}-01$ & 105 & $-8.96 \mathrm{E}-02$ & $8.52 \mathrm{E}-02$ & $9.13 \mathrm{E}-02$ \\
\hline 58 & $-3.57 \mathrm{E}-02$ & $4.98 \mathrm{E}-02$ & $9.26 \mathrm{E}-02$ & 106 & $-7.29 \mathrm{E}-02$ & $6.72 \mathrm{E}-02$ & $8.14 \mathrm{E}-02$ \\
\hline 59 & $-2.67 \mathrm{E}-02$ & $3.14 \mathrm{E}-02$ & 7.32E-02 & 107 & $-5.45 \mathrm{E}-02$ & $5.24 \mathrm{E}-02$ & $6.64 \mathrm{E}-02$ \\
\hline 60 & $-1.70 \mathrm{E}-02$ & $1.85 \mathrm{E}-02$ & $4.90 \mathrm{E}-02$ & 108 & $-3.47 \mathrm{E}-02$ & $4.20 \mathrm{E}-02$ & $4.72 \mathrm{E}-02$ \\
\hline 61 & $-6.71 \mathrm{E}-03$ & $1.25 \mathrm{E}-02$ & 2.13E-02 & 109 & $-1.36 \mathrm{E}-02$ & $3.70 \mathrm{E}-02$ & $2.48 \mathrm{E}-02$ \\
\hline 62 & 4.07E-03 & $1.39 \mathrm{E}-02$ & $-8.15 \mathrm{E}-03$ & 110 & $8.34 \mathrm{E}-03$ & $3.83 \mathrm{E}-02$ & $6.90 \mathrm{E}-04$ \\
\hline 63 & $1.51 \mathrm{E}-02$ & $2.32 \mathrm{E}-02$ & $-3.73 \mathrm{E}-02$ & 111 & $3.09 \mathrm{E}-02$ & $4.60 \mathrm{E}-02$ & $-2.36 \mathrm{E}-02$ \\
\hline 64 & $2.62 \mathrm{E}-02$ & $4.01 \mathrm{E}-02$ & $-6.40 \mathrm{E}-02$ & 112 & $5.36 \mathrm{E}-02$ & $6.02 \mathrm{E}-02$ & $-4.63 \mathrm{E}-02$ \\
\hline 65 & $3.72 \mathrm{E}-02$ & $6.38 \mathrm{E}-02$ & $-8.62 \mathrm{E}-02$ & 113 & $7.59 \mathrm{E}-02$ & $8.02 \mathrm{E}-02$ & $-6.56 \mathrm{E}-02$ \\
\hline 66 & $4.77 \mathrm{E}-02$ & $9.28 \mathrm{E}-02$ & $-1.02 \mathrm{E}-01$ & 114 & $9.74 \mathrm{E}-02$ & $1.05 \mathrm{E}-01$ & $-7.99 \mathrm{E}-02$ \\
\hline 67 & $5.76 \mathrm{E}-02$ & $1.25 \mathrm{E}-01$ & $-1.10 \mathrm{E}-01$ & 115 & $1.18 \mathrm{E}-01$ & $1.33 \mathrm{E}-01$ & $-8.79 \mathrm{E}-02$ \\
\hline 68 & $6.65 \mathrm{E}-02$ & $1.59 \mathrm{E}-01$ & $-1.10 \mathrm{E}-01$ & 116 & $1.36 \mathrm{E}-01$ & $1.61 \mathrm{E}-01$ & $-8.87 \mathrm{E}-02$ \\
\hline 69 & $7.42 \mathrm{E}-02$ & $1.91 \mathrm{E}-01$ & $-1.01 \mathrm{E}-01$ & 117 & $1.51 \mathrm{E}-01$ & $1.89 \mathrm{E}-01$ & $-8.22 \mathrm{E}-02$ \\
\hline 70 & $8.04 \mathrm{E}-02$ & $2.19 \mathrm{E}-01$ & $-8.42 \mathrm{E}-02$ & 118 & $1.64 \mathrm{E}-01$ & 2.13E-01 & $-6.88 \mathrm{E}-02$ \\
\hline 71 & $8.50 \mathrm{E}-02$ & $2.41 \mathrm{E}-01$ & $-6.03 \mathrm{E}-02$ & 119 & $1.73 \mathrm{E}-01$ & $2.32 \mathrm{E}-01$ & $-4.95 \mathrm{E}-02$ \\
\hline 72 & $8.78 \mathrm{E}-02$ & $2.55 \mathrm{E}-01$ & $-3.14 \mathrm{E}-02$ & 120 & $1.79 \mathrm{E}-01$ & $2.44 \mathrm{E}-01$ & $-2.59 \mathrm{E}-02$ \\
\hline 73 & $8.88 \mathrm{E}-02$ & $2.59 \mathrm{E}-01$ & $1.07 \mathrm{E}-14$ & 121 & $1.81 \mathrm{E}-01$ & 2.49E-01 & $6.24 \mathrm{E}-14$ \\
\hline 74 & $8.78 \mathrm{E}-02$ & $2.55 \mathrm{E}-01$ & $3.14 \mathrm{E}-02$ & 122 & $1.79 \mathrm{E}-01$ & $2.44 \mathrm{E}-01$ & $2.59 \mathrm{E}-02$ \\
\hline 75 & $8.50 \mathrm{E}-02$ & $2.41 \mathrm{E}-01$ & 6.03E-02 & 123 & $1.73 \mathrm{E}-01$ & $2.32 \mathrm{E}-01$ & $4.95 \mathrm{E}-02$ \\
\hline 76 & $8.04 \mathrm{E}-02$ & $2.19 \mathrm{E}-01$ & $8.42 \mathrm{E}-02$ & 124 & $1.64 \mathrm{E}-01$ & $2.13 \mathrm{E}-01$ & $6.88 \mathrm{E}-02$ \\
\hline 77 & $7.42 \mathrm{E}-02$ & $1.91 \mathrm{E}-01$ & $1.01 \mathrm{E}-01$ & 125 & $1.51 \mathrm{E}-01$ & $1.89 \mathrm{E}-01$ & $8.22 \mathrm{E}-02$ \\
\hline 78 & $6.65 \mathrm{E}-02$ & $1.59 \mathrm{E}-01$ & $1.10 \mathrm{E}-01$ & 126 & $1.36 \mathrm{E}-01$ & $1.61 \mathrm{E}-01$ & 8.87E-02 \\
\hline 79 & $5.76 \mathrm{E}-02$ & $1.25 \mathrm{E}-01$ & $1.10 \mathrm{E}-01$ & 127 & $1.18 \mathrm{E}-01$ & $1.33 \mathrm{E}-01$ & 8.79E-02 \\
\hline 80 & $4.77 \mathrm{E}-02$ & $9.28 \mathrm{E}-02$ & $1.02 \mathrm{E}-01$ & 128 & $9.74 \mathrm{E}-02$ & $1.05 \mathrm{E}-01$ & 7.99E-02 \\
\hline 81 & $3.72 \mathrm{E}-02$ & $6.38 \mathrm{E}-02$ & 8.62E-02 & 129 & 7.59E-02 & $8.02 \mathrm{E}-02$ & $6.56 \mathrm{E}-02$ \\
\hline 82 & $2.62 \mathrm{E}-02$ & $4.01 \mathrm{E}-02$ & $6.40 \mathrm{E}-02$ & 130 & $5.36 \mathrm{E}-02$ & $6.02 \mathrm{E}-02$ & 4.63E-02 \\
\hline 83 & $1.51 \mathrm{E}-02$ & $2.32 \mathrm{E}-02$ & $3.73 \mathrm{E}-02$ & 131 & $3.09 \mathrm{E}-02$ & $4.60 \mathrm{E}-02$ & $2.36 \mathrm{E}-02$ \\
\hline 84 & $4.07 \mathrm{E}-03$ & $1.39 \mathrm{E}-02$ & $8.15 \mathrm{E}-03$ & 132 & $8.34 \mathrm{E}-03$ & $3.83 \mathrm{E}-02$ & $-6.90 \mathrm{E}-04$ \\
\hline 85 & $-6.71 \mathrm{E}-03$ & $1.25 \mathrm{E}-02$ & $-2.13 \mathrm{E}-02$ & 133 & $-1.36 \mathrm{E}-02$ & $3.70 \mathrm{E}-02$ & $-2.48 \mathrm{E}-02$ \\
\hline 86 & $-1.70 \mathrm{E}-02$ & $1.85 \mathrm{E}-02$ & $-4.90 \mathrm{E}-02$ & 134 & $-3.47 \mathrm{E}-02$ & $4.20 \mathrm{E}-02$ & $-4.72 \mathrm{E}-02$ \\
\hline 87 & $-2.67 \mathrm{E}-02$ & $3.14 \mathrm{E}-02$ & $-7.32 \mathrm{E}-02$ & 135 & $-5.45 \mathrm{E}-02$ & $5.24 \mathrm{E}-02$ & $-6.64 \mathrm{E}-02$ \\
\hline 88 & $-3.57 \mathrm{E}-02$ & $4.98 \mathrm{E}-02$ & $-9.26 \mathrm{E}-02$ & 136 & $-7.29 \mathrm{E}-02$ & $6.72 \mathrm{E}-02$ & $-8.14 \mathrm{E}-02$ \\
\hline 89 & $-4.39 \mathrm{E}-02$ & $7.23 \mathrm{E}-02$ & $-1.06 \mathrm{E}-01$ & 137 & $-8.96 \mathrm{E}-02$ & $8.52 \mathrm{E}-02$ & $-9.13 \mathrm{E}-02$ \\
\hline 90 & $-5.12 \mathrm{E}-02$ & $9.73 \mathrm{E}-02$ & $-1.13 \mathrm{E}-01$ & 138 & $-1.05 \mathrm{E}-01$ & $1.05 \mathrm{E}-01$ & $-9.58 \mathrm{E}-02$ \\
\hline 91 & $-5.76 \mathrm{E}-02$ & $1.23 \mathrm{E}-01$ & $-1.13 \mathrm{E}-01$ & 139 & $-1.18 \mathrm{E}-01$ & $1.26 \mathrm{E}-01$ & $-9.48 \mathrm{E}-02$ \\
\hline 92 & $-6.30 \mathrm{E}-02$ & $1.48 \mathrm{E}-01$ & $-1.06 \mathrm{E}-01$ & 140 & $-1.29 \mathrm{E}-01$ & $1.45 \mathrm{E}-01$ & $-8.84 \mathrm{E}-02$ \\
\hline 93 & $-6.75 \mathrm{E}-02$ & $1.71 \mathrm{E}-01$ & $-9.28 \mathrm{E}-02$ & 141 & $-1.38 \mathrm{E}-01$ & $1.63 \mathrm{E}-01$ & $-7.72 \mathrm{E}-02$ \\
\hline 94 & $-7.09 \mathrm{E}-02$ & $1.90 \mathrm{E}-01$ & $-7.46 \mathrm{E}-02$ & 142 & $-1.45 \mathrm{E}-01$ & $1.78 \mathrm{E}-01$ & $-6.18 \mathrm{E}-02$ \\
\hline 95 & $-7.34 \mathrm{E}-02$ & $2.05 \mathrm{E}-01$ & $-5.21 \mathrm{E}-02$ & 143 & $-1.50 \mathrm{E}-01$ & $1.90 \mathrm{E}-01$ & $-4.31 \mathrm{E}-02$ \\
\hline 96 & $-7.49 \mathrm{E}-02$ & $2.14 \mathrm{E}-01$ & $-2.68 \mathrm{E}-02$ & 144 & $-1.53 \mathrm{E}-01$ & $1.97 \mathrm{E}-01$ & $-2.21 \mathrm{E}-02$ \\
\hline
\end{tabular}




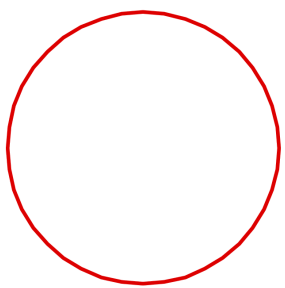

axial mode

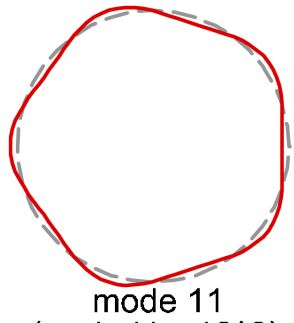

(scaled by $10^{\wedge} 3$ )
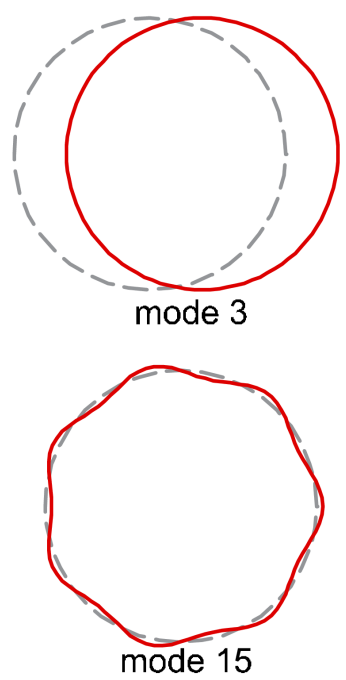

(scaled by $10^{\wedge} 4$ )
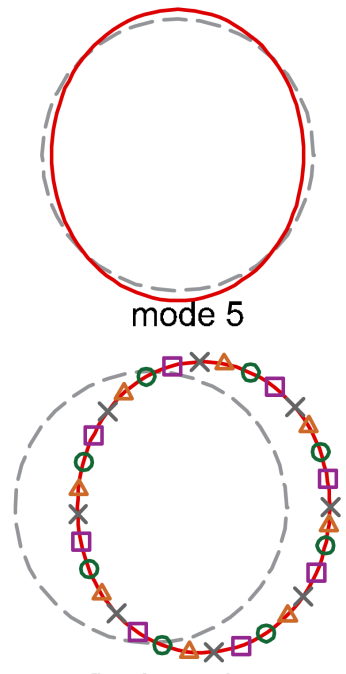

final result

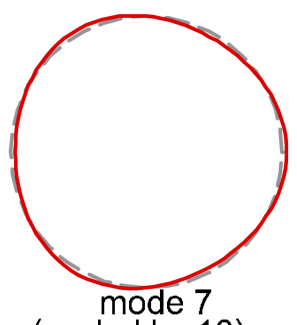

(scaled by 10)

cross-section middle wall

$=$ GBT solution

$x \times x$ shell 63

$\Delta \Delta \Delta$ shell 93

마 shell 181

৩o shell 281

Figure 6.11: Results of top cross-section: transversal displacement of mixed shell-GBT element model. The sum of all modal deformation factors, at the top point, leads to the GBT solution. The solutions achieved from the full shell element models are also presented.

Table 6.4: Mean differences, in \%, and standard deviation (SD) of displacement field between GBT and shell element models at the top point

\begin{tabular}{ccccccc}
\hline element & \multicolumn{2}{c}{ u } & \multicolumn{2}{c}{ v } & \multicolumn{2}{c}{ w } \\
& diff. & SD & diff. & SD & diff. & SD \\
\hline Shell 63 & $0.16 \%$ & $0.34 \%$ & $0.7 \%$ & $0.12 \%$ & $0.6 \%$ & $0.3 \%$ \\
Shell 93 & $0.08 \%$ & $0.24 \%$ & $0.62 \%$ & $0.07 \%$ & $0.55 \%$ & $0.18 \%$ \\
Shell 181 & $0.17 \%$ & $0.17 \%$ & $0.93 \%$ & $0.94 \%$ & $0.84 \%$ & $0.86 \%$ \\
Shell 281 & $0.62 \%$ & $0.07 \%$ & $0.54 \%$ & $0.19 \%$ & $0 \%$ & $0 \%$ \\
\hline
\end{tabular}

of shell type $93 \mathrm{w}=-45.126 \mathrm{~mm}$. Also, the difference between GBT and shell elements is almost imperceptibly; for this angle, it is $\mathrm{w}=-44.633 \mathrm{~mm}$. A difference of $1.10 \%$ above of shell element type 93 . Moreover, these difference are also found in the comparison between the pure GBT and shell models in Chapter 5, which indicates no perturbation in the results due to the coupling process developed here.

The diagrams of longitudinal displacement is plotted below as well. Similar to transversal displacements, the differences among the models are almost imperceptibly. The highest difference is between GBT and Shell element type 181: $-1.257 \%$ at angle $\boldsymbol{\theta}=90^{\circ}$.

Since the Poisson's effect plays an important role in the displacement field, especially where the longitudinal stress is high, it is interesting to compare the results obtained near the base of the structure. Here, one selects the coupling cross-section to explore 

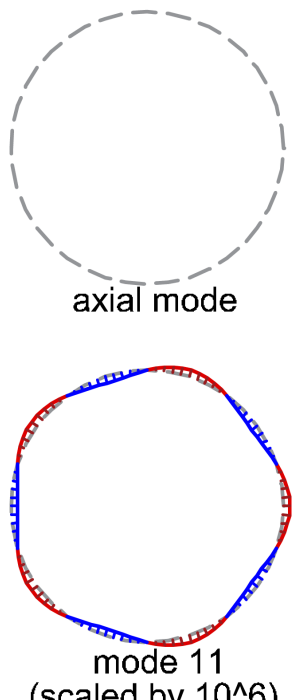

(scaled by $10^{\wedge} 6$ )

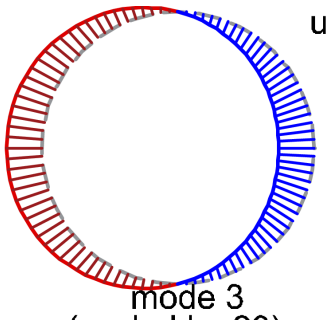

(scaled by 20)

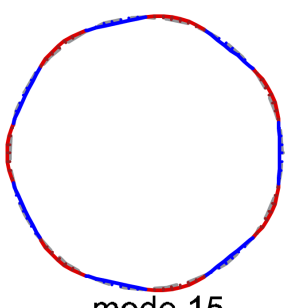

(scaled by $10^{\wedge} 7$ )

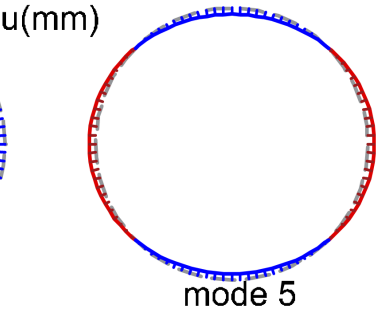

(scaled by $10^{\wedge} 2$ )

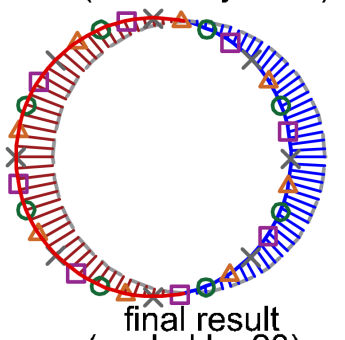

(scaled by 20 )

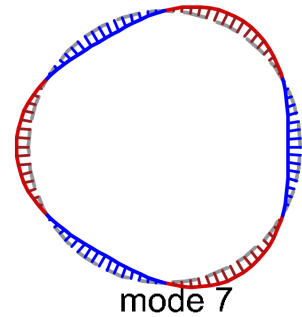

(scaled by $10^{\wedge} 4$ )

cross-section

middle wall

$=$ GBT solution

$\mathrm{XXX}$ shell 63

$\triangle \Delta \Delta$ shell 93

마 shell 181

๑॰ shell 281

Figure 6.12: Comparison of results among mixed shell-GBT and fully shell models at top cross-section: longitudinal displacement.

the Poisson's effect in mixed GBT-shell models. Figures 6.13 and 6.14 present the transversal and longitudinal displacement, respectively:

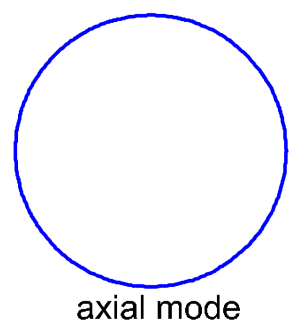

axial mode

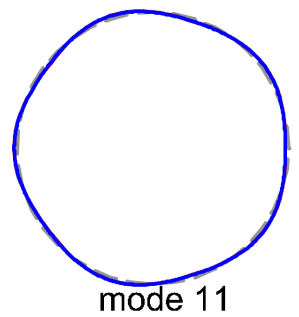

(scaled by $10^{\wedge} 5$ )

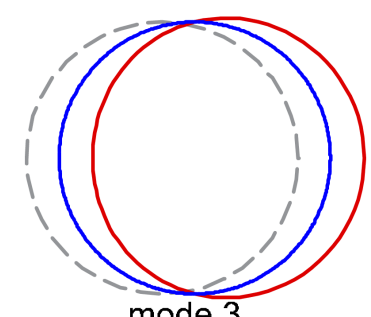

mode 3

(scaled by $10^{\wedge} 3$ )

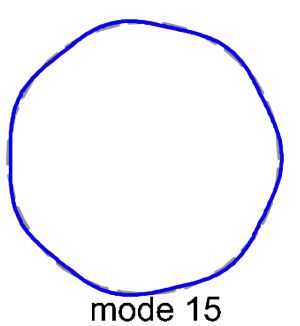

(scaled by $10^{\wedge} 6$ )

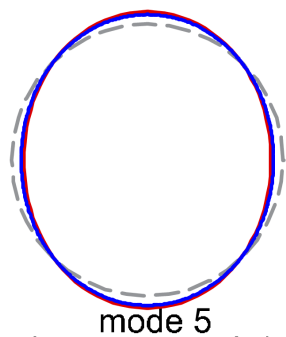

(scaled by 10^3)

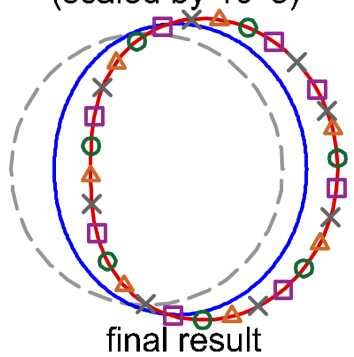

(scaled by $10^{\wedge} 3$ )

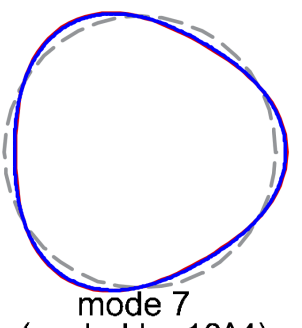

(scaled by $10^{\wedge} 4$ ) cross-section

- middle wall

_ GBT with

Poisson's effect

GBT without

Poisson's effect

$x \times x$ shell 63

$\Delta \Delta \Delta$ shell 93

마 shell 181

॰ shell 281

Figure 6.13: Comparison of results among mixed shell-GBT and fully shell models at the coupling cross-section: transversal displacement.

The differences among the shell models are indistinguishable and the highest difference as obtained between mixed GBT-shell and shell 63 models: $0.73 \%$, at angle $\theta=108^{\circ}$. 
Table 6.5 presents the detailed outcomes of radial and longitudinal displacements from these different models:

Table 6.5: Mean differences, in \%, and standard deviation (SD) of displacement field between GBT and shell elementsk models at the point $x=1 \mathrm{~m}$

\begin{tabular}{ccccccccc}
\hline element & \multicolumn{2}{c}{$\mathrm{u}$} & \multicolumn{2}{c}{$\mathrm{v}$} & \multicolumn{2}{c}{ w w/o Poisson } & \multicolumn{2}{c}{ w with Poisson } \\
& diff. & SD & diff. & SD & diff. & SD & diff. & SD \\
\hline Shell 63 & $0.57 \%$ & $1 \%$ & $0.14 \%$ & $0.11 \%$ & $85.47 \%$ & $29.03 \%$ & $0.07 \%$ & $0.21 \%$ \\
Shell 93 & $0.56 \%$ & $0.92 \%$ & $0.25 \%$ & $0.04 \%$ & $85.53 \%$ & $28.9 \%$ & $0.1 \%$ & $0.1 \%$ \\
Shell 181 & $0.65 \%$ & $1.09 \%$ & $0.23 \%$ & $0.13 \%$ & $85.58 \%$ & $29.09 \%$ & $0.14 \%$ & $0.25 \%$ \\
Shell 281 & $0.56 \%$ & $0.93 \%$ & $0.25 \%$ & $0.04 \%$ & $85.52 \%$ & $28.9 \%$ & $0.1 \%$ & $0.1 \%$ \\
\hline
\end{tabular}

There is one detail in figure 6.13 that is related to the observation of shear deformation in figure 5.16. The figure of this chapter shows that GBT reaches almost the same result of shell elements model; the difference is around $0.12 \%$. On the other hand, the figure in Chapter 5 presents a difference around 2.0\%. This increase in the quality of results of GBT is not an outcome from itself, but from the capacity of membranes element, at the structure's both, to handle shear deformations.
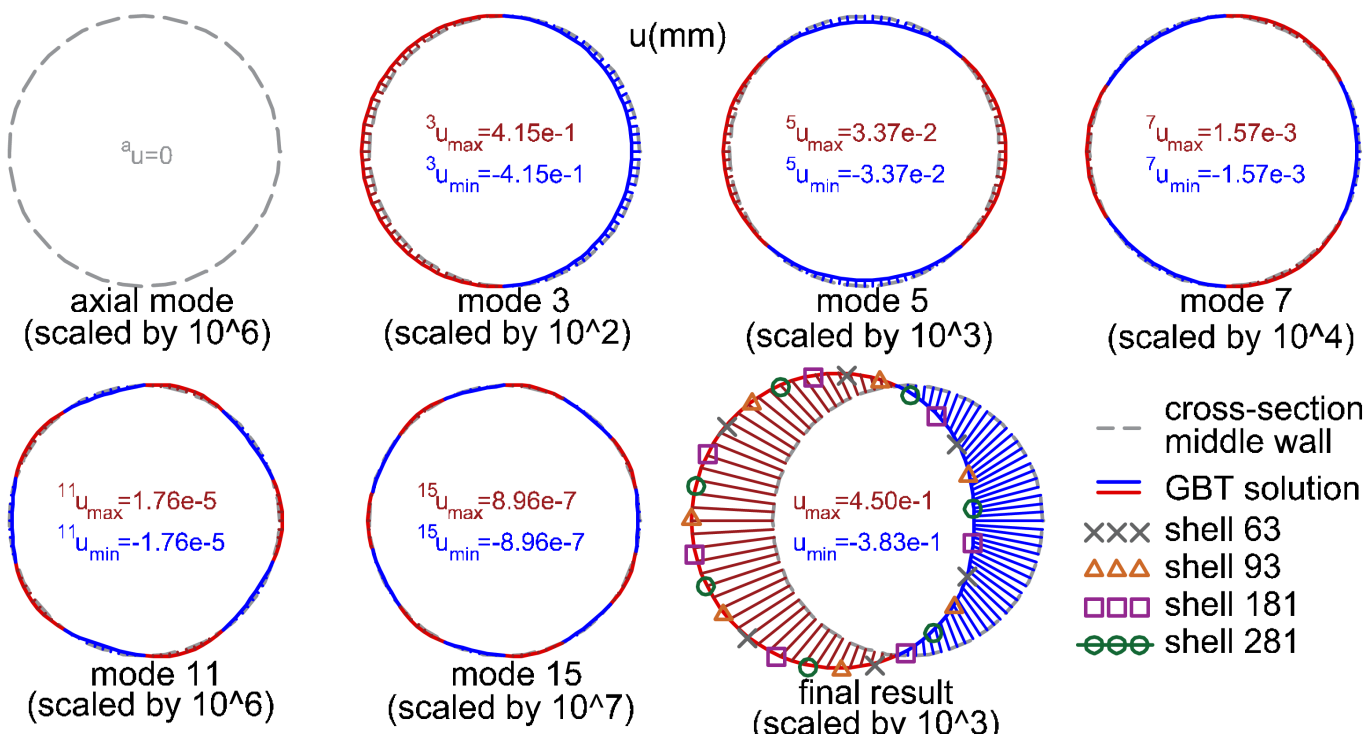

cross-section

-- middle wall

$=$ GBT solution

XXX shell 63

$\Delta \Delta \Delta$ shell 93

प्वप shell 181

๑॰ shell 281

Figure 6.14: Comparison of results among mixed shell-GBT and fully shell models at the coupling cross-section: longitudinal displacement.

Lastly, the comparison of longitudinal displacement at the coupling cross-section shows no course results in mixed GBT-Shell model to the other ones. The highest difference is $1.1 \%$, at angle $\theta=108^{\circ}$ between GBT and the shell models with elements with linear interpolation function, Shell 63 and Shell 181. 


\subsubsection{Analysis of stress field}

The comparisons among the mixed GBT-shell and the full-shell element models are placed at the longitudinal position of $x=525 \mathrm{~mm}$. This point is in the GBT domain in the mixed model and at the center of the first elements after the coupling cross-section in the full-shell element models. Following the same sequence of Chapter 5 , the analysis of stress field starts in internal bending moments:

Table 6.6: Longitudinal bending moment, $M_{x}$, at $x=525 \mathrm{~mm}$ : mean differences (in the table's upper-right side) and their standard deviation (in the table's lower-left side) among all models

\begin{tabular}{cccccc}
\hline$M_{x}$ & GBT & Shell-63 & Shell-93 & Shell-181 & Shell-281 \\
\hline GBT & - & $-9.22 \%$ & $-0.59 \%$ & $-9.45 \%$ & $79.57 \%$ \\
Shell 63 & $2.09 \%$ & - & $-8.57 \%$ & $0.25 \%$ & $95.65 \%$ \\
Shell 93 & $4.64 \%$ & $2.3 \%$ & - & $-8.8 \%$ & $77.55 \%$ \\
Shell 181 & $2.07 \%$ & $0.02 \%$ & $2.31 \%$ & - & $95.38 \%$ \\
Shell 281 & $19.48 \%$ & $21.33 \%$ & $22.18 \%$ & $28.31 \%$ & - \\
\hline
\end{tabular}
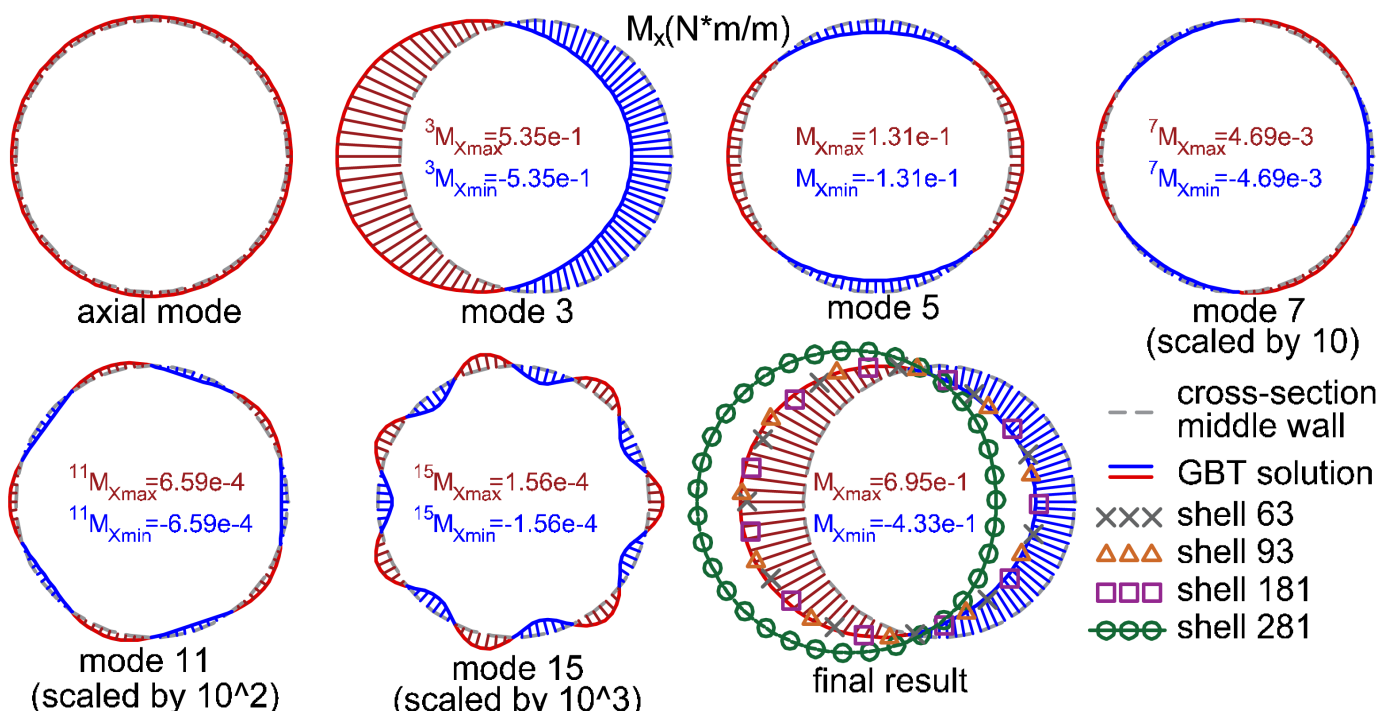

$=$ GBT solution

$x \times x$ shell 63

$\Delta \Delta \Delta$ shell 93

प्रव shell 181

๑॰ shell 281

Figure 6.15: Comparison of results among mixed shell-GBT and fully shell models at point $x=525 \mathrm{~mm}$ : longitudinal bending moment, $M_{x}$

Similar to table 5.7, in Chapter 5, GBT approaches to the solution of Shell-93. In general, there is an increase in the mean difference among GBT and all shell models. However, the standard deviation decreases, especially concerning the models with Shell63 and Shell-181. Also, the particular results of model Shell-281 are kept.

Although shell-93 gives the closest result to GBT in terms of the longitudinal bending moment, it provides the farthest one concerning the transversal bending moment. Ta- 
ble 6.7 presents similar results to Table 5.8, i.e., there is no significant interference due to the coupling approach proposed here.

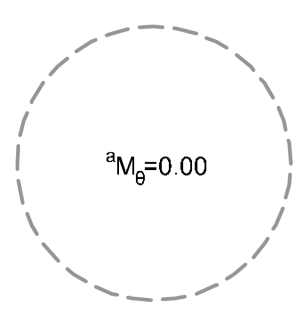

axial mode

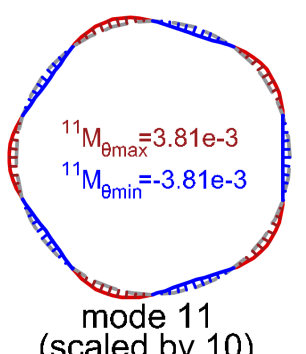

(scaled by 10)

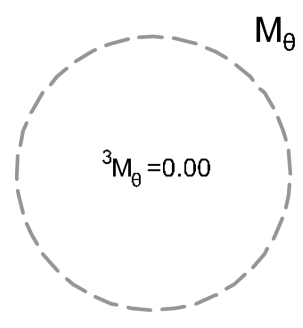

mode 3

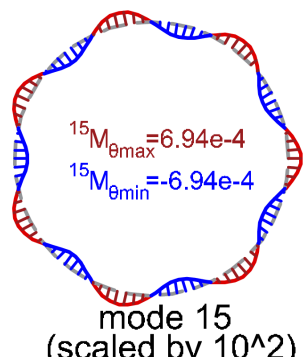

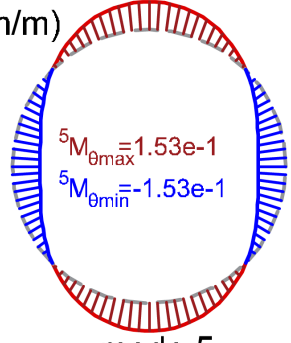

mode 5

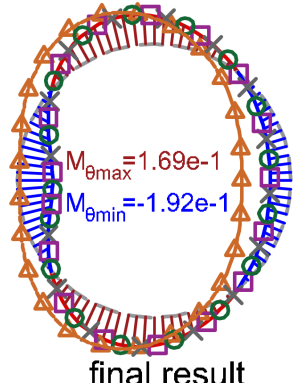

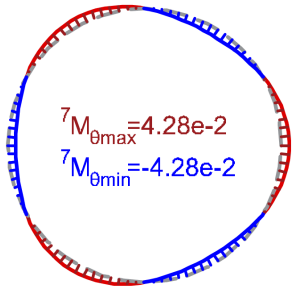

mode 7

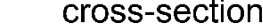

middle wall

$=$ GBT solution

$x \times x$ shell 63

$\triangle \Delta \Delta$ shell 93

마 shell 181

- shell 281

Figure 6.16: Comparison of results among mixed shell-GBT and fully shell models at point $x=525 \mathrm{~mm}$ : transversal bending moment, $M_{\theta}$.

Table 6.7: Transversal bending moment, $M_{\theta}$, at $x=525 \mathrm{~mm}$ : mean differences (in the table's upper-right side) and their standard deviation (in the table's lower-left side) among all models

\begin{tabular}{cccccc}
\hline$M_{\theta}$ & GBT & Shell-63 & Shell-93 & Shell-181 & Shell-281 \\
\hline GBT & - & $0.52 \%$ & $41.17 \%$ & $0.59 \%$ & $0.61 \%$ \\
Shell 63 & $0.48 \%$ & - & $-77.16 \%$ & $-0.06 \%$ & $0.09 \%$ \\
Shell 93 & $192.69 \%$ & $129.06 \%$ & - & $-77.22 \%$ & $-77.04 \%$ \\
Shell 181 & $0.89 \%$ & $0.49 \%$ & $131.32 \%$ & - & $0.03 \%$ \\
Shell 281 & $0.29 \%$ & $0.3 \%$ & $128.97 \%$ & $0.78 \%$ & - \\
\hline
\end{tabular}

Concerning the twist bending moment, $M_{\theta x}$, the same behavior from Chapter 5 is observed: GBT's results diverge from the Shell-93 ones and converges to the other models. Moreover, as well as the longitudinal bending moment, there is a increasing in the mean difference among GBT and all shell models, simultaneously with a decrease in standard deviation.

Since the cross-section analysis of this example is closer to the support conditions than in the example of Chapter 5, it is not a surprise that the divergence among the outcome's shear forces is higher than the previous chapter.

Table 6.9 confirms the analysis of Chapter 5: the longitudinal shear force of Shell-281 

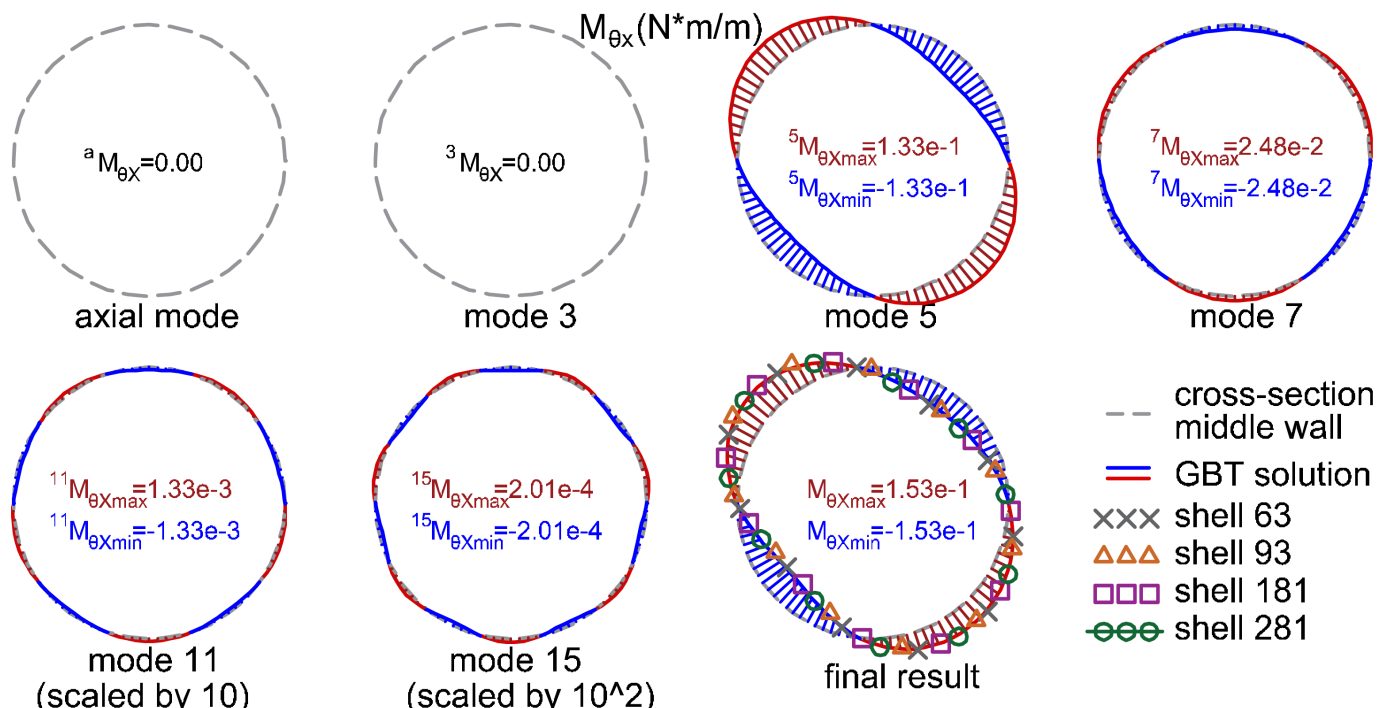

$x \times x$ shell 63

$\Delta \Delta \Delta$ shell 93

ㅁㅁ shell 181

- shell 281

Figure 6.17: Comparison of results among mixed shell-GBT and fully shell models at point $x=525 \mathrm{~mm}$ : twist moment, $M_{\theta x}$.

Table 6.8: Twist bending moment, $M_{\theta x}$, at $x=525 \mathrm{~mm}$ : mean differences (in the table's upper-right side) and their standard deviation (in table's lower-left side) among all models

\begin{tabular}{cccccc}
\hline$M_{\theta x}$ & GBT & Shell-63 & Shell-93 & Shell-181 & Shell-281 \\
\hline GBT & - & $6.7 \%$ & $6.77 \%$ & $6.08 \%$ & $7 \%$ \\
Shell 63 & $5.1 \%$ & - & $3.67 \%$ & $1.07 \%$ & $0.22 \%$ \\
Shell 93 & $18.41 \%$ & $19.34 \%$ & - & $1.1 \%$ & $3.97 \%$ \\
Shell 181 & $6.05 \%$ & $8.2 \%$ & $11 \%$ & - & $1.27 \%$ \\
Shell 281 & $6.35 \%$ & $1.11 \%$ & $20.39 \%$ & $9.12 \%$ & - \\
\hline
\end{tabular}

is the closest solution from GBT and Shell-181 has the worst results' agreement with GBT.

Table 6.9: Longitudinal plate's shear, $Q_{x}$, at $x=525 \mathrm{~mm}$ : mean differences (in the table's upper-right side) and their standard deviation (in the table's lower-left side) among all models

\begin{tabular}{ccccc}
\hline$Q_{x}$ & GBT & Shell-93 & Shell-181 & Shell-281 \\
\hline GBT & - & $7.29 \%$ & $55.43 \%$ & $7.21 \%$ \\
Shell 93 & $17.05 \%$ & - & $-22 \%$ & $1.34 \%$ \\
Shell 181 & $401.58 \%$ & $544 \%$ & - & $-69.95 \%$ \\
Shell 281 & $8.59 \%$ & $10.1 \%$ & $70.59 \%$ & - \\
\hline
\end{tabular}

However, the transversal shear force, $Q_{\theta}$, has the opposite behavior. GBT gets closer 

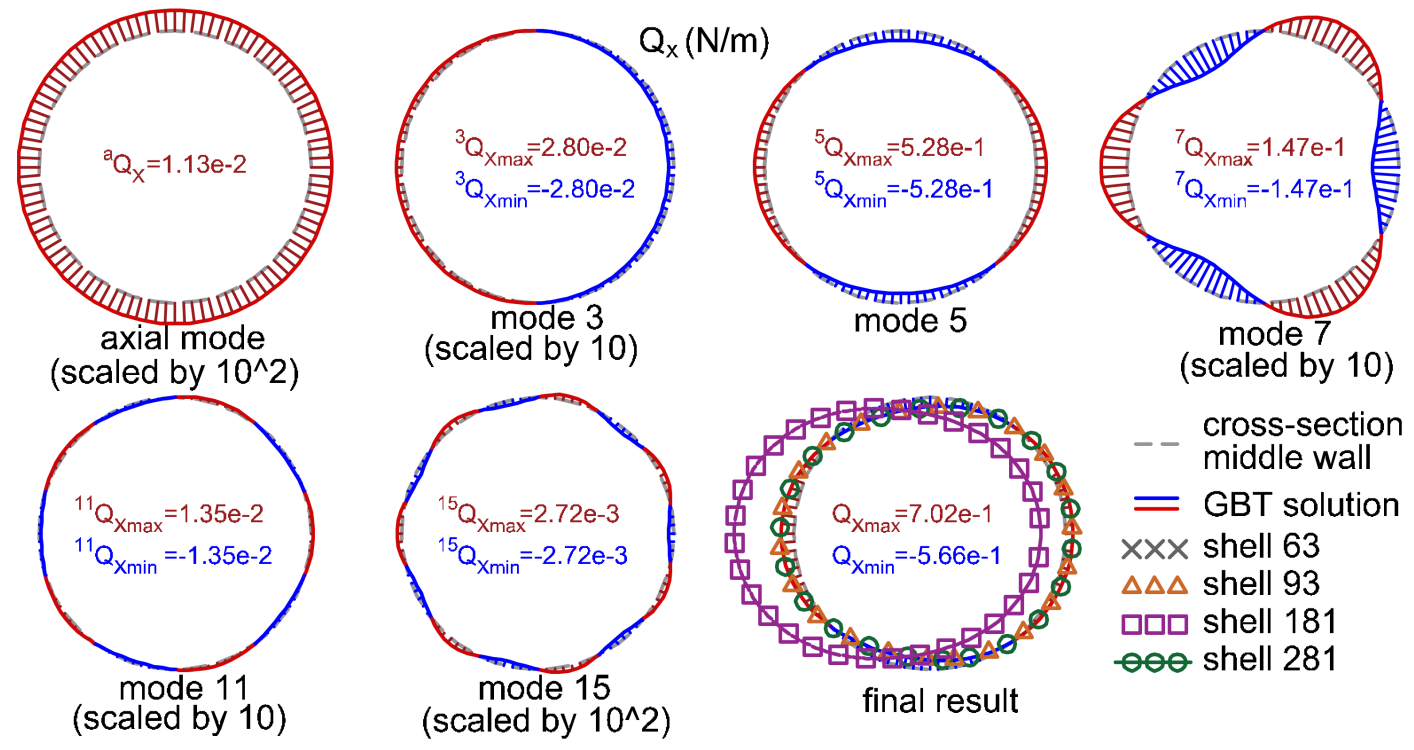

Figure 6.18: Comparison of results among mixed shell-GBT and fully shell models at point $x=525 \mathrm{~mm}$ : longitudinal shear force, $Q_{x}$.
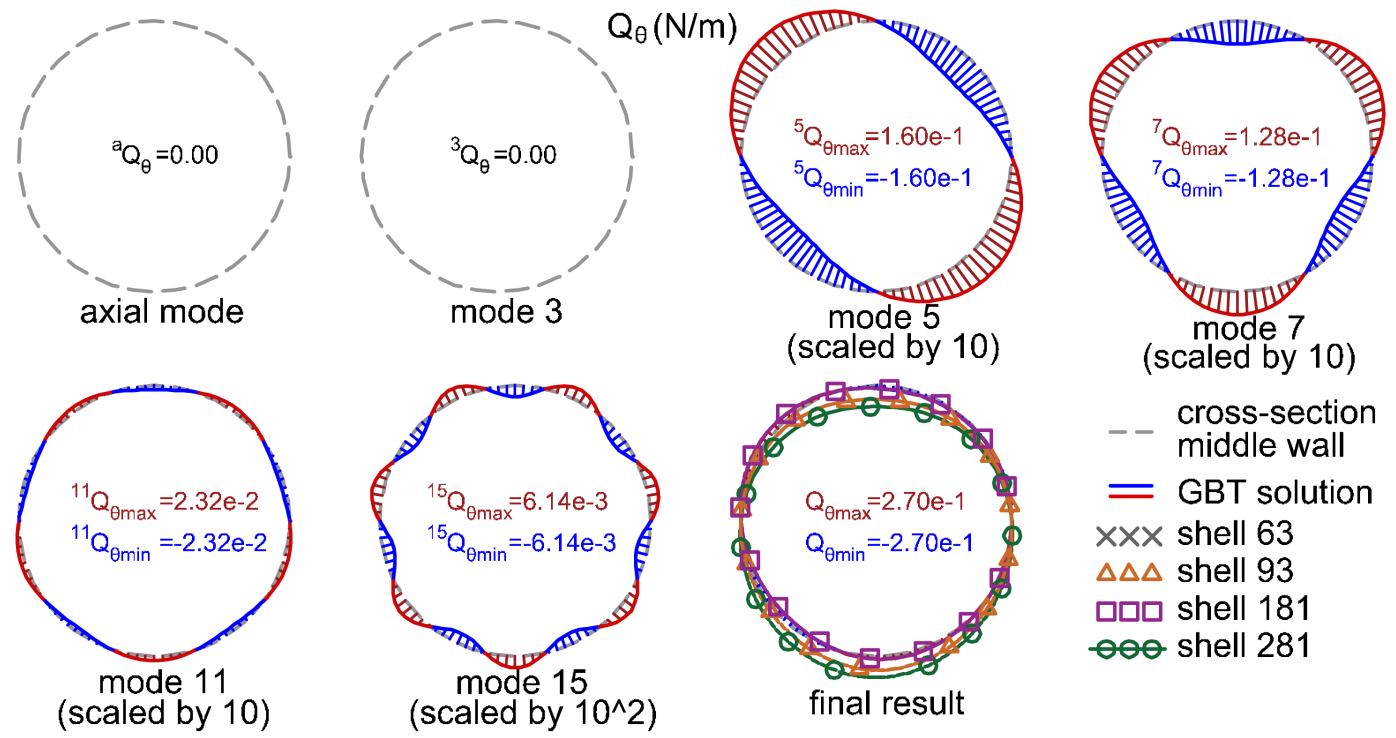

$=$ GBT solution

$x \times x$ shell 63

$\triangle \Delta \Delta$ shell 93

마 shell 181

oo shell 281

Figure 6.19: Comparison of results among mixed shell-GBT and fully shell models at point $x=525 \mathrm{~mm}$ : transversal shear force, $Q_{\theta}$.

to the solution of Shell-181 model and deviates from Shell-93 and Shell-281 models, as presented in figure 6.19 and table 6.10. Furthermore, one can observe that the diagram of solution from Shell-93 and Shell-281 is almost a shift from the diagram of GBT or Shell-181, which indicates a lack of shear force concerning mode 3 .

Concerning the internal membrane forces, the coupling approach introduces no pertur- 
Table 6.10: Transversal plate's shear, $Q_{\theta}$, at $x=525 \mathrm{~mm}$ : mean differences (in the table's upper-right side) and their standard deviation (in the table's lower-left side) among all models

\begin{tabular}{ccccc}
\hline$Q_{\theta}$ & GBT & Shell-93 & Shell-181 & Shell-281 \\
\hline GBT & - & $73.27 \%$ & $1.91 \%$ & $23.34 \%$ \\
Shell 93 & $362.26 \%$ & - & $-151.6 \%$ & $87.48 \%$ \\
Shell 181 & $6.67 \%$ & $87.84 \%$ & - & $247.31 \%$ \\
Shell 281 & $496.43 \%$ & $51.87 \%$ & $852.02 \%$ & - \\
\hline
\end{tabular}

bation in the results of longitudinal and shear forces. Tables 6.11 and 6.12 present almost the same values of the respective tables in Chapter 5: 5.12 and 5.14.
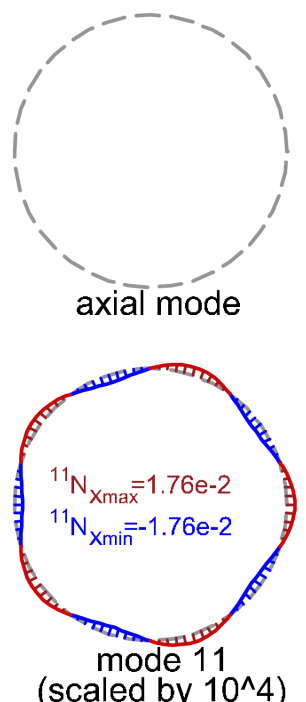

(scaled by $10^{\wedge} 4$ )
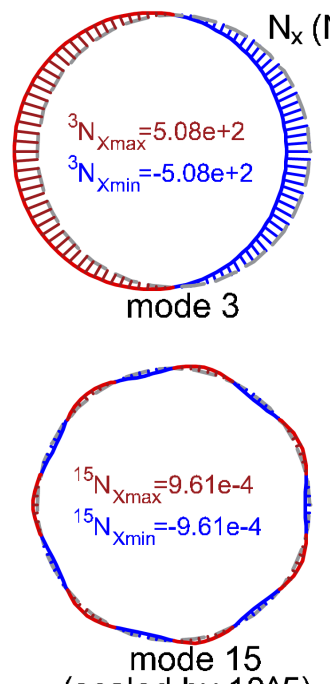

(scaled by $10^{\wedge} 5$ )
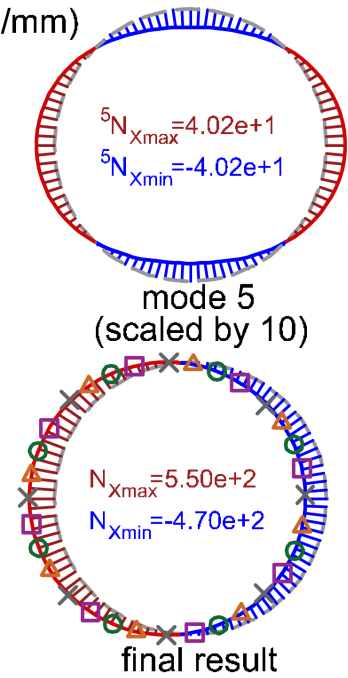

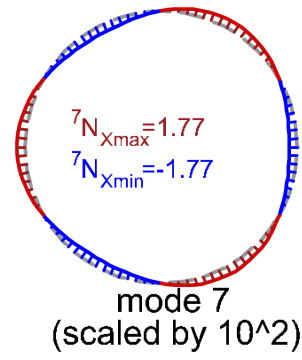

cross-section middle wall

$=$ GBT solution

$x \times x$ shell 63

$\Delta \Delta \Delta$ shell 93

ㅁㅁ shell 181

๑) shell 281

Figure 6.20: Comparison of results among mixed shell-GBT and fully shell models at point $x=525 \mathrm{~mm}$ : longitudinal membrane force, $N_{x}$.

Table 6.11: Longitudinal membrane force, $N_{x}$, at $x=525 \mathrm{~mm}$ : mean differences (in the table's upper-right side) and their standard deviation (in the table's lower-left side) among all models.

\begin{tabular}{cccccc}
\hline$N_{x}$ & GBT & Shell-63 & Shell-93 & Shell-181 & Shell-281 \\
\hline GBT & - & $0.02 \%$ & $-0.03 \%$ & $0.05 \%$ & $-0.03 \%$ \\
Shell 63 & $0.17 \%$ & - & $0.05 \%$ & $-0.03 \%$ & $-0.05 \%$ \\
Shell 93 & $0.13 \%$ & $0.04 \%$ & - & $0.08 \%$ & $0.01 \%$ \\
Shell 181 & $0.19 \%$ & $0.02 \%$ & $0.06 \%$ & - & $-0.08 \%$ \\
Shell 281 & $0.13 \%$ & $0.04 \%$ & $0 \%$ & $0.06 \%$ & - \\
\hline
\end{tabular}




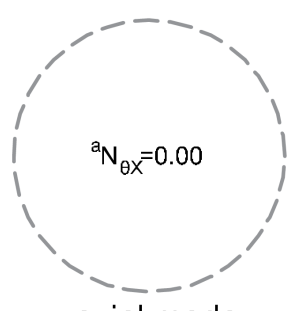

axial mode

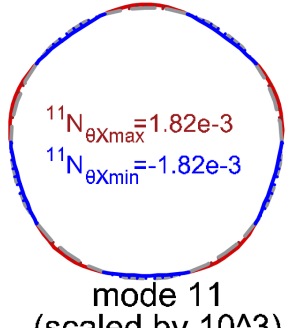

(scaled by $10^{\wedge} 3$ )
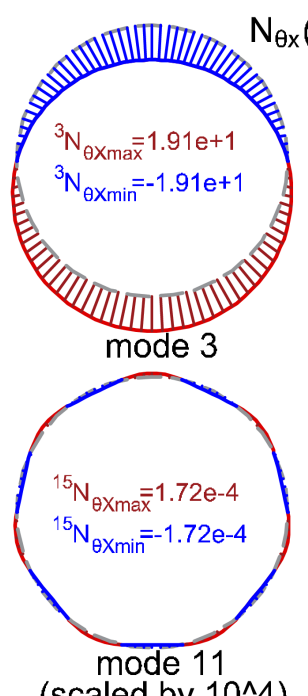

(scaled by 10^4)

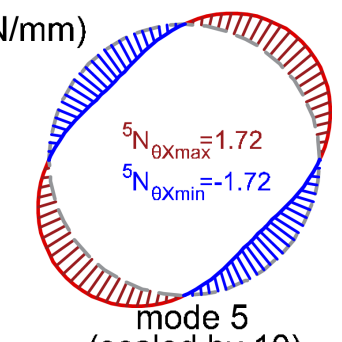

(scaled by 10)

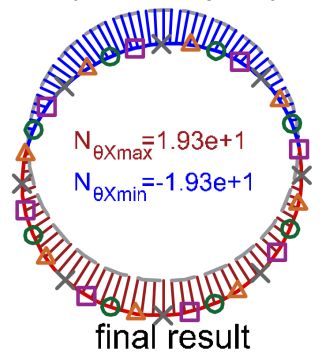

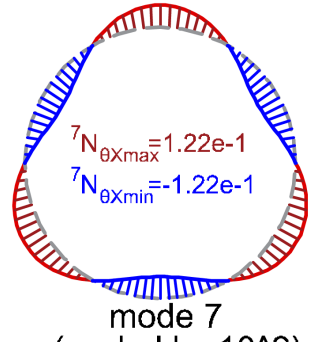

(scaled by $10^{\wedge} 2$ )

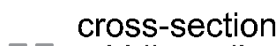

- middle wall

$=$ GBT solution

$x \times x$ shell 63

$\Delta \Delta \Delta$ shell 93

ㅁप shell 181

•

Figure 6.21: Comparison of results among mixed shell-GBT and fully shell models at point $x=525 \mathrm{~mm}$ : shear membrane force, $N_{\theta x}$.

Table 6.12: Shear membrane force, $N_{\theta x}$, at $x=525 \mathrm{~mm}$ : mean differences (in the table's upper-right side) and their standard deviation (in the table's lower-left side) among all models.

\begin{tabular}{cccccc}
\hline$N_{\theta x}$ & GBT & Shell-63 & Shell-93 & Shell-181 & Shell-281 \\
\hline GBT & - & $0.02 \%$ & $-0.01 \%$ & $0.02 \%$ & $-0.01 \%$ \\
Shell 63 & $0.15 \%$ & - & $0.03 \%$ & $0.01 \%$ & $-0.03 \%$ \\
Shell 93 & $0.12 \%$ & $0.03 \%$ & - & $0.03 \%$ & $0 \%$ \\
Shell 181 & $0.17 \%$ & $0.02 \%$ & $0.05 \%$ & - & $-0.03 \%$ \\
Shell 281 & $0.13 \%$ & $0.02 \%$ & $0.01 \%$ & $0.04 \%$ & - \\
\hline
\end{tabular}

However, the approximation of the cross-section analysis to the support conditions is reflected in the membrane's result in the transversal direction, $N_{\theta}$ : Shell-181 presents higher results than any other model. Moreover, there is no consensus among all models on the values of this internal force. Nevertheless, one can note that the values presented in figure 6.22 are really small compared to the values of the other membrane forces given in figures 6.20 and 6.21 .

A general evaluation of stress fields shows that the proposed formulation of coupling between GBT and shell elements has imperceptible differences for the internal forces $N_{x}$ and $N_{\theta x}$. The same evaluation can be built for the plate's bending moments $M_{x}$ and $M_{\theta}$. However, for these internal forces, one model of full shell elements leads to divergent results. The GBT results are approximately the same as the other models' results.

The mean difference and its standard deviation of twist bending moment, $M_{\theta x}$, have al- 

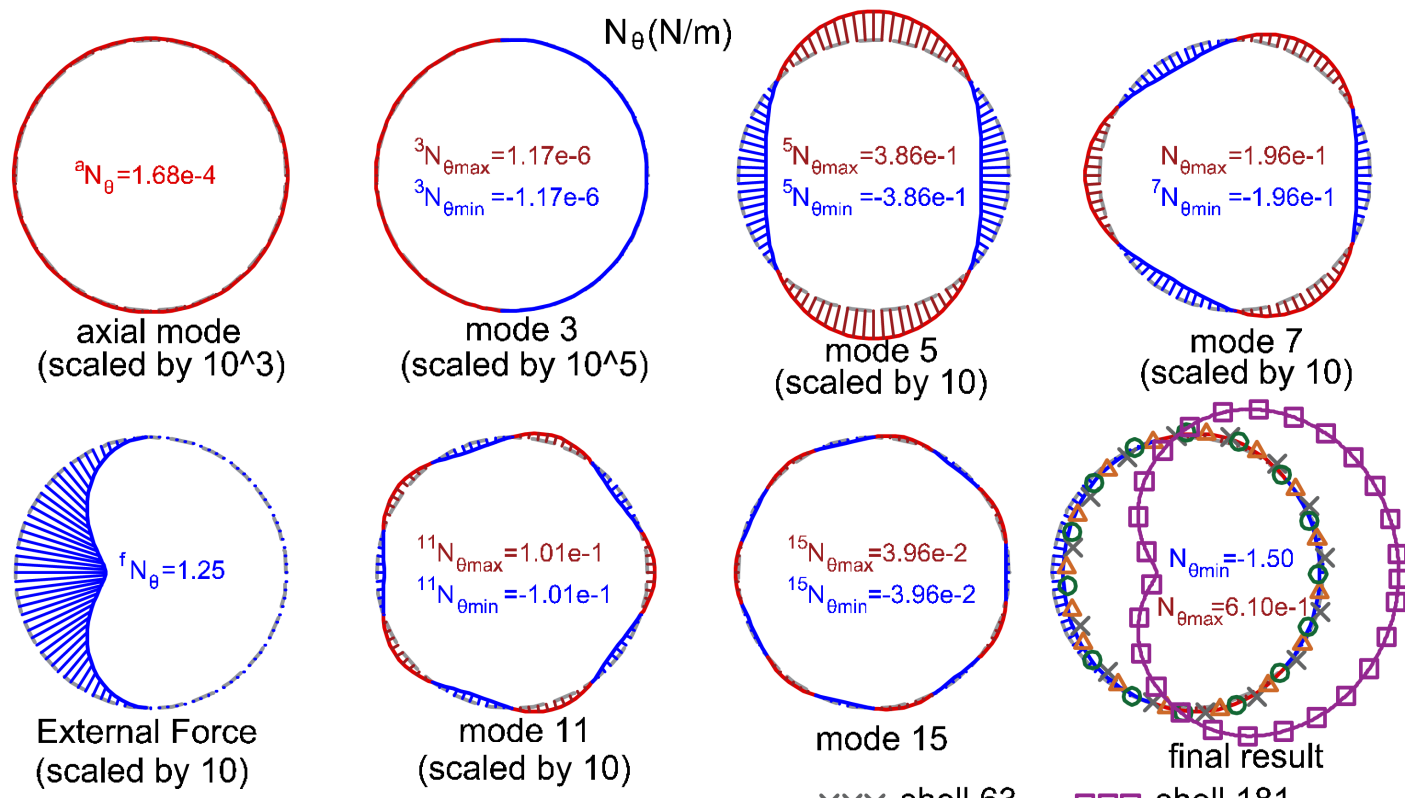

- cross-section middle wall $=$ GBT solution

Figure 6.22: Comparison of results among mixed shell-GBT and fully shell models at point $x=525 \mathrm{~mm}$ : transversal membrane force, $N_{\theta}$

Table 6.13: Membrane tangential force, $N_{\theta}$, at $x=525 \mathrm{~mm}$ : mean differences (in the table's upper-right side) and their standard deviation (in the table's lower-left side) among all models

\begin{tabular}{cccccc}
\hline$N_{\theta}$ & GBT & Shell-63 & Shell-93 & Shell-181 & Shell-281 \\
\hline GBT & - & $39.35 \%$ & $22.54 \%$ & $862.3 \%$ & $35.73 \%$ \\
Shell 63 & $137.35 \%$ & - & $14.87 \%$ & $-82.61 \%$ & $-82.2 \%$ \\
Shell 93 & $33.48 \%$ & $115.82 \%$ & - & $320.36 \%$ & $26.09 \%$ \\
Shell 181 & $3.25 \%$ & $0.87 \%$ & $2041.77 \%$ & - & $-86.47 \%$ \\
Shell 281 & $36.05 \%$ & $110.91 \%$ & $64.73 \%$ & $0.79 \%$ & - \\
\hline
\end{tabular}

most no perturbation when compared to results obtained in the evaluation of full GBT, developed in Chapter 5. The longitudinal shear force, $Q_{x}$, has a similar performance, but in this case, it has an increase of the mean difference. The reason for this increase is the position of the cross-section under analysis, which is closer to the support than the analyzed cross-section of the example presented in the previous chapter.

The transversal shear force and the transversal membrane force, $Q_{\theta}$ and $N_{\theta}$, respectively, cannot be concluded based on the perturbation of the coupling procedure, as all models have no consensus on the final results. Nevertheless, these internal forces are much lower than any other internal force. In fact, they have residual participation in the total stress field. 


\section{Non-linear analysis of mixed Shell-GBT models}

This chapter presents the introduction to non-linear geometrical analysis in GBT. After a brief historical review, it will bring the main concepts in non-linear GBT. The departure from linear analysis leads directly to the coupling among deformation modes. I.e., in non-linear analysis, each mode cannot be solved from the others independently. However, the uncoupling modes remain relevant to the linear analysis due to the fact that i) they still have a clear kinematic meaning; ii) they will lead to the Tensors of Coupling, which indicates how a coupler mode creates a link between two coupled modes. Since the geometric properties of the cross-section are the only source of these tensors, they have a unique potential of predicting coupling instability in the initial stages of the structural design.

Also, this chapter develops different types of non-linear stiffness matrices, as well as the consistent internal forces of GBT in the non-linear analysis. These matrices and internal forces, based on the shape functions developed in Chapter 5, have the benefits of using high derivatives of hyperbolic-trigonometric functions to describe any stress field in GBT's high modes. Moreover, they have a recursive property, useful in the development of quadratic terms in non-linear analysis. I.e., due to the similarity among shape functions and their derivatives, a stiffness matrix of a particular shape function can be adjusted and re-used in other terms of the non-linear analysis.

Here, the approach of splitting the stiffness matrix into two parts - the kernel and the coefficient completeness matrices - shows another benefit: a systematic technique of developing and applying the initial stress and displacement stiffness matrices.

The numerical examples of this chapter highlight not only the application of non-linear analysis in the mixed Shell-GBT models, but also the limitation from assumptions of no shear and transversal membrane strains energy of GBT to handle non-linear effects. Full shell element models, based on commercial packages, cross-check the results of these examples. 


\subsection{Introduction - Brief historical development of non-linear GBT}

Non-linear analysis in GBT have been proving to be an alternative and powerful tool in describing and modeling complex problems in thin-walled beams. The first non-linear analysis in GBT was developed to solve coupled stability problems by Zhang[143, 144, 180] and Richard Schardt, [137, 138], and later by Davies and his co-workers [47, 48]. These publications introduce the concept of third-order coupling tensor ${ }^{i j k} \mathbb{X}$ and its physical meaning.

However, the focus of these works is the linear instability analysis[21, 42], based on eigenvalue problem, which applies trigonometric functions to describe the strain and stress fields of thin-walled beams in the longitudinal direction. Although these amplification functions can satisfy the simple support boundary conditions of a beam under concentrated load at the nodes, they have limitations in other loading condition or/and complex modal coupling configurations.

At the beginning of this century, non-linear GBT started to have a dedicated researching group in the Technical University of Lisbon, where Dinar Camotim and his colleagues developed several contributions in this subject: [30, 51, 155, 162].

In particular, an astonishing work from Silvestre [152] is a turning point in the understanding of the GBT non-linear analysis. This work not only highlights the importance of the fourth-order tensor ${ }^{i j k l} \mathbb{X}$ to achieve precise and feasible results in non-linear geometrical analysis, but also it is the first work to observe the relevance of shear and transversal elongation modes in this type of analysis. Such observation is directly related to the results and conclusion achieved in this Chapter.

Also in Portugal but at the University of Coimbra, Simão et.al.[164, 165] developed a unified energy formulation of non-linear GBT. Similar to publications from Schardt, the development of these publications had a focus in buckling analysis.

Among the contributions of these publications, there is the development of non-linear analysis in different types of applied force and boundary conditionals [7, 22, 110]. Also, Silvestre et al used Hermitian [10, 40, 152] and Langrage [34, 35, 118] cubic polynomials to describe the displacement and stress fields. These polynomials have the benefits to describe the internal forces, and consequently the initial stress, in compacted and directly form. On the other hand, it is necessary to have enough discretization of the thin-walled beam to reach a smooth initial stress field between the elements.

Concerning large deformation and rotation, the first study of GBT appears by Madeo et.al.[106], which extends the corotational method into GBT analysis. Gonçalves et.al[81] also studied this method, and recently, both groups detailed this procedure in [132].

Lastly, Silvestre in [2] is the first to address the non-linear physical analysis in GBT, which shows the requirement of a quite large number of models to describe this type 
of non-linearity. Gonçalves et.al[70, 88] also confirm this feature. Later, this subject is applied in elastic-plastic with large displacement $[1,3,4,163]$ and Direct Strength Method, DSM, [9, 119]. Duan et.al also developed a GBT elastic-plastic formulation based on B-Splines curves $[54,56]$ and impact load analysis[57]. The non-linear physical GBT is beyond of this dissertation's scope.

\subsection{Geometric stiffness in GBT}

The starting point in the development of non-linear GBT's analysis is a review of the principle of virtual work and how an incremental virtual displacement leads to, among other matrices, the initial stress stiffness matrix [86, 87]. The next subsections present, in the sequence: i) the assumptions in strains and its incremental variation; ii) the variation of longitudinal and transversal strain energy, respectively; iii)the linear and quadratic initial stress stiffness matrices, and iv)the linear and quadratic initial displacement stiffness matrices.

\subsubsection{Principle of Virtual Works applied in non-linear GBT}

As a first step in non-linear GBT analysis, one can consider the fiber of a generic segment of a thin-walled beam, as shown in figure 7.1, under small strains:

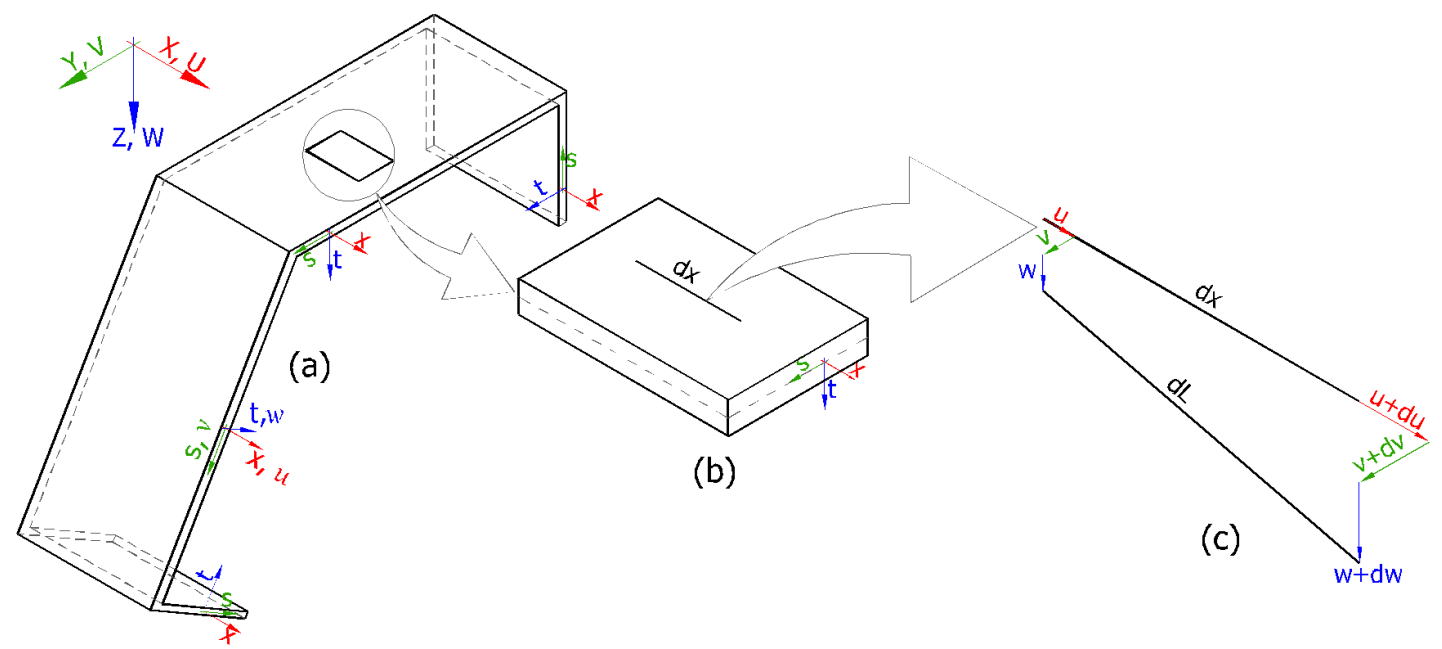

Figure 7.1: Fiber elongation of a thin-walled beam segment

By keeping the assumption of Lagrangian strain [32], it is possible to obtain from an infinitesimal fiber, as shown above, the following expressions:

$$
\begin{aligned}
& \varepsilon_{x}=\frac{d l x-d x}{d x}=\frac{\sqrt{(d x+d u)^{2}+d v^{2}+d w^{2}}-d x}{d x}=\sqrt{1+2 \frac{d u}{d x}+\frac{d u^{2}}{d x^{2}}+\frac{d v^{2}}{d x^{2}}+\frac{d w^{2}}{d x^{2}}}-1 \\
& \varepsilon_{s}=\frac{d l s-d s}{d s}=\frac{\sqrt{(d s+d v)^{2}+d u^{2}+d w^{2}}-d s}{d s}=\sqrt{1+2 \frac{d v}{d s}+\frac{d v^{2}}{d s^{2}}+\frac{d u^{2}}{d s^{2}}+\frac{d w^{2}}{d s^{2}}}-1
\end{aligned}
$$


Here, one approximates the square-root in equations 7.1 and 7.2 by a Taylor series. Truncating it in the linear part, one obtains:

$$
\begin{gathered}
\varepsilon_{x}=\left(1+\frac{1}{2}\left(2 u_{, x}+u_{, x}^{2}+v_{, x}^{2}+w_{, x}^{2}\right)\right)-1=u_{, x}+\frac{1}{2}\left(u_{, x}^{2}+v_{, x}^{2}+w_{, x}^{2}\right) \\
\varepsilon_{s}=\left(1+\frac{1}{2}\left(2 v_{, s}+v_{, s}^{2}+u_{, s}^{2}+w_{, s}^{2}\right)\right)-1=v_{, s}+\frac{1}{2}\left(u_{, s}^{2}+v_{, s}^{2}+w_{, s}^{2}\right)
\end{gathered}
$$

The terms $u_{x}^{2}$ and $v_{s}^{2}$ are small if compared with the other terms. Therefore, a new approximation neglects these terms [32]. Further, each displacement is divided into two states: the first one shows $\bar{u}, \bar{v}$ and $\bar{w}$, which concern the initial displacement state. The second one brings $\stackrel{+}{u}, \stackrel{+}{v}$ and $\stackrel{+}{w}$, concerning the incremental displacement state. Thus, one reaches:

$$
\begin{aligned}
& \varepsilon_{x}=\bar{u}_{, x}+\vec{u}_{, x}^{+}+\frac{1}{2}\left[\left(\overline{v_{, x}}+\dot{v}_{, x}^{+}\right)^{2}+\left(\bar{w}_{, x}+\stackrel{+}{w}, x^{2}\right]=\bar{\varepsilon}_{x}+\stackrel{+}{\varepsilon}_{x}+{\stackrel{+}{\varepsilon_{x}}}^{+}\right. \\
& \varepsilon_{s}=\bar{v}_{, s}+\stackrel{+}{v}_{, s}+\frac{1}{2}\left[\left(\bar{u}_{, s}+\stackrel{+}{u}, s^{2}+\left(\bar{w}_{, s}+\stackrel{+}{w}_{, s}\right)^{2}\right]=\bar{\varepsilon}_{s}+\stackrel{+}{\varepsilon}_{s}+\stackrel{+}{\varepsilon}_{s}^{+}\right.
\end{aligned}
$$

where:

$$
\begin{aligned}
& \bar{\varepsilon}_{x}=\bar{u}_{, x}+\frac{\bar{v}_{, x}^{2}+\bar{w}_{, x}^{2}}{2} \\
& \stackrel{+}{\varepsilon}_{x}=\stackrel{+}{u}_{, x}+\bar{v}_{, x} \stackrel{+}{v}, x+\bar{w}_{, x} \stackrel{+}{w}_{, x} \\
& \stackrel{+}{+}_{x}^{+}=\frac{{\stackrel{+}{v_{, x}}}^{2}+\stackrel{+}{w, x}^{2}}{2} \\
& \bar{\varepsilon}_{s}=\bar{v}_{, s}+\frac{\bar{u}_{, s}^{2}+\bar{w}_{, s}^{2}}{2} \\
& \stackrel{+}{\varepsilon}_{s}=\stackrel{+}{v}_{, s}+\bar{u}_{, s} \stackrel{+}{u}, s+\bar{w}_{, s} \stackrel{+}{w}_{, s} \\
& \stackrel{++}{\mathcal{E}_{s}}=\frac{\stackrel{+}{u}, s^{2}+\stackrel{+}{w}_{, s}}{2}
\end{aligned}
$$

It is important to note that the strain component to the initial displacement, $\bar{\varepsilon}$, has no variation. Hence, the variation of the strain can only affect the incremental component:

$$
\begin{aligned}
& \delta \varepsilon_{x}=\delta \dot{\varepsilon}_{x}^{+}+\delta{\stackrel{+}{\varepsilon_{x}}}^{+} \\
& \delta{\stackrel{+}{\varepsilon_{x}}}^{+} \delta u_{u_{, x}}^{+}+\bar{v}_{, x} \delta v_{, x}^{+}+\bar{w}_{, x} \delta w_{, x}^{+} \\
& \boldsymbol{\delta} \dot{\varepsilon}_{x}^{++}={ }_{v, x}^{+} \boldsymbol{\delta} v_{, x}^{+}+\stackrel{+}{w, x}^{+} \boldsymbol{\delta}{ }_{w}^{+} \\
& \delta \varepsilon_{s}=\delta \dot{\varepsilon}_{s}^{+}+\delta \dot{\varepsilon}^{+}+ \\
& \delta \dot{\varepsilon}_{s}^{+}=\delta v_{, s}^{+}+\bar{u}_{, s} \delta u_{, s}^{+}+\bar{w}_{, s} \delta w_{, s}^{+}
\end{aligned}
$$




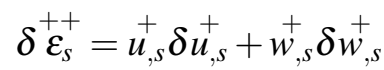

These variations are applied to minimize the functional of the energy system, given in eq. 2.22. Thus, it leads to same expression in eq. 2.23. However, for non-linear analysis, the variation of internal strain energy, $\delta U_{\text {int }}$, must be based on equations 7.13 and 7.16.

Although this new incremental variation links strains and stresses between membranes and plates, the superposition of these strains and stress, given in equations 7.19 and 7.20, are still valid. I.e., the strain and stresses over the thickness dimension maintain the constant and linear distributions related to membrane and plate behavior, respectively. However, the incremental variation builds crossing constitutive relationships between membrane's strain and plate's stress, as well as the plate's strain and membrane's stress

$$
\begin{gathered}
\varepsilon_{x}=\varepsilon_{x}^{M}+\varepsilon_{x}^{P} \\
\sigma_{x}=\sigma_{x}^{M}+\sigma_{x}^{P}
\end{gathered}
$$

Consequently, much more complex expressions are obtained to internal strain energy and its variation. In order to minimize this complexity, each strain and its incremental (from eq. 7.7 to 7.18 ) are presented below, in respect to membrane and plate parts. The evaluation of these expressions are based on GBT's displacements assumptions, given in equations $2.15,2.16$ and 2.17 :

$$
\begin{aligned}
& \bar{\varepsilon}_{x}^{M}=\bar{u}_{, x}+\frac{\bar{v}_{, x}^{2}+\bar{w}_{, x}^{2}}{2} \\
& \stackrel{+}{\varepsilon_{x}^{M}}=u_{, x}^{+}+\bar{v}_{, x} \stackrel{+}{v}_{, x}+\bar{w}_{, x} \stackrel{+}{w}_{, x} \\
& \stackrel{++}{\varepsilon_{x}^{M}}=\frac{{\stackrel{+}{v_{, x}}+{ }^{2}}_{w_{, x}}^{2}}{2} \\
& \delta \varepsilon_{x}^{+}=\delta \dot{u}_{, x}^{+}+\bar{v}_{, x} \delta_{v_{, x}}^{+}+\bar{w}_{, x} \delta_{w_{, x}^{+}}^{+}
\end{aligned}
$$

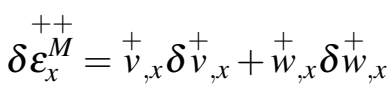

$$
\begin{aligned}
& \bar{\varepsilon}_{x}^{P}=-t \bar{w}_{, x x}+\frac{t^{2} \bar{w}_{, x s}^{2}}{2} \\
& \stackrel{+}{\varepsilon_{x}^{P}}=-t \stackrel{+}{w}, x x_{,}+t^{2} \bar{w}_{, x s} \stackrel{+}{w}, x s \\
& \stackrel{++}{\varepsilon_{x}^{P}}=\frac{t^{2}{ }_{w, x s}^{+2}}{2} \\
& \delta \stackrel{+}{P}_{x}^{P}=-t \delta \stackrel{+}{w}, x x^{+}+t^{2} \bar{w}_{, x s} \delta_{w_{, x s}}^{+} \\
& \delta \varepsilon_{x}^{P}=t^{2} w_{, x s}^{+} \delta w_{, x s}^{+}
\end{aligned}
$$




$$
\begin{aligned}
& \bar{\varepsilon}_{s}^{M}=\frac{\bar{u}_{, s}^{2}+\bar{w}_{, s}^{2}}{2} \\
& {\stackrel{+}{\varepsilon_{s}}}^{M}=\bar{u}_{, s} \stackrel{+}{u}, s+\bar{w}_{, s} \stackrel{+}{w}_{, s} \\
& \stackrel{++}{M}_{s}^{+}=\frac{\stackrel{+}{u}, s^{2}+\stackrel{+}{w}, s^{2}}{2} \\
& \delta \varepsilon_{s}^{\stackrel{+}{M}}=\bar{u}_{, s} \delta \stackrel{+}{u}, s^{+}+\bar{w}_{, s} \delta \stackrel{+}{w_{, s}} \\
& \delta \stackrel{++}{\varepsilon_{s}^{M}}=\stackrel{+}{u}, s \delta \dot{u}_{, s}^{+}+\stackrel{+}{w}_{, s} \delta \stackrel{+}{w}, s^{+} \\
& \bar{\varepsilon}_{s}^{P}=-t \bar{w}_{, s s}+\frac{t^{2} \bar{w}_{, x s}^{2}}{2} \\
& {\stackrel{+}{\varepsilon_{s}^{P}}}^{P}=-t \stackrel{+}{w}_{, s s}+t^{2} \bar{w}_{, s x} \stackrel{+}{w}_{, s x} \\
& \stackrel{+}{P}_{s}^{P}=\frac{t^{2}{ }_{w, s x}^{+}}{2} \\
& \delta \stackrel{+}{\varepsilon}_{s}^{P}=-t \delta_{w_{, s s}^{+}}^{+}+t^{2} \bar{w}_{, s x} \delta \stackrel{+}{w}_{, s x} \\
& \delta \varepsilon_{s}^{P}=t^{2} \stackrel{+}{w}, s x^{+} \dot{w}_{, s x}^{+}
\end{aligned}
$$

\subsubsection{Variation of longitudinal internal strain energy}

To develop the expression of internal strain energy in non-linear GBT analysis, one can start from the total energy for longitudinal strain in linear analysis, which is given by the two first integrals in eq. 2.24:

$$
U_{x}=\int_{V}\left[\int_{\varepsilon_{x}^{M}} \sigma_{x}^{M} \mathrm{~d} \varepsilon_{x}^{M}+\int_{\varepsilon_{x}^{P}} \sigma_{x}^{P} \mathrm{~d} \varepsilon_{x}^{P}\right] \mathrm{d} V
$$

However, in non-linear analysis, this expression must be defined in a more general way based on equations 7.19 and 7.20 :

$$
U_{x}=\int_{V} \int_{\varepsilon_{x}} \sigma_{x} \mathrm{~d} \varepsilon_{x} \mathrm{~d} V=\int_{V}\left[\int_{\varepsilon_{x}^{M}}\left(\sigma_{x}^{M}+\sigma_{x}^{P}\right) \mathrm{d} \varepsilon_{x}^{M}+\int_{\varepsilon_{x}^{P}}\left(\sigma_{x}^{M}+\sigma_{x}^{P}\right) \mathrm{d} \varepsilon_{x}^{P}\right] \mathrm{d} V
$$

Introducing the material constitutive relationships in this expression, given in equations 2.10 and 2.11 , one can reach:

$$
U_{x}=\int_{V} \frac{E}{1-\mu^{2}}\left[\frac{\varepsilon_{x}^{P^{2}}}{2}+\mu \varepsilon_{x}^{P} \varepsilon_{s}^{P}+\varepsilon_{x}^{M}\left(\varepsilon_{x}^{P}+\mu \varepsilon_{s}^{P}\right)\right]+E \frac{\varepsilon_{x}^{M^{2}}}{2} \mathrm{~d} V
$$

From this expression, the variations $[63,117]$ of membrane's and plate's strain in longitudinal directions lead respectively to: 


$$
\delta U_{x}^{M}=\int_{V} \delta \varepsilon_{x}^{M} E\left[\frac{\varepsilon_{x}^{P}+\mu \varepsilon_{s}^{P}}{1-\mu^{2}}+\varepsilon_{x}^{M}\right] \mathrm{d} V \quad(7.44) \quad \delta U_{x}^{P}=\int_{V} \delta \varepsilon_{x}^{P} \frac{E}{1-\mu^{2}}\left[\varepsilon_{x}^{P}+\mu \varepsilon_{s}^{P}+\varepsilon_{x}^{M}\right] \mathrm{d} V
$$

And the variation of the transversal strain, due to Poison effect leads to:

$$
\delta U_{x s}^{P}=\int_{V} \delta \varepsilon_{s}^{P} \frac{\mu E}{1-\mu^{2}}\left[\varepsilon_{x}^{P}+\varepsilon_{x}^{M}\right] \mathrm{d} V
$$

Now, one can expand the above expressions based on strain definitions of equations 7.5 and 7.6 and their variations from equations 7.13 to 7.18. For instance, the introduction of these terms in eq. 7.44 leads to:

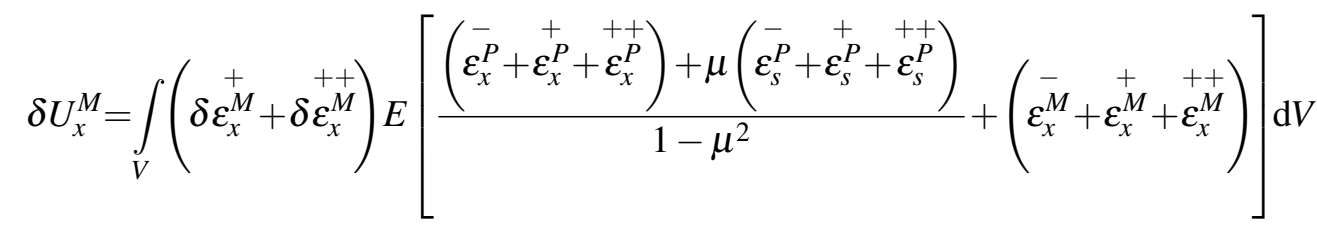

One can reorganize this integral in a sum of six parts:

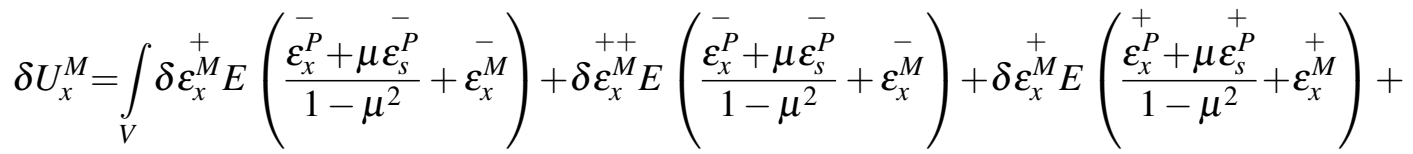

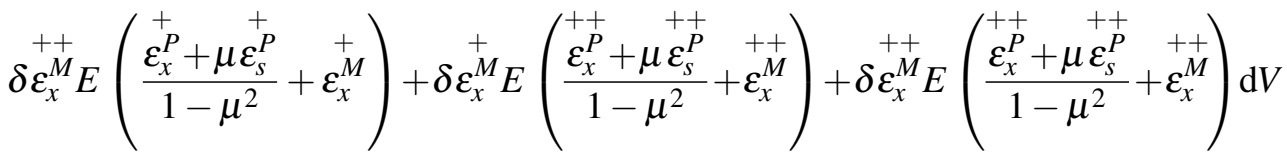

Here, the first term corresponds to the virtual work of the initial stress at the initial stage. This term gives the internal forces. It plays a central role in the iteration procedure since the difference between internal and external forces leads to unbalanced ones. The second term represents the virtual work of the initial stress in the incremental stages, which leads to the linear and quadratic stiffness matrices. The third term is the virtual work due to initial displacement that provides the linear and quadratic initial displacement matrices. Finally, the fourth, fifth and sixth terms are much smaller than the others. Thus, one can neglect them.

The variation of internal energy concerning the longitudinal plate's strains can be developed in the same way, and reach a similar expression. The introduction of equations 7.13 to 7.18 into eq. 7.45 leads to: 


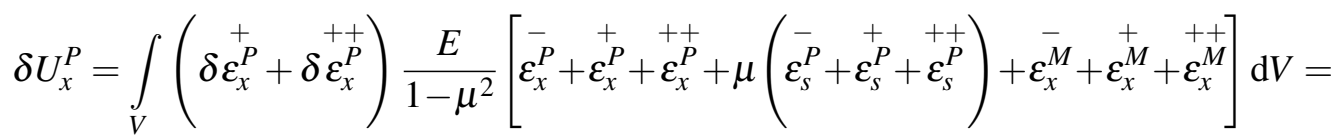

$$
\begin{aligned}
& \int_{V} \delta \stackrel{+}{\delta \varepsilon_{x}^{P}} \frac{E}{1-\mu^{2}}\left(\bar{\varepsilon}_{x}^{P}+\mu \bar{\varepsilon}_{s}^{P}+\bar{\varepsilon}_{x}^{M}\right)+\stackrel{+}{\delta} \varepsilon_{x}^{P} \frac{E}{1-\mu^{2}}\left(\bar{\varepsilon}_{x}^{P}+\mu \bar{\varepsilon}_{s}^{P}+\bar{\varepsilon}_{x}^{M}\right)+ \\
& \stackrel{+}{\delta \varepsilon_{x}^{P}} \frac{E}{1-\mu^{2}}\left(\stackrel{+}{\varepsilon_{x}^{P}}+\mu \stackrel{+}{\varepsilon_{s}^{P}}+\stackrel{+}{\varepsilon_{x}^{M}}\right)+\stackrel{++}{\delta \varepsilon_{x}^{P}} \frac{E}{1-\mu^{2}}\left(\stackrel{+}{\varepsilon_{x}^{P}}+\mu \stackrel{+}{\varepsilon_{s}^{P}}+\stackrel{+}{\varepsilon_{x}^{M}}\right)+ \\
& \delta \varepsilon_{x}^{P} \frac{E}{1-\mu^{2}}\left(\stackrel{++}{\varepsilon_{x}^{P}}+\mu \stackrel{++}{\varepsilon_{s}^{P}}+\stackrel{++}{\varepsilon_{x}^{M}}\right)+\stackrel{++}{\varepsilon_{x}^{P}} \frac{E}{1-\mu^{2}}\left(\stackrel{++}{\varepsilon_{x}^{P}}+\mu \stackrel{++}{+\varepsilon_{s}^{P}}+\stackrel{++}{\varepsilon_{x}^{M}}\right) \mathrm{d} V
\end{aligned}
$$

In above integral, the first, second and third terms lead to the internal forces of the plate behavior and the initial stress and displacement matrices in the incremental plate's strains, respectively.

Following the same approach, one evaluates the variation of the plate's longitudinal strain due to the Poisson effect:

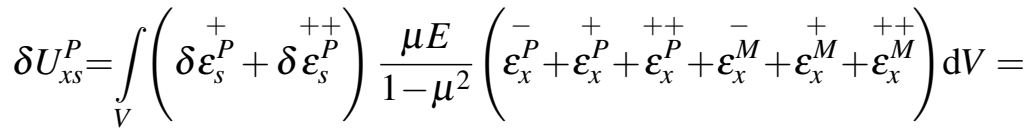

$$
\begin{aligned}
& \int_{V} \delta \stackrel{+}{\varepsilon_{s}^{P}} \frac{\mu E}{1-\mu^{2}}\left(\stackrel{-}{\varepsilon_{x}^{P}}+\bar{\varepsilon}_{x}^{M}\right)+\stackrel{++}{\delta \varepsilon_{s}^{P}} \frac{\mu E}{1-\mu^{2}}\left(\vec{\varepsilon}_{\varepsilon_{x}^{P}}^{+}+\vec{\varepsilon}_{x}^{M}\right)+\stackrel{+}{\delta \varepsilon_{s}^{P}} \frac{\mu E}{1-\mu^{2}}\left(\stackrel{+}{\varepsilon_{x}^{P}}+\stackrel{+}{\varepsilon_{x}^{M}}\right) \\
& +\stackrel{++}{\delta} \varepsilon_{s}^{P} \frac{\mu E}{1-\mu^{2}}\left(\stackrel{+}{\varepsilon_{x}^{P}}+\stackrel{+}{\varepsilon_{x}^{M}}\right)+\stackrel{+}{\delta \varepsilon_{s}^{P}} \frac{\mu E}{1-\mu^{2}}\left(\stackrel{++}{\varepsilon_{x}^{P}}+\stackrel{++}{\varepsilon_{x}^{M}}\right)+\stackrel{++}{\delta \varepsilon_{s}^{P}} \frac{\mu E}{1-\mu^{2}}\left(\stackrel{++}{\varepsilon_{x}^{P}}+\stackrel{++}{\varepsilon_{x}^{M}}\right) \mathrm{d} V
\end{aligned}
$$

\subsubsection{Variation of transversal internal strain energy}

The evaluation of transversal internal strain energy in non-linear GBT is similar to the longitudinal direction. However, as shown later in the development of this internal strain energy, the assumption of no membrane transversal strain leads only to quadratic terms.

Nevertheless, the evaluation of internal strain energy itself, in this direction, has a high contrast between the linear and non-linear analysis. Here, this strain has components from the derivatives in the transversal direction of the displacements in the thickness and longitudinal directions, as presented in equations 7.31, 7.32 and 7.33.

Therefore, the simple internal strain energy in the linear case, which only considers the plate's behavior:

$$
U_{s}=\int_{V} \frac{E}{1-\mu^{2}}\left[\frac{\varepsilon_{s}^{P^{2}}}{2}+\mu \varepsilon_{x}^{P} \varepsilon_{s}^{P}\right] \mathrm{d} V
$$


is replaced by a more general expression, but similar to longitudinal direction (eq. 7.43):

$$
U_{s}=\int_{V} \frac{E}{1-\mu^{2}}\left[\frac{\varepsilon_{s}^{P^{2}}}{2}+\mu \varepsilon_{x}^{P} \varepsilon_{s}^{P}+\varepsilon_{s}^{M}\left(\varepsilon_{s}^{P}+\mu \varepsilon_{x}^{P}\right)\right]+E \frac{\varepsilon_{s}^{M^{2}}}{2} \mathrm{~d} V
$$

and its variation in respect to membrane and plate strains in longitudinal and transversal directions are, respectively:

$$
\begin{gathered}
\delta U_{s}^{M}=\int_{V} \delta \varepsilon_{s}^{M} E\left[\frac{\varepsilon_{s}^{P}+\mu \varepsilon_{x}^{P}}{1-\mu^{2}}+\varepsilon_{s}^{M}\right] \mathrm{d} V \\
\delta U_{s}^{P}=\int_{V} \delta \varepsilon_{s}^{P} \frac{E}{1-\mu^{2}}\left[\varepsilon_{s}^{P}+\mu \varepsilon_{x}^{P}+\varepsilon_{s}^{M}\right] \mathrm{d} V \\
\delta U_{s x}^{P}=\int_{V} \delta \varepsilon_{x}^{P} \frac{\mu E}{1-\mu^{2}}\left(\varepsilon_{s}^{P}+\varepsilon_{s}^{M}\right) \mathrm{d} V
\end{gathered}
$$

Consequently, the final variations of this internal energy lead to similar expressions of longitudinal direction. As an example, one obtains the variation of transversal membrane strain from the introduction of equations 7.13 to 7.18 into eq. 7.53:

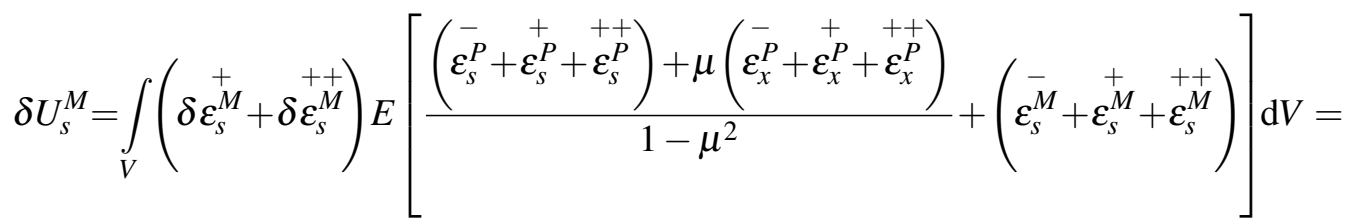

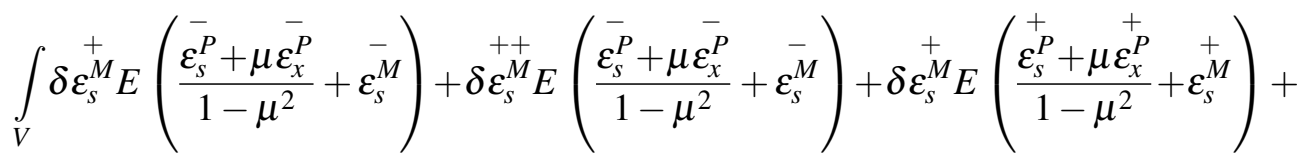

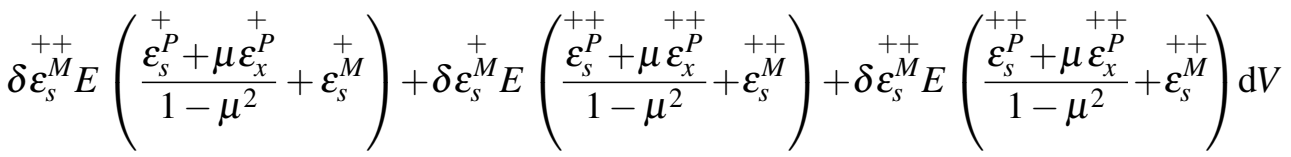

The variation of transversal plate strain (eq. 7.54) has the same evaluation:

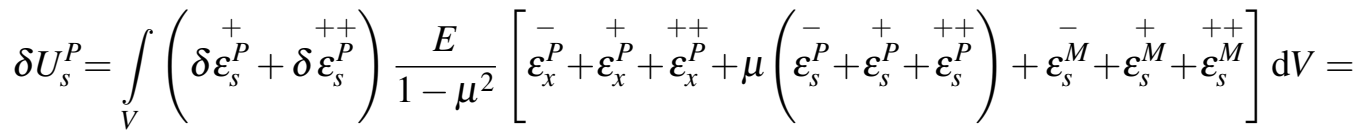

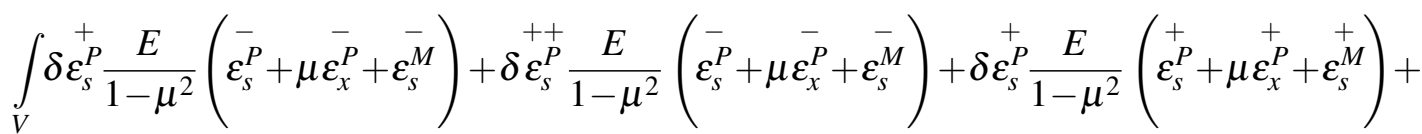

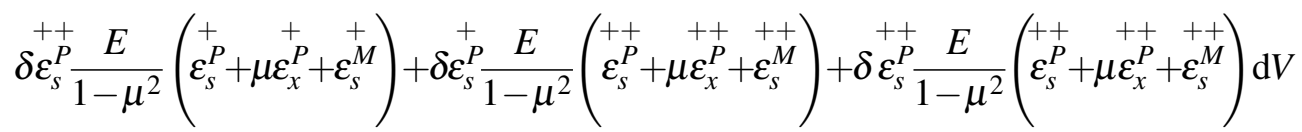


And the last evaluation of internal strain energy is related to Poisson's effect of longitudinal direction into incremental transversal strains, given in eq. 7.55:

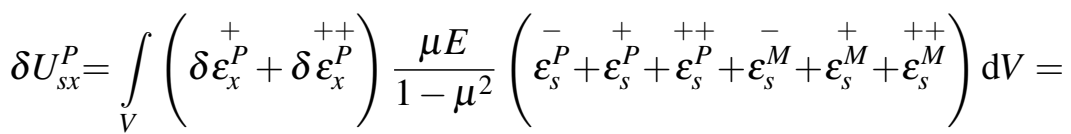

$$
\begin{aligned}
& \int_{V} \delta \stackrel{+}{\delta \varepsilon_{x}^{P}} \frac{\mu E}{1-\mu^{2}}\left(\bar{\varepsilon}_{s}^{P}+\vec{\varepsilon}_{s}^{M}\right)+\stackrel{+}{\delta \varepsilon_{x}^{P}} \frac{\mu E}{1-\mu^{2}}\left(\bar{\varepsilon}_{s}^{P}+\bar{\varepsilon}_{s}^{M}\right)+\stackrel{+}{\delta \varepsilon_{x}^{P}} \frac{\mu E}{1-\mu^{2}}\left(\stackrel{+}{\varepsilon_{s}^{P}}+\stackrel{+}{\varepsilon_{s}^{M}}\right)+ \\
& \delta \stackrel{++}{\varepsilon_{x}^{P}} \frac{\mu E}{1-\mu^{2}}\left(\stackrel{+}{\varepsilon_{s}^{P}}+\stackrel{+}{\varepsilon_{s}^{M}}\right)+\stackrel{+}{\stackrel{+}{\varepsilon_{x}^{P}}} \frac{\mu E}{1-\mu^{2}}\left(\stackrel{++}{\varepsilon_{s}^{P}}+\stackrel{++}{\varepsilon_{s}^{M}}\right)+\stackrel{++}{\delta \varepsilon_{x}^{P}} \frac{\mu E}{1-\mu^{2}}\left(\begin{array}{c}
++ \\
\varepsilon_{s}^{P}
\end{array}+\stackrel{++}{\varepsilon_{s}^{M}}\right) \mathrm{d} V
\end{aligned}
$$

Similar to equations $7.48,7.49$, and 7.50, the first terms in the equations $7.56,7.57$ and 7.58 represent the internal forces, the second and third terms represent the virtual work of the initial stress and displacement, respectively. All other terms are neglected.

\subsubsection{Linear and Quadratic Initial Stresses Stiffness - Longitudinal Direction}

As mentioned earlier, the second terms in equations 7.48, 7.49, 7.50, 7.56, 7.57 and 7.58 represent the virtual work of initial stress. Each of these terms can also split into two parts: linear and quadratic. To illustrate this division, one can introduce eq. 7.21 and 7.25 at the membrane part of second term in eq. 7.48:

$$
\begin{aligned}
& \int_{V} \delta \varepsilon_{x}^{++} E \varepsilon_{x}^{\bar{M}} \mathrm{~d} V=\int_{V}\left({ }_{v_{, x}}^{+} \delta v_{, x}^{+}+{ }_{w, x}^{+} \delta w_{, x}^{+}\right) E\left(\overline{u_{, x}}+\frac{1}{2}\left(\bar{v}_{, x}^{2}+\bar{w}_{, x}^{2}\right)\right) \mathrm{d} V= \\
& \int_{V} E \overline{u_{, x}}\left({ }_{v_{, x}^{+}}^{+} \delta v_{, x}^{+}+\stackrel{+}{w, x}^{+} \stackrel{+}{w, x}^{+}\right) \mathrm{d} V+\int_{V} \frac{E}{2}\left(\bar{v}_{, x}^{2}+\bar{w}_{, x}^{2}\right)\left(\stackrel{+}{v_{, x}} \boldsymbol{\delta} v_{, x}^{+}+\stackrel{+}{w_{, x}} \boldsymbol{\delta} \stackrel{+}{w, x}^{+}\right) \mathrm{d} V
\end{aligned}
$$

The first resultant integral in eq. 7.59 is the linear part of the virtual work of the initial stress, which leads to the initial stress stiffness matrix due to the membrane part. The studies of Richard Schardt, [137, 138] consider only this part in non-linear GBT. The development of this integral is carried out by the introduction of GBT's separation of variable assumptions, given in equations 2.12, 2.13 and 2.14, which splits the domain of integration in longitudinal and transversal directions, $x$ and $s$, respectively. Also, the concept of superposition modes, given in equations 2.18, 2.19 and 2.20, is introduced:

$$
\begin{aligned}
& \int_{V} E \overline{u_{, x}}\left({ }_{v_{, x}}^{+} \delta \nu_{, x}^{+}+{ }_{w, x}^{+} \delta w_{, x}^{+}\right) \mathrm{d} V=E \sum_{i=1}^{n} \sum_{j=1}^{n} \sum_{k=1}^{n} \int_{V}^{i} \bar{u}(s)^{i} \bar{V}_{, x x}(x) \\
& \left({ }^{j}{ }_{v}^{+}(s){ }^{j} V_{, x}^{+}(x){ }^{k}{ }_{v}^{+}(s) \delta^{k}{ }_{, x}^{+}(x)+{ }^{j}{ }_{w}^{+}(s)^{j} V_{, x}^{+}(x){ }^{k}{ }_{w}^{+}(s) \delta^{k}{ }_{V, x}^{+}(x)\right) \mathrm{d} V=E t^{i j k} \mathbb{X}_{3}^{M M i} \mathbb{V}_{X 3}^{j k}
\end{aligned}
$$


where:

$$
{ }^{i j k} \mathbb{X}_{3}^{M M}=\int_{s}^{i} \bar{u}(s)\left({ }^{j}{ }_{v}^{+}(s){ }^{k}+(s)+{ }^{j}+(s){ }^{k}+(s)\right) \mathrm{d} s(7.61) \quad{ }^{i} \mathbb{V}_{X 3}^{j k}=\int_{x}^{i} \bar{V}_{, x x}(x)^{j} V_{, x}^{+}(x) \delta^{k} V_{, x}^{+}(x) \mathrm{d} x
$$

${ }^{i j k} \mathbb{X}_{3}^{M M}$ is the third order coupling tensor, obtained only from the cross-section properties. The following section details this feature. The index ${ }^{M M}$ indicates the virtual work of initial Membrane strain, in the longitudinal direction, in the incremental Membrane strain in the same direction. The integral ${ }^{i} \mathbb{V}_{X 3}^{j k}$ leads to the correspondent matrices of the variation in the longitudinal direction. The left- and right-upper indices emphasize the initial stage mode $i$ and the incremental stages $j$ and $k$, respectively.

The evaluation of eq. 7.60 considered a uniform thickness, $t$, in all segments of the crosssection. Consequently, if a cross-section has different thicknesses in different segments, then the coupling tensor $\mathbb{X}$ must be developed for each group of segments with the same thickness.

The second resultant integral in eq. 7.59 represents the quadratic part, neglected by many applications. Nevertheless, its evaluation is:

$$
\begin{aligned}
& \int_{V} \frac{E}{2}\left(\bar{v}_{, x}^{-2}+\bar{w}_{, x}^{2}\right)\left(v_{, x}^{+} \delta v_{, x}^{+}+w_{, x}^{+} \delta w_{, x}^{+}\right) \mathrm{d} V= \\
& \quad \frac{E}{2} \sum_{i=1}^{n} \sum_{j=1}^{n} \sum_{k=1}^{n} \sum_{l=1}^{n} \int_{V}\left({ }^{i} \bar{v}(s)^{i} \bar{V}_{, x}(x)^{j} \bar{v}(s)^{j} \bar{V}_{, x}(x)+{ }^{i} \bar{w}(s)^{i} \bar{V}_{, x}(x)^{j} \bar{w}(s)^{j} \bar{V}_{, x}(x)\right) \\
& \left({ }^{k}{ }_{v}^{+}(s)^{k} V_{, x}^{+}(x)^{l}{ }^{+}(s) \delta^{l} V_{, x}^{+}(x)+{ }^{k}{ }_{w}^{+}(s)^{k} V_{, x}^{+}(x)^{l}{ }^{+}(s) \delta^{l} V_{, x}^{+}(x)\right) \mathrm{d} V=\frac{E t_{i j k l} \mathbb{X}_{4}^{M M i j} \mathbb{V}_{X 4}^{k l}}{2}
\end{aligned}
$$

with:

$$
\begin{gathered}
{ }^{i j k l} \mathbb{X}_{4}^{M M}=\int_{s}\left({ }^{i} \bar{v}(s){ }^{j} \bar{v}(s)+{ }^{i} \bar{w}(s){ }^{j} \bar{w}(s)\right)\left({ }^{k}+(s)^{l}{ }^{+}(s)+{ }^{k}{ }_{w}^{+}(s)^{l}{ }^{+}(s)\right) \mathrm{d} s \\
{ }^{i j} \mathbb{V}_{X 4}^{k l}=\int_{x}{ }^{i} \bar{V}_{, x}(x)^{j} \bar{V}_{, x}(x)^{k} \stackrel{V}{, x}_{, x}^{+}(x) \delta^{l} \stackrel{V}{V, x}^{+}(x) \mathrm{d} x
\end{gathered}
$$

Here, ${ }^{i j k l} \mathbb{X}_{4}^{M M}$ is the fourth order coupling tensor between $\underline{\text { Membrane-Membrane strains }}$ and its respective variation matrices are ${ }^{i j} \mathbb{V}_{X 4}^{k l}$, that is based on the initial stage of modes $i$ and $j$.

The virtual work of the initial plate's strains in the incremental membrane's strains behavior follows the same evaluation. Introducing equations 7.33, 7.26 and 7.36 into second terms in the equation 7.48 leads to: 


$$
\begin{aligned}
& \int_{V} \delta \varepsilon_{x}^{++} E \frac{\overline{\varepsilon_{x}^{P}}+\mu \varepsilon_{s}^{P}}{1-\mu^{2}} \mathrm{~d} V=\int_{V}\left({ }_{v}^{+}{ }_{, x}^{+} \bar{v}_{, x}+\stackrel{+}{w}, x_{w_{, x}}^{+}\right) \frac{E}{1-\mu^{2}}\left(\frac{t^{2} \bar{w}_{, x s}^{2}}{2}-t \bar{w}_{, x x}+\mu\left(\frac{t^{2} \bar{w}_{, x s}^{2}}{2}-t \bar{w}_{, s s}\right)\right) \mathrm{d} V \\
& =\frac{E(1+\mu)}{2\left(1-\mu^{2}\right)} \sum_{i=1}^{n} \sum_{j=1}^{n} \sum_{k=1}^{n} \sum_{l=1}^{n} \int_{V} t^{2 i} \bar{w}_{, s}(s)^{i} \bar{V}_{, x}(x)^{j} \bar{w}_{, s}(s)^{j} \bar{V}_{, x}(x) \\
& \left({ }^{k}{ }^{+}(s){ }^{k} \stackrel{+}{V}_{, x}(x){ }^{l} \stackrel{+}{v}(s){ }^{l} \delta \stackrel{+}{V}_{, x}(x)+{ }^{k}{ }_{w}^{+}(s)^{k} \stackrel{+}{V}_{, x}(x){ }^{l} \stackrel{+}{w}(s) \delta^{l} V_{, x}^{+}(x)\right)=(1+\mu) \frac{K_{i j k l}}{2} \mathbb{X}_{4}^{M P i j} \mathbb{V}_{X 4}^{k l}
\end{aligned}
$$

where $K$ is the plate stiffness, given in eq. 2.41 and:

$$
{ }^{i j k l} \mathbb{X}_{4}^{M P}=\int_{s}\left({ }^{i} \bar{v}(s)^{j} \bar{v}(s)+{ }^{i} \bar{w}(s){ }^{j} \bar{w}(s)\right)^{k}{ }^{+}, s(s)^{l}{ }^{+}{ }_{, s}(s) \mathrm{d} s
$$

Once the virtual work about the incremental membrane's strain is evaluated, the next evaluation concerns the virtual work of incremental plate's strains in longitudinal directions and its Poisson's effect, given in the second terms in equations 7.49 and 7.50, respectively. The evaluation of both expressions can be carried out simultaneously, since the incremental strains $\delta \varepsilon_{x}^{++}$and $\delta \varepsilon_{s}^{P}$ are the same, as shown in equations 7.30 and 7.40. Therefore, the introduction of equations 7.30, 7.40, 7.26, 7.36 and 7.21 into second integral in equations 7.49 and 7.50 leads to:

$$
\begin{gathered}
\int_{V} \delta \varepsilon_{x}^{++} \frac{E}{1-\mu^{2}}\left(\mu \overline{\varepsilon_{s}^{P}}+\overline{\varepsilon_{x}^{P}+\bar{\varepsilon}_{x}^{M}}\right) \mathrm{d} V+\int_{V} \delta \varepsilon_{s}^{++} \frac{\mu E}{1-\mu^{2}}\left(\overline{\varepsilon_{x}^{P}+\bar{\varepsilon}_{x}^{M}}\right) \mathrm{d} V=\int_{V} t^{2} w_{, x s}^{+} \delta w_{, x s}^{+} \frac{E}{1-\mu^{2}} \\
\left(\mu\left(-t \bar{w}_{, s s}+\frac{t^{2} \bar{w}_{, x s}^{2}}{2}\right)+(1+\mu)\left(-t \bar{w}_{, x x}+\frac{t^{2} \bar{w}_{, x s}^{2}}{2}+\bar{u}_{, x}+\frac{\bar{v}_{, x}^{2}+\bar{w}_{, x}^{2}}{2}\right)\right) \mathrm{d} V
\end{gathered}
$$

One can observe that the linear part concerning the initial strains of plate leads to an odd function. Thus, the integral over the thickness vanishes it. Therefore, the only remained in the linear part of the virtual work is the membrane's initial stress over the incremental plate strain:

$$
\begin{aligned}
& \int_{V} t^{2} w_{, x s}^{+} \delta w_{, x s}^{+} \frac{E(1+\mu)}{1-\mu^{2}} \bar{u}, x_{,} \mathrm{d} V=\frac{E(1+\mu)}{1-\mu^{2}} \sum_{i=1}^{n} \sum_{j=1}^{n} \sum_{k=1}^{n} \\
& \int_{V}{ }^{i} \bar{u}(s)^{i} \bar{V}_{, x x}(x) t^{2 j} \dot{w}_{, s}^{+}(s)^{j}{ }_{V, x}^{+}(x) \delta^{k}{ }^{+}{ }_{, s}(s)^{k}{ }_{V, x}^{+}(x) \mathrm{d} V=(1+\mu) K^{i j k} \mathbb{X}_{3}^{M P i} \mathbb{V}_{X 3}^{j k}
\end{aligned}
$$

Here, $\mathbb{V}_{X 3}$ are the variation matrices give in eq. 7.62 and ${ }^{i j k} \mathbb{X}_{3}^{M P}$ is the third-order tensor of coupling of initial Membrane strain and the incremental Plate strain:

$$
{ }^{i j k} \mathbb{X}_{3}^{M P}=\int_{s}{ }^{i} \bar{u}(s)^{j}{ }_{w, s}^{+}(s)^{k}{ }_{w, s}^{+}(s) \mathrm{d} s
$$


Usually, in thin-walled structures, the linear coupling part of membrane initial strains and plate's incremental strains has a secondary role to archive a consistent displacement and stress fields. The quadratic part of this coupling is even smaller. Generally, they are neglected as well. Nevertheless, the evaluation of this term is present below:

$$
\begin{aligned}
& \int_{V} t^{2} w_{, x s}^{+} \delta w_{, x s}^{+} \frac{E(1+\mu)}{1-\mu^{2}} \frac{{\overline{v_{, x}}}^{2}+\bar{w}_{, x}^{2}}{2} \mathrm{~d} V=\frac{E(1+\mu)}{2\left(1-\mu^{2}\right)} \sum_{i=1}^{n} \sum_{j=1}^{n} \sum_{k=1}^{n} \sum_{l=1}^{n} \int_{V} t^{2}\left({ }^{i} \bar{v}(s)^{i} \bar{V}_{, x}(x)^{j} \bar{v}(s)^{j} \bar{V}_{, x}(x)+\right. \\
& \left.{ }^{i} \bar{w}(s)^{i} \bar{V}_{, x}(x){ }^{j} \bar{w}(s)^{j} \bar{V}_{, x}(x)\right)^{k}{ }_{w, s}^{+}(s){ }^{k} V_{, x}^{+}(x){ }^{l}{ }_{w}^{+}(s) \delta^{l} V_{, x}^{+}(x) \mathrm{d} V=(1+\mu) \frac{K_{i j k l}}{2} \mathbb{X}_{4}^{M P i j} \mathbb{V}_{X 4}^{k l}
\end{aligned}
$$

The last evaluation concerns the quadratic term of coupling between the initial plate's strains and itself:

$$
\begin{aligned}
& \int_{V} t^{2} w_{, x s}^{+} \delta w_{, x s}^{+} \frac{E(1+2 \mu)}{1-\mu^{2}} \frac{t^{2} \bar{w}_{, x s}^{2}}{2} \mathrm{~d} V=\frac{E(1+2 \mu)}{2\left(1-\mu^{2}\right)} \sum_{i=1}^{n} \sum_{j=1}^{n} \sum_{k=1}^{n} \\
& \int_{V} t^{4 i} \bar{w}_{, s}(s)^{i} \bar{V}_{, x}(x)^{j} \bar{w}_{, s}(s)^{j} \bar{V}_{, x}(x)^{k}{ }_{w, s}^{+}(s)^{k} V_{, x}^{+}(x)^{l^{+}}{ }_{, s}(s) \delta^{l} V_{, x}^{+}(x) \mathrm{d} V=(1+2 \mu) \frac{3 t^{2} K_{i j k l}}{40} \mathbb{X}_{4}^{P P i j} \mathbb{V}_{X 4}^{k l}
\end{aligned}
$$

where:

$$
{ }^{i j k l} \mathbb{X}_{4}^{P P}=\int{ }^{i} \bar{w}_{, s}(s){ }^{j} \bar{w}_{, s}(s)^{k^{+}{ }_{, s}(s)}{ }^{l}{ }^{+}{ }_{, s}(s) \mathrm{d} s
$$

One can notice that the quadratic coupling between plates' strains is small in thinwalled beams since it involves the fifth order of thickness. To clarify all possibilities in the virtual work of longitudinal direction, table 7.1 presents a summary of it.

Table 7.1: Summary of virtual work of the initial stress in the incremental stages in the longitudinal direction.

\begin{tabular}{c|c|c|c}
\hline \multirow{2}{*}{ Relation } & initial & \multicolumn{2}{|c}{ incremental strain in: } \\
\cline { 3 - 4 } & stress in: & Membrane: & Plate: \\
\hline Linear & Membrane & eq. 7.60: $E t^{i j k} \mathbb{X}_{3}^{M M i} \mathbb{V}_{X 3}^{j k}$ & eq. 7.69: $(1+\mu) K^{i j k} \mathbb{X}_{3}^{M P i} \mathbb{V}_{X 3}^{j k}$ \\
\hline \multirow{2}{*}{ Quadratic } & Membrane & eq. 7.63: $\frac{E t}{2} i j k l \mathbb{X}_{4}^{M M i j} \mathbb{V}_{X 4}^{k l}$ & eq. 7.71: $(1+\mu) \frac{K}{2}{ }^{i j k l} \mathbb{X}_{4}^{M P i j} \mathbb{V}_{X 4}^{k l}$ \\
& Plate & eq. 7.66: $(1+\mu) \frac{K}{2}{ }^{i j k l} \mathbb{X}_{4}^{M P i j} \mathbb{V}_{X 4}^{k l}$ & eq. 7.72: $(1+2 \mu) \frac{3 t^{2} K i j k l}{40} \mathbb{X}_{4}^{P P i j} \mathbb{V}_{X 4}^{k l}$ \\
\hline
\end{tabular}

\subsubsection{Linear and Quadratic Initial Stresses Stiffness - Transversal Direction}

The initial stresses stiffness matrices in transversal direction have a more straightforward evaluation than the longitudinal direction. GBT's assumption of non-transversal elongation eliminates the linear part of the membrane's strain. Also, as shown in the 
longitudinal direction evaluation, there is not linear part due to plate's strains. Hence, the transversal direction has only quadratic stiffness matrices, as shown in the evaluation of incremental membrane strain, given in the second term of eq. 7.56:

$$
\begin{aligned}
& \int_{V} \delta \varepsilon_{s}^{++} E\left(\frac{\bar{\varepsilon}_{s}^{P}+\mu \varepsilon_{x}^{P}}{1-\mu^{2}}+\overline{\varepsilon_{s}^{M}}\right) \mathrm{d} V= \\
& \quad \int_{V}\left(\stackrel{+}{u}, s^{+} \dot{u}_{, s}^{+}+\stackrel{+}{w}, s^{+} \delta \stackrel{w}{, s}^{+}\right) E\left(\frac{-t \bar{w}_{, s s}+\frac{t^{2} \bar{w}_{, x s}^{2}}{2}+\mu\left(-t \bar{w}_{, x x}+\frac{t^{2} \bar{w}_{, x s}^{2}}{2}\right)}{1-\mu^{2}}+\frac{\bar{u}_{, s}^{2}+\bar{w}_{, s}^{2}}{2}\right) \mathrm{d} V
\end{aligned}
$$

One can introduce in the above expression, in part concerning the initial membrane strain, the equations 7.35 and 7.31 and reach the quadratic expression of variation of initial and incremental membrane's strains in transversal direction:

$$
\begin{gathered}
\int_{V} \delta \varepsilon_{s}^{++} E \varepsilon_{s}^{M} \mathrm{~d} V=\int_{V}\left(u_{, s}^{+} \delta u_{, s}^{+}+{ }_{w, s}^{+} \delta w_{, s}^{+}\right) E \frac{\bar{u}_{, s}^{2}+\bar{w}_{, s}^{2}}{2} \mathrm{~d} V= \\
\frac{E}{2} \sum_{i=1}^{n} \sum_{j=1}^{n} \sum_{k=1}^{n} \sum_{l=1}^{n} \int_{V}\left({ }^{i} \bar{u}_{, s}(s)^{i} \bar{V}_{, x}(x)^{j} \bar{u}_{, s}(s)^{j} \bar{V}_{, x}(x)+{ }^{i} \bar{w}_{, s}(s)^{i} \bar{V}(x)^{j} \bar{w}_{, s}(s)^{j} \bar{V}(x)\right) \\
\left({ }^{k} u_{, s}^{+}(s)^{k}{ }_{V}^{+}, x(x)^{l}{ }^{+}{ }_{, s}(s) \delta^{l} V_{, x}^{+}(x)+{ }^{k}{ }_{w, s}^{+}(s)^{k}{ }_{V}^{+}(x)^{l}{ }^{+}{ }_{, s}(s) \delta^{l}{ }_{V}^{+}(x)\right) \mathrm{d} V= \\
\frac{E t}{2}\left({ }^{i j k l} \mathbb{S}_{u 4}^{M M i j} \mathbb{V}_{X 4}^{k l}+2^{i j k l} \mathbb{S}_{u w 4}^{M M i j} \mathbb{V}_{X S 4}^{k l}+{ }^{i j k l} \mathbb{X}_{4}^{P P i j} \mathbb{V}_{S 4}^{k l}\right)
\end{gathered}
$$

where:

$$
\begin{aligned}
& { }^{i j k l} \mathbb{S}_{u 4}^{M M}=\int_{s}{ }^{i} \bar{u}_{, s}(s)^{j} \bar{u}_{, s}(s)^{k} \stackrel{u}{u}, s^{+}(s)^{l}{ }^{+}, s(s) \mathrm{d} s \\
& { }^{i j k l} \mathbb{S}_{u w 4}^{M M}=\int_{s}{ }^{i} \bar{u}_{, s}(s)^{j} \bar{u}_{, s}(s)^{k}{ }_{w, s}^{+}(s)^{l}{ }_{w}^{+}(s) \mathrm{d} s \\
& { }^{i j} \mathbb{V}_{X S 4}^{k l}=\int_{L}^{i} \bar{V}_{, x}(x)^{j} \bar{V}_{, x}(x)^{k}{ }^{+}(x) \delta^{l} V^{+}(x) \mathrm{d} x \\
& { }^{i j} \mathbb{V}_{S 4}^{k l}=\int_{L}{ }^{i} \bar{V}(x)^{j} \bar{V}(x)^{k}{ }^{+}(x) \delta^{l}{ }^{+}(x) \mathrm{d} x
\end{aligned}
$$

For the part of the initial plate's strain, the introduction of equations 7.36 and 7.26 leads to the quadratic relationship: 


$$
\begin{aligned}
& \int_{V} \delta \varepsilon_{s}^{++} E \frac{\bar{\varepsilon}_{s}^{P}+\mu \varepsilon_{x}^{P}}{1-\mu^{2}} \mathrm{~d} V=\int_{V}\left(u_{, s}^{+} \delta u_{, s}^{+}+{ }_{w}^{+}{ }_{, s} \delta w_{, s}^{+}\right) \frac{E(1+\mu)}{2\left(1-\mu^{2}\right)} t^{2} \bar{w}_{, x s}^{2} \mathrm{~d} V= \\
& \frac{E(1+\mu)}{2\left(1-\mu^{2}\right)} \sum_{i=1}^{n} \sum_{j=1}^{n} \sum_{k=1}^{n} \sum_{l=1}^{n} \int_{V} t^{2 i} \bar{w}_{, s}(s)^{i} \bar{V}_{, x}(x)^{j} \bar{w}_{, s}(s)^{j} \bar{V}_{, x}(x)
\end{aligned}
$$

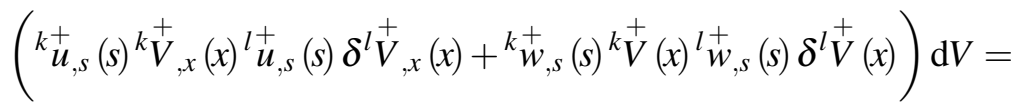

$$
\begin{aligned}
& \frac{K(1+\mu)}{2}\left({ }^{i j k l} \mathbb{S}_{u w 4}^{M M i j} \mathbb{V}_{X 4}^{k l}+{ }^{i j k l} \mathbb{X}_{4}^{P P i j} \mathbb{V}_{X S 4}^{k l}\right)
\end{aligned}
$$

Similar to longitudinal direction, the evaluation of incremental plate's strains is developed simultaneously for both terms $\delta \dot{\varepsilon}_{x}^{P}$ and $\delta \dot{\varepsilon}_{s}^{P}$. Thereby, the introduction of equations $7.40,7.26$ and 7.36 into the second terms of equations 7.57 and 7.58 leads to:

$$
\begin{aligned}
& \int_{V} \delta \varepsilon_{s}^{+} \frac{E}{1-\mu^{2}}\left(\bar{\varepsilon}_{s}^{P}+\mu \bar{\varepsilon}_{x}^{P}+\varepsilon_{s}^{M}\right) \mathrm{d} V+\int_{V} \delta \dot{\varepsilon}_{x}^{+} \frac{\mu E}{1-\mu^{2}}\left(\bar{\varepsilon}_{s}^{P}+\bar{\varepsilon}_{s}^{M}\right) \mathrm{d} V= \\
& \int_{V} t^{2} w_{, s x}^{+} \delta w_{, s x}^{+} \frac{E}{1-\mu^{2}}\left((1+\mu)\left(\frac{\bar{u}_{, s}^{2}+\bar{w}_{, s}^{2}}{2}-t \bar{w}_{, s s}+\frac{t^{2} \bar{w}_{, x s}^{2}}{2}\right)+\mu\left(-t \bar{w}_{, x x}+\frac{t^{2} \bar{w}_{, x s}^{2}}{2}\right)\right) \mathrm{d} V
\end{aligned}
$$

From the above expression, one reaches the following quadratic relationship between the initial membrane and incremental plate strains:

$$
\begin{aligned}
& \int_{V} t^{2} w_{, s x}^{+} \delta w_{, s x}^{+} \frac{E(1+\mu)}{1-\mu^{2}} \frac{\bar{u}_{, s}^{2}+\bar{w}_{, s}^{2}}{2} \mathrm{~d} V= \\
& \frac{E(1+\mu)}{2\left(1-\mu^{2}\right)} \sum_{i=1}^{n} \sum_{j=1}^{n} \sum_{k=1}^{n} \sum_{l=1}^{n} \int_{V}\left({ }^{i} \bar{u}_{, s}(s){ }^{i} \bar{V}_{, x}(x){ }^{j} \bar{u}_{, s}(s)^{j} \bar{V}_{, x}(x)+{ }^{i} \bar{w}_{, s}(s)^{i} \bar{V}(x)^{j} \bar{w}_{, s}(s)^{j} \bar{V}(x)\right) \\
& t^{2 k_{w}^{+}}{ }_{, s}(s){ }^{k} V_{, x}^{+}(x){ }^{l}{ }_{w, s}^{+}(s) \delta^{l} V_{, x}^{+}(x) \mathrm{d} V=(1+\mu) \frac{K}{2}\left({ }^{i j k l} \mathbb{S}_{u w 4}^{M M i j} \mathbb{V}_{X 4}^{k l}+{ }^{i j k l} \mathbb{X}_{4}^{P P i j} \mathbb{V}_{S X 4}^{k l}\right)
\end{aligned}
$$

with:

$$
{ }^{i j} \mathbb{V}_{S X 4}^{k l}=\int_{L}^{i} \bar{V}(x)^{j} V^{+}(x)^{k}{ }_{, x}^{+}(x) \delta^{l_{V}^{+}}{ }_{, x}(x) \mathrm{d} x
$$

It is essential to highlight that if different shape modes have different shape functions, then: $\mathbb{V}_{S X 4} \neq \mathbb{V}_{X S 4}$. Finally, the quadratic relationship between the initial and incremental plate's strains is: 


$$
\begin{aligned}
& \int_{V} t^{2}{ }^{+}, s x \\
& \delta w_{, s x}^{+} \frac{E(1+2 \mu)}{2\left(1-\mu^{2}\right)} t^{2} \bar{w}_{, s x}^{2} \mathrm{~d} V=\frac{E(1+2 \mu)}{2\left(1-\mu^{2}\right)} \sum_{i=1}^{n} \sum_{j=1}^{n} \sum_{k=1}^{n} \sum_{l=1}^{n} \\
& \int_{V} t^{4 i} \bar{w}_{, s}(s)^{i} \bar{V}_{, x}(x)^{j} \bar{w}_{, s}(s)^{j} \bar{V}_{, x}(x)^{k^{+}}{ }_{, s}^{+}(s)^{k}{ }_{V}^{+}, x(x){ }^{l_{w}^{+}}(s) \delta^{l} V_{, x}^{+}(x) \mathrm{d} V=(1+2 \mu) \frac{3 t^{2} K_{i j k l}}{40} \mathbb{X}_{4}^{P P i j} \mathbb{V}_{X 4}^{k l}
\end{aligned}
$$

To clarify all possibilities in the virtual work of longitudinal direction, table 7.2 presents a summary of it.

Table 7.2: Summary of virtual work of the initial stress in the incremental stages in the transversal direction.

\begin{tabular}{c|c|c|c}
\hline \multirow{2}{*}{ Relation } & initial & \multicolumn{2}{|c}{ incremental strain in: } \\
\cline { 3 - 4 } & stress in: & Membrane: & Plate: \\
\hline \multirow{2}{*}{ Quadratic } & Membrane & eq. 7.75: $\frac{E t}{2}\left({ }^{i j k l} \mathbb{S}_{u 4}^{M M i j} \mathbb{V}_{X 4}^{k l}+\right.$ & eq. 7.82: $(1+\mu) \frac{K}{2}$ \\
& Plate & $\left.2^{i j k l} \mathbb{S}_{u w 4}^{M M i j} \mathbb{V}_{X S 4}^{k l}+{ }^{i j k l} \mathbb{X}_{4}^{P P i j} \mathbb{V}_{S 4}^{k l}\right)$ & $\left({ }^{i j k l} \mathbb{S}_{u w 4}^{M M i j} \mathbb{V}_{X 4}^{k l}+{ }^{i j k l} \mathbb{X}_{4}^{P P i j} \mathbb{V}_{S X 4}^{k l}\right)$ \\
& & $\left({ }^{i j k l} \mathbb{S}_{u w 4}^{M M i j} \mathbb{V}_{X 4}^{k l}+{ }^{i j k l} \mathbb{X}_{4}^{P P i j} \mathbb{V}_{X S 4}^{k l}\right)$ & eq. 7.84: $(1+2 \mu) \frac{3 t^{2} K}{40}{ }^{i j k l} \mathbb{X}_{4}^{P P i j} \mathbb{V}_{X 4}^{k l}$ \\
\hline
\end{tabular}

\subsubsection{Linear and Quadratic Initial Displacements Stiffness - Lon- gitudinal Direction}

The development of Linear and Quadratic Initial Displacements Stiffness Matrices in GBT is based on the third terms in the integrals of equations $7.48,7.49,7.50,7.56,7.57$ and 7.58 .

The evaluations of these expressions are similar to the case of initial stress stiffness matrices. For instance, the variation of longitudinal membrane strain correspondent to its incremental strains, given the third term of integral in eq. 7.48, has the following evaluation with the introduction of equations 7.8 and 7.14 :

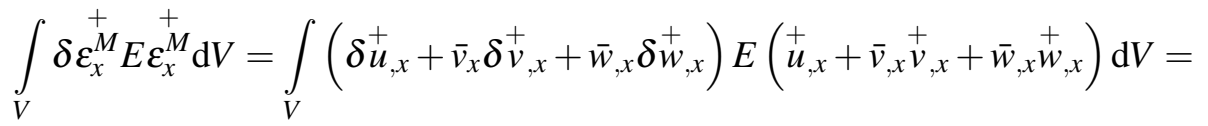

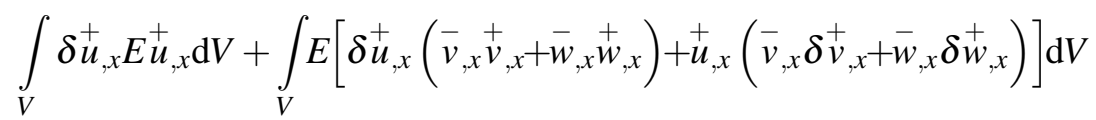

$$
\begin{aligned}
& +\int_{V}\left(\bar{v}_{, x} \stackrel{+}{v}, x_{,}+\bar{w}_{, x} \stackrel{+}{w}, x^{\prime}\right) E\left(\bar{v}_{, x} \delta \stackrel{+}{v}, x+\bar{w}_{, x} \boldsymbol{\delta}_{w}^{+}, x\right) \mathrm{d} V
\end{aligned}
$$


The first resulting integral in eq. 7.85 represents the virtual work of the incremental displacement at the incremental stage. The second and the third integral above are, respectively, the linear and quadratic part of the virtual work of the initial displacement at the incremental stage. Evaluating the linear part, one obtains:

$$
\begin{aligned}
& \int_{V} E\left[\delta_{u_{, x}}^{+}\left(\bar{v}_{, x} \stackrel{+}{v}, x_{,}+\bar{w}_{, x} \stackrel{+}{w}_{, x}\right)+\stackrel{+}{u}, x_{,}\left(\bar{v}_{, x} \delta \stackrel{+}{v}, x^{+} \bar{w}_{, x} \boldsymbol{\delta}^{+}, x\right)\right] \mathrm{d} V=
\end{aligned}
$$

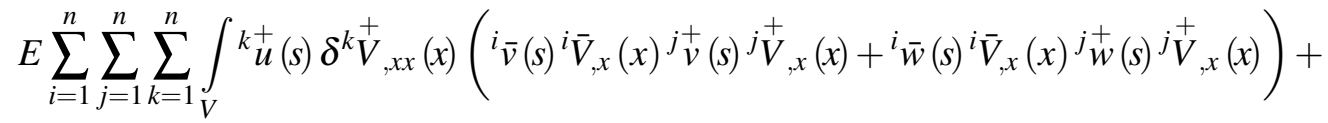

$$
\begin{aligned}
& j_{u}^{+}(s)^{j}{ }^{+}, x x(x)\left({ }^{i} \bar{v}(s)^{i} \bar{V}_{, x}(x)^{k} \stackrel{+}{v}(s)^{k} \stackrel{+}{V}_{, x}(x)+{ }^{i} \bar{w}(s)^{i} \bar{V}_{, x}(x)^{k}{ }_{w}^{+}(s)^{k}{ }^{+} V_{, x}(x)\right) \mathrm{d} V= \\
& E t\left({ }^{k i j} \mathbb{X}_{3}^{M M i} \mathbb{V}_{3 u}^{j k}+{ }^{j i k} \mathbb{X}_{3}^{M M i} \mathbb{V}_{3 v w}^{j k}\right)
\end{aligned}
$$

Where:

$$
\begin{gathered}
{ }^{i} \mathbb{V}_{3 u}^{j k}=\int_{x}{ }^{i} \bar{V}_{, x}(x)^{j} \stackrel{V}{, x}_{, x}^{+}(x) \delta^{k} V_{, x x}^{+}(x) \mathrm{d} x \\
{ }^{i} \mathbb{V}_{3 v w}^{j k}=\int_{x}^{i} \bar{V}_{, x}(x)^{j} V_{, x x}^{+}(x) \delta^{k} V_{, x}^{+}(x) \mathrm{d} x
\end{gathered}
$$

Here, one can observe two properties. The first one is in regards to the integral in the longitudinal direction: if the mode shapes $j$ and $k$ have the same shape function, then $\mathbb{V}_{3 u}$ leads to a transpose matrix of $\mathbb{V}_{3 v w}$. The second observation is about the tensors ${ }^{k i j} \mathbb{X}_{3}^{M M}$ and ${ }^{j i k} \mathbb{X}_{3}^{M M}$. These tensors are the same as the tensor in eq. 7.61 , but the different order of reading. The following section details this feature.

Concerning the quadratic part of eq. 7.85 , its evaluation leads to:

$$
\begin{aligned}
& \int_{V}\left(\bar{v}_{, x} \stackrel{+}{v}, x_{,}+\bar{w}_{, x} \stackrel{+}{w}, x^{\prime}\right) E\left(\bar{v}_{, x} \boldsymbol{\delta}_{\vec{v}, x}^{+}+\bar{w}_{, x} \delta \stackrel{+}{w}, x^{+}\right) \mathrm{d} V= \\
& E \sum_{i=1}^{n} \sum_{j=1}^{n} \sum_{k=1}^{n} \sum_{l=1}^{n} \int_{V}\left({ }^{i} \bar{v}(s){ }^{i} \bar{V}_{, x}(x){ }^{k}{ }_{\nu}^{+}(s){ }^{k}{ }_{V}^{+}, x(x)+{ }^{i} \bar{w}(s){ }^{i} \bar{V}_{, x}(x){ }^{k}{ }_{w}^{+}(s){ }^{k} V_{, x}^{+}(x)\right) \\
& \left({ }^{j} \bar{v}(s)^{j} \bar{V}_{, x}(x)^{l} \stackrel{+}{v}(s) \delta^{l} V_{, x}^{+}(x)+{ }^{j} \bar{w}(s)^{j} \bar{V}_{, x}(x)^{l}{ }^{+}(s) \delta^{l} V_{, x}^{+}(x)\right) \mathrm{d} V=E t^{i k j l} \mathbb{X}_{4}^{M M i j} \mathbb{V}_{X 4}^{k l}
\end{aligned}
$$

Similar to the linear part, eq. 7.89 involves the same tensor of initial quadratic stress, given in eq. 7.64, but with an interchanged order of reading.

For the virtual work correspondent to incremental plate and membrane displacement, the linear part from the plate's strain is vanished, due to the integral of the odd functions over the thickness dimension. Thus, the introduction of equations 7.27 and 7.37 
in the plate's part in the third integral of eq. 7.48 leads to:

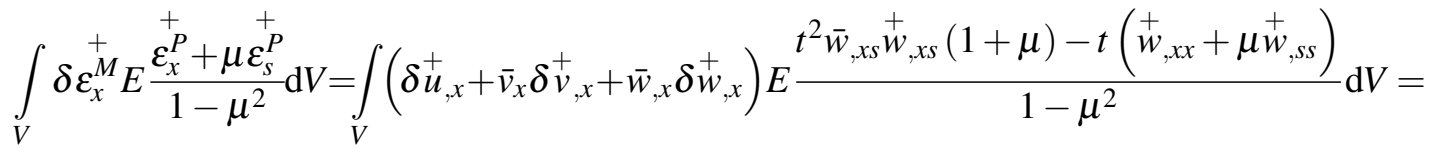

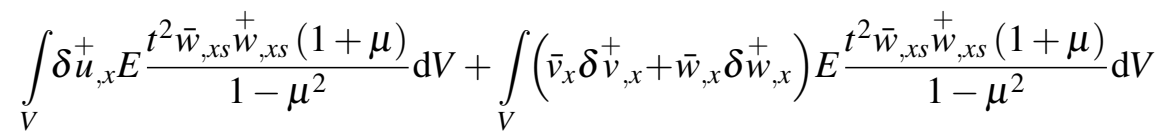

The first and second integrals above are the initial linear and quadratic displacement, respectively. They couple the plate and variation membrane's strains. The evaluation of each term is:

$$
\begin{aligned}
& \int_{V} \delta \dot{u}_{, x}^{+} E \frac{t^{2} \bar{w}_{, x s} \stackrel{+}{w}, x s^{(1+\mu)}}{1-\mu^{2}} \mathrm{~d} V=\frac{E(1+\mu)}{1-\mu^{2}} \sum_{i=1}^{n} \sum_{j=1}^{n} \sum_{k=1}^{n} \\
& \int_{V}{ }^{+} u(s) \delta^{k} V_{, x x}^{+}(x) t^{2 i} \bar{w}_{, s}(s)^{i} \bar{V}_{, x}(x)^{j}{ }_{w}^{+}, s(s)^{j} V_{, x}^{+}(x) \mathrm{d} V=\frac{E t}{1-\mu}{ }^{k i j} \mathbb{X}_{3}^{M P i} \mathbb{V}_{3 u}^{j k} \\
& \int_{V}\left(\bar{v}_{x} \delta_{v}^{+}, \bar{w}_{, x} \delta_{w}^{+}, x\right) E \frac{t^{2} \bar{w}_{, x s} \stackrel{+}{w}, x s^{2}(1+\mu)}{1-\mu^{2}} \mathrm{~d} V= \\
& \frac{E(1+\mu)}{1-\mu^{2}} \sum_{i=1}^{n} \sum_{j=1}^{n} \sum_{k=1}^{n} \sum_{k=1}^{l} \int{ }_{V}\left({ }_{\bar{v}}(s)^{i} \bar{V}_{, x}(x)^{l}{ }^{+}(s) \delta^{l} V_{, x}^{+}(x)^{i} \bar{w}(s)^{i} V_{, x}(x)^{l}{ }^{+}(s) \delta^{l} V_{, x}(x)\right) \\
& t^{2 j} \bar{w}_{, s}(s)^{j} \bar{V}_{, x}(x)^{k_{w}^{+}}{ }_{, s}(s)^{j}{ }_{V, x}^{+}(x) \mathrm{d} V=K(1+\mu)^{i l j k} \mathbb{X}_{4}^{M P i j} \mathbb{V}_{X 4}^{k l}
\end{aligned}
$$

The next evaluations are related to the variation of longitudinal plate's strains and its Poisson's effect. Similar to the evaluation of initial stress terms, the development of both expressions is carried out simultaneously, since the incremental strains are similar (as given in equations 7.29 and 7.39). Thereby, the introduction of expressions $7.29,7.39,7.227 .27$, and 7.37 into the sum of third terms of equations 7.49 and 7.50 leads to:

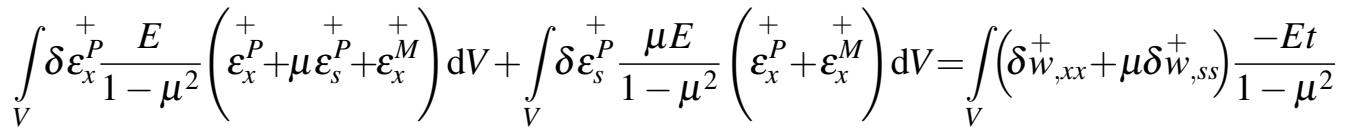

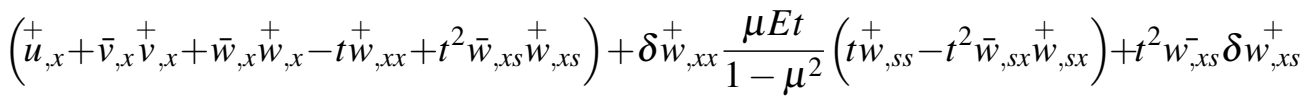

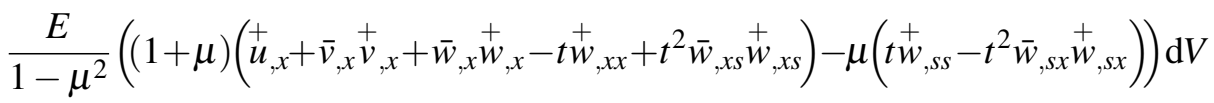


And by eliminating terms involving integrals of odd functions in the thickness domain, the above expression is simplified to:

$$
\begin{aligned}
& \int_{V} \delta^{+}, x x \frac{E t^{2}}{1-\mu^{2}}\left(\stackrel{+}{w}, x x^{+}+\stackrel{+}{w}_{, s s}\right) \mathrm{d} V+\int_{V} \delta \stackrel{+}{w}, s s^{+} \frac{\mu E t^{2}}{1-\mu^{2}} \stackrel{+}{w}, x x \mathrm{~d} V+ \\
& \int_{V} \bar{w}_{, x s} \delta_{w_{, x s}}^{+} \frac{E t^{2}}{1-\mu^{2}}\left((1+\mu)\left(\stackrel{+}{u}, x_{,}+\bar{v}_{, x} \stackrel{+}{v}, x_{,}+\bar{w}_{, x} \stackrel{+}{w}, x^{\prime}\right)+(1+2 \mu) t^{2} \bar{w}_{, x s} \stackrel{+}{w}, x s\right) \mathrm{d} V
\end{aligned}
$$

In the three resulting integrals above, the first and the second one represents the virtual work of the incremental plate's displacement, at the same stage, in the longitudinal and transversal direction, respectively. Therefore, they are archived directly by the linear stiffness matrix at the corresponding stage. The third resulting integral represents all virtual work of initial displacement. One can split this integral into three terms: i) linear between plate and membrane; ii) quadratic between plate and membrane; iii) quadratic between plates. The developments of each term are: $6++6$

$$
\begin{aligned}
& \int_{V} \bar{w}_{, x s} \delta \stackrel{w}{w}, x s^{+} \frac{E t^{2}}{1-\mu^{2}}(1+\mu) \stackrel{+}{u}, x \mathrm{~d} V=K(1+\mu) \sum_{i=1}^{n} \sum_{j=1}^{n} \sum_{k=1}^{n} \\
& \int_{V}{ }^{i} \bar{w}_{, s}(s)^{i} \bar{V}_{, x}(x)^{k}{ }_{w}^{+}, s(s) \delta^{k} \stackrel{V}{, x}^{j}{ }_{u}^{+}(s)^{j}{ }_{V, x x}^{+}(x) \mathrm{d} V=K(1+\mu)^{j i k} \mathbb{X}_{3}^{M P i} \mathbb{V}_{3 v w}^{j k} \\
& \int_{V} \bar{w}_{, x s} \delta_{w_{, x s}}^{+} \frac{E t^{2}}{1-\mu^{2}}(1+\mu)\left(\bar{v}_{, x} \stackrel{+}{v}, x_{,}+\bar{w}_{, x} \stackrel{+}{w}, x^{\prime}\right) \mathrm{d} V=K(1+\mu) \sum_{i=1}^{n} \sum_{j=1}^{n} \sum_{k=1}^{n} \sum_{l=1}^{n}
\end{aligned}
$$

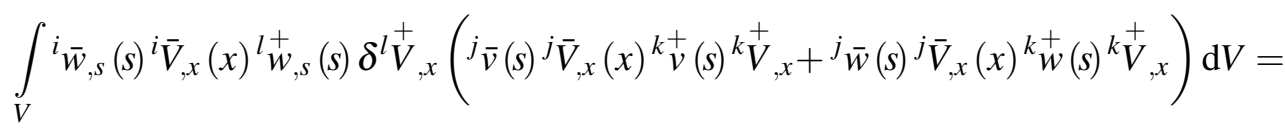

$$
\begin{aligned}
& K(1+\mu)^{j k i l} \mathbb{X}_{4}^{M P i j} \mathbb{V}_{X 4}^{k l} \\
& \int_{V} \bar{w}_{, x s} \delta w_{, x s}^{+} \frac{E t^{4}}{1-\mu^{2}}(1+2 \mu) \bar{w}_{, x s} \stackrel{+}{w}, x s \mathrm{~d} V=(1+2 \mu) \frac{3 t^{2} K}{40} \sum_{i=1}^{n} \sum_{j=1}^{n} \sum_{k=1}^{n} \sum_{l=1}^{n}
\end{aligned}
$$

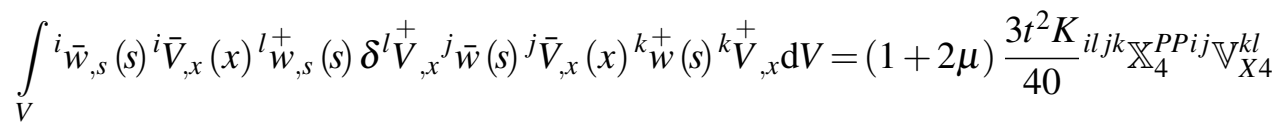

Similar to virtual work of initial stress, the coupling among plate's strains reach the fifth power of thickness. Therefore, one can neglect this coupling in thin-walled applications.

The table below presents a summary of all coupling relationships due to the virtual work of initial displacement in the longitudinal direction. 
Table 7.3: Summary of virtual work of the initial displacement in the incremental stages in the longitudinal direction.

\begin{tabular}{c|c|c|c}
\hline \multirow{2}{*}{ Relation } & initial & \multicolumn{2}{|c}{ incremental strain in: } \\
\cline { 3 - 4 } & displ. in: & Membrane: & Plate: \\
\hline \multirow{2}{*}{ Linear } & Membrane & eq. 7.86: & eq. $7.95: K(1+\mu)^{j i k} \mathbb{X}_{3}^{M P i} \mathbb{V}_{3 v w}^{j k}$ \\
& Plate & $\begin{array}{c}\left.k i j \mathbb{X}_{3}^{M M i} \mathbb{V}_{3 u}^{j k}+{ }^{j i k} \mathbb{X}_{3}^{M M i} \mathbb{V}_{3 v w}^{j k}\right) \\
\text { eq. 7.91: } \frac{E t}{1-\mu}{ }^{k i j} \mathbb{X}_{3}^{M P i} \mathbb{V}_{3 u}^{j k}\end{array}$ & \\
\hline \multirow{2}{*}{ Quadratic } & Membrane & eq. 7.89: $E t^{i k j l i j \mathbb{X}_{4}^{M M i j} \mathbb{V}_{X 4}^{k l}}$ & eq. 7.96: $K(1+\mu)^{j k i l} \mathbb{X}_{4}^{M P i j} \mathbb{V}_{X 4}^{k l}$ \\
& Plate & eq 7.92: $K(1+\mu)^{i l j k} \mathbb{X}_{4}^{M P i j} \mathbb{V}_{X 4}^{k l}$ & eq. $7.97:(1+2 \mu) \frac{3 t^{2} K i l j k}{40} \mathbb{X}_{4}^{P P i j} \mathbb{V}_{X 4}^{k l}$ \\
\hline
\end{tabular}

\subsubsection{Linear and Quadratic Initial Displacements Stiffness - transver-}

\section{sal Direction}

The last evaluation of incremental virtual work is related to the initial displacement in transversal direction, which is given in the third terms of equations 7.56, 7.57 and 7.58. Starting with the incremental part of membrane strain, eq. 7.56, one can insert the expressions 7.34, 7.37, 7.27 and 7.32 and finds:

$$
\begin{aligned}
& \int_{V} \delta \varepsilon_{s}^{+} E\left(\frac{\stackrel{+}{\varepsilon_{s}^{P}}+\mu \stackrel{+}{\varepsilon_{x}^{P}}}{1-\mu^{2}}+\stackrel{+}{M}_{s}^{M}\right) \mathrm{d} V=\int_{V}\left(\bar{u}_{, s} \delta u_{, s}^{+}+\bar{w}_{, s} \delta_{w_{, s}}^{+}\right) E
\end{aligned}
$$

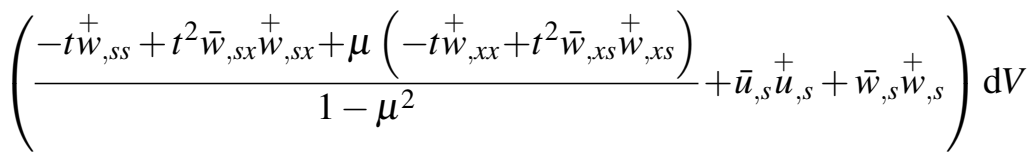

It is possible to observe from the above expression that all linear terms vanish. Consequently, there are only quadratic terms:

$$
\int_{V} \delta \varepsilon_{s}^{+} E\left(\frac{\stackrel{+}{\varepsilon_{s}^{P}}+\mu \dot{\varepsilon}_{x}^{P}}{1-\mu^{2}}+\dot{\varepsilon}_{s}^{+}\right) \mathrm{d} V=\int_{V}\left(\bar{u}_{, s} \delta_{u_{, s}}^{+}+\bar{w}_{, s} \delta \stackrel{+}{w_{, s}}\right) E\left(\frac{t^{2} \bar{w}_{, s x} \stackrel{+}{w_{, s x}}(1+\mu)}{1-\mu^{2}}+\bar{u}_{, s} \stackrel{+}{u}, s^{+} \bar{w}_{, s}{ }^{+}, s\right) \mathrm{d} V
$$


The evaluation of membrane's part leads to:

$$
\begin{aligned}
& \int_{V} \delta \varepsilon_{s}^{+} E \varepsilon_{s}^{M} \mathrm{~d} V=\int_{V}\left(\bar{u}_{, s} \delta \dot{u}_{, s}^{+}+\bar{w}_{, s} \delta \stackrel{+}{w}, s\right) E\left(\bar{u}_{, s} \stackrel{+}{u}, s^{+}+\bar{w}_{, s} \stackrel{+}{w}, s\right) \mathrm{d} V= \\
& E \sum_{i=1}^{n} \sum_{j=1}^{n} \sum_{k=1}^{n} \sum_{l=1}^{n} \int_{V}\left({ }^{i} \bar{u}_{, s}(s)^{i} \bar{V}_{, x}(x)^{l}{ }^{+}{ }_{, s}(s) \delta^{l} \stackrel{V}{, x}_{,}^{+}+{ }^{i} \bar{w}_{, s}(s)^{i} \bar{V}(x)^{l}{ }^{+}{ }_{, s}(s) \delta^{l}{ }_{V}^{+}\right)
\end{aligned}
$$

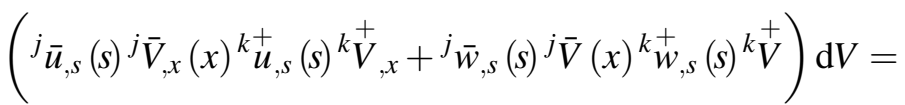

$$
\begin{aligned}
& E t\left({ }^{i l j k} \mathbb{S}_{u 4}^{M M i j} \mathbb{V}_{X 4}^{k l}+2^{i l j k} \mathbb{S}_{u w 4}^{M M i j} \mathbb{V}_{X S 4}^{k l}+{ }^{i l j k} \mathbb{X}_{4}^{P P i j} \mathbb{V}_{S 4}^{k l}\right)
\end{aligned}
$$

And the plate's part to:

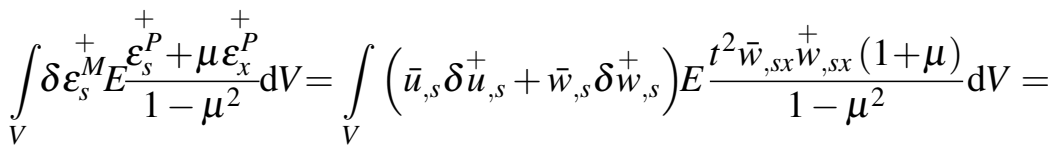

$$
\begin{aligned}
& K(1+\mu) \sum_{i=1}^{n} \sum_{j=1}^{n} \sum_{k=1}^{n} \sum_{l=1}^{n} \int_{V}\left({ }^{i} \bar{u}_{, s}(s)^{i} \bar{V}_{, x}(x){ }^{l}{ }^{+}, s(s) \delta^{l}{ }_{V}^{+}, x+{ }^{i} \bar{w}_{, s}(s)^{i} \bar{V}(x)^{l}{ }^{+}{ }_{, s}(s) \delta^{l}{ }_{V}^{+}\right) \\
& \left.{ }^{j} \bar{w}_{, s}(s){ }^{j} \bar{V}_{, x}(x){ }^{k}{ }_{w}^{+}, s\right)^{k}{ }_{V, x}^{+} \mathrm{d} V=K(1+\mu)\left({ }^{i l j k} \mathbb{S}_{u w 4}^{M M} \mathbb{V}_{X 4}+{ }^{i l j k} \mathbb{X}_{4}^{P P} \mathbb{V}_{X S 4}\right)
\end{aligned}
$$

The incremental parts of plate strain, equations 7.57 and 7.58 , are developed simultaneously. The introduction of expressions $7.39,7.29,7.37,7.27$ and 7.32 in the sum of these equations achieves:

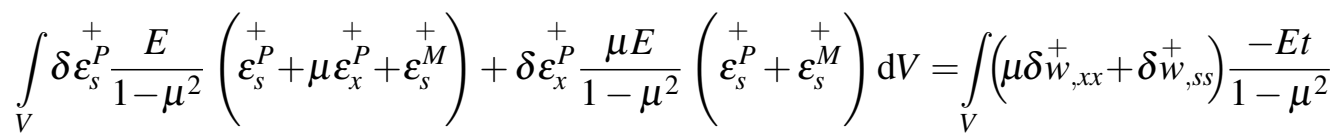

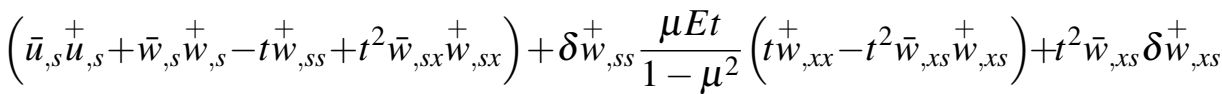

$$
\begin{aligned}
& \frac{E}{1-\mu^{2}}\left((1+\mu)\left(\bar{u}_{, s} \stackrel{+}{u}, s_{,}+\bar{w}_{, s} \stackrel{+}{w}_{, s}-t \stackrel{+}{w}_{, s s}+t^{2} \bar{w}_{, s x} \stackrel{+}{w}, s x^{\prime}\right)-\mu\left(t \stackrel{+}{w}, x x-t^{2} \bar{w}_{, x s} \stackrel{+}{w}, x s\right)\right) \mathrm{d} V
\end{aligned}
$$

The elimination of the terms, which involves integrals of odd functions in the thickness domain, simplifies the above expression into:

$$
\begin{aligned}
& \int_{V}\left(\mu \delta_{w}^{+}, x x+\delta \delta_{w, s s}^{+}\right) \frac{E t^{2}}{1-\mu^{2}} \stackrel{+}{w}, s s^{+}+\delta_{w}^{+}, \frac{\mu E t^{2}}{1-\mu^{2}} \stackrel{+}{w}, x x^{+}+ \\
& t^{2} \bar{w}_{, x s} \delta \stackrel{+}{w}, x s^{+} \frac{E}{1-\mu^{2}}\left((1+\mu)\left(\bar{u}_{, s} \stackrel{+}{u}, s^{+}+\bar{w}_{, s} \stackrel{+}{w}, s^{+}+t^{2} \bar{w}_{, x s} \stackrel{+}{w}, x s^{+}\right)+\mu t^{2} \bar{w}_{, s x} \stackrel{+}{w}, s x^{+}\right) \mathrm{d} V=
\end{aligned}
$$

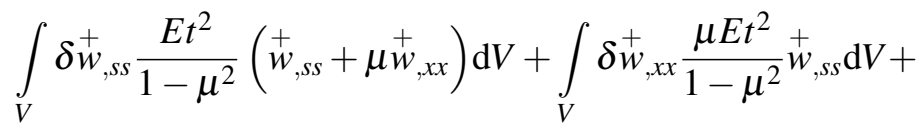

$$
\begin{aligned}
& \int_{V} \bar{w}_{, x s} \delta \delta^{+}, \frac{E t^{2}}{1-\mu^{2}}\left((1+\mu)\left(\bar{u}_{, s} \stackrel{+}{u}, s^{+}+\bar{w}_{, s} \stackrel{+}{w}, s^{\prime}\right)+(1+2 \mu) t^{2} \bar{w}_{, x s} \stackrel{+}{w}, x s\right) \mathrm{d} V
\end{aligned}
$$


The interpretation of this expression is entirely analogous to expression in the longitudinal direction, eq. 7.94. Thus, the first and the second resulting integrals are the virtual work, at the incremental stage, of the plate's displacement in transversal and longitudinal direction, respectively. Thus, they lead to the linear stiffness matrix at the corresponding stage. The third resulting integral is the virtual work of initial transversal displacement. One divides and evaluates this integral in two terms: i) quadratic between plates, which has the same evaluation of eq.: 7.97 and ii) quadratic between plate and membrane, from which the evaluation is:

$$
\begin{aligned}
& \int_{V} \bar{w}_{, x s} \delta \stackrel{+}{w}, x s^{\frac{E t^{2}}{1-\mu^{2}}}(1+\mu)\left(\bar{u}_{, s} \stackrel{+}{u}, s_{,}+\bar{w}_{, s} \stackrel{+}{w}, s^{\prime}\right) \mathrm{d} V=K(1+\mu) \sum_{i=1}^{n} \sum_{j=1}^{n} \sum_{k=1}^{n} \sum_{l=1}^{n} \\
& \int_{V}{ }^{i} \bar{w}_{, s}(s)^{i} \bar{V}_{, x}(x)^{l}{ }^{+}{ }_{, s}(s) \delta^{l} V_{, x}^{+}\left({ }^{j} \bar{u}_{, s}(s){ }^{j} \bar{V}_{, x}(x){ }^{k}{ }_{u, s}^{+}(s){ }^{k} V_{, x}^{+}+{ }^{j} \bar{w}_{, s}(s){ }^{j} \bar{V}(x){ }^{k}{ }_{w, s}^{+}(s)^{k}{ }^{+}\right) \mathrm{d} V= \\
& K(1+\mu)\left({ }^{i l j k} \mathbb{S}_{u w 4}^{M M i j} \mathbb{V}_{X 4}^{k l}+{ }^{i l j k} \mathbb{X}_{4}^{P P i j} \mathbb{V}_{S X 4}^{k l}\right)
\end{aligned}
$$

Table 7.4 presents the summary of all possibilities in the virtual work of initial displacement in transversal direction:

\begin{tabular}{|c|c|c|c|}
\hline \multirow{2}{*}{ Relation } & \multirow{2}{*}{$\begin{array}{l}\text { initial } \\
\text { displ. in: }\end{array}$} & \multicolumn{2}{|c|}{ incremental strain in: } \\
\hline & & Membrane: & Plate: \\
\hline \multirow[t]{2}{*}{ Quadratic } & Membrane & $\begin{array}{c}\text { eq. 7.100: } \quad E t\left({ }^{i l j k} \mathbb{S}_{u 4}^{M M i j} \mathbb{V}_{X 4}^{k l}\right. \\
\left.+2^{i l j k} \mathbb{S}_{u w 4}^{M M i j} \mathbb{V}_{X S 4}^{k l}+{ }^{i l j k} \mathbb{X}_{4}^{P P i j} \mathbb{V}_{S 4}^{k l}\right)\end{array}$ & $\begin{array}{c}\text { eq. 7.104: } \quad K(1+\mu) \\
\left({ }^{i l j k} \mathbb{S}_{u w 4}^{M M i j} \mathbb{V}_{X 4}^{k l}+{ }^{i l j k} \mathbb{X}_{4}^{P P i j} \mathbb{V}_{S X 4}^{k l}\right)\end{array}$ \\
\hline & Plate & $\begin{array}{c}\text { eq. 7.101: } \quad K(1+\mu) \\
\left({ }^{i l j k} \mathbb{S}_{u w 4}^{M M i j} \mathbb{V}_{X 4}^{k l}+{ }^{i l j k} \mathbb{X}_{4}^{P P i j} \mathbb{V}_{X S 4}^{k l}\right)\end{array}$ & $\begin{array}{l}\text { eq. 7.97: } \quad(1+2 \mu) \\
\frac{3 t^{2} K}{40}{ }^{i l j k} \mathbb{X}_{4}^{P P i j} \mathbb{V}_{X 4}^{k l}\end{array}$ \\
\hline
\end{tabular}

Table 7.4: Summary of virtual work of the initial displacement in the incremental stages in transversal direction.

\subsection{Internal forces evaluation in non-linear GBT}

In the non-linear analysis, the evaluation of internal forces plays a central role, since all incremental/iterative procedure is based on the balance of this force and the external one. In the particular case of GBT, the evaluation of internal forces, based on the first integrals from equation 7.48 to 7.50 and from equation 7.56 to 7.58 , will lead again to the coupling tensors.

For instance, the introduction of equations 7.24, 7.26, 7.36 and 7.21 into the first integral of eq. 7.48 results in the internal force due to the membrane strain behavior in the 
longitudinal direction:

$$
\begin{aligned}
& \int_{V} \delta \varepsilon_{x}^{+} E\left(\frac{\bar{\varepsilon}_{x}^{P}+\mu \varepsilon_{s}^{P}}{1-\mu^{2}}+\bar{\varepsilon}_{x}^{M}\right) \mathrm{d} V=\int_{V} \delta \dot{u}_{, x}^{+}+\left(\bar{v}_{, x} \delta \bar{v}_{, x}^{+}+\bar{w}_{, x} \delta w_{, x}^{+}\right) E \\
&\left(\frac{-t \bar{w}_{, x x}+\frac{t^{2} \bar{w}_{, x s}^{2}}{2}+\mu\left(-t \bar{w}_{, s s}+\frac{t^{2} \bar{w}_{, x s}^{2}}{2}\right)}{1-\mu^{2}}+\bar{u}_{, x}+\frac{\bar{v}_{, x}^{2}+\bar{w}_{, x}^{2}}{2}\right) \mathrm{d} V
\end{aligned}
$$

From the above expression, one realizes that: i) the integral ovter the thickness domains eliminates the linear terms $t \bar{w}_{, x x}$ and $t \bar{w}_{, s s}$; ii) the integral $\int_{V} \delta \boldsymbol{u}_{, x}^{+} \bar{u}_{, x} \mathrm{~d} V$ is nothing more than longitudinal membrane internal force of the linear analysis of the initial membrane displacement; iii) longitudinal membrane internal forces, due to initial plate displacements are always quadratic. The evaluation of the terms related only to membrane behavior leads to:

- linear membrane internal force due to initial membrane displacements:

$$
\begin{aligned}
& \int_{V} \delta u_{, x} E \\
& \frac{\bar{v}_{, x}^{2}+\bar{w}_{, x}^{2}}{2} \mathrm{~d} V=\frac{E}{2} \sum_{i=1}^{n} \sum_{j=1}^{n} \sum_{k=1}^{n} \int_{V}{ }^{i} u(s)^{i} \delta \stackrel{V}{, x x}_{, x}(x) \\
&\left({ }^{j} \bar{v}(s)^{j} \bar{V}_{, x}(x){ }^{k} \bar{v}(s){ }^{k} \bar{V}_{, x}(x)+{ }^{j} \bar{w}(s)^{j} \bar{V}_{, x}(x)^{k} \bar{w}(s)^{k} \bar{V}_{, x}(x)\right) \mathrm{d} V=\frac{E}{2} t^{i j k} \mathbb{X}_{3}^{M M}{ }^{j k} \mathbb{V}_{X 3}^{i}
\end{aligned}
$$

Here, one must be aware that the integral ${ }^{j k} \mathbb{V}_{X 3}^{i}$ is related to two initial displacement modes, and not just one as presented in the evaluations of initial linear stresses and displacements matrices. Thus, the result of this integral is a vector and not a matrix.

- linear membrane internal force due to initial membrane stress:

$$
\begin{aligned}
& \int_{V}\left(\bar{v}_{, x} \boldsymbol{\delta} \dot{v}_{, x}+\bar{w}_{, x} \boldsymbol{\delta}_{w_{, x}}^{+}\right) E \bar{u}_{, x} \mathrm{~d} V=E \sum_{i=1}^{n} \sum_{j=1}^{n} \sum_{k=1}^{n} \int_{V}\left({ }^{i} \bar{v}(s)^{i} \bar{V}_{, x}(x)^{j}{ }_{v}^{+}(s)^{j} \boldsymbol{\delta}^{+}, x(x)+\right. \\
& \left.{ }^{i} \bar{w}(s)^{i} \bar{V}_{, x}(x)^{j}{ }^{+}(s)^{j} \delta \stackrel{V}{V}, x^{+}(x)\right){ }^{k} \bar{u}(s)^{k} \bar{V}_{, x x}(x) \mathrm{d} V=E t^{k i j} \mathbb{X}_{3}^{M M i k} \mathbb{V}_{X 3}^{j}
\end{aligned}
$$

- quadratic membrane internal force due to initial membrane stress:

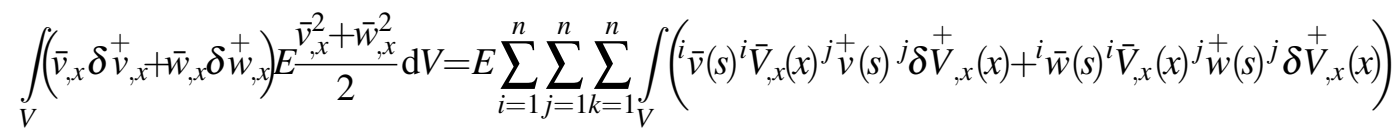

$$
\begin{aligned}
& \left({ }^{k} \bar{v}(s)^{k} \bar{V}_{, x}(x)^{l} \bar{v}(s)^{l} \bar{V}_{, x}(x)+{ }^{k} \bar{w}(s)^{k} \bar{V}_{, x}(x)^{l} \bar{w}(s)^{l} \bar{V}_{, x}(x)\right) \mathrm{d} V=\frac{E t_{i j k l}}{2} \mathbb{X}_{4}^{M M i k l} \mathbb{V}_{X 4}^{j}
\end{aligned}
$$

And the evaluation of terms involving the plate strains leads to: 
- linear membrane internal force due to initial plate displacements:

$$
\int_{V} \delta u_{, x}^{+} \frac{E}{2}(1+\mu) \frac{t^{2} \bar{w}_{, x s}^{2}}{1-\mu^{2}} \mathrm{~d} V=(1+\mu) \frac{K_{i j k}}{2} \mathbb{X}_{3}^{M P j k} \mathbb{V}_{X 3}^{i}
$$

- quadratic membrane internal force due to initial plate stress:

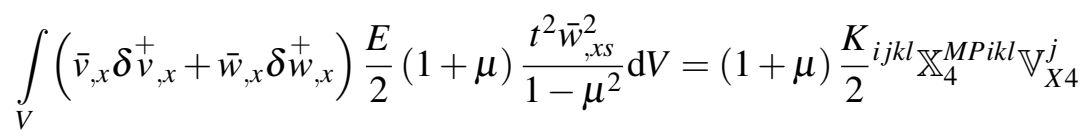

One obtains the longitudinal plate internal forces in a similar way. The evaluation of the first integral in eq. 7.49 leads to the following components:

- linear plate internal force due to initial membrane displacements:

$$
\int_{V} t^{2} \bar{w}_{, x s} \delta_{w_{, x s}}^{+} \frac{E}{\left(1-\mu^{2}\right)} \bar{u}_{, x} \mathrm{~d} V=K^{k i j} \mathbb{X}_{3}^{M P i k} \mathbb{V}_{X 3}^{j}
$$

- quadratic plate internal force due to initial membrane displacements:

$$
\int_{V} t^{2} \bar{w}_{, x s} \delta w_{, x s}^{+} \frac{E}{\left(1-\mu^{2}\right)} \frac{\bar{v}_{, x}^{2}+\bar{w}_{, x}^{2}}{2} \mathrm{~d} V=\frac{K^{k i j l}}{2} \mathbb{X}_{4}^{M P i k l} \mathbb{V}_{X 4}^{j}
$$

- quadratic plate internal force due to initial plate displacements:

$$
\int_{V} t^{2} \bar{w}_{, x s} \delta w_{, x s}^{+} \frac{E}{\left(1-\mu^{2}\right)}(1+\mu) t^{2} \bar{w}_{, x s}^{2} \mathrm{~d} V=3(1+\mu) \frac{K t^{2}}{20}{ }_{k i j l} \mathbb{X}_{4}^{P P i k l} \mathbb{V}_{X 4}^{j}
$$

The integral of the first term in eq. 7.56 achieves the respective membrane transversal internal forces:

- transversal quadratic membrane internal force due to initial membrane displacements:

$$
\int_{V} \delta \varepsilon_{s}^{+} E \varepsilon_{s}^{M} \mathrm{~d} V=\frac{E t}{2\left(1-\mu^{2}\right)}\left[{ }^{i j k l} \mathbb{S}_{u 4}^{M M i k l} \mathbb{V}_{X 4}^{j}+{ }^{i j k l} \mathbb{S}_{u w 4}^{M M i k l} \mathbb{V}_{X S 4}^{j}+{ }^{k l i j} \mathbb{S}_{u w 4}^{M M i k l} \mathbb{V}_{S X 4}^{j}+{ }^{i j k l} \mathbb{X}_{4}^{P P i k l} \mathbb{V}_{S 4}^{j}\right](7
$$

- transversal quadratic membrane internal force due to initial plate displacements:

$$
\int_{V} \delta \varepsilon_{s}^{+} E^{-} \frac{-\overline{\varepsilon_{s}^{P}}+\mu \varepsilon_{x}^{P}}{1-\mu^{2}} \mathrm{~d} V=(1+\mu) \frac{K}{2}\left[{ }^{i j k l} \mathbb{S}_{u w 4}^{M M i k l} \mathbb{V}_{X 4}^{j}+{ }^{i j k l} \mathbb{X}_{4}^{P P i k l} \mathbb{V}_{S X 4}^{j}\right]
$$

And the integral of the first term in eq. 7.57 achieves the respective plate transversal internal forces: 
- transversal quadratic plate internal force due to initial membrane displacements:

$$
\int_{V} \delta \varepsilon_{s}^{+} \frac{E}{1-\mu^{2}} \varepsilon_{s}^{-} \mathrm{d} V=\frac{K}{2}\left[{ }^{k l i j} \mathbb{S}_{u w 4}^{M M i k l} \mathbb{V}_{X 4}^{j}+{ }^{i j k l} \mathbb{X}_{4}^{P P i k l} \mathbb{V}_{X S 4}^{j}\right]
$$

- transversal quadratic plate internal force due to initial plate displacements:

$$
\int_{V} \delta \varepsilon_{s}^{+} \frac{E}{1-\mu^{2}}\left(\bar{\varepsilon}_{s}^{P}+\mu \bar{\varepsilon}_{x}^{P}\right) \mathrm{d} V=3(1+\mu) \frac{K t^{2}}{40}{ }^{i j k l} \mathbb{X}_{4}^{P P i k l} \mathbb{V}_{X 4}^{j}
$$

The internal forces due to Poisson's effect have the same evaluation. Starting with eq. 7.50, one obtains the following cases:

- transversal linear and quadratic plate internal force due to initial membrane displacements:

$$
\int_{V} \delta \varepsilon_{s}^{+} \frac{\mu E}{1-\mu^{2}} \varepsilon_{x}^{M} \mathrm{~d} V=\mu K\left[{ }^{k i j} \mathbb{X}_{3}^{M P k i} \mathbb{V}_{X 3}^{j}+\frac{1}{2}{ }^{k l i j} \mathbb{X}_{4}^{M P i k l} \mathbb{V}_{X 4}^{j}\right]
$$

- transversal quadratic plate internal force due to initial plate displacements:

$$
\int_{V} \delta \varepsilon_{s}^{P} \frac{\mu E}{1-\mu^{2}} \varepsilon_{x}^{M} \mathrm{~d} V=\frac{3 \mu K t^{2}}{40}{ }^{k l i j} \mathbb{X}_{4}^{M P k l i} \mathbb{V}_{X 4}^{j}
$$

Finally, the evaluation of the first integral of eq. 7.58 leads to:

- transversal quadratic plate internal force due to initial membrane:

$$
\int_{V} \delta \varepsilon_{x}^{+} \frac{\mu E}{1-\mu^{2}} \varepsilon_{s}^{-} \mathrm{d} V=\frac{\mu K}{2}\left[{ }^{k l i j} \mathbb{S}_{u w 4}^{M M k l i} \mathbb{V}_{X 4}^{j}+{ }^{k l i j} \mathbb{X}_{4}^{P P k l i} \mathbb{V}_{S 4}^{j}\right]
$$

- transversal quadratic plate internal force due to initial plate:

$$
\int_{V} \delta \varepsilon_{x}^{+} \frac{\mu E}{1-\mu^{2}} \bar{\varepsilon}_{s}^{P} \mathrm{~d} V=\frac{3 \mu K t^{2}}{40}{ }^{k l i j} \mathbb{X}_{4}^{M P k l i} \mathbb{V}_{X 4}^{j}
$$

\subsection{Properties of GBT's coupling tensors}

The coupling tensors of GBT have two interesting properties that deserve special attention.

The first property is the practical nature of these tensors, which provides a map of all possible coupling effects of a cross-section. For instance, the third-order tensor of initial stress from the membrane to membrane strains, ${ }^{i j k} X_{3}^{M M}$, indicates how initial stress from the coupler mode i can build the relationship between the coupled displacement mode 
$\mathrm{j}$ and the coupled force mode $\mathrm{k}$. It is important to note that one obtains this tensor only based on geometric properties of the cross-section. Therefore, GBT can provide a priori qualitative analysis of the coupling problems of a thin-walled beam. For example: in a hypothetical application, the major external forces is the bending moment (mode 3 ) and symmetric distortion mode, such as mode 5. With this information, one can search for an optimal cross-section which has the minimal value possible for the ${ }^{i 35} X_{3}$ or ${ }^{i 53} X_{3}$. Although one must perform a fully non-linear analysis for total quantification of the second or higher-order effects, this tensor property is helpful in firsts stages in the design of thin-walled structures.

The second property of GBT's coupling tensors is their symmetric. The third-order tensors always hold the property ${ }^{i j k} X_{3}={ }^{i k j} X_{3}$ and the fourth-order the property ${ }^{i j k l} X_{4}=$ ${ }^{i j l k} X_{4}={ }^{j i k l} X_{4}={ }^{j i l k} X_{4}$. Consequently, there is a reduction of the numerical effort in their evaluation as well as computational implementation. GBT's tensors not only lead to a symmetric system of data, but are also reused in different manners. The tensor used to set up the initial matrix is the same to set up the corespondent initial displacement matrix. The only difference is the order of reading the tensors, as mentioned in the previous sections. Also, the coupling tensors are required in the evaluation of internal forces in each incremental/iteration step, as showed in the next section.

\subsection{Development of geometric stiffness matrices in GBT}

After the development and analysis of GBT's coupling tensors, it is necessary to develop for each term in these tensors to the respective stiffness matrix. Here, the non-linear stiffness matrices present main differences from the linear stiffness matrices developed in Chapter 5. This difference concerns the amount of shape functions. Linear analysis requires only 2 shape functions to develop the stiffness matrix. However, in non-linear analysis, it is necessary 3 or 4 shapes functions, of different modes, to achieve the stiffness matrix.

One can develop the non-linear stiffness matrices based on Hermitian shape functions, which has an extensive literature (such as [61, 179]), or based on hyperbolictrigonometric shape functions. Since the last option not only has smoother highderivative functions but also they are the exact solution of the linear analysis, the hyperbolic-trigonometric are adopted. This choice directly leads to arduous and extensive expression terms in the stiffness matrices. Therefore, the next subsection presents some strategies to simplify and optimize the evaluations of stiffness matrices of these functions.

\subsubsection{Simplification of the multiplication among several shape func- tions}

Although the expression in eq. 5.111 is a pair of different modes $i \neq j$, the GBT's nonlinear analysis cannot use this matrix directly. In fact, non-linear analysis requires coupling among three or four modes. However, the hyperbolic-trigonometric functions have 
several mathematical properties that enable the use of eq. 5.111. These mathematical properties convert a multiplication between two simple modal hyperbolic-trigonometric functions into a sum of two linear combined modal hyperbolic-trigonometric functions:

$$
\begin{gathered}
\operatorname{Cos}\left({ }^{i} \beta\right) \operatorname{Cos}\left({ }^{j} \beta\right)=0.5\left[\operatorname{Cos}\left({ }^{i} \beta+{ }^{j} \beta\right)+\operatorname{Cos}\left({ }^{i} \beta-{ }^{j} \beta\right)\right] \\
\operatorname{Cos}\left({ }^{i} \beta\right) \operatorname{Sin}\left({ }^{j} \beta\right)=0.5\left[\operatorname{Sin}\left({ }^{i} \beta+{ }^{j} \beta\right)-\operatorname{Sin}\left({ }^{i} \beta-{ }^{j} \beta\right)\right] \\
\operatorname{Sin}\left({ }^{i} \beta\right) \operatorname{Sin}\left({ }^{j} \beta\right)=0.5\left[-\operatorname{Cos}\left({ }^{i} \beta+{ }^{j} \beta\right)+\operatorname{Cos}\left({ }^{i} \beta-{ }^{j} \beta\right)\right] \\
\operatorname{Sin}\left({ }^{i} \beta\right) \operatorname{Cos}\left({ }^{j} \beta\right)=0.5\left[\operatorname{Sin}\left({ }^{i} \beta+{ }^{j} \beta\right)+\operatorname{Sin}\left({ }^{i} \beta-{ }^{j} \beta\right)\right] \\
\operatorname{Cosh}\left({ }^{i} \beta\right) \operatorname{Cosh}\left({ }^{j} \beta\right)=0.5\left[\operatorname{Cosh}\left({ }^{i} \beta+{ }^{j} \beta\right)+\operatorname{Cosh}\left({ }^{i} \beta-{ }^{j} \beta\right)\right] \\
\operatorname{Cosh}\left({ }^{i} \beta\right) \operatorname{Sinh}\left({ }^{j} \beta\right)=0.5\left[\operatorname{Sinh}\left({ }^{i} \beta+{ }^{j} \beta\right)-\operatorname{Sinh}\left({ }^{i} \beta-{ }^{j} \beta\right)\right] \\
\operatorname{Sinh}\left({ }^{i} \beta\right) \operatorname{Sinh}\left({ }^{j} \beta\right)=0.5\left[\operatorname{Cosh}\left({ }^{i} \beta+{ }^{j} \beta\right)-\operatorname{Cosh}\left(\left({ }^{i} \beta-{ }^{j} \beta\right)\right]\right. \\
\operatorname{Sinh}\left({ }^{i} \beta\right) \operatorname{Cosh}\left({ }^{j} \beta\right)=0.5\left[\operatorname{Sinh}\left({ }^{i} \beta+{ }^{j} \beta\right)+\operatorname{Sinh}\left({ }^{i} \beta-{ }^{j} \beta\right)\right]
\end{gathered}
$$

Thus, in non-linear analysis, one can decompose the multiplication among several shape functions into the summation of pair multiplication of several linear combinations among the modes. For instance:

$$
{ }^{i} V(x){ }^{j} V(x){ }^{k} V(x)=0.5\left[{ }^{i+j} V(x)+{ }^{i \pm j} V(x)+{ }^{i-j} V(x)+{ }^{i \mp j} V(x)\right]{ }^{k} V(x) V(x)
$$

Here, modes $i+j, i \pm j, i-j$ and $i \mp j$ are the possible modes from the linear combinations between modes $i$ and $j$. The pairs $\alpha$ and $\beta$ of these modes are: - mode $i+j: \stackrel{+}{\alpha}$ and $\stackrel{+}{\beta}$; - mode $i \pm j: \stackrel{+}{\alpha}$ and $\bar{\beta}$; - mode $i-j: \bar{\alpha}$ and $\bar{\beta}$; - mode $i \mp j: \bar{\alpha}$ and $\stackrel{+}{\beta}$.

\subsubsection{Setup of GBT's non-linear stiffness matrices}

The simplification of the previous subsection optimize and systematize the process in the setup of the non-linear GBT's stiffness matrices. As an example of this procedure, one can evaluate the integral of $\mathbb{V}_{X 3}$, given in eq. 7.62 , based on equations 5.106 and 5.108:

$$
\begin{aligned}
& \mathbb{V}_{X 3}=\int_{x}{ }^{i} \bar{V}_{, x x}(x)^{j} \stackrel{+}{V, x}_{, x}^{+}(x) \delta^{k} \stackrel{+}{V}, x^{+}(x) \mathrm{d} x= \\
& \int_{L}^{i}\left[T x_{N H a}\right]^{i}\left[S h_{N H a c c}\right]_{, x x}{ }^{i}[\bar{\vartheta}]^{k}\left[S h_{N H a c c}\right]_{, x}^{T}{ }^{k}\left[T x_{N H a}\right]^{T}\left[T x_{N H a}\right] \mathrm{d} x^{j}\left[S h_{N H a c c}\right]_{, x}^{j}[\stackrel{+}{\vartheta}]
\end{aligned}
$$

In the above expression, it is important to highlight the meaning of each index. The initial stress in mode $i$ is the coupler mode that connects the forces in coupled mode $j$ to the virtual displacement of coupled-mode k. To simplify this expression, it is important to divide the vector ${ }^{i}\left[T x_{N H a}\right]$ into its components:

$\left[\begin{array}{llllll}\cosh \left({ }^{i} \alpha x\right) \cos \left({ }^{i} \beta x\right) & \cosh \left({ }^{i} \alpha x\right) \sin \left({ }^{i} \beta x\right) & \sinh \left({ }^{i} \alpha x\right) \sin \left({ }^{i} \beta x\right) & \sinh \left({ }^{i} \alpha x\right) \cos \left({ }^{i} \beta x\right) & x & 1\end{array}\right]$. 
It is clear that each part must be preceded by the correct completeness constant and

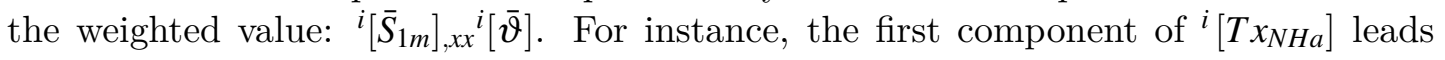
to:

$$
{ }_{1} V_{X 3}=\left[\bar{S}_{1 m}\right]_{, x x}{ }^{i}[\bar{\vartheta}]^{k}\left[S h_{N H a c c}\right]_{, x}^{T} \int_{L} \cosh \left({ }^{i} \alpha x\right) \cos \left({ }^{i} \beta x\right)^{k}\left[T x_{N H a}\right]^{T j}\left[T x_{N H a}\right] \mathrm{d} x^{j}\left[S h_{N H a c c}\right]_{, x}^{j}[\vartheta](7 .
$$

Then, one can transform each term in the multiplication $\cosh \left({ }^{i} \alpha x\right) \cos \left({ }^{i} \beta x\right)^{k}\left[T x_{N H a}\right]^{T}$ into a sum of the combined modes $i$ and $k$, as presented from eq. 7.122 to 7.129. Thus, each row of the original stiffness matrix is a linear combination of the rows of the combined stiffness matrices obtained from eq. 5.112. For the above multiplication, which involves $\cosh \left({ }^{i} \alpha x\right) \cos \left({ }^{i} \beta x\right)$, one obtains the following linear combination:

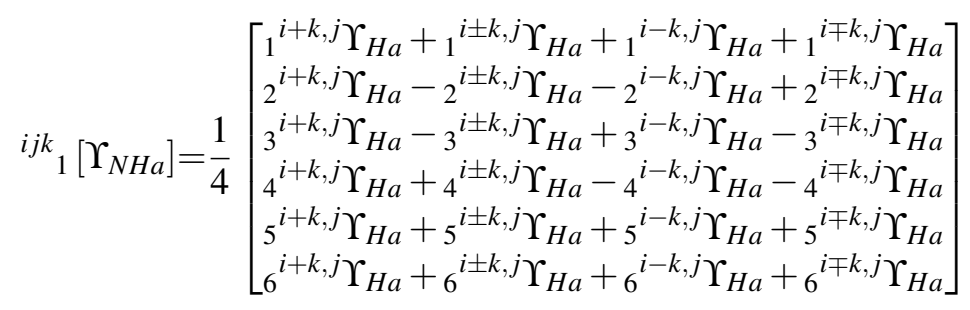

For the others terms $\cosh \left({ }^{i} \alpha x\right) \sin \left({ }^{i} \beta x\right), \sinh \left({ }^{i} \alpha x\right) \sin \left({ }^{i} \beta x\right)$ and $\sinh \left({ }^{i} \alpha x\right) \cos \left({ }^{i} \beta x\right)$, one achieves the respective matrices:

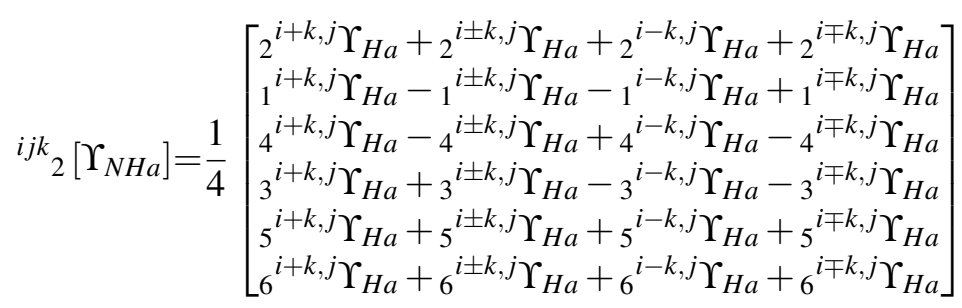

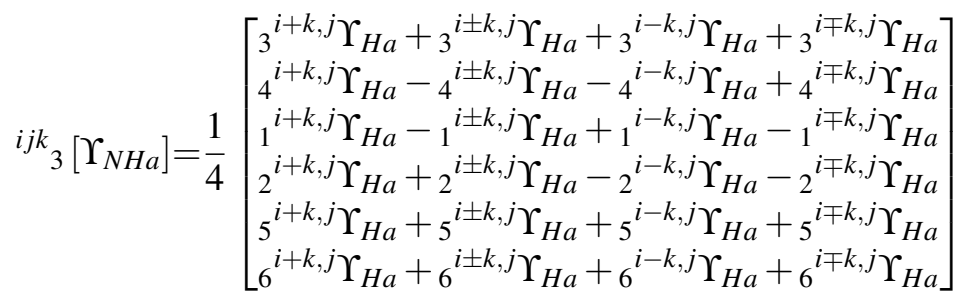

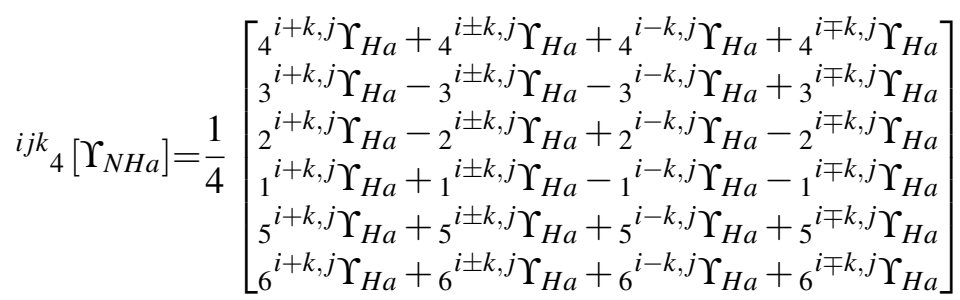

Concerning the linear and constant terms of the vector ${ }^{i}\left[\operatorname{Tx}_{N H a}\right]$, the development of their stiffness matrices is not necessary, since the second derivative eliminates these 
terms. For the first derivative, ${ }^{i} \bar{V}_{, x}(x)$, one obtains the non-linear stiffness matrix related to variation of $x$ term directly from eq. 5.111.

The final remark is about the quadratic stiffness matrices. These matrices involve the coupling of four modes, which requires the combination of modes $i, j$ and $l$. Thus, one must apply the simplification procedure twice. I.e., one obtains each quadratic stiffness matrix from a linear combination of matrices involves three modes. Each row of these matrices with three modes is also a linear combination of the matrices of two combined modes, as shown above. Consequently, one builds the stiffness matrices of three modes coupling from the combination of four stiffness matrices. Proportionately, one creates the stiffness matrices of four modes coupling from the combination of 16 stiffness matrices.

\subsection{Numerical example of non-linear analysis in coupling GBT and shell elements}

The goal of this example is the study of the non-linear effects in a GBT's element due to the load conditions transmitted from a structural connection modelled with shell elements. Thus, this example analyses the displacement field of a hollow circular column under different load conditions in the linear and non-linear analyses. This column is part of a structure, which is a combination of the components found in examples of Chapters 4, 5 and 6 . The $10 \mathrm{~m}$ height steel column, with hollow circular cross-section of Chapter 5 , has in its tip a connection to two steel gutter beams, as presented in figure. 7.2. The adopted steel properties are: Young Modulus, Young Modulus, $E=205,000 \mathrm{~N} / \mathrm{mm}^{2}$, Poisson's ratio, $\mu=0.0$, Shear Modulus, $G=102,500 \mathrm{~N} / \mathrm{mm}^{2}$.

The steel gutter beams have the profile of Chapter 4's example but in scale 1/10. Concerning the connection, it is a shell super-element model, developed in ANSYS ${ }^{\circledR}$ software with elements Shell-181 (linear interpolation elements based on Mindlin-Reissner Theory). The discretizations of the circular hollow and opened thin-walled cross-sections have 100 and 27 nodes, respectively (as presented in figure. 7.2.e). The connection's shell model has 1,916 elements and 12,120 degrees of freedom, in total. However, based on Super-element technique [105], the final stiffness matrix of the connection is reduced to 474 degrees of freedom. While, the GBT's discretization uses a single element for the column and each. Due to the symmetric of geometry, boundary conditions and load cases presented below, only symmetric and bi-symmetric modes are required to model the beams and the columns respectively. Therefore, one adopts for the column modes "a" and 1 (with 2 degrees of freedom each one) and modes: 5, 9, 13, 17, 21, 25, 29, 33 and 37 (with 6 degrees of freedom each one). The adopted mode of the beam are 3,6 and 7 (as presented in figures. A.2 and A.3), each mode has 6 degrees of freedom as well. As a total, there are 94 GBT's degrees of freedom.

Thus, the unconstrained and uncoupled model has 568 degrees of freedom. However, the boundary conditions and the coupling equations reduce the amount of free coupled 


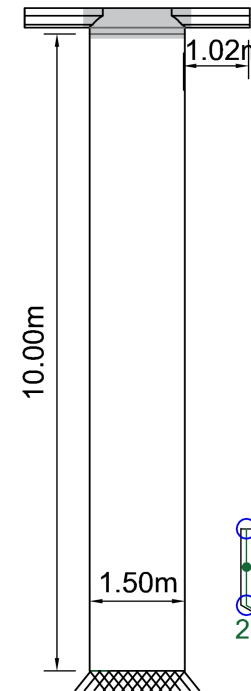

(a)

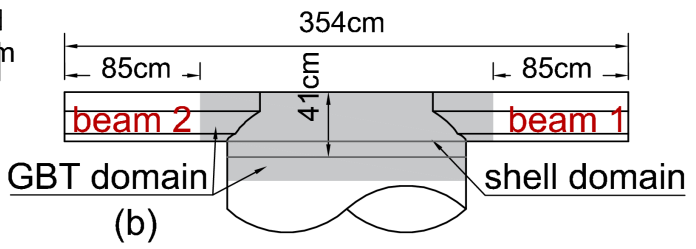

(b)

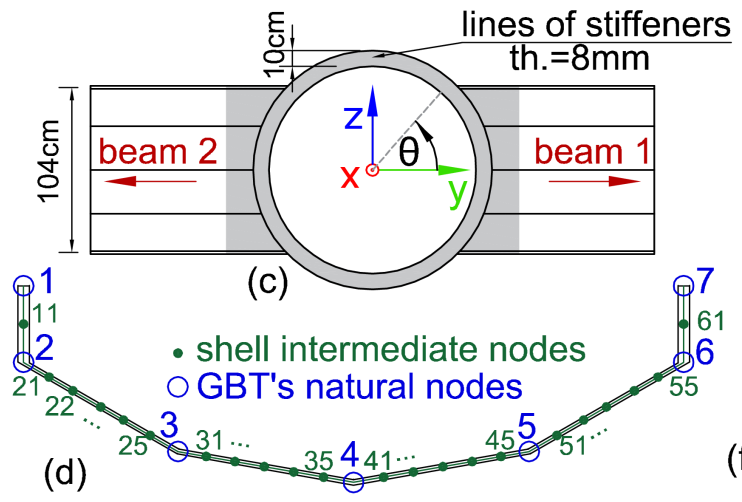

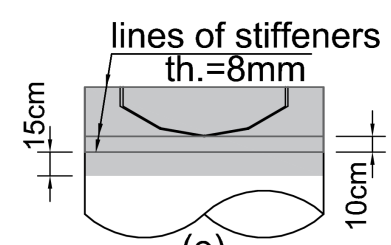

(e)

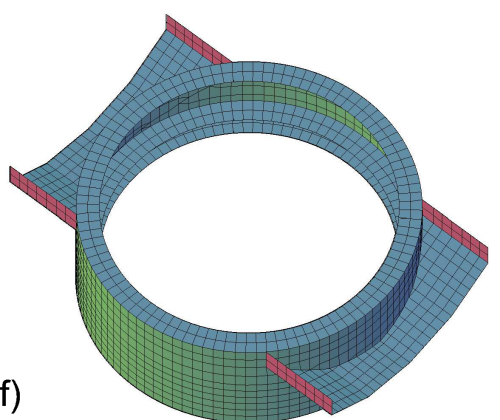

Figure 7.2: Coupling structure among thin-walled circular hollow section and two open thin-walled cross-sections: a)frontal elevation; b)connection's frontal elevation; c)top view; d)beams' discretization; e)connections left view; f) shell model's perspective.

degrees of freedom to 174. From this amount, 100 degrees of freedom concern to nodes in the shell connection, which are left free to receive load conditions.

In order to evaluate the performance of GBT's non-linear analysis in the column, this example uses two load cases. The first one is a periodic load function: $f_{x}(\theta)=-50-$ $20(\operatorname{Cos}(\theta)+(2 \theta))$, at the 100 degrees of freedom in the connection's super-element. The second load case has a surface distributed force of $0.1 \mathrm{kN} / \mathrm{cm}^{2}$ on the beams, as well as a concentrated load of $45 \mathrm{kN}$ at the 100 degrees of freedom in the connection's super-element.

\subsubsection{Setup of the mixed GBT-shell finite element model}

As a first step in the setup of the mixed GBT-shell model, one obtains the GBT crosssection properties of GBT for linear and non-linear.

For linear analysis, Table 7.5 presents the deformation modes of the hollow circular cross-section. The differences between the values of this table and Table 5.2 are related to Poisson's ratio, which is null in this example. Also, due to the symmetric conditions of load and geometry, only the bi-symmetrical modes of the hollow circular crosssection are adopted. Concerning the beams' cross-section, Table 7.6 shows not only the values of the symmetric modes used in this analysis, but also the modal forces in the second load case. One obtains these values from the modal eigenvectors presented in Table. A.97 of the appendix. In fact, the beams' cross-section of this example is proportional to the cross-section shown in Chapter 4. 
Table 7.5: Generalized properties: hollow circular cross-section

\begin{tabular}{cccc}
\hline Mode & $\begin{array}{c}C \\
\mathrm{~cm}^{4}\end{array}$ & $\begin{array}{c}D \\
\mathrm{~cm}^{4}\end{array}$ & $\begin{array}{c}B \\
\mathrm{~cm}^{-1}\end{array}$ \\
\hline $\mathrm{a}$ & 0 & 0 & 515.22 \\
5 & 397616.3 & 0.01 & 0.05 \\
9 & 397743.5 & 1.36 & 19.78 \\
13 & 398294.9 & 16.63 & 545.3 \\
17 & 399779.3 & 95.76 & 5584 \\
21 & 402909.3 & 369.49 & 33664.6 \\
25 & 408600.9 & 1110.11 & 145646.3 \\
29 & 417973.8 & 2809.68 & 501745.9 \\
33 & 432351.3 & 6275.54 & 1463736 \\
37 & 453260.2 & 12743.28 & 3761815.8 \\
\hline
\end{tabular}

Table 7.6: Generalized properties: Beams' cross-section

\begin{tabular}{ccccc}
\hline Mode & $\begin{array}{c}C \\
\mathrm{~cm}^{4}\end{array}$ & $\begin{array}{c}D \\
\mathrm{~cm}^{4}\end{array}$ & $\begin{array}{c}B \\
\mathrm{~cm}^{-1}\end{array}$ & $\begin{array}{c}\text { Load } \\
\frac{\mathrm{kN}}{c m}\end{array}$ \\
\hline 1 & 132.8 & 0 & 0 & 0 \\
3 & 12482.3 & 0 & 0 & -1.36 \\
6 & 24.19 & 0.0037 & 0 & 0.019 \\
7 & 28.29 & 0.0592 & 0.43 & 0.066 \\
$6-7$ & -4.63 & -0.0126 & -0.01 & 0 \\
\hline
\end{tabular}

One also uses these eigenvectors to setup the coupling equations between the degrees of freedom of beams' modes and the shell elements. Since the discretization of the beams' cross-section in shell elements uses more nodes than the GBT discretization, one needs to apply the coupling equations with interpolation functions, equations $6.5,6.9$ and 6.10. Based on these equations and eigenvectors, together with the nodal discretization of the beams' cross-section (figure 7.2.d), one obtains the coupling matrices (equations 7.137, 7.138 and 7.139) between the nodal displacement in the global coordinate system and GBT modes of Beam 1. For the coupling matrices for Beam 2, due to its orientation, one must multiply by -1 the matrices related to the global displacement $y$ and $z$. Concerning the coupling matrices between the hollow circular cross-section and the shell elements, they follow the same procedure presented in the example of Chapter 6 . The only difference here is the number of nodes in the discretization of the cross-section. 


\begin{tabular}{|c|c|c|c|c|}
\hline & & & & \\
\hline$y_{1}$ & 1 & -17.95 & 1 & -0.44 \\
\hline & 1 & -11.95 & 0.13 & 0.06 \\
\hline$y_{2}$ & 1 & -5.95 & -0.73 & 0.57 \\
\hline$y_{2}$ & 1 & -3.63 & -0.61 & 0.34 \\
\hline$y_{2}$ & 1 & -1.48 & -0.5 & 0.12 \\
\hline$y_{22}$ & 1 & 1.05 & -0.37 & -0.14 \\
\hline$y_{24}$ & & 3.11 & -0.26 & -0.35 \\
\hline$y_{25}$ & & 5.53 & -0.14 & -0.59 \\
\hline$y_{3}$ & & 8.05 & -0.01 & -0.85 \\
\hline$y_{31}$ & & 8.83 & 0.07 & -0.55 \\
\hline$y_{32}$ & & 9.62 & 0.15 & -0.25 \\
\hline$y_{33}$ & 1 & 10.43 & 0.24 & 0.06 \\
\hline$y_{34}$ & 1 & 11.25 & 0.32 & 0.37 \\
\hline$y_{35}$ & & 12.08 & 0.41 & 0.69 \\
\hline$y_{4}$ & 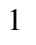 & 12.91 & 0.49 & 1 \\
\hline$y_{41}$ & 1 & 12.08 & 0.41 & 0.69 \\
\hline$y_{42}$ & 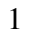 & 11.25 & 0.32 & 0.37 \\
\hline$y_{43}$ & 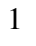 & 10.43 & 0.24 & 0.06 \\
\hline$y_{44}$ & & 9.62 & 0.15 & -0.25 \\
\hline$y_{45}$ & & 8.83 & 0.07 & -0.55 \\
\hline$y_{5}$ & & 8.05 & -0.01 & -0.85 \\
\hline$y_{51}$ & & 5.53 & -0.14 & -0.59 \\
\hline$y_{52}$ & & 3.11 & -0.26 & -0.35 \\
\hline$y_{53}$ & . & 1.05 & -0.37 & -0.14 \\
\hline$y_{54}$ & L & -1.48 & -0.5 & 0.12 \\
\hline$y_{55}$ & 1 & -3.63 & -0.61 & 0.34 \\
\hline$y_{6}$ & 1 & -5.95 & -0.73 & 0.57 \\
\hline$y_{61}$ & 1 & -11.95 & 0.13 & 0.06 \\
\hline$y_{7}$ & & -17.95 & 1 & -0.44 \\
\hline
\end{tabular}

$(7.137)$

\begin{tabular}{|c|c|c|}
\hline & ${ }^{6} v$ & ${ }^{7} v$ \\
\hline 4 & -0.21 & 0.49 \\
\hline 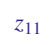 & -0.16 & 0.3 \\
\hline$z_{2}$ & -0.11 & 0.11 \\
\hline$z_{21}$ & -0.09 & 0.03 \\
\hline$z_{22}$ & -0.08 & -0.03 \\
\hline$z_{23}$ & -0.06 & -0.09 \\
\hline$z_{24}$ & -0.04 & -0.12 \\
\hline 225 & -0.03 & -0.14 \\
\hline$z_{3}$ & -0.01 & -0.12 \\
\hline$z_{31}$ & -0.01 & -0.11 \\
\hline$z_{32}$ & -0.01 & -0.08 \\
\hline$z_{33}$ & -0.0034 & -0.05 \\
\hline$z_{34}$ & -0.0015 & -0.03 \\
\hline$z_{35}$ & -0.0004 & -0.01 \\
\hline$z_{4}$ & 0 & 0 \\
\hline$z_{41}$ & 0.0004 & 0.01 \\
\hline$z_{42}$ & 0.0015 & 0.03 \\
\hline$z_{43}$ & 0.0034 & 0.05 \\
\hline$z_{44}$ & 0.01 & 0.08 \\
\hline$z_{45}$ & 0.01 & 0.11 \\
\hline$z_{5}$ & 0.01 & 0.12 \\
\hline$z_{51}$ & 0.03 & 0.14 \\
\hline 25 & 0.04 & 0.12 \\
\hline$z 53$ & 0.06 & 0.09 \\
\hline 25 & 0.08 & 0.03 \\
\hline$z_{5}$ & 0.09 & -0.03 \\
\hline$z_{6}$ & 0.11 & -0.11 \\
\hline$z_{6}$ & 0.16 & -0.3 \\
\hline$z 7$ & 0.21 & -0.49 \\
\hline
\end{tabular}

$(7.138)$

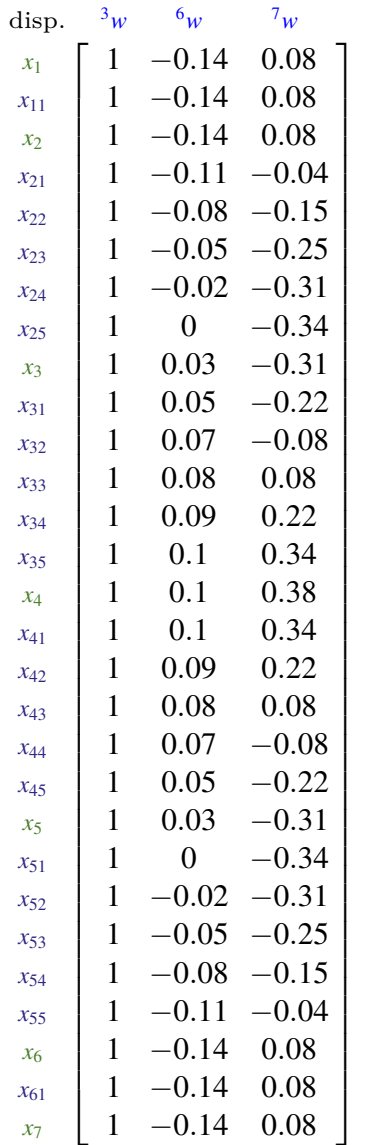

(7.139)

Concerning the non-linear analysis, the matrix in eq. 7.140 presents all terms related to the third-order tensor due to membrane behavior, ${ }^{i j k} \mathbb{X}_{3}^{M M}$. One obtains these terms from the evaluation of the integral in eq. 7.61 with the cross-section modal functions given in Table. 3.1. From the matrix in eq. 7.140, one realizes how the modal representation in GBT is useful in non-linear analysis: From all possibilities of third-order coupling (in this case $10^{3}$ ) only 117 cases exist. Also, if one excludes the symmetric cases, the coupling cases are 63 . Therefore, it is possible to plot all levels of the tensor ${ }^{i j k} \mathbb{X}_{3}^{M M}$ in a two-dimension matrix.

The fourth-order tensor, ${ }^{i j k l} \mathbb{X}_{4}^{M M}$, shows the same prosperity. From the all $10^{4}$ possible cases, there are only 1569 . If one considers the symmetric of the tensor, the number of coupling cases are 880. Since the exhibition of these cases is quite long for a quite tiny contribution in the final displacement field, the results of the integral in eq. 7.64 are not plotted here.

The final evaluation, in the setup of the mixed GBT-shell model, concerns in the assessment of the GBT stiffness matrices. Each beam and the column have only one element in discretization, with the lengths of 985 and $85 \mathrm{~cm}$, respectively. The procedure to achieve the numerical values of the linear stiffness matrices follows the same steps 
detailed in Chapter 5.

\begin{tabular}{|c|c|c|c|c|c|c|c|c|c|}
\hline$j \backslash k$ & 5 & 9 & 13 & 17 & 21 & 25 & 29 & 33 & 37 \\
\hline \multirow{4}{*}{5} & {$[i=1$} & $i=5$ & $i=9$ & $i=13$ & $i=17$ & $i=21$ & $i=25$ & $i=29$ & \\
\hline & 1413.7 & 190852 & 413512 & 720996 & 1113302 & 1590431 & 2152384 & 2799159 & $i=33$ \\
\hline & $i=9$ & $i=13$ & $i=17$ & $i=21$ & $i=25$ & $i=29$ & $i=33$ & $i=37$ & 3530757 \\
\hline & 31809 & 148440 & 349895 & 636173 & 1007273 & 1463197 & 2003943 & 2629513 & \\
\hline \multirow{4}{*}{9} & & $i=1$ & $i=5$ & $i=9$ & $i=13$ & $i=17$ & $i=21$ & & \\
\hline & & 19227 & 1590431 & 2799159 & 4347179 & 6234491 & 8461094 & $i=25$ & $i=29$ \\
\hline & & $i=17$ & $i=21$ & $i=25$ & $i=29$ & $i=33$ & $i=37$ & 11026990 & 13932178 \\
\hline & & 636173 & 1463197 & 2629513 & 4135121 & 5980022 & 8164214 & & \\
\hline \multirow{4}{*}{13} & & & $i=1$ & $i=5$ & $i=9$ & $i=13$ & & & \\
\hline & & & 94154 & 6234491 & 9701631 & 13932178 & $i=17$ & $i=21$ & $i=25$ \\
\hline & & & $i=25$ & $i=29$ & $i=33$ & $i=37$ & 18926132 & 24683493 & 31204262 \\
\hline & & & 3339906 & 5980022 & 9383545 & 13550475 & & & \\
\hline \multirow{4}{*}{17} & & & & $i=1$ & $i=5$ & & & & \\
\hline & & & & 294053 & 17176658 & $i=9$ & $i=13$ & $i=17$ & $i=21$ \\
\hline & & & & $i=33$ & $i=37$ & 24683493 & 33547497 & 43768669 & 55347009 \\
\hline & & & & 10687698 & 16752543 & & & & \\
\hline \multirow[b]{2}{*}{21} & & & & & $i=1$ & $i=5$ & $i=9$ & $i=13$ & $i=17$ \\
\hline & & & & & 713927 & 38488437 & 52325189 & 68282516 & 86360419 \\
\hline \multirow[b]{2}{*}{25} & & & & & & $i=1$ & $i=5$ & $i=9$ & $i=13$ \\
\hline & & & & & & 1475920 & 75259208 & 98225036 & 124244492 \\
\hline \multirow{2}{*}{29} & & & & & & & $i=1$ & $i=5$ & $i=9$ \\
\hline & & & & & & & 2729321 & 133596228 & 168999228 \\
\hline \multirow{2}{*}{33} & & & & & & & & $i=1$ & $i=5$ \\
\hline & & & & & & & & 4650562 & 220624627 \\
\hline 37 & sym. & & & & & & & & $\begin{array}{c}i=1 \\
\end{array}$ \\
\hline & & & & & & & & & \\
\hline
\end{tabular}

\subsubsection{Finite element solution}

After the setup of the mixed GBT-shell model, one can perform the finite element solution. Since ANSYS ${ }^{\circledR}$ provides the stiffness matrix of the connection's super-element only in linear analyses, this example adopts the modified Newton-Raphson method to solve the non-linear analysis. Thus, each iteration reuses the linear stiffness matrices. Nevertheless, the achievement of the internal forces uses widely the non-linear terms developed in this chapter, as explained in section 7.3.

Since, the linear analysis is the first iteration of the non-linear analysis in this example, Tables 7.7.a and 7.7.b show the column's accumulative modal displacements of both analyses for load cases 1 and 2, respectively. The initial degrees of freedom 1 and 2 are null for all GBT's modes.

Concerning the number of iterations, the non-linear procedure in mixed GBT-shell models uses the same amount of iterations of the ANSYS $^{\circledR}$ solution. 
Table 7.7: Accumulative modal displacements

\begin{tabular}{|c|c|c|c|c|c|c|c|c|}
\hline Mode & \multicolumn{4}{|c|}{ Iterations Load case 1} & \multicolumn{4}{|c|}{ Iterations Load case 2} \\
\hline DOF & 1 & 2 & 3 & 4 & 1 & 2 & 3 & 4 \\
\hline \begin{tabular}{c|c}
$a-2$ \\
\end{tabular} & $-1.86 \mathrm{E}-06$ & $-1.86 \mathrm{E}-06$ & $-1.90 \mathrm{E}-06$ & $-1.93 \mathrm{E}-06$ & $6.36 \mathrm{E}-06$ & $6.34 \mathrm{E}-06$ & $4.87 \mathrm{E}-06$ & $3.56 \mathrm{E}-06$ \\
\hline $1-2$ & $-1.70 \mathrm{E}+00$ & $-1.70 \mathrm{E}+00$ & $-1.74 \mathrm{E}+00$ & $-1.77 \mathrm{E}+00$ & $-1.61 \mathrm{E}+00$ & $-1.64 \mathrm{E}+00$ & $-3.10 \mathrm{E}+00$ & $-4.43 \mathrm{E}+00$ \\
\hline $5-3$ & $-1.70 \mathrm{E}-03$ & $-2.19 \mathrm{E}-03$ & $-2.31 \mathrm{E}-03$ & $-2.35 \mathrm{E}-03$ & $-1.41 \mathrm{E}-01$ & $-1.46 \mathrm{E}-01$ & $-1.47 \mathrm{E}-01$ & $-1.52 \mathrm{E}-01$ \\
\hline $5-4$ & $1.09 \mathrm{E}-04$ & $1.28 \mathrm{E}-04$ & $1.32 \mathrm{E}-04$ & $1.34 \mathrm{E}-04$ & $7.50 \mathrm{E}-04$ & $8.88 \mathrm{E}-04$ & $9.20 \mathrm{E}-04$ & $1.09 \mathrm{E}-03$ \\
\hline $5-5$ & $-1.41 \mathrm{E}-02$ & $-1.82 \mathrm{E}-02$ & $-1.92 \mathrm{E}-02$ & $-1.96 \mathrm{E}-02$ & $-1.61 \mathrm{E}-01$ & $-1.90 \mathrm{E}-01$ & $-1.97 \mathrm{E}-01$ & $-2.34 \mathrm{E}-01$ \\
\hline $5-6$ & $-2.99 \mathrm{E}-05$ & $-3.73 \mathrm{E}-05$ & $-3.91 \mathrm{E}-05$ & $-3.97 \mathrm{E}-05$ & $-4.02 \mathrm{E}-04$ & $-4.61 \mathrm{E}-04$ & $-4.80 \mathrm{E}-04$ & $-5.90 \mathrm{E}-04$ \\
\hline $9-3$ & $-1.22 \mathrm{E}-04$ & $-1.34 \mathrm{E}-04$ & $-1.36 \mathrm{E}-04$ & $-1.37 \mathrm{E}-04$ & $-4.53 \mathrm{E}-04$ & $-4.29 \mathrm{E}-04$ & $-4.07 \mathrm{E}-04$ & $-3.96 \mathrm{E}-04$ \\
\hline $9-4$ & $6.29 \mathrm{E}-06$ & $7.10 \mathrm{E}-06$ & $7.28 \mathrm{E}-06$ & 7.37E-06 & $7.34 \mathrm{E}-06$ & $5.57 \mathrm{E}-06$ & $4.06 \mathrm{E}-06$ & $3.43 \mathrm{E}-06$ \\
\hline $9-5$ & $-7.13 \mathrm{E}-05$ & $-6.50 \mathrm{E}-05$ & $-6.01 \mathrm{E}-05$ & $-5.81 \mathrm{E}-05$ & $-8.13 \mathrm{E}-05$ & $4.48 \mathrm{E}-05$ & $1.36 \mathrm{E}-04$ & $2.66 \mathrm{E}-04$ \\
\hline $9-6$ & $-8.16 \mathrm{E}-07$ & $-1.39 \mathrm{E}-06$ & $-1.72 \mathrm{E}-06$ & $-1.93 \mathrm{E}-06$ & $-1.12 \mathrm{E}-06$ & $1.91 \mathrm{E}-07$ & $1.68 \mathrm{E}-06$ & $3.16 \mathrm{E}-06$ \\
\hline $13-3$ & $6.32 \mathrm{E}-06$ & $6.92 \mathrm{E}-06$ & $6.96 \mathrm{E}-06$ & $6.93 \mathrm{E}-06$ & $-1.76 \mathrm{E}-04$ & $-1.94 \mathrm{E}-04$ & $-2.32 \mathrm{E}-04$ & $-3.37 \mathrm{E}-04$ \\
\hline $13-4$ & $-3.34 \mathrm{E}-07$ & $-3.90 \mathrm{E}-07$ & $-3.97 \mathrm{E}-07$ & -3.98 & $9.89 \mathrm{~F}$ & 1.13 & $1.34 \mathrm{E}-05$ & -05 \\
\hline $13-5$ & $-7.22 \mathrm{E}-08$ & $-2.61 \mathrm{E}-07$ & $-2.97 \mathrm{E}-07$ & $-3.24 \mathrm{E}-07$ & $2.14 \mathrm{E}-06$ & $9.78 \mathrm{E}-06$ & $-6.41 \mathrm{E}-06$ & $-3.03 \mathrm{E}-05$ \\
\hline $13-6$ & $-1.94 \mathrm{E}-09$ & $-2.17 \mathrm{E}-09$ & $-9.07 \mathrm{E}-10$ & $4.77 \mathrm{E}-10$ & $5.70 \mathrm{E}-08$ & $8.34 \mathrm{E}-08$ & $1.18 \mathrm{E}-06$ & $4.14 \mathrm{E}-06$ \\
\hline \begin{tabular}{l|}
$17-3$ \\
\end{tabular} & $1.01 \mathrm{E}-07$ & $6.96 \mathrm{E}-09$ & $-3.48 \mathrm{E}-08$ & $-5.86 \mathrm{E}-08$ & $-4.72 \mathrm{E}-05$ & $-5.08 \mathrm{E}-05$ & $-5.17 \mathrm{E}-05$ & $-5.60 \mathrm{E}-05$ \\
\hline $17-4$ & 3.01E-08 & $3.86 \mathrm{E}-08$ & $4.23 \mathrm{E}-08$ & 4.45 & $1.96 \mathrm{E}-06$ & 2.17 & -06 & -06 \\
\hline $17-5$ & $6.34 \mathrm{E}-12$ & $-6.73 \mathrm{E}-10$ & $1.45 \mathrm{E}-09$ & $2.32 \mathrm{E}-09$ & $2.46 \mathrm{E}-09$ & $1.13 \mathrm{E}-06$ & $1.33 \mathrm{E}-06$ & $2.05 \mathrm{E}-06$ \\
\hline $17-6$ & $-7.44 \mathrm{E}-13$ & $-1.82 \mathrm{E}-11$ & $-9.39 \mathrm{E}-12$ & $-2.75 \mathrm{E}-12$ & $-3.36 \mathrm{E}-11$ & $6.72 \mathrm{E}-09$ & $8.24 \mathrm{E}-09$ & $1.32 \mathrm{E}-08$ \\
\hline 21-3 & $1.51 \mathrm{E}-06$ & $1.65 \mathrm{E}-06$ & $1.67 \mathrm{E}-06$ & $1.68 \mathrm{E}-06$ & $1.57 \mathrm{E}-06$ & $2.30 \mathrm{E}-06$ & $2.33 \mathrm{E}-06$ & $2.95 \mathrm{E}-06$ \\
\hline $21-4$ & $-4.93 \mathrm{E}-08$ & $-5.96 \mathrm{E}-08$ & $-6.12 \mathrm{E}-08$ & $-6.14 \mathrm{E}-08$ & $-4.42 \mathrm{E}-07$ & $-5.68 \mathrm{E}-07$ & $-5.83 \mathrm{E}-07$ & $-6.69 \mathrm{E}-07$ \\
\hline $21-5$ & $1.29 \mathrm{E}-13$ & $-1.80 \mathrm{E}-08$ & $-2.02 \mathrm{E}-08$ & $-2.09 \mathrm{E}-08$ & $8.65 \mathrm{E}-13$ & $-1.49 \mathrm{E}-08$ & $-2.39 \mathrm{E}-08$ & $-4.51 \mathrm{E}-08$ \\
\hline $21-6$ & $1.06 \mathrm{E}-14$ & $-1.07 \mathrm{E}-10$ & $-1.25 \mathrm{E}-10$ & $-1.30 \mathrm{E}-10$ & $5.04 \mathrm{E}-14$ & $-2.35 \mathrm{E}-11$ & $-5.82 \mathrm{E}-11$ & $-4.15 \mathrm{E}-10$ \\
\hline \begin{tabular}{l|}
$25-3$ \\
\end{tabular} & $1.72 \mathrm{E}-06$ & $1.91 \mathrm{E}-06$ & $1.95 \mathrm{E}-06$ & $1.97 \mathrm{E}-06$ & \begin{tabular}{|l|}
$-3.42 \mathrm{E}-07$ \\
\end{tabular} & $-2.10 \mathrm{E}-07$ & $3.45 \mathrm{E}-07$ & $9.88 \mathrm{E}-07$ \\
\hline $25-4$ & $-3.62 \mathrm{E}-09$ & $-6.17 \mathrm{E}-09$ & $-6.26 \mathrm{E}-09$ & $-5.90 \mathrm{E}-09$ & $-3.89 \mathrm{E}-08$ & $-4.21 \mathrm{E}-08$ & $-1.60 \mathrm{E}-08$ & $1.47 \mathrm{E}-09$ \\
\hline $25-5$ & $5.35 \mathrm{E}-17$ & $-1.37 \mathrm{E}-08$ & $-1.55 \mathrm{E}-08$ & $-1.62 \mathrm{E}-08$ & $1.69 \mathrm{E}-17$ & $-3.03 \mathrm{E}-09$ & $-4.99 \mathrm{E}-09$ & $-1.16 \mathrm{E}-08$ \\
\hline $25-6$ & $5.11 \mathrm{E}-18$ & $-8.45 \mathrm{E}-11$ & $-9.82 \mathrm{E}-11$ & $-1.03 \mathrm{E}-10$ & $5.16 \mathrm{E}-19$ & $-1.95 \mathrm{E}-11$ & $-3.28 \mathrm{E}-11$ & $-7.43 \mathrm{E}-11$ \\
\hline $29-3$ & $-4.72 \mathrm{E}-07$ & $-5.36 \mathrm{E}-07$ & $-5.56 \mathrm{E}-07$ & $-5.68 \mathrm{E}-07$ & $-6.24 \mathrm{E}-06$ & $-6.99 \mathrm{E}-06$ & $-7.51 \mathrm{E}-06$ & $-8.80 \mathrm{E}-06$ \\
\hline $29-4$ & $1.85 \mathrm{E}-08$ & $2.18 \mathrm{E}-08$ & $2.27 \mathrm{E}-08$ & $2.33 \mathrm{E}-08$ & $8.64 \mathrm{E}-08$ & $1.01 \mathrm{E}-07$ & $1.20 \mathrm{E}-07$ & $1.54 \mathrm{E}-07$ \\
\hline $29-5$ & $-1.99 \mathrm{E}-21$ & $2.74 \mathrm{E}-09$ & $3.14 \mathrm{E}-09$ & $3.32 \mathrm{E}-09$ & $-1.93 \mathrm{E}-20$ & $3.19 \mathrm{E}-08$ & $3.68 \mathrm{E}-08$ & 7.67E-08 \\
\hline $29-6$ & $-2.23 \mathrm{E}-22$ & $1.67 \mathrm{E}-11$ & $1.96 \mathrm{E}-11$ & $2.08 \mathrm{E}-11$ & $-2.40 \mathrm{E}-21$ & $1.96 \mathrm{E}-10$ & $2.31 \mathrm{E}-10$ & $4.82 \mathrm{E}-10$ \\
\hline $33-3$ & $5.02 \mathrm{E}-07$ & $5.67 \mathrm{E}-07$ & $5.88 \mathrm{E}-07$ & $6.02 \mathrm{E}-07$ & \begin{tabular}{|l|}
$-1.93 \mathrm{E}-06$ \\
\end{tabular} & $-2.14 \mathrm{E}-06$ & $-1.70 \mathrm{E}-06$ & $-1.44 \mathrm{E}-06$ \\
\hline $33-4$ & $-1.76 \mathrm{E}-08$ & $-2.00 \mathrm{E}-08$ & $-2.08 \mathrm{E}-08$ & $-2.13 \mathrm{E}-08$ & $-2.89 \mathrm{E}-08$ & $-4.13 \mathrm{E}-08$ & $-6.02 \mathrm{E}-08$ & $-9.01 \mathrm{E}-08$ \\
\hline $33-5$ & $1.52 \mathrm{E}-24$ & $-2.05 \mathrm{E}-09$ & $-2.33 \mathrm{E}-09$ & $-2.47 \mathrm{E}-09$ & $-4.82 \mathrm{E}-24$ & $6.18 \mathrm{E}-09$ & 7.02E-09 & $1.14 \mathrm{E}-08$ \\
\hline $33-6$ & $4.56 \mathrm{E}-26$ & $-1.26 \mathrm{E}-11$ & $-1.45 \mathrm{E}-11$ & $-1.54 \mathrm{E}-11$ & $-1.34 \mathrm{E}-25$ & $3.85 \mathrm{E}-11$ & $4.45 \mathrm{E}-11$ & $7.26 \mathrm{E}-11$ \\
\hline \begin{tabular}{l|}
$37-3$ \\
\end{tabular} & $3.54 \mathrm{E}-07$ & $4.10 \mathrm{E}-07$ & $4.28 \mathrm{E}-07$ & $4.39 \mathrm{E}-07$ & $1.92 \mathrm{E}-07$ & $2.57 \mathrm{E}-07$ & $5.74 \mathrm{E}-07$ & $9.82 \mathrm{E}-07$ \\
\hline $37-4$ & $3.32 \mathrm{E}-08$ & $4.07 \mathrm{E}-08$ & 4.30E-08 & $4.43 \mathrm{E}-08$ & 2.39E-08 & 3.03E-08 & $6.06 \mathrm{E}-08$ & $1.05 \mathrm{E}-07$ \\
\hline $37-5$ & $1.37 \mathrm{E}-25$ & $-1.09 \mathrm{E}-09$ & $-1.27 \mathrm{E}-09$ & $-1.35 \mathrm{E}-09$ & $1.86 \mathrm{E}-27$ & $-9.60 \mathrm{E}-10$ & $-1.24 \mathrm{E}-09$ & $-3.64 \mathrm{E}-09$ \\
\hline $37-6$ & $1.21 \mathrm{E}-27$ & $-6.79 \mathrm{E}-12$ & $-8.01 \mathrm{E}-12$ & $-8.55 \mathrm{E}-12$ & $1.30 \mathrm{E}-27$ & $-5.97 \mathrm{E}-12$ & $-7.77 \mathrm{E}-12$ & $-2.29 \mathrm{E}-11$ \\
\hline
\end{tabular}

\subsubsection{Analysis of displacement field}

Following the same procedure of Chapters 5 and 6 , the analysis of the displacement fields uses a full shell element model developed in ANSYS $^{\circledR}$ as a control model. However, this example compares only the results between the GBT and shell-181 models. As presented in the two previous chapters, the difference in the displacement fields among the shell models are almost imperceptible. Moreover, shell-181 is the same element type applied in the development of the connection's super-element. The mesh of the 
full shell model follows the same discretization of the connection's super-element. Thus, the full model has 42,200 elements and 135,828 degrees of freedom.

Tables from 7.8 to 7.11 and figures 7.3 to 7.10 show the results of the displacement fields of the cross-sections at the levels $250,500,750$ and $985 \mathrm{~cm}$. The plotted results in figures 7.3, 7.4, 7.7 and 7.8 do not show the value of the uniform longitudinal displacement. This representation is necessary, since the uniform longitudinal displacement is much larger than the periodic warping displacement.

Table 7.8: Mean differences, in \%, and standard deviation (SD) of displacement field between mixed GBT-Shell and Shell-181 model - linear analysis load case 1

\begin{tabular}{ccccccc}
\hline Section & \multicolumn{2}{c}{$\mathrm{u}$} & \multicolumn{2}{c}{$\mathrm{v}$} & \multicolumn{2}{c}{$\mathrm{w}$} \\
Level $(\mathrm{m})$ & diff. & SD & diff. & SD & diff. & SD \\
\hline 9.85 & $-0.03 \%$ & $0.01 \%$ & $12.59 \%$ & $1.36 \%$ & $11.28 \%$ & $10.71 \%$ \\
7.50 & $-0.03 \%$ & $0.01 \%$ & $4.31 \%$ & $0.28 \%$ & $4.03 \%$ & $2.19 \%$ \\
5.00 & $-0.03 \%$ & $0.01 \%$ & $5.1 \%$ & $0.07 \%$ & $5.26 \%$ & $0.56 \%$ \\
2.50 & $-0.03 \%$ & $0.01 \%$ & $8.36 \%$ & $0.06 \%$ & $8.49 \%$ & $0.48 \%$ \\
\hline
\end{tabular}

Table 7.9: Mean differences, in \%, and standard deviation (SD) of displacement field between mixed GBT-Shell and Shell-181 model - Non-linear analysis load case 1

\begin{tabular}{ccccccc}
\hline Section & \multicolumn{2}{c}{$\mathrm{u}$} & \multicolumn{2}{c}{$\mathrm{v}$} & \multicolumn{2}{c}{$\mathrm{w}$} \\
Level $(\mathrm{m})$ & diff. & SD & diff. & SD & diff. & SD \\
\hline 9.85 & $-/ 5.38 \%$ & $0.1 \%$ & $14.93 \%$ & $1.96 \%$ & $14.84 \%$ & $7.93 \%$ \\
7.50 & $-5.37 \%$ & $0.04 \%$ & $19.25 \%$ & $0.35 \%$ & $19.12 \%$ & $1.91 \%$ \\
5.00 & $-5.37 \%$ & $0.03 \%$ & $20.47 \%$ & $2.18 \%$ & $21.84 \%$ & $17.22 \%$ \\
2.50 & $-5.38 \%$ & $0.11 \%$ & $23.86 \%$ & $3.87 \%$ & $21.88 \%$ & $25.13 \%$ \\
\hline
\end{tabular}

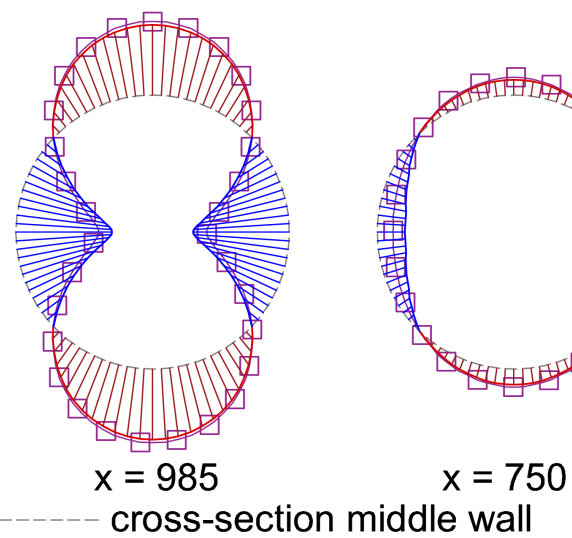

$\mathrm{u}(\mathrm{mm})$ scale

cross-section middle wall

\section{factor 5000}
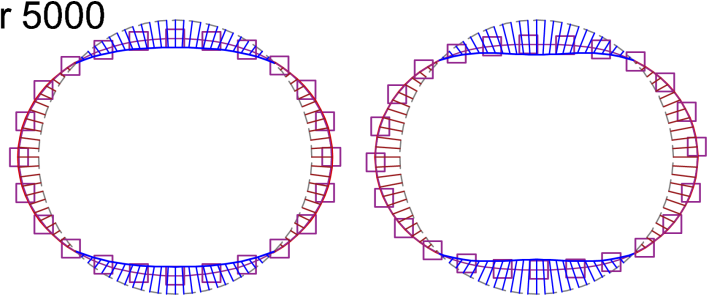

Figure 7.3: Results of longitudinal displacement, without mean value, of mixed shellGBT and full shell-181 model: Linear analysis - Load Case 1.

From tables 7.8 and 7.10, one realizes that the highest mean difference in the displacement fields in the linear analyses is $12.6 \%$ for the transversal displacement at the level $985 \mathrm{~cm}$ in the load case 1 . Meanwhile, for the same level, mean difference in load case 

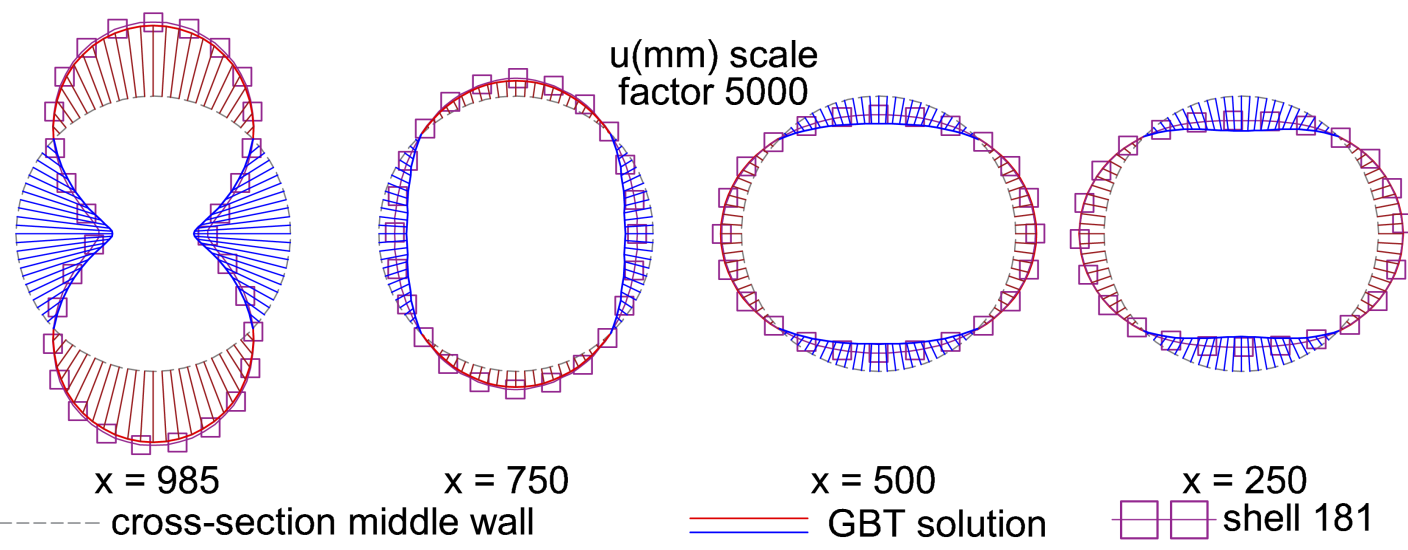

Figure 7.4: Results of longitudinal displacement, without mean value, of mixed shellGBT and full shell-181 model: Non-linear analysis - Load Case 1.

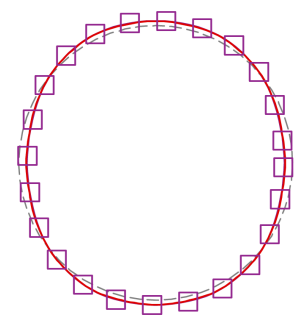

$\mathrm{x}=985$

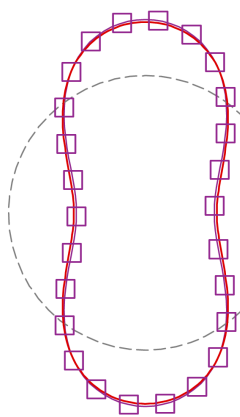

$\mathrm{x}=750$

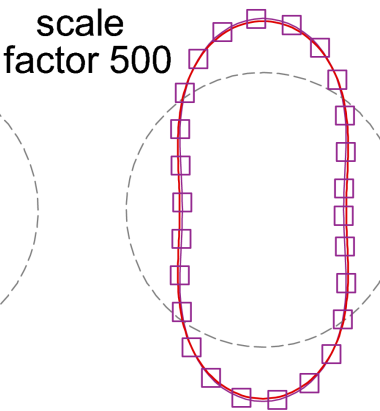

$x=500$

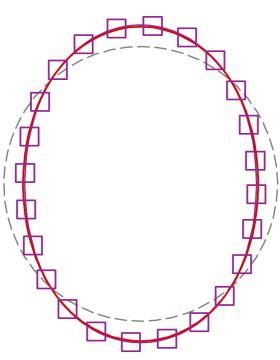

$x=250$

GBT solution

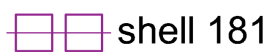

Figure 7.5: Results of transversal displacement of mixed shell-GBT and full shell-181 model: Linear analysis - Load Case 1.

Table 7.10: Mean differences, in \%, and standard deviation (SD) of displacement field between mixed GBT-Shell and Shell-181 model - linear analysis load case 2

\begin{tabular}{ccccccc}
\hline Section & \multicolumn{2}{c}{$\mathrm{u}$} & \multicolumn{2}{c}{$\mathrm{v}$} & \multicolumn{2}{c}{$\mathrm{w}$} \\
Level $(\mathrm{m})$ & diff. & SD & diff. & SD & diff. & SD \\
\hline 9.85 & $-0.02 \%$ & $0.09 \%$ & $0.6 \%$ & $0.02 \%$ & $-0.42 \%$ & $0.75 \%$ \\
7.50 & $-0.02 \%$ & $0.1 \%$ & $1.14 \%$ & $0.01 \%$ & $1.25 \%$ & $0.04 \%$ \\
5.00 & $-0.02 \%$ & $0.11 \%$ & $2.11 \%$ & $0.01 \%$ & $2.21 \%$ & $0 \%$ \\
2.50 & $-0.02 \%$ & $0.13 \%$ & $5.04 \%$ & $0.01 \%$ & $5.14 \%$ & $0.05 \%$ \\
\hline
\end{tabular}

2 is lower than $1 \%$. The magnitude of the longitudinal displacement explains this contrast: load case 1 has a tiny transversal displacement, near zero. Such small numerical approximation leads to significant mean differences. Since the transversal displacement in load case 2 has an order ten times bigger than load case 1, it reduces this numerical approximation effect. Also, tables 7.8 and 7.3 show an agreement between the results for the longitudinal displacements. 


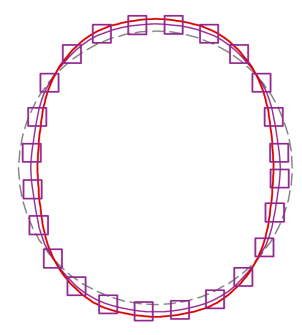

$x=985$

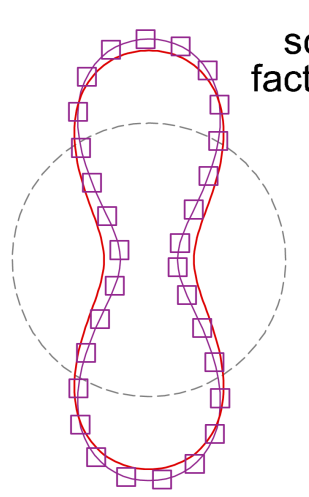

$x=750$

cross-section middle wall

scale

factor 500

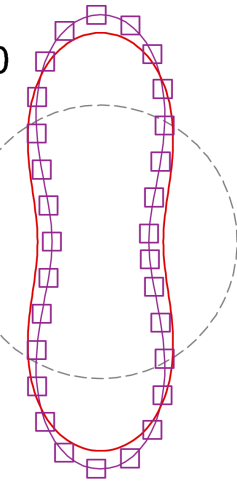

$x=500$

GBT solution

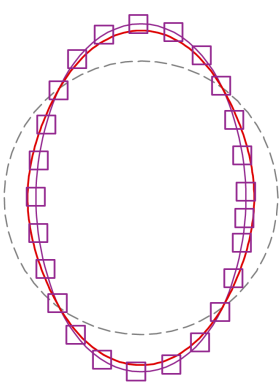

$x=250$

Figure 7.6: Results of transversal displacement of mixed shell-GBT and full shell-181 model: Non-linear analysis - Load Case 1.

Table 7.11: Mean differences, in \%, and standard deviation (SD) of displacement field between mixed GBT-Shell and Shell-181 model - Non-linear analysis load case 2

\begin{tabular}{ccccccc}
\hline Section & \multicolumn{2}{c}{$\mathrm{u}$} & \multicolumn{2}{c}{$\mathrm{v}$} & \multicolumn{2}{c}{$\mathrm{w}$} \\
Level $(\mathrm{m})$ & diff. & SD & diff. & SD & diff. & SD \\
\hline 9.85 & $-63.69 \%$ & $0.77 \%$ & $3.93 \%$ & $1.02 \%$ & $0.37 \%$ & $2.84 \%$ \\
7.50 & $-63.69 \%$ & $0.1 \%$ & $-4.29 \%$ & $4.35 \%$ & $-6.29 \%$ & $19.04 \%$ \\
5.00 & $-63.69 \%$ & $0.64 \%$ & $-4.79 \%$ & $3.69 \%$ & $-4.58 \%$ & $19.31 \%$ \\
2.50 & $-63.73 \%$ & $1.5 \%$ & $1.51 \%$ & $5.79 \%$ & $22.65 \%$ & $26.1 \%$ \\
\hline
\end{tabular}

However, this convergence of the results of the longitudinal displacement is lost in the non-linear analysis, as presented in tables 7.9 and 7.11. For load case 1 the mean difference increases, in absolute values, from $0.03 \%$ to $5.4 \%$. For the load case 2, the growth is even more drastic, from $.02 \%$ to $63.7 \%$. From the standard deviations (SD) in these tables, as well as from the periodic warping in figures 7.4 and 7.8 , one realizes that such increase in the mean difference is related to the uniform longitudinal displacement.

Such divergence in the results not only highlights the limitation of the proposed mixed GBT-shell model in non-linear analysis, but also gives an indicator of this contrast: for the load case with higher transversal displacements, the divergence of the longitudinal displacements in the non-linear analysis is higher. This observation refers directly to the initial assumptions of GBT of non-shear and non-transversal membrane deformation energy. Thus, these assumptions, in non-linear analysis, lead to incorrect internal forces related to transversal displacements. Since the current formulation of non-linear GBT neglects the third-order coupling tensor of the transversal membrane, the relationship between GBT's amplification function $V(x)$ and $V_{, x}(x)$ (for transversal and longitudinal displacement, respectively) links indirectly the transversal displacement and the internal forces in longitudinal direction.

Here, it rises the opportunity to develop future studies in the mixed GBT-shell model, 

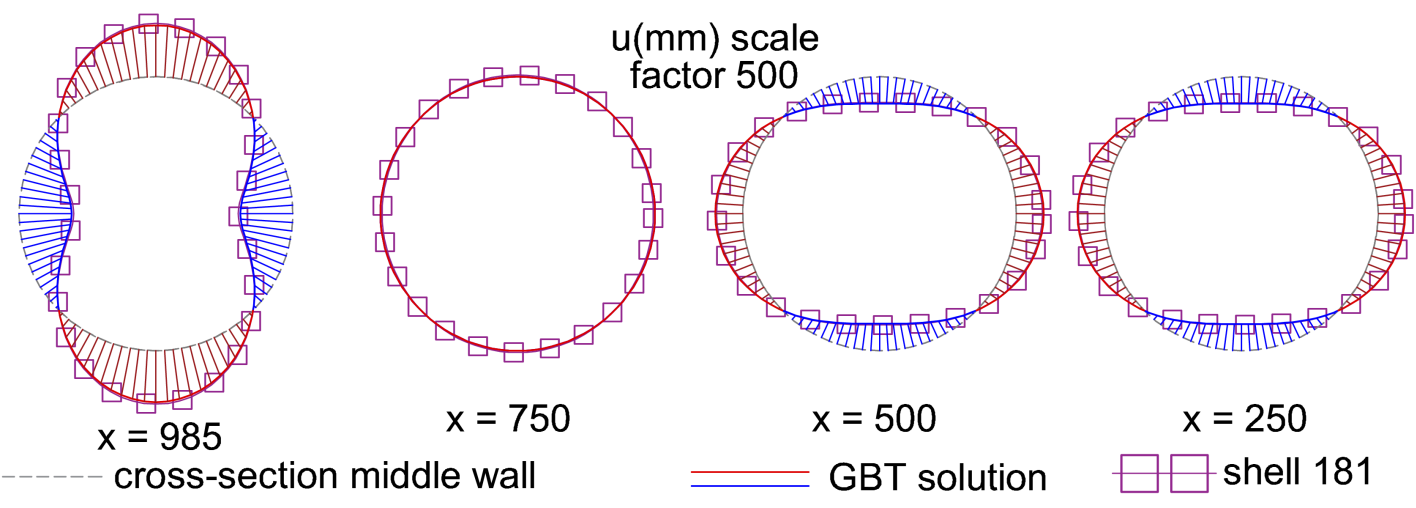

Figure 7.7: Results of longitudinal displacement of mixed shell-GBT and full shell-181 model: Linear analysis - Load Case 2.
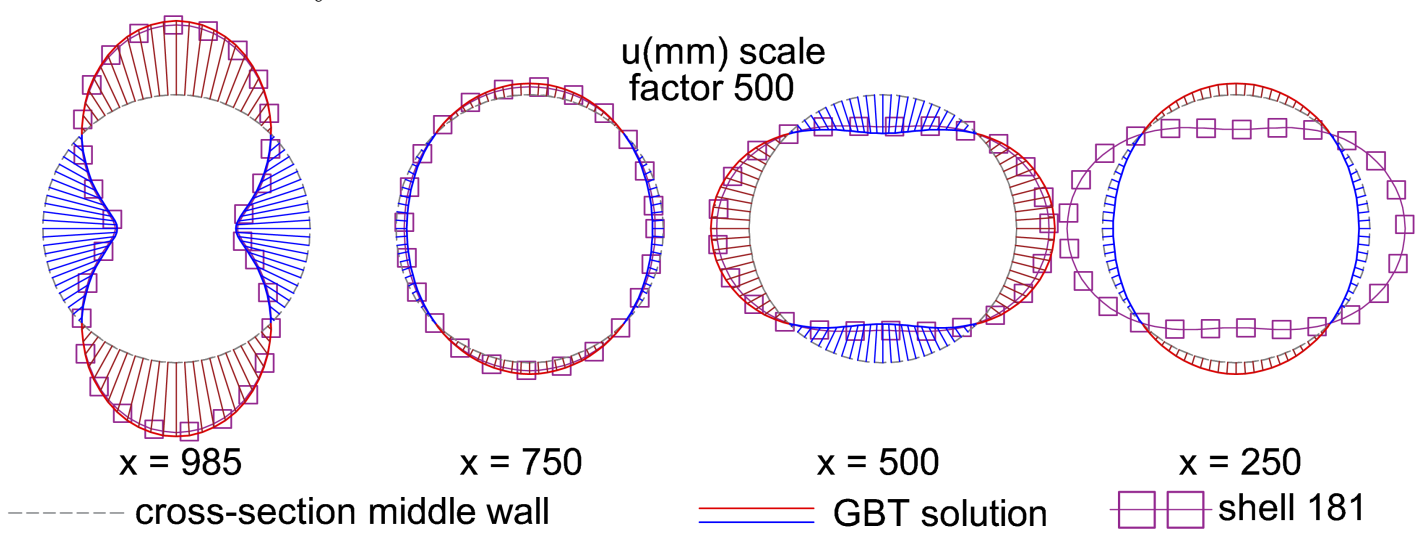

Figure 7.8: Results of longitudinal displacement, without mean value, of mixed shellGBT and full shell-181 model: Non-linear analysis - Load Case 2.

in order to incorporate the shear and transversal membrane elongation in the linear and non-linear analyses. 


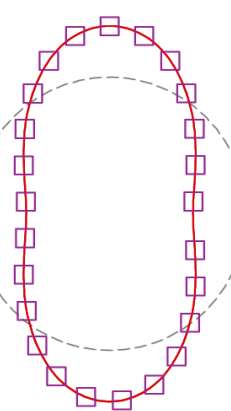

$x=985$

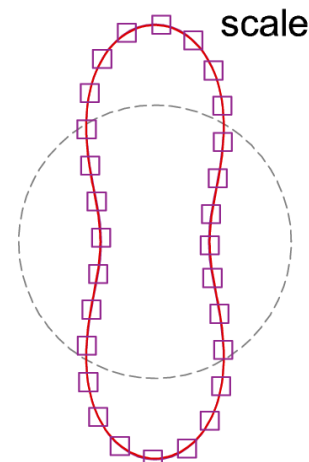

$x=750$

cross-section middle wall

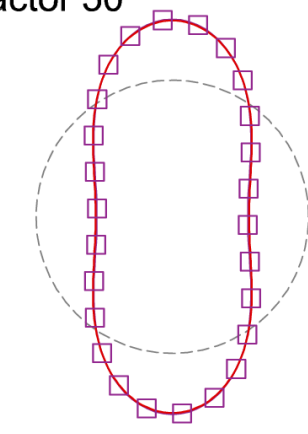

$x=500$

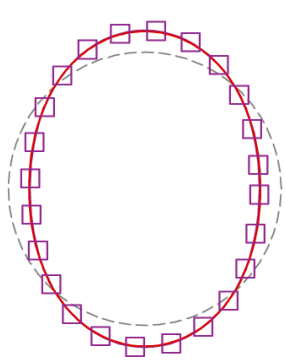

$x=250$

GBT solution

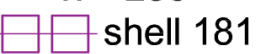

Figure 7.9: Results of transversal displacement of mixed shell-GBT and full shell-181 model: Linear analysis - Load Case 2.

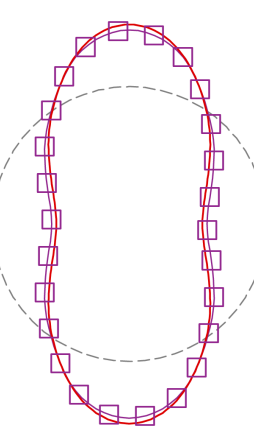

$\mathrm{x}=985$

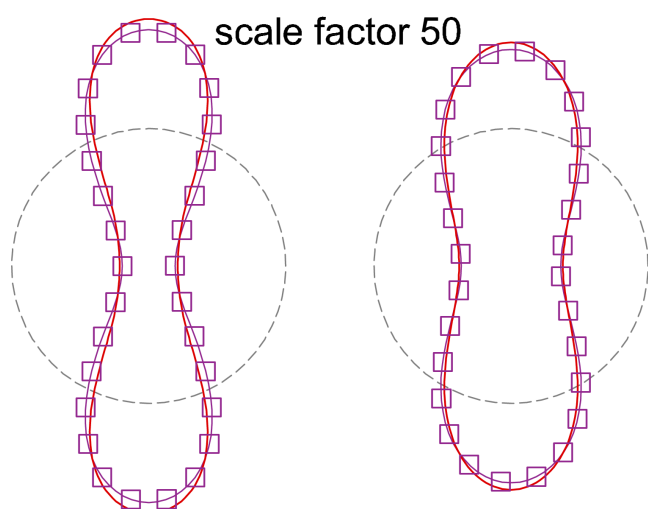

$x=750$

cross-section middle wall

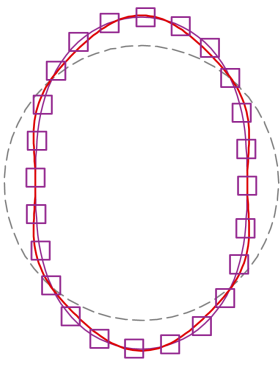

$x=250$

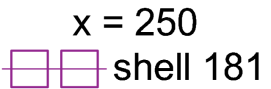

Figure 7.10: Results of transversal displacement, without mean value, of mixed shellGBT and full shell-181 model: Non-linear analysis - Load Case 2. 


\section{Conclusion and future research}

\subsection{Conclusion}

This dissertation presented the application of Finite Elements Method, FEM, in Generalized Beam Theory (GBT). Such application combines the benefits of the versatility of FEM's solution with the high numerical performance of GBT.

Starting from the GBT's assumptions in Chapter 2, this dissertation presents the possibility of achieving the separation of variables in structural beam assessments. Thus, this division leads to two steps. The first one is related to cross-section analysis, which is independent of boundary and loading conditions. The second one is related to the solution of the longitudinal beam's axis. In the perspective of FEM, this separation allows not only parallel processing between the cross-section analysis and the modelling process, but also uses uni-dimensional elements, which are easier to model.

Unfortunately, GBT has an arduous and elaborate process for the cross-section analysis, as presented in Chapter 4. Therefore, this dissertation first introduces the study of hollow circular cross-section in Chapter 3, which requires only a decomposition in Fourier-Series. Thus, this type of cross-section provides a faster overview of the GBT. Moreover, Chapter 3 has a minor contribution in the original GBT circular hollow cross-section to incorporate radial deformations due to Poisson's effect. The example in Chapter 5 shows that this minor contribution reduces the difference between GBT and shell finite elements models from 35\% (original GBT formulation) to 10\% (with Poisson's effect).

For the complex process of a general segmented cross-section, Chapter 4 has another minor contribution to the procedures recently presented, that uses FEM in this step. This contribution allows the separation of the pure plate and membrane-plate deformation modes before the performance of the quadratic eigenvalue problem. Consequently, one obtains a reduced problem, which requires a lower numerical effort, as well as a solution with an explicit decomposition between modes with and without membrane's 
warping mechanisms. A detailed example, presented in Appendix, is the same one found in Richard Schardt's book [136], which is the primary reference in GBT. Thus, the comparison between the results shows: i) the simplified version of the presented contribution has the values of the original work of Richard Schardt; ii) the quadratic eigenvalue problem leads to results with lower longitudinal stiffness and higher shear stiffness, especially for higher modes.

The major contributions in this dissertation are the exact GBT's finite element formulation and the coupling between these elements with shell elements, presented in Chapters 5 and 6 , respectively.

The exact solution formulation is not only attractive in GBT due to minimal discretization, but also in the numerical effort's point of view. Since each node of a GBT's element has several mode shapes with one or two degrees of freedom, the total amount of unknown in a fine discretized GBT model is comparable to a usual shell FEM model. Therefore, the exact solution highlights the compact feature of GBT. Moreover, the shape functions involved in the exact solution, hyperbolic-trigonometric functions, are smooth. This property of having derivatives for any order at any point of the element domain leads to reliable internal forces, as well as to a systematic approach to developing the stiffness matrices. The example in this chapter provides not only a detailed implementation of the stiffness matrices based on hyperbolic-trigonometric functions, but also compares the results among these functions, the Hermitian shape functions and full shell element models. The highest difference among the GBT and the shell modes is $15 \%$ related to tangential displacement.

In Chapter 6, the major contribution concerns the study of coupling between GBT and shell elements. Based on GBT's displacement field definition, the Master-Slave method defines the GBT's degrees of freedom as master ones and creates not only coupling with shell elements, but also among the deformation modes of GBT themselves.

The resulting mixed finite elements models have the benefits of using the versatility of shell elements to model structural connections and the numerical performance of GBT elements to model beams. Although these mixed models remove the isolation of the GBT's implementation from its own elements, they create displacement constraints in shell element part. Thus, the solution in the neighbourhood of a coupling crosssection is limited to the GBT's assumptions. The numerical example in this chapter shows the results' convergence of the displacement field among the proposed mixed model and others created with different types of shell elements. The analysis of the stress field among all models, in the neighbourhood of the coupling cross-section, shows convergence if the results concern the most relevant internal forces, such as longitudinal membrane force in this example. For this force, the highest difference is below $0.1 \%$. However, internal forces with low values present divergent results not only between the mixed and shell models, but also among the shell models. For instance, transversal plate shear forces have a difference between the mixed and shell-93 models of $22 \%$, but the difference between shell-93 and shell-281 is around $26 \%$. Nevertheless, these internal forces have residual values when compared to relevant internal forces. 
The last chapter in this dissertation explores the performance of mixed GBT-shell models in non-linear analysis. Based on incremental strain formulation, Chapter 7 achieves the non-linear stiffness matrices of initial stresses and displacements, as well as the expressions of the internal forces. The development of these matrices and internal forces shows that the original assumptions of GBT, of non-transversal and non-shear membrane strains energy, neglects the third-order coupling tensors related to these strains. Thereby, the transversal strains and displacements involve only fourth-order tensors, which have a minor effect since they are limited to plate's behaviour. The last example of this dissertation shows the consequences due to the absence of the third-order tensor: the initial transversal displacements leads to unrealistic uniform longitudinal internal force and, consequently, to unreliable longitudinal displacements as well. For instance, the last model in this dissertation has a ratio of the radial displacement by the radius of $1 / 80$ that, with the modal amplification functions, $V(x)$, and their derivative, $V_{, x}(x)$, link the transversal deformations solely with the longitudinal strains/stresses. Consequently, for this example, the longitudinal displacement and internal forces in the longitudinal direction are $64 \%$ higher than the results of the control model. Here, it stands out the limitation of GBT in non-linear analysis under the original assumptions of this theory.

\subsection{Future research}

Although this dissertation developed the coupling between GBT and shell finite elements, some questions arise during this development. Thus, one can list some future research and studies:

- Study of structural preserving isospectral flow, in GBT's quadratic eigenvalue problems, to reduce the residual coupling among the GBT's modes;

- Analysis and validation of GBT's complementary modes related to the membrane's transversal elongation and shear deformation;

- Development of exact finite elements of these complementary modes and the classification concerning the longitudinal amplification function;

- Coupling of complementary modes of GBT and shell elements;

- Development of non-linear analysis of these complementary modes. This development leads to the coupling of plate and membrane, based on third-order tensors; 


\section{Bibliography}

[1] Abambres, M., Camotim, D., and Silvestre (2013a). Gbt-based first-order analysis of elastic-plastic thin-walled steel members exhibiting strain-hardening. The IES Journal Part A: Civil and Structural Engineering, 6:119-134.

[2] Abambres, M., Camotim, D., and Silvestre, N. (2013b). Physically non-linear gbt analysis of thin-walled members. Computers and Structures, 129:148-165.

[3] Abambres, M., Camotim, D., and Silvestre, N. (2014a). Gbt-based elastic-plastic post-buckling analysis of stainless steel thin-walled members. Thin-Walled Structures, 83:85-102.

[4] Abambres, M., Camotim, D., Silvestre, N., and Rasmussen, K. (2014b). Gbt-based structural analysis of elastic-plastic thin-walled members. Computers and Structures, 136:1-23.

[5] ANSYS, I. (2009). Ansys theoretical manual, Software, Version 12. Canonsburg,PA 15317.

[6] Basaglia, C. and Camotim, D. (2013a). Buckling, post-buckling, strength and dsm design of cold-formed steel cont. lipped channel beams. Journal of Structural Engineering (ASCE), 139:657-668.

[7] Basaglia, C. and Camotim, D. (2013b). Enhanced generalised beam theory buckling formulation to handle transverse load application effects. Intern. Journal of Solids and Structures, 50:531-547.

[8] Basaglia, C. and Camotim, D. (2015). Buckling analysis of thin-walled steel structural systems using generalised beam theory (gbt). International Journal of Structural Stability and Dynamics, 15:10 -17. 
[9] Basaglia, C., Camotim, D., and Coda, H. (2014). Behaviour, failure and dsm design of cold-formed steel beams:influence of the load point of application. Thin-Walled Structures, 81:78-88.

[10] Basaglia, C., Camotim, D., Gonçales, R., and Graça, A. (2013a). Gbt-based assessment of the buckling behaviour of cold-formed steel purlins restrained by sheeting. Thin-Walled Structures, 72:217-229.

[11] Basaglia, C., Camotim, D., and Silvestre, N. (2007). Plane and spatial analysis of the global behaviour of thin-walled steel frames using generalised beam theory. South-American Journal of Structural Engineering, 4:9-44.

[12] Basaglia, C., Camotim, D., and Silvestre, N. (2008). Global buckling analysis of plane and space thin-walled frames in the context of gbt. Thin-Walles Structures, 46:79-101.

[13] Basaglia, C., Camotim, D., and Silvestre, N. (2009a). Gbt-based local, distortional and global buckling analysis of thin-walled steel frames. Thin-Walled Structures, $47: 1246-1264$.

[14] Basaglia, C., Camotim, D., and Silvestre, N. (2009b). Post-buckling behaviour of thin-walled steel members and frames: Application of generalised beam theory (gbt). South-American Journal of Structural Engineering, 6:37-68.

[15] Basaglia, C., Camotim, D., and Silvestre, N. (2010). Gbt-based buckling analysis of thin-walled steel frames with arbitrary loading and support conditions. International Journal of Structural Stability and Dynamics, 10:363-385.

[16] Basaglia, C., Camotim, D., and Silvestre, N. (2012). Torsion warping transmission at thin-walled frame joints: Kinematics, modelling and structural response. Journal of Constructional Steel Research, 69:39-53.

[17] Basaglia, C., Camotim, D., and Silvestre, N. (2013b). Post-buckling analysis of thin-walled steel frames using generalised beam theory (gbt). Thin-Walled Structures, 62:229-242.

[18] Basaglia, C., Camotim, D., and Silvestre, N. (2015). Buckling and vibration analysis of cold-formed steel chs members and frames using generalised beam theory. International Journal of Structural Stability and Dynamics, 15:1540021:1-25.

[19] Bathe, K. (1996). Finite Element Procedures. Prentice-Hall.

[20] Bathe, K. and Almeida, C. (1980). A simple and effective pipe elbow element linear analysis. Journal of Applied Mechanics, 47:93-100.

[21] Bazant, Z. and L., C. (2003). Stability of Structures - Elastic, Inelastic, Fracture, and Damage Theories. Dover Publications INC. 
[22] Bebiano, R., Basaglia, C., Camotim, D., and Gonçalves, R. (2018a). Gbt buckling analysis of generally loaded thin-walled members with arbitrary flat-walled crosssection. Thin-Walled Structures, 123:11-24.

[23] Bebiano, R., Camotim, D., and Gonçalves, R. (2018b). Gbtul 2.0 - a secondgeneration code for the gbt-based buckling and vibration analysis of thin-walled members. Thin-Walled Structures, 124:235-253.

[24] Bebiano, R., Camotim, D., and Silvestre, N. (2013). Dynamic analysis of thinwalled members using generalised beam theory (gbt). Thin-Walled Structures, $72: 188-205$.

[25] Bebiano, R., Eisenberger, M., Camotim, D., and Gonçalves, R. (2017). Gbt-based buckling analysis using the exact element method. International Journal of Structural Stability and Dynamics, 17(10):1750125.

[26] Bebiano, R., Eisenberger, M., Camotim, D., and Gonçalves, R. (2018c). Gbtbased vibration analysis using the exact element method. International Journal of Structural Stability and Dynamics, 18(5):1850068.

[27] Bebiano, R., Gonçales, R., and Camotim, D. (2015). A cross-section analysis procedure to rationalise and automate the performance of gbt-based structural analyses. Thin-Walled Structures, 92:29-47.

[28] Bebiano, R., Gonçales, R., Camotim, D., and Eisenberger, M. (2016a). Gbtbased buckling analysis using the exact element method. Proceedings of the 7 th International Conference on Coupled Instabilities in Metal Structures, 4:1-24.

[29] Bebiano, R., Gonçales, R., Camotim, D., Garcea, G., Bilotta, A., Manta, D., Leonetti, L., and Magisano, D. (2016b). Deformation modes of thin-walled members: A comparison between the method of generalized eigenvectors and generalized beam theory. Thin-Walled Structures, 100:192-212.

[30] Bebiano, R., Silvestre, N., and Camotim, D. (2007). Gbt formulation to analyze the buckling behavior of thin-walled members subjected to non-uniform bending. International Journal of Structural Stability and Dynamics, pages 23-54.

[31] Bebiano, R., Silvestre, N., and Camotim, D. (2008). Local and global vibration of thin-walled members subjected to compression and non-uniform bending. J. of Sound and Vibration, 315:509-535.

[32] Borst, R., Crisfield, A., Remmers, J., and Verhoosel, C. (2012). Non-linear Finite Element Analysis of Solids and Structures. John Wiley and Sons, Inc.

[33] Camotim, D. and Basaglia, C. (2013). Buckling analysis of thin-walled structures using generalised beam theory(gbt): State-of-the-art report. Steel Construction, $6: 117-131$. 
[34] Camotim, D., Basaglia, C., and Silvestre, N. (2010). Gbt buckling analysis of thinwalled steel frames: A state-of-the-art report. Thin-Walled Structures, 48:726-743.

[35] Camotim, D., Basaglia, C., and Silvestre, N. (2011a). Non-linear gbt formulation for open-section thin-walled members with arbitrary support conditions. Computers and Structures, 89:1906-1919.

[36] Camotim, D., Basaglia, C., and Silvestre, N. (2019). Gbt-based buckling analysis of steel cylindrical shells under combinations of compression and external pressure. Thin-Walled Structures, 144:106274.

[37] Camotim, D., Dinis, P., and Martins, A. (2017). Behaviour and dsm design of stiffened lipped channel columns undergoing local-distortional interaction. Journal of Constructional Steel Research, 128:99-118.

[38] Camotim, D., Dinis, P., and Silvestre, N. (2012). Post-buckling behaviour and direct strength design of lipped channel columns experiencing local/distortional interaction. Journal of Constructional Steel Research, 73:12-30.

[39] Camotim, D., Silva, N., Silvestre, N., Correia, J., and Brando, F. (2011b). Firstorder, buckling and post-buckling behaviour of gfrp pultruded beams. part 2: Numerical simulation. Computer and Structures, 89:2065-2078.

[40] Camotim, D., Silvestre, N., Basaglia, C., and Bebiano, R. (2008). Gbt-based buckling analysis of thin-walled members with non-standard support conditions. ThinWalled Structures, 46:800-815.

[41] Carrera, E., Giunta, G., and Petrolo, M. (2011). Beam Structures - Classical and Advanced Theories. John Wiley and Sons, Inc.

[42] Chai, H. and Sung, C. (2011). Stability of Structures - Principles and Applications. Elsevier Inc.

[43] Cheung, Y. (1976). Finite Strip Method in Structural Analysis. Pergamon Press.

[44] Chu, M. and Del Buono, N. (2008a). Total decoupling of general quadratic pencils, part i: Theory. Journal of Sound and Vibration, 309:96-111.

[45] Chu, M. and Del Buono, N. (2008b). Total decoupling of general quadratic pencils, part ii: Structure preserving isospectral flows. Journal of Sound and Vibration, 309:96-111.

[46] Davies, J. (1998). Generalised beam theory (gbt) for coupled instability problems. In Rondal, J., editor, Coupled Instabilities in Metal Structures, pages 151-223. SpringerWienNewYork.

[47] Davies, J., Leach, P., and Heiny, D. (1994). Second-order generalised beam theory. Journal of Constructional Steel Research, 31:221-241. 
[48] Davies, J. and Leach.P. (1996). An experimental verification of the generalised beam theory applied to interactive buckling problems. Thin-Walled Structures, $25: 61-79$.

[49] Davies, J. M. (1986). An exact finite element for beam on elastic foundation problems. Journal of Structural Mechanics, 14(4):489-499.

[50] Dinis, P., Camotim, D., and Silvestre, N. (2006). Gbt formulation to analyse the buckling behaviour of thin-walled members with arbitrarily 'branched' open crosssection. Thin-Walled Structures, 44:20-38.

[51] Dinis, P., Camotim, D., and Silvestre, N. (2010). On the local and global buckling behaviour of angle, t-section and cruciform thin-walled members. Thin-Walled Structures, 48:786-797.

[52] Dinis, P., Camotim, D., and Silvestre, N. (2012). On the mechanics of thin-walled angle column instability. Thin-Walled Structures, 52:80-89.

[53] Ádány, S., Silvestre, N., Schafer, B., and Camotim, D. (2009). Gbt and cfsm: Two modal approaches to the buckling analysis of unbranched thin-walled members. Advanced Steel Construction - an InternaQonal Journal, 5:195-223.

[54] Duan, L. and Zhao, J. (2019). Gbt deformation modes for thin-walled crosssections with circular rounded corners. Thin-Walled Structures, 136:64-89.

[55] Duan, L., Zhao, J., Liu, S., and Zhang, D. (2016a). A b-splines-based gbt formulation for modeling fire behavior of restrained steel beams. Journal of Constructional Steel Research, 116:65-78.

[56] Duan, L., Zhao, J., Liu, S., and Zhang, D. (2016b). A b-splines based nonlinear gbt formulation for elastoplastic analysis of prismatic thin-walled members. Engineering Structures, 110:325-346.

[57] Duan, L., Zhao, J., Liu, S., and Zhang, D. (2018). A nonlinear explicit dynamic gbt formulation for modeling impact response of thin-walled steel members. Journal of Southeast University (English Edition), 2:237-250.

[58] Falk, L. (1960). Das jacobiverfahren für reellsymmetrische matrizenpaare i, ii. Elektronische Datenverarbeitung, 1:30-43.

[59] Fujitani, Y. and Fujii, D. (1998a). A structural analysis method of framed structures with thin-walled open section members based on the bending-torsional theory of beams. Computational Mechanics: New Trends and Applications, 61:749-763.

[60] Fujitani, Y. and Fujii, D. (1998b). A structural analysis method of framed structures with thin-walled open section members based on the bending-torsional theory of beams. In S.Idelsohn, E. O. and Dvorkin, E., editors, Proceedings of Computational Mechanics: New Trends and Applications 1998, pages 1-10. CIMNE. 
[61] Galishinikova, V., Dunaiski, P., and Pahl, J. (2009). Geometrically Nonlinear Analysis of Plane Trusses and Frames. Sun Press.

[62] Garvey, S. (2002). Co-ordinate transformation for second ordem systems. part i: General transformations. Journal of Sound and Vibration, 258:885-909.

[63] Gelfand, I. and Fomin, S. (1991). Calculus of Variations. Dover Publications, INC.

[64] Gonçalves, R., Bebiano, R., and Camotim, D. (2014). On the shear deformation modes in the framework of generalised beam theory. Thin-Walled Structures, 84:325334.

[65] Gonçalves, R. and Camotim, D. (016.). On the first-order and buckling behaviour of thin-walled regular polygonal tubes. Steel Construction - Design and Research, 9(4):279-290.

[66] Gonçalves, R. and Camotim, D. (2004). Gbt local and global buckling analysis of aluminium and stainless steel columns. Computers and Structures, 82:1473-1484.

[67] Gonçalves, R. and Camotim, D. (2007). Thin-walled member plastic bifurcation analysis using generalised beam theory. Advances in Engineering Software, 38:637646.

[68] Gonçalves, R. and Camotim, D. (2010). Steel-concrete composite bridge analysis using generalised beam theory. teel and Composite Structures, 10:223-243.

[69] Gonçalves, R. and Camotim, D. (2011). Generalised beam theory-based finite elements for elastoplastic thin- walled metal members. Thin-Walled Structures, 49:1237-1245.

[70] Gonçalves, R. and Camotim, D. (2012). Geometrically non-linear generalised beam theory for elastoplastic thin-walled metal members. Thin-Walled Structures, 51:121129.

[71] Gonçalves, R. and Camotim, D. (2013a). Buckling behaviour of thin-walled regular polygonal tubes subjected to bending or torsion. Thin-Walled Structures, 73:185197.

[72] Gonçalves, R. and Camotim, D. (2013b). Elastic buckling of uniformly compressed thin-walled regular polygonal tubes. Thin-Walled Structures, 71:35-45.

[73] Gonçalves, R. and Camotim, D. (2013c). On the behaviour of thin-walled steel regular polygonal tubular members. Thin-Walled Structures, 62:191-205.

[74] Gonçalves, R. and Camotim, D. (2014). The vibration behaviour of thin-walled regular polygonal tubes. Thin-Walled Structures, 84:177-188.

[75] Gonçalves, R. and Camotim, D. (2015). On distortion of symmetric and periodic open-section thin-walled members. Thin-Walled Structures, 94:314-324. 
[76] Gonçalves, R. and Camotim, D. (2016). Gbt deformation modes for curved thinwalled cross-sections based on a mid-line polygonal approximation. Thin-Walled Structures, 103:231-243.

[77] Gonçalves, R. and Camotim, D. (2017). Improving the efficiency of gbt displacement-based finite elements. Thin-Walled Structures, 111:165-175.

[78] Gonçalves, R., Dinis, P., and Camotim, D. (2009). Gbt formulation to analyse the first-order and buckling behaviour of thin-walled members with arbitrary crosssections. Thin-Walled Structures, 47:583-600.

[79] Gonçalves, R., Le Grognec, P., and Camotim, D. (2010a). Gbt-based semianalytical solutions for the plastic bifurcation of thin-walled members. Intern. Journal of Solids and Structures, 47:34- 50 .

[80] Gonçalves, R., Peres, N., Bebiano, R., and Camotim, D. (2015). Global-localdistortional vibration of thin-walled rectangular multi-cell beams. Intern. Journal of Structural Stability and Dynamics, 15:1540022:1-20.

[81] Gonçalves, R., Ritto-Corrêa, M., and Camotim, D. (2010b). A large displacement and finite rotation thin-walled beam formulation including cross-section deformation. Computer Methods in Applied Mechanics and Engineering, 199:1627-1643.

[82] Gonçalves, R., Ritto-Corrêa, M., and Camotim, D. (2010c). A new approach to the calculation of cross-section deformation modes in the framework of generalized beam theory. Computational Mechanics, 46:759-781.

[83] Greiner, R. (1983). Zur ingenieurmässigen Berechnung und Konstruktion zylindrischer Behälter aus Stahl unter allgemeiner Belastung. Wissenschaft und Praxis.

[84] Grenberg, M. (1988). Advanced Engineering Mathematics. Prentice-Hall International Editions.

[85] Hancok, G. (1998). Finite strip buckling and nonlinear analyses and distortional buckling analysis of thin-walled structural members. In Rondal, J., editor, Coupled Instabilities in Metal Structures, pages 225-289. SpringerWienNewYork.

[86] Harte, R. and Eckstein, U. (1986). Derivation of geometrically nonlinear finite shell elements via tensor notation. Numercal Methods in Engineering, 23:367-384.

[87] Harte, R. and Krätig, W. (1986). Tensor-orientierte formulierung nichtlinearer, finiter schalenelemente. Archive of Applied Mechanics, 56:114-129.

[88] Henrique, D., D., C., and Gonçales, R. (2015). A physically non-linear gbt-based finite element for steel and steel-concrete beams including shear lag effects. ThinWalled Structures, 90:202-215.

[89] Henriques, D., Gonçalves, R., and Camotim, D. (016.). Gbt-based finite element to assess the buckling behaviour of steel-concrete composite beams. Thin-Walled Structures, 107:207-220. 
[90] Huei-Huang, L. (2018). Finite Element Simulations with ANSYS Workbench 19. SDC Publications.

[91] Hughes, T. (2000). The Finite Element Method - Linear Static and Dynamic Finite Element Analysis. Dover Publications INC.

[92] Jelenic, G. and Crisfield, M. (1996). Non-linear 'master-slave' relationships for joints in 3-d beams with large rotations. Computer methods in applied mechanics and engineering, 135:211-228.

[93] Jelenic, G. and Crisfield, M. (2003). Master-slave approach for the modelling of joints with dependent degrees of freedom in flexible mechanisms. Communications in Numerical Methods in engineering, 19:689-702.

[94] Jönsson, J. (1997). Determination of shear stresses, warping functions and section properties of thin-walled beams using finite elements. Thin-Walled Structures, 68:393-410.

[95] Jönsson, J. (1999a). Distortional theory of thin-walled beams. Thin-Walled Structures, 33:269-303.

[96] Jönsson, J. (1999b). Distortional warping functions and shear distributions in thin-walled beams. Computers and Structures, 33:245-268.

[97] Jönsson, J. and Andreassen, M. (2011). Distortional eigenmodes and homogeneous solutions for semi-discretized thin-walled beams. Thin-Walled Structures, 49:691707.

[98] Jönsson, J. and Andreassen, M. (2012a). Distortional buckling modes of semidiscretized thin-walled columns. Thin-Walled Structures, 51:53-63.

[99] Jönsson, J. and Andreassen, M. (2012b). Distortional solutions for loaded semidiscretized thin-walled beams. Thin-Walled Structures, 50:116-127.

[100] Jönsson, J. and Andreassen, M. (2013). A distortional semi-discretized thinwalled beam element. Thin-Walled Structures, 62:142-157.

[101] Kindmann, R. and Kraus, M. (2011). Steel Structures design using FEM. Berlin: Ernst adn Sohn.

[102] Kreyszig, E. (1993). Advanced Engineering Mathematics. John Wiley and Sons, Inc.

[103] Lancaster, P. and Zaballa, I. (2009). Diagonalizable quadratic eigenvalue problems. Mechanical Systems and Signal Processing, 23:1134-1144.

[104] Leach, P. (1994). The calculation of modal cross-section properties for use in the generaliyed beam theory. Thin-Walled Structures, 19:61-79. 
[105] Madenci, E. and Guven, I. (2015). The Finite Element Method and Applications in Engineering Using ANSYS®. Springer US.

[106] Madeo, A., Garcea, G., and Casciaro, R. (2012). The implicit corotational method and its use in the derivation of nonlinear structural models for beams and plates. Journal of Mechanics of Materials and Structures, 6:509-538.

[107] Magbi, M. and F., N. (2003). Exact finite element for nonuniform torsion of open sections. Journal of Structural Engineering, 129:2:215-223.

[108] Martins, A., Camotim, D., Gonçalves, R., and Dinis, P. (2018a). Enhanced geometrically non-linear generalized beam theory (gbt) formulation: derivation, numerical implementation and illustration. ASCE Journal of Engineering Mechanics, 144(6):4018036.

[109] Martins, A., Camotim, D., Gonçalves, R., and Dinis, P. (2018b). Gbt-based assessment of the mechanics of distortional-global interaction in thin-walled lipped channel beams. Thin-Walled Structures, 124:32-47.

[110] Martins, A., Camotim, D., Gonçalves, R., and Dinis, P. (2018c). On the mechanics of distortional-global interaction in fixed-ended columns. Thin-Walled Structures, 123:162-184.

[111] Martins, A., Camotim, D., Gonçalves, R., and Dinis, P. (2018d). On the mechanics of local-distortional interaction in thin-walled lipped channel columns. ThinWalled Structures, 125:187-202.

[112] Mesacasa, E., Dinis, P., Camotim, D., and Malite, M. (2014.). Mode interaction in thin-walled equal-leg angle columns. Thin-Walled Structures, 81:138-149.

[113] Miranda, S., Guitiérrez, A., Miletta, R., and Ubertini, F. (2013). A generalized beam theory with shear deformation. Thin-Walled Structures, 67:88-100.

[114] Möller, R. (1984). Zur berechnung prismatischer strukturen mit beliebigen nicht fortreuen quersnit. Institut für Statik, Technische Hochschule Darmstadt, 2:14951517.

[115] Mörschardt, S. (1990). Die verallgemeinerte technische biegetheorie für faltwerke mit kragteilen. Institut für Statik, Technische Hochschule Darmstadt, 51:259-275.

[116] Muresan, A., Nedelcu, M., and Gonçalves, R. (2019). Gbt-base fe formulation to analyse the buckling behaviour of isotropic conical shells with circular cross-section. Thin-Walled Structures, 134:84-101.

[117] Myskis, A. (1975). Advanced Mathematics for Engineers. MIR Publishers.

[118] Natário, P. and Silvestre, N.and Camotim, D. (2012). Localized web buckling analysis of beams subjected to concentrated loads using gbt. Thin-Walled Structures, 61:27-41. 
[119] Natário, P., Silvestre, N., and Camotim, D. (2016). Direct strength prediction of web crippling failure of beams under etf loading. Thin-Walled Structures, 94B:360374.

[120] Nedelcu, M. (2010). Gbt formulation to analyse the behaviour of thin-walled members with variable cross-section. Thin-Walled Structures, 48:629-638.

[121] Nedelcu, M. (2011). Gbt formulation to analyse the buckling behaviour of isotropic conical shells. Thin-Walled Structures, 49:812-818.

[122] Nedelcu, M. and Cucu, H. (2014). Buckling modes identification from fea of thin-walled members using only gbt cross-sectional deformation modes. Thin-Walled Structures, 81:150-158.

[123] Neumann, J. v. (1927). Zur hilbertschen beweistheorie. Mathematische Zeitschrift, 26:1-46.

[124] Peres, N., Gonçalves, R., and Camotim, D. (2016). First-order generalised beam theory for curved thin-walled members with circular axis. Thin-Walled Structures, 107:345-361.

[125] Peres, N., Gonçalves, R., and Camotim, D. (2018a). Application of generalised beam theory to curved members withcircular axis. Stahlbau, 87(4):345-354.

[126] Peres, N., Gonçalves, R., and Camotim, D. (2018b). Gbt-based cross-section deformation modes for curved thin-walled members with circular axis. Thin-Walled Structures, 127:769-780.

[127] Piccardo, G., Ranzi, G., and Luongo, A. (2013). A complete dynamic approach to the generalized beam theory cross-section analysis including extension and shear modes. Mathematics and Mechanics of Solids, 19:900-924.

[128] Piccardo, G., Ranzi, G., and Luongo, A. (2014). A direct approach for the evaluation of the conventional modes within the gbt formulation. Thin-Walled Structures, 74:133-145.

[129] Pilkey, W. (2002). Analyis and Design of Elastic Beams Beam - Computational Methods. John Wiley and Sons, Inc.

[130] Polyanin, A. and Zaitev, V. (2004). Handbook of Nonlinear Partial Differential Equations. Chapman and Hall CRC.

[131] Rubin, H. (1988). Ein einfaches allgemeingültiges lösungskonzept für lineare differentialgleichungen beliebiger ordnung mit konstanten koeffizienten und mit analytischer störfunktion. ZAMM, 68:433-443.

[132] Ruggerinia, A., Madeo, A., Gonçalves, R., Camotim, D., Ubertinia, F., and Miranda, S. (2019). Gbt post-buckling analysis based on the implicit corotational method. International Journal of Solids and Structures, 163:40-60. 
[133] Saal, G. (1980). Zur berechnung offener kreiszylinderschalen mit beliebigen randbedingungen an der längs- und querändern. Der Stahlbau, 49:97-110.

[134] Schafer, B. and Zhanjie, L. (2013). Constrained finite strip method for thin-walled members with general end boundary conditions. Journal of Engineering Mechanics, 139:1566-1576.

[135] Schardt, R. (1966). Eine erweiterung der technischen biegelehre für die berechnung biegesteifer prismatischer faltwerke. Stahlbau, 6:161-171.

[136] Schardt, R. (1989). Verllgemeinerte Technische Biegetheorie. Springer-Vertrag.

[137] Schardt, R. (1994a). Generalized beam theory - an adequate method of coupled stability problems. Thin-Walled Structures, 19:161-180.

[138] Schardt, R. (1994b). Lateral torsional and distortional buckling of channel-and hat-sections. Journal of Constructional Steel Research, 31:243-265.

[139] Schardt, R. and Okur, H. (1971). Hilfswerte für die losüng der differentialgleichung ay"" (x)-by" $(x)+c y(x)=p(x)$. Der Stahlbau, 40:6-17.

[140] Schardt, R. and Schardt, C. (2001). Anwendungen der verallgemeinerten technischen biegetheorie im leichtbau. Stahlbau, 70:710-717.

[141] Schardt, R. and Steingass, J. (1970). Eine erweiterung der technischen biegelehre für dieberechnung dünnwandiger geschlossener kreiszylinderschalen. Stahlbau, 39:6573.

[142] Schardt, R. and Strehl, C. (1976). Theoretische grundlagen für die bestimmung derschubsteifigkeit von trapezblechscheiben - vergleich mit anderen berechnungsansät-zen und versuchsergebnissen. Stahlbau, 45:97-108.

[143] Schardt, R. and Zhang, X. (1988). Biegetragfähigkeit eines balkens mit dünnwandigem u-querschnitt. THD-Schriftenreihe Wissenschaft und Technik, 40.

[144] Schardt, R. and Zhang, X. (1989). Die anwendung der verallgemeinerten technischen biegetheorie im nichlinearen beulbereich. In Stein, E., editor, Nichtlineare Berechnungen im Konstruktiven Ingenieurbau, pages 482-504. Springer-Verlag.

[145] Silva, N., Silvestre, N., and Camotim, D. (2010a). Gbt formulation to analyse the buckling behaviour of frp composite open-section thin-walled columns. Composite Structures, 93:79-92.

[146] Silva, N. F., Degenhardt, R., Camotim, D., and Silvestre, N. (2010b). On the use of generalised beam theory to assess the buckling and post-buckling behaviour of laminated cfrp cylindrical stiffened panels. International Journal of Structural Stability and Dynamics, 10:737-760.

[147] Silvestre, N. (2007). Generalized beam theory to analyse the buckling hebaviour of circular cylindrical shells and tubes. Thin-Walled Structures, 45:185-198. 
[148] Silvestre, N. (2008). Buckling behaviour of elliptical cylindrical shells and tubes under compression. International Journal of Solids and Structures, 45:4427-4447.

[149] Silvestre, N. and Camotim, D. (2002a). First order generalised beam theory for arbitrary orthotropic materials. Thin-Walled Structures, 40:755-789.

[150] Silvestre, N. and Camotim, D. (2002b). Second order generalised beam theory for arbitrary orthotropic materials. Thin-Walled Structures, 40:791-820.

[151] Silvestre, N. and Camotim, D. (2003a). Gbt buckling analysis of pultruded frp lipped channed members. Computer and Structures, 81:1889-1904.

[152] Silvestre, N. and Camotim, D. (2003b). Nonlinear generalized beam theory for cold-formed steel members. Interational Journal of Structural Stability and Dynamics, 3:461-490.

[153] Silvestre, N. and Camotim, D. (2004a). Distortional buckling formulae for coldformed steel c and z-section members: Part i - derivaqon. Thin-Walled Structures, 42:1567-1597.

[154] Silvestre, N. and Camotim, D. (2004b). Distortional buckling formulae for coldformed steel c and z-section members: Part ii - validaqon and applicaqon. ThinWalled Structures, 42:1599- 1629.

[155] Silvestre, N. and Camotim, D. (2004c). Distortional buckling formulae for coldformed steel rack-section members. Steel and Composite Structures, 4:49-75.

[156] Silvestre, N. and Camotim, D. (2006a). Gbt-based local and global vibration analysis of loaded composite open section thin-walled members. International Journal of Structural Stability and Dynamics, 6:1-29.

[157] Silvestre, N. and Camotim, D. (2006b). Local-plate and distortional post-buckling behavior of cold-formed steel lipped channel columns with intermediate stiffeners. Journal of Structural Engineering (ASCE), 132:529-540.

[158] Silvestre, N. and Camotim, D. (2006c). Vibration behaviour of axially compressed cold-formed steel members. Steel and Composite Structures, 6:221-236.

[159] Silvestre, N. and Camotim, D. (2013a). Generalized beam theory to analyze the vibration of open-section thin- walled composite members. Journal of Engineering Mechanics (ASCE), 139:992-1024.

[160] Silvestre, N. and Camotim, D. (2013b). A shear deformable generalized beam theory for the analysis of thin- walled composite members. Journal of Engineering Mechanics (ASCE), 139:1010-1024.

[161] Silvestre, N., Camotim, D., and Silva, N. (2011). Generalised beam theory revisited: from the kinematical assumptions to the deformation mode determination. International Journal of Structural Stability and Dynamics, 11:969-997. 
[162] Silvestre, N. and Gardner, L. (2011). Elastic local post-buckling of elliptical tubes. Journal of Constructional Steel Research, 67:281-292.

[163] Silvestre, N., Young, B., and Camotim, D. (2008). Non-linear behaviour and loadcarrying capacity of cfrp strengthened lipped channel steel columns. Engineering Structures, 30:2613-2630.

[164] Simão, P. and Simões da Silva, L. (2004). A unified energy formulation for the stability analysis of open and closed thin-walled members in the framework of the generalized beam theory. Thin-Walled Structures, 42:1495-1517.

[165] Simão, P. and Simões da Silva, L. (2017). Post-buckling analysis of nonlinear shear-deformable prismatic columns using a gbt consistent energy formulation. Computers and Structures, 190:186-204.

[166] Sobel, L. (1977). In-plane bending of elbows. Journal of Computers and Structures, 7:701-715.

[167] Soriano, H. (2003). Método de Elementos Finitos em Análise de Estruturas. Editora da Universidade de São Paulo.

[168] Stolarski, T., Nakasone, Y., and Yoshimoto, S. (2007). Engineering Analysis with ANSYS Software. Butterworth-Heinemann.

[169] Taig, G., Ranzi, G., and D'Annibale, F. (2014). An unconstrained dynamic approach for the generalised beam theory. Continuum Mechanics and Thermodynamics, 27:879-904.

[170] Timoshenko, S. and J.N., G. (2016). Theory of Elasticity. McGraw Hill Education Private Limited.

[171] Tong, G., Yan, X., and Zhang, L. (2005). Warping and bimoment transmissiion through diagonally stiffened beam-to-column joints. Journal of Constructional Steel Research, 61:749-763.

[172] Turner, M. J., Martin, H. C., and Weikel, B. C. (1964). Further developments and applications of the stiffness method. Pergamon Press.

[173] U.C., D., A.A., S., and J., S. (2010). Tensor Calculus. Alpha Science International Ltd.

[174] Uhlmann, W. (1970). Die berechung von im grundriss gekrümmten biegesteifen faltwerken mit offnem im längsrichtung unveränderlichen querschnitt. Der Stahlbau, 39:193-199.

[175] Vieira, L., Gonçalves, R., and Camotim, D. (2018). On the local buckling of rhs members under axial force and biaxial bending. Thin-Walled Structures, 129:43739.

[176] Vlasov, V. (1961). Thin-Walled Elastic Beams. Israel Program for Scientific Translations. 
[177] von Karman, T. (1911). Über die formänderung dünnwanndiger rohre, innsbesondere federnder ausgleichrohre. Zeitschrift des Vereines deutscher Ingenieure, 55:1889-1895.

[178] Wang, C., Reddy, J., and Lee, K. (2000). Shear Deformable Beams and Plates Relationships with Classical Solutions. Elsevier Inc.

[179] Wriggers, P. (2008). Nonlinear Finite Element Methods. Springer Science and Business Media.

[180] Zhang, X. (1989). Local and overall buckling interaction of columns with thinwalled channel-sections. In Kanok-Nukulchai, W., editor, Structural engineering and construction : achievements, trends, and challenges : proceedings of the Second East Asia-Pacific Conference on Structural Engineering and Construction, Chiang Mai, Thailand 11-13, pages 1997-2012. Bangkok, Thailand : EASEC-2 Secretariat, Division of Structural Engineering and Construction, Asian Institute of Technology. 


\section{Appendix A: Numerical example - GBT's analysis of a generic segmented thin-walled cross-section}

\section{A.1. Numerical example}

The presented numerical example is the same open cross-section found in the example of the second chapter of Schardt's book [136]. Thus, it is possible to evaluate and crosscheck the results of the presented approach and the original procedure of GBT.

The open cross-section is a concrete roof, as plotted in figure. A.1, in which the material parameters are: Young Modulus $=21,000 M N / \mathrm{m}^{2}$, Poisson's ratio $=0$, Shear Modulus $=10,500 \mathrm{MN} / \mathrm{m}^{2}$.

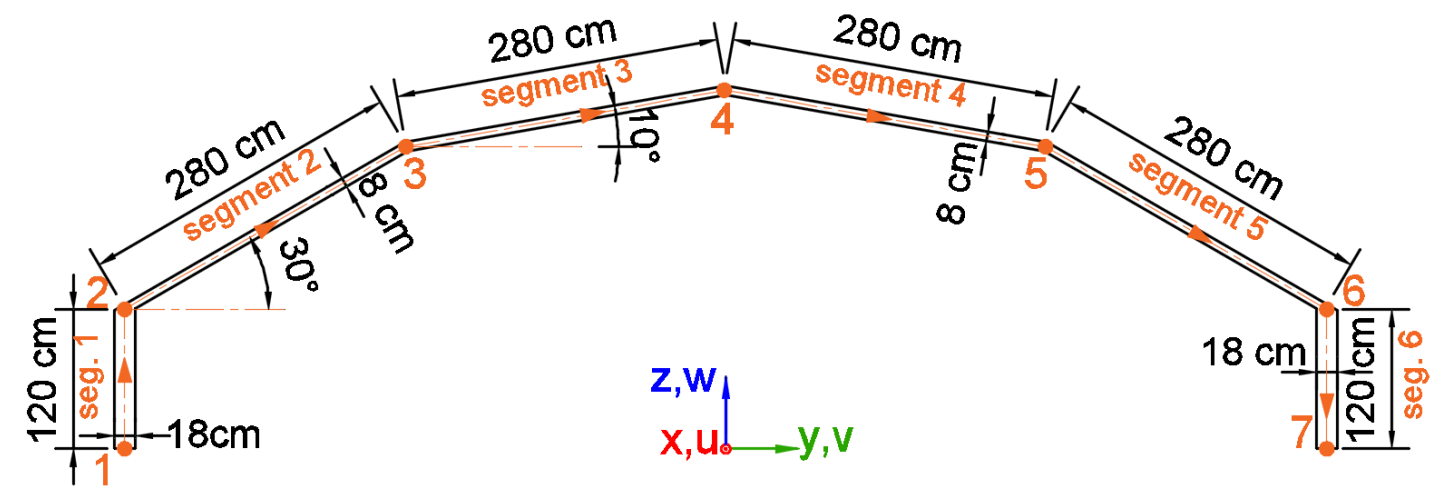

Figure A.1: Concrete Roof extracted from R. Schadt [136].

\section{A.1.1. Step 1 - Setup of Cross-section stiffness matrices}

In local coordinate systems, segments 1 and 6 , as well as segments $2,3,4$ and 5, share the same geometry and material property. Hence, one builds up the cross-section stiffness matrices due to membrane behavior. 
- longitudinal membrane stiffness matrix: based on eq. 4.25, one achieves the following local stiffness matrices for segments $1 ; 6$ and for segments 2-5, respectively:

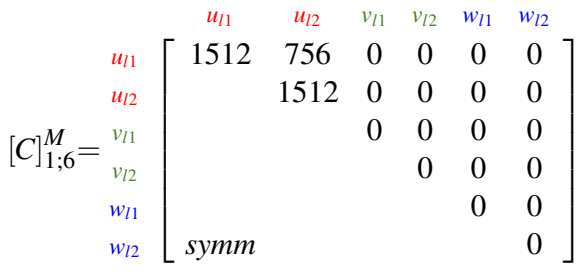

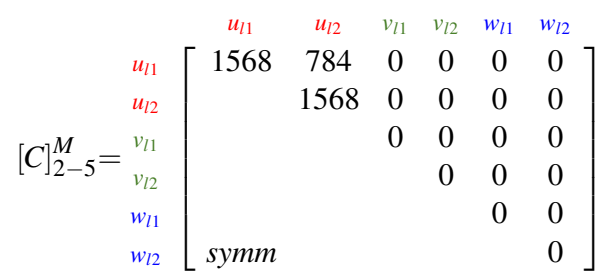

- shear membrane stiffness matrix: this stiffness matrix has terms related to the longitudinal displacement, eq. 4.29, terms concerning the transverse displacement, eq. 4.26, and terms concerning both longitudinal and transverse displacement, equations 4.29 and 4.34. Thus, the local stiffness matrices are:

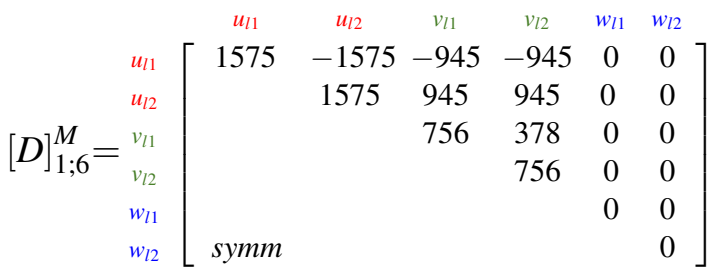

$$
\begin{aligned}
& {[D]_{2-5}^{M}=\begin{array}{l}
u_{l 1} \\
u_{l 1} \\
v_{l / 2} \\
w_{l 1} \\
w_{l 2}
\end{array}\left[\begin{array}{cccccc}
u_{l 1} & u_{l 2} & v_{l 1} & v_{l 2} & w_{l 1} & w_{l 2} \\
300 & -300 & -420 & -420 & 0 & 0 \\
& 300 & 420 & 420 & 0 & 0 \\
& & 784 & 392 & 0 & 0 \\
& & & 784 & 0 & 0 \\
& & & & 0 & 0 \\
\text { symm } & & & & & 0
\end{array}\right]}
\end{aligned}
$$

- transverse elongation membrane stiffness matrix: eq. 4.30 provides the terms of this stiffness matrix:

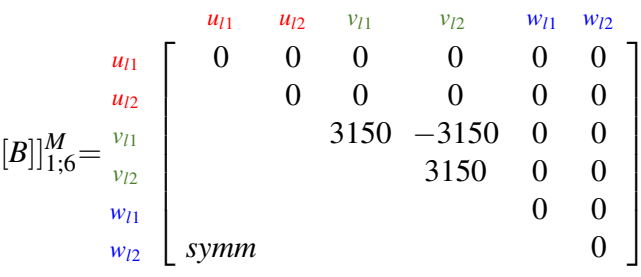

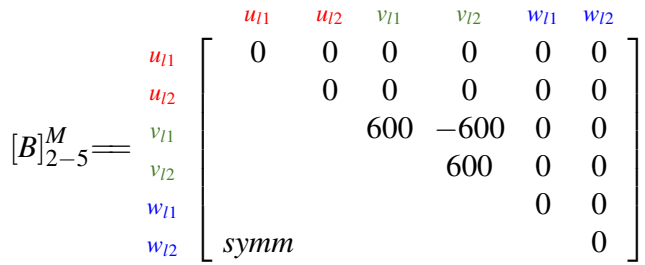


After the setup of the local stiffness matrices, one must transform them into the global coordinate system, as presented in equations 4.46 and 4.47. Since this transformation is well-known in finite element applications, the numerical values are not presented. Nevertheless, these values are indirectly audited in the global cross-sections stiffness matrices, as presented below. The assembly of these global matrices is guided by the mapping matrix, which informs of each local degree of freedom the respective one in the global coordinate system:

$u_{l 1}$
$u_{l 2}$
$v_{l 1}$
$v_{l 2}$
$w_{l 1}$
$w_{l 2}$
$\phi_{l 1}$
$\phi_{l 2}$$\left[\begin{array}{cccccc}u_{1} & u_{2} & u_{3} & u_{4} & u_{5} & u_{6} \\ u_{2} & u_{3} & u_{4} & u_{5} & u_{6} & u_{7} \\ v_{1} & v_{2} & v_{3} & v_{4} & v_{5} & v_{6} \\ v_{2} & v_{3} & v_{4} & v_{5} & v_{6} & v_{7} \\ w_{1} & w_{2} & w_{3} & w_{4} & w_{5} & w_{6} \\ w_{2} & w_{3} & w_{4} & w_{5} & w_{6} & w_{7} \\ \phi_{1} & \phi_{2} & \phi_{3} & \phi_{4} & \phi_{5} & \phi_{6} \\ \phi_{2} & \phi_{3} & \phi_{4} & \phi_{5} & \phi_{6} & \phi_{7}\end{array}\right]$

The above mapping of the degrees of freedom presents also the relationships of the longitudinal rotations for future in plate behavior stiffness matrices. Based on this mapping, the assembly of the global cross-section stiffness matrices of membrane behavior leads to:

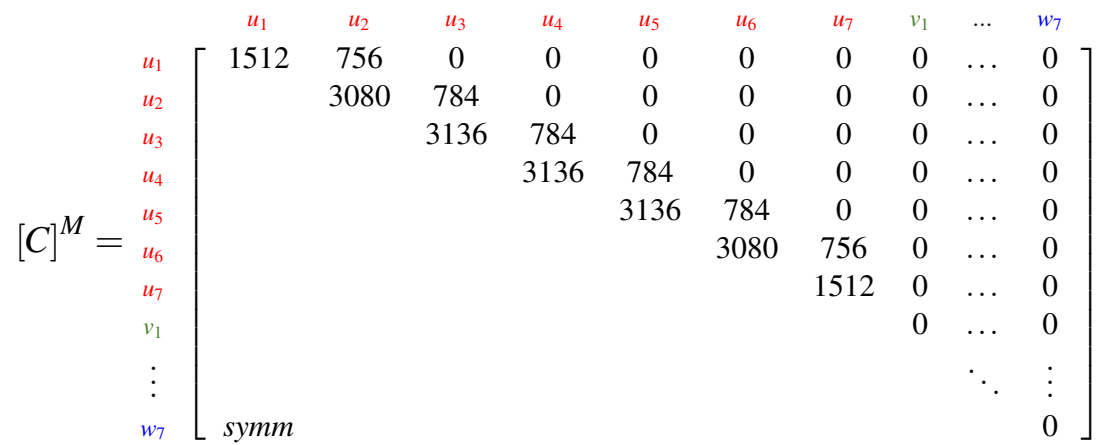

In this matrix, the null values of all terms involving the transverse displacements, $v$ and $w$, highlight the assumption of a simplified material constitutive relationship, given in eq. 2.10. Reciprocally, the global transverse membrane stiffness matrix presents null 
values concerning the longitudinal displacements degrees of freedom:

$$
\begin{aligned}
& {[B]^{M}=}
\end{aligned}
$$

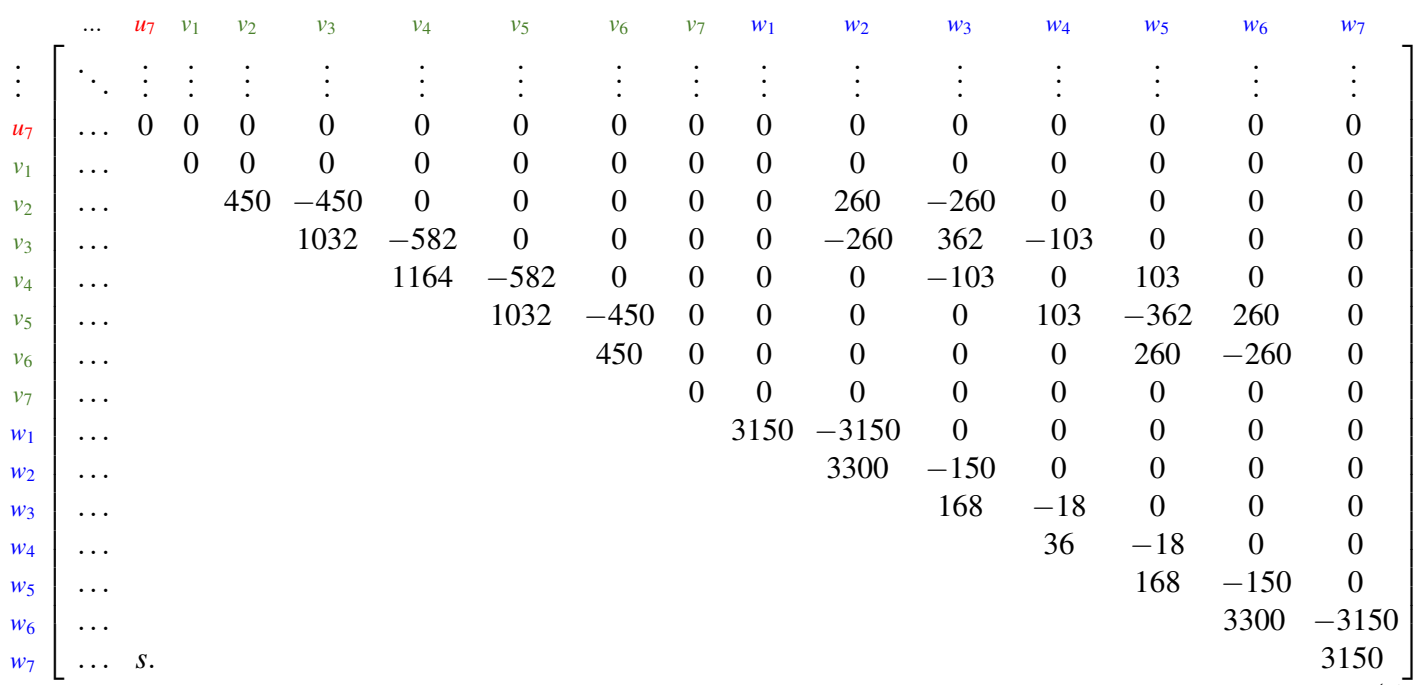

The global shear stiffness matrix due to membrane behavior presents a property concerning segments with an end free node: the degree of freedom of the correspondent transverse displacement has no participation. One realizes it in rows and columns $V_{1}$ and $V_{7}$ of eq. A.10 and check the same rows and columns in expressions A.8 and A.9. 


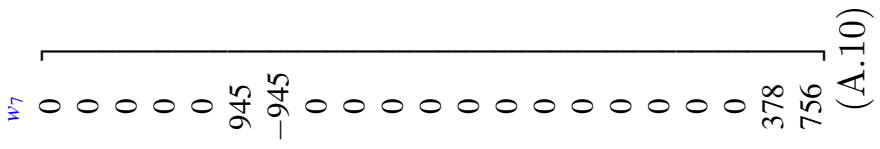

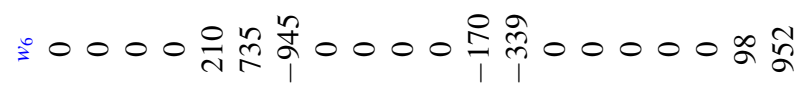

$$
\begin{aligned}
& \text { m000n }
\end{aligned}
$$

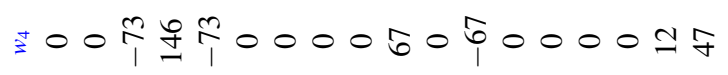

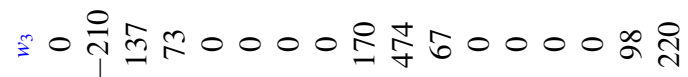

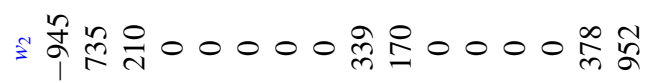

$$
\begin{aligned}
& \text { 三集过000000000000k } \\
& =00000000000000
\end{aligned}
$$

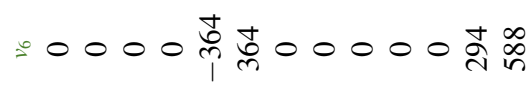

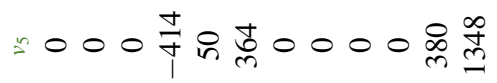

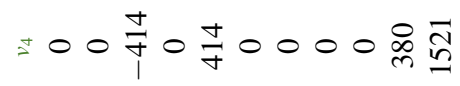

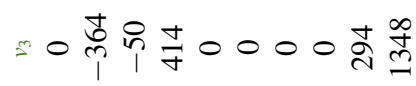

$$
\begin{aligned}
& \because \text { 常 } \\
& =00000000 \\
& \text { s00000望盀 }
\end{aligned}
$$

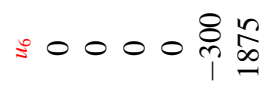

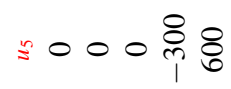

$$
\begin{aligned}
& \text { క0 } \\
& \text { ऽ } \\
& \cong \stackrel{n}{n} \stackrel{n}{n}=
\end{aligned}
$$

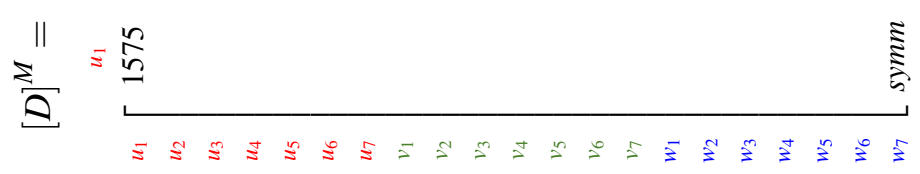


The cross-section stiffness matrices due to plate behavior follows a similar assembling:

- longitudinal plate stiffness matrix: based on eq. 4.36 , one reaches the following matrices:

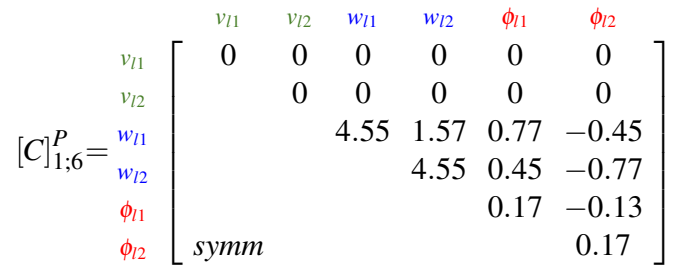

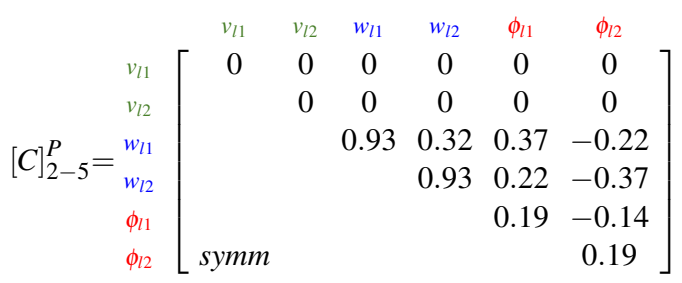

- shear plate stiffness matrix: it is obtained directly by eq. 4.39. Thus, the local stiffness matrices are:

$$
\begin{aligned}
& {[D]_{1 ; 6}^{P}={ }_{v_{l 1}} \begin{array}{c}
v_{l 1} \\
v_{l 2} \\
w_{l 2} \\
\phi_{l 1} \\
\phi_{l 2}
\end{array}\left[\begin{array}{cccccc}
v_{l 1} & v_{l 2} & w_{l 1} & w_{l 2} & \phi_{l 1} & \phi_{l 2} \\
& 0 & 0 & 0 & 0 & 0 \\
& 0 & 0 & 0 & 0 & 0 \\
& & 20.41 & -20.41 & 2.04 & 2.04 \\
& & & 20.41 & -2.04 & -2.04 \\
& & & & 3.27 & -0.82 \\
\text { symm } & & & & 3.27
\end{array}\right]} \\
& {[D]_{2-5}^{P}=\begin{array}{c}
v_{l 1} \\
v_{l 2} \\
w_{l 1} \\
w_{l 2} \\
\phi_{l 1} \\
\phi_{l 2}
\end{array}\left[\begin{array}{cccccc}
v_{l 1} & v_{12} & w_{l 1} & w_{l 2} & \phi_{11} & \phi_{12} \\
0 & 0 & 0 & 0 & 0 & 0 \\
& 0 & 0 & 0 & 0 & 0 \\
& & 0.77 & -0.77 & 0.18 & 0.18 \\
& & & 0.77 & -0.18 & -0.18 \\
& & & & 0.67 & -0.17 \\
\text { symm } & & & & & 0.67
\end{array}\right]}
\end{aligned}
$$

- transverse elongation plate stiffness matrix: lastly, eq. 4.42 provides the transverse bending stiffness matrices, which for segments 1 and 6 leads to:

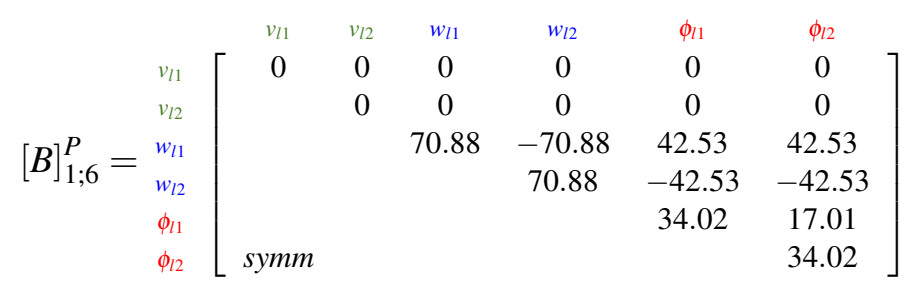


and for segments $2,3,4$ and 5 to:

$$
[B]_{2-5}^{P}=\begin{gathered}
v_{11} \\
v_{12} \\
w_{l 1} \\
w_{12} \\
\phi_{11} \\
\phi_{12}
\end{gathered}\left[\begin{array}{cccccc}
v_{11} & v_{12} & w_{11} & w_{12} & \phi_{11} & \phi_{12} \\
0 & 0 & 0 & 0 & 0 & 0 \\
& 0 & 0 & 0 & 0 & 0 \\
& & 0.49 & -0.49 & 0.69 & 0.69 \\
& & & 0.49 & -0.69 & -0.69 \\
& & & & 1.28 & 0.64 \\
& & & & & \\
& & & & & 1.28
\end{array}\right]
$$

To transform each plate stiffness matrix of each segment, one must apply equations 4.44 and 4.45. Furthermore, the assembly of the global plate stiffness matrices is driven by the mapping matrix in A.7. Thereby, one achieves the following matrices: 


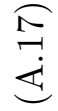

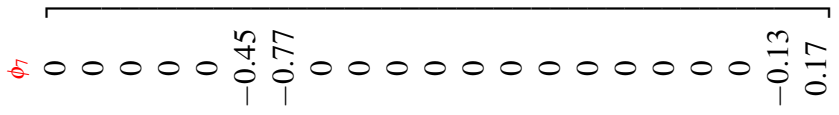

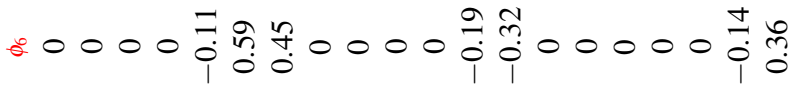

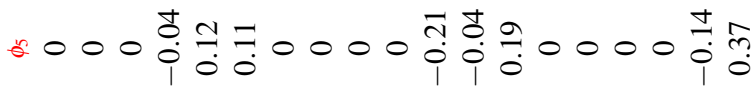

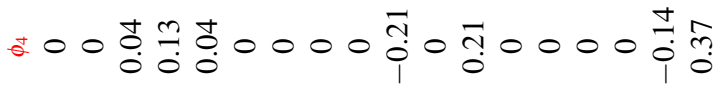

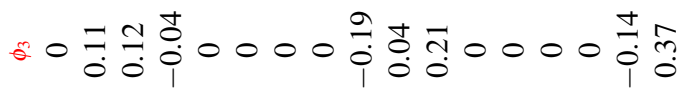

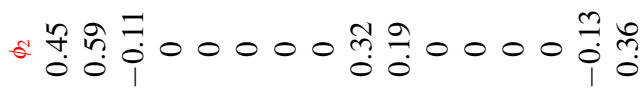

$$
\begin{aligned}
& =\frac{1}{\hat{0}} \stackrel{i}{i} 000000000000 \frac{0}{0} \\
& 100000000000000
\end{aligned}
$$

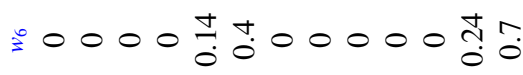

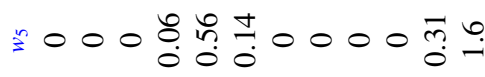

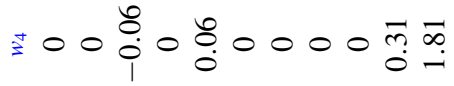

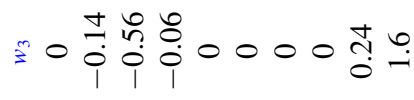

$$
\begin{aligned}
& \cong 0 \underset{i}{t} \frac{1}{0} 0000000 \\
& \equiv 00000000 \\
& \text { =00000望 } \\
& =0000 \stackrel{\infty}{\stackrel{\infty}{+}+\frac{1}{\dot{r}}}
\end{aligned}
$$

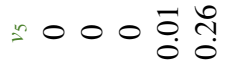

$$
\begin{aligned}
& \text { × } 00 \stackrel{0}{0}: 0 \\
& =0 \stackrel{\infty}{0} \stackrel{0}{0} \stackrel{0}{0}
\end{aligned}
$$

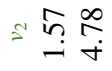

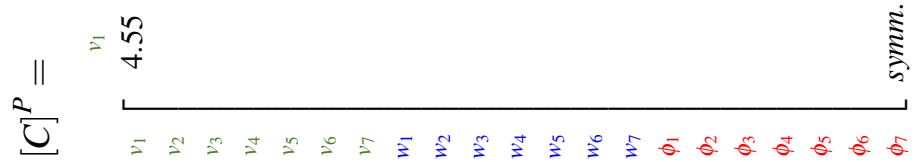




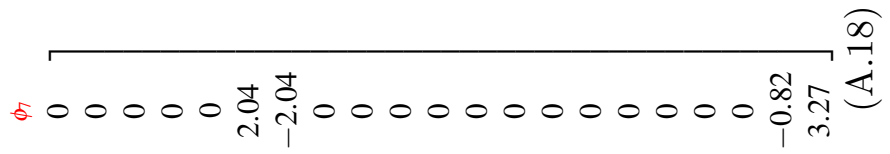

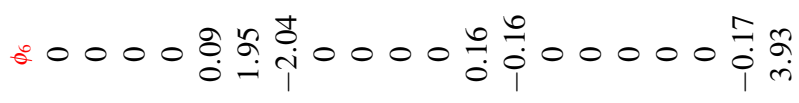

$$
\begin{aligned}
& \text { 。 }
\end{aligned}
$$

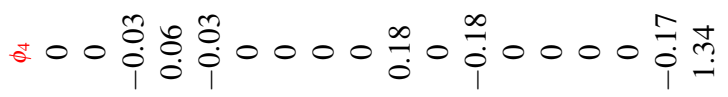

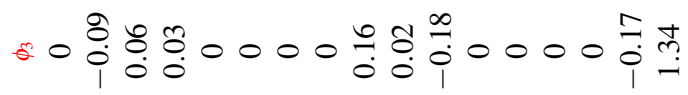

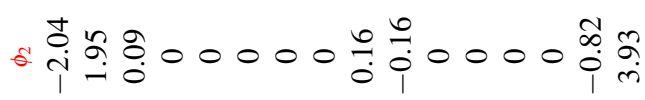

$$
\begin{aligned}
& =\underset{i}{i} \underset{4}{4} 000000000000 \hat{m} \\
& \equiv 00000000000000
\end{aligned}
$$

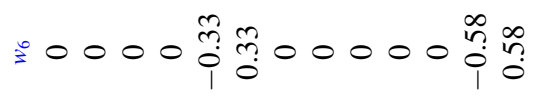

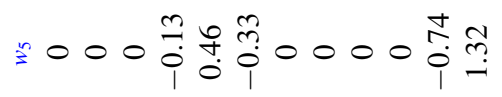

$$
\begin{aligned}
& =00 \frac{m}{0} \circ \frac{m}{i} 0000 \stackrel{\frac{1}{i}}{\frac{R}{q}} \\
& \text { ?ం } \\
& \cong 0 \prod_{i}^{m} \stackrel{m}{0} 00000 \stackrel{\infty}{n} \\
& \equiv 00000000
\end{aligned}
$$

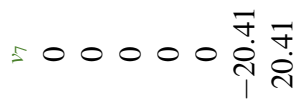

$$
\begin{aligned}
& =0000 \frac{9}{i} \stackrel{i}{\dot{1}} \\
& \text { - } 000 \frac{1}{0} \tilde{0}
\end{aligned}
$$

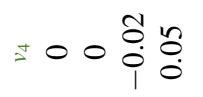

$$
\begin{aligned}
& =0 \frac{1}{0} \hat{0}
\end{aligned}
$$

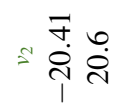

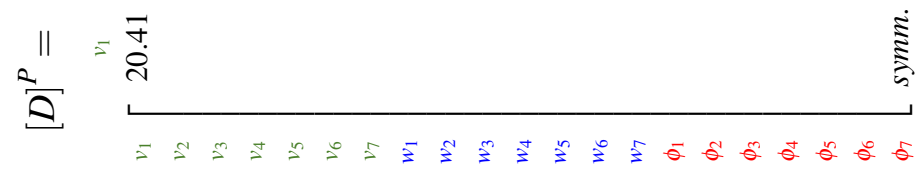




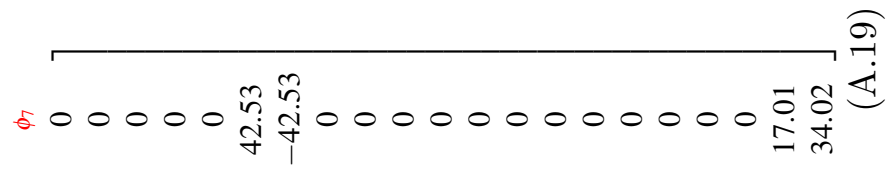

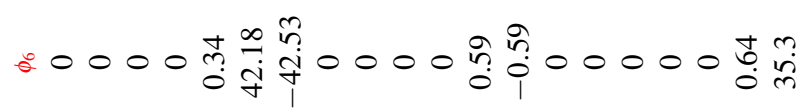

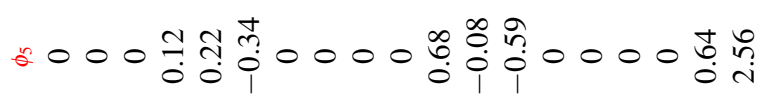

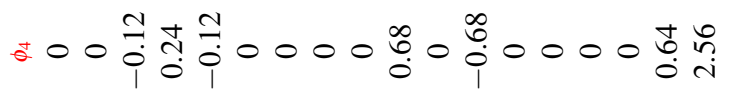

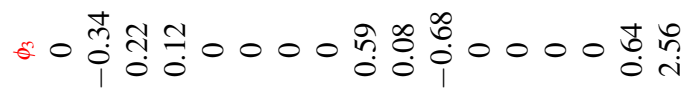

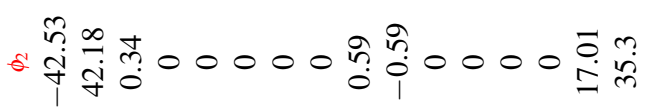

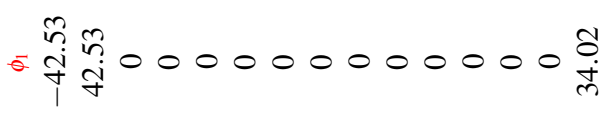

$$
\begin{aligned}
& \$ 00000000000000
\end{aligned}
$$

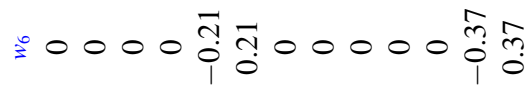

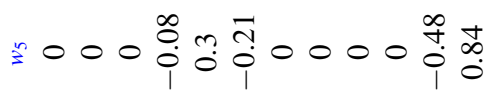

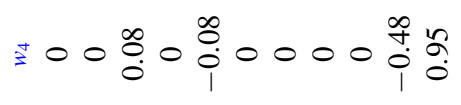

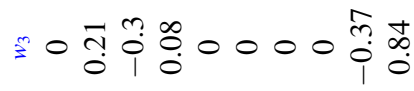

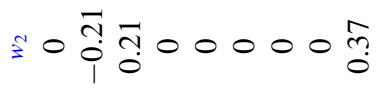

$$
\begin{aligned}
& \equiv 00000000
\end{aligned}
$$

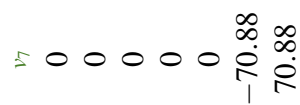

$$
\begin{aligned}
& =0000 \frac{1}{0}= \\
& =000 \frac{1}{0} \frac{0}{0}
\end{aligned}
$$

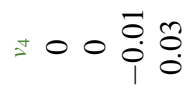

$$
\begin{aligned}
& =0 \frac{1}{0} \frac{1}{0} \\
& =\stackrel{\infty}{\infty}
\end{aligned}
$$

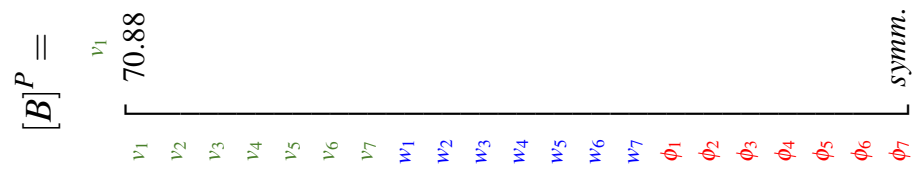




\section{A.1.2. Step 2 - transverse elongation constrains}

As one obtains the global cross-section stiffness matrices, the next step is the assembly of multi-freedom constraints concerning the transverse elongation. As mentioned in sec. 4.4.1, these constraints are not only necessary in plate stiffness matrices, in which the plate theory does not involve these degrees of freedom, but they are also necessary due to GBT's assumption of non-transverse strain: $\varepsilon_{s}=0$

Equation. 4.49 provides the coupling in the element local coordinate system. Thus, one must apply the following transformation to achieve the multi-freedom constraints in the cross-section global coordinate system:

$$
\left[\begin{array}{cccc}
\cos [\theta] & 0 & \sin [\theta] & 0 \\
0 & \cos [\theta] & 0 & \sin [\theta] \\
-\sin [\theta] & 0 & \cos [\theta] & 0 \\
0 & -\sin [\theta] & 0 & \cos [\theta]
\end{array}\right]\left[\begin{array}{llll}
1 & -1 & 0 & 0
\end{array}\right]\left[\begin{array}{c}
v_{i} \\
v_{f} \\
w_{i} \\
w_{f}
\end{array}\right]_{g}=0
$$

Where $\theta$ is the angle of the segment from the global coordinate system to the local one. After this transformation in each segment, one collects all multi-freedom constraints equations into a single matrix formulation:

$$
\begin{aligned}
& 0=[1,1 N]\left[\begin{array}{c}
v \\
w
\end{array}\right]=
\end{aligned}
$$

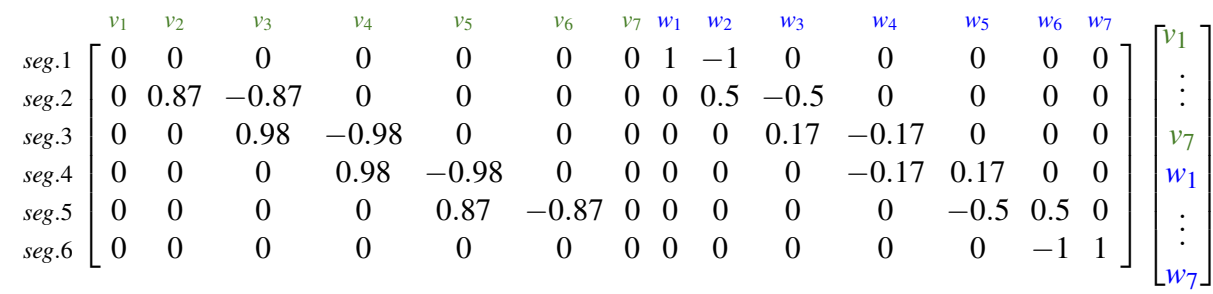

From the above matrix, one starts a recursive approach to achieve the transformation matrix $\left[{ }_{1} U\right]$. This approach is based on selecting one coupling equation from matrix $\left[{ }_{1,1} N\right]$ to build an intermediate transformation matrix $[1, i U]$, applied back to the matrix $\left[{ }_{1,1} N\right]$ in order to find a new reduced coupling matrix $\left[{ }_{1,2} N\right]$. From this matrix, the approach is restarted to reach the next intermediate transformation matrix $[1, i+1 U]$. Then, the approach continues until it covers all constraint equations. Once this process is done, the product among all intermediate matrices leads to the final transformation matrix $\left[{ }_{1} U\right]$ :

$$
\left[{ }_{1} U\right]=\prod_{i=1}^{n}[1, i]
$$

For instance, if one selects the last row in matrix $\left[{ }_{1,1} N\right]$ in eq. A.21 and the degree of freedom $w_{7}$ as a slave one, then one obtains the following intermediate transformation matrix, $[1, i U]$ : 


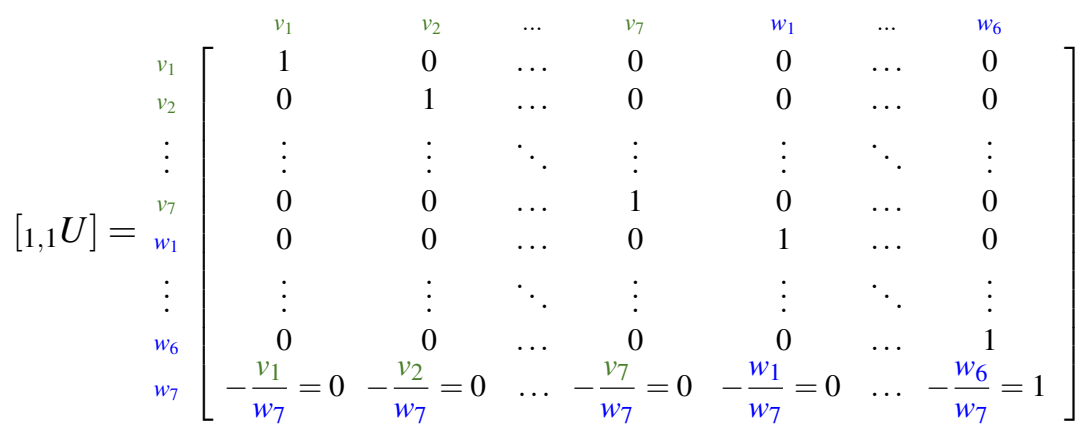

From these intermediate transformation matrix, one transforms the initial multi-freedom constrain matrix into:

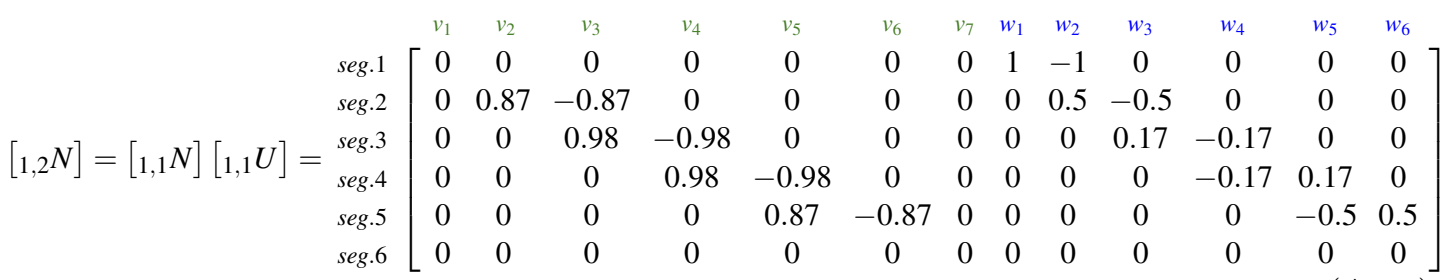

Since the last row of $[1,1 N]$ was chosen, $[1,2 N]$ must eliminate this row, as presented above. Continuing the approach, if one selects the fifth row of $\left[{ }_{1,2} N\right]$ and $w_{6}$ as slave degree of freedom, then the following $\left[{ }_{1, i+2} U\right]$ is set up:

$$
[1,2 U]=\begin{gathered}
v_{1} \\
v_{2} \\
\vdots \\
v_{6} \\
\vdots \\
w_{5} \\
w_{6}
\end{gathered}\left[\begin{array}{ccccccc}
v_{1} & v_{2} & \ldots & v_{5} & v_{6} & \ldots & w_{5} \\
1 & 0 & \ldots & 0 & 0 & \ldots & 0 \\
0 & 1 & \ldots & 0 & 0 & \ldots & 0 \\
\vdots & \vdots & \ddots & \vdots & \vdots & \ddots & \vdots \\
0 & 0 & \ldots & 1 & 0 & \ldots & 0 \\
0 & 0 & \ldots & 0 & 1 & \ldots & 0 \\
\vdots & \vdots & \ddots & \vdots & \vdots & \ddots & \vdots \\
-\frac{v_{1}}{w_{6}}=0 & -\frac{v_{2}}{w_{6}}=0 & \ldots & 0 & 0 & \ldots & 1 \\
w_{6} & =-1.732 & -\frac{v_{7}}{w_{6}}=1.732 & \ldots & -\frac{v_{5}}{w_{6}}=1
\end{array}\right]
$$

Thus, the new intermediate coupling matrix $[1,3 N]$ is:

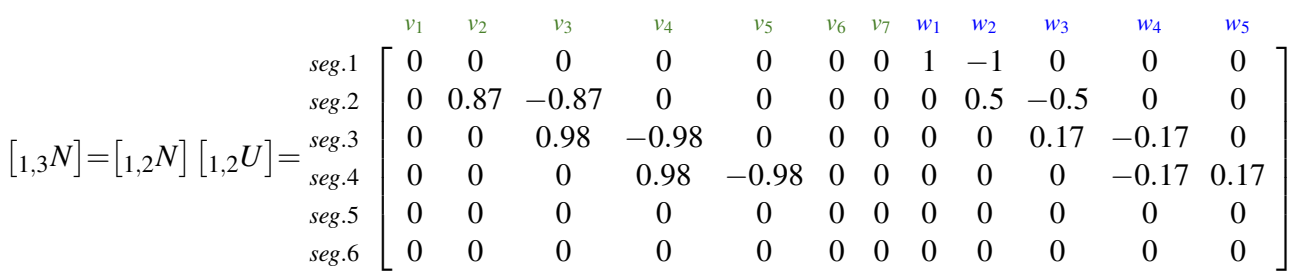

Repeating this process until the last, one achieves by the eq. A.22 the transverse elongation constraint: 


$$
\begin{aligned}
& {\left[{ }_{1} U\right]=\prod_{i=1}^{n}\left[{ }_{1, i} U\right]=\left[{ }_{1,1} U\right]\left[{ }_{1,2} U\right]\left[{ }_{1,3} U\right]\left[{ }_{1,4} U\right]\left[{ }_{1,5} U\right]\left[{ }_{1,6} U\right]=}
\end{aligned}
$$

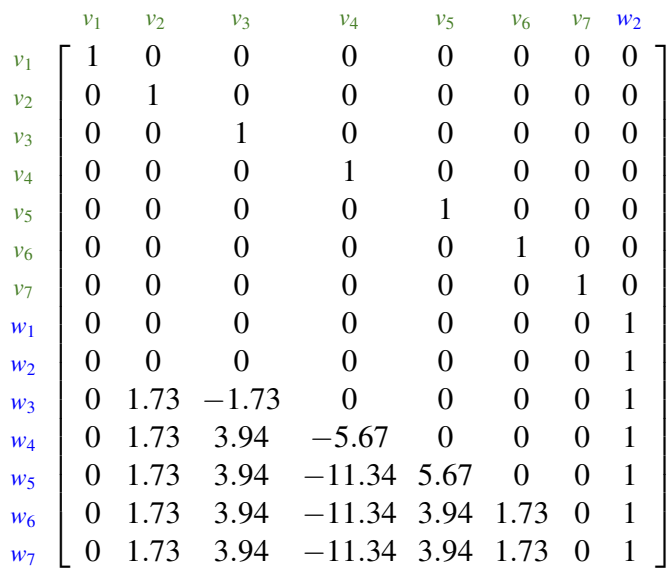

Once $\left[{ }_{1} U\right]$ was obtained, then it is possible to apply eq. 4.51 to verify this transformation matrix. Thereby, one finds the constrained form of shear membrane cross-section stiffness matrix by eq. 4.52 , which leads to A.28.

Here, one observes that all membrane stiffness matrices due to membrane behavior have no terms related to the degrees of freedom $v_{1}$ and $v_{7}$. End segments for open cross-section do not have any stiffness in the transverse direction of the segment at the end nodes. Hence, one can eliminate the corresponding row and columns of these degrees of freedom in membrane stiffness matrices.

$$
\begin{aligned}
& {\left[{ }_{1} D\right]^{M}=}
\end{aligned}
$$

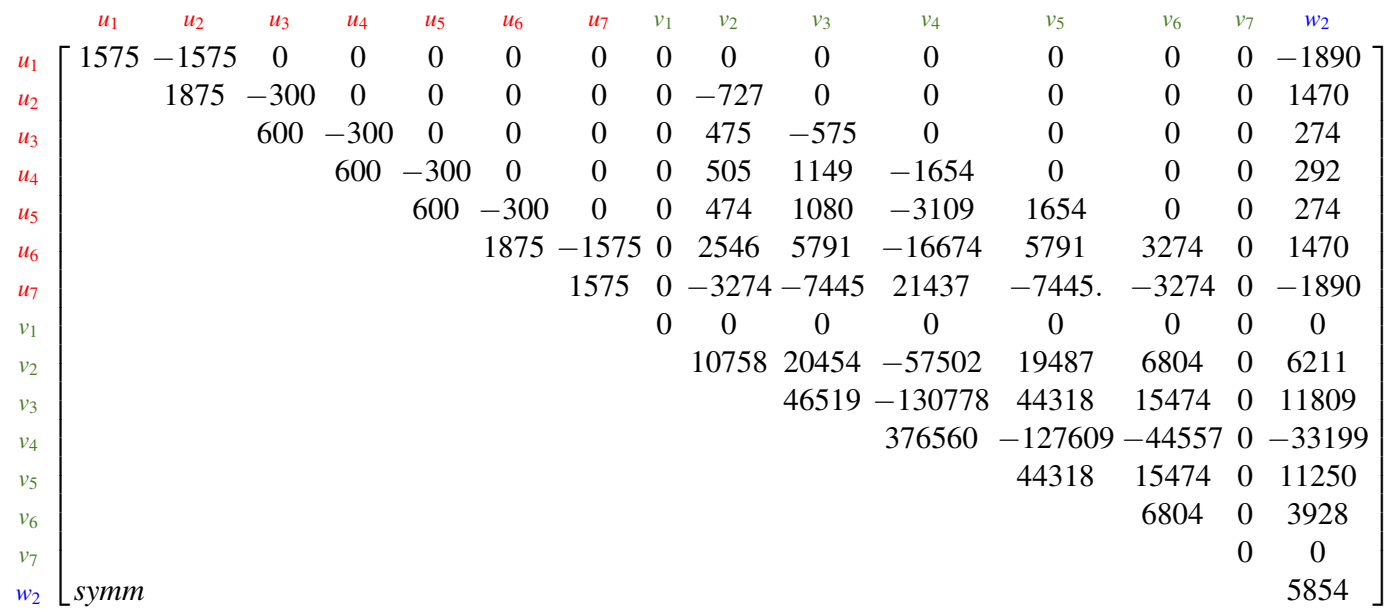




\section{A.1.3. Step 3 - Pure elongation membrane mode}

The isolation of the single pure membrane mode, the longitudinal elongation one, is straight forward. It is only necessary to set up the transformation matrix $\left[{ }_{2} U\right]$ based on a unit longitudinal displacement:

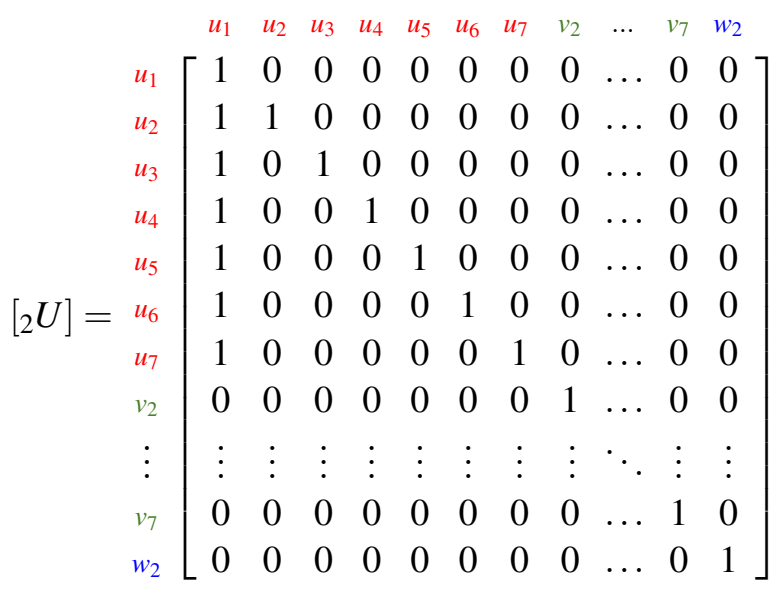

This transformation applied in equations 4.57 and 4.58 leads to the matrices $\left[{ }_{2} C\right]^{M}$ and $\left[{ }_{2} D\right]^{M}$, given in equations A.30 and A.31, respectively.

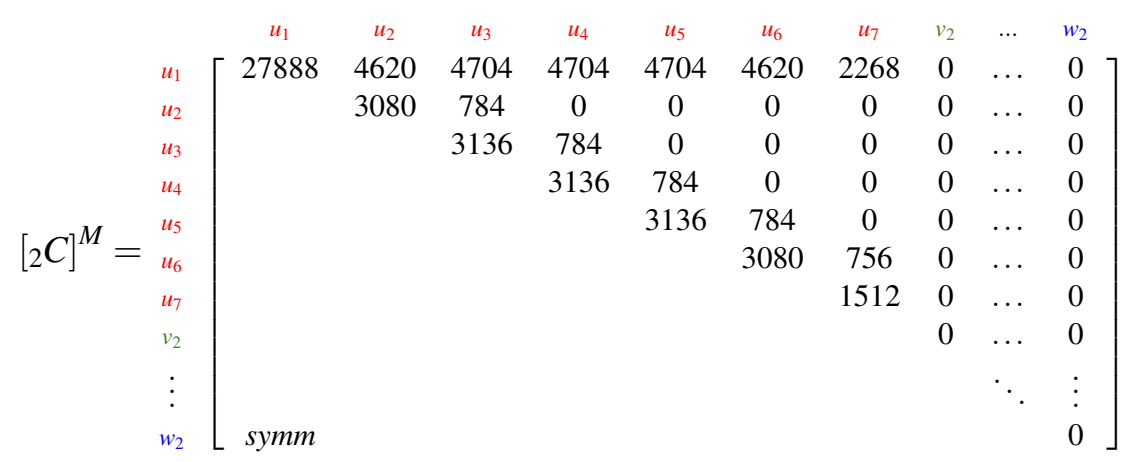

Here, one observes that the first term in first row and column is, in fact, the stiffness product $E A=21000 \mathrm{MN} / \mathrm{m}^{2} * 1.328 \mathrm{~m}^{2}$ of the cross-section.

From $\left[{ }_{2} D\right]^{M}$ one realizes that $\left[{ }_{2} U\right]$ removes the shear membrane stiffness of the longitudinal elongation mode. I.e., there is any term in the first row/column of $\left.{ }_{2} D\right]^{M}$ : 


$$
\begin{aligned}
& {\left[{ }_{2} D\right]^{M}=}
\end{aligned}
$$

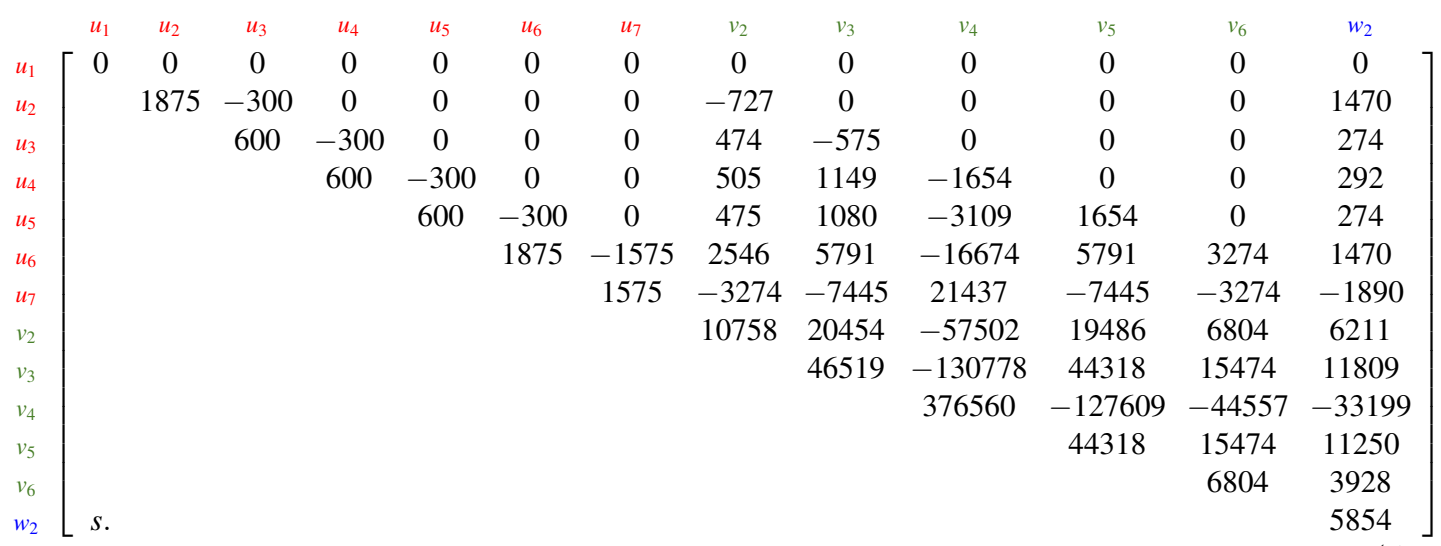

Hence, it is possible to isolate this mode from all others by the transformation $\left[{ }_{3} U\right]$. Based on the coupling equation. 4.59, one uses the first row of matrix $\left[{ }_{2} C\right]^{M}$ to achieve the transformation row in $\left[{ }_{3} U\right]:\left[\begin{array}{lll}-\frac{u_{2}}{u_{1}}=-\frac{4620}{27888} & -\frac{u_{3}}{u_{1}}=-\frac{4704}{27888} & \cdots\end{array}\right]$

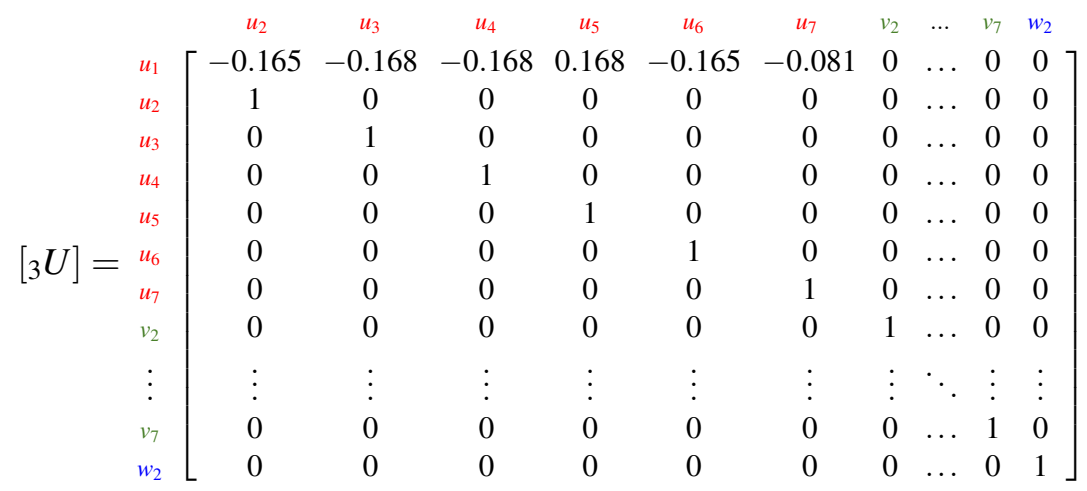

Applying equations 4.60 and 4.61, one reaches the membrane stiffness matrices without the pure longitudinal elongation mode:

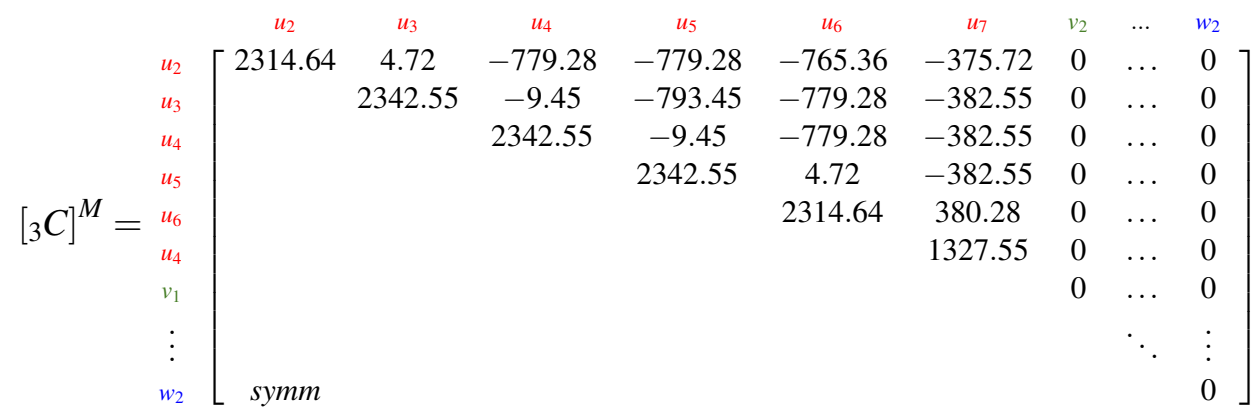

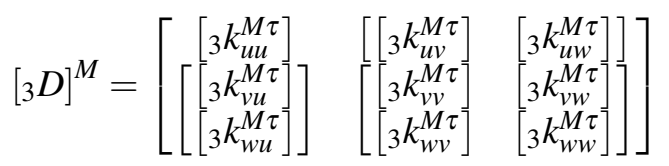


where:

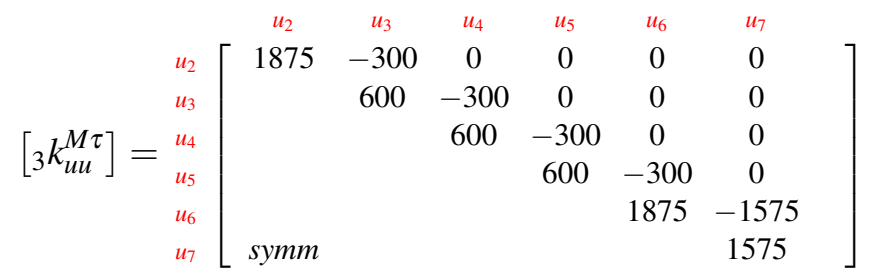

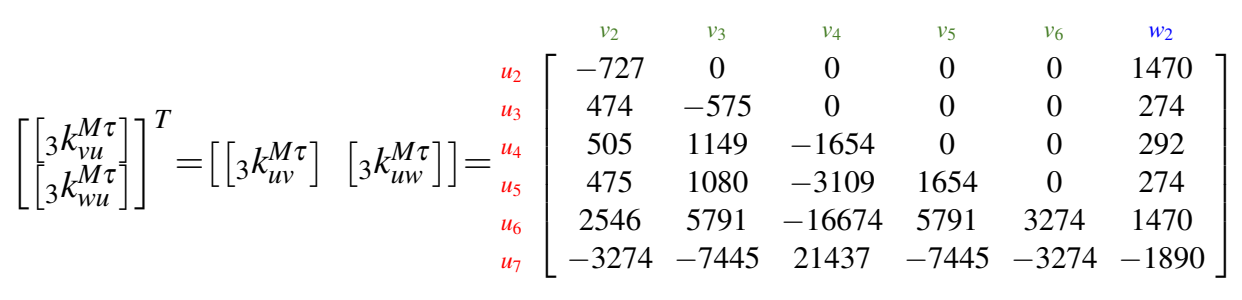

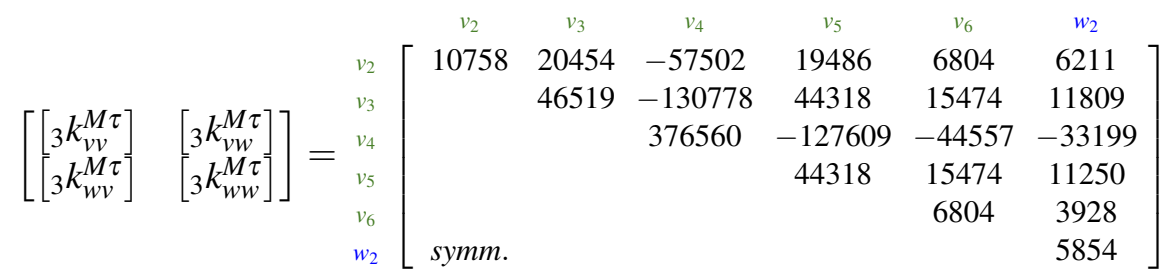

\section{A.1.4. Step 4 - Membrane shear strain constraint}

Inserting equations A.36 and A.35 into eq. 4.66, one achieves the membrane shear multi-freedom constraint:

$$
-\left[\left[{ }_{3} k_{u v}^{M \tau}\right]\left[{ }_{3} k_{u w}^{M \tau}\right]\right]^{-1}\left[{ }_{3} k_{u u}^{M \tau}\right]=\begin{gathered}
v_{2} \\
v_{3} \\
v_{4} \\
v_{5} \\
v_{6} \\
v_{1}
\end{gathered}\left[\begin{array}{cccccc}
u_{2} & u_{3} & u_{4} & u_{5} & u_{6} & u_{7} \\
-0.89 & -0.41 & 0 & 0 & 0 & 0 \\
0 & 0.7 & -0.52 & 0 & 0 & 0 \\
0 & 0 & 0 & -0.18 & 0 & 0 \\
0 & 0 & 0 & 0.41 & 0.18 & 0 \\
-0.83 & 0 & 0 & 0 & 0 & 0
\end{array}\right]
$$

The above matrix leads to the matrix $\left[{ }_{4} U\right]$, as presented in eq. 4.67. Moreover, one verifies this transformation by: i) eq. 4.68 , which must vanish any stiffness due to membrane's shear strains, $\left[{ }_{4} C\right]^{M}=0$; ii) eq. 4.69, which must keep $\left[{ }_{4} C\right]^{M}=\left[{ }_{3} C\right]^{M}$. Thus, at this stage, the cross-section stiffness matrices due to membrane behavior are limited only to longitudinal matrix.

\section{A.1.5. Step 5 - Pure plate modes}

After all transformation in membrane stiffness, one must extract and isolate the pure plate modes. As exposed in section 4.4.2, the extraction of pure plate mode is straight forward. It is only necessary to set up a transformation matrix, which isolates the degrees of freedom of longitudinal rotation $\phi$, and the orthogonal transverse displacement at the extreme node of free end segments, $v_{1}$ and $v_{7}$ in this example. Thus, the transformation matrix $\left[{ }_{4} U\right]$ is: 


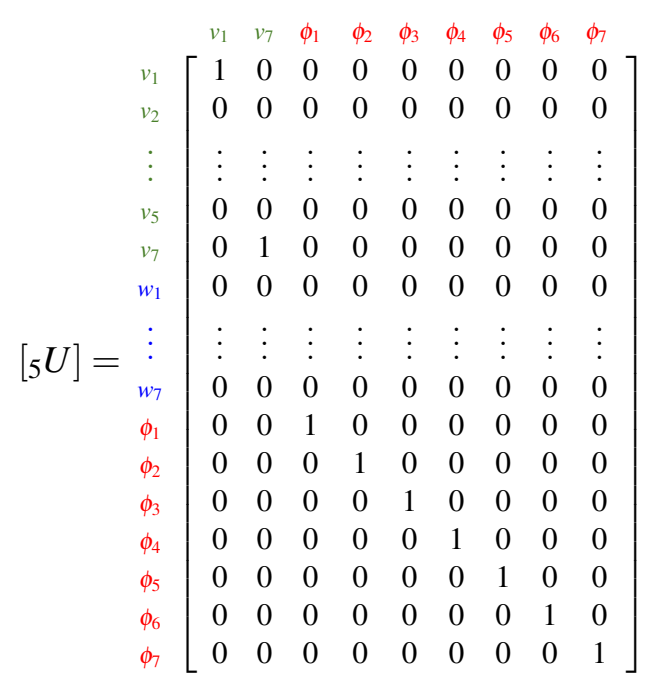

The introduction of above transformation matrix into equations 4.70, 4.71 and 4.72 leads to pure plate behavior stiffness matrices, as presented below. They are directly addressed to the quadratic eigenvalue problem.

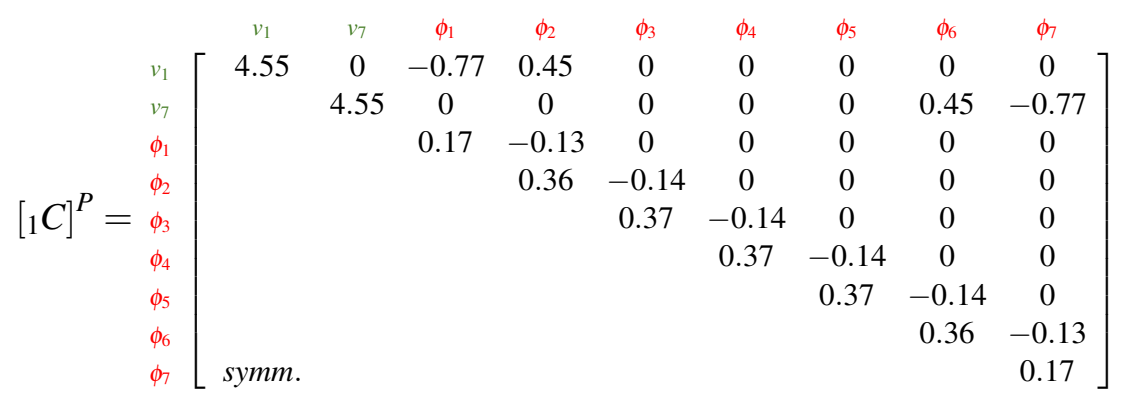

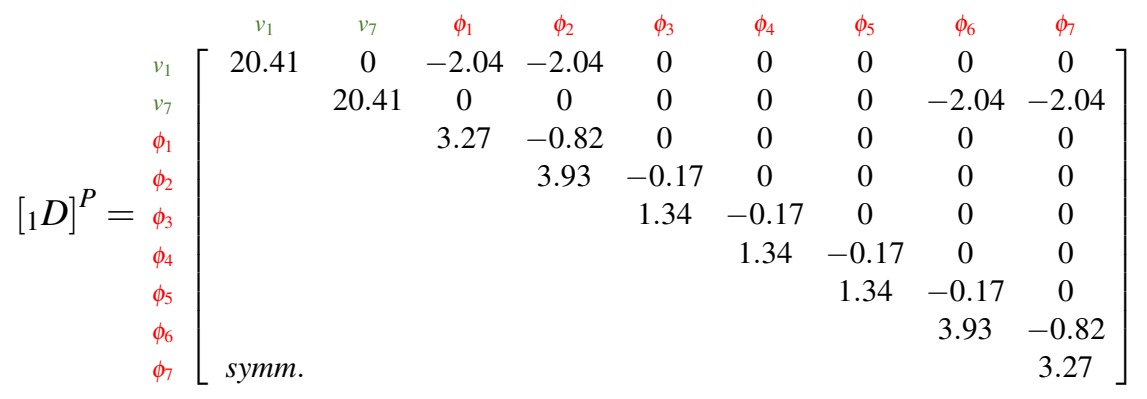

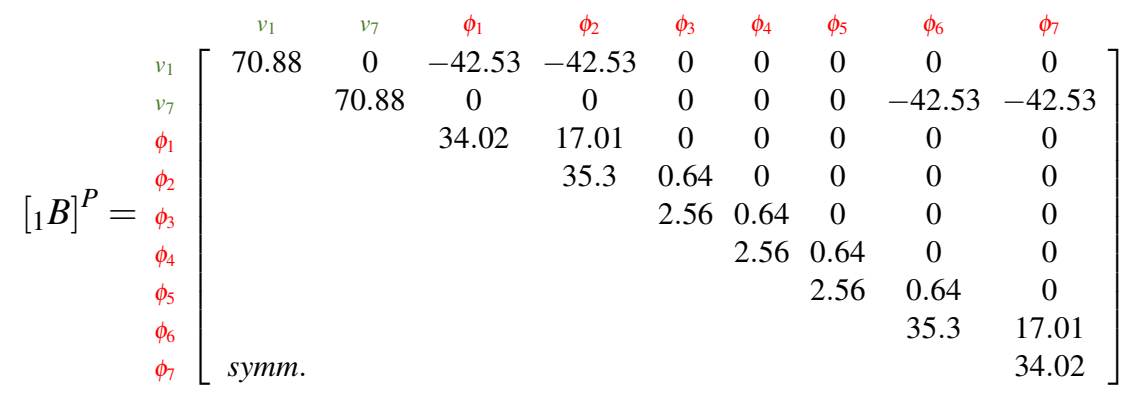




\section{A.1.6. Step 6 - Membrane-plate modes}

The isolation of membrane-plate modes is complementary to pure plate modes extracted in the previous step. Therefore, one expects 9 coupling equations in this example: 4 concerning the assumption of null transverse bending moment at any point of the extreme segments (2 coupling equations for each segment); 5 concerning the equilibrium of transverse bending moment at the internal nodes.

Starting with the extreme segments, one obtains eq. 4.73 directly from last two rows of transverse stiffness matrix, given in eq. A.15. Thus, the coupling equation in the local coordinate system for segments 1 and 6 is:

$$
\left[\begin{array}{l}
0 \\
0
\end{array}\right]=\left[\begin{array}{cccccc}
v_{11} & v_{12} & w_{11} & w_{12} & \phi_{l 1} & \phi_{12} \\
0 & 0 & 42.53 & -42.53 & 34.02 & 17.01 \\
0 & 0 & 42.53 & -42.53 & 17.01 & 34.02
\end{array}\right]\left[\begin{array}{llllll}
v_{1} & v_{2} & w_{1} & w_{2} & \phi_{1} & \phi_{2}
\end{array}\right]_{l}^{T}
$$

Applying the transformation from local to the global coordinate system, together with the mapping among the local and global degrees of freedom, one achieves the following global coupling equations:

- For segment 1:

$$
\left[\begin{array}{l}
0 \\
0
\end{array}\right]=\left[\begin{array}{cccccc}
-42.53 & 42.53 & 0 & 0 & 34.02 & 17.01 \\
-42.53 & 42.53 & 0 & 0 & 17.01 & 34.02
\end{array}\right]\left[\begin{array}{llllll}
v_{1} & v_{2} & w_{1} & w_{2} & \phi_{1} & \phi_{2}
\end{array}\right]^{T}
$$

- For segment 6:

$$
\left[\begin{array}{l}
0 \\
0
\end{array}\right]=\left[\begin{array}{cccccc}
42.53 & -42.53 & 0 & 0 & 34.02 & 17.01 \\
42.53 & -42.53 & 0 & 0 & 17.01 & 34.02
\end{array}\right]\left[\begin{array}{llllll}
v_{6} & v_{7} & w_{6} & w_{7} & \phi_{6} & \phi_{7}
\end{array}\right]^{T}
$$

Concerning the internal nodes, the coupling equations of nodes 2 and 6 have a more straightforward implementation. The displacements in segments 2 and 5 must lead to zero transverse bending moments at these nodes. Hence, one applies the transformation and mapping from local to the global coordinate system over the fifth and last rows of the matrix in eq. A.16, to find the coupling equation of node 2 and 6 , respectively:

- For node 2:

$$
0=\left[\begin{array}{cccccc}
v_{2} & v_{3} & w_{2} & w_{3} & \phi_{2} & \phi_{3} \\
-0.343 & 0.343 & 0.594 & -0.594 & 1.28 & 0.64
\end{array}\right]\left[\begin{array}{llllll}
v_{2} & v_{3} & w_{2} & w_{3} & \phi_{2} & \phi_{3}
\end{array}\right]^{T}
$$

- For node 6:

$$
0=\left[\begin{array}{cccccc}
v_{5} & v_{6} & w_{5} & w_{6} & \phi_{5} & \phi_{6} \\
0.343 & -0.343 & 0.594 & -0.594 & 1.28 & 0.64
\end{array}\right]\left[\begin{array}{llllll}
v_{5} & v_{6} & w_{5} & w_{6} & \phi_{5} & \phi_{6}
\end{array}\right]^{T}
$$

The other three coupling equations, of internal nodes 3,4 and 5, involve the transverse stiffness matrices of all linked segments at a particular node to obtain the equilibrium. For instance, the above relationship between segments 2 and 3 defines the equilibrium of transverse bending moment at node 3 :

$$
\begin{aligned}
& {\left[\begin{array}{cccccc}
v_{2} & v_{3} & w_{2} & w_{3} & \phi_{2} & \phi_{3} \\
-0.343 & 0.343 & 0.594 & -0.594 & 0.64 & 1.28
\end{array}\right]\left[\begin{array}{llllll}
v_{2} & v_{3} & w_{2} & w_{3} & \phi_{2} & \phi_{3}
\end{array}\right]^{T}=} \\
& \text { - }\left[\begin{array}{cccccc}
v_{3} & v_{4} & w_{3} & w_{4} & \phi_{3} & \phi_{4} \\
-0.12 & 0.12 & 0.675 & -0.675 & 1.28 & 0.64
\end{array}\right]\left[\begin{array}{llllll}
v_{3} & v_{4} & w_{3} & w_{4} & \phi_{3} & \phi_{4}
\end{array}\right]^{T}
\end{aligned}
$$


Which leads to the following coupling equation:

$$
\left[\begin{array}{ccccccccc}
v_{2} & v_{3} & v_{4} & w_{2} & w_{3} & w_{4} & \phi_{2} & \phi_{3} & \phi_{4} \\
-0.343 & 0.224 & 0.119 & 0.594 & 0.081 & -0.675 & 0.64 & 2.56 & 0.64
\end{array}\right]\left[\begin{array}{lllllllll}
v_{2} & v_{3} & v_{4} & w_{2} & w_{3} & w_{4} & \phi_{2} & \phi_{3} & \phi_{4}
\end{array}\right]^{T}=0
$$

Following the same procedure, one obtains the following coupling equations for nodes 4 and 5, respectively:

$$
\begin{aligned}
& {\left[\begin{array}{ccccccccc}
v_{3} & v_{4} & v_{5} & w_{3} & w_{4} & w_{5} & \phi_{3} & \phi_{4} & \phi_{5} \\
-0.119 & 0.238 & -0.119 & 0.675 & 0 & -0.675 & 0.64 & 2.56 & 0.64
\end{array}\right]\left[\begin{array}{lllllllll}
v_{3} & v_{4} & v_{5} & w_{3} & w_{4} & w_{5} & \phi_{3} & \phi_{4} & \phi_{5}
\end{array}\right]^{T}=0}
\end{aligned}
$$

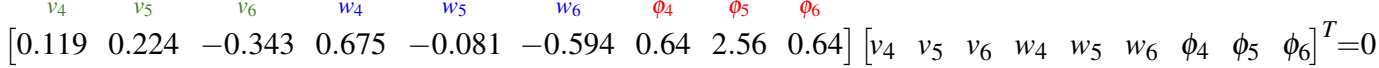

Similar to Step 2, one can collect all these 9 coupling equations into a single coupling matrix. This matrix leads to the same recursive approach to reach the transformation matrix $\left[{ }_{6} U\right]$. However, one remark is necessary here: the slave degrees of freedom must be the ones that have no terms in membrane stiffness matrices. In this example, they are $v_{1}, v_{7}$ and from $\phi_{1}$ until $\phi_{7}$. Thus, the transformation matrix $\left[{ }_{6} U\right]$ is:

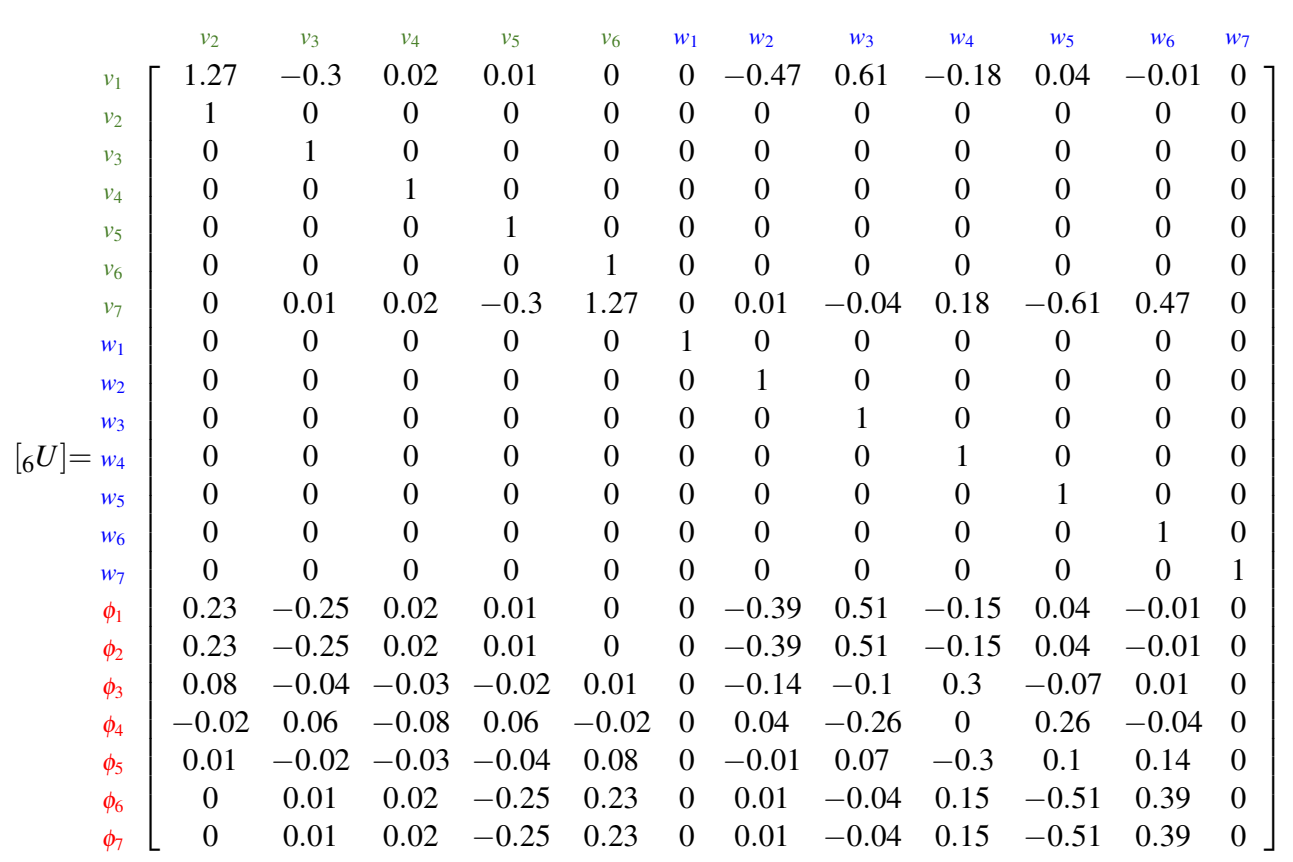

Since the plate assumptions do not consider the transverse elongation of each segment, one must also apply the transformation $\left[{ }_{1} U\right]$, eq. A.27, in the stiffness matrices of plate behavior. Here, one must be aware that the degrees of freedom $v_{1}$ and $v_{7}$ are already removed in transformation matrix $\left[{ }_{6} U\right]$. Fortunately, as transverse displacements of extreme nodes, these degrees of freedom can have their corresponding columns in matrix $\left[{ }_{1} U\right]$ removed without any costs. Thus, one has the following modified $\left[{ }_{1} U^{P}\right]$ matrix: 


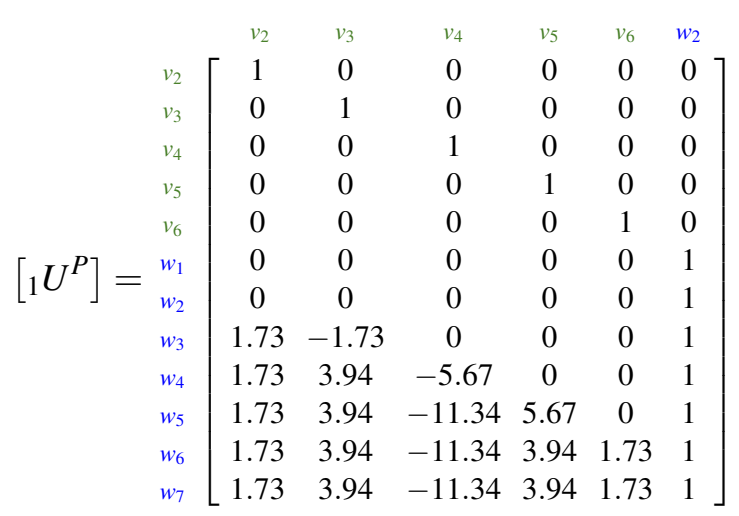

Applying the above transformations, $\left[{ }_{6} U\right]$ and $\left[{ }_{1} U^{P}\right]$, into plate stiffness matrices (equations A.17, A.18 and A.19), one achieves:

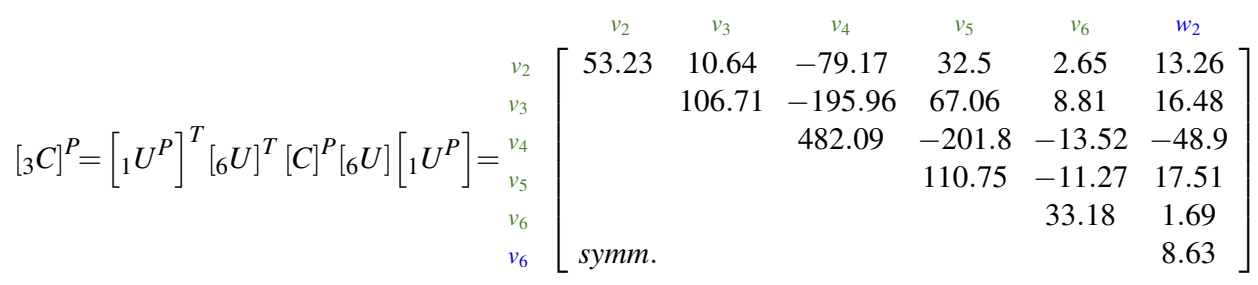

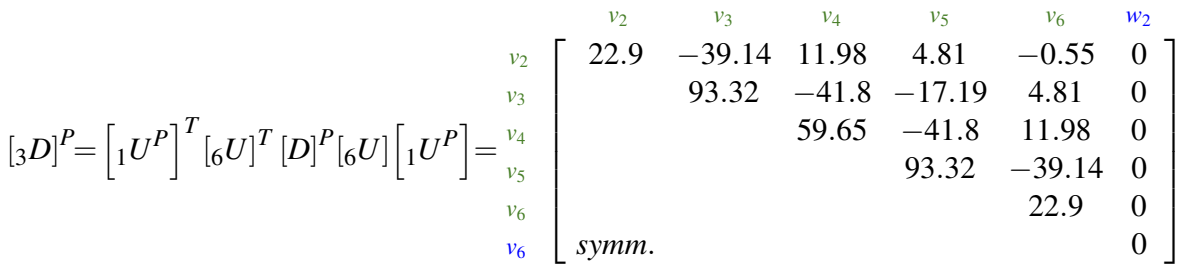

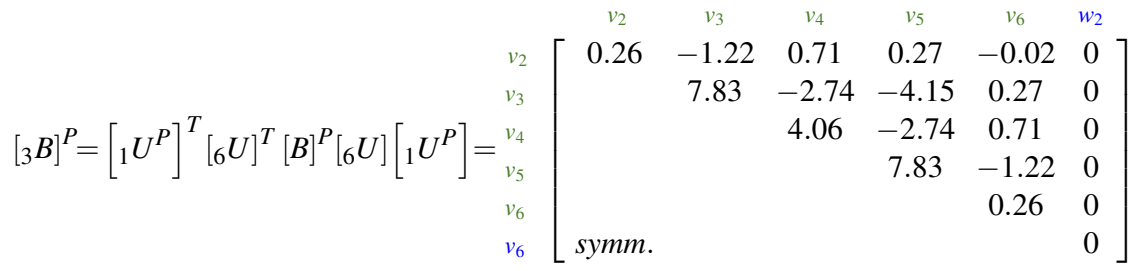

The three above stiffness matrices are related to transverse displacements. However, the membrane stiffness matrix is related to longitudinal displacement. Therefore, it is necessary the transformation from transverse to longitudinal displacement in plate stiffness matrices, which is already defined by the membrane shear multi-freedom constraint, in eq. A.38. For convenience, this multi-freedom constraint matrix is labeled as $\left[{ }_{7} U\right]$ :

$$
\left[{ }_{7} U\right]=-\left[\left[{ }_{3} k_{u v}^{M \tau}\right] \quad\left[{ }_{3} k_{u w}^{M \tau}\right]\right]^{-1}\left[{ }_{3} k_{u u}^{M \tau}\right]
$$

The introduction of the above transformation into equations 4.82, 4.83 and 4.84, with the respective matrices A.54, A.55 and A.56 leads to: 


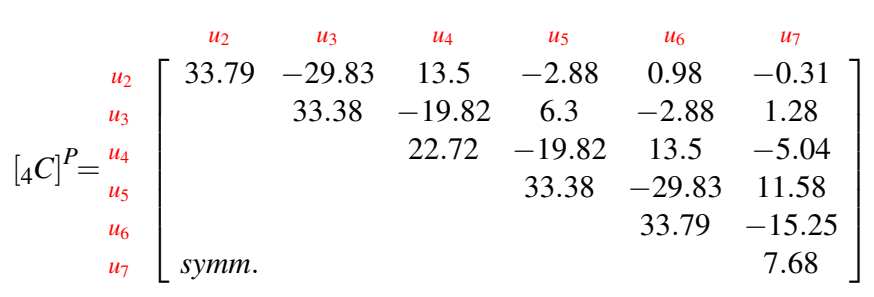

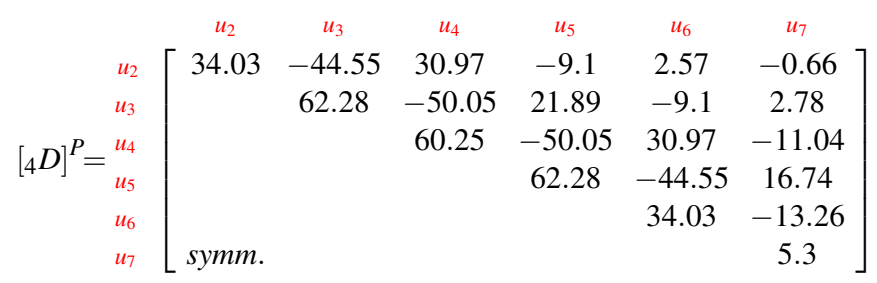

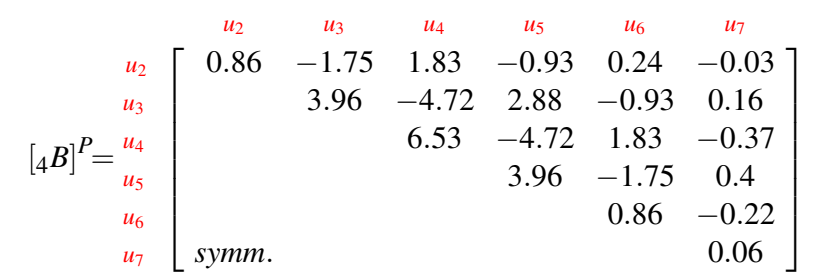

Thus, one can merge the membrane and plate longitudinal strains stiffness matrices into a single matrix, as presented in eq. 4.85:

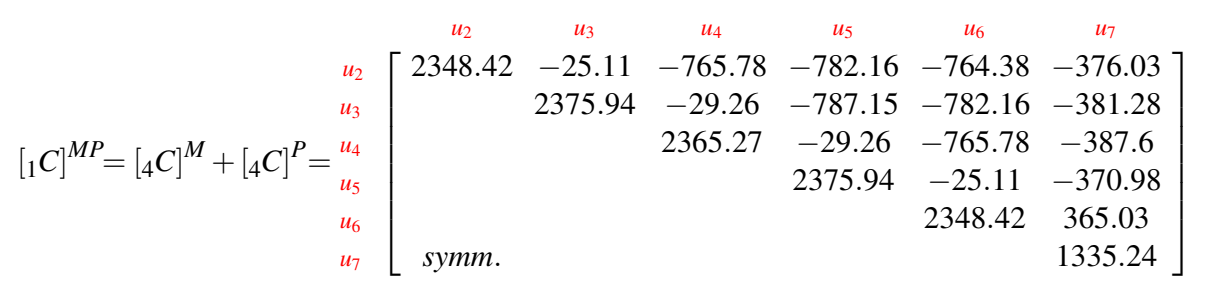

$$
\begin{aligned}
& {\left[{ }_{1} D\right]^{M P}=\left[{ }_{4} D\right]^{M}+\left[{ }_{4} D\right]^{P}=\left[{ }_{4} D\right]^{P} \quad(\text { A. } 62) \quad\left[{ }_{1} B\right]^{M P}=\left[{ }_{1} B\right]^{M}+\left[{ }_{4} B\right]^{P}=\left[{ }_{4} B\right]^{P}}
\end{aligned}
$$

\section{A.1.7. Step 7 - Pure longitudinal bending modes and Vlasov mode}

As exposed in section 4.4.3, the pure bending modes are extracted by the definition of unit transverse displacements. They need to be orthogonal to each other, but not necessarily in the main direction of bending. Moreover, one can present these unit transverse displacements already in the most compacted form, after the constraints of transverse elongation and the elimination of pure plate modes. Then, the unit vectors are collected into matrix $\left[\mathbb{V}_{v w}\right]$ :

$$
\left[\mathbb{V}_{v w}\right]^{T}=\left[\begin{array}{cccccc}
v_{2} & v_{3} & v_{4} & v_{5} & v_{6} & w_{2} \\
1 & 1 & 1 & 1 & 1 & 0 \\
0 & 0 & 0 & 0 & 0 & 1
\end{array}\right]
$$

By applying eq. 4.90, one converts the above matrix into terms of the longitudinal displacement:

$$
\left.\left[{ }_{8} U\right]={ }_{7} U\right]^{-1}\left[\mathbb{V}_{v w}\right]^{T}=\left[\begin{array}{cccccc}
u_{2} & u_{3} & u_{4} & u_{5} & u_{6} & u_{7} T^{T} \\
0 & -2.42 & -5.18 & -7.94 & -10.36 & -10.36 \\
-1.2 & -2.6 & -3.09 & -2.6 & -1.2 & 0
\end{array}\right]
$$


The transformation $\left[{ }_{8} U\right]$ applied in longitudinal strain stiffness matrix, $\left[{ }_{1} C\right]^{M P}$, leads to well-known second order tensor of bending, eq. 4.91:

$$
[\mathbb{I}]=\left[{ }_{8} U\right]^{T}\left[{ }_{1} C\right]^{M P}\left[{ }_{8} U\right]=\left[\begin{array}{cc}
420394.40 & 0 \\
0 & 26212.9
\end{array}\right]
$$

Here, one realizes that the off-diagonal terms are zero. Consequently, the above tensor is already in the main directions and the transformation $\left[T_{f}\right]$, eq. 4.93 , is an identity matrix. Thus, the vectors of the longitudinal displacements of mode 2 and 3 ( $\left[{ }^{2} U\right]$ and $\left[{ }^{2} U\right]$ ) are equal to the columns of matrix $\left.{ }_{8} U\right]$.

To obtain the shear center, one adopts: i) the node 1 as a trial shear center; ii) the global coordinate system as the temporary one. Hence, the vectors ${ }^{2}\left[\mathbb{V}_{i}\right],{ }^{3}\left[\mathbb{V}_{i}\right]$ and ${ }^{4}\left[\mathbb{V}_{i}\right]$, in figure 4.6.b, define the origin angle and the orientation of radius vector. Based on this definition of radius vector, one finds the following transverse displacement vector for mode 4:

$$
{ }^{4}[\mathbb{V}]^{T}=\left[\begin{array}{cccccc}
v_{2} & v_{3} & v_{4} & v_{5} & v_{6} & w_{2} \\
0 & -1.4 & -1.886 & -1.4 & 0 & 0
\end{array}\right]
$$

Converting this transverse displacement into longitudinal direction by the inverse of matrix $\left[{ }_{7} U\right]$, the transformation matrix $\left[{ }_{9} U\right]$ is obtained:

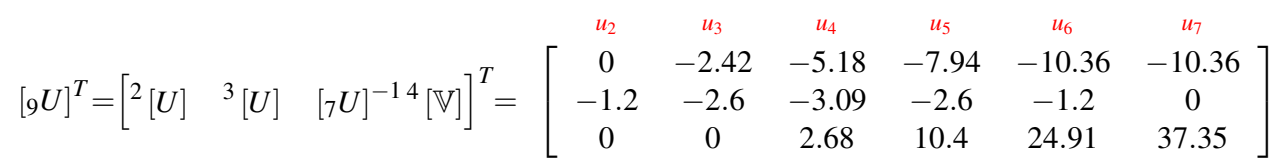

The introduction of the above transformation into eq. 4.97 leads to the second order tensor with Vlasov's warping stiffness:

$$
\left[\mathbb{I}_{\text {Vlasov }}\right]=\left[{ }_{9} U\right]^{T}\left[{ }_{1} C\right]^{M P}\left[{ }_{9} U\right]=\left[\begin{array}{ccc}
420394.40 & 0 & -1120305 \\
& 26212.9 & 135844 \\
\text { symm. } & & 3790837
\end{array}\right]
$$

The first and second rows of the tensor above, in equations 4.98 and 4.99 respectively, achieve the position of the shear center by its radius vector: $r v_{c s}=-1120305 / 420394=$ -2.66 and $r w_{c s}=135844 / 26212.9=5.18$. These radius vectors, in global coordinate orientation, reach the point $y=5.18$ and $z=2.66$. They are also applied in eq. 4.100 to find the longitudinal displacement according to Vlasov beam theory:

$$
{ }^{4}[U]^{T}=\left[\begin{array}{cccccc}
u_{2} & u_{3} & u_{4} & u_{5} & u_{6} & u_{7} \\
6.22 & 7.01 & 4.86 & 2.72 & 3.51 & 9.73
\end{array}\right]
$$

So, the transformation matrix $\left[{ }_{10} U\right]$, given in eq. 4.101 is:

$$
\left[{ }_{10} U\right]=\begin{aligned}
& u_{2} \\
& u_{3} \\
& u_{4} \\
& u_{5} \\
& u_{6} \\
& u_{7}
\end{aligned}\left[\begin{array}{cccccc}
u_{2} & u_{3} & u_{4} & u_{5} & u_{6} & u_{7} \\
-2.42 & -2.6 & 7.01 & 0 & 0 & 0 \\
-5.18 & -3.09 & 4.86 & 0 & 0 & 0 \\
-7.94 & -2.6 & 2.72 & 1 & 0 & 0 \\
-10.36 & -1.2 & 3.51 & 0 & 1 & 0 \\
-10.36 & 0 & 9.73 & 0 & 0 & 1
\end{array}\right]
$$


Applying this transformation and matrices $\left[{ }_{1} D\right]^{M P},\left[{ }_{1} B\right]^{M P}$ and $\left[{ }_{1} C\right]^{M P}$ into equations 4.102, 4.103 and 4.104, one achieves:

$$
\begin{aligned}
& {\left[{ }_{2} D\right]^{M P}=\begin{array}{c}
u_{2} \\
u_{3} \\
u_{4} \\
u_{5} \\
u_{6} \\
u_{6} \\
u_{7}
\end{array}\left[\begin{array}{cccccc}
u_{2} & u_{3} & u_{4} & u_{5} & u_{6} & u_{7} \\
0 & 0 & 0 & 0 & 0 & 0 \\
& 0 & 0 & 0 & 0 & 0 \\
& & 69.06 & 29.18 & -27.77 & 12.25 \\
& & & 62.28 & -44.55 & 16.74 \\
\text { symm. } & & & 34.03 & -13.26 \\
& & & & 5.3
\end{array}\right]} \\
& {\left[{ }_{2} B\right]^{M P}={ }^{u_{4}}{ }^{u_{3}} \begin{array}{cccccc}
u_{2} \\
u_{5} \\
u_{6} \\
u_{7}
\end{array}\left[\begin{array}{cccccc}
u_{2} & u_{3} & u_{4} & u_{5} & u_{6} & u_{7} \\
0 & 0 & 0 & 0 & 0 & 0 \\
& 0 & 0 & 0 & 0 & 0 \\
& & 0 & 0 & 0 & 0 \\
& & & 3.96 & -1.75 & 0.4 \\
& & & & 0.86 & -0.22 \\
\text { symm. } & & & & 0.06
\end{array}\right]}
\end{aligned}
$$

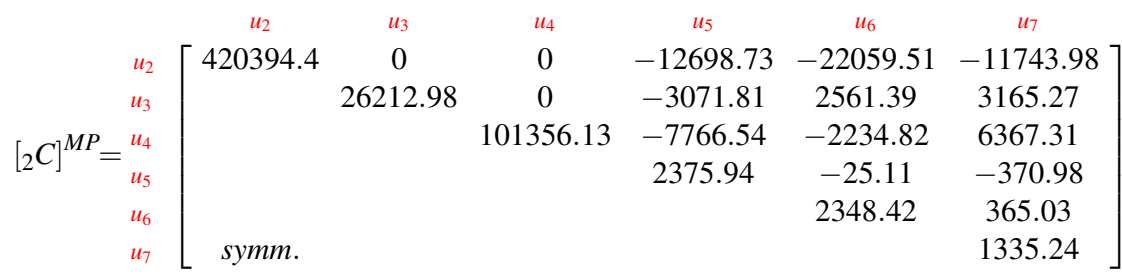

As it was expected, the first two rows/columns in matrices $\left[{ }_{2} D\right]^{M P}$ and $\left[{ }_{2} B\right]^{M P}$ are eliminated. In fact, there is no participation of shear or transverse strain energy in Bernoulli-Euler beam theory. Also, these two rows/columns are almost decoupled from the rest of the matrices. Only matrix $\left.{ }_{2} C\right]^{M P}$ has coupling terms, which leads to the next transformation $\left[{ }_{11} U\right]$, given in eq. 4.106:

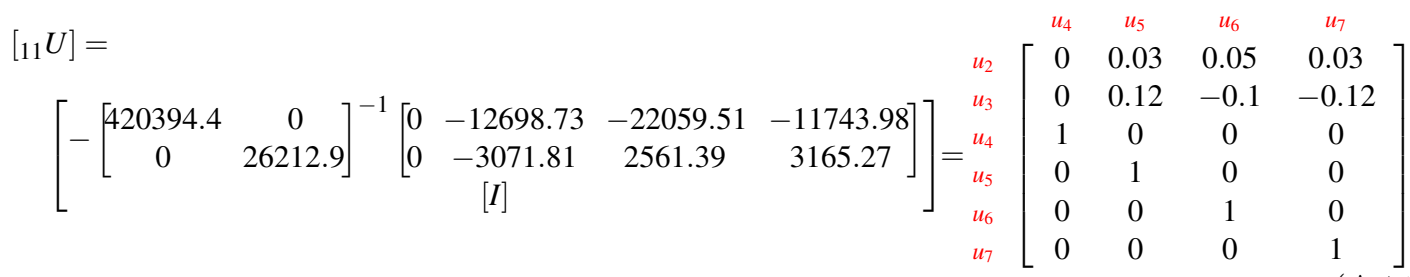

This transformation and equations 4.109- 4.110 lead the cross-section stiffness matrices to:

$$
\begin{aligned}
& {\left[{ }_{3} C\right]^{M P}=u_{u_{5}}^{u_{5}} \begin{array}{cccc}
u_{4} & u_{5} & u_{6} & u_{7} \\
u_{6} & u_{7}
\end{array}\left[\begin{array}{cccc}
101356.13 & -7766.54 & -2234.82 & 6367.31 \\
& 1632.37 & -391.3 & -354.8 \\
& & 940.6 & -560.51 \\
& & & 624.95
\end{array}\right]} \\
& {\left[{ }_{3} D\right]^{M P}=\begin{array}{c}
u_{4} \\
u_{5} \\
u_{6} \\
u_{7}
\end{array}\left[\begin{array}{cccc}
u_{4} & u_{5} & u_{6} & u_{7} \\
& 29.18 & -27.77 & 12.25 \\
& 62.28 & -44.55 & 16.74 \\
& & 34.03 & -13.26 \\
& & & 5.3
\end{array}\right]}
\end{aligned}
$$




$$
\left[{ }_{3} B\right]^{M P}={ }_{u_{5}}^{u_{5}} \begin{array}{cccc}
u_{6} \\
u_{7}
\end{array}\left[\begin{array}{cccc}
u_{4} & u_{5} & u_{6} & u_{7} \\
& 0 & 0 & 0 \\
& 3.96 & -1.75 & 0.4 \\
& & 0.86 & -0.22 \\
& & & 0.06
\end{array}\right]
$$

The first rows in each of the above matrices show how mode 4 has no transverse distortion. However, this mode is still coupled to the other modes and it requires the solution of the quadratic eigenvalue problem.

\section{A.1.8. Step 8 - Symmetric and anti-symmetric modes}

Since the present example has one symmetric axis, it is interesting to perform the FalkLangemeyer method to split the GBT modes into symmetric and anti-symmetric. As exposed in section 4.5.1, the Falk-Langemeyer method is applied in matrices $\left[{ }_{3} C\right]^{P M}$ and $\left[{ }_{3} B\right]^{P M}$, leading to the following transformation matrix:

$$
\left[{ }_{12} U\right]={ }_{{ }_{4}}^{u_{4}} u_{5}\left[\begin{array}{cccc}
u_{4} & u_{5} & u_{6} & u_{7} \\
u_{6} & 0.31 & 0.3 & -0.03 \\
u_{7}
\end{array}\left[\begin{array}{cccc}
0 & -1.32 & -0.47 & 0.23 \\
0 & -5.14 & -2.51 & 0.6 \\
0 & -8.41 & -6.29 & 1
\end{array}\right]\right.
$$

Equations 4.112, 4.114 and 4.113 lead to:

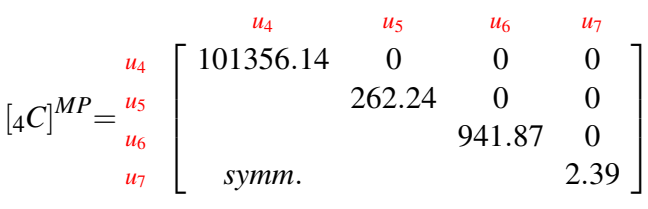

$$
\begin{aligned}
& {\left[{ }_{4} B\right]^{M P}={ }_{{ }_{u}}^{u_{6}}\left[\begin{array}{cccc}
u_{4} \\
u_{7}
\end{array}\left[\begin{array}{cccc}
u_{4} & u_{5} & u_{6} & u_{7} \\
& 0 & 0 & 0 \\
& 0.23 & 0 & 0 \\
& & 0.05 & 0 \\
s y . & & & 0.02
\end{array}\right]\right.}
\end{aligned}
$$

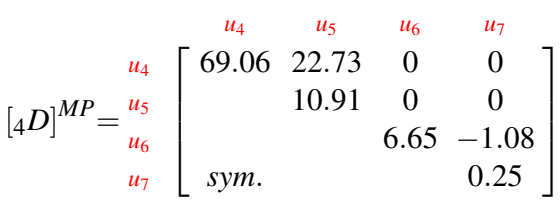

Eq. A.82 shows that the current order of terms in the matrices leads to the subdivision of symmetric and anti-symmetric modes. Since the Vlasov torsion mode is anti-symmetric and its inertia is related to the degree of freedom $u_{4}$, then one can expect an antisymmetric mode related to the degree of freedom $u_{5}$. Consequently, the degrees of freedom $u_{6}$ and $u_{7}$ lead to symmetric modes.

Furthermore, eq. A.82 requires, in this example, no re-order in the rows and columns. Thus, the transformation matrix $\left[{ }_{13} U\right]$ is an identity matrix and equations $4.115,4.116$ and 4.117 lead to: $\left[{ }_{5} C\right]^{M P}=\left[{ }_{4} C\right]^{M P},\left[{ }_{5} D\right]^{M P}=\left[{ }_{4} D\right]^{M P}$ and $\left[{ }_{5} B\right]^{M P}=\left[{ }_{4} B\right]^{M P}$, respectively. 


\section{A.1.9. Step 9 - Quadratic eigenvalue problem}

From the cross-section stiffness matrices $\left[{ }_{5} C\right]^{M P},\left[{ }_{5} D\right]^{M P}$ and $\left[{ }_{5} B\right]^{M P}$, one extracts two quadratic eigenvalue problems. Starting from the first two rows/columns of these matrices, one has the first quadratic eigenvalue problem:

$$
Q(\lambda) u:=\left(\lambda^{2}\left[\begin{array}{cc}
101356.14 & 0 \\
0 & 262.24
\end{array}\right]-\lambda\left[\begin{array}{cc}
69.06 & 22.73 \\
22.73 & 10.91
\end{array}\right]+\left[\begin{array}{cc}
0 & 0 \\
0 & 0.23
\end{array}\right]\right)[u]=0
$$

Based on the pencil given in eq. 4.119, one solves the linearized problem. Here, the software Matlab ${ }^{\circledR}$ is used to achieve the following complex eigenvectors and eigenvalues, respectively:

$$
\begin{aligned}
& \left.{ }_{[A} U\right]^{M P}={ }_{u_{5}} u_{4}\left[\begin{array}{cccc}
u_{a 1} & u_{a 2} & u_{a 3} & u_{a 4} \\
-1.0 & -0.0077+0.000135 i & -0.0077-0.000135 i & -0.997 \\
0.0 & -0.7041-0.710032 i & -0.7041+0.710032 i & -0.0697
\end{array}\right] \\
& {\left[{ }_{A} \lambda\right]^{M P}=\left[\begin{array}{cccc}
\lambda_{a 1} & \lambda_{a 2} & \lambda_{a 3} & \lambda_{a 4} \\
0.0 & 0.0208+0.021 i & 0.0208-0.021 i & 0.000697
\end{array}\right]}
\end{aligned}
$$

Among the above eigenvectors and eigenvalues, it is easy to identify the pairs of conjugated modes: $u_{a 1}$ with $u_{a 4}$ and $u_{a 2}$ with $u_{a 3}$. The last pair share both the same real part and the imaginary part in absolute values, which defines it as a conjugate pair. Based on these vectors, one can reach the system of ordinary differential equations by the transformation given in equations 4.121, 4.122 and 4.123. For instance, the stiffness matrix due to longitudinal strain is transformed into:

$$
\begin{aligned}
& {\left[{ }_{6 A} C\right]^{M P}=\frac{1}{2}\left[\begin{array}{cc}
u_{a 1} \\
u_{a 2}
\end{array}\left[\begin{array}{cc}
u_{4} & u_{5} \\
-1.0 & 0.0 \\
-0.0077+0.000135 i & -0.7041-0.710032 i
\end{array}\right]\left[{ }_{5 A} C\right]^{M P}{ }^{u_{4}} u_{5}\left[\begin{array}{ccc}
u_{a 4} & u_{a 3} \\
-0.997 & -0.0077-0.000135 i \\
-0.0697 & -0.7041+0.710032 i
\end{array}\right]\right.} \\
& \left.+{ }_{u_{a 3}}^{u_{a 4}}\left[\begin{array}{cc}
u_{4} & u_{5} \\
-0.997 & -0.0697 \\
-0.0077-0.000135 i & -0.7041+0.710032 i
\end{array}\right]\left[{ }_{5 A} C\right]^{M P}{ }^{u_{4}}{ }_{u_{5}}\left[\begin{array}{cc}
u_{a 1} & u_{a 2} \\
-1.0 & -0.0077+0.000135 i \\
0.0 & -0.7041-0.710032 i
\end{array}\right]\right]
\end{aligned}
$$

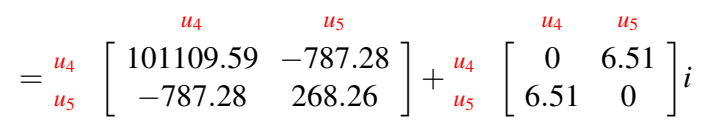

And the other stiffness matrices become:

$$
\begin{aligned}
{\left[{ }_{6 A} D\right]^{M P} } & ={ }_{u_{4}}\left[\begin{array}{cc}
u_{4} & u_{5} \\
-10.48 & -16.79 \\
-16.79 & 11.15
\end{array}\right]+{ }_{u_{5}}\left[\begin{array}{cc}
u_{4} & u_{5} \\
0 & 0.25 \\
0.25 & 0
\end{array}\right] i \\
\left.{ }_{6 A} B\right]^{M P} & ={ }_{u_{5}}\left[\begin{array}{cc}
u_{4} & u_{5} \\
0 & -0.01 \\
-0.01 & 0.23
\end{array}\right]+{ }_{u_{5}}\left[\begin{array}{cc}
u_{4} & u_{5} \\
0 & 0.01 \\
0.01 & 0
\end{array}\right] i
\end{aligned}
$$

Following the same procedure to symmetric modes, one defines the following quadratic eigenvalue problem as:

$$
Q(\lambda) u:=\left(\lambda^{2}\left[\begin{array}{cc}
941.87 & 0 \\
0 & 2.39
\end{array}\right]-\lambda\left[\begin{array}{cc}
6.65 & -1.08 \\
-1.08 & 0.25
\end{array}\right]+\left[\begin{array}{cc}
0.05 & 0 \\
0 & 0.02
\end{array}\right]\right)[u]=0
$$


Which has the corresponding eigenvector and eigenvalues:

$$
\begin{aligned}
& \left.{ }_{B} U\right]^{M P}={ }_{u_{7}} u_{6}\left[\begin{array}{cccc}
u_{a 1} & u_{a 2} & u_{a 3} & u_{a 4} \\
-0.5926-0.7036 i & -0.5926+0.7036 i & 0.0070-0.0121 i & 0.0045+0.0121 i \\
-0.1522+0.3615 i & -0.1522-0.3615 i & -0.998+0.0669 i & -0.998-0.0669 i
\end{array}\right] \\
& \left.{ }_{B} \lambda\right]^{M P}=\left[\begin{array}{llll}
0.0038+0.0061 i & 0.0038-0.0061 i & 0.0523+0.0687 i & 0.0523-0.0687 i
\end{array}\right]
\end{aligned}
$$

Here, the following conjugated pairs stand out: $u_{a 1}$ with $u_{a 2}$ and $u_{a 3}$ with $u_{a 4}$. Applying these conjugated vectors into equations $4.121,4.122$ and 4.123 , one achieves the following stiffness matrices of symmetric modes:

$$
\begin{aligned}
& {\left[{ }_{6 B} C\right]^{M P}={ }_{u_{7}}^{u_{6}}\left[\begin{array}{cc}
u_{6} & u_{7} \\
497.37 & 4.56 \\
4.56 & 2.57
\end{array}\right]} \\
& {\left[{ }_{6 B} D\right]^{M P}={ }_{u_{7}}^{u_{6}}\left[\begin{array}{cc}
u_{6} & u_{7} \\
-0.02 & -0.51 \\
-0.51 & 0.27
\end{array}\right]} \\
& {\left[{ }_{6 B} B\right]^{M P}={ }_{u_{7}}^{u_{6}}\left[\begin{array}{cc}
0.041 & 0.0036 \\
0.0036 & 0.0192
\end{array}\right]}
\end{aligned}
$$

For these symmetric modes, the stiffness matrices are only in real domain.

After one obtains the stiffness matrices and their respective displacement vectors, it is important to scale these vectors. The reference to this example uses this action, imposing a unit value at the maximum longitudinal displacement. Therefore, it is compulsory here to achieve cross-validation among the results. However, to perform this scaling, it is necessary to recover all displacements of each eigenvector in the original coordinate system.

\section{A.1.10. Step 10 - eigenvectors in original coordinate system}

Equations 4.124 and 4.125 provide the recovery of eigenvectors in the original coordinate system. For the first equation, one applies the vectors ${ }^{2}[U]$ and ${ }^{3}[U]$ found in the first two columns of matrix $\left[{ }_{9} U\right]$, given in eq. A.68. The second equation is applied twice: for real and imaginary matrices below:

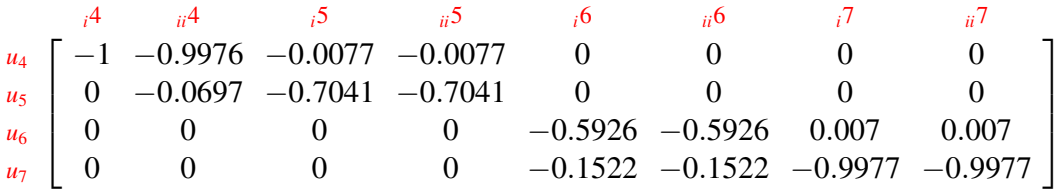

$$
\begin{aligned}
& \begin{array}{l}
u_{4} 5 \\
u_{5} \\
u_{5} \\
u_{6} \\
u_{7}
\end{array}\left[\begin{array}{cccccc}
0.0001 & -0.0001 & 0 & 0 & 0 & 0 \\
-0.71 & 0.71 & 0 & 0 & 0 & 0 \\
0 & 0 & -0.7036 & 0.7036 & -0.0121 & 0.0121 \\
0 & 0 & 0.3615 & -0.3615 & 0.0669 & -0.0669
\end{array}\right] i
\end{aligned}
$$

In the above matrices, the sub-indexes $i()$ and $i i()$ indicate the first and second vectors of the conjugated pairs, respectively. Thus, one finds the vectors for the real part, as 
presented in eq. A.97. The vectors related to modes 1, 2, 3 and 4 are already scaled. The initial longitudinal and transverse displacements are based on the unit displacement according to rod, Bernoulli-Euler and Vlasov theory. Figure. A.2 illustrates the transverse deformation of these modes.

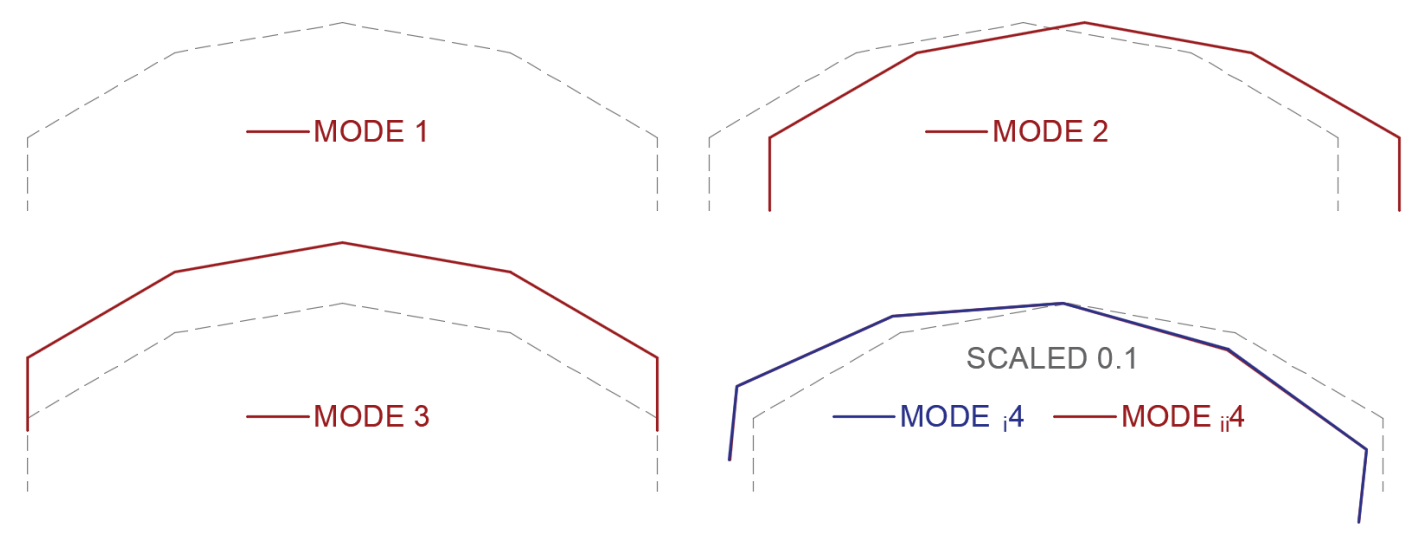

Figure A.2: transverse modal displacements: modes 1 to 4

Here, it is possible to see that the two real possible deformation shapes of mode 4 are practically the same. 


\begin{tabular}{|c|c|c|c|c|c|c|c|c|}
\hline & 1 & 2 & 3 & $i^{4}$ & $i{ }_{i 4}^{4}$ & ${ }_{i} 5={ }_{i i} 5$ & ${ }_{i} 6={ }_{i i} 6$ & ${ }_{i} 7={ }_{i i} 7$ \\
\hline$u_{1}$ & 1 & 5.1823 & 1.7953 & 4.8649 & 4.863 & 0.1379 & 0.2562 & -0.0092 \\
\hline$u_{2}$ & 1 & 5.1823 & 0.5953 & -1.3539 & -1.3626 & -0.1313 & -0.1865 & 0.0119 \\
\hline 13 & 1 & 2.7575 & -0.8047 & -2.1472 & -2.1304 & 0.1006 & -0.0075 & -0.0176 \\
\hline 4 & 1 & 0 & -1.2909 & 0 & 0 & 0 & 0.1342 & 0.0208 \\
\hline 45 & 1 & -2.7575 & -0.8047 & 2.1472 & 2.1304 & -0.1006 & -0.0075 & -0.0176 \\
\hline$u_{6}$ & 1 & -5.1823 & 0.5953 & 1.3539 & 1.3626 & 0.1313 & -0.1865 & 0.0119 \\
\hline$u_{7}$ & 1 & -5.1823 & 1.7953 & -4.8649 & -4.863 & -0.1379 & 0.2562 & -0.0092 \\
\hline$\nu_{1}$ & 0 & 1 & 0 & -3.8649 & -3.9123 & -0.6036 & -0.5133 & 0.1017 \\
\hline$v_{2}$ & 0 & 1 & 0 & -2.6649 & -2.6787 & -0.2252 & -0.2868 & 0.0223 \\
\hline$v_{3}$ & 0 & 1 & 0 & -1.2649 & -1.2515 & 0.0945 & -0.0415 & -0.0254 \\
\hline$v_{4}$ & 0 & 1 & 0 & -0.7787 & -0.7726 & 0.0365 & 0 & 0 \\
\hline$v_{5}$ & 0 & 1 & 0 & -1.2649 & -1.2515 & 0.0945 & 0.0415 & 0.0254 \\
\hline$v_{6}$ & 0 & 1 & 0 & -2.6649 & -2.6787 & -0.2252 & 0.2868 & -0.0223 \\
\hline$v_{7}$ & 0 & 1 & 0 & -3.8649 & -3.9123 & -0.6036 & 0.5133 & -0.1017 \\
\hline$w_{1}$ & 0 & 0 & 1 & 5.1823 & 5.188 & 0.2244 & 0.3689 & -0.0176 \\
\hline$w_{2}$ & 0 & 0 & 1 & 5.1823 & 5.188 & 0.2244 & 0.3689 & -0.0176 \\
\hline$w_{3}$ & 0 & 0 & 1 & 2.7575 & 2.716 & -0.3294 & -0.056 & 0.0651 \\
\hline 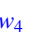 & 0 & 0 & 1 & 0 & 0 & 0 & -0.2913 & -0.079 \\
\hline$w_{5}$ & 0 & 0 & 1 & -2.7575 & -2.716 & 0.3294 & -0.056 & 0.0651 \\
\hline$w_{6}$ & 0 & 0 & 1 & -5.1823 & -5.188 & -0.2244 & 0.3689 & -0.0176 \\
\hline$w_{7}$ & 0 & 0 & 1 & -5.1823 & -5.188 & -0.2244 & 0.3689 & -0.0176 \\
\hline$\phi_{1}$ & 0 & 0 & 0 & -1 & -1.028 & -0.3153 & -0.1887 & 0.0662 \\
\hline$\phi_{2}$ & 0 & 0 & 0 & -1 & -1.028 & -0.3153 & -0.1887 & 0.0662 \\
\hline$\phi_{3}$ & 0 & 0 & 0 & -1 & -1.0022 & -0.0545 & -0.1482 & -0.0302 \\
\hline$\phi_{4}$ & 0 & 0 & 0 & -1 & -0.9764 & 0.2064 & 0 & 0 \\
\hline$\phi_{5}$ & 0 & 0 & 0 & -1 & -1.0022 & -0.0545 & 0.1482 & 0.0302 \\
\hline$\phi_{6}$ & 0 & 0 & 0 & -1 & -1.028 & -0.3153 & 0.1887 & -0.0662 \\
\hline$\varphi_{7}$ & 0 & 0 & 0 & -1 & -1.028 & -0.3153 & 0.1887 & -0.0662 \\
\hline
\end{tabular}

As exposed above, the GBT modes 5, 6 to 7 require the scaling procedure. Hence, one obtains the vectors for these high mode presented in eq. A.98. The graphical representation of the transverse deformation of these vectors is shown in figure A.3.
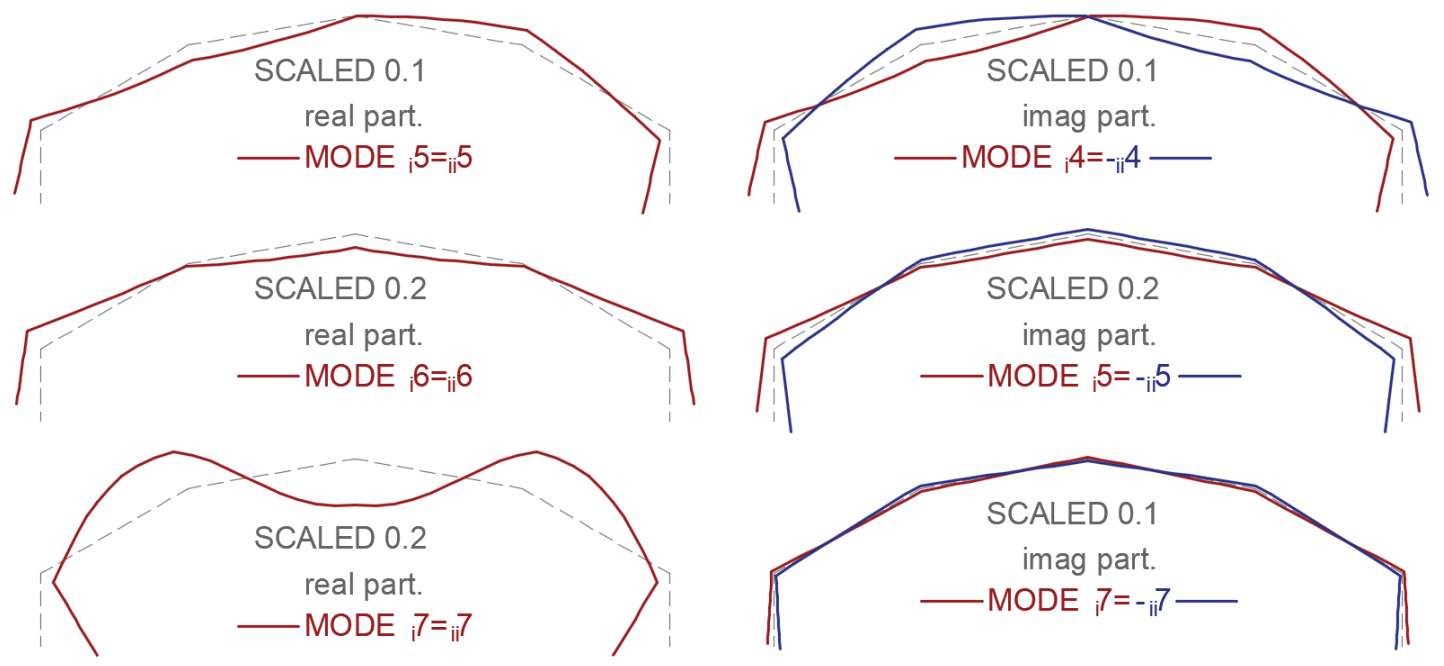

Figure A.3: transverse modal displacements: modes 5 to 7 


\begin{tabular}{|c|c|c|c|c|c|c|c|}
\hline & ${ }_{i} 5={ }_{i i} 5$ & ${ }_{i} 6={ }_{i i} 6$ & ${ }_{i} 7={ }_{i i} 7$ & & ${ }_{i} 5=-{ }_{i i} 5$ & ${ }_{i} 6=-i i 6$ & $i 7=-i i 7$ \\
\hline$u_{1}$ & 1 & 1 & -0.443 & $u_{1}$ & 0.7293 & 1.2003 & 0.2721 \\
\hline & -0.952 & -0.7279 & 0.5696 & $u_{2}$ & -0.8823 & -0.8846 & -0.2154 \\
\hline & 0.7289 & -0.0291 & -0.8458 & $u_{3}$ & 0.8582 & 0.0029 & 0.0522 \\
\hline & 0 & 0.5237 & 1 & $u_{4}$ & 0 & 0.5743 & 0.0563 \\
\hline$t_{5}$ & -0.7289 & -0.0291 & -0.8458 & $u_{5}$ & -0.8582 & 0.0029 & 0.0522 \\
\hline 16 & 0.952 & -0.7279 & 0.5696 & $u_{6}$ & 0.8823 & -0.8846 & -0.2154 \\
\hline 47 & -1 & 1 & -0.443 & $u_{7}$ & -0.7293 & 1.2003 & 0.2721 \\
\hline$v_{1}$ & -4.3754 & -2.0035 & 4.8869 & $v_{1}$ & -4.1904 & -2.5812 & -0.8252 \\
\hline$y_{2}$ & -1.6323 & -1.1195 & 1.0709 & $v_{2}$ & -1.4932 & -1.3691 & -0.3449 \\
\hline$v_{3}$ & 0.6854 & -0.1619 & -1.2204 & $v_{3}$ & 0.7637 & -0.1374 & 0.0464 \\
\hline$v_{4}$ & 0.2643 & 0 & 0 & $v_{4}$ & 0.3112 & 0 & 0 \\
\hline$v_{5}$ & 0.6854 & 0.1619 & 1.2204 & $v_{5}$ & 0.7637 & 0.1374 & -0.0464 \\
\hline 16 & -1.6323 & 1.1195 & -1.0709 & $v_{6}$ & -1.4932 & 1.3691 & 0.3449 \\
\hline 7 & -4.3754 & 2.0035 & -4.8869 & $v_{7}$ & -4.1904 & 2.5812 & 0.8252 \\
\hline 1 & 1.6267 & 1.4399 & -0.8439 & $w_{1}$ & 1.343 & 1.7374 & 0.4063 \\
\hline$w_{2}$ & 1.6267 & 1.4399 & -0.8439 & $w_{2}$ & 1.343 & 1.7374 & 0.4063 \\
\hline$w_{3}$ & -2.3877 & -0.2187 & 3.1248 & $w_{3}$ & -2.5659 & -0.3959 & -0.2715 \\
\hline 4 & 0 & -1.1369 & -3.7963 & $w_{4}$ & 0 & -1.1752 & -0.0084 \\
\hline$v_{5}$ & 2.3877 & -0.2187 & 3.1248 & $w_{5}$ & 2.5659 & -0.3959 & -0.2715 \\
\hline$w_{6}$ & -1.6267 & 1.4399 & -0.8439 & $w_{6}$ & -1.343 & 1.7374 & 0.4063 \\
\hline$w_{7}$ & -1.6267 & 1.4399 & -0.8439 & $w_{7}$ & -1.343 & 1.7374 & 0.4063 \\
\hline$\phi_{1}$ & -2.2859 & -0.7367 & 3.18 & $\phi_{1}$ & -2.2477 & -1.0101 & -0.4003 \\
\hline$\phi_{2}$ & -2.2859 & -0.7367 & 3.18 & $\phi_{2}$ & -2.2477 & -1.0101 & -0.4003 \\
\hline$\phi_{3}$ & -0.3948 & -0.5786 & -1.45 & $\phi_{3}$ & -0.3407 & -0.6193 & -0.038 \\
\hline$\phi_{4}$ & 1.4963 & 0 & 0 & $\phi_{4}$ & 1.5662 & 0 & 0 \\
\hline$\phi_{5}$ & -0.3948 & 0.5786 & 1.45 & $\phi_{5}$ & -0.3407 & 0.6193 & 0.038 \\
\hline$\phi_{6}$ & -2.2859 & 0.7367 & -3.18 & $\phi_{6}$ & -2.2477 & 1.0101 & 0.4003 \\
\hline $4 /$ & -2.2859 & 0.7367 & -3.18 & $\phi_{7}$ & -2.2477 & 1.0101 & 0.4003 \\
\hline
\end{tabular}

One can obtain, from the above vectors, the generalized cross-sections properties. Applying these vectors together with global stiffness matrices of membrane and plate (equations A.8- A.9 and A.17- A.19, respectively) into equations 4.121, 4.122 and 4.123, the results in table A.1 stand out. This table also presents the results extracted from [136]. Also, table A.2 presents the residual coupled cross-section properties between modes 4 and 5, as well as modes 6 and 7 .

Table A.1: Generalized cross-section properties

\begin{tabular}{c|ccc|ccc|ccc}
\hline Mode & \multicolumn{3}{|c}{ This example } & \multicolumn{3}{c}{ Schardt [136] } & \multicolumn{3}{c}{ Difference \% } \\
& $\mathrm{C}$ & $\mathrm{D}$ & $\mathrm{B}$ & $\mathrm{C}$ & $\mathrm{D}$ & $\mathrm{B}$ & $\mathrm{C}$ & $\mathrm{D}$ & $\mathrm{B}$ \\
\hline 1 & 1.328 & 0 & 0 & 1.328 & 0 & 0 & $0 \%$ & $0 \%$ & $0 \%$ \\
2 & 20.019 & 0 & 0 & 20.018 & 0 & 0 & $0 \%$ & $0 \%$ & $0 \%$ \\
3 & 1.248 & 0 & 0 & 1.248 & 0 & 0 & $0 \%$ & $0 \%$ & $0 \%$ \\
4 & 4.815 & 0.007 & 0 & 4.808 & 0.007 & 0 & $0.1 \%$ & $0 \%$ & $0 \%$ \\
5 & 0.671 & 0.056 & 12.309 & 0.405 & 0.035 & 7.885 & $39.7 \%$ & $37.3 \%$ & $35.9 \%$ \\
6 & 0.578 & 0.009 & 0.632 & 0.235 & 0.003 & 0.242 & $59.4 \%$ & $61.5 \%$ & $61.7 \%$ \\
7 & 0.283 & 0.059 & 44.321 & 0.22 & 0.047 & 39.054 & $22.3 \%$ & $20.9 \%$ & $11.9 \%$ \\
\hline
\end{tabular}

Table A.1 presents almost the same result for modes 1 to 4 between the presented 
Table A.2: Generalized cross-section properties due to coupling modes

\begin{tabular}{c|ccc}
\hline Mode & C & D & B \\
\hline $4-5$ & $0.2718+0.0022 \mathrm{i}$ & $0.0116+0.0002 \mathrm{i}$ & $0.0417+0.042 \mathrm{i}$ \\
$6-7$ & 0.0407 & -0.0091 & 0.671 \\
\hline
\end{tabular}

alternative approach and the original work of Schardt. However, for modes 5 to 7 , there is an increase in the difference among the results. These differences have two causes: i) the numerical approach to solve the quadratic eigenvalue problem. Here, it is used the solution of the quadratic eigenvalue problem, instead of the simplification to generalized eigenvalue problem between matrices $[C]$ and $[B]$; ii) Schardt's example has no participation of plate's stiffness matrix of longitudinal strain, $[C]^{P}$. It is assumed as null. Nevertheless, Schardt includes this plate's longitudinal strain stiffness and presents only the results concerning the longitudinal stiffness, leading to the differences presented in table A.3

Table A.3: Longitudinal generalized stiffness, including plate's longitudinal strain stiffness

\begin{tabular}{c|c|c|c|c|c|c|c}
\hline C & \multicolumn{7}{|c}{ Mode } \\
& 1 & 2 & 3 & 4 & 5 & 6 & 7 \\
\hline This example & 1.328 & 20.0188 & 1.2482 & 4.8147 & 0.6713 & 0.3145 & 0.2658 \\
[136]-Ch.3 & 1.328 & 20.019 & 1.248 & 4.826 & 0.424 & 0.268 & 0.227 \\
Difference \% & $0 \%$ & $0 \%$ & $0 \%$ & $-0.2 \%$ & $36.8 \%$ & $14.8 \%$ & $14.6 \%$ \\
\hline
\end{tabular}

Table A.4: Generalized cross-section properties - without $[C]^{P}$ and QEP

\begin{tabular}{c|ccc|ccc|ccc}
\hline Mode & \multicolumn{3}{|c}{ This example } & \multicolumn{3}{c}{ Schardt [136] } & \multicolumn{3}{c}{ Difference \% } \\
& $\mathrm{C}$ & $\mathrm{D}$ & $\mathrm{B}$ & $\mathrm{C}$ & $\mathrm{D}$ & $\mathrm{B}$ & $\mathrm{C}$ & $\mathrm{D}$ & $\mathrm{B}$ \\
\hline 1 & 1.328 & 0 & 0 & 1.328 & 0 & 0 & $0 \%$ & $0 \%$ & $0 \%$ \\
2 & 20.019 & 0 & 0 & 20.018 & 0 & 0 & $0 \%$ & $0 \%$ & $0 \%$ \\
3 & 1.248 & 0 & 0 & 1.248 & 0 & 0 & $0 \%$ & $0 \%$ & $0 \%$ \\
4 & 4.826 & 0.007 & 0 & 4.808 & 0.007 & 0 & $0.4 \%$ & $0 \%$ & $0 \%$ \\
5 & 0.421 & 0.035 & 7.899 & 0.405 & 0.035 & 7.885 & $3.8 \%$ & $0.2 \%$ & $0.2 \%$ \\
6 & 0.238 & 0.003 & 0.242 & 0.235 & 0.003 & 0.242 & $1.4 \%$ & $0 \%$ & $0 \%$ \\
7 & 0.232 & 0.049 & 39.031 & 0.22 & 0.047 & 39.054 & $5.1 \%$ & $4.6 \%$ & $-0.1 \%$ \\
\hline
\end{tabular}

Alternatively, one can compare the results of Schardt [136] setting the plate's stiffness matrix of longitudinal strain, in eq. A.17, as a null matrix. Furthermore, Step 9 is skipped in the present approach and the vectors of Step 8 lead to the recovery of eigenvector in the original coordinate system. Thus, one obtains the results for the generalized cross-section properties (showed in table A.4), and their eigenvectors are directly cross-checked with results found in [136]-Chapter 2, as presented in table A.5. 
Table A.5: Differences among the eigenvectors from the proposed approach and [136]

\begin{tabular}{c|c|c|c|c|c|c|c}
\hline Displ. & \multicolumn{7}{|c}{ Difference $\%$} \\
\hline & 1 & 2 & 3 & 4 & 5 & 6 & 7 \\
\hline$u_{1}$ & $0 \%$ & $0 \%$ & $0 \%$ & $0 \%$ & $-0.3 \%$ & $0 \%$ & $-0.1 \%$ \\
$u_{2}$ & $0 \%$ & $0 \%$ & $0 \%$ & $0.1 \%$ & $0 \%$ & $-0.1 \%$ & $0.1 \%$ \\
$u_{3}$ & $0 \%$ & $0 \%$ & $-0.1 \%$ & $0 \%$ & $-0.2 \%$ & $-3.3 \%$ & $0 \%$ \\
$u_{4}$ & $0 \%$ & $0 \%$ & $-0.1 \%$ & $0 \%$ & $0 \%$ & $-0.1 \%$ & $0 \%$ \\
$u_{5}$ & $0 \%$ & $0 \%$ & $-0.1 \%$ & $0 \%$ & $-0.2 \%$ & $-3.3 \%$ & $0 \%$ \\
$u_{6}$ & $0 \%$ & $0 \%$ & $0 \%$ & $0.1 \%$ & $0 \%$ & $-0.1 \%$ & $0.1 \%$ \\
$u_{7}$ & $0 \%$ & $0 \%$ & $0 \%$ & $0 \%$ & $-0.3 \%$ & $0 \%$ & $-0.1 \%$ \\
$v_{1}$ & $0 \%$ & $0 \%$ & $0 \%$ & $0 \%$ & $-0.1 \%$ & $0 \%$ & $0 \%$ \\
$v_{2}$ & $0 \%$ & $0 \%$ & $0 \%$ & $0 \%$ & $-0.1 \%$ & $0 \%$ & $0 \%$ \\
$v_{3}$ & $0 \%$ & $0 \%$ & $0 \%$ & $0 \%$ & $-0.1 \%$ & $-0.1 \%$ & $0 \%$ \\
$v_{4}$ & $0 \%$ & $0 \%$ & $0 \%$ & $-0.1 \%$ & $-0.1 \%$ & $0 \%$ & $0 \%$ \\
$v_{5}$ & $0 \%$ & $0 \%$ & $0 \%$ & $0 \%$ & $-0.1 \%$ & $-0.1 \%$ & $0 \%$ \\
$v_{6}$ & $0 \%$ & $0 \%$ & $0 \%$ & $0 \%$ & $-0.1 \%$ & $0 \%$ & $0 \%$ \\
$v_{7}$ & $0 \%$ & $0 \%$ & $0 \%$ & $0 \%$ & $-0.1 \%$ & $0 \%$ & $0 \%$ \\
$w_{1}$ & $0 \%$ & $0 \%$ & $0 \%$ & $0 \%$ & $-0.1 \%$ & $0 \%$ & $-0.1 \%$ \\
$w_{2}$ & $0 \%$ & $0 \%$ & $0 \%$ & $0 \%$ & $-0.1 \%$ & $0 \%$ & $-0.1 \%$ \\
$w_{3}$ & $0 \%$ & $0 \%$ & $0 \%$ & $0 \%$ & $-0.1 \%$ & $-0.3 \%$ & $0 \%$ \\
$w_{4}$ & $0 \%$ & $0 \%$ & $0 \%$ & $0 \%$ & $0 \%$ & $0 \%$ & $0 \%$ \\
$w_{5}$ & $0 \%$ & $0 \%$ & $0 \%$ & $0 \%$ & $-0.1 \%$ & $-0.3 \%$ & $0 \%$ \\
$w_{6}$ & $0 \%$ & $0 \%$ & $0 \%$ & $0 \%$ & $-0.1 \%$ & $0 \%$ & $-0.1 \%$ \\
$w_{7}$ & $0 \%$ & $0 \%$ & $0 \%$ & $0 \%$ & $-0.1 \%$ & $0 \%$ & $-0.1 \%$ \\
\hline Mean Diff. & $0 \%$ & $0 \%$ & $0 \%$ & $0 \%$ & $-0.1 \%$ & $-0.4 \%$ & $0 \%$ \\
Standard Deviation. & $0 \%$ & $0 \%$ & $0 \%$ & $0 \%$ & $0.1 \%$ & $1 \%$ & $0.1 \%$ \\
\hline
\end{tabular}

\section{A.1.11. Step 11 - Analysis of pure plate modes}

Similar to membrane-plate modes, the pure plate modes uses first the Falk-Langemeyer method (Step 8) to split the modes into symmetric and anti-symmetric. Based on the stiffness matrices found in Step 5, the Falk-Langemeyer method leads to the following transformation matrix:

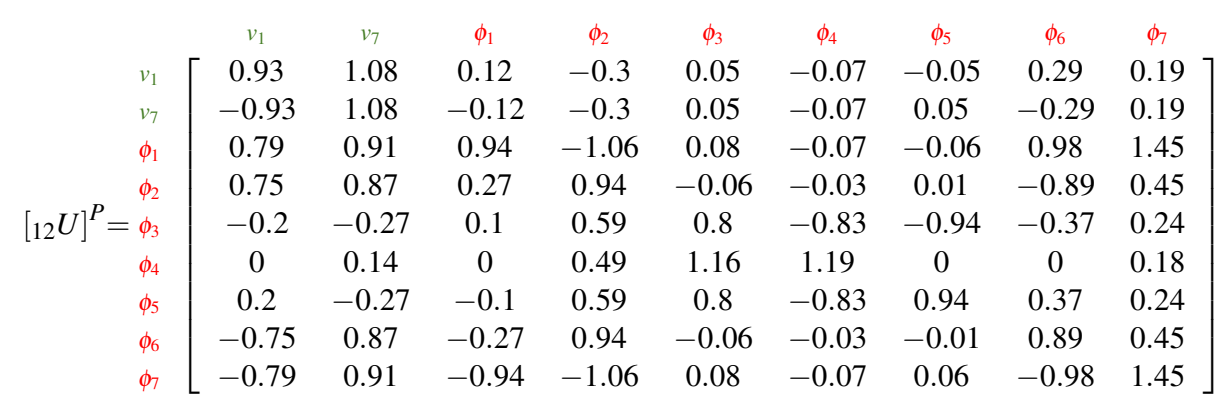

The transformation above, when applied in eq. 4.71, leads to a shear strain stiffness matrix, inviting to the following re-order transformation: 


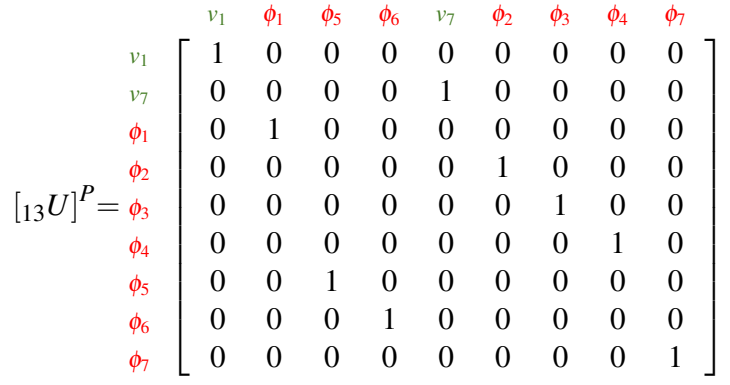

By applying this transformations into matrices given in equations A.40, A.41 and A.42, one obtains the matrices presented in equations A.101, A.102 and A.103, respectively. Each matrix in these equations can split into two parts that will lead to symmetric and anti-symmetric modes. Hence, one solves the quadratic eigenvalue for each part and the transformation given in eq. 4.126 recovers the eigenvectors in the original coordinate system. The real and imaginary part of the resulting eigenvectors are shown in A.104 and A.105, respectively.

$$
\begin{aligned}
& \left.\left[{ }_{5} C\right]^{P}=\left[{ }_{13} U^{P}\right]^{T}\left[{ }_{12} U^{P}\right]^{T}{ }_{1} C\right]^{P}\left[{ }_{12} U^{P}\right]\left[{ }_{13} U^{P}\right]=
\end{aligned}
$$

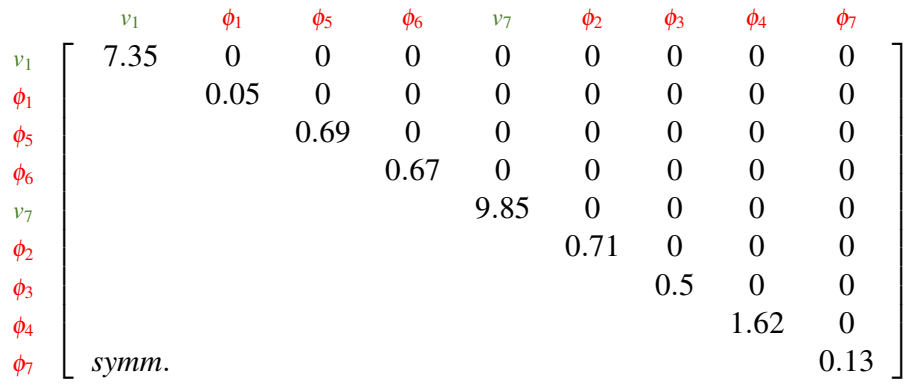

$$
\begin{aligned}
& {\left[{ }_{5} D\right]^{P}=\left[{ }_{13} U^{P}\right]^{T}\left[{ }_{12} U^{P}\right]^{T}\left[{ }_{1} D\right]^{P}\left[{ }_{12} U^{P}\right]\left[{ }_{13} U^{P}\right]=} \\
& \begin{array}{l}
v_{1} \\
\phi_{1} \\
\phi_{5} \\
\phi_{6} \\
v_{7} \\
\phi_{2} \\
\phi_{3} \\
\phi_{4} \\
\phi_{7}
\end{array}\left[\begin{array}{ccccccccc}
v_{1} & \phi_{1} & \phi_{5} & \phi_{6} & v_{7} & \phi_{2} & \phi_{3} & \phi_{4} & \phi_{7} \\
& 4.17 & -1 & 8.82 & 0 & 0 & 0 & 0 & 0 \\
& 4.91 & -0.51 & 5.02 & 0 & 0 & 0 & 0 & 0 \\
& & 2.51 & -0.45 & 0 & 0 & 0 & 0 & 0 \\
& & & 18.83 & 0 & 0 & 0 & 0 & 0 \\
& & & & 40.8 & -10.79 & 1.25 & -1.31 & 7.31 \\
& & & & & 21.66 & -0.38 & 0.64 & -7.79 \\
\text { symm. } & & & & & & 3.11 & -0.07 & 1.16 \\
& & & & & & & 4.54 & -0.7 \\
& & & & & & & & 11.78
\end{array}\right]
\end{aligned}
$$




$$
\begin{aligned}
{\left[{ }_{5} B\right]^{P}=\left[{ }_{13} U^{P}\right]^{T}\left[{ }_{12} U^{P}\right]^{T}\left[{ }_{1} B\right]^{P}\left[{ }_{12} U^{P}\right]\left[{ }_{13} U^{P}\right]=} \\
\\
v_{1} \\
\phi_{1} \\
\phi_{5} \\
\phi_{6} \\
v_{7} \\
\phi_{2} \\
\phi_{3} \\
\phi_{4} \\
\phi_{7}
\end{aligned}\left[\begin{array}{ccccccccccc}
{ }_{1} \\
\hline
\end{array}\right.
$$

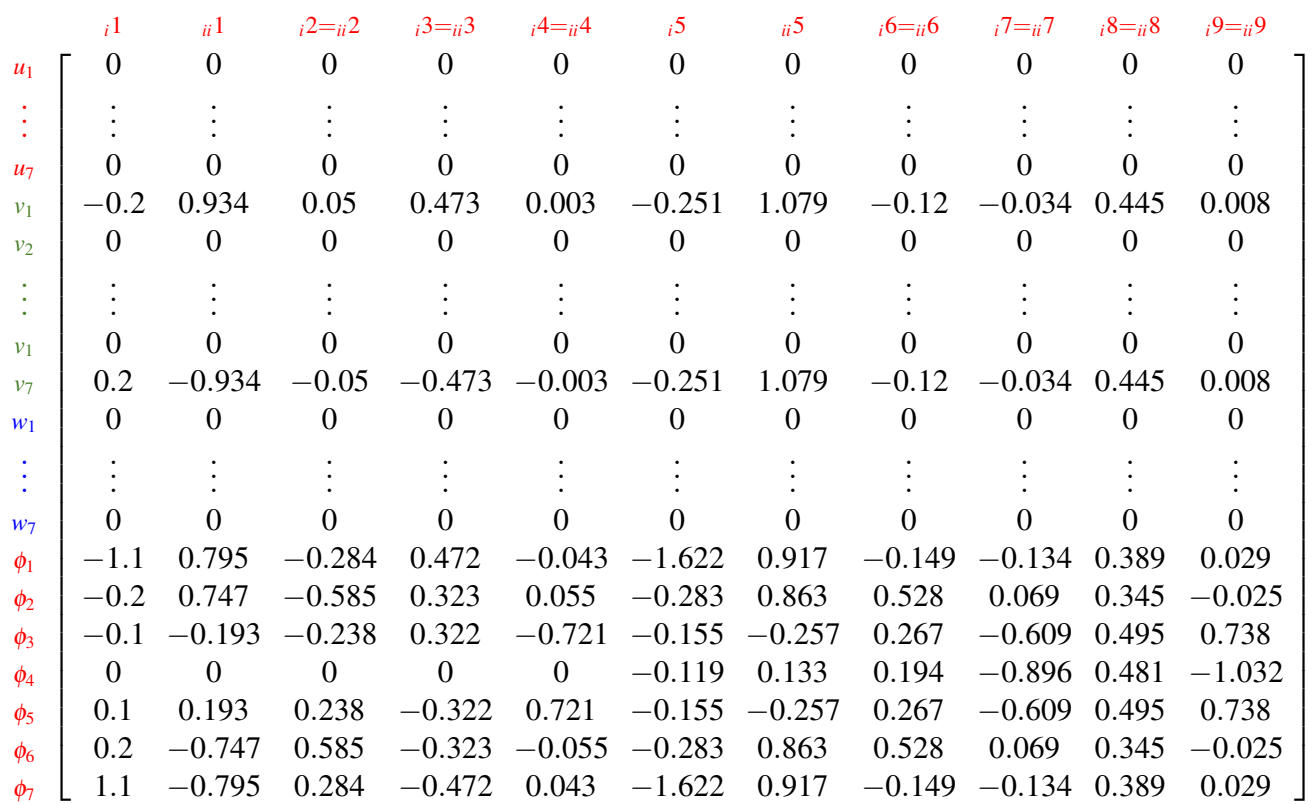




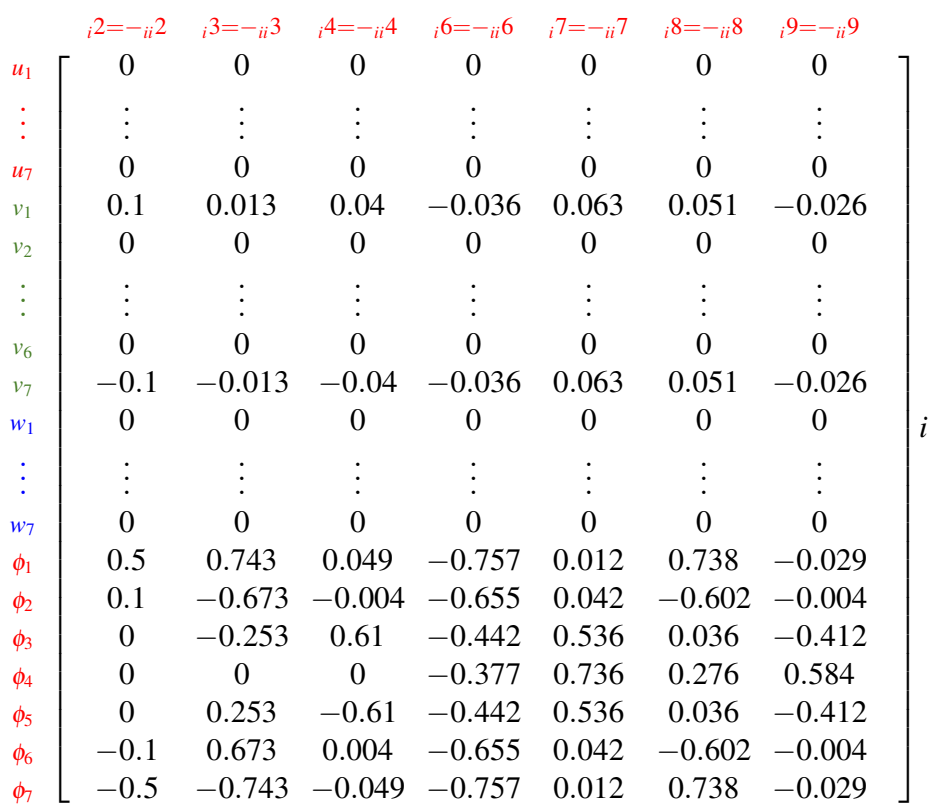

The above vectors are plotted in figures A.4 and A.5. Furthermore, one obtains the generalized cross-section properties, replacing the stiffness matrices of membrane-plate with the pure plate in equations 4.121, 4.122 and 4.123. These generalized crosssection properties are presented in equations A.106, A.107 and A.108. In contrast to the model of membrane-plate behavior, the pure plate modes have a relevant coupling among the modes. Here rises the possibility of future studies in decoupling approach of these modes $[44,45]$.

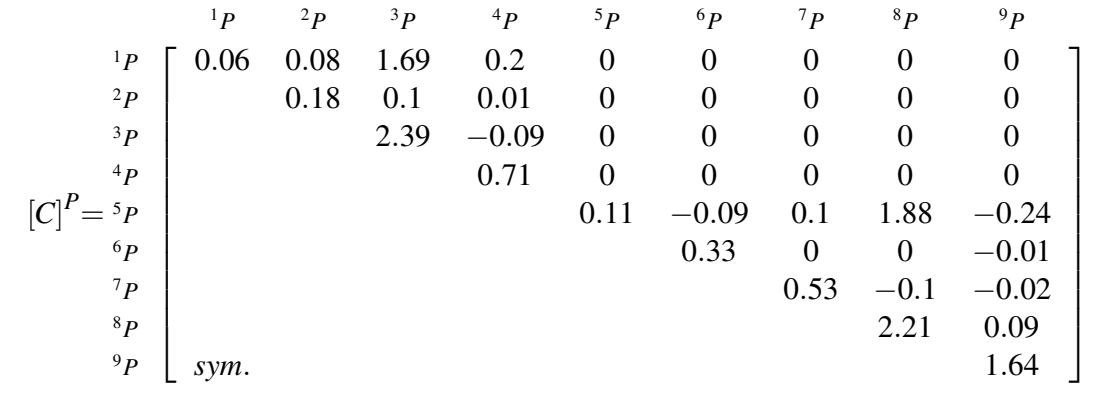

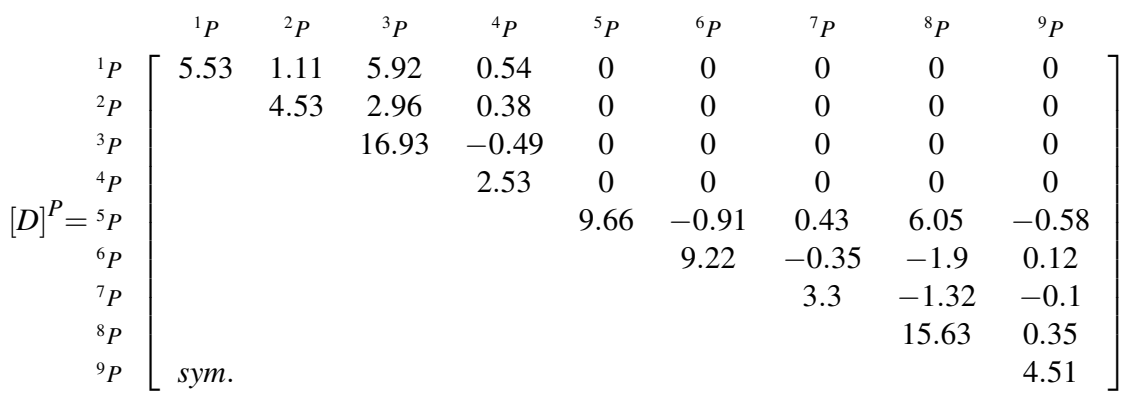



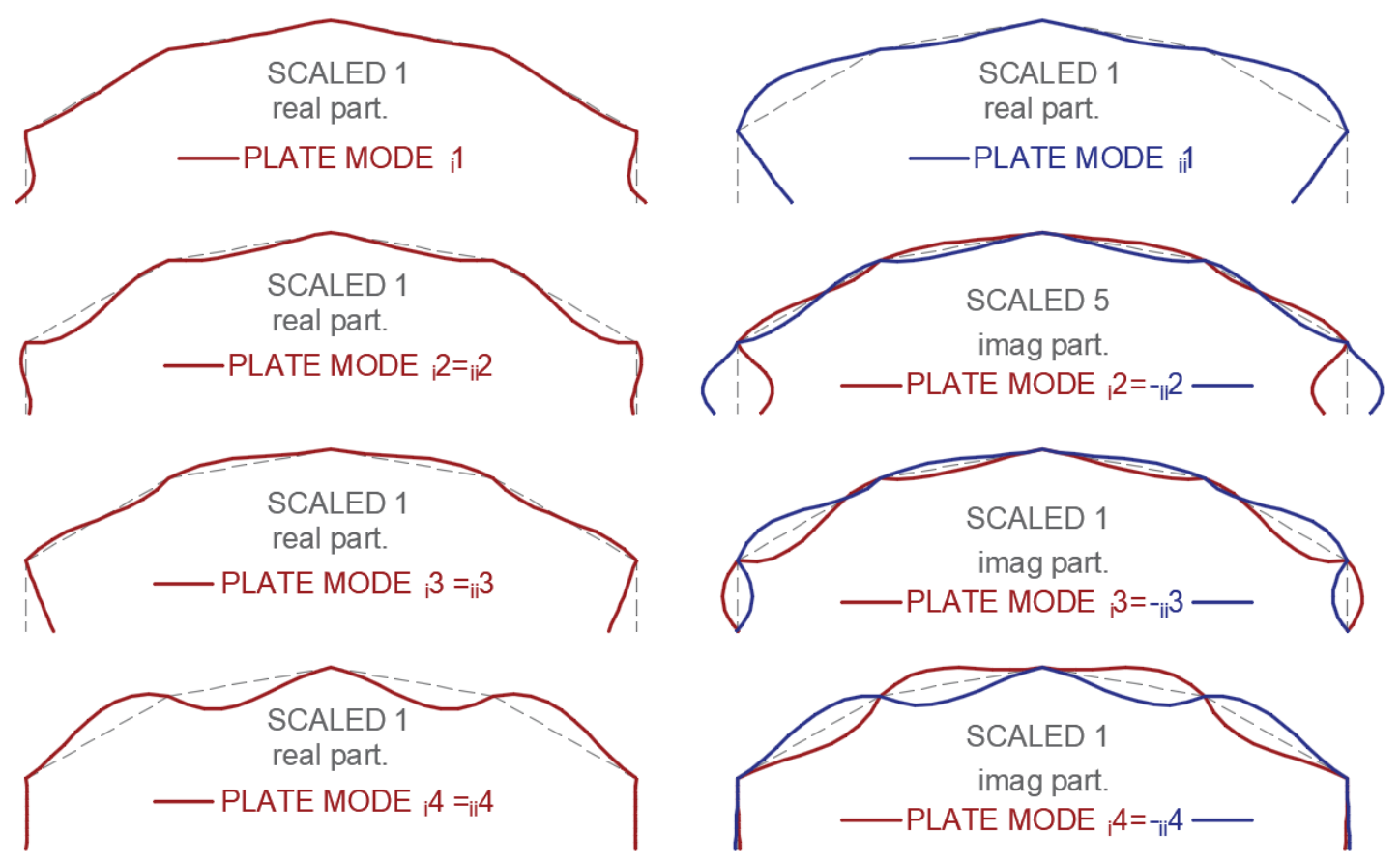

Figure A.4: transverse modal displacements of plate symmetric modes: 1 to 4

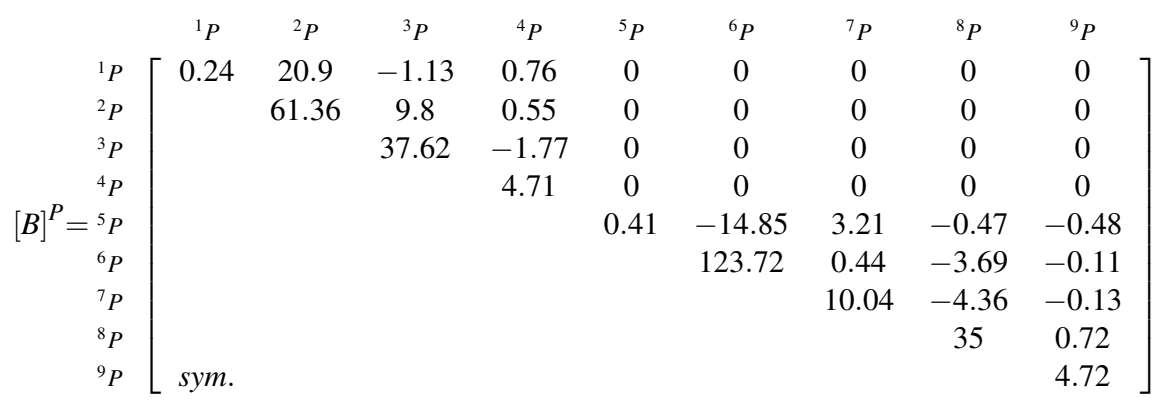



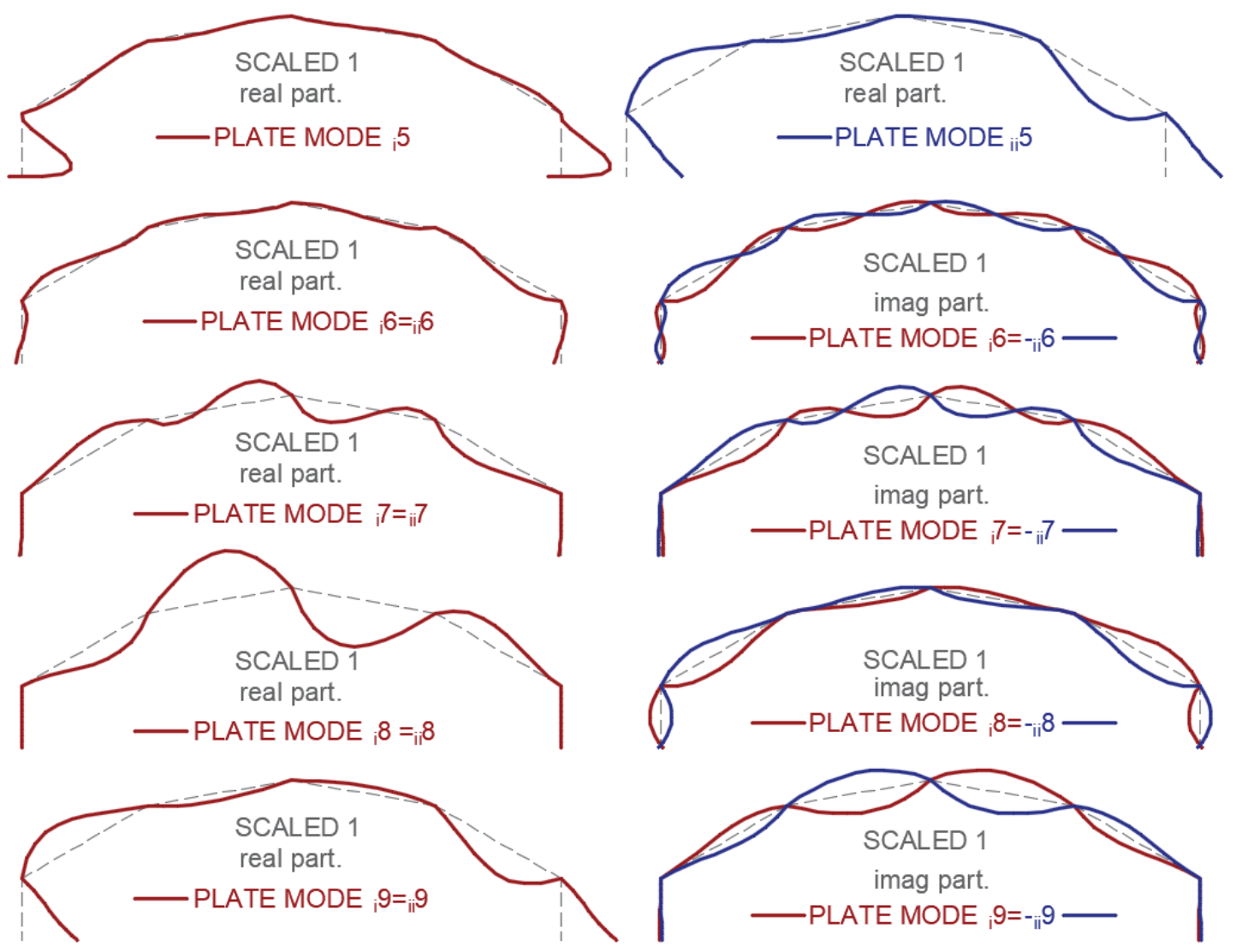

Figure A.5: transverse modal displacements of plate anti-symmetric modes: 5 to 9 